\title{
Designing New Heterometallic [2 $x$ 2] Grids using Pyrazolate-bridged Ligands
}

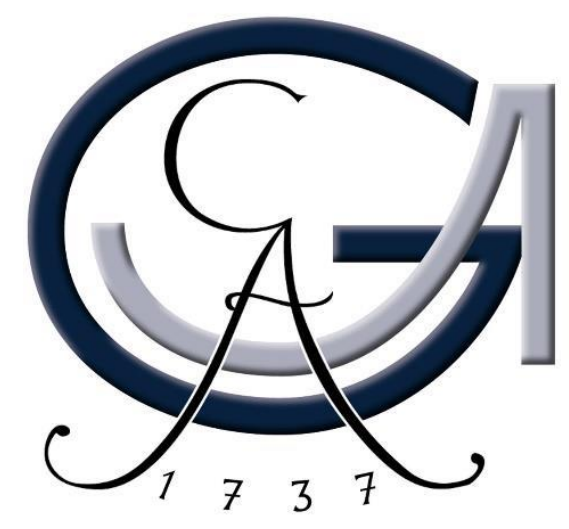

Dissertation

for the award of the degree

"Ph.D. Division of Mathematics and Natural Sciences"

of the Georg-August-Universität Göttingen

within the doctoral program of chemistry

of the Georg-August University School of Science (GAUSS)

\author{
submitted by \\ Joanne Wong \\ Toronto, Canada
}

Göttingen 



\section{Thesis Committee}

Prof. Dr. Franc Meyer, Institut für Anorganische Chemie, Georg-August-Universität Göttingen

Prof. Dr. Sven Schneider, Institut für Anorganische Chemie, Georg-August-Universität Göttingen

Prof. Dr. Dirk Schwarzer, Max-Planck-Institut für Biophysikalische Chemie

\section{Members of the Examination Board}

Reviewer: Prof. Dr. Franc Meyer, Institut für Anorganische Chemie, Georg-AugustUniversität Göttingen

Second reviewer: Prof. Dr. Sven Schneider, Institut für Anorganische Chemie, Georg-AugustUniversität Göttingen

Third reviewer: Prof. Dr. Dirk Schwarzer, Max-Planck-Institut für Biophysikalische Chemie

\section{Further Members of the Examination Board:}

Fourth reviewer: Prof. Dr. Marina Bennati, Max-Planck-Institute for Biophysical Chemistry

Fifth reviewer: Arbeitskreis Jun.-Prof. Dr. Nathalie Kunkel, Institut für Anorganische Chemie, Georg-August-Universität Göttingen

Sixth reviewer: Dr. Christian Sindlinger, Institut für Anorganische Chemie, Georg-AugustUniversität Göttingen

Date of Examination: Oct 24, 2019. 
1.1 Supramolecular Chemistry and the Information Storage Paradigm .......................................... 1

1.2 - Origins of Spin Crossover Complexes ………………………………………...................... 2

1.3 - Role of Cooperativity in SCO Complexes …………................................................................ 3

1.4 - Mössbauer Spectroscopy for Iron Containing SCO Complexes .................................................. 4

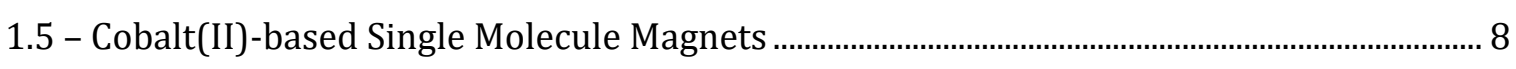

1.6 - Homonuclear $[2$ x 2] Grids ................................................................................................

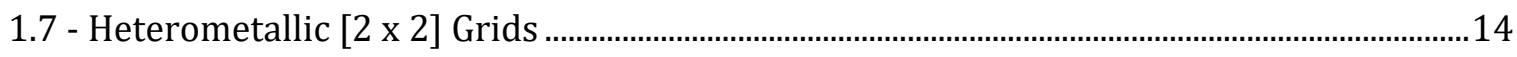

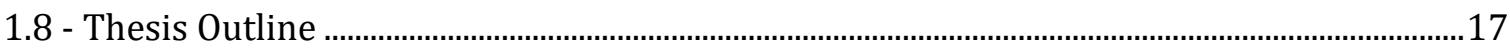

Chapter 2 - Femtosecond Investigations on Optically Excited Homonuclear Fe Grids 19

2.1 - Introduction ..........................................................................................................................19

2.2 - Pump-Probe Spectroscopy

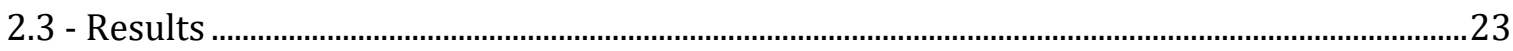

2.3.1 - Synthesis of the pyrazolate-bridge Ligand \& Homonuclear Fe Grids ................................2

2.3.2 - Mössbauer Spectroscopy on Homonuclear Fe Grids...............................................................2

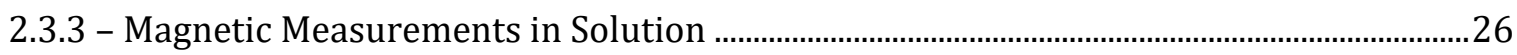

2.3.4 - Variable Temperature UV-Vis Spectroscopy ………………………………..........................27

2.3.5 - Transient Absorption Mid IR and UV-vis Pump-Probe Spectroscopy …….......................28

Chapter 3 - Heterometallic $\mathrm{Ru}_{2} \mathrm{Co}_{2}$ [2 x 2] Grid with Localized Single Molecule Magnet

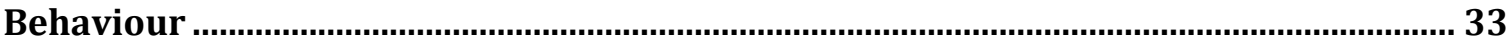

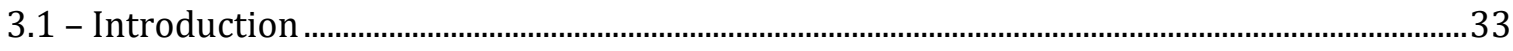

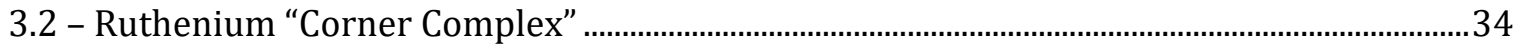

3.2.1 - Synthesis of the $\left[\mathrm{HL}_{2} \mathrm{H}_{2} \mathrm{Ru}\right]^{2+}$ "Corner Complex" ...................................................................... 34

3.2.2 -X-ray Crystallography of the $\mathrm{Ru}^{\mathrm{II}}$ "Corner Complex" ................................................................ 34

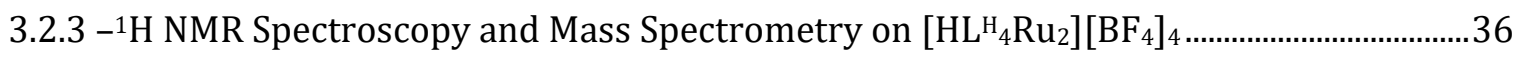

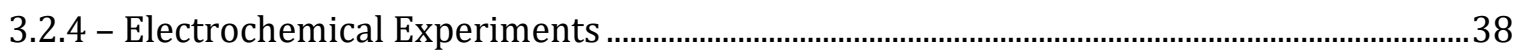

3.2.5 - UV-Vis Spectroelectrochemistry ............................................................................................

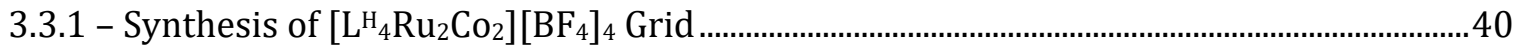

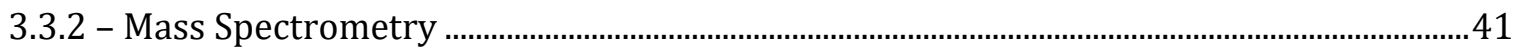

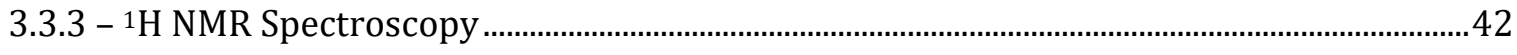

3.3.4 - X-ray Crystallography ....................................................................................................... 43

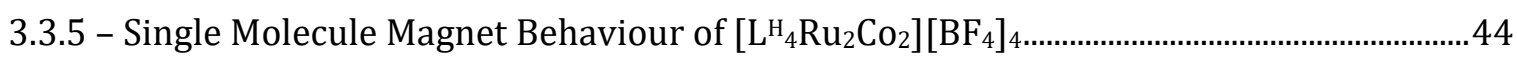

3.3.6 - Probing the Redox Properties of $\left[\mathrm{LH}_{4} \mathrm{Ru}_{2} \mathrm{Co}_{2}\right]\left[\mathrm{BF}_{4}\right]_{4}$ with Cyclic Voltammetry ............... 47

3.3.7 - UV-Vis Spectroscopy and Spectroelectrochemistry on $\left[\mathrm{L}_{4} \mathrm{Ru}_{2} \mathrm{Co}_{2}\right]\left[\mathrm{BF}_{4}\right]_{4} \ldots \ldots \ldots \ldots \ldots \ldots \ldots . . . . . . . . . .48$

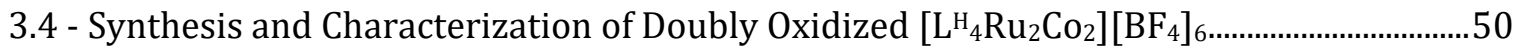

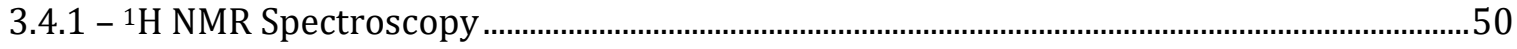

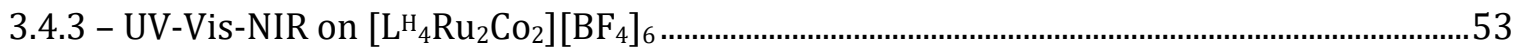


Chapter 4 - Synthesis and Characterization of Heterometallic $\left[\mathrm{L}_{4} \mathrm{Ru}_{2} \mathrm{Fe}_{2}\right]\left[\mathrm{BF}_{4}\right]_{4}$ Grid

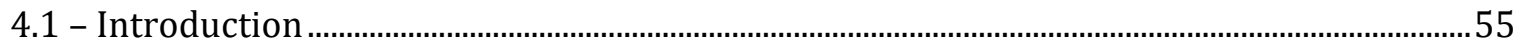

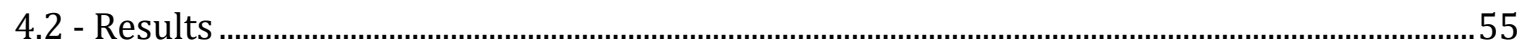

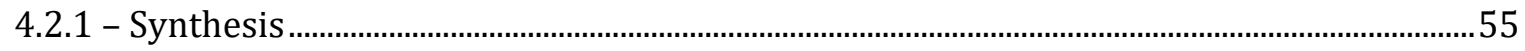

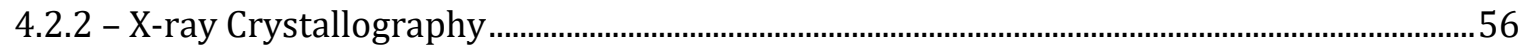

4.2.4 - Cyclic Voltammetry and UV-vis Spectroelectrochemistry...............................................5

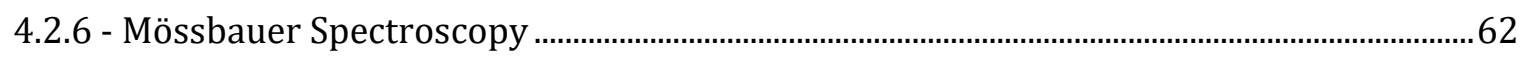

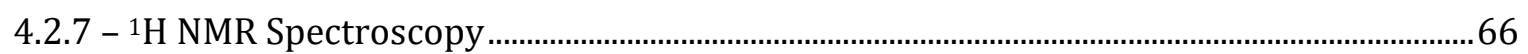

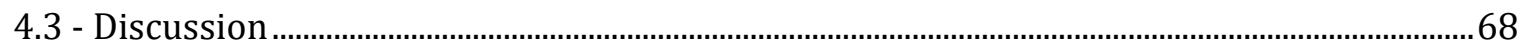

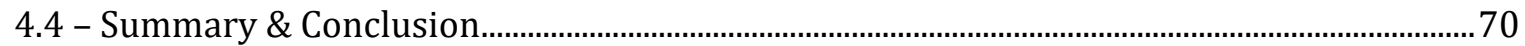

Chapter 5 - Highly Distorted Dinuclear Fe"I and CoII Complexes ......................................... 71

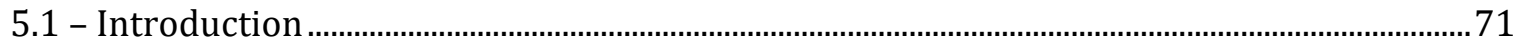

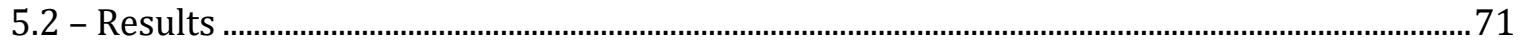

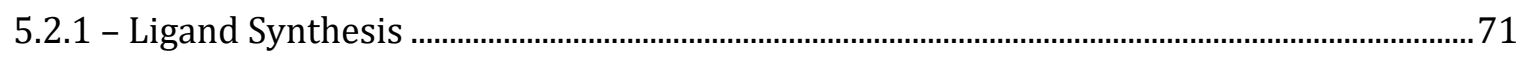

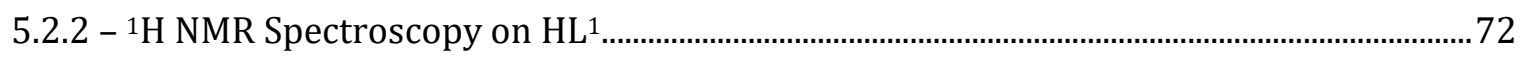

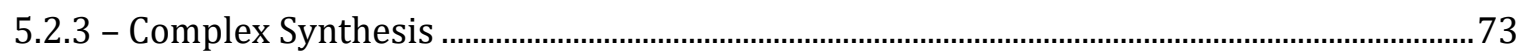

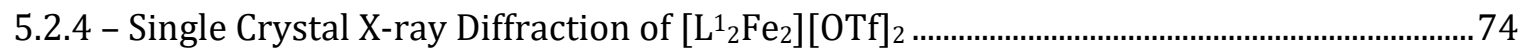

5.2.5 - Mössbauer Spectroscopy \& Magnetic Susceptibility Measurements ...................................75

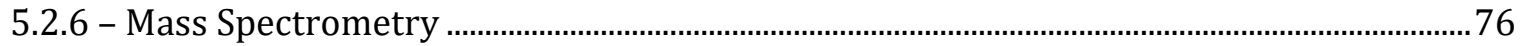

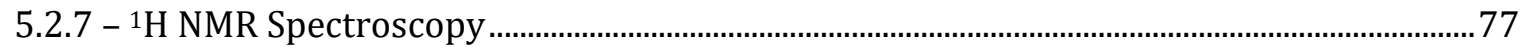

5.2 .8 - Cyclic Voltammetry and Spectroelectrochemistry................................................................

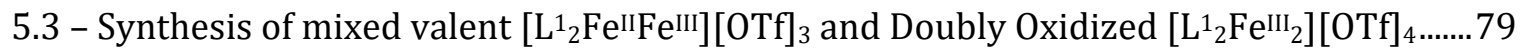

5.3.1 - X-ray Crystallography on the Mixed valent [ $\left.\mathrm{L}_{2}^{1} \mathrm{Fe}^{\mathrm{II}} \mathrm{Fe}^{\mathrm{III}}\right][\mathrm{OTf}]_{3}$ Complex...........................80

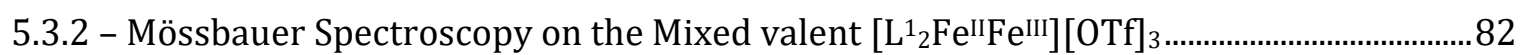

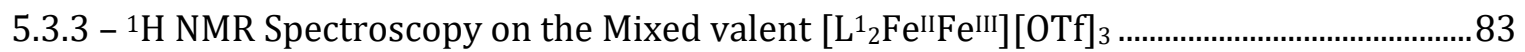

5.4 - Preliminary Work on an Analogous Dinuclear $\left[\mathrm{L}_{2}{ }_{2} \mathrm{Co}_{2}\right]\left[\mathrm{BF}_{4}\right]_{2}$ Complex .............................85

5.4.1 - X-ray Crystallography ...............................................................................................................

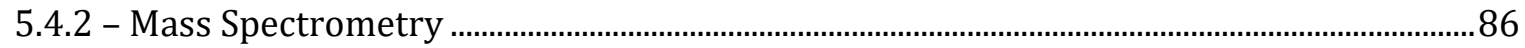

5.4 .3 - ${ }^{1}$ H NMR Spectroscopy ………………………….....................................................................

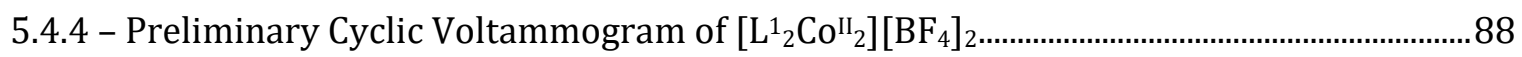

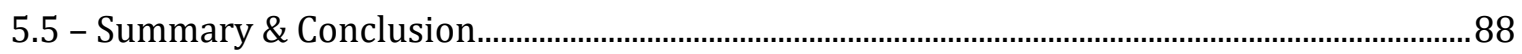

Chapter 6 - [2 x 2] Grid-like Arrays with Asymmetric Pyrazolate-bridged Ligands .... 91

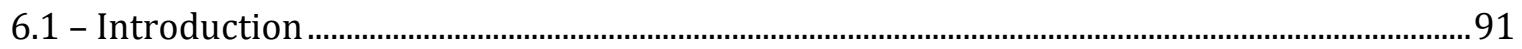

6.2.1 - Ligand Synthesis and Characterization ……….....................................................................92

6.2.2 - Complex Synthesis ................................................................................................................93

6.2.3 - X-ray Crystallography ............................................................................................................94 


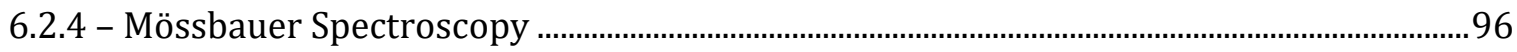

6.2.5 - Investigations in Solution: Mass Spectrometry \& 1H NMR Spectroscopy ……................. 97

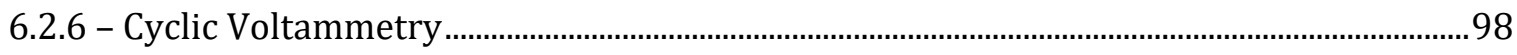

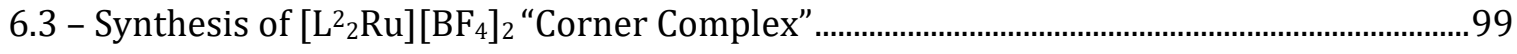

6.3.1 - Solution Investigations: Mass Spectrometry \& ${ }^{1} \mathrm{H}$ NMR Spectroscopy........................... 100

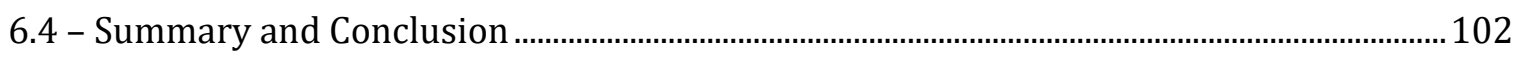

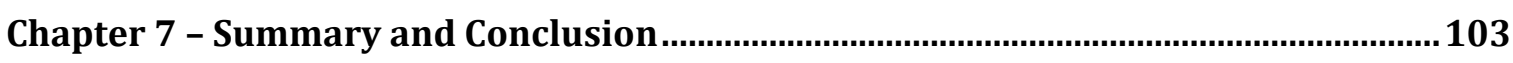

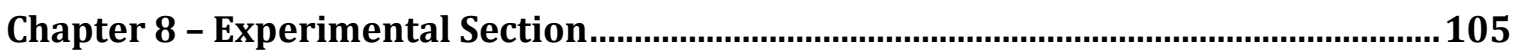

8.1 - Materials and Methods.......................................................................................................... 105

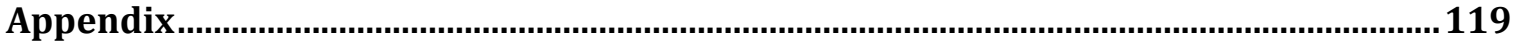

A1 - NMR Spectroscopy for the Ligands and Ligand Precursors ..................................................119

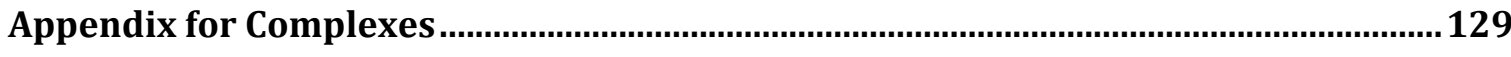

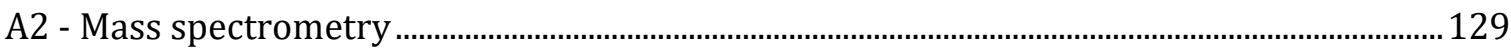

A3 - NMR Spectroscopy for Complexes …………….................................................................. 130

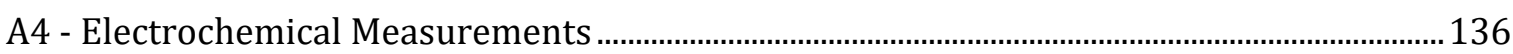

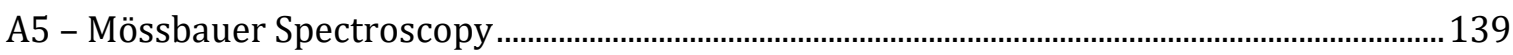

A6 - Magnetic Measurements....................................................................................................... 140

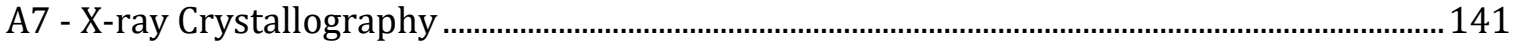

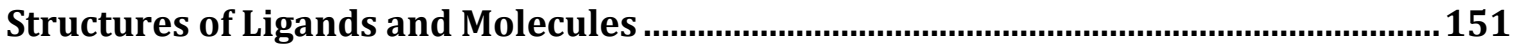

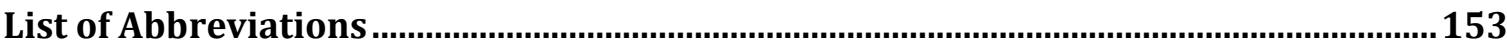

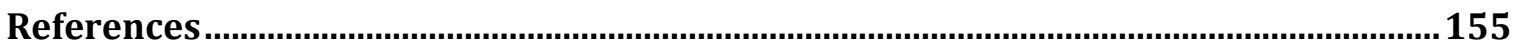

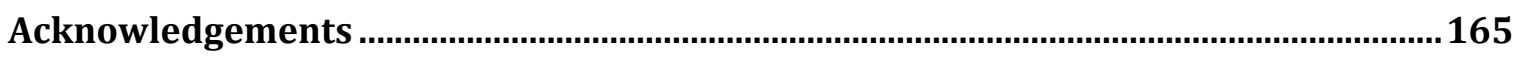




\section{Chapter 1 - Introduction}

\subsection{Supramolecular Chemistry and the Information Storage Paradigm}

The field of supramolecular chemistry pioneered by Nobel Prize Laureate Jean-Marie Lehn specializes in molecular systems involving non-covalent interactions including hydrogen bonding, van der Waals forces, electrostatic forces, and donor-acceptor interactions. ${ }^{1}$ Originally, the field started with selective binding of alkali metals to crown ethers and cryptands, ${ }^{2,3}$ but eventually expanded towards synthetic molecular receptors and substrates as well as self-assembled oliogonuclear and polynuclear complexes. ${ }^{4}$ The ability to manipulate the non-covalent interactions for molecular recognition provides a means for building complex structural architectures with specific preprogrammed functions. For instance, careful ligand design along with appropriate metal ion selection afforded a variety of molecular structures ranging from double and triple helical complexes, ${ }^{5-7}$ as well as rack-8 and grid-like arrays. ${ }^{9-11}$ More complex structures involving multicomponent systems with several different ligand strands and metal ions have also been developed, as observed in multicompartamental cylindrical cages. ${ }^{12}$

From an information storage point of view, supramolecular chemistry, in particular selfassembled [ $\mathrm{n} \times \mathrm{n}]$ grid-like arrays, have gained significant interest over the recent years. The requirements for molecular storage devices involve the need for two or more bistable states that can be accessed by an external stimulus such as temperature, light, or pressure. ${ }^{13}$ The supramolecular structure of [ $\mathrm{n} \times \mathrm{n}$ ] grid-like arrays, in which a set of metal ions are locked in between mutually perpendicular ligand strands, provides several attractive features for this purpose. ${ }^{11,14}$ Firstly, a variety of unique chemical and physical properties including multiple stable redox states, spin crossover and magnetic coupling have been well documented.9,11 Secondly, the two dimensional network of metal ions in a matrix-like array resembles those found in crossbar switches found in electronic information processing applications and may also be extended onto surfaces, as observed in molecular wires. ${ }^{15}$

An alternative possibility for molecular information storage with grid-like arrays involves the so-called Quantum Cellular Automata (QCA), which functions on the basis of energetically degenerate structured cells composed of different redox centers or spins states (i.e. Fe ${ }^{\mathrm{II}}$, Fe ${ }^{\mathrm{III}}$ or HS-Fe"I, LS-FeII). ${ }^{16,17}$ The two degenerate states can be interconverted by electron transfer within the cell such that electrostatic interactions between the neighbouring cells lift the degeneracies affording two distinguishable states labeled " 0 " and " 1 " (Figure 1.1). Unlike crossbar switches, QCA functions strictly on Coulomb interactions and thus current is not generated affording less power dissipation lost. Application of this model was first implemented by Lent using quantum dots, ${ }^{18}$ but can be extended to spin crossover (SCO) complexes with a mixed valent [HS-LS-HS-LS] configuration. In this case, spin crossover must occur at each of the metal centers to form the other degenerate [LS-HS-LS-HS] state, while the overall [2HS-2LS] spin-state of the cell remains unchanged. 


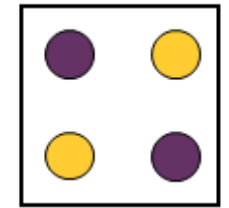

"0"

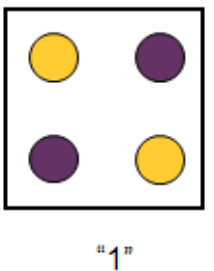

Possible implementations

(a) $\bigcirc=\mathrm{Fe}^{\| l} \bigcirc=\mathrm{Fe}^{\mathrm{ll}}$

(b)

$=\mathrm{HS}-\mathrm{Fe}$ II

Figure 1.1. Possible implementations for a four dot Quantum Cellular Automata.

Accordingly, [2 x 2] grid-like arrays display a multitude of attractive features for QCA as well as potential for other interesting redox and magnetic properties including spin crossover and single molecule magnet behaviour. Prior to discussing the recent advances in homonuclear and heterometallic [ $2 \mathrm{x} 2$ ] grid-like motifs, a brief introduction to the origins of spin crossover and single molecule magnets will be discussed in this chapter.

\section{2 - Origins of Spin Crossover Complexes}

The spin crossover phenomena occurs in molecules capable of switching from a low-spin (LS) configuration to a high-spin (HS) configuration when induced by an external stimulus such as light, temperature, pressure, electric field, or guest molecules. ${ }^{19,20}$ This phenomena typically occurs in $\mathrm{d}^{4}-\mathrm{d}^{7}$ octahedral complexes, with the most common associated with Fe-based molecules . ${ }^{20-22}$ In the absence of ligands, the Fe ${ }^{\mathrm{II}}$ cation exhibits five degenerate $3 \mathrm{~d}$ orbitals. Upon coordination of ligand(s) to form an octahedral environment, the degeneracy of the $3 \mathrm{~d}$ orbitals is lifted to form two new sets of orbitals. The lower energy orbitals, $t_{2 g}$, are comprised of the $d_{x y}, d_{x z}$, and $d_{y z}$ orbitals, while the higher energy level, $e_{g}$, consist of the $d_{z}{ }^{2}$ and $d_{x}{ }^{2} y^{2}$ orbitals. The energy difference between the $t_{2 g}$ and $e_{g}$ orbital sets in an octahedral environment, $\Delta_{0}$, is determined by the ligand field induced by the coordinating ligands (Figure 1.2). ${ }^{23}$

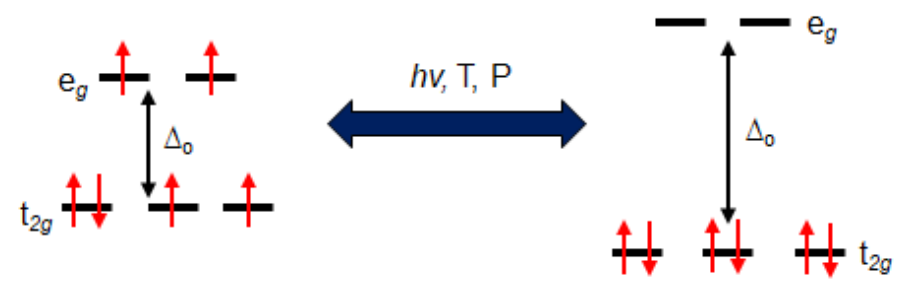

Figure 1.2. Schematic representation of SCO in an FeII complex induced by an external stimulus such as light, temperature or pressure. $\Delta_{0}$ is the energy gap between the two sets of orbitals in an octahedral environment.

Consequently, two ground-states are possible depending on the ligand field and the pairing energy $(P)$ associated with population of two electrons on the same orbital. ${ }^{24,25}$ In the case of strong-field ligands giving rise to a large energy gap, $\Delta_{0}$, the preference for pairing of the electrons is much greater than population of the $\mathrm{e}_{\mathrm{g}}$ orbitals $\left(\Delta_{\mathrm{o}}>P\right)$, and thus a diamagnetic LS-Fe"l state is favoured. On the other hand, with weak field ligands corresponding to a small energy gap $\Delta_{0}$, the preference for the electrons to occupy the d-orbitals according to Hund's rule is favoured $\left(\Delta_{0}<P\right)$, giving rise to a HS-Fe"l state. If the ligand field $\left(\Delta_{0}\right)$ and pairing energy $(P)$ are of the same order of magnitude, an external stimuli may induce a spin transition between the two states. ${ }^{20}$ 
Since the high-spin and low-spin states are accompanied by distinct changes in the molecular structure, a wide variety of techniques can be used to detect the SCO phenomena. For instance, occupation of the antibonding orbitals in the high-spin state affords longer metalligand bond lengths than the low-spin state. ${ }^{19}$ Thus, the structural changes observed in SCO complexes can be measured using temperature dependent X-ray crystallography. However, the changes in bond lengths for Fe ${ }^{\text {II }}$ SCO complexes are often more pronounced than SCO involving $\mathrm{Fe}^{\mathrm{III}}$ ions due to the electron hole in the $\mathrm{t}_{2 \mathrm{~g}}$ orbital. Furthermore, with the presence of Fe atoms, Mössbauer (MB) spectroscopy 26,27 can also be used to quantify the different spinstates at different temperatures, which will be discussed in more detail in section 1.4. A more direct method commonly used to monitor SCO complexes is variable temperature magnetic susceptibility measurements, in which different $\chi_{\mathrm{M}} T$ values would be observed for a diamagnetic low-spin Fe II complex $(S=0)$ versus a paramagnetic high-spin FeII complex $(S=2)$.

In many cases, the LS state assumes the ground-state at low temperatures. However, at higher temperatures, the HS state assumes the thermodynamic ground-state governed by Gibbs free energy (eq. 1.1), where $\Delta H$ and $\Delta S$ is the difference in enthalpy and entropy of the HS and LS state, respectively. ${ }^{13}$ At temperatures below the transition temperature $\left(\mathrm{T}_{1 / 2}\right), \Delta H$ is dominant and thus the LS state is the thermodynamic ground-state. However, at higher temperatures, $\Delta S$ becomes much larger due to the contributions from the electronic entropy component, $\Delta S_{\text {el, }}$ and the vibrational entropy component, $\Delta S_{\text {vib. }}$. While a small contribution is made from the increase in spin multiplicity in $\Delta S_{\text {el }}\left(13.38 \mathrm{~J} \mathrm{~mol}^{-1} \mathrm{~K}^{-1}\right.$ for HS-Fe $\left.{ }^{\mathrm{II}}\right)$, studies have shown $\Delta \mathrm{S}_{\text {vib }}$ is favoured for the HS state and at higher temperatures, ranging up to $40-80 \mathrm{~J} \mathrm{~mol}^{-1} \mathrm{~K}^{-1} .^{28}$ Consequently, the SCO phenomena is typically called an entropy driven process.

$$
\Delta G=\Delta H-T \Delta S
$$

\section{3 - Role of Cooperativity in SCO Complexes}

Since the first spin crossover complex discovered by Cambi and Szego in 1931,29 numerous other SCO complexes have been reported with distinctly different SCO profiles. ${ }^{19,21}$ A schematic representation of possible SCO curves plotted in the form of HS fraction as a function of temperature is given in Figure 1.3.21 The majority of these SCO curves are a result of cooperative effects originating from elongation or shortening of the Fe- $\mathrm{N}$ bond distances in SCO materials. ${ }^{28,30}$ In the case of a gradual conversion from the LS to HS state (Figure 1.3a), minimal cooperativity is present in the SCO material. This is typically detected for complexes in solution, in which the SCO curve follows a simple Boltzmann distribution. ${ }^{21}$ In contrast, large structural changes associated with solid-state SCO complexes may give rise to abrupt spin transitions signifying a high degree of cooperativity (Figure 1.3b). In some cases, SCO may be abrupt and hysteretic such that the spin crossover temperature $\mathrm{T}_{1 / 2}$ is different during the warming and cooling phase (Figure 1.3c). ${ }^{13}$ The existence of hysteresis has gained substantial interest especially in the field of molecular information storage devices since an intrinsic memory effect is implemented in the system. Although quite rare among mononuclear $\mathrm{Fe}^{\mathrm{II}}$ complexes, SCO may occur in two steps, in which an intermediate phase transition is thermodynamically stable over a small temperature range (Figure 1.3d). ${ }^{31-33}$ This phenomena can be extended to polynuclear systems such as [ $\mathrm{n} \mathrm{n}]$ grid-like arrays where a spin-transition at one site may induce a spin-transition at another site affording a step-wise SCO curve. To date, a systematic approach to design and/or predict SCO behaviour with high cooperativity and hysteresis does not exist. However, incorporation of hydrogen bonds and 
$\pi$-stacking have demonstrated enhanced cooperativity for mononuclear Fe ${ }^{\mathrm{II}}$ complexes in the solid-state. ${ }^{34}$ The goal many SCO chemists continue to strive for is to design ligands with the appropriate $\Delta_{\mathrm{o}}$ for SCO as well as enforce cooperativity between the metal centers through the use of strong bonding interactions.
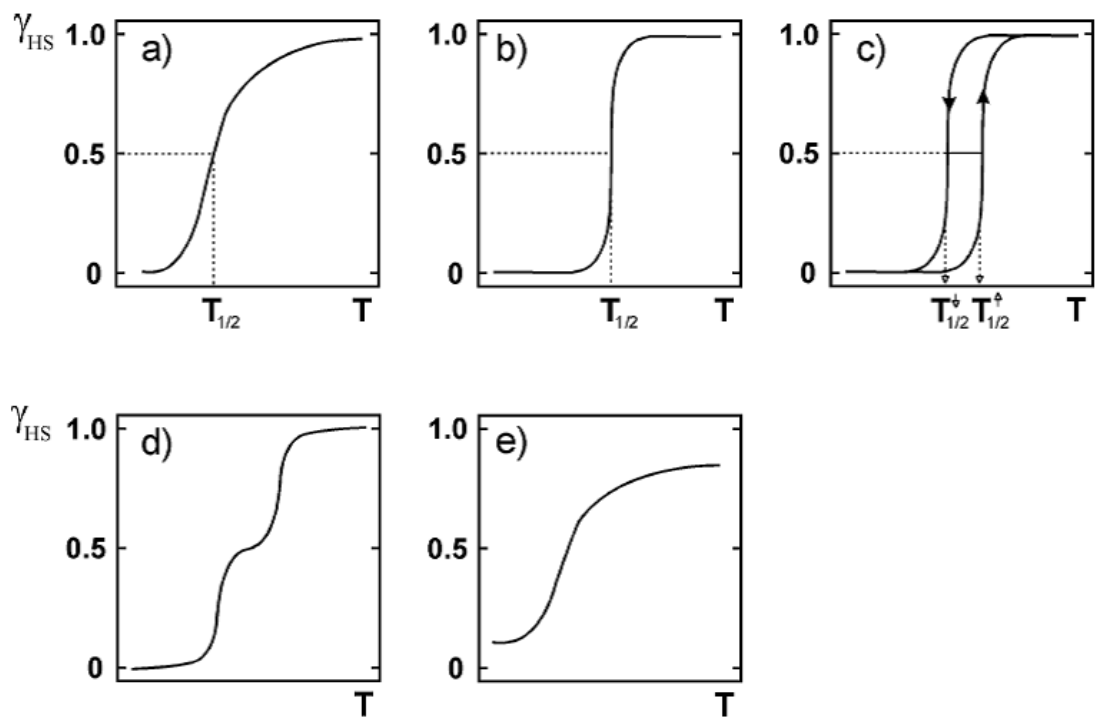

Figure 1.3. Schematic representation of possible SCO curves: (a) gradual, (b) abrupt, (c) with hysteresis, (d), step-wise, and (e) incomplete. Reprinted with permission from Chakrabarty, R. et. al. Copyright (2011) American Chemical Society. ${ }^{19}$

\section{4 - Mössbauer Spectroscopy for Iron Containing SCO Complexes}

As mentioned previously, MB spectroscopy is a valuable tool for investigating iron containing SCO complexes. The technique discovered by Rudolf L. Mössbauer in 1958 relies on the recoilless emission and absorption of gamma radiation to probe the hyperfine interactions between the electrons and nuclear moments, later termed the Mössbauer Effect. ${ }^{26}$ If an excited state nucleus (source) decays, gamma radiation is emitted which is subsequently absorbed by a second nucleus (sample) if the emitted energy is in resonance with the energy required to excite the second nucleus (Figure 1.4). For many free nuclei, absorption or emission of gamma radiation is accompanied by a recoil energy, $E_{R}$, due to conservation of momentum, which inherently leads to a decrease in energy in the emitting source. The same recoil energy occurs at the absorbing nuclei albeit in the opposite direction. ${ }^{35}$ This results in an increase in energy of the same magnitude at the absorbing nuclei. Consequently, the emitted $\gamma$-radiation from the source is no longer identical to the energy required to excite the second nucleus. 


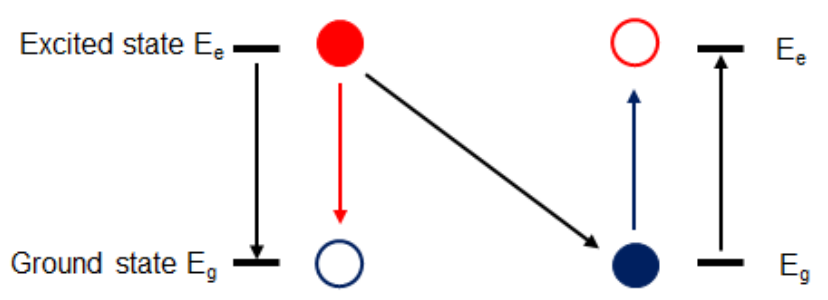

Figure 1.4. Decay of the excited state emits gamma radiation which is subsequently absorbed by a second nucleus if the energies are identical.

In order to achieve recoilless emission and absorption, the experiment must be carried out either in the solid-state or in frozen solution. For a single atom, the recoil energy $E_{R}$ is given in Equation 1.2, where $E_{\gamma}$ corresponds to the emitted $\gamma$-radiation, $\mathrm{m}$ is the mass of the emitting particle or atom, and $\mathrm{c}$ is the velocity of light. In the case of a single atom, the recoil energy is large since the mass is dependent only on a single nucleus. However, atoms embedded in a solid matrix will significantly increase the overall effective mass, $\mathrm{m}$, and thus drastically decrease the recoil energy $E_{R} .{ }^{26}$ The degree of recoilless absorption and emission can be measured by the so-called Debye-Waller factor, f. According to the Debye model, this factor is dependent on the bonding interactions in the crystal lattice and transition energies, in which molecules with stronger bonds and smaller transition energies correspond to larger $f$ values. Moreover, the Debye Waller factor is inversely proportional to the temperature, such that at lower temperatures, $\mathrm{f}$ is larger.

$$
E_{R}=\frac{E_{\gamma}^{2}}{2 m c^{2}}
$$

For ${ }^{57} \mathrm{Fe} \mathrm{MB}$ spectroscopy, a radioactive ${ }^{57} \mathrm{Co}$ is used as the source, which upon electron capture from the K-shell, decays initially to the $136 \mathrm{keV}$ nuclear level corresponding to the ${ }^{57} \mathrm{Fe}$ with nuclear quantum number $I=5 / 2$ (Figure 1.5). This excited state (life time $\sim 10 \mathrm{~ns}$ ) further decays to the $14.4 \mathrm{keV}$ level $(I=3 / 2)$ with $85 \%$ probability or directly to the ground state $(I=1 / 2)$ with $15 \%$ probability. ${ }^{26}$ The combination of the half-life of the $I=3 / 2$ level $(100$ ns) and the emitted $\gamma$-quanta (14.4 keV) are ideal for ${ }^{57} \mathrm{Fe} \mathrm{MB}$ spectroscopy. 


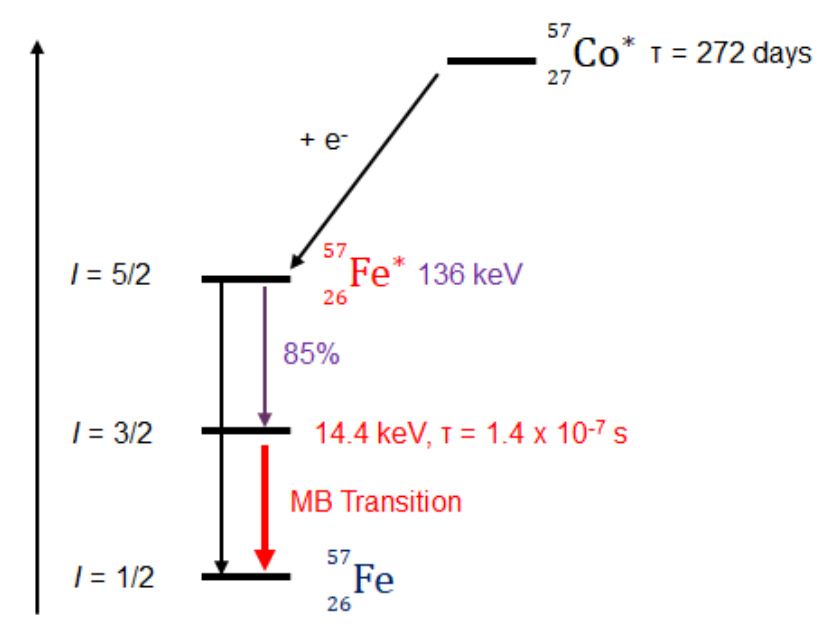

Figure 1.5. Simplified nuclear decay scheme and transitions for ${ }^{57} \mathrm{Fe} \mathrm{MB}$ spectroscopy.

If the nuclei of the emitting (source) and absorbing (sample) nuclei are identical, a single line would be observed. However, the resonance effect may be perturbed by the interaction of the ${ }^{57} \mathrm{Fe}$ nucleus with the surrounding environment giving rise to three main hyperfine interactions: the isomer shift, the quadrupole splitting, and the magnetic dipole splitting. ${ }^{26}$ The isomer shift is defined by the interaction between the protons of the nucleus and electrons (predominately s-electrons) with the nuclear field, also known as the electric monopole interaction. This interaction may shift the isomer shift, $\delta$, either to more positive or negative values relative to the source (i.e. $\alpha$-Fe). For Fe ${ }^{\mathrm{II}}(S=1 / 2)$, the presence of six $d$ electrons exerts a high degree of shielding on the $s$-electrons, which reduces the $s$-electron density at the nucleus pushing the isomer shift to more positive values. In contrast, for $\mathrm{Fe}^{\mathrm{VI}}$ $(S=1$ ) species with only two $d$-electrons, the shielding effect is much less affording a much higher s-electron density at the nucleus, and thus more negative isomer shift values. ${ }^{26}$ The isomer shift $\delta$ may also be affected indirectly by shielding effects, presence of strong $\pi$ acceptors, electronegativity of the ligands, as well as the metal-to-ligand bond distances. Based on the isomer shift, the oxidation state, spin state, bond properties as well as electronegativity can be determined.

The second parameter, quadrupole splitting $\left(\Delta E_{Q}\right)$, involves the interaction between the nuclear quadrupole moment and an inhomogeneous electric field gradient (EFG). A nuclear quadrupole moment is observed for systems with $I>1 / 2$ which may lift the degeneracies of the nuclear states. For instance, in the presence of an inhomogeneous EFG, the excited state of ${ }^{57} \mathrm{Fe}(I=3 / 2)$ will split into two substates with magnetic quantum numbers, $\mathrm{m}_{l}= \pm 1 / 2$ and $\mathrm{m}_{\mathrm{l}}= \pm 3 / 2$. The difference between the transitions from the ground-state $(I=1 / 2)$ to the excited states $\left(I=3 / 2, \mathrm{~m}_{1}= \pm 1 / 2\right.$ and $\left.I=3 / 2, \mathrm{~m}_{1}=3 / 2\right)$ gives rise to two different lines in the spectrum. The separation between the two lines is known as the quadruple splitting $\Delta E_{\mathrm{Q}}$. In general, there are two components that affect the quadrupole splitting: the lattice contribution and the valence contribution. The lattice contribution arises from non-cubic symmetry of the charged ligands surrounding the Mössbauer atom. The valence contribution arises from anisotropic population of the d-orbitals. For instance, for HS-Fe ${ }^{I I I}$ ions, the $d$-electrons are distributed evenly among the $d$-electrons (i.e. one in each orbital), affording an electric field gradient of zero. On the other hand, $\mathrm{HS}-\mathrm{Fe}^{\mathrm{II}}$ ions typically have large quadruple splitting parameters due to the Jahn-Teller distortion, which gives rise to the anisotropic distribution of the electrons in the $d$-orbitals. The effect of different $\sigma$-bonding and $\pi$-backbonding may 
also affect the quadruple splitting. Using the obtained quadrupole splitting parameter, information regarding the molecular symmetry, oxidation state, spin-state and bond properties can be determined.

The last hyperfine interaction observed in a MB spectrum is the magnetic dipole splitting arising from the interaction between the nuclear magnetic dipole moment, $\mu$, and the magnetic field at the nucleus. The ground state $(I=1 / 2)$ and excited state $(I=3 / 2)$ are split into $2 I+1$ substates with quantum numbers $m_{1}$ (Figure 1.6). Thus, in the case of the excited state $I$ $=3 / 2$, four states characterized by $m_{1}=+3 / 2,+1 / 2,-1 / 2$, and $-3 / 2$ are observed. Similarly, the ground state $I=1 / 2$ is split into two states characterized by $m_{l}=-1 / 2$ and $m_{l}=+1 / 2$. For a typical ${ }^{57} \mathrm{Fe}$ Mössbauer spectrum with magnetic dipole interactions, six lines are observed governed by the selection rules for magnetic dipole transitions: $\Delta I= \pm 1$ and $\Delta \mathrm{m}_{1}=0, \pm 1$. Through simulation of the spectra based on theoretical models, the relaxation time, zero-field splitting (ZFS) parameters, and g-values may also be determined.

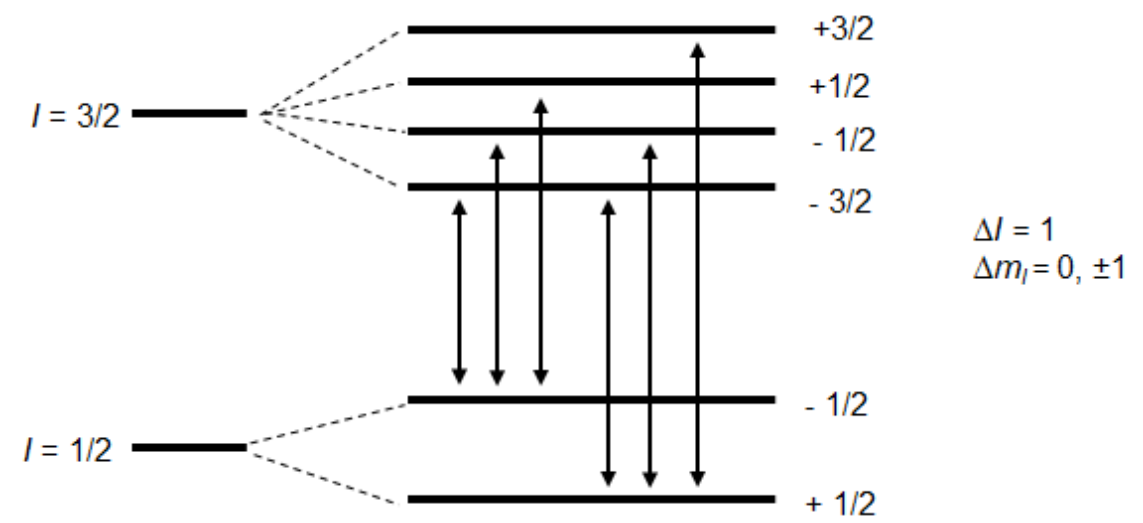

Figure 1.6. The splitting of the ground state and excited state of ${ }^{57} \mathrm{Fe}$ from magnetic dipole interactions affording six lines in the ${ }^{57} \mathrm{Fe} \mathrm{MB}$ spectrum.

For iron-containing SCO complexes, MB spectroscopy has proven to be a highly valuable tool used for monitoring the changes in the electronic structure at various temperatures. For instance, the Meyer group reported a step-wise SCO $\mathrm{Fe}^{I_{4}}{ }_{4}$ grid confirmed by variable temperature magnetic susceptibility measurements and MB spectroscopy (Figure 1.7). ${ }^{36}$ At $295 \mathrm{~K}$, the presence of a [HS-HS-HS-HS] configuration was confirmed by the presence of one doublet with an isomer shift of $\delta=0.92 \mathrm{mms}^{-1}$ and quadruple splitting of $\Delta E_{\mathrm{Q}}=2.02 \mathrm{mms}^{-1} \mathrm{in}$ the MB spectrum. Upon lowering the temperature to $133 \mathrm{~K}$, step-wise SCO was observed in which one HS-Fe ${ }^{\mathrm{II}}$ ion undergoes SCO to LS-Fe ${ }^{\mathrm{II}}$, giving an overall [3HS-1LS] state, confirmed by a second doublet with a relative area of $28 \%$ and an isomer shift of $\delta=0.38 \mathrm{mms}^{-1}$ $\left(\Delta E_{\mathrm{Q}}=0.90 \mathrm{mms}^{-1}\right)$, consistent with LS-Fe ${ }^{\mathrm{II}}$ ions. Further cooling to $5 \mathrm{~K}$ afforded a further decrease in the $\mathrm{HS}-\mathrm{Fe}^{\mathrm{II}}$ ions, but full conversion was never obtained. Nevertheless, MB spectroscopy has demonstrated to be a powerful technique in conjunction with variable temperature magnetic susceptibility measurements to determine the spin-state of the Fe ions during SCO. 

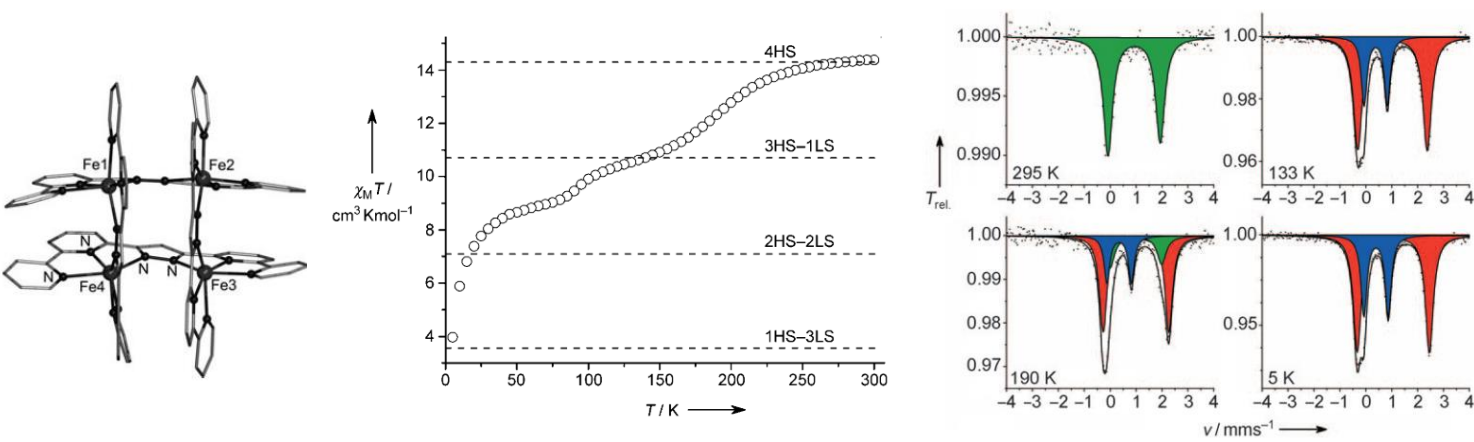

Figure 1.7. Molecular structure of $\mathrm{Fe}_{4}$ grid with the corresponding variable temperature magnetic susceptibility and MB spectra illustrating the change in spin-state at various temperatures. ${ }^{36}$

\section{5 - Cobalt(II)-based Single Molecule Magnets}

Single molecule magnets (SMMs) are defined by molecules with paramagnetic metal ions, which retain their magnetization for long periods of time in the absence of an external field at low temperatures. ${ }^{37,38}$ In principle, the molecular nature of SMMs hold potential for various applications including high-density information storage devices. ${ }^{39,40}$ However, practical applications are limited due to the extremely low operating temperatures (usually at liquid helium temperatures), in which the intrinsic memory effect is often lost upon warming. ${ }^{41}$ However, from a fundamental point of view, SMMs are a unique class of magnetic materials that have been extensively studied since the discovery of the first single molecule magnet, $\left[\mathrm{Mn}_{12} \mathrm{O}_{12}(\mathrm{OAc})_{16}\left(\mathrm{H}_{2} \mathrm{O}\right)_{4}\right] \cdot 2 \mathrm{HOAc} \cdot 4 \mathrm{H}_{2} \mathrm{O}$ (" $\mathrm{Mn}_{12}$ acetate"), which has a thermal barrier for spin reversal of $\mu_{\text {eff }}=51 \mathrm{~cm}^{-1} .38,42 \mathrm{~A}$ variety of other molecules exhibiting SMM and single ion magnet (SIM) behaviour based on 3d-transition metals (i.e. $\mathrm{Fe}, \mathrm{Co}, \mathrm{Ni}$, and $\mathrm{Mn}$ ) have been developed, ${ }^{43}$ in which one particular linear $\mathrm{Fe}^{\mathrm{I}}$ compound displayed a thermal barrier to spin reversal of $\mu_{\text {eff }}=226 \mathrm{~K} .44$ Another method involves incorporation of lanthanides due to their large unquenched molecular orbital momentum and large spin-orbit coupling, which are both essential for SIM anisotropies. In particular, molecules containing DyIII ions have displayed remarkably high relaxation energy barriers ${ }^{45-48}$ with a record of $\mu_{\text {eff }}=1277 \mathrm{~cm}^{-1}$ for $\left[\left(\mathrm{Cp}^{\mathrm{ttt}}\right)_{2} \mathrm{Dy}\right]\left[\mathrm{B}-\left(\mathrm{C}_{6} \mathrm{~F}_{5}\right)_{4}\right](\mathrm{Cpttt}=1,2,4$-tri(tert-butyl $)$ cyclopentadienide $){ }_{49}$

A variety of methods are used to characterize the properties of an SMM including the blocking temperature, $\mathrm{T}_{\mathrm{B}}$, the coercive magnetic field $H_{\mathrm{c}}$, and the effective energy barrier for spin reversal, $U_{\text {eff }}$ (mentioned above). The blocking temperature refers to the highest temperature a molecule exhibits hysteresis, but is strongly dependent on the sweep rate of the magnetic field. Hence, direct comparisons must be done cautiously. ${ }^{41}$ The coercive field corresponds to the field at which remnant magnetization is reduced to zero. The last, and by far, the most commonly used parameter is the $U_{\text {eff, }}$ which describes the thermal energy barrier for spin reversal. For molecules with a high $U_{\text {eff, }}$ slow relaxation of the spins coupled with a hysteretic effect is typically observed. This thermal energy barrier can be described by equation 1.3 and 1.4 for SMMs with integer or non-integer spins, respectively. Here, $D$ corresponds to the axial zero-field splitting (ZFS) parameter and $S$ corresponds to the total spin of the system.

$$
\begin{gathered}
U_{e f f}=|D| S^{2} \\
U_{e f f}=|D|\left(S^{2}-\frac{1}{4}\right)
\end{gathered}
$$


Based on the above equation, the simplified approach in designing SMMs with a large $U_{\text {eff }}$ involves increasing the overall ground spin state $S$ and ZFS parameter $D$. In order to achieve a non-zero ground spin state, the choice of metal centers (i.e. mixed valent) and bridging ligands are essential. For instance, metal ions in different oxidation states may induce an overall magnetic moment even if the overall exchange coupling is antiferromagnetic such as $\mathrm{Mn}^{\mathrm{III}} / \mathrm{Mn}^{\mathrm{IV}}$ in $\mathrm{Mn}_{12}$ acetate.50 Moreover, bridging ligands with angles of approximately $90^{\circ}$ induces an accidental orthogonality between the metal centers affording ferromagnetic exchange, as observed in cubanes with a $\left\{\mathrm{Ni}_{4} \mathrm{O}_{4}\right\}$ core, which afforded an $S=4$ ground state. ${ }^{51,52}$ Although this method revealed SMM behaviour in several complexes, the correlation between $S$ and $D$ have been well established such that increasing the overall ground spin-state does not increase the ZFS parameter $D$ by a factor of $S^{2}$ since $D$ is also proportional to $1 / \mathrm{S}^{2}$ (eq. 1.5).53,54 Thus, focus on increasing $D$ is a more viable strategy, especially in the weak field limit as observed for $\mathrm{Co}^{\mathrm{II}}$ ions.

$$
D=\frac{1}{S^{2}}
$$

The second strategy focuses on increasing the ZFS parameter, $D$. By definition, ZFS refers to lifting the degeneracies of the spin states in the absence of a magnetic field. This is typically accompanied by an axial or equatorial (rhombic) distortion commonly described by ZFS parameters $D$ and $E$, respectively. The simplest example is the triplet state, $S=1$ with $M_{s}=+1$, $0,-1$ microstates. In the absence of a magnetic field, the three microstates are degenerate. However, if the molecules are compressed along the $z$-axis or elongated along the $x$-axis, the degeneracy of the microstates are lifted as shown in Figure 1.8, giving rise to positive $D$ and $E$ values. In contrast, negative $D$ values signifies elongation along the $z$-axis. The ZFS parameters can be obtained by EPR spectroscopy as well as magnetic susceptibility measurements.
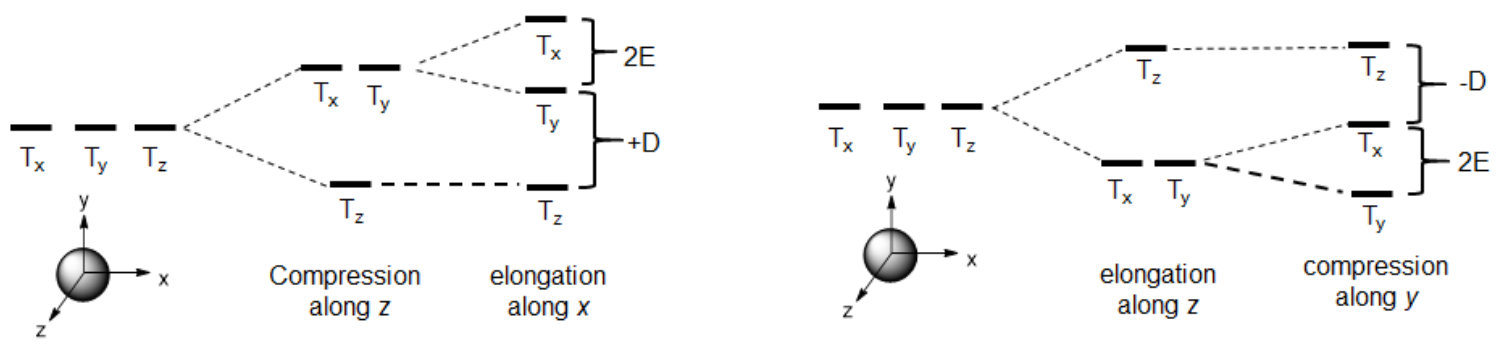

Figure 1.8. Schematic representation for zero-field splitting (ZFS) in an $S=1$ triplet state arising from distortions along the $x^{-}, y^{-}$, and $z$-axis.

In pursuit of intrinsically large ZFS parameters, lanthanide ions have gained significant interest due to the large diffused $4 \mathrm{f} / 5 \mathrm{f}$ magnetic orbitals resulting in stronger magnetic exchange and spin-orbit coupling. ${ }^{55}$ The presence of spin-orbit coupling often lifts the degeneracies of the orbitals giving rise to large ZFS parameters required for single molecule magnet behaviour. While numerous SMMs utilize lanthanide ions for their intrinsic large unquenched magnetic momentum, ${ }^{56}$ the focus of this section will be on $3 \mathrm{~d}$-transition metal SMMs, in particular $\mathrm{Co}^{\mathrm{II}}$ ions, which has been used extensively throughout this work. 
In general, $\mathrm{Co}^{\mathrm{II}}$ ions have a first order orbital momentum contribution which may lead to a large anisotropy depending on the ligand field and coordination environment. In many cases, $\mathrm{Co}^{\mathrm{II}}$ ions are not in strictly octahedral coordination spheres, which lifts the degeneracy of the ${ }^{4} \mathrm{~T}_{1 \mathrm{~g}}$ state. In the case of elongation or compression of the axial or equatorial bonds (tetragonal distortion), the ${ }^{4} \mathrm{~T}_{1 \mathrm{~g}}$ state is split into a new ${ }^{4} \mathrm{~A}_{2 \mathrm{~g}}$ ground state and an excited ${ }^{4} \mathrm{E}_{\mathrm{g}}$ state (Figure 1.9). ${ }^{57}$ When spin-orbit coupling takes into effect, six Kramer's doublets are observed, with the ${ }^{4} \mathrm{~A}_{2 \mathrm{~g}}$ state splitting further into a $M= \pm 3 / 2$ ground state and $M= \pm 1 / 2$ excited state (or vice versa, depending on the system). ${ }^{58}$ Typically, the separation between the ${ }^{4} \mathrm{Eg}$ and ${ }^{4} \mathrm{~A}_{2 \mathrm{~g}}$ state are large such that population of only the lowest energy Kramer's doublet is observed (i.e. only from the ${ }^{4} \mathrm{~A}_{2 \mathrm{~g}}$ state). The separation between the $M= \pm 3 / 2$ ground state and $M= \pm$ $1 / 2$ excited state is $2 D$. However, a further approximation can be made at low temperatures, in which only the lowest lying Kramer's doublet is populated.59 Hence, $|2 D|$ can be used to approximate the thermal barrier to spin inversion between the $+3 / 2$ and $-3 / 2$ state (or $-1 / 2$ and $+\frac{1}{2}$ state).

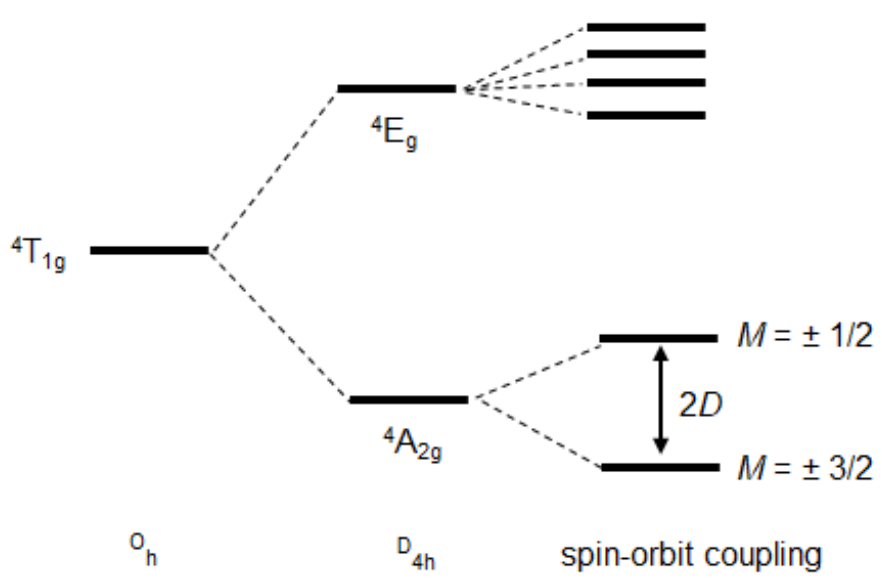

Figure 1.9. The combined effect of tetragonal distortion and spin-orbit coupling for a $\mathrm{Co}^{\mathrm{II}}$ ion.

Typically, in order to study the slow magnetic relaxation of SMMs, alternative current (ac) magnetic susceptibility is used to monitor the molecule's magnetic moment's response to an oscillating magnetic field. At higher frequencies, the magnetic moment may lag behind the applied field and thus two components are observed: the real component (in-phase, $\chi^{\prime}$ ) and imaginary component (out-of-phase, $\chi$ "). If the maximum of $\chi^{\prime}$ and $\chi^{\prime \prime}$ changes as a function of temperature at different frequencies this signifies slow relaxation and a barrier to spin reversal. The average relaxation time, $\tau$, can be determined from the $\chi$ " curve at each temperature and the barrier to spin reversal $U_{\text {eff }}$ can be determined based on the Arrheniuslike equation (eq. 1.6), in which a plot of $\ln (\tau)$ versus $(1 / \mathrm{T})$ can be used to determine the energy barrier $\left(\Delta E / K_{\mathrm{B}}\right)$ and the relaxation time $\tau_{0}$.

$$
\tau=\tau_{0} \exp ^{\frac{\Delta E}{k_{B} T}}
$$

For 6-coordinated $\mathrm{Co}^{\mathrm{II}}$ ions, $|D|$ can be exceptionally large with thermal barriers up to $U_{\text {eff }}=67 \mathrm{~cm}^{-1}$, as reported in a $\mathrm{Co}^{\mathrm{II}}$ complex [Co(hfpip) $\left.{ }_{2}\left\{\mathrm{D} 2 \mathrm{py}_{2}(\mathrm{TBA})\right\}\right]_{2}$, with hexafluoro-4-(4tert-butylphenylimino)-2-pentanoate (hfpip) and diazo-dipyridyl ligands [D2py2(TBA)].60 For comparison, the original $\mathrm{Mn}_{12}$ acetate has a thermal energy barrier of $U_{\text {eff }}=51 \mathrm{~cm}^{-1}$. Larger barriers $\left(U_{\text {eff }}=104-181 \mathrm{~cm}^{-1}\right)$ in $3 \mathrm{~d}$-transition metal based SMMs have been achieved with a family of linear two-coordinate $\mathrm{Fe}^{\mathrm{II}}$ complexes. ${ }^{61}$ 
To date, numerous $\mathrm{Co}^{\mathrm{II}}$ complexes with varying molecular structures have been shown to exhibit SMM behaviour including $\mathrm{Co}_{4}$ cubanes, ${ }^{62-65} \mathrm{Co}_{4}$ molecular squares, ${ }^{66} \mathrm{Co}_{7}$ discs, ${ }^{67-70}$ and $\mathrm{Co}_{5}$ square pyramids. ${ }^{71} \mathrm{~A}$ particular $\mathrm{Co}_{4}$ molecular square consisting of four $\mathrm{Co}_{4}$ ions linked together by $\mu$-bridging alkoxide and $\mathrm{Co}-\mathrm{N}-\mathrm{N}$-Co ligands (Figure 1.10) displayed ferromagnetic coupling between the $\mathrm{Co}^{\mathrm{II}}$ ions, confirmed by magnetic susceptibility measurements. ${ }^{66}$ Furthermore, ac magnetic measurements revealed a frequency dependence as a function of temperature typically observed in superparamagnets and slow relaxing molecule clusters. ${ }^{41}$ Using the Arrhenius law, an energy barrier of $\Delta E / k_{\mathrm{B}}=39 \mathrm{~K}\left(27.0 \mathrm{~cm}^{-1}\right)$ and a relaxation time of $\tau_{0}=5.4 \times 10^{-9} \mathrm{~s}$ was obtained.
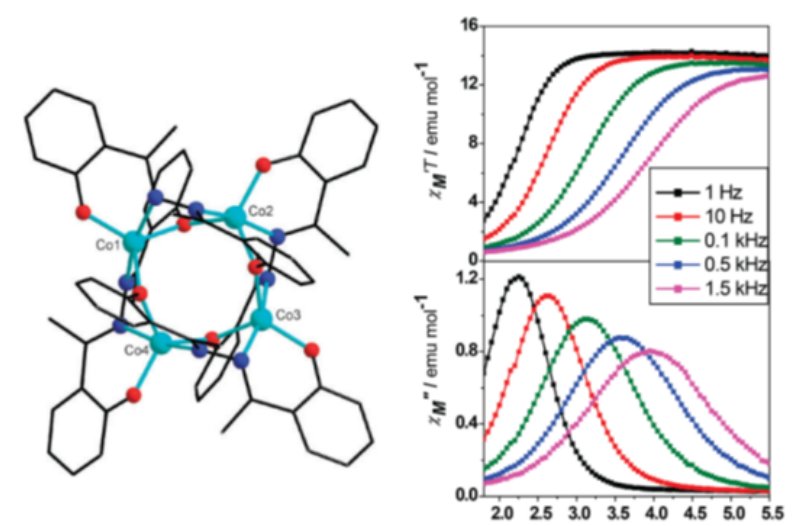

Figure 1.10. Molecular structure of the $\left[\mathrm{Co}_{4} \mathrm{~L}_{4}\right]$ square and ac magnetic susceptibility illustrating the in-phase $(\chi)$ and out-of-phase $(\chi \prime)$ dependence on temperature and frequency. Reprinted (adapted) with permission from Wu, D. et. al. Copyright (2009) American Chemical Society. ${ }^{66}$

\section{6 - Homonuclear [2 x 2] Grids}

In pursuit of new material with novel properties such as those described above (SCO and $\mathrm{SMM}$ ), homoleptic [ $\mathrm{n} \times \mathrm{n}$ ] grids are attractive candidates since the molecular properties of the aggregate are a direct consequence of the metal ions, donors, and/or bridging groups. Since grid formation depends strictly on the orientation of the ligands and the metal binding affinity, typically rigid, planar polydentate ligands are used (Chart 1.1). To date, ligands with nitrogen donors such as pyridines, pyrimidine, ${ }^{72}$ imidazole and pyrazole moieties are the most common,11,14,73 however carbohydrazide 1-5 and thiocarbohydrazide 1-6 based ligands have also been observed for grid-like formation. ${ }^{73,74}$ 


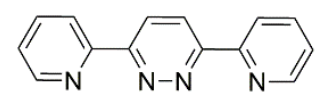

1-1

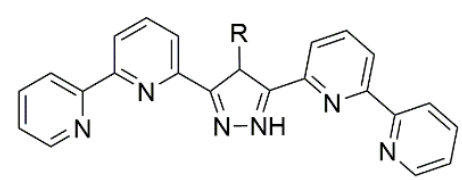

$1-3$<smiles>[R]/C(=N\N=C(/O)N/N=C(\[R])c1ccccn1)c1ccccn1</smiles>

1-5

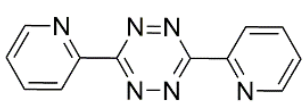

$1-2$

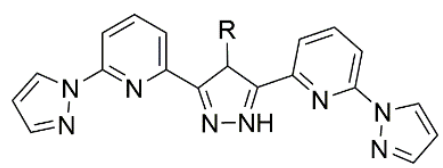

1-4

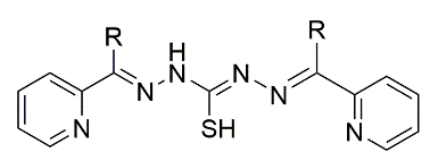

1-6

Chart 1.1. Selected ditopic ligands for [ $\mathrm{n} \times \mathrm{n}]$ grid-like assembly.

The first reported [ 2 x 2] grid-like array consists of four 3,6-bis(2'-pyridyl)pyridazine (dppn) 1-1 ligands with four copper(I) ions. ${ }^{75}$ Each copper(I) ion is coordinated to two mutually perpendicular ligand strands in a distorted tetrahedral environment. The distortion from a square to a rhombus most likely originates from $\pi$-stacking between the parallel pairs of dppn ligands. More importantly, the spontaneous self-assembly of the $\mathrm{Cu}_{4}$ grid was studied in solution, in which the formation of the $\mathrm{Cu}_{4}$ grid appears to be controlled thermodynamically. In the presence of excess dppn ligand (3-fold compared to $\mathrm{Cu}$ ), an equilibrium between the mononuclear $\left[\mathrm{Cu}(\mathrm{dppn})_{2}\right]^{+}$and the $\left[\mathrm{Cu}_{4}(\mathrm{dppn})_{4}\right]^{4+}$ are observed in solution. Upon removal of the solvent, ${ }^{1} \mathrm{H}$ NMR spectroscopy revealed the formation of the $\left[\mathrm{Cu}_{4}(\mathrm{ddpn})\right]^{4+}$ grid and liberation of four dppn ligands, as shown according to scheme 1.1.

$$
4\left[\mathrm{Cu}(\mathrm{dppn})_{2}\right]\left[\mathrm{CF}_{3} \mathrm{SO}_{3}\right] \rightleftharpoons\left[\mathrm{Cu}_{4}(\mathrm{dppn})_{4}\right]\left[\mathrm{CF}_{3} \mathrm{SO}_{3}\right]_{4}+4 \mathrm{dppn}
$$

Scheme 1.1. Dissolution of $\left[\mathrm{Cu}(\mathrm{dppn})_{2}\right]\left[\mathrm{CF}_{3} \mathrm{SO}_{3}\right]$ afforded the $\mathrm{Cu}_{4}$ molecular square along with four dppn ligands, confirmed by ${ }^{1} \mathrm{H}$ NMR spectroscopy.

Since the first homonuclear [2 $\times 2$ ] grid-like arrays, considerable advances have been made to fine-tune the ligands for the desired molecular properties. In terms of SMM behaviour, the majority is limited to lanthanide-based grids, ${ }^{76-80}$ however, the Meyer group recently reported a redox-induced SMM in a mixed valent $\left[\mathrm{L}^{1-4} \mathrm{Co}_{2}{ }_{2} \mathrm{Co}{ }^{I I I} 2\right]\left[\mathrm{BF}_{4}\right]_{4}$ grid using a derivative of the known compartmental pyrazolate-bridged ligand 1-4.81 While the homonuclear $\left[\mathrm{L}^{1-4} \mathrm{Co}_{4}{ }_{4}\right]\left[\mathrm{BF}_{4}\right]_{4}$ grid displayed antiferromagnetic coupling between the $\mathrm{Co}^{\mathrm{II}}$ ions, upon oxidation to the mixed valent congener $\left[\mathrm{L}^{1-4} \mathrm{Co}_{2}{ }_{2} \mathrm{Co}^{\mathrm{III}}{ }_{2}\right]\left[\mathrm{BF}_{4}\right]_{6}$, two diagonally opposed LS-Co ${ }^{\mathrm{III}}$ ions are magnetically switched off, such that the remaining $\mathrm{HS}-\mathrm{Co}^{\mathrm{II}}$ ions are magnetically isolated giving rise to SMM behaviour.

Another important feature displayed by several [2 x 2] grid-like arrays is their potential for cooperative SCO. While cooperativity is not guaranteed simply with the presence of multiple 
metal ions, it provides an avenue for scientists to investigate the effect of a spin transition at one site on the entire molecule. To that end, the Meyer group recently reported another family of $\left[\mathrm{L}_{4} \mathrm{Br}_{4} \mathrm{Fe}_{4}\right] \mathrm{X}_{4}$ grids $\left(\mathrm{X}=\mathrm{PF}_{6}, \mathrm{BF}_{4}, \mathrm{ClO}_{4}, \mathrm{Br}\right)$ with a bromine substituent at the 4-position of the pyrazole backbone of 1-3 $(\mathrm{R}=\mathrm{Br}) .{ }^{82}$ Six new compounds with varying solvents and counterions were characterized, each displaying different spin-states as well as SCO properties. The structural distortions in each compound were characterized by Continuous Symmetry Measures (CSM), 83 in which the bond distances and angles of a molecule were compared to an ideal octahedron. For small $\mathrm{S}\left(\mathrm{O}_{\mathrm{h}}\right)$ values, deviations from an ideal octahedral environment are small, while high $\mathrm{S}\left(\mathrm{O}_{\mathrm{h}}\right)$ values indicate a highly distorted coordination sphere. To no surprise, LS-FeII ions typically have small $\mathrm{S}\left(\mathrm{O}_{\mathrm{h}}\right)$ values while $\mathrm{HS}-\mathrm{Fe}^{\mathrm{II}}$ ions are typically associated with high $\mathrm{S}\left(\mathrm{O}_{\mathrm{h}}\right)$ values. Generally, $\mathrm{Fe}^{\mathrm{II}}$ ions which display high $\mathrm{S}\left(\mathrm{O}_{\mathrm{h}}\right)$ values are typically locked in the HS state and thermal spin-transitions are highly unlikely.

Using the $\mathrm{S}\left(\mathrm{O}_{\mathrm{h}}\right)$ values obtained from CSM calculations of the six $\left[\mathrm{L}_{4} \mathrm{Br}_{4} \mathrm{Fe}_{4}\right] \mathrm{X}_{4}\left(\mathrm{X}=\mathrm{PF}_{6}, \mathrm{BF}_{4}\right.$, $\mathrm{ClO}_{4}, \mathrm{Br}$ ) grids with different counterions and solvent lattice molecules, a high degree of cooperativity was observed in the grid imparted by the strain effects during a spin-transition throughout the entire molecule. In particular, a spin-transition at one site (Figure 1.11, blue) induces a lowering of the $\mathrm{S}\left(\mathrm{O}_{\mathrm{h}}\right)$ value for the metal ion at the opposite corner (Figure 1.11, green). At the same time, further SCO is severely hampered in the [2HS-2LS] state due to the high distortion of the coordination polyhedral of the HS-Fell ions.

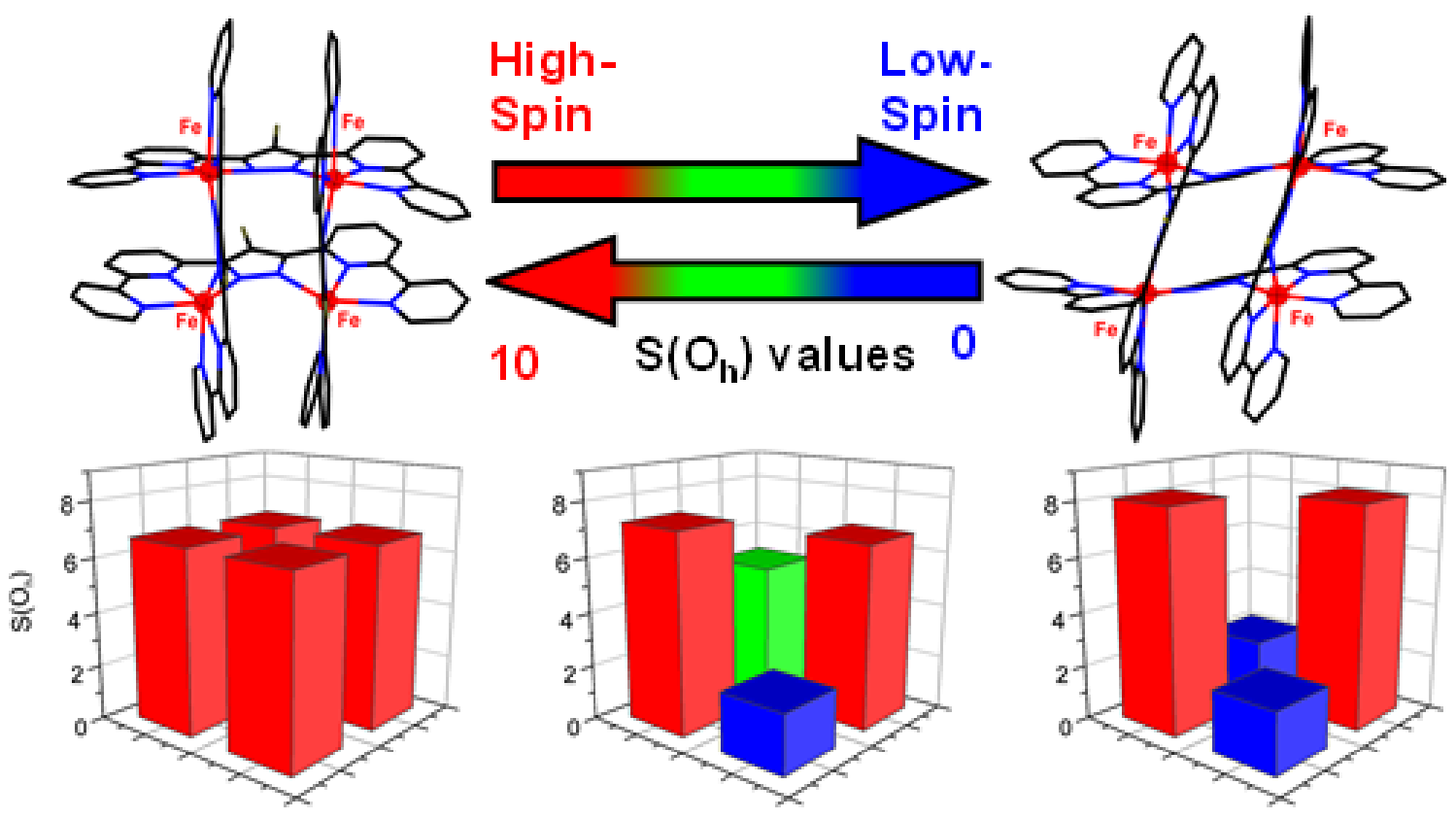

Figure 1.11. Continuous symmetry measures on various $\left[\mathrm{L}_{4} \mathrm{Fe}_{4}\right] \mathrm{X}_{4}$ grids $\left(\mathrm{X}=\mathrm{PF}_{6}, \mathrm{BF}_{4}, \mathrm{ClO}_{4}\right.$, $\mathrm{Br}$ ) illustrating the cooperativity effect within the grid. In particular, high $\mathrm{S}\left(\mathrm{O}_{\mathrm{h}}\right)$ values typically correspond to $\mathrm{HS}-\mathrm{Fe} \mathrm{e}^{\mathrm{II}}$, while low $\mathrm{S}\left(\mathrm{O}_{\mathrm{h}}\right)$ values correspond to $\mathrm{LS}-\mathrm{Fe}^{\mathrm{II}}$ ions. During a spin transition at one Fe center, the metal center on the opposite corner is ultimately affected and prone to become LS while further SCO from the [2HS-2LS] state is severely hampered.82 


\section{7 - Heterometallic [2 x 2] Grids}

Using the appropriate ditopic ligand and metal ions, homonuclear grids can be easily synthesized by a one-pot reaction via self-assembly. While a variety of interesting properties have been exhibited with homonuclear [ $\mathrm{n} \mathrm{x} \mathrm{n}]$ grids, heterometallic grids involving two or more different metal ions provide the possibility for multiple stable redox states as well as site specific responses. However, heterometallic grids are synthetically more challenging since different isomers, syn or anti, are equally possible and metal ion scrambling may occur. Consequently, in order to selectively insert a metal ion in a grid-like array, careful ligand design with the appropriate binding pockets for specific metal ions must be judiciously engineered. For this purpose, asymmetric ligands with bidentate and tridentate binding moieties are typically employed (Chart 1.2).10 Utilizing the preferential coordinating number of the metal ions, the octahedral cavity created from two tridentate binding sites of two mutually perpendicular ligands 1-7 was occupied by $\mathrm{Zn}^{\mathrm{II}}$ ions while the tetrahedral cavity created by the bidentate binding sites of two orthogonal ligand strands 1-7 was occupied by $\mathrm{Cu}^{\mathrm{I}}$ ions forming the expected heterometallic [2 $\mathrm{x}$ 2] grid-like architecture. ${ }^{84}$ Hydrazone ligands developed by Thompson have also been used for several heterometallic [ $\mathrm{n} \times \mathrm{n}]$ grids. One particular system using ligand $\mathbf{1 - 8}$ afforded a unique $\left[\mathrm{Fe}^{\mathrm{III}} \mathrm{Cu}^{\mathrm{II}}\right]^{4+}$ grid, in which one of the corners is occupied by an $\mathrm{Fe}^{\mathrm{III}}$ atom and the remaining sites are occupied by $\mathrm{Cu}^{\mathrm{II}}$ ions. ${ }^{85}$ While the $\mathrm{Cu} \cdots \mathrm{Cu}$ ions are antiferromagnetically coupled, the $\mathrm{Cu} \cdots \mathrm{Fe}$ interactions display weak ferromagnetic coupling, affording a ground state of $S=3$. Similarly, using ligand 1-9, a neutral $\left[\mathrm{Mn}_{2} \mathrm{Cu}_{2}{ }_{2}\right]$ grid was obtained with a ferrimagnetic ground state of $S=4 .{ }^{86}$ Hence, using asymmetric ligands with specific sites for different metal ions, a non-zero ground state can also be achieved using heterometallic grid-like arrays ideal for potential SMM behaviour.

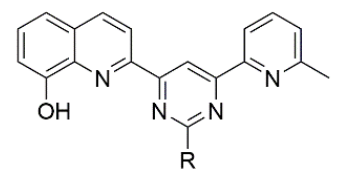

$1-7$

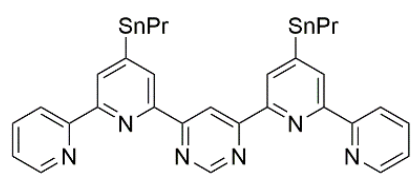

$1-10$<smiles>NC(=NN=C(O)c1ccccn1)c1ccccn1</smiles>

$1-8$<smiles>OCc1cccc(/C=N/N=C(\O)c2cccc(/C(O)=N/N=C/c3cccc(CO)n3)n2)n1</smiles>

$1-11$<smiles>CC(=Nc1ccccn1)NC(=O)C(C)=NO</smiles>

$1-9$

$1-12$

Chart 1.2. Selected ligands used for the synthesis of heterometallic [ $\mathrm{x} n$ ] grid-like arrays. 
In the case of symmetric ligands, a complementary method was developed by Lehn, in which a step-wise approach coupled with protection/deprotection is employed (Figure 1.12). ${ }^{87,88}$ In the case of 1-10, protection of one of the peripheral pyridine moieties with a methyl moiety was employed to give $\mathbf{1 - 1 0 b}$. Addition of $\mathrm{RuCl}_{3}$ to $\mathbf{1 - 1 0 b}$ afforded the kinetically stable $\mathrm{Ru}^{\mathrm{II}}$ "corner complex" 1-13b, ${ }^{87}$ which was subsequently deprotected with dabco to form 1-13. Introduction of a more labile metal such as $\mathrm{Fe}^{\mathrm{II}}, \mathrm{Co}^{\mathrm{II}}$, or $\mathrm{Ni}{ }^{\mathrm{II}}$ into a solution of 1-13 afforded the desired heterometallic [2 $\mathrm{x} 2]$ grid. In some cases, protection/deprotection is not required due to the kinetic stability of the first metal ion relative to the second metal ion. This was demonstrated with the OsII "corner complex" with ligand 1-10, in which exclusive formation of $\left[\mathrm{L}^{1-10_{2} \mathrm{Os}}\right]^{2+} \mathbf{1 - 1 4}$ was obtained without protection of the pyridine moiety. Naturally, using the above methodology, three different metal ions can also be incorporated into the grid-like array. Addition of two equivalents of Fe $\mathrm{II}^{\mathrm{II}}$ ions to an equimolar solution of $\left[\mathrm{L}^{1-10_{2} \mathrm{Os}}\right]^{2+}$ and $\left[\mathrm{L}^{1-10_{2}} \mathrm{Ru}\right]^{2+}$ afforded the $\mathrm{Os}^{\mathrm{II}}$ and $\mathrm{Ru}^{\mathrm{II}}$ ions at opposite corners and the remaining sites were occupied by $\mathrm{Fe}^{\mathrm{II}}$ ions. ${ }^{87}$ However, mixtures of $\left[\mathrm{L}^{1-10_{4} \mathrm{OsRuFe}}\right]^{4+},\left[\mathrm{L}^{1-10}{ }_{4} \mathrm{Ru}_{2} \mathrm{Fe}_{2}\right]^{4+}$ and $\left[\mathrm{L}^{1-10_{4}} \mathrm{Os}_{2} \mathrm{Fe}_{2}\right]^{4+}$ in the ratio of 2:1:1 were observed in fast-atom-bombardment mass spectrometry (FAB-MS) and ${ }^{1} \mathrm{H}$ NMR spectroscopy.

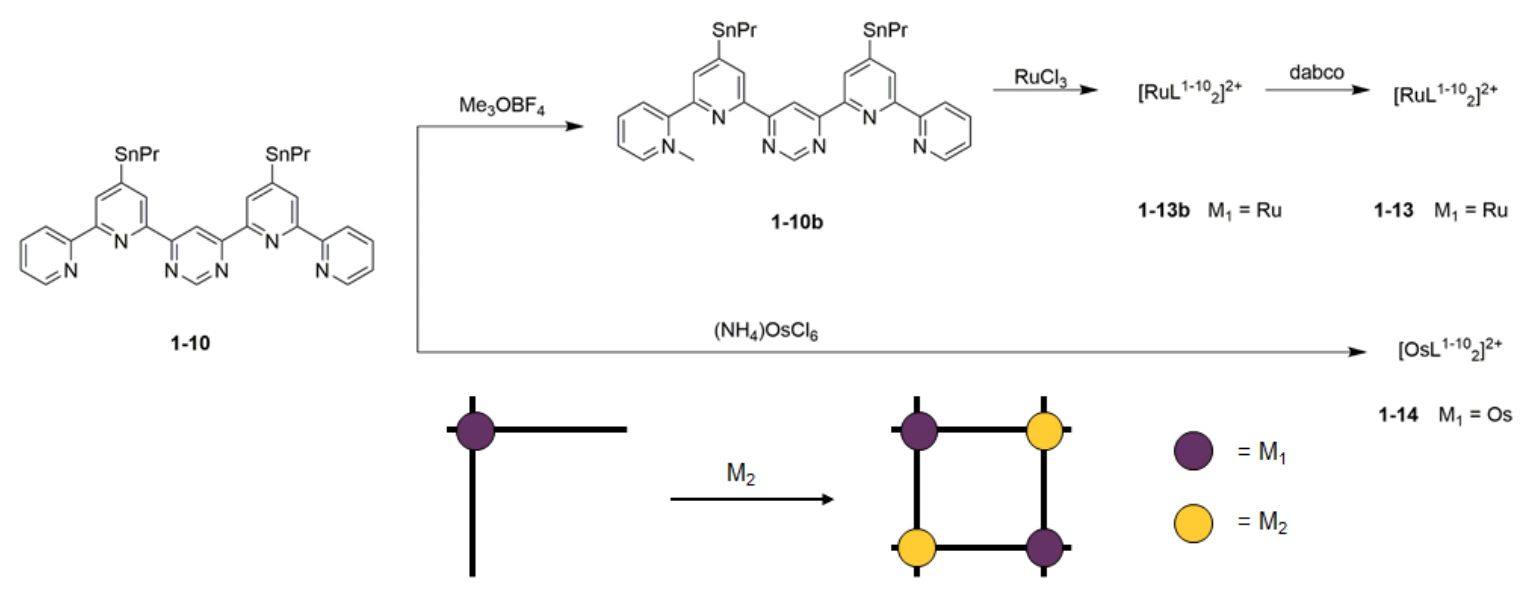

Figure 1.12. Step-wise approach coupled with protection/deprotection developed by Lehn for the synthesis of heterometallic [2 $\times 2]$ grids.

Recently, in pursuit of SMM behaviour in supramolecular clusters, ligands with much larger cavities were designed in order to incorporate lanthanide ions into heterometallic grids. Indeed, using ligand 1-11, a [DyIII $\mathrm{Cu}_{4}{ }_{4}$ ] grid was isolated with the DyIII ions positioned in the $\mathrm{O}-\mathrm{N}-\mathrm{N}-\mathrm{O}$ binding pocket of the corners of the grid, while the $\mathrm{Cu}^{\mathrm{II}}$ ions occupy the N-N-N pockets in between the DyII ions. ${ }^{80}$ Analysis of the ac magnetic susceptibility measurement of the [Dy $\mathrm{DIII}_{4} \mathrm{Cu}_{4}$ ] grid afforded an energy barrier of $U_{\text {eff }}=32.2 \mathrm{~K}$ and a relaxation time of $\tau_{0}=8.1 \times 10^{-9} \mathrm{~s}$. While this field is still much in its infancy, incorporation of lanthanide ions into grid-like arrays have demonstrated the potential for significant improvements in the field of molecular magnetism in [ $\mathrm{n} \mathrm{n}$ ] grids.

In extension to [2 x 2] grids, heterometallic [3 $\mathrm{x} 3$ ] grid-like arrays can also be synthesized by following these methods. ${ }^{89}$ Although quite rare, a series of heterometallic [3 $\mathrm{x} 3$ ] grids using tritopic ligands such as 1-12 with two different coordination sites were reported. ${ }^{73}$ Initially, the homometallic $\mathrm{Mn}^{\mathrm{II}_{9}}[3 \times 3$ ] grid was synthesized, in which electrochemistry demonstrated the metal centers at the corner of the grid are easily oxidized, signifying a low degree of 
stability at the corners of the grid. Consequently, addition of $\mathrm{Cu}^{\mathrm{II}}$ ions afforded a metal substitution at the four corners to give a new heterometallic $\left[\mathrm{L}^{1-12} \mathrm{Mn}_{5} \mathrm{Cu}_{4}\right]$ grid. ${ }^{9}$

Overall, since the first [ $2 \times 2$ ] grid was reported, considerable progress has been made to synthesize molecules with a preprogrammed architecture with complementary physical properties. To date, a variety of homonuclear grids have been reported and an easily expandable class of ligands (pyrazoles, pyridines, hydrazones etc) are continuously being developed for this purpose. In the case of heterometallic grids, despite the synthetic challenges, significant progress has also been made in this domain. The ability to selectively introduce metal ions at certain sites provides a means to design and fine-tune electrochemical, photochemical, and magnetic properties. From an application perspective, understanding the structure property correlations will help for future rational design of potential molecular-based materials. 


\section{8 - Thesis Outline}

The field of self-assembled homonuclear and heterometallic [ $\mathrm{n} x \mathrm{n}$ ] grids are quite diverse in terms of the type of ligands, size, and molecular properties. In the Meyer group, the family of rigid pyrazolate-bridged compartmental ligands $1-3^{90}$ has been used for numerous [2 $\left.\mathrm{x} 2\right]$ grid-like assemblies. Using this ligand, a series of $\left[\mathrm{L}_{4} \mathrm{Fe}^{\mathrm{II}_{4}}\right]^{4+}$ grids have been synthesized with various substituents on the pyrazole backbone $(\mathrm{R}=\mathrm{H}, \mathrm{Me}, \mathrm{Br}) \cdot{ }^{36,82,91}$ As already mentioned, the parent $\left[\mathrm{LH}_{4} \mathrm{Fe}_{4} \mathrm{II}_{4}\right]\left[\mathrm{BF}_{4}\right]_{4}$ grid $(\mathrm{R}=\mathrm{H})$ displayed step-wise $\mathrm{SCO}$ and the cooperativity effects were explained using the diverse spin-states exhibited by the $\left[\mathrm{L}_{4} \mathrm{Br}_{4} \mathrm{Fe}^{\mathrm{II}} 4\right]^{4+}(\mathrm{R}=\mathrm{Br})$ grid with different counterions and lattice solvent molecules. In hopes of stabilizing the [2HS-2LS] state for applications in quantum cellular automata, the ligand field was increased by substitution of a methyl moiety on the pyrazole backbone. ${ }^{91}$ Indeed, this afforded a large stability of the [2HS-2LS] state from $7-250 \mathrm{~K}$ and step-wise SCO was no longer observed. At the same time, new $\mathrm{Fe}_{4}{ }_{4}$ and $\mathrm{Co}_{4}{ }_{4}$ grids were developed using the ligand 1-4, in which the mixed valent $\left[\mathrm{L}^{1-4}{ }_{4} \mathrm{Co}_{2} \mathrm{Co}_{2} \mathrm{III}_{2}\right]\left[\mathrm{BF}_{4}\right]_{6}$ grid displayed SMM behaviour. ${ }^{81}$

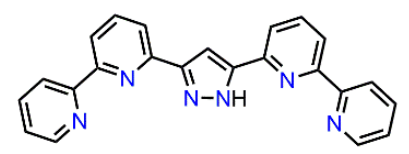

$\mathrm{HL}^{\mathrm{H}}$

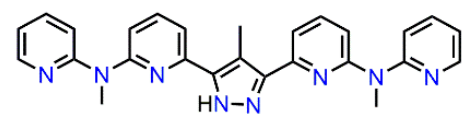

$\mathrm{HL}^{1}$

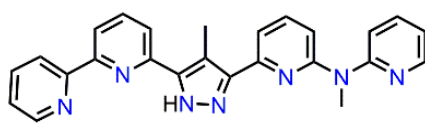

$\mathrm{HL}^{2}$

Chart 1.3. Ligands used for this work.

Due to the diverse magnetic properties exhibited by the series of $\mathrm{Fe}_{4}{ }_{4}$ grids, the structural changes accompanied by optical excitation may provide insight on the cooperativity effects between the metal ions during optical excitation. Moreover, with the presence of several metal ions, new relaxation pathways differing from typical mononuclear Fe SCO complexes may be realized. Since the excitations most likely occur on short time-scales (i.e. femtosecond - nanoseconds), transient absorption spectroscopy is a versatile technique to investigate these changes. To that end, in collaboration with the group of Prof. Dr. Dirk Schwarzer (MPI for Biophysical Chemistry), UV-vis pump/broadband-mid-IR probe spectroscopy will be used to investigate the electronic structure of the optically excited states on the previously reported homonulcear $\left[\mathrm{L}_{4} \mathrm{Fe}_{4}{ }_{4}\right]\left[\mathrm{BF}_{4}\right]_{4}$ grids $(\mathrm{R}=\mathrm{H}, \mathrm{Br})$ in solution. The results will be discussed in Chapter 2.

While a series of homometallic [2 $\mathrm{x}$ 2] grids have already been developed using the ligand 1-3 $(\mathrm{R}=\mathrm{H}, \mathrm{Br}, \mathrm{Me})$, the quest for new heterometallic [ $2 \times 2]$ grids may provide new interesting properties. Although single molecule magnet behaviour in [2 2 2] grids are rare due to antiferromagnetic coupling between the metal ions to form a diamagnetic ground state $\mathrm{S}_{\mathrm{T}}=0$, SMM behaviour was observed in the mixed valent $\left[\mathrm{L}^{1-4} \mathrm{Co}^{\mathrm{II}} \mathrm{Co}^{\mathrm{III}}\right]\left[\mathrm{BF}_{4}\right]_{6}$ grid arising from magnetically isolated $\mathrm{HS}-\mathrm{Co}^{\mathrm{II}}$ ions. A similar strategy may be employed by introducing diamagnetic $\mathrm{Ru}^{\mathrm{II}}$ ions at opposite corners of the grid following the step-wise approach developed by Lehn. Consequently, the magnetically active HS-CoII ions at the remaining corners of the grid may give rise to SMM behaviour. The results of the $\left[\mathrm{LH}_{4} \mathrm{Ru}_{2} \mathrm{Co}_{2}\right]\left[\mathrm{BF}_{4}\right]_{4}$ grid along with the mixed valent $\left[\mathrm{LH}_{4} \mathrm{Ru}_{2} \mathrm{Co}_{2}\right]\left[\mathrm{BF}_{4}\right]_{6}$ grid will be discussed in Chapter 3. Similarly, $\mathrm{Fe}^{\mathrm{II}}$ ions may also be incorporated into the [2 $\mathrm{x} 2$ ] grid-like array. Naturally, with the presence of $\mathrm{Fe}^{\mathrm{II}}$ ions, interesting magnetic properties including SCO may be realized. Moreover, the 
intervalence charge transfer (IVCT) between the metal ions will also be investigated (Chapter 4).

In pursuit of new [ $\mathrm{n} \times \mathrm{n}$ ] grids, a new ligand $\mathbf{H L}^{\mathbf{1}}$ was synthesized with additional electron donating groups ( $\mathrm{NMe}$ ) inserted in between the pyridine moieties of $\mathbf{H L}^{\mathbf{H}}$. While these types of ligands are typically used for enhanced luminescent properties in $\mathrm{Ru}^{\mathrm{II}}$ complexes, ${ }^{92}$ incorporation of $\mathrm{Co}^{\text {II }}$ ions have shown to be in the regime of SCO under mild pressures. ${ }^{93}$ Consequently, Fe ${ }^{I I}$ and $\mathrm{Co}^{\mathrm{II}}$ ions were used. Moreover, the ligand $\mathbf{H L}^{\mathbf{1}}$ can be easily functionalized with different electron donating or electron withdrawing groups on the pyrazole backbone to tune the ligand field. However, only the unsubstituted derivative (hydrogen on the pyrazole backbone) was studied in the course of this work (Chapter 5).

The last chapter entails the synthesis of an asymmetric ligand in pursuit of developing another class of heterometallic [ $2 \times 2$ ] grids. The ligand $\mathbf{H L}^{2}$ is composed of a hybrid of both precursors used in $\mathbf{H L}^{\mathbf{H}}$ and $\mathbf{H L}^{\mathbf{1}}$, namely two bipyridine moieties on one end and two bipyridine groups connected by an NMe moiety on the other end. While the overall goal is to create new heterometallic grids, homonuclear grid formation must be determined initially. The preliminary results for the homometallic $\mathrm{Fe}_{4}$ grids will be discussed in Chapter 6 . 


\section{Chapter 2 - Femtosecond Investigations on Optically Excited Homonuclear Fe Grids}

\section{1 - Introduction}

Spin crossover (SCO) complexes are prime candidates for information storage devices due to their bistable nature. Interconversion between the low-spin (LS) state and the high-spin (HS) state can be triggered by an external stimulus such as light, temperature, or pressure. While, to date, numerous SCO complexes have been reported with the majority being Fe based,19,21 the mechanistic pathway behind the optical excitation and the structural changes associated with SCO have only been recently investigated with the help of ultrafast spectroscopy. ${ }^{94-97}$ In particular, FeII spin crossover (SCO) complexes have been extensively investigated by femtosecond (fs) transient absorption (or pump-probe) spectroscopy in solution. In general, optical excitation from the ${ }^{1} \mathrm{~A}_{1}$ ground state to the ${ }^{1,3} \mathrm{MLCT}$ state is followed by relaxation to the quintet ${ }^{5} \mathrm{~T}_{2}$ state. ${ }^{97}$ However, using various ultrafast spectroscopic techniques, an intermediate ${ }^{3}$ T state was also identified (Figure 2.1). ${ }^{95}$

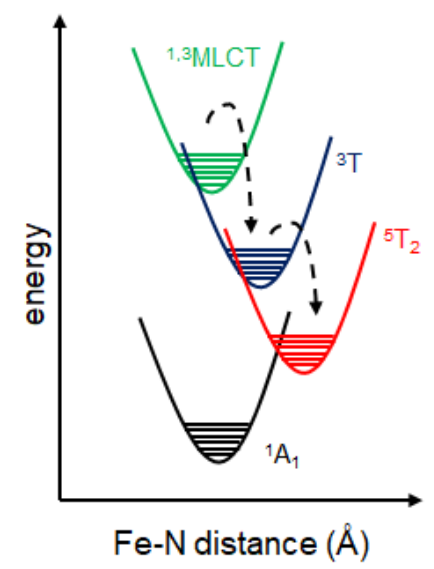

Figure 2.1. Schematic relaxation cascade from the excited ${ }^{1,3} \mathrm{MLCT}$ state to the HS quintet ${ }^{5} \mathrm{~T}_{2}$ state. Reproduced from Zhang W. et. al. ${ }^{97}$

In extension to mononuclear systems, polynuclear complexes containing several metal ions have also gained significant interest due to the potential for cooperativity between elastically coupled metal ions in a rigid framework. In particular, the mechanistic pathway as well as structural dynamics associated with optical excitation of tetranuclear [2 x 2] grids may lead to different relaxation pathways compared to mononuclear Fe complexes. For instance, in addition to MLCT transitions, metal-to-metal charge transfer, also known as intervalence charge transfer (IVCT) may also be possible. In some cases, electron transfer between two metal ions may induce a spin transition, also called the charge transfer induced spintransition (CTIST), which were observed for various Prussian Blue Analogues (PBAs). ${ }^{98}$ These systems, consisting of Fe and Co ions bridged by a cyanide (CN) moiety, can be interconverted thermally and by light irradiation. ${ }^{99}$ During this process, an electron is transferred from the LS-Fe ${ }^{I I}$ ion in diamagnetic LS-FeII-CN-LS-Co ${ }^{I I I}$ to the LS-Co ${ }^{I I I}$ ion, which induces a spintransition to form paramagnetic LS-FeIII-CN-HS-CoII. These compounds are also potential candidates for ultrafast spectroscopy, however, the focus of this chapter will be on homonuclear [2 x 2] grid-like arrays. 
In pursuit of oligonuclear SCO complexes, the Meyer group has developed a series of $\left[\mathrm{LR}_{4} \mathrm{Fe}_{4}\right]^{4+}$ grids $(\mathrm{R}=\mathrm{H}, \mathrm{Me}, \mathrm{Br}$ ) using the known compartmental pyrazolate-bridged ligand 2-1a-c (Chart 2.1). ${ }^{36,82,90,91}$ As already mentioned in chapter 1 , the parent $\left[\mathrm{LH}_{4} \mathrm{Fe}_{4}\right]\left[\mathrm{BF}_{4}\right]_{4}$ grid $(\mathrm{R}=\mathrm{H})$ exhibited step-wise spin crossover (SCO) from the [4HS] state at room temperature to the [3HS-1LS] state at $133 \mathrm{~K}$ and further incomplete SCO to the [2HS-2LS] state below $133 \mathrm{~K} .{ }^{36} \mathrm{In}$ contrast, variable temperature magnetic susceptibility measurements on the methylated $\left[\mathrm{LMe}_{4} \mathrm{Fe}_{4}\right]\left[\mathrm{BF}_{4}\right]_{4}$ grid revealed a stabilization of the [2HS-2LS] configuration from 7-250 K in the solid-state. ${ }^{91}$ In extension to the parent $(\mathrm{R}=\mathrm{H})$ and methylated $(\mathrm{R}=\mathrm{Me})\left[\mathrm{L}_{4} \mathrm{Fe}_{4}\right]\left[\mathrm{BF}_{4}\right]_{4}$ grids, a family of brominated $\left[\mathrm{LBr}_{4} \mathrm{Fe}_{4}\right] \mathrm{X}_{4}$ grids $\left(\mathrm{X}=\mathrm{PF}_{6}, \mathrm{BF}_{4}, \mathrm{ClO}_{4}, \mathrm{Br}\right)$ were synthesized which displayed different spin configurations depending on the anions and solvent lattice molecules. Using Continuous Symmetry Measures (CSM) for the different $\left[\mathrm{LBr}_{4} \mathrm{Fe}_{4}\right] \mathrm{X}_{4}$ grids, the cooperative nature of the Fe ions was established in which a spin transition at one site induces a second spin transition at the opposite corner. ${ }^{82}$ Furthermore, a trinuclear defect $\left[\mathrm{LH}_{2}\left(\mathrm{HL}^{\mathrm{H}}\right)_{2} \mathrm{Fe}_{3}\right]\left[\mathrm{BF}_{4}\right]_{4}$ grid using the parent ligand 2-1a $(\mathrm{R}=\mathrm{H})$ was also synthesized by using stoichiometric amounts of $\mathrm{Fe}^{\mathrm{II}}$ salt. 100 The overall spin-state of $\left[\mathrm{L}_{2}(\mathrm{HL})_{2} \mathrm{Fe}_{3}\right]\left[\mathrm{BF}_{4}\right]_{4}$ was confirmed by X-ray crystallography (Fe-N: $1.96 \AA$ (LS), Fe-N: $2.18 \AA$ (HS)) and Mössbauer (MB) spectroscopy, revealing the presence of a [1HS:2LS] configuration in the solid-state. Surprisingly, the presence or absence of solvent molecules in $\left[\mathrm{L}_{2}\left(\mathrm{HL}^{\mathrm{H}}\right)_{2} \mathrm{Fe}_{3}\right]\left[\mathrm{BF}_{4}\right]_{4}$ demonstrated a profound effect on the magnetic properties. In the presence of acetonitrile, the spin-state remains [2LS-1HS] from 2-380 K. However, removal of the solvent afforded an abrupt and hysteretic SCO at $355 \mathrm{~K}$. Although transient absorption X-ray crystallography (Prof. Dr. Simone Techert, DESY) were conducted on single crystals of 2-2a-c and 2-3, the results are not reported in this work.
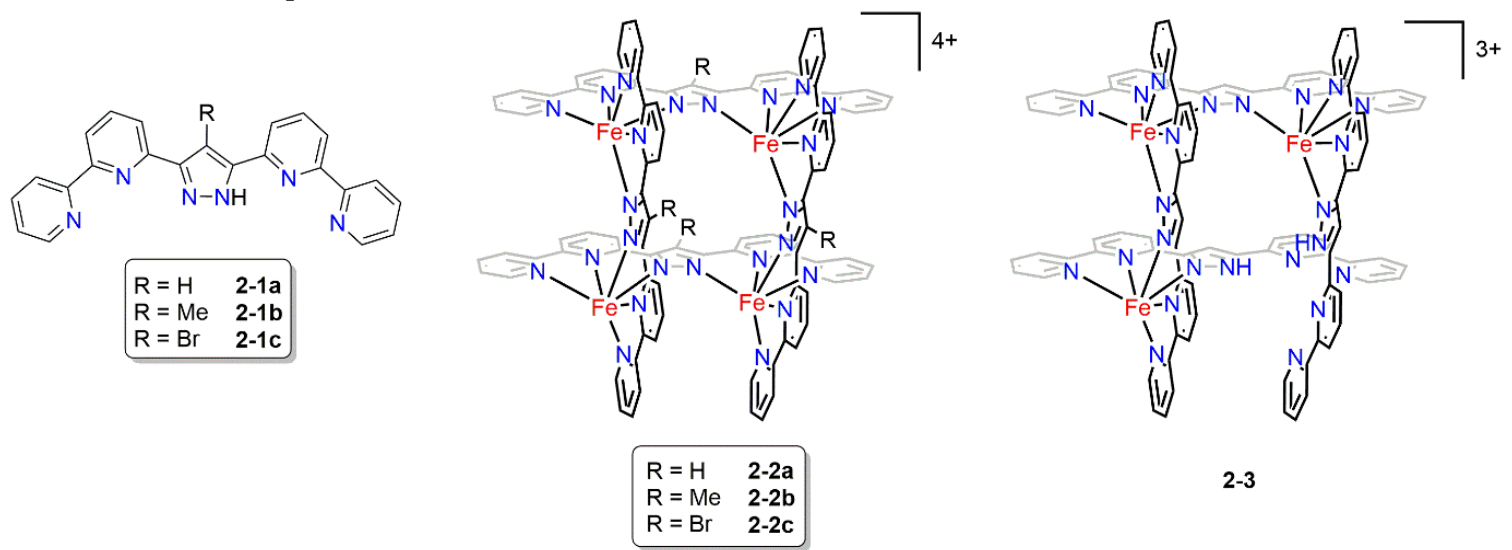

2-3

Chart 2.1. Schematic representation of the pyrazolate-bridged ligand 2-1a-c, $\left[\mathrm{L}_{4}{ }_{4} \mathrm{Fe}_{4}\right]^{4+}$ 2-2a-c and the trinuclear defect $\mathrm{Fe}_{3}$ grid 2-3.

The diversity of the magnetic properties and established cooperative nature of the Fe grids in the solid-state provides an avenue for scientists to investigate the structural dynamics of oligonuclear SCO complexes. However, prior to femtosecond transient absorption measurements, several things must be addressed. Firstly, since the transient absorption measurements are conducted in solution, the ground-state must also be determined in solution by variable temperature magnetic susceptibility measurements. Secondly, an optically excited state must be accessible from the ground-state. In this specific instrument setup (Prof. Dr. Dirk Schwarzer, Max Planck Institute), only a single-photon excitation can be employed (i.e. [3HS-1LS] $\rightarrow$ [4HS] or [2HS-2LS] $\rightarrow$ [3HS-1LS]). Consequently, only selected Fe 
grids with a [2HS-2LS] or [3HS-1LS] spin configuration were pursued for femtosecond transient absorption spectroscopy in solution at room temperature.

Although the brominated $\left[\mathrm{L}_{4} \mathrm{Fe}_{4}\right]\left[\mathrm{BF}_{4}\right]_{4}$ grid displayed a thermally stable [2HS-2LS] configuration from $30-250 \mathrm{~K}$ in the solid-state, magnetic measurements in MeCN revealed gradual SCO commencing at approximately $50 \mathrm{~K}$ (discussed in section 2.3.3). Similarly, the parent $\left[\mathrm{LH}_{4} \mathrm{Fe}_{4}\right]\left[\mathrm{BF}_{4}\right]_{4}$ grid displayed step-wise SCO in the solid-state, however, variable temperature magnetic susceptibility revealed a remarkable difference in solution, in which step-wise SCO was no longer present. Instead, gradual SCO was observed similar to $\left[\mathrm{LBr}_{4} \mathrm{Fe}_{4}\right]\left[\mathrm{BF}_{4}\right]_{4}$ in acetonitrile. Although incomplete gradual SCO was observed for both $\left[\mathrm{L}_{4} \mathrm{Fe}_{4}\right]\left[\mathrm{BF}_{4}\right]_{4}$ grids $(\mathrm{R}=\mathrm{H}, \mathrm{Br})$, the overall spin configuration at room temperature may be estimated using the $\chi_{\mathrm{M}} \mathrm{T}$ value at $295 \mathrm{~K}$. Finally, $\left[\mathrm{L}_{2}\left(\mathrm{HL}_{2}\right)_{2} \mathrm{Fe}_{3}\right]\left[\mathrm{BF}_{4}\right]_{4}$ is also of interest due to its intrinsic abrupt hysteretic SCO behaviour in the solid-state. Unfortunately, this effect vanishes in solution, and once again only gradual SCO is observed.

Although femtosecond IR/UV-vis transient absorption spectroscopy was conducted on all three grids in acetonitrile, analysis of the spectra was exceptionally challenging due to the onset of incomplete SCO below room temperature. Additionally, the large size of the Fe grids requires extensive theoretical calculations for vibrational band assignments as well as excited state calculations. Nevertheless, the magnetic measurements in solution on the parent $\left[\mathrm{LH}_{4} \mathrm{Fe}_{4}\right]\left[\mathrm{BF}_{4}\right]_{4}$ grid, the brominated $\left[\mathrm{LBr}_{4} \mathrm{Fe}_{4}\right]\left[\mathrm{BF}_{4}\right]_{4}$ grid, and the trinuclear defect $\mathrm{Fe}_{3}$ grid $\left[\mathrm{LH}_{2}\left(\mathrm{HL}_{2}\right)_{2} \mathrm{Fe}_{3}\right]\left[\mathrm{BF}_{4}\right]_{4}$ will be discussed followed by the preliminary transient absorption IR/UV-Vis spectra of all three Fe grids. However, prior to discussing these results, a basic overview of transient absorption spectroscopy will be introduced.

\section{2 - Pump-Probe Spectroscopy}

Information regarding spatial distribution as well as structural changes during optical excitations are often studied using relaxation dynamics. Especially for nanomaterials, in which the relaxation times following optical excitation typically fall into the femtosecond range, ultrafast transient absorption, or pump-probe spectroscopy has become a versatile technique used to investigate these materials in real time.101 In transient absorption spectroscopy, a sample is excited by irradiation with a pump pulse followed by a probe pulse with a time delay, $\Delta \mathrm{t}$ (Figure 2.2a). A difference in absorbance spectra $(\triangle A$ or $\triangle O D$ ) at a certain wavelength (pump pulse) between the excited state and ground state is obtained at different time delays affording a map of $\Delta A$ as a function of time, $t$. Using this, the life times of the excited states can be derived. 
(a)
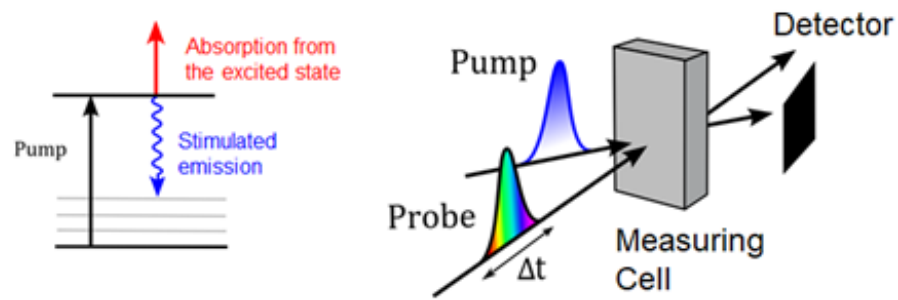

(b)

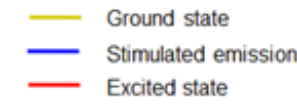

A

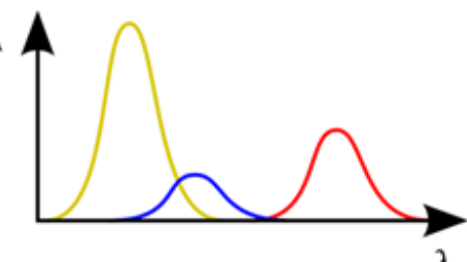

(c) $\Delta \mathrm{A}$

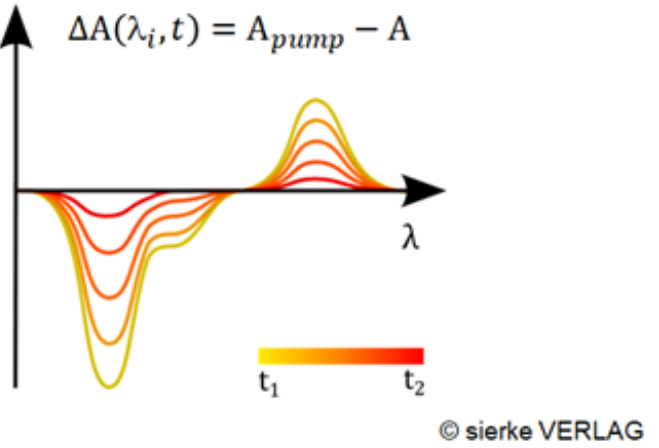

Figure 2.2. (a) Schematic representation of pump-probe spectroscopy. (b) Illustration of a typical absorbance spectrum of a sample at the ground-state (yellow), stokes-shifted stimulated emission (blue) and excited state (red). (c) The difference in absorbance $\Delta A$ of the excited and ground-state is plotted as a function of wavelength. ${ }^{102}$

In general, the changes in $\Delta A$ originate from several processes: ground-state bleaching, stimulated emission, excited state absorption, and product absorption. Ground-state bleaching refers to the excitation of a fraction of the molecules affording negative $\Delta A$ values corresponding to the depletion of the ground-state at that specific wavelength. For instance, prior to optical excitation, the ground-state displays a band at a certain wavelength, $\lambda$ (Figure $2.2 \mathrm{~b}$, yellow line). Following optical excitation with the pump pulse, a probe pulse at time delay $t_{1}$ results in a ground-state bleach $(-\Delta \mathrm{A})$ at the same wavelength $\lambda$ (Figure 2.2c). After a certain time, $\mathrm{t}_{2}, \Delta A$ gradually returns to zero, suggesting full conversion from the excited state to the ground state. In some cases, stimulated emission from the probe pulse may cause a photon from the excited state to relax to the ground-state affording negative $\Delta A$ values, which may be Stokes-shifted with respect to the ground-state bleach (Figure 2.2b, blue line). However, the Stokes shift may be extremely small such that it merges with the ground-state bleach and only one band is observed in the difference in absorbance spectrum $\Delta A .{ }^{101}$

In response to bleaching of the ground-state, an excited state absorption is generally observed at a different wavelength with a positive $\Delta A$ value (Figure 2.2c). From the example above, bleaching of the ground-state affords a new band at higher wavelengths, $\lambda$, corresponding to the new excited state species. Another possibility involves absorption of the sample, in which upon optical excitation, the molecule undergoes a reaction to form new species such as triplet states or isomerization processes. In these cases, a new long-lived species is formed affording new bands in the positive $\Delta A$ spectrum. 


\section{3 - Results}

\subsection{1 - Synthesis of the pyrazolate-bridge Ligand \& Homonuclear Fe Grids}

As mentioned previously, compounds 2-2a-c and 2-3 were used to study transient absorption X-ray crystallography. However, only $\left.\left[\mathrm{L}^{\mathrm{R} F e}\right]_{4}\right]\left[\mathrm{BF}_{4}\right]_{4}(\mathrm{R}=\mathrm{H}, \mathrm{Br})$ and the defect $\left[\mathrm{L}_{2}\left(\mathrm{HL}^{\mathrm{H}}\right)_{2} \mathrm{Fe}_{3}\right]\left[\mathrm{BF}_{4}\right]_{4}$ grid were used for transient absorption spectroscopy in solution. Therefore, only the synthesis of $\mathbf{H L}^{\mathbf{H}}$ and $\mathbf{H} \mathbf{L}^{\mathrm{Br}}$ will be discussed in the follow section. The parent ligand 2-1a $(\mathrm{R}=\mathrm{H})$ was synthesized according to literature procedures with minimal changes (Scheme 2.1). ${ }^{90}$ The synthesis commences with oxidation of commercially available 2,2'-bipyridine 2-4 with hydrogen peroxide to form the $\mathrm{N}$-oxide 2-5. Once activated, a nitrile group can be incorporated ortho to one of the nitrogen atoms on the pyridine ring using trimethylsilyl cyanide and benzoyl chloride affording a white powder 2-6, which was subsequently used for two separate precursors: the ester 2-7 and the ketone 2-8. For the ester 2-7, the cyano-precursor 2-6 undergoes methanolysis with sodium methoxide (generated in situ) in methanol, while acetylation of 2-6 to obtain 2-8 was achieved using methylmagnetisum bromide as a Grignard reagent. Condensation of the ester 2-7 and ketone 2-8 with sodium $t$-butoxide $\left(\mathrm{NaO} \mathrm{t}^{\mathrm{Bu}}\right.$ ) in dry 1,4-dioxane followed by acidic workup afforded the diketone 2-9. Lastly, reaction of the diketone 2-9 with hydrazine monohydrate afforded the desired pyrazolate-bridged ligand $\mathbf{2 - 1 a}\left(\mathbf{H L}^{\mathrm{H}}\right)$. In order to incorporate a bromide moiety onto the pyrazole backbone, elemental bromine was added to a solution of $\mathbf{H L}^{\mathbf{H}}$ in dichloromethane and sodium carbonate $\left(\mathrm{Na}_{2} \mathrm{CO}_{3}\right)$. The reaction was stirred at room temperature for 16 hours to give $\mathbf{H L}^{\mathrm{Br}}$ as a fine yellow powder $\mathbf{2 - 1 \mathbf { c }}{ }^{82}$

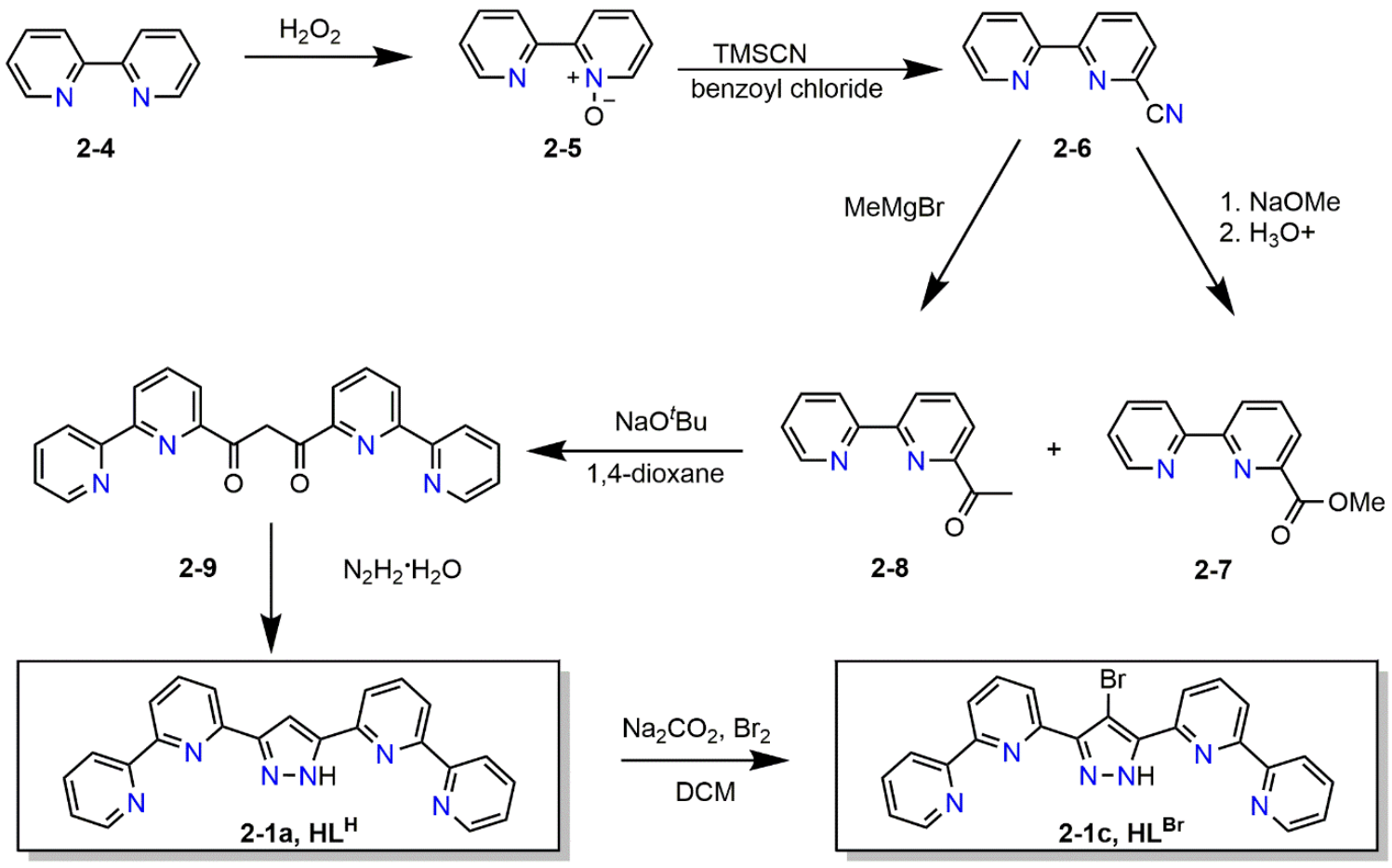

Scheme 2.1. Synthetic scheme for the ligands $H L^{H}$ 2-1a and $H L^{B r} \mathbf{2 - 1 c}$.

The tetranuclear $\left[\mathrm{L}_{4} \mathrm{Fe}_{4}\right]\left[\mathrm{BF}_{4}\right]_{4}(\mathrm{R}=\mathrm{H}, \mathrm{Me}, \mathrm{Br})$ grids were also synthesized according to literature procedures. ${ }^{36,82}$ Since $\mathbf{2 - 2 a - c}$ were synthesized following similar procedures, only the synthesis of the parent $\left[\mathrm{LH}_{4} \mathrm{Fe}_{4}\right]\left[\mathrm{BF}_{4}\right]_{4}$ grid will be discussed (Scheme 2.2). The parent ligand 2-1a was deprotonated with an excess of $\mathrm{NaO}^{t} \mathrm{Bu}$ in a degassed solution of MeCN:THF 
(1:1) followed by addition of stoichiometric amounts of $\mathrm{Fe}^{\mathrm{II}}\left(\mathrm{BF}_{4}\right)_{2}$. The reaction was stirred for 16 hours at room temperature, the product precipitated with diethyl ether and crystallized by slow diffusion of diethyl either into a solution of 2-2a in acetonitrile to give brown crystalline material.

For the synthesis of the defect $\mathrm{Fe}_{3}$ grid $\left[\mathrm{LH}_{2}\left(\mathrm{HL}^{\mathrm{H}}\right)_{2} \mathrm{Fe}_{3}\right]\left[\mathrm{BF}_{4}\right]_{4}$, stoichiometric amounts of base and $\mathrm{Fe}^{\mathrm{II}}$ salt were required to produce the $\mathrm{Fe}_{3}$ grid exclusively. ${ }^{100}$ To that end, a ratio of 4:3:2 of $\mathbf{H L}^{\mathbf{H}}, \mathrm{Fe}^{\mathrm{II}}\left(\mathrm{BF}_{4}\right)_{2}$, and $\mathrm{NaO}^{t} \mathrm{Bu}$ was employed in dry acetonitrile. Consequently, one corner of the grid remains protonated and devoid of a metal ion. The reaction was stirred at room temperature for 16 hours followed by crystallization by slow diffusion of diethyl ether into a solution of the product in acetonitrile to give $\left[\mathrm{LH}_{2}(\mathrm{HL})_{2} \mathrm{Fe}_{3}\right]\left[\mathrm{BF}_{4}\right]_{4}$ as dark red crystalline material.

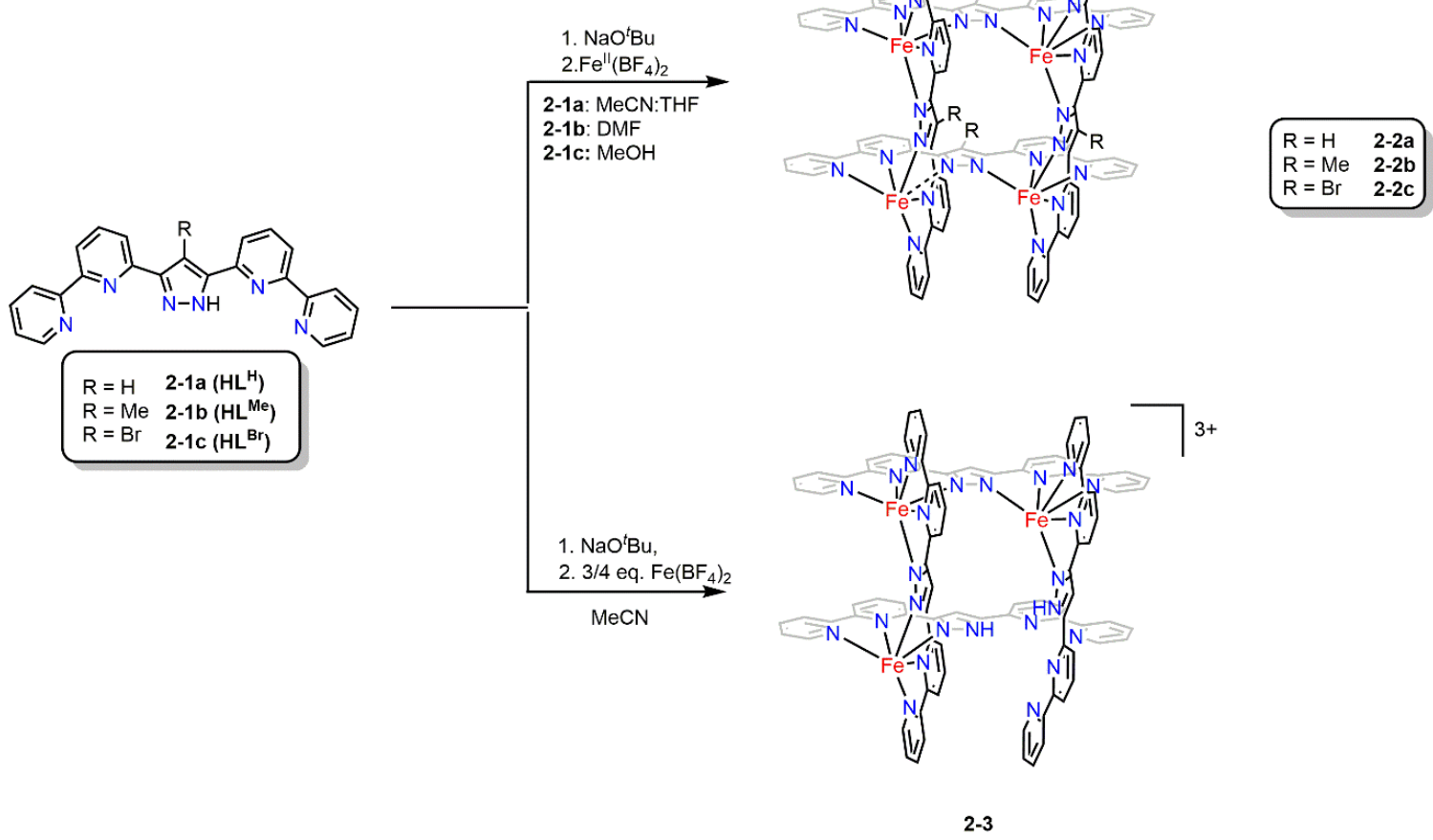

Scheme 2.2. Synthetic scheme for $\left[\mathrm{L}_{4} \mathrm{Fe}_{4}\right]\left[\mathrm{BF}_{4}\right]_{4}$ grids $(\mathrm{R}=\mathrm{H}, \mathrm{Me}, \mathrm{Br})$ and trinuclear defect $\mathrm{Fe}_{3}$ grid $\left[\mathrm{L}_{2}\left(\mathrm{HL}^{\mathrm{H}}\right)_{2} \mathrm{Fe}_{3}\right]\left[\mathrm{BF}_{4}\right]_{4}$.

\subsection{2 - Mössbauer Spectroscopy on Homonuclear Fe Grids}

Prior to the pump-probe IR/UV-vis measurements, the electronic structures of the homonuclear Fe grids were determined by Mössbauer (MB) spectroscopy in the solid-state and in frozen solution. All three Fe grids displayed minimal differences between the two measurements, suggesting negligible contributions from intermolecular interactions on the spin-state at $80 \mathrm{~K}$. Since the transient absorption measurements were conducted in solution, only the MB spectra in frozen solution are shown in Figure 2.3. All parameters are tabulated in Table 2.1 

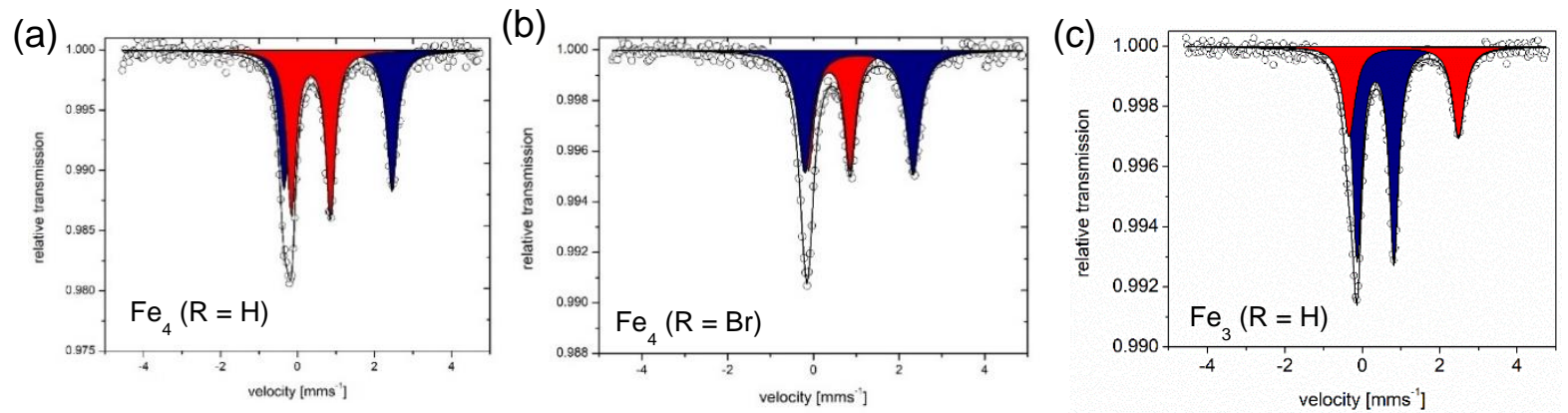

Figure 2.3. $\mathrm{MB}$ spectrum of (a) the parent $\left[\mathrm{LH}_{4} \mathrm{Fe}_{4}\right]\left[\mathrm{BF}_{4}\right]_{4}$ grid $(\mathrm{R}=\mathrm{H})$, brominated $\left[\mathrm{LBr}_{4} \mathrm{Fe}_{4}\right]\left[\mathrm{BF}_{4}\right]_{4}$ grid $(\mathrm{R}=\mathrm{Br})$ and $(\mathrm{c})\left[\mathrm{L}_{2}\left(\mathrm{HL}^{\mathrm{H}}\right)_{2} \mathrm{Fe}_{3}\right]\left[\mathrm{BF}_{4}\right]_{4}$ at $80 \mathrm{~K}$ in frozen acetonitrile.

Table 2.1. MB parameters for $\left[\mathrm{L}_{4} \mathrm{Fe}_{4}\right]\left[\mathrm{BF}_{4}\right]_{4}$, $\left[\mathrm{L}^{\mathrm{Br}}{ }_{4} \mathrm{Fe}_{4}\right]\left[\mathrm{BF}_{4}\right]_{4}$, and $\left[\mathrm{L}_{2}\left(\mathrm{HL}^{\mathrm{H}}\right)_{2} \mathrm{Fe}_{3}\right]\left[\mathrm{BF}_{4}\right]_{4}$.

\begin{tabular}{ccccc}
\hline Compound & Temperature $(\mathrm{K})$ & $\delta\left[\mathrm{mms}^{-1}\right]$ & $\Delta E_{\mathrm{Q}}\left[\mathrm{mms}^{-1}\right]$ & Area \\
\hline$\left[\mathrm{L}_{4} \mathrm{Fe}_{4}\right]\left[\mathrm{BF}_{4}\right]_{4}$ & \multirow{2}{*}{80} & 1.05 & 2.81 & 48 \\
& & 0.35 & 1.01 & 52 \\
{$\left[\mathrm{LBr}_{4} \mathrm{Fe}_{4}\right]\left[\mathrm{BF}_{4}\right]_{4}$} & \multirow{2}{*}{80} & 1.07 & 2.53 & 56 \\
& & 0.37 & 0.98 & 44 \\
{$\left[\mathrm{~L}_{2}(\mathrm{HL})_{2} \mathrm{Fe}_{3}\right]\left[\mathrm{BF}_{4}\right]_{4}$} & \multirow{2}{*}{80} & 1.07 & 2.83 & 36 \\
& & 0.34 & 0.95 & 64 \\
\hline
\end{tabular}

The Mössbauer spectrum of the parent $\left[\mathrm{LH}_{4} \mathrm{Fe}_{4}\right]\left[\mathrm{BF}_{4}\right]_{4}$ grid revealed two doublets with an isomer shift of $\delta=1.05 \mathrm{mms}^{-1}\left(\Delta E_{\mathrm{Q}}=2.81 \mathrm{mms}^{-1}\right)$ and $\delta=0.35 \mathrm{mms}^{-1}\left(\Delta E_{\mathrm{Q}}=1.01 \mathrm{mms}^{-1}\right)$ with a relative area of 48:52 (HS:LS) consistent with two HS-Fe ${ }^{\mathrm{II}}$ and two LS-Fe ${ }^{\mathrm{II}}$ ions, respectively. All parameters are consistent with those reported in the literature. ${ }^{36}$ Although the MB spectrum of the brominated $\left[\mathrm{LBr}_{4} \mathrm{Fe}_{4}\right]\left[\mathrm{BF}_{4}\right]_{4}$ grid revealed subtle differences between the solid-state and frozen solution MB spectra of the same sample, the ratio between the HS-FeII ions and LS-Fe"I ions varied significantly from one synthesis to another. For instance, although a ratio of 50:50 (HS-Fe ${ }^{I I}$ : LS-Fe ${ }^{I I}$ ) was reported, ${ }^{100}$ the relative area of the two quadrupole doublets for $\left[\mathrm{L}^{\mathrm{Br} F e} e_{4}\right]\left[\mathrm{BF}_{4}\right]_{4}$ can vary up to $70: 30$ (HS-Fe'I $\mathrm{LS}-\mathrm{Fe}^{\mathrm{II}}$ ) even with repeated crystallizations. For the purpose of the transient absorption measurements, crystalline material exhibiting a ratio of approximately 50:50 (HS:LS) was used.

Similar to the preceding $\mathrm{MB}$ spectra, the $\mathrm{MB}$ spectrum of the defect $\mathrm{Fe}_{3}$ grid $\left[\mathrm{L}_{2}\left(\mathrm{HL}^{\mathrm{H}}\right)_{2} \mathrm{Fe}_{3}\right]\left[\mathrm{BF}_{4}\right]_{4}$ displayed negligible differences in the solid-state and in frozen solution. Once again, two doublets were observed with an isomer shift of $\delta=1.07 \mathrm{mms}^{-1}\left(\Delta E_{\mathrm{Q}}=2.83\right.$ $\left.\mathrm{mms}^{-1}\right)$ and $\delta=0.34 \mathrm{mms}^{-1}\left(\Delta E_{\mathrm{Q}}=0.95 \mathrm{mms}^{-1}\right)$, consistent with HS-Fe ${ }^{\mathrm{II}}$ and LS-Fell, respectively. The relative area of 36:64 (HS:LS) suggests a spin configuration of [1HS:2LS] at $80 \mathrm{~K}$, consistent with the reported molecular structure. 


\subsection{3 - Magnetic Measurements in Solution}

Since the transient absorption measurements were conducted at room temperature in solution, the ground state spin state pattern was determined by variable temperature magnetic susceptibility measurements in solution. As mentioned in the introduction, the parent $\left[\mathrm{LH}_{4} \mathrm{Fe}_{4}\right]\left[\mathrm{BF}_{4}\right]_{4}$ grid exhibited step-wise SCO in the solid-state, while the brominated $\left[\mathrm{LBr}_{4} \mathrm{Fe}_{4}\right]\left[\mathrm{BF}_{4}\right]_{4}$ grid displayed a magnetic susceptibility value of $\chi_{\mathrm{M}} \mathrm{T}=7.44 \mathrm{~cm}^{3} \mathrm{~K} \mathrm{~mol}^{-1}$ between $50 \mathrm{~K}-250 \mathrm{~K}$ in the solid-state, corresponding to a [2HS-2LS] configuration. ${ }^{22}$ Above $250 \mathrm{~K}$, a small increase of $\chi_{\mathrm{M}} \mathrm{T}$ was observed, signifying the onset of gradual SCO behaviour. Below $50 \mathrm{~K}$, a steep decrease in the $\chi_{\mathrm{M}} \mathrm{T}$ values originate from the ZFS of the $\mathrm{HS}^{-\mathrm{Fe}^{\mathrm{II}}}(S=2)$ ions. In comparison, variable temperature magnetic susceptibility measurements on the brominated $\left[\mathrm{LBr}_{4} \mathrm{Fe}_{4}\right]\left[\mathrm{BF}_{4}\right]_{4}$ grid in acetonitrile also afforded gradual SCO. However, the SCO temperature is much lower commencing at $50 \mathrm{~K}$. Full conversion to the [3HS-1LS] configuration occurs at approximately $330 \mathrm{~K}$. Similar to the solid-state measurement, a significant decrease in $\chi_{\mathrm{M}} \mathrm{T}$ is observed below $50 \mathrm{~K}$ as a result of ZFS for HS-Fell ions (Figure 2.4a, orange). Since this phenomenon occurs in all the Fe grids, this low temperature feature will not be mentioned in each case. Using the magnetic susceptibility value at $295 \mathrm{~K}$ $\left(\chi_{M} \mathrm{~T}=8.86 \mathrm{~cm}^{3} \mathrm{~mol}^{-1} \mathrm{~K}\right)$ along with $\chi_{\mathrm{M}} \mathrm{T}(3 \mathrm{HS}-1 \mathrm{LS})=9.3 \mathrm{~cm}^{3} \mathrm{~mol}^{-1} \mathrm{~K}(100 \% 3 \mathrm{HS}-1 \mathrm{LS})$ and $\chi_{\mathrm{M}} \mathrm{T}(2 \mathrm{HS}-2 \mathrm{LS})=6.2 \mathrm{~cm}^{3} \mathrm{~mol}^{-1} \mathrm{~K}(0 \% 3 \mathrm{HS}-1 \mathrm{LS})$, a ratio of 86:14 (3HS-1LS : 2HS-2LS) was determined. Therefore, the overall HS:LS ratio for $\left[\mathrm{LBr}_{4} \mathrm{Fe}_{4}\right]\left[\mathrm{BF}_{4}\right]_{4}$ is $72: 28 \pm 5 \%$ (HS:LS) at $295 \mathrm{~K}$.
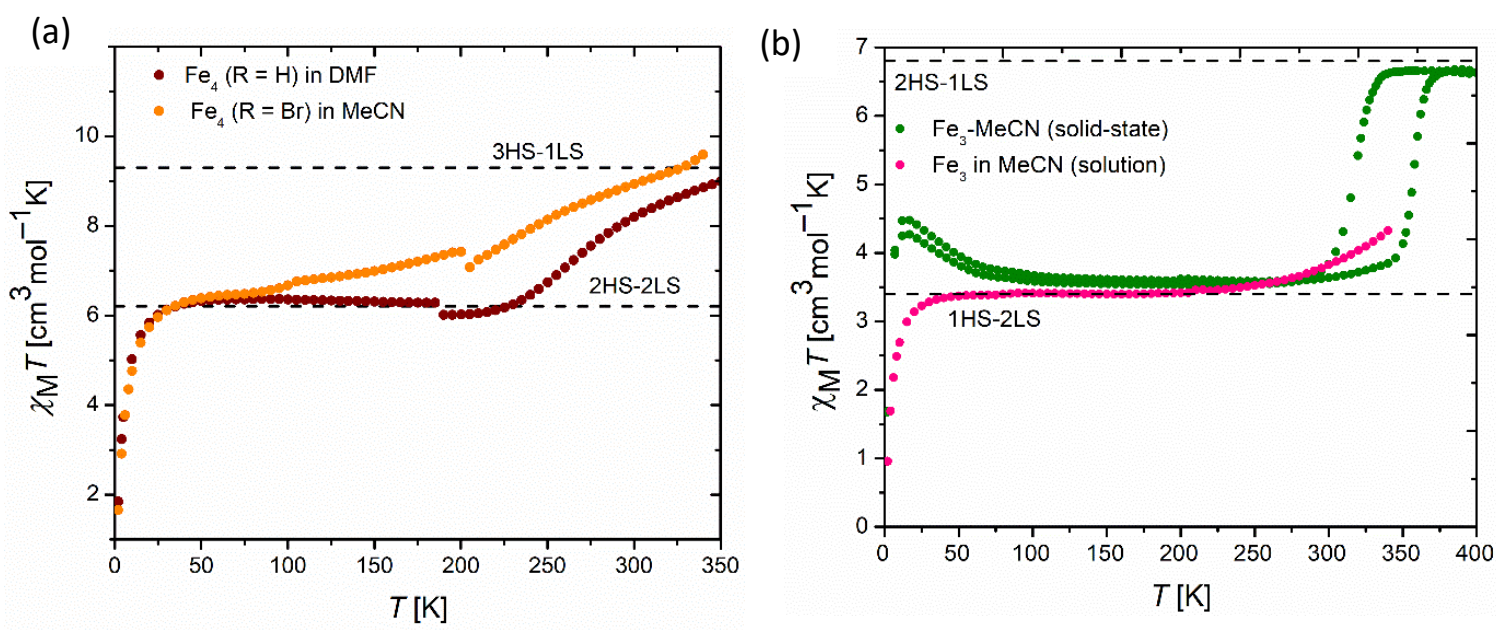

Figure 2.4. (a) Variable magnetic susceptibility of the parent $\left[\mathrm{LH}_{4} \mathrm{Fe}_{4}\right]\left[\mathrm{BF}_{4}\right]_{4}$ grid in DMF and brominated $\left[\mathrm{LBr}_{4} \mathrm{Fe}_{4}\right]\left[\mathrm{BF}_{4}\right]_{4}$ grid in MeCN. (b) Variable magnetic susceptibility of the defect $\mathrm{Fe}_{3}$ grid 2-3 in the solid-state and in solution.

In the case of the parent $\left[\mathrm{LH}_{4} \mathrm{Fe}_{4}\right]\left[\mathrm{BF}_{4}\right]_{4}$ grid, a similar gradual $\mathrm{SCO}$ behaviour was observed in DMF. The measurements were also conducted in acetonitrile illustrating comparable results, however, only the magnetic measurements in DMF are shown due to solubility reasons (Figure 2.4a, brown). Nevertheless, a similar trend of gradual SCO is observed, although at much higher temperatures $(\sim 220 \mathrm{~K})$. At $350 \mathrm{~K}$, the $\chi_{\mathrm{M}} \mathrm{T}$ approaches full conversion to the [3HS-1LS] state, however, full conversion was not obtained due to the temperature limit of the experiment. Following procedures similar to $\left[\mathrm{LBr}_{4} \mathrm{Fe}_{4}\right]\left[\mathrm{BF}_{4}\right]_{4}$, the ground state for $\left[\mathrm{LH}_{4} \mathrm{Fe}_{4}\right]\left[\mathrm{BF}_{4}\right]_{4}$ at $295 \quad \mathrm{~K} \quad\left(\chi_{\mathrm{M}} \mathrm{T}=8.09 \mathrm{~cm}^{3} \mathrm{~mol}^{-1} \mathrm{~K}^{-1}\right)$ consisting of a ratio of 61:39 (3HS-1LS : 2HS-2LS) affords an overall ratio of 72:28 HS:LS at $295 \mathrm{~K}$. 
The final compound studied by magnetic susceptibility measurements in solution was the defect $\left[\mathrm{LH}_{2}\left(\mathrm{HL}^{\mathrm{H}}\right)_{2} \mathrm{Fe}_{3}\right]\left[\mathrm{BF}_{4}\right]_{4}$ grid. As mentioned previously, SCO for this complex is highly dependent on the solvation of the molecule. In the presence of acetonitrile molecules in the crystal lattice, the $\mathrm{Fe}_{3}$ grid retains a $\chi_{\mathrm{M}} \mathrm{T}$ value of $3.1 \mathrm{~cm}^{3} \mathrm{~K} \mathrm{~mol}^{-1}$ from $200-400 \mathrm{~K}$, consistent with a [1HS-2LS] configuration. ${ }^{100}$ However, removal of solvents in the crystal lattice afforded an abrupt and hysteretic SCO at $355 \mathrm{~K}$ with a $\chi_{\mathrm{M}} \mathrm{T}$ value of $6.34 \mathrm{~cm}^{3} \mathrm{~K} \mathrm{~mol}^{-1}$, consistent with SCO of one iron atom to form the [2HS-1LS] state (Figure 2.4b). Unsurprisingly, the magnetic properties of $\left[\mathrm{LH}_{2}\left(\mathrm{HL}^{\mathrm{H}}\right)_{2} \mathrm{Fe}_{3}\right]\left[\mathrm{BF}_{4}\right]_{4}$ in acetonitrile reflect the [1HS-2LS] configuration from $50-250 \mathrm{~K}$ similar to the solvated congener and the onset of gradual SCO commences above $250 \mathrm{~K}$. Once again, the overall spin configuration at room temperature was determined by using the $\chi_{\mathrm{M}} \mathrm{T}$ value at $295 \mathrm{~K}\left(3.78 \mathrm{~cm}^{3} \mathrm{~mol}^{-1} \mathrm{~K}\right)$ affording a ratio of $88: 12$ (1HS:2LS : 2HS-1LS) for $\left[\mathrm{LH}_{2}(\mathrm{HL})_{2} \mathrm{Fe}_{3}\right]\left[\mathrm{BF}_{4}\right]_{4}$, which translates into a ratio of $37: 63 \pm 5 \%$ of $\mathrm{HS}: \mathrm{LS}$ at $295 \mathrm{~K}$.

\subsection{4 - Variable Temperature UV-Vis Spectroscopy}

In addition to variable temperature magnetic susceptibility measurements, optical spectroscopy is also a well-established technique used to monitor SCO complexes due to the vibrant colour changes exhibited by numerous Fe ${ }^{\mathrm{II}}$ complexes. ${ }^{19,103,104}$ Although only gradual SCO was observed for the Fe grids in solution, variable temperature UV-vis spectroscopy of 2-2a,c and 2-3 in MeCN was conducted to determine if any significant changes occur between $-25^{\circ} \mathrm{C}$ to $35^{\circ} \mathrm{C}$ (limit of the femtosecond IR/UV-vis pump-probe instrument). Since the UVvis spectra of all the Fe grids displayed little differences, only the variable temperature UVvis spectra of the brominated $\left[\mathrm{LBr}_{4} \mathrm{Fe}_{4}\right]\left[\mathrm{BF}_{4}\right]_{4}$ grid are shown (Figure 2.5).

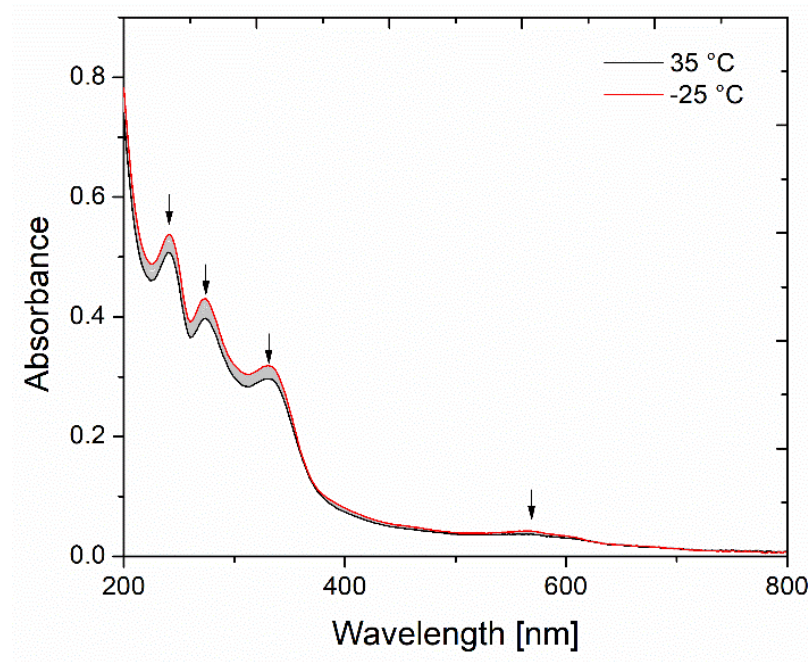

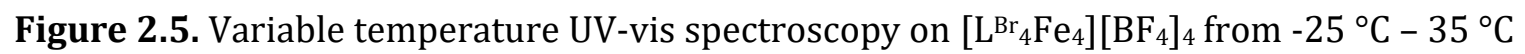
in $\mathrm{MeCN}$. 
At $35^{\circ} \mathrm{C}$, three intense bands at $235 \mathrm{~nm}, 265 \mathrm{~nm}$, and $310 \mathrm{~nm}$ were observed corresponding to the ligand $\pi-\pi^{*}$ transition. $36,82,100$ The weak broad band at $549 \mathrm{~nm}$ is assigned to a MLCT transition. Upon lowering the temperature, a small decrease in the absorbance of all bands was observed. Since only subtle changes were observed in the variable temperature UV-Vis spectra, transient absorption spectroscopy was only conducted at room temperature.

\subsection{5 - Transient Absorption Mid IR and UV-vis Pump-Probe Spectroscopy}

In order to investigate the electronic structure of the optically excited states, pump probe IR and UV-vis spectroscopy were conducted in acetonitrile at room temperature. Unlike the simple schematic spectra depicted in the introduction, the vibrational bands in the pumpprobe IR measurements of the $\mathrm{Fe}_{4}$ grids are more challenging to decipher since the bands cannot be distinctly assigned to a specific group of the molecule. Nevertheless, optical excitation at $400 \mathrm{~nm}$ on $\left[\mathrm{LH}_{4} \mathrm{Fe}_{4}\right]\left[\mathrm{BF}_{4}\right]_{4}$ revealed an immediate bleaching of the ground state at $1610 \mathrm{~cm}^{-1}$ and the rise of a new band at $1595 \mathrm{~cm}^{-1}$ (Figure 2.6a). After 270 picoseconds, this band is shifted to slightly higher wavenumbers and with a smaller intensity. The signals at $1440 \mathrm{~cm}^{-1}$ and $1565 \mathrm{~cm}^{-1}$ appear relatively unaffected during the optical excitation, signifying these bands are present in the ground-state as well as in the excited state species. Surprisingly, the signal at $1462 \mathrm{~cm}^{-1}$ displayed an immediate bleaching of the ground-state, but the long-lived species (yellow band) has the exact same wavenumber as the ground-state, suggesting the excited state and ground state may not be subjected to large structural deviations. Using the change in optical density at five different frequencies $(1446,1462,1557$, 1565 , and $1610 \mathrm{~cm}^{-1}$ ), the curve was modelled following a biexponential function affording two time constants, $\tau_{1}=15.4 \mathrm{ps}$ and a long lived species with a lifetime of $\tau_{2}>1000 \mathrm{ps}$.

(a)

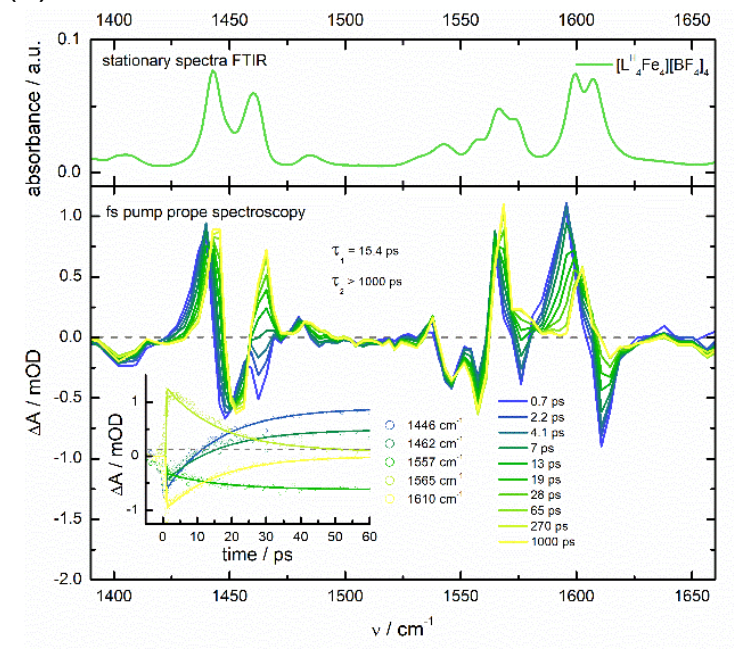

(b)

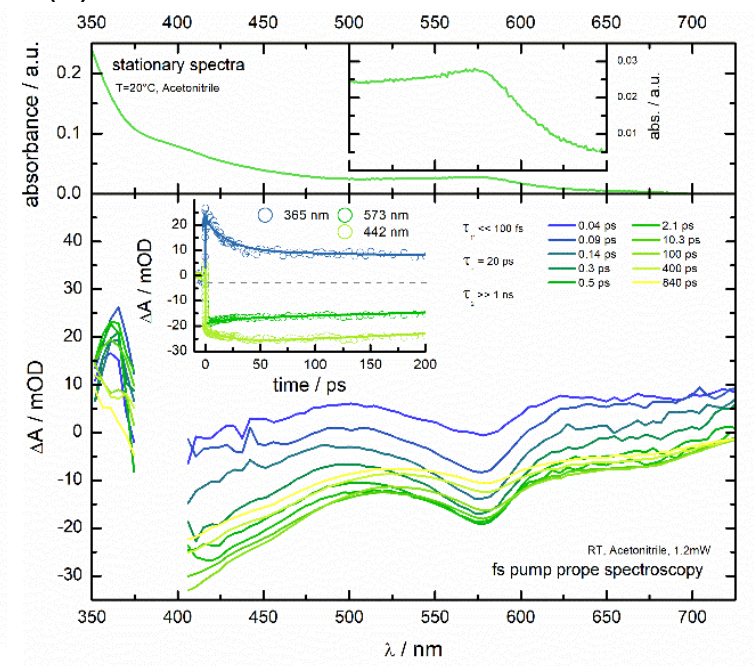

Figure 2.6. (a) Femtosecond IR transient absorption and (b) UV-vis spectroscopy on the parent $\left[\mathrm{LH}_{4} \mathrm{Fe}_{4}\right]\left[\mathrm{BF}_{4}\right]_{4}$ grid in acetonitrile at room temperature.

The time constants of the optically excited states were also determined using pump-probe UV-vis spectroscopy (Figure 2.6b). Once again, the sample was excited at $400 \mathrm{~nm}$ revealing a bleaching of the MLCT band at $573 \mathrm{~nm}$. The presence of additional bands were not observed between $350-750 \mathrm{~nm}$. Using the change in optical density at three different wavelengths $(365,573$, and $442 \mathrm{~nm})$, the data was fitted using a triexponential function affording three different life times, $\tau_{1^{\prime}}<<100 \mathrm{fs}, \tau_{1}=20 \mathrm{ps}$, and $\tau_{2}>1 \mathrm{~ns}$. Due to the higher sensitivity of pump- 
probe UV-vis spectroscopy, a short-lived species in the femtosecond range was also detected. Nevertheless, the relaxation times obtained from both spectroscopic techniques (IR and UVVis) are comparable. Unfortunately, the identity of the excited species cannot be determined without the help of theoretical calculations. All relaxation times from the pump-probe IR/UVvis spectroscopy are tabulated in Table 2.2.

Table 2.2. Summary of the life times of the excited states of the Fe grids.

\begin{tabular}{cccccc}
\hline Complex & \multicolumn{2}{c}{$\begin{array}{l}\text { IR pump-probe } \\
\text { measurements }\end{array}$} & \multicolumn{2}{c}{ UV-vis pump-probe measurements } \\
\hline & $\tau_{1}[\mathrm{ps}]$ & $\tau_{2}[\mathrm{ps}]$ & $\tau_{1^{\prime}}[\mathrm{fs}]$ & $\tau_{1}[\mathrm{ps}]$ & $\tau_{2}[\mathrm{~ns}]$ \\
\hline $\mathbf{2 - 2 a}$ & 15.4 & $>1000$ & $<<100$ & 20 & $>>1$ \\
$\mathbf{2 - 2 c}$ & 12.6 & $>1000$ & $<<100$ & 19 & 0.6 \\
$\mathbf{2 - 3}$ & - & - & $<<100$ & 19 & 2.5 \\
\hline
\end{tabular}

The effect of the bromo-substituent on the relaxation times of the excited states of the $\left[\mathrm{LBr}_{4} \mathrm{Fe}_{4}\right]\left[\mathrm{BF}_{4}\right]_{4}$ grid was investigated using pump-probe IR spectroscopy. With the presence of the bromide moiety on the pyrazole backbone, an additional band at $1518 \mathrm{~cm}^{-1}$ was observed in the IR spectrum. Optical excitation at $400 \mathrm{~nm}$ afforded a ground-state bleach at this band and a new absorption band at marginally lower wavenumbers at $1511 \mathrm{~cm}^{-1}$ (Figure 2.7a). Similar to the parent $\left[\mathrm{LH}_{4} \mathrm{Fe}_{4}\right]\left[\mathrm{BF}_{4}\right]_{4}$ grid, several new absorption bands were observed similar wavelengths as the ground-state bleach, suggesting minimal structural changes after optical excitation. The change in optical density at six different wavelengths were fitted with a biexponential function affording a short-lived species with a life time of $\tau_{1}=12.6 \mathrm{ps}$ and a long lived species $\left(\tau_{2}>1000 \mathrm{ps}\right)$. The time constants of the excited states were further confirmed with pump-probe UV-vis spectroscopy at $400 \mathrm{~nm}$, in which a ground-state bleach was once again observed at $573 \mathrm{~nm}$. The change in optical density at three wavelengths $(365$, 618, and $718 \mathrm{~nm}$ ) was fitted with a triexponential function affording time constants of $\tau_{1^{\prime}}<<100 \mathrm{fs}, \tau_{1}=19 \mathrm{~s}$, and $\tau_{2}=600 \mathrm{ps}$ (Figure $2.7 \mathrm{~b}$ ). The life time of all transient species are reminiscent to the parent $\left[\mathrm{LH}_{4} \mathrm{Fe}_{4}\right]\left[\mathrm{BF}_{4}\right]_{4}$ grid, suggesting the bromo-substituent has little effect on the relaxation dynamics of the excited states. 
(a)

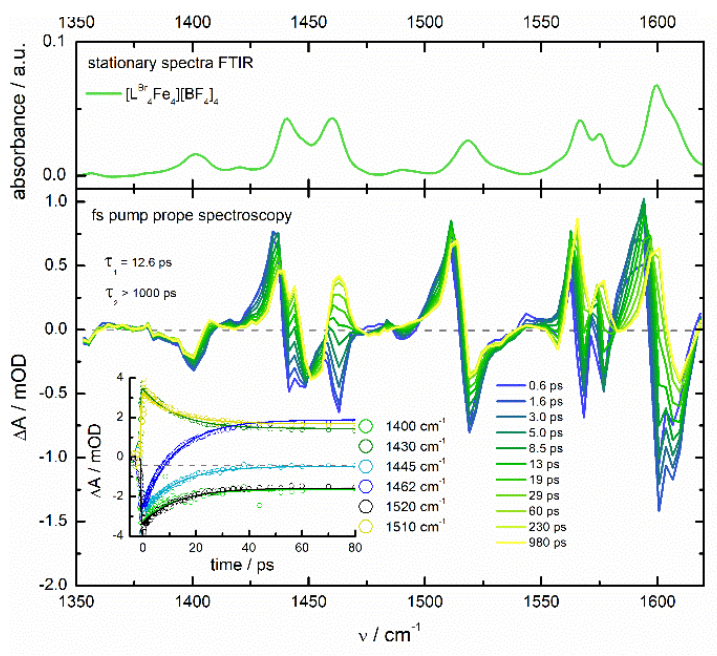

(b)

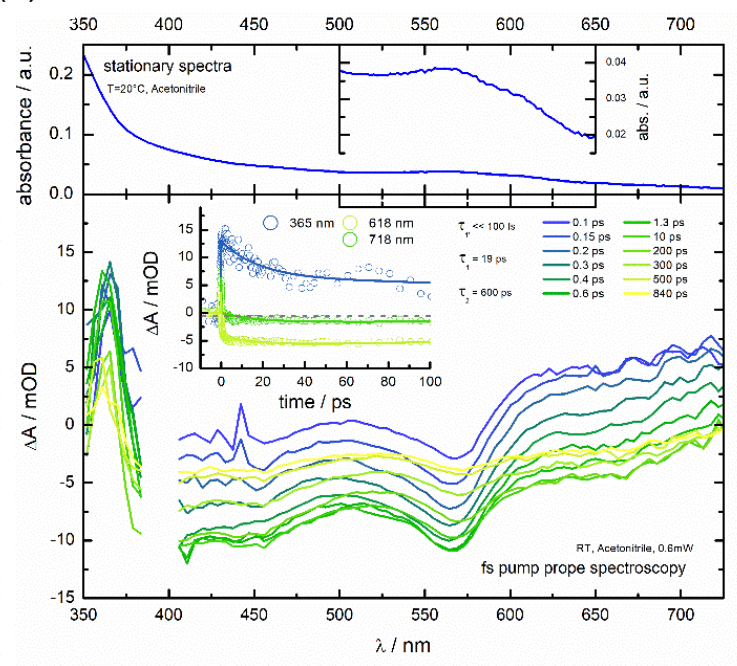

Figure 2.7. (a) Femtosecond IR and (b) UV-vis transient absorption spectroscopy on the brominated $\left[\mathrm{LBr}_{4} \mathrm{Fe}_{4}\right]\left[\mathrm{BF}_{4}\right]_{4}$ grid in acetonitrile at room temperature.

The last compound investigated using pump-probe spectroscopy was the defect $\left[\mathrm{L}_{2}\left(\mathrm{HL}^{\mathrm{H}}\right)_{2} \mathrm{Fe}_{3}\right]\left[\mathrm{BF}_{4}\right]_{4}$ grid. Although transient absorption IR spectroscopy was not obtained at this time, pump-probe UV-vis spectroscopy was conducted in acetonitrile revealing a groundstate bleach at $555 \mathrm{~nm}$ (Figure 2.8). However, new adsorption bands were not observed between $350-750 \mathrm{~nm}$. The change in optical density at $422 \mathrm{~nm}$ and $360 \mathrm{~nm}$ were fitted with a triexponential function affording three time constants, $\tau_{1^{\prime}}<<100 \mathrm{fs}, \tau_{1}=19 \mathrm{ps}$ and $\tau_{2}=2.5$ ns, corresponding to a short-lived species followed by a transient species in the picosecond range and finally a long-lived species. Once again, without theoretical calculations, definitive assignment of the transient species cannot be deduced. Nevertheless, based on the pumpprobe IR and UV-vis spectroscopy measurements of the three Fe grids, the relaxation cascade can be summarized as follows: optical excitation affords a short-lived species in the sub-100 femtosecond range followed by a transient species with a life time between 15-20 ps. This state further relaxes into a long-lived species. While a variety of hypotheses can be made, further experiments and theoretical calculations must be done to definitively elucidate the mechanistic pathway. 


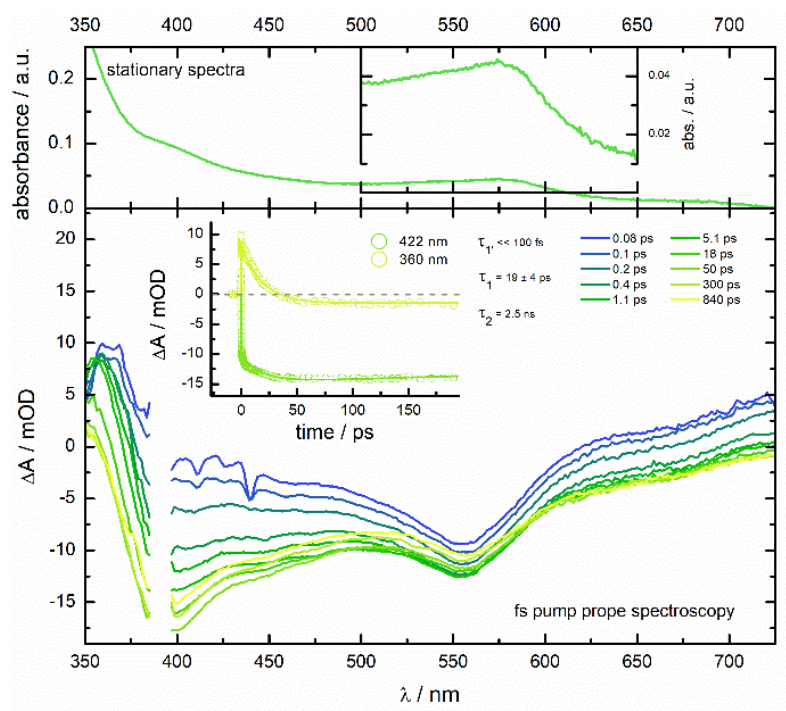

Figure 2.8. Femtosecond UV-vis transient absorption spectroscopy on $\left[\mathrm{L}_{2}\left(\mathrm{HL}^{\mathrm{H}}\right)_{2} \mathrm{Fe}_{3}\right]\left[\mathrm{BF}_{4}\right]_{4}$ in acetonitrile at room temperature.

\section{4 - Summary \& Conclusion}

In summary, the electronic structures of the homonuclear Fe grids in solution were determined by Mössbauer spectroscopy and variable temperature magnetic susceptibility measurements. The spin configuration of the Fe grids was confirmed by Mössbauer spectroscopy in frozen acetonitrile at $80 \mathrm{~K}$, revealing little differences compared to their solid-state counterparts. Variable temperature magnetic susceptibility measurements in solution revealed gradual SCO between $50 \mathrm{~K}-250 \mathrm{~K}$ for all Fe grids, differing substantially from the solid-state measurements. While in principle the Fe grids are ideal candidates for studying the nature of the optical excitation and the structural changes accompanied by optical excitation, analysis of the pump-probe spectra is rather challenging for several reasons. Firstly, SCO occurs well below room temperature and thus precise determination of the ground-state at $295 \mathrm{~K}$ has a large margin of error $( \pm 5 \%)$. Secondly, the Fe grids do not exhibit any signature bands in the IR spectrum which can be monitored (i.e. distinctive new bands in the excited-state absorption spectrum). Thirdly, due to the large size of the Fe grids, theoretical calculations for the vibrational assignments as well as for elucidating the electronic and magnetic structures of potential transient species remains a challenge. Nevertheless, based on the preliminary transient absorption spectroscopy on the Fe grids, the family of Fe grids behave quite similarly despite the different substitution on the pyrazole backbone. While a schematic reaction pathway can be deduced by the relaxation times, the exact nature of the transient species will require detailed theoretical calculations. 


\section{Chapter 3 - Heterometallic $\mathrm{Ru}_{2} \mathrm{Co}_{2}[2$ x 2] Grid with Localized Single Molecule Magnet Behaviour}

\section{1 - Introduction}

In the preceding chapters, a family of pyrazolate-bridged $\mathrm{Fe}_{4}$ grids developed by the Meyer group were introduced and their unique magnetic and physical properties including ferromagnetic and antiferromagnetic coupling, multiple stable redox states, and cooperative spin crossover. ${ }^{36,82,91}$ The latter was heavily investigated for the homonuclear $\mathrm{Fe}_{4}$ grid as well as the so-called defect $\mathrm{Fe}_{3}$ grid ${ }^{100}$ (where only three metal centers are assembled in a grid-like array) by time-resolved UV-vis pump-broadband mid-IR probe spectroscopy, in hopes of understanding the nature of the optical excitations. In order to complement these studies, new heterometallic [2 $\mathrm{x} 2$ ] grids were pursued, where the presence of two different metal centers holds potential for more interesting chemical and physical properties.

To date, numerous homonuclear grids have been synthesized, however, heterometallic grids are less frequently reported. The synthetic challenges associated with heterometallic [2 x 2] grids arise from the need for rigid and planar ligands with well-defined binding pockets for different metal ions to prevent scrambling of the metal centers. While a variety of heteronuclear supramolecular structures have been developed using this method, ${ }^{73,105-107}$ metals with similar coordination geometries (i.e. octahedral) cannot be employed as a result. Consequently, a complementary method pioneered by Lehn utilizes the kinetic stability of the metal ions in a step-wise manner to develop heterometallic [2 2 2] grids. ${ }^{87,108}$ In order for this to be effective, the first metal ion introduced must be kinetically inert compared to the second metal ion to prevent scrambling of the metal centers.

While there has been considerable progress since the first heterometallic grid, the properties of [ $\mathrm{n} \times \mathrm{n}$ ] grids using $3 \mathrm{~d}$ transition metals in the field of molecular magnetism, in particular single molecule magnets (SMMs), 38 tend to be limited, with some notable exceptions.66,81 The challenge with $3 \mathrm{~d}$ transition metals lies in mediating the antiferromagnetic coupling between the metal centers, where strong intramolecular magnetic interactions typically lead to a diamagnetic ground state $S_{T}=0.90,109$ However, in some cases, insertion of large bulky substituents to enforce large separations between the metal ions has effectively prevented strong intermolecular interactions of the metal ions leading to single ion magnet (SIM) behaviour. One particular example involves insertion of a $\gamma$-cyclodextrin subunit to implement large Co …Co separations $(\sim 11 \AA)$ in a heterometallic dodanuclear sandwich-type complex, $\left[\mathrm{Co}_{4} \mathrm{Li}_{8}\right]$, where the magnetically isolated $\mathrm{Co}^{\text {II }}$ ions displayed a positive zero-field splitting (ZFS) parameter and field-induced slow magnetic relaxation typical of single ion magnets (SIM). ${ }^{110}$

More recently, the Meyer group introduced the usage of inert diamagnetic centers in a mixed valent $\mathrm{Co}_{2} \mathrm{Co}_{2} \mathrm{III}_{2}\left[\begin{array}{lll}2 & \mathrm{x} & 2\end{array}\right]$ grid to prevent intermolecular interactions between the two paramagnetic cobalt centers.81 Prior to oxidation, the homovalent $\mathrm{Co}^{{ }^{I I}} 4$ grids are antiferromagnetically coupled affording a diamagnetic ground state $S_{T}=0$. However, upon oxidation to the mixed valent $\mathrm{Co}_{2} \mathrm{Co}_{2} \mathrm{CoI}_{2}$ grid, two diagonally positioned high-spin Co ${ }^{\mathrm{II}}$ ions are

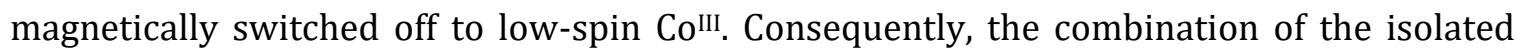
high-spin $\mathrm{Co}^{\mathrm{II}}$ ions and the highly distorted octahedral environment afforded single molecule magnet behaviour exclusively in the mixed valent [ $2 \times 2$ ] $\mathrm{Co}_{2} \mathrm{II}_{2} \mathrm{CoII}_{2}$ grid, but not in the homovalent $\mathrm{Co}{ }_{4} 4$ grid. 
Following a similar strategy, a new heterometallic $\left[\mathrm{L}_{4} \mathrm{Ru}_{2} \mathrm{Co}_{2}\right]\left[\mathrm{BF}_{4}\right]_{4}$ grid was synthesized using the known compartmental pyrazolate-bridged ligand. ${ }^{90}$ Ruthenium ions were selected based on their kinetic inertness, multiple stable redox states, and potential as a diamagnetic chemical spacer to prevent antiferromagnetic coupling between the other metal ions. At the same time, cobalt ions were chosen for their intrinsic large magnetic anisotropy that may lead to SMM behaviour, as observed in numerous other cobalt complexes. ${ }^{58}$

Herein, we report the synthesis of a kinetically stable ruthenium "corner complex", which selectively enforces an anti-arrangement of the four metal ions, such that the ruthenium and cobalt ions are situated at opposite corners of the [2 $\mathrm{x} 2]$ grid. Consequently, the magnetically active HS-CoII spins are, once again, isolated in highly distorted environments giving rise to SMM behaviour. Furthermore, the redox properties and characterization of the doubly oxidized $\left[\mathrm{LH}_{4} \mathrm{Ru}_{2} \mathrm{Co}_{2}\right]\left[\mathrm{BF}_{4}\right]_{6}$ grid will also be discussed in the following sections. The results of this chapter have been published and thus some parts are adapted from manuscript.111

\section{2 - Ruthenium "Corner Complex"}

\subsection{1 - Synthesis of the $\left[\mathrm{HL}_{2} \mathrm{H}_{2} \mathrm{Ru}\right]^{2+}$ "Corner Complex"}

As mentioned in the introduction, prior to the self-assembly of the $[2 \times 2]\left[\mathrm{LH}_{4} \mathrm{Ru}_{2} \mathrm{Co}_{2}\right]\left[\mathrm{BF}_{4}\right]_{4}$ grid, a kinetically inert $\mathrm{Ru}^{\mathrm{II}}$ "corner complex", $\left[\mathrm{HL}_{2} \mathrm{Ru}{ }^{2+}\right.$ with the previously reported pyrazolate-bridged ligand, $\mathbf{H L}^{\mathbf{H}}$, must be synthesized. Although both $\left[\mathrm{HL}_{2} \mathrm{H}_{2} \mathrm{Ru}\right]\left[\mathrm{BF}_{4}\right]_{2} \mathbf{3 - 1 a}$ and $\left[\mathrm{HL}_{2} \mathrm{Ru}\right][\mathrm{OTf}]_{2}$ 3-1b were synthesized, only $\left[\mathrm{HL}_{2} \mathrm{Ru}\right]\left[\mathrm{BF}_{4}\right]_{2}$ was used for the subsequent synthesis of $\left[\mathrm{LH}_{4} \mathrm{Ru}_{2} \mathrm{Co}_{2}\right]\left[\mathrm{BF}_{4}\right]_{4}$. The synthesis commences by combining an excess of the pyrazolate-bridged ligand, $\mathbf{H L}^{\mathbf{H}}$, with $\mathrm{Ru}(\mathrm{DMSO})_{4} \mathrm{Cl}_{2}$ in a degassed solution of acetone:ethanol (1:1), as shown in Scheme 3.1. The reaction was heated at reflux for 16 hours followed by precipitation and crystallization by slow diffusion of diethyl ether into a solution of 3-1 in acetonitrile to give red crystalline material.
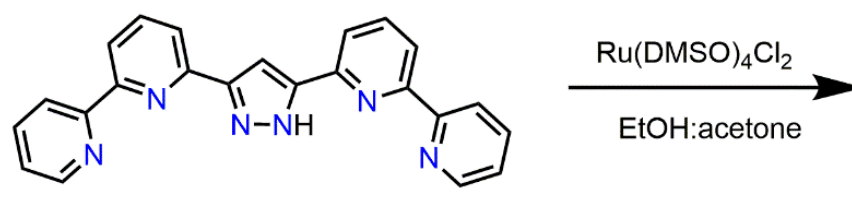

$\mathrm{HL}^{\mathrm{H}}$

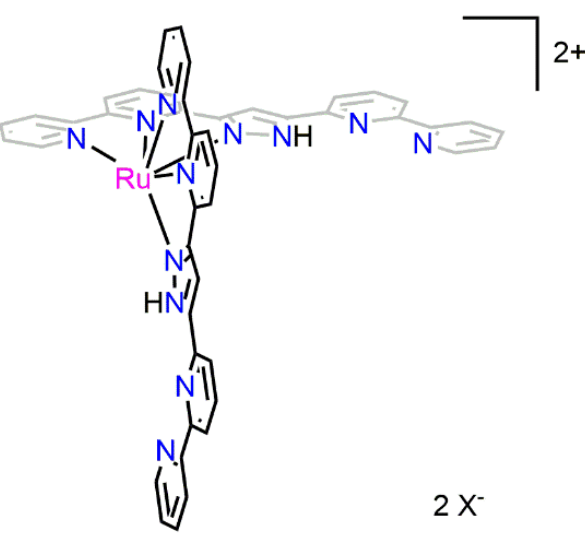

3-1a: $X=\mathrm{BF}_{4}^{-}$ 3-1b: $X=$ OTf $^{-}$

Scheme 3.1. Synthetic scheme for the Ru" "corner complex".

\subsection{2 -X-ray Crystallography of the Ru "Corner Complex"}

The $\mathrm{Ru}^{\mathrm{II}}$ "corner complex" crystallizes as a dimer in the monoclinic space group $C 2 / c$ with hydrogen bond interactions $(2.062 \AA-2.128 \AA$ ) between the protonated sites of the pyrazole moiety and the adjacent pyridine ring of another ligand strand (Figure 3.1). The ruthenium ions are coordinated to six nitrogen atoms in an $\{\mathrm{N} 6\}$ environment arising from two mutually 
perpendicular tridentate ligand strands. Analysis of the average Ru-N bond lengths of $1.98 \AA$ - $2.08 \AA$ fall well within range of typical $\mathrm{Ru}^{\mathrm{II}}$ ions coordinated to bipyridine systems. ${ }^{112}$ Selected bond lengths and distances are tabulated in Table 3.1.

The distortion from an ideal octahedral environment was determined by Continuous Symmetry Measures (CSM), ${ }^{83}$ in which the difference between the bond lengths and angles of the metal coordination sphere are compared to an ideal octahedral environment. Typically, a small $\mathrm{S}\left(\mathrm{O}_{\mathrm{h}}\right)$ value corresponds to an ideal octahedron, whereas a large $\mathrm{S}\left(\mathrm{O}_{\mathrm{h}}\right)$ value signifies high distortion in the coordination sphere. Generally, strong distortions from an ideal octahedral environment results in a trigonal prism coordination sphere. The trigonal prism distortion parameter, $\mathrm{S}(\mathrm{itp})$ can also be calculated by CSM, in which large $\mathrm{S}\left(\mathrm{O}_{\mathrm{h}}\right)$ values typically correspond to small S(itp) values. Using this method, the overall distortion was determined affording $\mathrm{S}\left(\mathrm{O}_{\mathrm{h}}\right)$ values of 2.98 for both ruthenium ions, indicating only minor distortions as expected for low-spin $\mathrm{Ru}^{\mathrm{II}}$ ions.

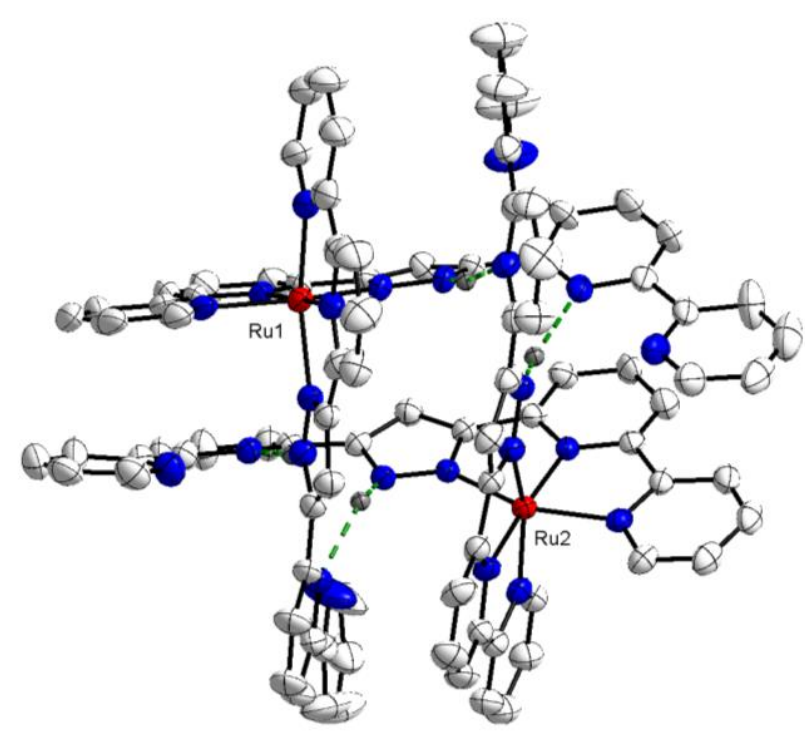

Figure 3.1. Molecular structure of the $\mathrm{Ru}^{\mathrm{II}}$ "corner complex" 3-1a, crystallizing as a hydrogen-bonded dimer (50\% probability thermal ellipsoids). Dash lines indicate hydrogen bonding between the nitrogen atoms of the pyridine and pyrazole moiety.

Table 3.1. Relevant bond lengths and Continuous Symmetry Measures for 3-1a.

\begin{tabular}{ccccc}
\hline & $\boldsymbol{d}_{\text {avg }}(\AA)$ & Spin-state & $\mathbf{S}\left(\mathbf{O}_{\mathbf{h}}\right)$ & S(itp) \\
\hline Ru1-N & 2.040 & LS & 2.98 & 10.91 \\
Ru2-N & 2.043 & LS & 2.98 & 11.17 \\
\hline
\end{tabular}




\subsection{3 -1 ${ }^{-1}$ NMR Spectroscopy and Mass Spectrometry on $\left[\mathrm{HL}_{4} \mathrm{Ru}_{2}\right]\left[\mathrm{BF}_{4}\right]_{4}$}

${ }^{1} \mathrm{H}$ NMR spectroscopy of 3-1a was conducted in MeCN- $\mathrm{d}_{3}$ affording 15 distinct signals in the diamagnetic region (Figure 3.2). The distinctive singlet at $8.1 \mathrm{ppm}$ corresponds to the hydrogen atom on the backbone of the pyrazole moiety, and the broad resonance at $15.1 \mathrm{ppm}$ can be assigned to the $\mathrm{NH}$ proton. The remaining signals are assigned according to ${ }^{1} \mathrm{H}-{ }^{1} \mathrm{H}$ COSY experiments. Although the X-ray structure revealed the presence of a dimer in the solid-state, the persistence of the dimer in solution was investigated using mass spectrometry and DOSY experiments (discussed in the following section). Similar to the first homonuclear $\left[\mathrm{Cu}_{4}(\mathrm{dppn})_{4}\right][\mathrm{OTf}]_{4}$ grid presented in chapter 1 , in which an equilibrium between the mononuclear $\left[\mathrm{Cu}(\mathrm{dppn})_{2}\right][\mathrm{OTf}]$ and $\left[\mathrm{Cu}_{4}(\mathrm{dppn})_{4}\right][\mathrm{OTf}]_{4}$ grid existed in the presence of excess ligand, an equilibrium between the mononuclear $\left[\mathrm{HL}_{2} \mathrm{Ru}\right]\left[\mathrm{BF}_{4}\right]_{2}$ and the dimer $\left[\mathrm{HL}_{4} \mathrm{Ru}_{2}\right]\left[\mathrm{BF}_{4}\right]_{4}$ was also observed depending on the concentration. For solutions with concentrations of less than $10^{-5} \mathrm{M}$, two species with $m / z=853$ and $m / z=458$, corresponding to the monomer $\left[\mathrm{HL}_{2} \mathrm{H}_{2} \mathrm{Ru}\right]^{2+}$ and dimer $\left[\mathrm{HL}^{\mathrm{H}}{ }_{4} \mathrm{Ru}_{2} \mathrm{NaF}\right]^{4+}$, respectively, were detected in the ESI mass spectrum. In more concentrated solutions $\left(10^{-4} \mathrm{M}\right)$, the equilibrium is shifted towards the dimer, as observed with a much higher intensity of the $m / z=458$ peak relative to the $m / z=853$ peak (Figure A21).
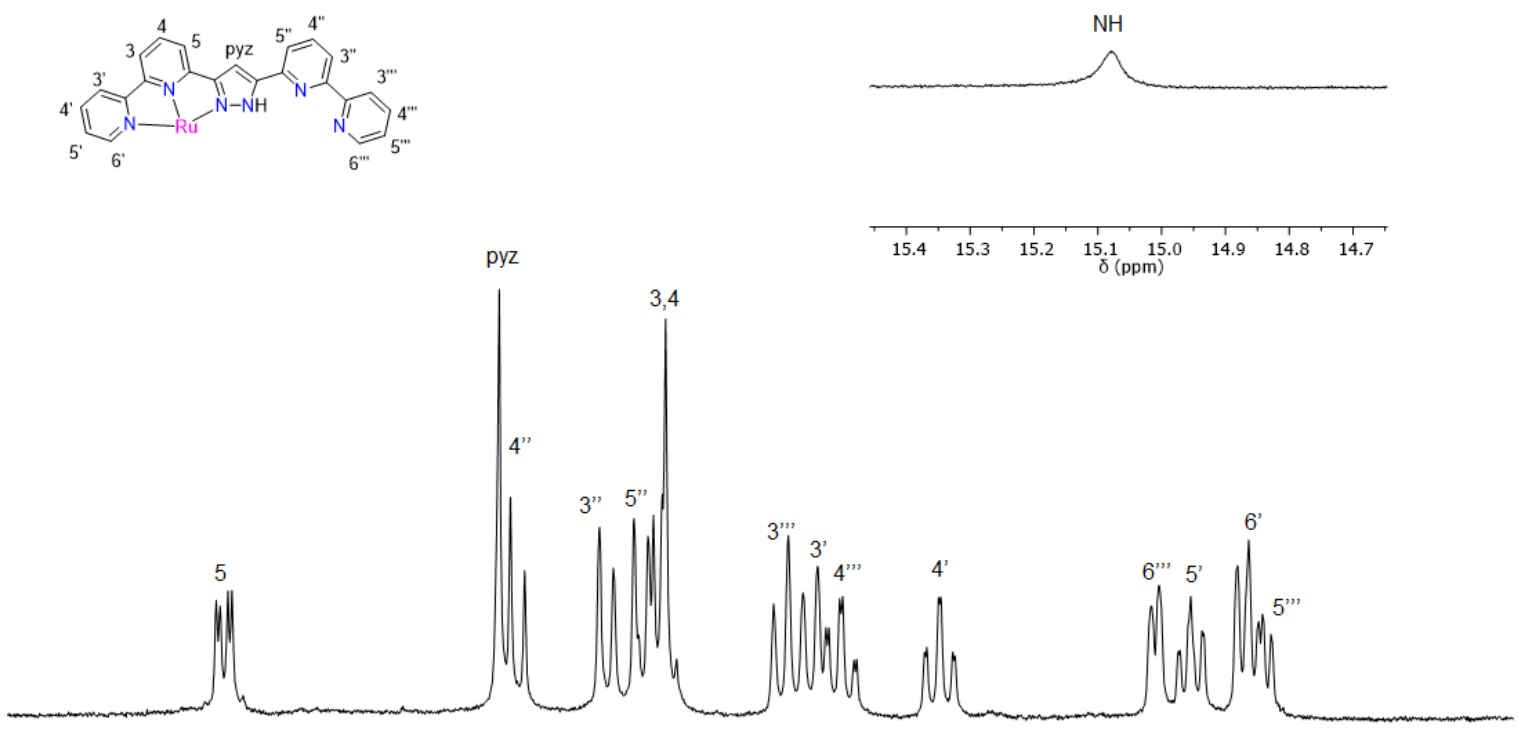

\begin{tabular}{llllllllllllllllllllllllllllllllllllllll}
\hline & 1.9 & 8.8 & 8.7 & 8.6 & 8.5 & 8.4 & 8.3 & 8.2 & 8.1 & 8.0 & 7.9 & 7.8 & 7.7 & 7.6 & 7.5 & 7.4 & 7.3 & 7.2 & 7.1 & 7.0 & 6.9 & 6.8 & 6.7 & 6.6 & 6.5 & 6.4 & 6.3 & 6.2
\end{tabular}

Figure 3.2. ${ }^{1} \mathrm{H}$ NMR spectrum of $\left[\mathrm{HL}_{2} \mathrm{Ru}\right]\left[\mathrm{BF}_{4}\right]_{2}$ 3-1a in $\mathrm{MeCN}-\mathrm{d}_{3}$ revealing the expected 15 peaks. All protons are assigned according to ${ }^{1} \mathrm{H}-{ }^{-1} \mathrm{H}$ COSY experiments.

To further confirm the presence of the monomer or dimer in solution, DOSY experiments were performed on the $\mathrm{Ru}^{\mathrm{II}}$ "corner complex" referenced to the ligand $\mathbf{H L}^{\mathbf{H}}$ in a mixture of $\mathrm{CDCl}_{3}: \mathrm{CD}_{3} \mathrm{CN}$ (1:1) at $323 \mathrm{~K}$ (Figure 3.3). At room temperature, dynamic exchange of the $\mathrm{NH}$ moiety on the ligand results in a broad NH resonance, which gives rise to a broad signal affording a higher margin of error in determining the diffusion coefficient. Consequently, the DOSY experiments were conducted at $323 \mathrm{~K}$. The diffusion coefficient was obtained by averaging the relaxation times of a set of resonances of each compound (Figure A27) to give 
diffusion coefficients of $7.15 \times 10^{-10} \mathrm{~m}^{2} \mathrm{~s}^{-1}$ and $1.33 \times 10^{-9} \mathrm{~m}^{2} \mathrm{~s}^{-1}$ for the $\mathrm{Ru}^{\mathrm{II}}$ "corner complex" and the ligand $\mathbf{H L}^{\mathbf{H}}$, respectively (Table 3.2). Using these values, the hydrodynamic radius of each compound was calculated following the Stokes Einstein equation (eq. 3.1), where $k_{B}$ is Boltzmann constant, $T$ is the temperature, $\eta$ is the viscosity of the solvent, and $r_{s}$ is the hydrodynamic radius. The ratio of the hydrodynamic volumes were determined to be 5.428, suggesting the presence of a dimer at concentrations greater than $10^{-3} \mathrm{M}$.

$$
D=\frac{k_{B} T}{6 \pi \eta r_{S}}
$$

(a)

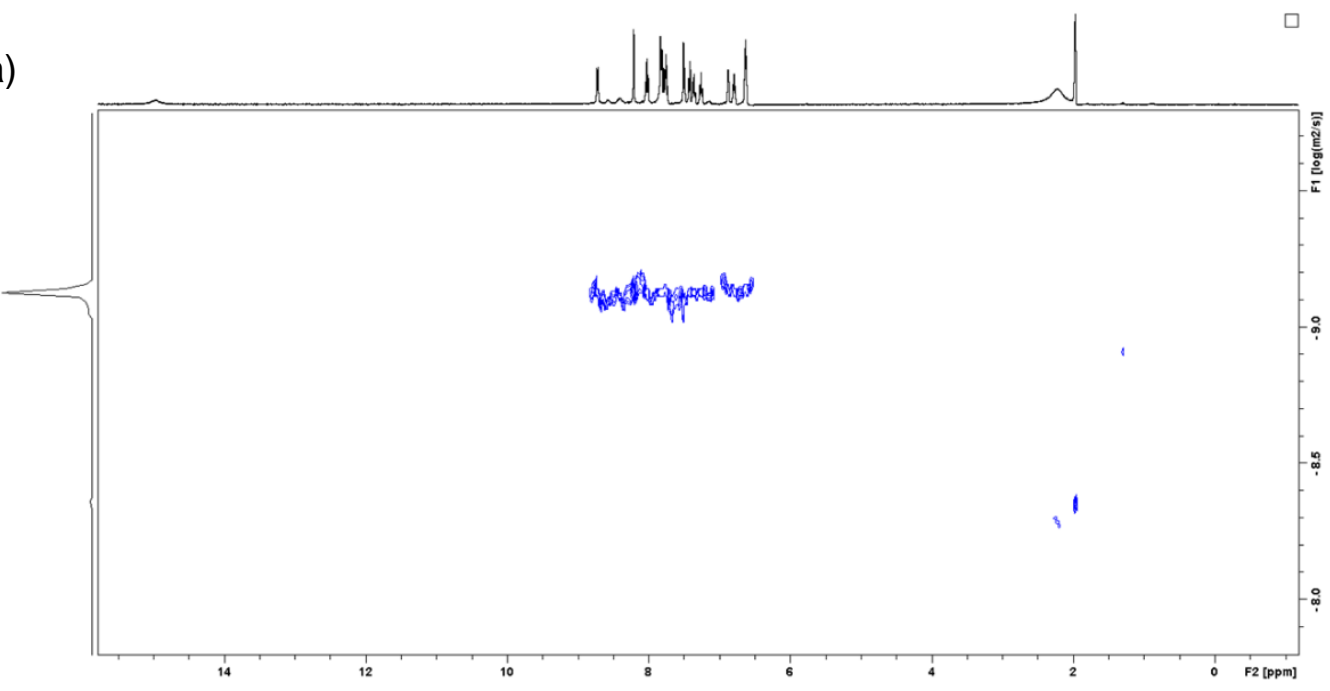

(b)

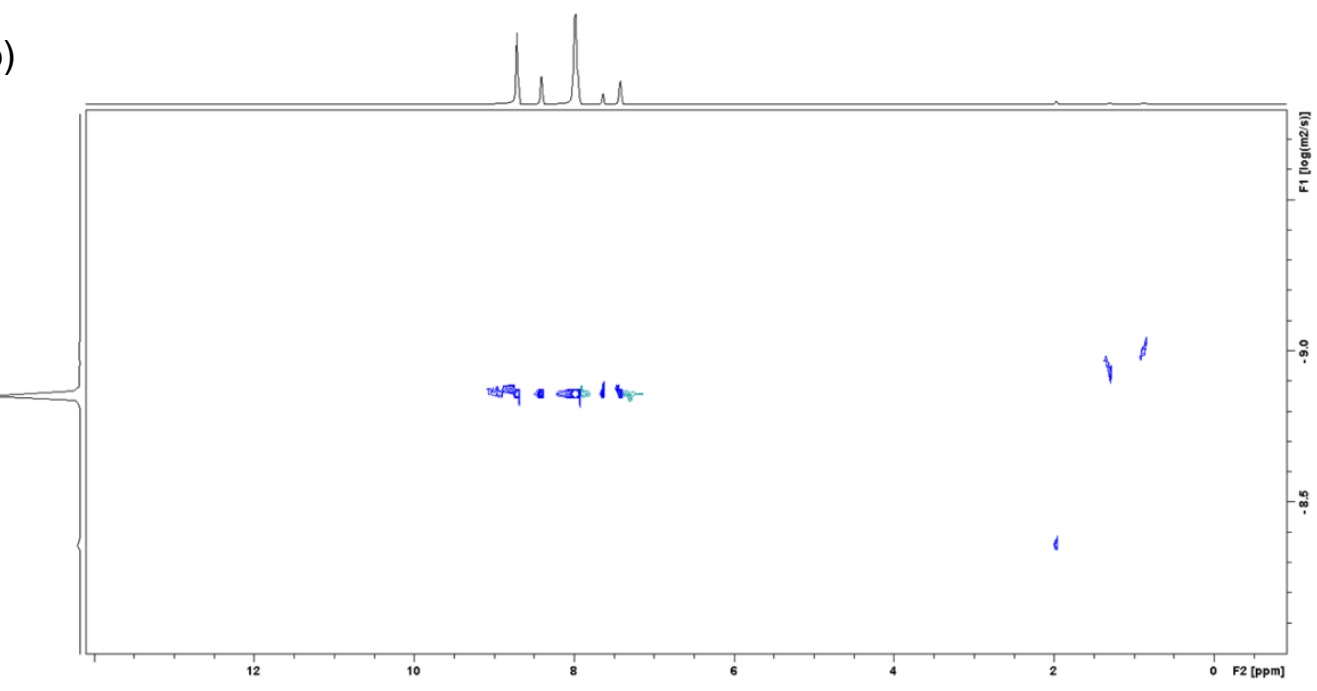

Figure 3.3. DOSY experiments on the (a) $\mathrm{Ru}^{\mathrm{II}}$ "corner complex" and the (b) ligand $\mathbf{H L}^{\mathbf{H}}$ in $\mathrm{CDCl}_{3}: \mathrm{CD}_{3} \mathrm{CN}$ at $323 \mathrm{~K}$. 
Table 3.2. Diffusion coefficient and calculated hydrodynamic volumes for 3-1a and $\mathbf{H L}^{\mathrm{H}}$.

\begin{tabular}{cccc} 
& $\boldsymbol{D}\left[\mathbf{m}^{\mathbf{2}} \mathbf{s}^{-\mathbf{1}}\right]$ & $\mathbf{r}_{\mathbf{s}}[\AA ⿻$ & $\mathbf{4} \boldsymbol{\pi} \mathbf{r}^{3}\left[\AA^{3}\right]$ \\
\hline $\mathbf{3 - 1 a}$ & $7.15 \times 10^{-10}$ & 7.086 & 4471.11 \\
$\mathbf{H L}^{\mathbf{H}}$ & $1.33 \times 10^{-9}$ & 4.032 & 823.704 \\
\hline
\end{tabular}

\subsection{4 - Electrochemical Experiments}

The redox properties of the $\mathrm{Ru}^{\mathrm{II}}$ "corner complex" were investigated by cyclic voltammetry in acetonitrile with $\left[\mathrm{Bu}_{4} \mathrm{~N}\right] \mathrm{PF}_{6}$ as the electrolyte (Figure 3.4). Two quasi-reversible waves are observed at $E_{1 / 2}=0.556 \mathrm{~V}$ and $E_{1 / 2}=1.017 \mathrm{~V}$ vs $\mathrm{Fc} / \mathrm{Fc}^{+}$corresponding to the sequential one-electron oxidations of the dimer; viz, the $\mathrm{Ru}^{\mathrm{II}} \mathrm{Ru}^{\mathrm{II}} / \mathrm{Ru}^{\mathrm{II}} \mathrm{Ru}^{\mathrm{III}}$ couple and $\mathrm{Ru}{ }^{\mathrm{II}} \mathrm{Ru}^{\mathrm{III}} / \mathrm{Ru}^{\mathrm{III}} \mathrm{Ru}^{\mathrm{III}}$ couple, respectively, consistent with other reported dinuclear $\mathrm{Ru}^{\mathrm{II}}$ complexes. ${ }^{113,114}$ Although the potential changes slightly with increasing scan rates, $E_{1 / 2}$ remains relatively constant and peak separations $\Delta E_{\mathrm{p}}$ for the first anodic wave range between $102-180 \mathrm{mV}$, while $\Delta E_{\mathrm{p}}$ for the second anoidic wave range between 96-108 $\mathrm{mV}$. However, at slower scan rates, the second anodic peak becomes less reversible as indicated by a significantly smaller current during the reverse scan (Figure A34), most likely arising from partial deprotonation of the NH moieties and possibly further transformations after the second oxidation.

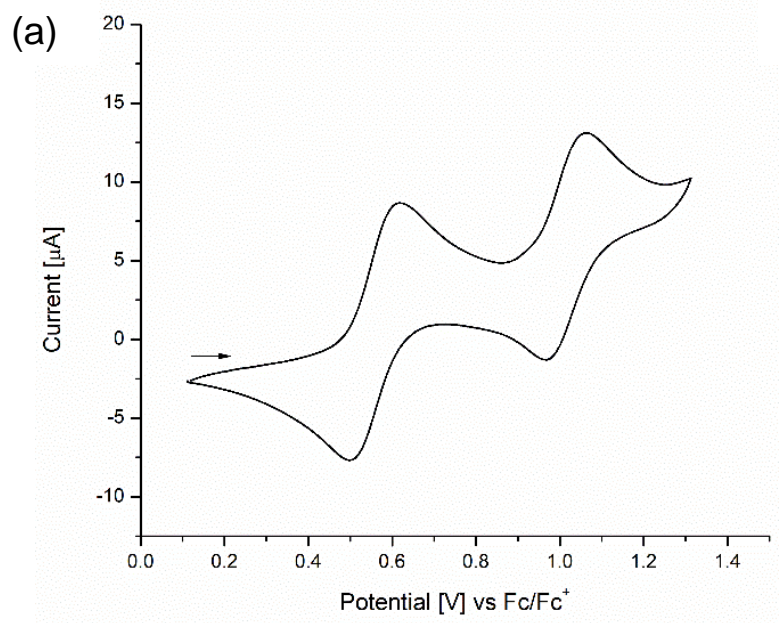

(b)

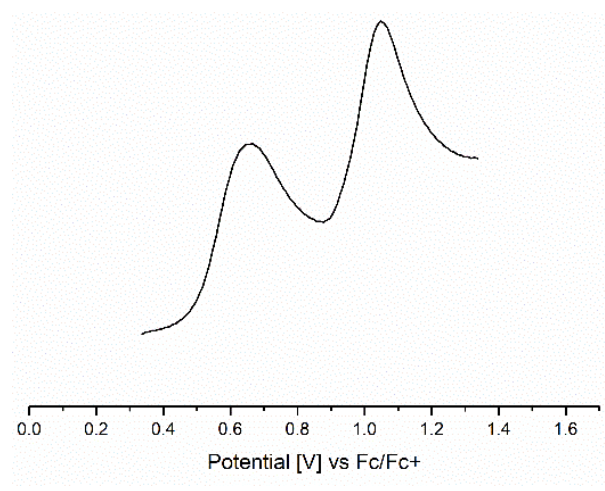

Figure 3.4. (a) Cyclic voltammogram and (b) square wave voltammogram of the $\mathrm{Ru}^{\mathrm{II}}$ "corner complex" at $100 \mathrm{mV} / \mathrm{s}$ in acetonitrile with $\left[\mathrm{Bu}_{4} \mathrm{~N}\right] \mathrm{PF}_{6}$ as the conducting salt. All potentials are referenced versus $\mathrm{Fc} / \mathrm{Fc}^{+}$.

\subsection{5 - UV-Vis Spectroelectrochemistry}

In order to monitor the electrochemical changes associated with the first oxidation of the $\mathrm{Ru}^{\mathrm{II}}$ "corner complex", UV-vis spectroelectrochemistry was conducted by applying a potential of $0.885 \mathrm{~V}$ vs $\mathrm{Fc} / \mathrm{Fc}^{+}$followed by the re-reduction to the original $\mathrm{Ru}^{\mathrm{II}}$ species at $-0.2 \mathrm{~V} \mathrm{vs} \mathrm{Fc} / \mathrm{Fc}^{+}$. Prior to applying a potential, the characteristic $\mathrm{Ru}^{\mathrm{II}}$ band ${ }^{115-117}$ at $440 \mathrm{~nm}$ along with a weak shoulder centered at $530 \mathrm{~nm}$ corresponding to the MLCT bands are observed. Oxidation to $\mathrm{Ru}^{\mathrm{III}}$ afforded a decrease in the band at $440 \mathrm{~nm}$ and a concomitant formation of a new broad band at $500 \mathrm{~nm}$ (Figure 3.5a). Subsequently, re-reduction to the original $\mathrm{Ru}^{\mathrm{II}}$ species was achieved by applying a potential of $-0.2 \mathrm{~V}$ vs $\mathrm{Fc} / \mathrm{Fc}^{+}$, displaying a similar spectrum (Figure 3.5b, orange) after 20 minutes suggesting full chemical reversibility. 
(a)

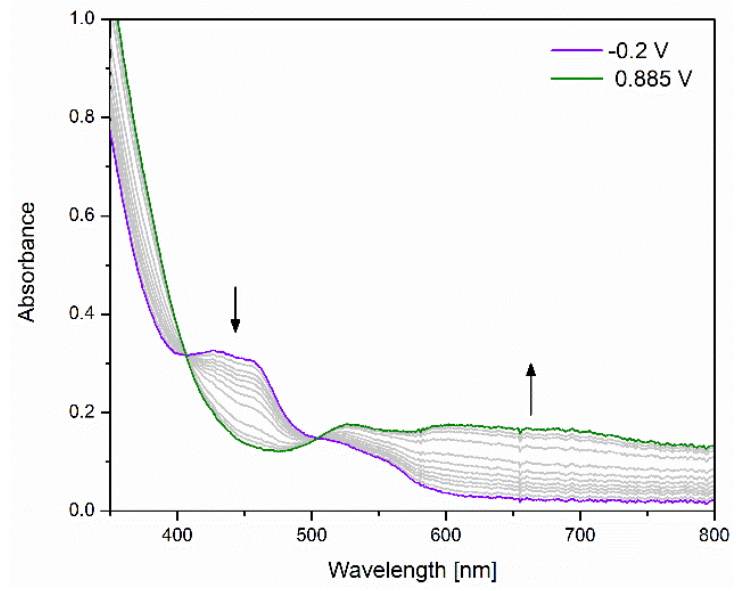

(b)

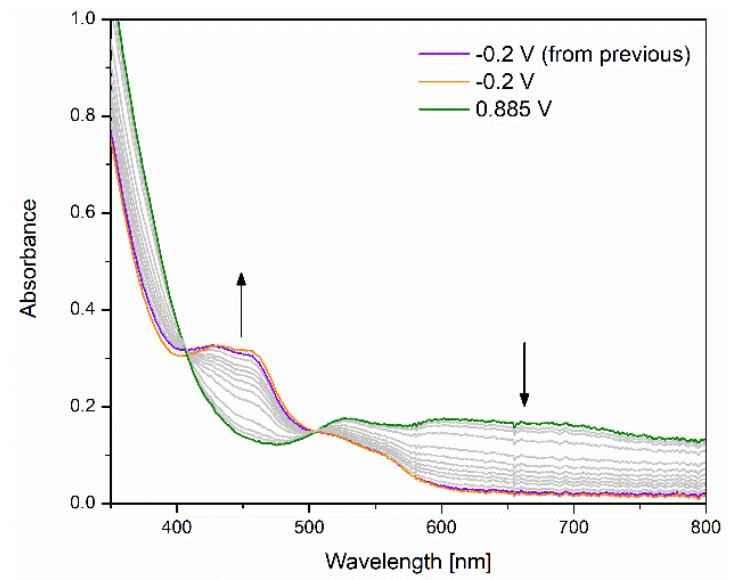

Figure 3.5. (a) UV-Vis spectroelectrochemistry of the first anodic wave ( $\mathrm{Ru}^{\mathrm{III}}$ ) by applying a potential of $0.885 \mathrm{~V}$ followed by (b) the re-reduction by applying a potential at $-0.2 \mathrm{~V}$. All potentials are referenced vs $\mathrm{Fc} / \mathrm{Fc}^{+}$.

The second anodic wave was also monitored by UV-vis spectroelectrochemistry by applying a potential of $1.4 \mathrm{~V}$ vs $\mathrm{Fc} / \mathrm{Fc}^{+}$. The band at $440 \mathrm{~nm}$ vanishes as expected, while the broad band centered at $640 \mathrm{~nm}$ continues to increase in intensity (Figure 3.6a). Surprisingly, the reverse process to obtain the original $\mathrm{Ru}^{\mathrm{II}}$ species afforded a stark broadening of the band at $440 \mathrm{~nm}$ (Figure 3.6b), most likely due to deprotonation of the $\mathrm{NH}$ moieties and possibly dissociation of the dimer after the second oxidation of the $\mathrm{Ru}^{\mathrm{II}}$ "corner complex"; viz, the second oxidation is not fully reversible.

(a)

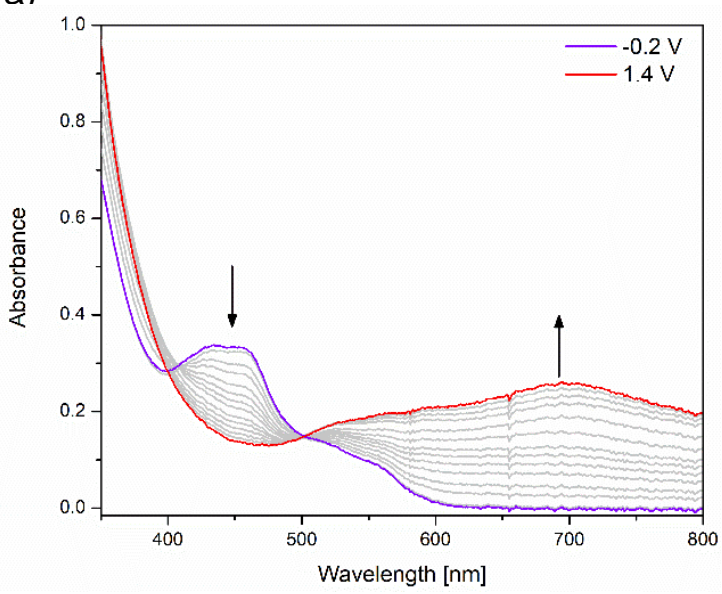

(b)

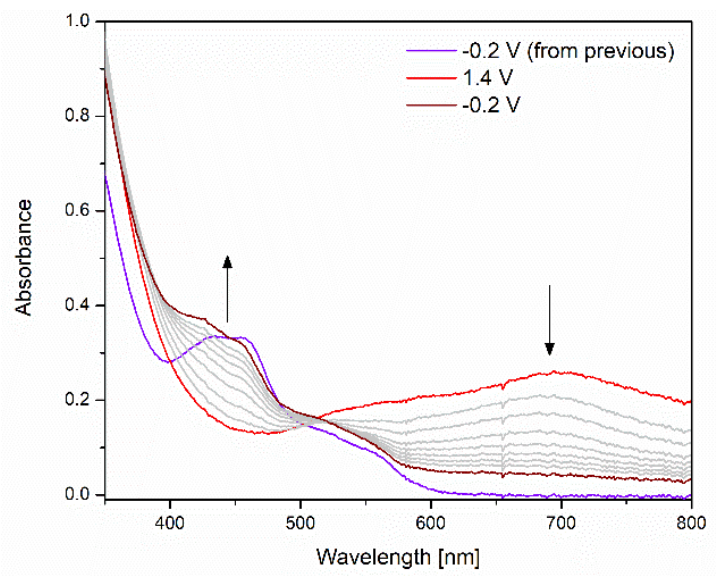

Figure 3.6. (a) UV-vis spectroelectrochemistry of the conversion of the $\mathrm{Ru}^{\mathrm{II}}$ species $(-0.2 \mathrm{~V})$ to the doubly oxidized species $(1.4 \mathrm{~V})$, followed by (b) the reversed process to form the original $\mathrm{Ru}^{\mathrm{II}}$ species. All potentials are referenced against $\mathrm{Fc} / \mathrm{Fc}^{+}$. 


\subsection{6 - EPR Spectroscopy on the Oxidized $\left[\mathrm{HL}_{4}{ }_{4} \mathrm{Ru}_{2}{ }^{\mathrm{III}}\right]^{5+}$ Complex}

Oxidation of the $\mathrm{Ru}^{\mathrm{II}}$ "corner complex" with an excess of $\mathrm{NOBF}_{4}$ afforded a dark purple solution. Due to the paramagnetic nature of the oxidized "corner complex" $\left[\mathrm{HL}_{4}{ }_{4} \mathrm{Ru}_{2}\right]^{5+}, \mathrm{EPR}$ spectroscopy was conducted on $\left[\mathrm{HL}_{4} \mathrm{Ru}_{2}\right]^{5+}$ in frozen acetonitrile (Figure 3.7). Simulation with EasySpin afforded two g-values of $\mathrm{g}_{1}=\mathrm{g}_{2}=2.40$ and $\mathrm{g}_{3}=1.78$ illustrating a uniaxial spectrum, consistent with other $\mathrm{Ru}^{\mathrm{III}}$ systems. ${ }^{118,119}$ Surprisingly, the oxidized $\left[\mathrm{HL}_{4}{ }_{4} \mathrm{Ru}_{2}\right]^{5+}$ appears to gradually revert to the original $\mathrm{Ru}^{\mathrm{II}}$ species suggested by the colour change from dark purple $\left(\left[\mathrm{HL}_{4}{ }_{4} \mathrm{Ru}_{2}\right]^{5+}\right)$ to light orange $\left(\left[\mathrm{HL}_{4} \mathrm{Ru}_{2}\right]^{4+}\right)$.
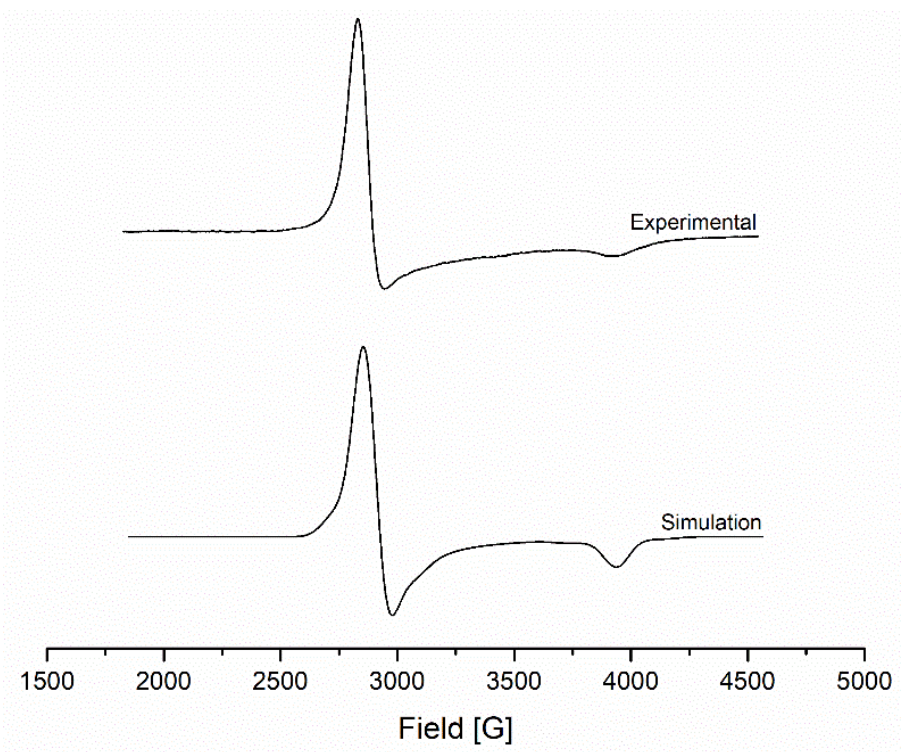

Figure 3.7. EPR spectrum of the oxidized $\left[\mathrm{HL}_{4} \mathrm{Ru}_{2}\right]^{5+}$ with five equivalents of $\mathrm{NOBF}_{4}$ in frozen acetonitrile.

\subsection{1 - Synthesis of $\left[\mathrm{LH}_{4} \mathrm{Ru}_{2} \mathrm{Co}_{2}\right]\left[\mathrm{BF}_{4}\right]_{4}$ Grid}

Once the "corner complex" 3-1a was obtained, the desired heterometallic $\left[\mathrm{LH}_{4} \mathrm{Ru}_{2} \mathrm{Co}_{2}\right]\left[\mathrm{BF}_{4}\right]_{4}$ grid 3-2 was synthesized by adding an excess of sodium $t$-butoxide $\left(\mathrm{NaO}{ }^{t} \mathrm{Bu}\right)$ to a solution of 3-1a in dry acetonitrile followed by one equivalent of $\mathrm{Co}^{\mathrm{II}}\left(\mathrm{BF}_{4}\right)_{2}$, affording a dark red solution (Scheme 3.2). The reaction was heated at reflux for 48 hours. Subsequently, the solution was filtered and precipitated with diethyl ether to give a brown solid, which was further purified by slow diffusion of diethyl either into a solution of $\left[\mathrm{L}_{4} \mathrm{Ru}_{2} \mathrm{Co}_{2}\right]\left[\mathrm{BF}_{4}\right]_{4}$ in acetonitrile. 


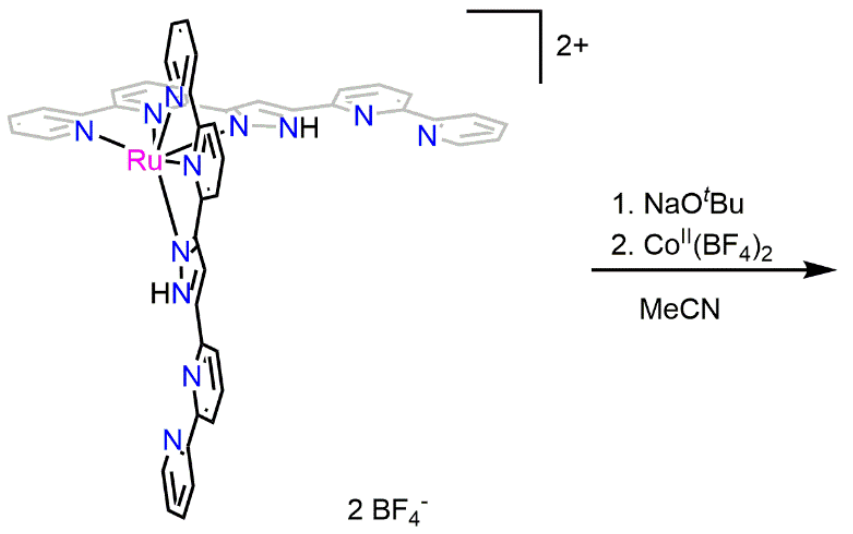

3-1a

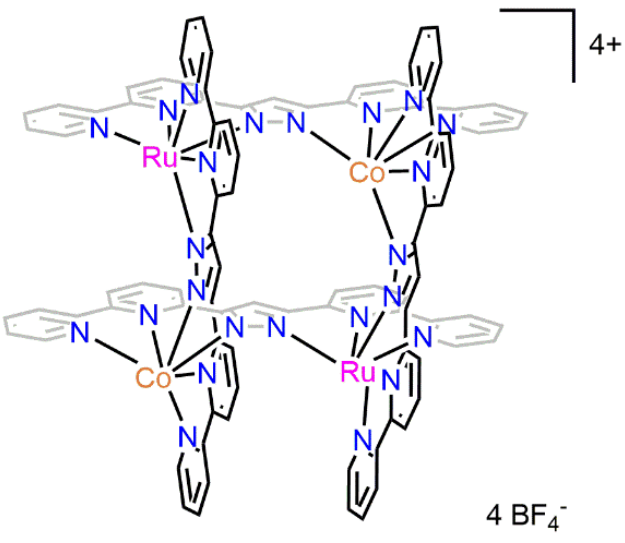

3-2

Scheme 3.2. Synthetic scheme of the $\left[\mathrm{L}_{4} \mathrm{Ru}_{2} \mathrm{Co}_{2}\right]\left[\mathrm{BF}_{4}\right]_{4}$ grid commencing from the stable $\mathrm{Ru}^{\mathrm{II}}$ "corner complex" 3-1a.

\subsection{2 - Mass Spectrometry}

The integrity of $\left[\mathrm{L}_{4} \mathrm{Ru}_{2} \mathrm{Co}_{2}\right]\left[\mathrm{BF}_{4}\right]_{4}$ was confirmed by mass spectrometry in acetonitrile affording a single prominent peak at $m / z=455$ corresponding to the tetracationic $\left[\mathrm{L}_{4} \mathrm{Ru}_{2} \mathrm{Co}_{2}\right]^{4+}$ grid (Figure 3.8). The distinctive $\mathrm{Ru}^{\mathrm{II}}$ isotopic pattern can be simulated accordingly, as shown in the inset of Figure 3.8. Consequently, based on ESI mass spectrometry, $\left[\mathrm{LH}_{4} \mathrm{Ru}_{2} \mathrm{Co}_{2}\right]\left[\mathrm{BF}_{4}\right]_{4}$ is stable and remains intact in acetonitrile.

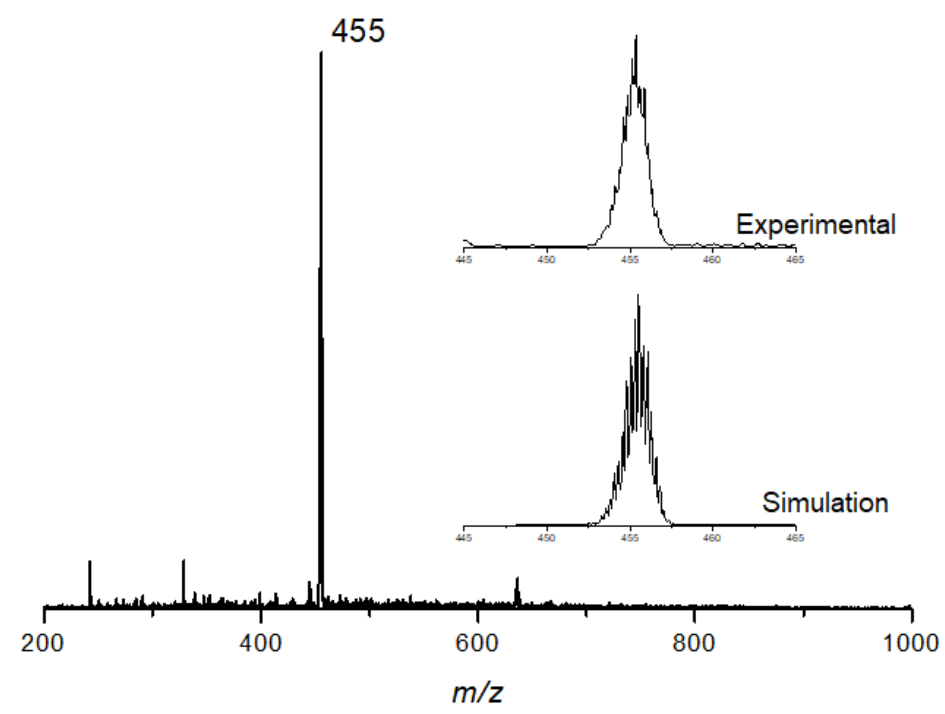

Figure 3.8. ESI-MS of $\left[\mathrm{LH}_{4} \mathrm{Ru}_{2} \mathrm{Co}_{2}\right]\left[\mathrm{BF}_{4}\right]_{4}$ in acetonitrile displaying one dominant peak at $m / z=455$ corresponding to $\left[\mathrm{LH}_{4} \mathrm{Ru}_{2} \mathrm{Co}_{2}\right]^{4+}$. Experimental and simulated isotopic distribution patterns are shown in the inset. 


\subsection{3 - 1H NMR Spectroscopy}

As mentioned in the introduction, the presence of two different metal ions in heterometallic [2 $\times 2$ 2] grids may give rise to scrambling of the metal centers. However, using the kinetically stable $\mathrm{Ru}^{\mathrm{II}}$ "corner complex", only the anti-isomer was observed, which was confirmed by the simplicity of the ${ }^{1} \mathrm{H}$ NMR spectrum. In the case of the syn-isomer, 28 signals would be observed excluding the hydrogen atom closest to the paramagnetic center. This was confirmed by previous ${ }^{1} \mathrm{H}$ NMR studies on similar $\mathrm{Co}^{\mathrm{II}_{4}}$ grids as well as an analogous $\left[\mathrm{L}_{4} \mathrm{Ru}_{2} \mathrm{Fe}_{2}\right]\left[\mathrm{BF}_{4}\right]_{4}$ grid discussed in Chapter 4. On the contrary, for the anti-isomer, only 14 signals would be observed by virtue of symmetry. This was confirmed by ${ }^{1} \mathrm{H}$ NMR spectroscopy in $\mathrm{MeCN}-\mathrm{d}_{3}$ at room temperature, which revealed 14 distinct signals from $-30 \mathrm{ppm}$ to $+120 \mathrm{ppm}$ (Figure 3.9). While 2D NMR spectroscopy was conducted on similar $\mathrm{Co}_{4}{ }_{4}$ grids, ${ }^{81}$ the broad linewidths of this particular grid prevented any 2D NMR experiments. Nevertheless, based on the symmetric nature of the ${ }^{1} \mathrm{H}$ NMR spectrum, one can conclude that the $\left[\mathrm{L}_{4} \mathrm{Ru}_{2} \mathrm{Co}_{2}\right]\left[\mathrm{BF}_{4}\right]_{4}$ grid remains intact in solution and only the trans-isomer is formed.

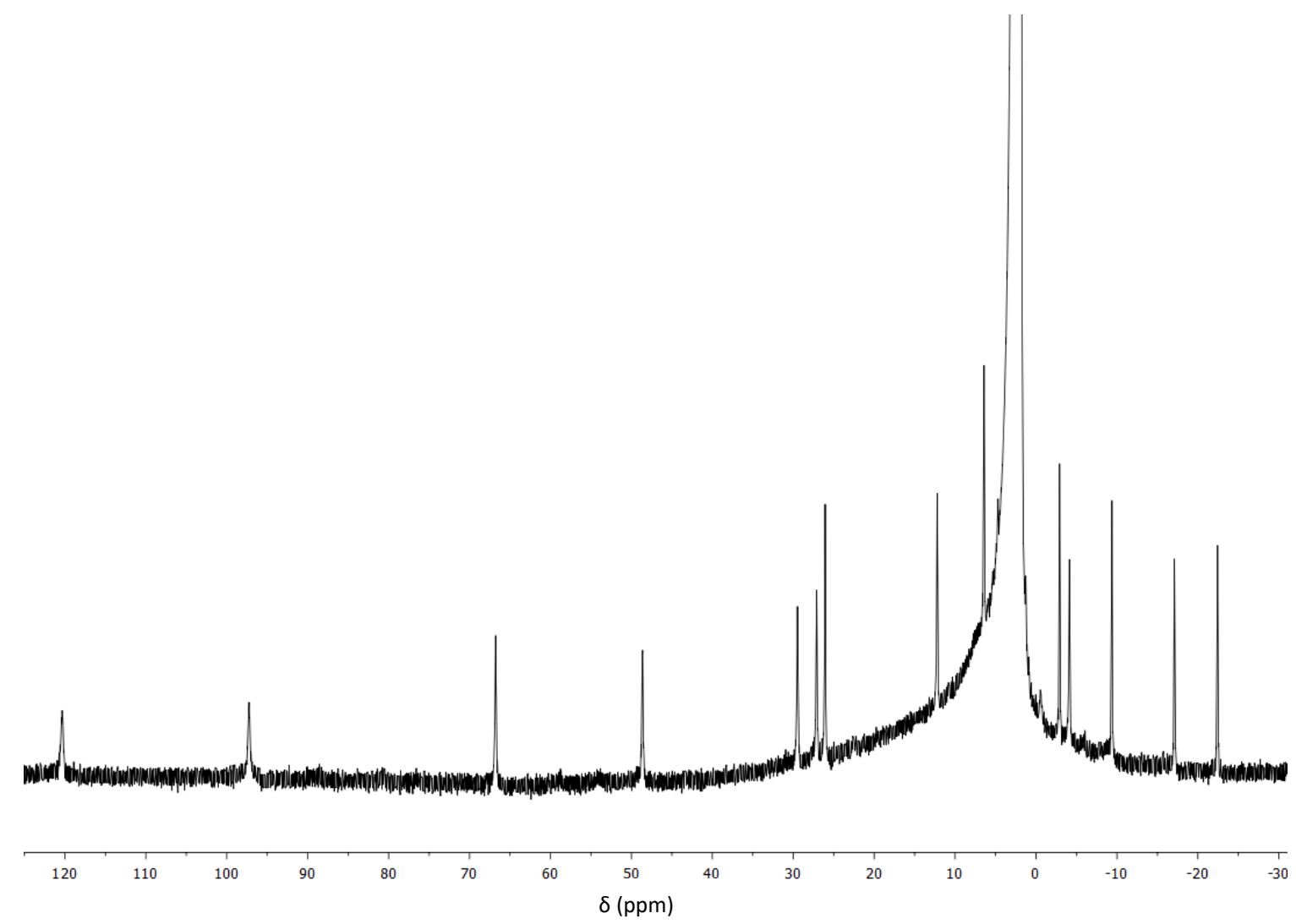

Figure 3.9. ${ }^{1} \mathrm{H} N M R$ spectrum of $\left[\mathrm{L}_{4} \mathrm{Ru}_{2} \mathrm{Co}_{2}\right]\left[\mathrm{BF}_{4}\right]_{4}$ in $\mathrm{MeCN}-\mathrm{d}_{3}$ at room temperature. 


\subsection{4 - X-ray Crystallography}

Crystals of $\left[\mathrm{L}_{4} \mathrm{Ru}_{2} \mathrm{Co}_{2}\right]\left[\mathrm{BF}_{4}\right]_{4}$ suitable for X-ray diffraction were obtained by slow diffusion of diethyl ether into a solution of 3-2 in acetonitrile revealing the expected [2 2 2] grid-like topology. $\left[\mathrm{L}_{4} \mathrm{Ru}_{2} \mathrm{Co}_{2}\right]\left[\mathrm{BF}_{4}\right]_{4}$ crystallizes in the monoclinic space group $P 2_{1} / \mathrm{n}$ with four $\mathrm{BF}_{4}^{-}$ anions to ensure electroneutrality of the species in the solid-state. Moreover, acetonitrile molecules are present in the crystal lattice. Similar to the previously reported mixed valent [2 $x 2$ ] grids, the ruthenium and cobalt ions are situated at opposite corners of the grid and are crystallographically independent. Both metal ions are coordinated to six nitrogen atoms from two perpendicularly bridging ligands forming an octahedral environment. Analysis of the average $\mathrm{Ru}-\mathrm{N}$ and $\mathrm{Co}-\mathrm{N}$ bond lengths of $2.05 \AA$ and $2.16 \AA$ suggests the presence of low-spin $\mathrm{Ru}^{\mathrm{II}}$ ions and high-spin $\mathrm{Co}^{\mathrm{II}}$ ions. The crystal structure of $\left[\mathrm{LH}_{4} \mathrm{Ru}_{2} \mathrm{Co}_{2}\right]\left[\mathrm{BF}_{4}\right]_{4}$ is shown in Figure 3.10 and relevant bond lengths are tabulated in Table 3.3.

The neighbouring metal centers (Ru1, Ru2, Co1, and Co2) are separated by approximately $4.55 \AA$ with angles of $100^{\circ}$ and $80^{\circ}$ forming a rhombus, reminiscent to the parameters found in the previously reported homonuclear $\mathrm{Fe}_{4}$-grids. The distortion of the octahedral environment for each metal center varies depending on the planarity of the ligand strands as described by the torsion angle between the pyrazole moiety and the terminal pyridine group (Figure 3.10b). This was further confirmed by Continuous Symmetry Measures (CSM), ${ }^{83}$ where a small $\mathrm{S}\left(\mathrm{O}_{\mathrm{h}}\right)$ value of 2.95 and 3.60 was determined for Ru1 and Ru2, respectively. In contrast, a large $\mathrm{S}\left(\mathrm{O}_{\mathrm{h}}\right)$ value of 6.57 and 7.11 were obtained for Co1 and Co2, respectively, indicative of a highly distorted octahedral environment. For comparison, the S(itp) values reflecting the distortion from a trigonal prism geometry were also computed and tabulated in Table 3.3. Based on the bond lengths and high $\mathrm{S}\left(\mathrm{O}_{\mathrm{h}}\right)$ value (corresponding to a low $\mathrm{S}$ (itp) value), the cobalt ions are most likely locked in the high-spin state and thermal switching to the low-spin state is highly unlikely.

(a)

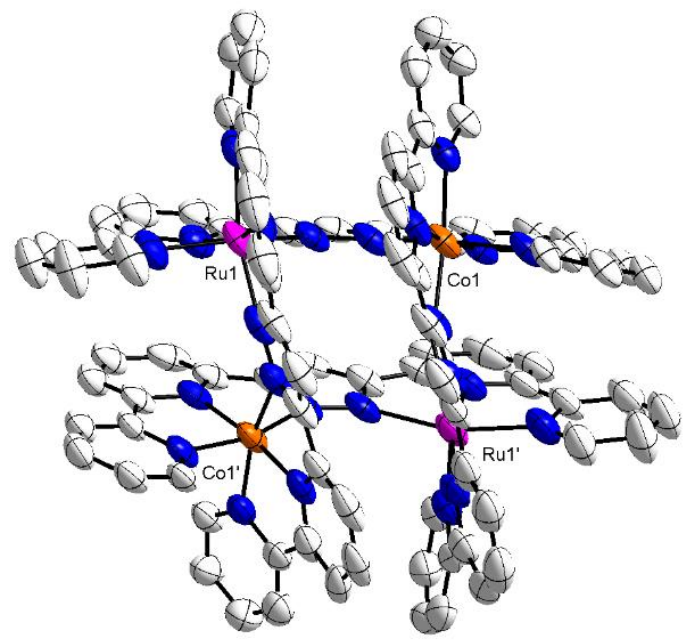

(b)

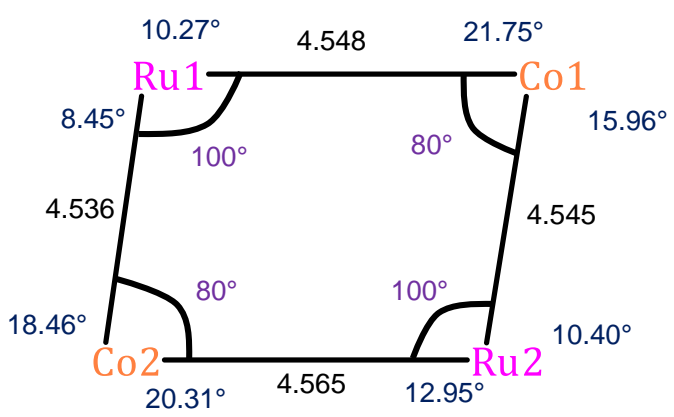

Figure 3.10. (a) Molecular structure of the cation $\left[\mathrm{L}_{4} \mathrm{Ru}_{2} \mathrm{Co}_{2}\right]\left[\mathrm{BF}_{4}\right]_{4}$ (50\% probability thermal ellipsoids). Counterions and hydrogen atoms are omitted for clarity. (b) Schematic representation of the Ru ‥Co distances (black), Ru-Co-Ru and Co-Ru-Co angles (purple), and torsion angles defined by the plane of the pyrazole moiety and terminal pyridine group of the same ligand strand (blue). 
Table 3.3. Relevant bond lengths and CSM for $\left[\mathrm{L}_{4} \mathrm{Ru}_{2} \mathrm{Co}_{2}\right]\left[\mathrm{BF}_{4}\right]_{4}$.

\begin{tabular}{ccccc}
\hline & $\boldsymbol{d}_{\text {avg }}(\AA)$ & Spin-state & $\mathbf{S}\left(\mathbf{O}_{\mathbf{h}}\right)$ & S(itp) \\
\hline Ru1-N & 2.04 & LS & 2.95 & 10.12 \\
Ru2-N & 2.06 & LS & 3.60 & 9.46 \\
Co1-N & 2.16 & HS & 6.57 & 5.47 \\
Co2-N & 2.16 & HS & 7.11 & 4.73
\end{tabular}

\subsection{5 - Single Molecule Magnet Behaviour of $\left[\mathrm{LH}_{4} \mathrm{Ru}_{2} \mathrm{Co}_{2}\right]\left[\mathrm{BF}_{4}\right]_{4}$}

In order to investigate the magnetic behaviour of $\left[\mathrm{LH}_{4} \mathrm{Ru}_{2} \mathrm{Co}_{2}\right]\left[\mathrm{BF}_{4}\right]_{4}$, variable temperature magnetic susceptibility measurements were conducted from $2-210 \mathrm{~K}$ (Figure 3.11a). The maximum temperature at $210 \mathrm{~K}$ was chosen based on the pour point of the oil that was used to prevent preferential orientation of the crystals to the magnetic field. Although a thermal spin transition was not observed in this temperature range, a large molar susceptibility of $\chi_{\mathrm{M}} \mathrm{T}=5.44 \mathrm{~cm}^{3} \mathrm{~mol}^{-1} \mathrm{~K}$ was observed for two $\mathrm{Co}^{\text {II }}$ high-spin ions $\left(2.72 \mathrm{~cm}^{3} \mathrm{~mol}^{-1} \mathrm{~K}\right.$ for one CoII ion) compared to the expected spin-only value of $1.875 \mathrm{~cm}^{3} \mathrm{~mol}^{-1} \mathrm{~K}$ for a $S=3 / 2$ system. This phenomenon is typically observed for highly distorted $\mathrm{Co}^{\mathrm{II}}$ complexes resulting in large contributions to the molecular susceptibility from the unquenched orbital angular momentum. ${ }^{59}$ Upon lowering the temperature, the $\chi_{\mathrm{M}} \mathrm{T}$ decreases substantially below $100 \mathrm{~K}$ reaching a minimum of $3.65 \mathrm{~cm}^{3} \mathrm{~mol}^{-1} \mathrm{~K}$ at $2 \mathrm{~K}$, suggestive of a sizable zero-field splitting (ZFS) parameter, $D$.
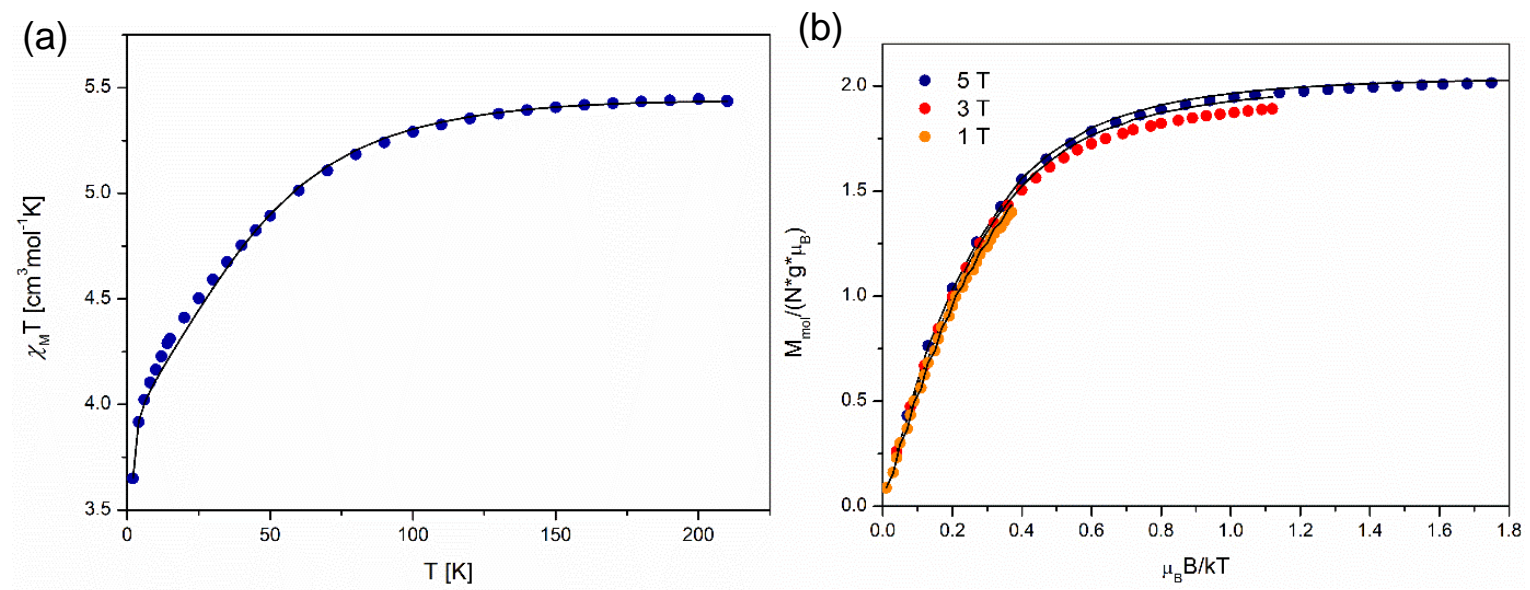

Figure 3.11. (a) Magnetic susceptibility measurements of $\left[\mathrm{LH}_{4} \mathrm{Ru}_{2} \mathrm{Co}_{2}\right]\left[\mathrm{BF}_{4}\right]_{4}$ from $210 \mathrm{~K}-2 \mathrm{~K}$. (b) In order to obtain the ZFS parameter, $D$, magnetization plots at different magnetic fields were measured. The solid lines represent the best fits (see text).

In order to determine the ZFS parameter more precisely, variable temperature variable field (VTVH) magnetization measurements were also conducted (Figure 3.11b). Magnetization of $\left[\mathrm{LH}_{4} \mathrm{Ru}_{2} \mathrm{Co}_{2}\right]^{4+}$ was measured from $0-2 \mathrm{~K}$ at 1,3 , and $5 \mathrm{~T}$ displaying three distinct magnetization curves at different fields, confirming the presence of zero-field-splitting. Variable temperature magnetic susceptibility $\chi_{M} \mathrm{~T}$ and VTVH magnetization data were simultaneously fitted following the spin Hamiltonian for one cobalt $(S=3 / 2)$ ion shown in equation 3.2. 
Using the spin Hamiltonian (Equation 3.2), $\mathrm{g}_{\mathrm{x}}$ and $\mathrm{g}_{\mathrm{y}}$ were determined to be 2.32, while a value of 2.64 was obtained for $\mathrm{g}_{\mathrm{z}}$. All three g-values are significantly larger than the spin-only case $(g=2.0)$, confirming the non-negligible angular orbital contribution of the $\mathrm{Co}^{\mathrm{II}}$ ions $(\mathrm{S}=3 / 2) .120$ Moreover, the large negative axial zero-field splitting value, $D$, was determined to be $D=-64 \mathrm{~cm}^{-1}$ (the rhombic zero field splitting parameter, $E$, was assumed to be negligible, where $E / D=0$ ), which suggest exclusive population of the Kramer's doublets with $M_{s}= \pm 3 / 2$ states at lower temperatures. Consequently, the thermal barrier to spin inversion between the $M_{S}=+3 / 2$ and $M_{S}=-3 / 2$ states can be approximated by $|2 D| \sim 128 \mathrm{~cm}^{-1}$, which is remarkably large and suggestive of single molecular magnet (SMM) behaviour.

$$
\widehat{H}=\mu_{B}\left(S_{x} g_{x} B_{x}+S_{y} g_{y} B_{y}+S_{z} g_{z} B_{z}\right)+D\left[\hat{S}_{z}^{2}-\frac{1}{3} S(S+1)+\frac{E}{D}\left(\hat{S}_{x}^{2}-\hat{S}_{y}^{2}\right)\right]
$$

Consequently, alternating current (ac) magnetic measurements were conducted from $2.0 \mathrm{~K}-5.8 \mathrm{~K}$ with an applied dc field of $H_{\mathrm{dc}}=3000 \mathrm{Oe}$, displaying a temperature dependent out-of-phase ( $\chi^{\prime \prime}$ ) signal (Figure 3.12). As the temperature increases from $2 \mathrm{~K}$ to $5.8 \mathrm{~K}$, the maxima is shifted to higher frequencies, suggestive of a barrier to spin reversal, a typical phenomenon for superparamagnets and/or slow relaxing molecular clusters.

(a)

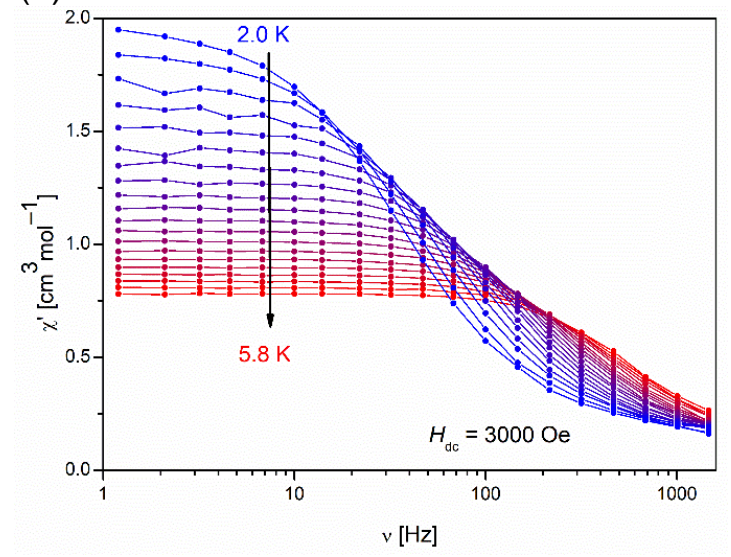

(b)

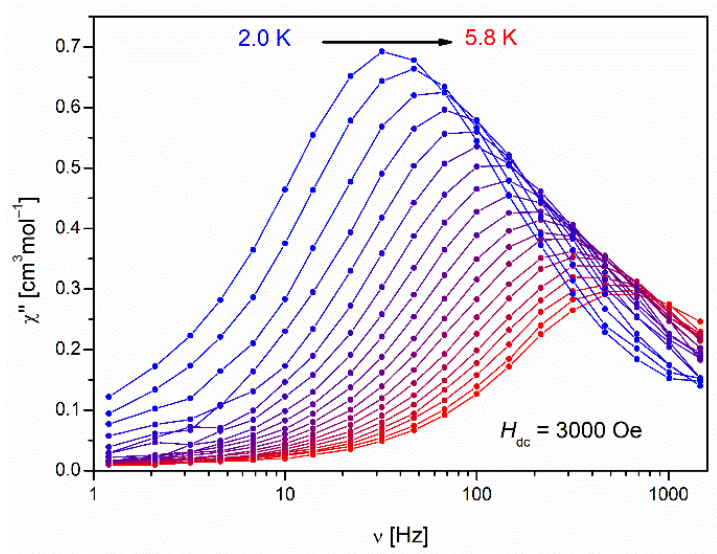

Figure 3.12. (a) in-phase $\left(\chi^{\prime}\right)$ and (b) out-of-phase $\left(\chi^{\prime \prime}\right)$ ac magnetic susceptibility measurements for $\left[\mathrm{LH}_{4} \mathrm{Ru}_{2} \mathrm{Co}_{2}\right]\left[\mathrm{BF}_{4}\right]_{4}$ displaying temperature dependent out-of-phase $(\chi ")$ signals over a temperature range of $2.0-5.8 \mathrm{~K}$.

An effective method to investigate the magnetization dynamics involves the usage of the socalled Cole-Cole plots or Argand diagrams, where the out-of-phase ( $\left.\chi^{\prime \prime}\right)$ signals are plotted against the in-phase $\left(\chi^{\prime}\right)$ signals. Magnetization relaxation times as well as the width of its distribution can be extracted from the frequency dependent ac susceptibility curves. For a single relaxation process, one can fit the data according to equation 3.3, where $\chi_{\mathrm{T}}$ and $\chi_{\mathrm{S}}$ is the isothermal and adiabatic susceptibility, respectively. If a molecule exhibits a single relaxation process, a perfect semicircle centered on the $\chi^{\prime}$ axis would be observed in the Cole-Cole diagram. Using the maximum frequency of absorption, $v$, the relaxation time $\tau$ can be determined according to equation 3.4.

$$
\begin{gathered}
\chi_{A C}(\omega)=\chi_{S}+\frac{\chi_{T}-\chi_{S}}{1+i \omega \tau} \\
\tau=\frac{1}{2 \pi v}
\end{gathered}
$$


Of course, there lies a possibility where $\chi$ " does not reach the maximum of the calculated value affording a flattened or stretched out semicircle, suggestive of the presence of one or more time constants or a distribution of relaxation times. In this case, equation 3.3 is modified to include a distribution factor, $\alpha$, shown in equation 3.5. This distribution factor, $\alpha$, ranges between 0 and 1 , where if $\alpha=0$, only one relaxation process is observed while if $\alpha=1$, the relaxation process is absent.

$$
\chi(\omega)=\chi_{s}+\frac{\chi_{T}-\chi_{S}}{1+(i \omega \tau)^{1-\alpha}}
$$

Thus, in order to determine the number of relaxation processes $\left[\mathrm{L}_{4} \mathrm{Ru}_{2} \mathrm{Co}_{2}\right]\left[\mathrm{BF}_{4}\right]_{4}$ exhibits, a Cole-Cole diagram with an applied dc field of $H_{\mathrm{dc}}=3000$ Oe between 2.0 and $5.8 \mathrm{~K}$ was constructed and fitted with the Debye model with the C-C Fit program (Figure 3.13a, black lines). The obtained distribution index $\alpha$ ranges from 0.1 to 0.2 , with small deviations between the temperature limits, confirming the presence of a single relaxation process.

$$
\tau=\tau_{o} e^{\left(\frac{U_{e f f}}{\mu_{B} T}\right)}
$$

The thermally activated relaxation times can be extracted from the Arrhenius plot (Figure 3.13b). The linear portion is fitted according to the Arrhenius law given in equation 3.6, where $\tau$ is the relaxation time, $U_{\text {eff }}$ is the effective energy barrier, $\mu_{\mathrm{B}}$ is the Bohr magneton, and $\tau_{0}$ is the pre-exponential factor. Using this equation, the relaxation time $\tau_{0}$ and energy barrier for spin inversion, $U_{\text {eff, }}$ was determined to be $\tau_{0}=3.1 \times 10^{-5} \mathrm{~s}$ and $U_{\text {eff }}=8.8 \mathrm{~cm}^{-1}$, which is consistent with typical $\mathrm{Co}^{\text {II }}$ SMMs. ${ }^{58}$ However, the relaxation time $\tau_{0}$ appears quite large and the energy barrier is relatively small compared to systems exhibiting Orbach relaxation, which suggests that the relaxation is most likely dominated by a Raman process. Consequently, the entire temperature range was fitted solely for Raman relaxation (Figure A43), which was also observed in other reported $\mathrm{Co}^{\mathrm{II}}$ SMMs. For comparison with other reported CoII SMMs, the Arrhenius plot is typically shown.

(a)

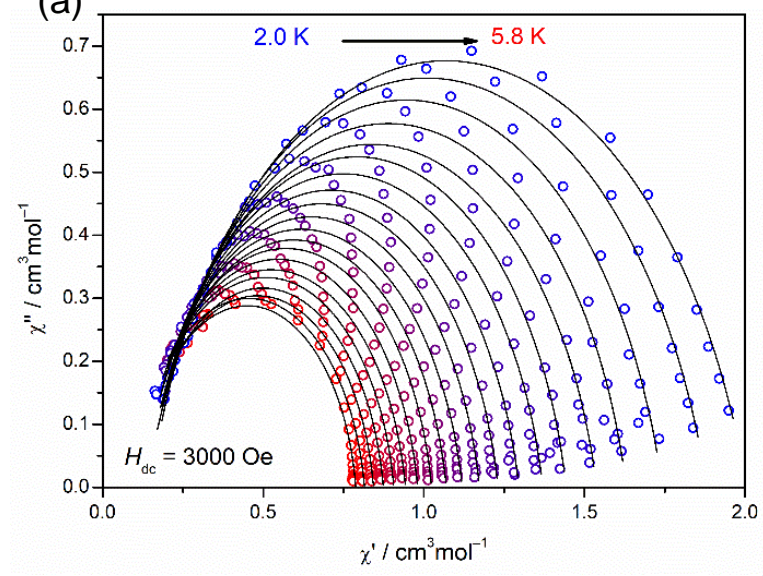

(b)

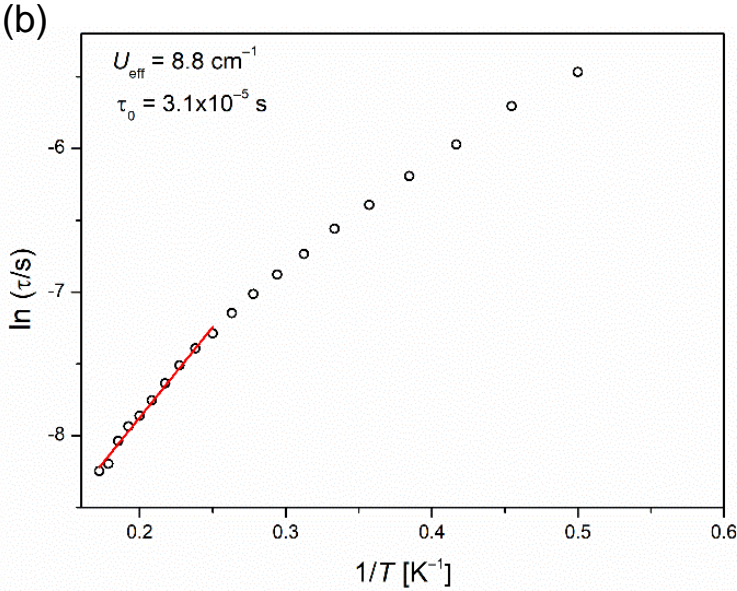

Figure 3.13. (a) Cole-Cole diagram with an applied dc field of $H_{\mathrm{dc}}=3000$ Oe between 2.0 and $5.8 \mathrm{~K}$. The black lines are the theoretical fit using the Debye model. (b) Relaxation times are calculated using the linear portion of the Arrhenius plot. 
Although $U_{\text {eff }}$ is quite low compared to current single molecular magnets (SMM), not many grid-like complexes exhibit this type of magnetic behaviour. Among those that are known, only lanthanide-based [2 $\mathrm{x} 2$ 2] grids (DyIII) have shown SMM behaviour due to their intrinsic weak intermolecular interactions between the metal centers. ${ }^{77,78}$ However, in terms of $3 \mathrm{~d}$ transition metal [ $2 \times 2$ ] grid-like complexes, aside from the previously reported mixed valent $\mathrm{Co}_{2} \mathrm{Co}_{2}{ }_{2}$ grid, this is the first heterometallic $3 \mathrm{~d}$-transition metal grid exhibiting SMM properties.

\subsection{6 - Probing the Redox Properties of $\left[\mathrm{LH}_{4} \mathrm{Ru}_{2} \mathrm{Co}_{2}\right]\left[\mathrm{BF}_{4}\right]_{4}$ with Cyclic Voltammetry}

With the presence of two different metal centers, the potential to have multiple stable redox states was investigated by cyclic voltammetry in acetonitrile with $\left[\mathrm{Bu}_{4} \mathrm{~N}\right] \mathrm{PF}_{6}$ as the conducting salt. As expected, four oxidation waves were observed corresponding to the oxidation of each of the metal centers in $\left[\mathrm{LH}_{4} \mathrm{Ru}_{2} \mathrm{Co}_{2}\right]\left[\mathrm{BF}_{4}\right]_{4}$ (Figure 3.14). A large separation between the second and third oxidative waves suggest a high thermodynamic stability of the doubly oxidized $\left[\mathrm{L}_{4} \mathrm{Ru}_{2} \mathrm{Co}_{2}\right]\left[\mathrm{BF}_{4}\right]_{6}$ grid $\left(K_{\mathrm{c}}=3.35 \times 10^{7}\right.$; calculated using $\left.E_{1_{2} / 2}\left(6^{+} / 7^{+}\right)-E_{\mathrm{pf}}\left(5^{+} / 6^{+}\right)\right)$, which was indeed chemically synthesized permitting a definitive assignment of the anodic waves. In particular, the first two anodic peaks are assigned to the oxidation of both $\mathrm{Co}^{\mathrm{II}}$ ions at opposite corners, while the latter two anodic peaks are assigned to oxidation of the $\mathrm{Ru}^{\mathrm{II}}$ ions.

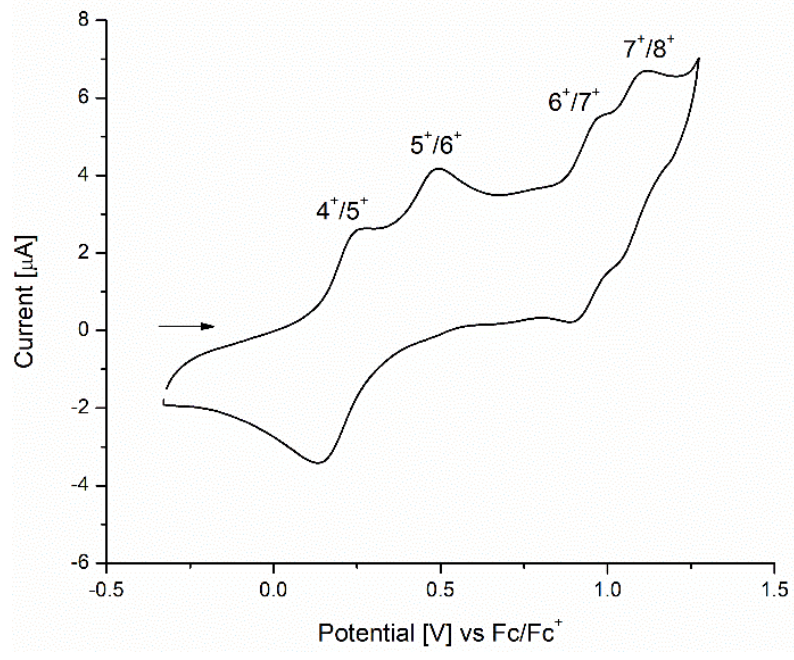

Figure 3.14. Cyclic voltammogram of $\left[\mathrm{LH}_{4} \mathrm{Ru}_{2} \mathrm{Co}_{2}\right]\left[\mathrm{BF}_{4}\right]_{4}$ in degassed acetonitrile with $\left[\mathrm{NBu}_{4}\right] \mathrm{PF}_{6}$ as the electrolyte at $100 \mathrm{mV} / \mathrm{s}$.

Table 3.4 Electrochemical potentials for $\left[\mathrm{LH}_{4} \mathrm{Ru}_{2} \mathrm{Co}_{2}\right]\left[\mathrm{BF}_{4}\right]_{4}$ in acetonitrile.

\begin{tabular}{ccccc}
\hline & $E_{a} p[\mathrm{mV}]$ & $E_{1 / 2}[\mathrm{mV}]$ & $\Delta E_{p}[\mathrm{mV}]$ & $E_{1 / 2}-E_{1 / 2}[\mathrm{mV}]$ \\
\hline $4^{+} / 5^{+}$ & 272 & - & $136^{\mathrm{b}}$ & - \\
$5^{+} / 6^{+}$ & 496 & - & - & - \\
$6^{+} / 7^{+}$ & 977 & 941 & 72 & 148 \\
$7^{+} / 8^{+}$ & 1121 & 1089 & 64 & \\
\hline
\end{tabular}

aAll chemical potentials are referenced to $\mathrm{Fc} / \mathrm{Fc}^{+}$. bUsing the broad cathodic wave at $E_{\mathrm{c}^{\mathrm{p}}}=136$ $\mathrm{mV}$.

Interestingly, as the polarity was reversed, only three cathodic waves were observe. Initially, the broad reductive wave at $136 \mathrm{mV}$ was attributed to a two-electron reduction from the two cobalt ions. Based on several reported $\mathrm{Co}^{{ }^{I I}} 4$ grid-like complexes as well as similar 
mononuclear cobalt complexes, the electron transfer of the $\mathrm{Co}^{\mathrm{III}} / \mathrm{Co}^{\mathrm{II}}$ redox couple is known to be kinetically hampered due to large structural reorganization during oxidation affording ill-resolved and broad waves. ${ }^{121-123}$ Using scan-rate dependent CV measurements, the quasireversible broad peak at $136 \mathrm{mV}$ can be separated into two peaks at extremely slow scan rates ( $2 \mathrm{mV} / \mathrm{s}$ ), supporting the slow electron transfer kinetics from $\mathrm{Co}^{\mathrm{III}}$ to $\mathrm{Co}^{\mathrm{II}}$ (Figure 3.15). Moreover, coulometry experiments were conducted to electrochemically generate the doubly oxidized $\left[\mathrm{L}_{4} \mathrm{Ru}_{2} \mathrm{Co}_{2}\right]^{6+}$ grid, followed by the re-reduction. However, the amount of charge passed through the cell during electrochemical oxidation did not reach the expected charge for a two-electron process after 24 hours. Consequently, coulometry experiments on the rereduction was never obtained.

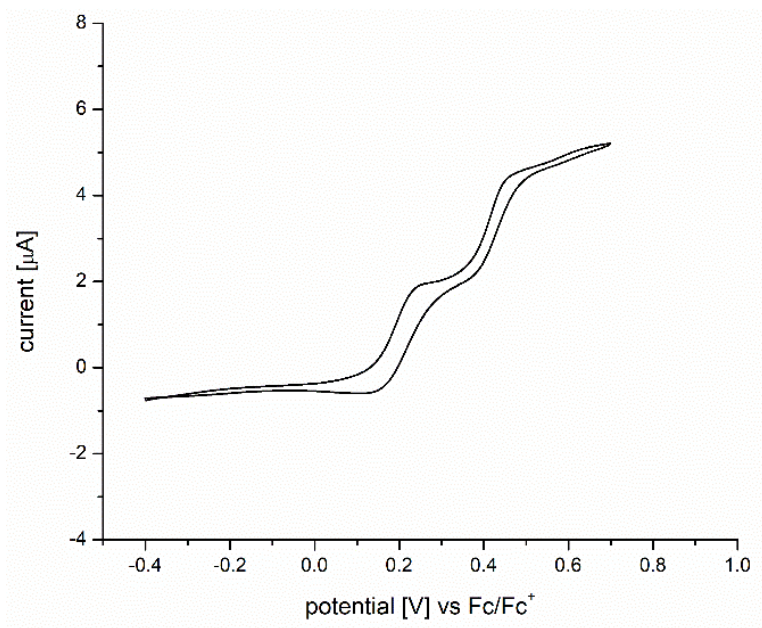

Figure 3.15. Cyclic voltammogram spanning the first two anodic waves from $-0.4-0.8 \mathrm{~V}$ at $2 \mathrm{mV} / \mathrm{s}$. All potentials are referenced to $\mathrm{Fc} / \mathrm{Fc}^{+}$.

\subsection{7 - UV-Vis Spectroscopy and Spectroelectrochemistry on $\left[\mathrm{L}_{4} \mathrm{Ru}_{2} \mathrm{Co}_{2}\right]\left[\mathrm{BF}_{4}\right]_{4}$}

UV-vis spectroscopy was conducted on a solution of $\left[\mathrm{L}_{4} \mathrm{Ru}_{2} \mathrm{Co}_{2}\right]\left[\mathrm{BF}_{4}\right]_{4}$ in acetonitrile displaying four bands at $320 \mathrm{~nm}, 388 \mathrm{~nm}, 505 \mathrm{~nm}$, and $620 \mathrm{~nm}$, whereas, in the case of the homonuclear $\mathrm{Fe}_{4}$ grids, only one band is observed in the visible $(400-800 \mathrm{~nm})$ region due to the four equivalent iron atoms. The most intense band at $320 \mathrm{~nm}$ corresponds to the ligandbased $\pi \rightarrow \pi^{*}$ transition, also observed in the $\mathrm{Fe}_{4}$ grids. ${ }^{36,82,91}$ The remaining three weaker bands are assigned to metal-to-ligand-charge-transfer (MLCT) bands arising from the different metal ions.

From the cyclic voltammetry experiments discussed in the previous section, four waves were observed. In order to probe the changes associated with electrochemical oxidation of $\left[\mathrm{LH}_{4} \mathrm{Ru}_{2} \mathrm{Co}_{2}\right]\left[\mathrm{BF}_{4}\right]_{4}$ to the doubly oxidized $\left[\mathrm{LH}_{4} \mathrm{Ru}_{2} \mathrm{Co}_{2}\right]\left[\mathrm{BF}_{4}\right]_{6}$ grid, the changes in the UV-vis spectrum were monitored by applying a voltage at $0.7 \mathrm{~V}$ vs $\mathrm{Fc} / \mathrm{Fc}^{+}$until full conversion (i.e. no further visible changes in the UV-vis spectra) followed by re-reduction to the original $\left[\mathrm{L}_{4} \mathrm{Ru}_{2} \mathrm{Co}_{2}\right]\left[\mathrm{BF}_{4}\right]_{4}$ grid by applying a potential of $-0.6 \mathrm{~V}$ vs Fc/Fc'. Once the first voltage $(0.7 \mathrm{~V}$ vs $\mathrm{Fc} / \mathrm{Fc}^{+}$) was applied, the intensity of both bands at $388 \mathrm{~nm}$ and $505 \mathrm{~nm}$ decrease significantly and the peak maxima are shifted slightly to $402 \mathrm{~nm}$ and $517 \mathrm{~nm}$, respectively. However, the small broad peak at $620 \mathrm{~nm}$ disappears completely (Figure 3.16a). Application of a potential of $-0.6 \mathrm{~V}$ for 15 minutes to re-reduce the doubly oxidized $\left[\mathrm{LH}_{4} \mathrm{Ru}_{2} \mathrm{Co}_{2}\right]\left[\mathrm{BF}_{4}\right]_{6}$ grid 
to the original complex $\left[\mathrm{L}_{4} \mathrm{Ru}_{2} \mathrm{Co}_{2}\right]\left[\mathrm{BF}_{4}\right]_{4}$ afforded only partial conversion (Figure $3.16 \mathrm{~b}$, green). This is consistent with the slow electron transfer observed in the cyclic voltammetry experiments.

(a)

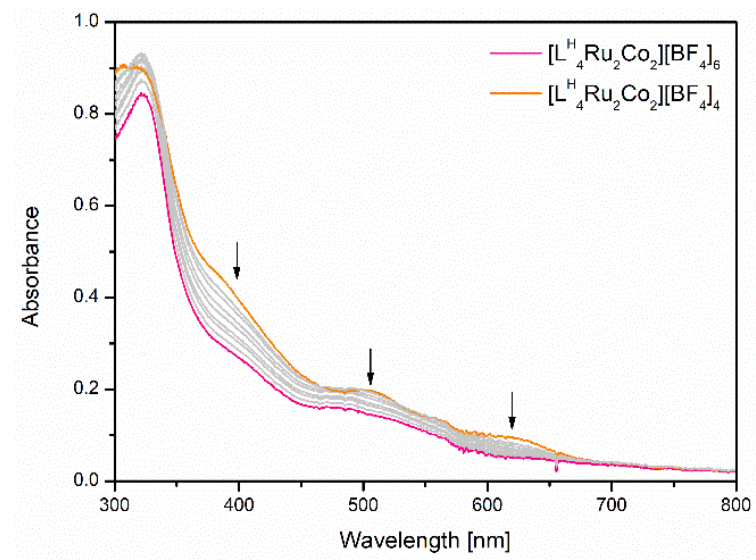

(b)

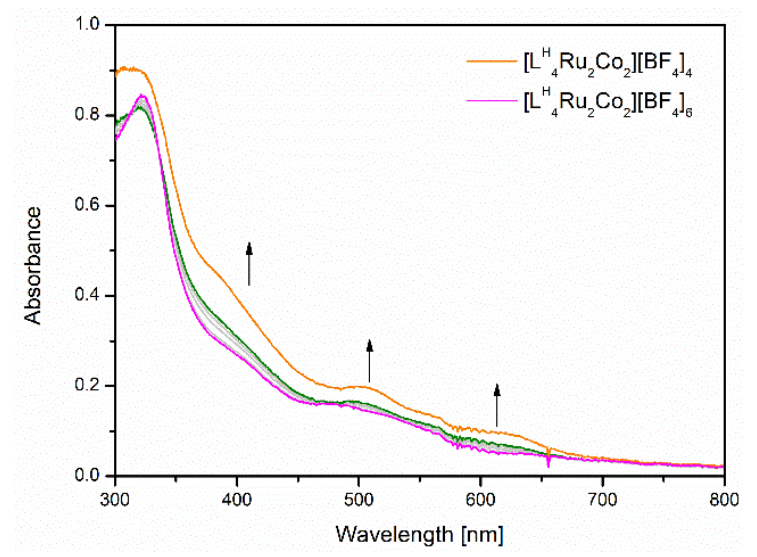

Figure 3.16. (a) Electrochemical oxidation of $\left[\mathrm{L}_{4} \mathrm{Ru}_{2} \mathrm{Co}_{2}\right]\left[\mathrm{BF}_{4}\right]_{4}$ to the doubly oxidized $\left[\mathrm{LH}_{4} \mathrm{Ru}_{2} \mathrm{Co}_{2}\right]\left[\mathrm{BF}_{4}\right]_{6}$ grid by application of a potential of $0.7 \mathrm{~V}$. (b) Re-reduction to $\left[\mathrm{L}_{4} \mathrm{Ru}_{2} \mathrm{Co}_{2}\right]\left[\mathrm{BF}_{4}\right]_{4}$ by application of a potential of $-0.6 \mathrm{~V}$. All potentials are referenced against $\mathrm{Fc} / \mathrm{Fc}^{+}$.

Naturally, the formation of the fully oxidized $\left[\mathrm{L}_{4} \mathrm{Ru}_{2} \mathrm{Co}_{2}\right]\left[\mathrm{BF}_{4}\right]_{8}$ grid can also be monitored following a similar procedure. Here, a voltage of $1.3 \mathrm{~V}$ was applied starting from the electrochemically produced doubly oxidized $\left[\mathrm{LH}_{4} \mathrm{Ru}_{2} \mathrm{Co}_{2}\right]\left[\mathrm{BF}_{4}\right]_{6}$ grid to form the fully oxidized $\left[\mathrm{LH}_{4} \mathrm{Ru}_{2} \mathrm{Co}_{2}\right]\left[\mathrm{BF}_{4}\right]_{8}$ grid (Figure 3.17a). A further decrease in the absorption of the bands at $402 \mathrm{~nm}$ and $517 \mathrm{~nm}$ concomitant with the rise of a new broad band at $652 \mathrm{~nm}$ was observed. This band is reminiscent of the broad band of the $\mathrm{Ru}^{\mathrm{II}}$ "corner complex", indicating that the latter two oxidative waves observed in the cyclic voltammogram correspond to the $\mathrm{Ru}^{\mathrm{II}} / \mathrm{Ru}^{\mathrm{III}}$ couples. Upon reversal by applying a voltage of $1.3 \mathrm{~V}$, the intensity of the MLCT bands increases nearly to its original intensity and the new band at $650 \mathrm{~nm}$ disappears suggesting full conversion of $\left[\mathrm{L}_{4} \mathrm{Ru}_{2} \mathrm{Co}_{2}\right]\left[\mathrm{BF}_{4}\right]_{8}$ to $\left[\mathrm{L}_{4} \mathrm{Ru}_{2} \mathrm{Co}_{2}\right]\left[\mathrm{BF}_{4}\right]_{6}$ (Figure $3.17 \mathrm{~b}$, pink).

(a)

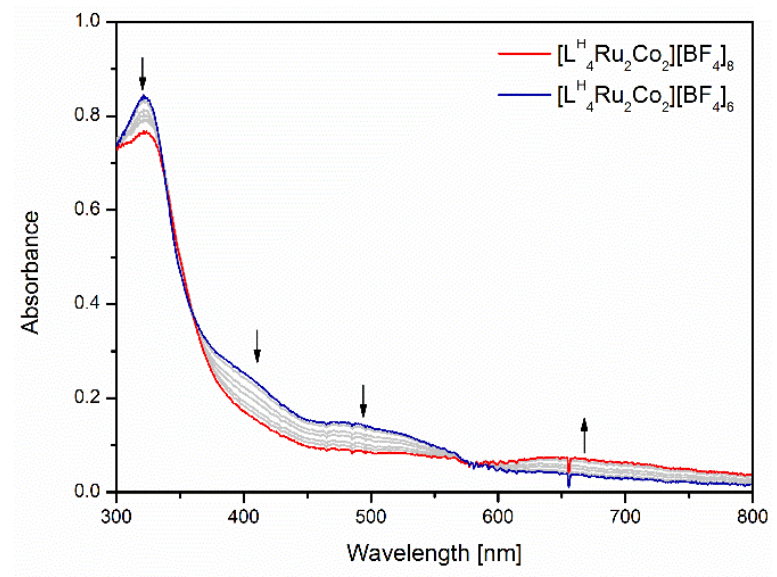

(b)

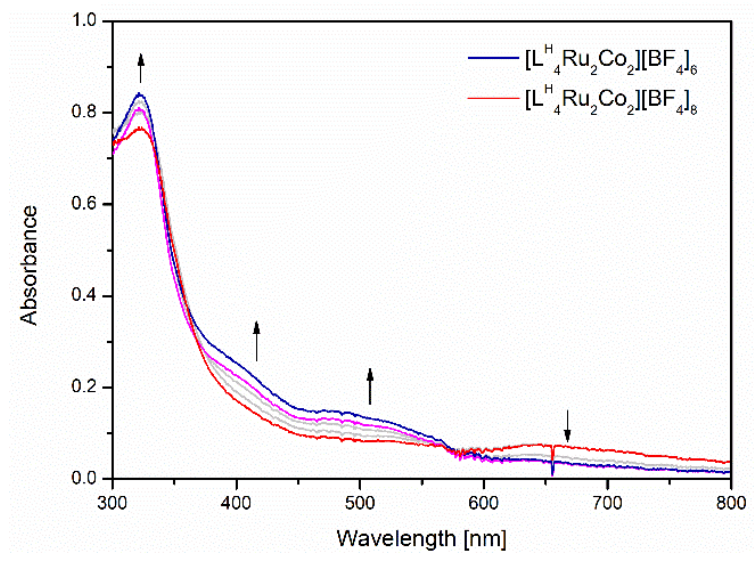

Figure 3.17. (a) A potential of $1.3 \mathrm{~V}$ was applied to obtain the fully oxidized $\left[\mathrm{L}_{4} \mathrm{Ru}_{2} \mathrm{Co}_{2}\right]\left[\mathrm{BF}_{4}\right]_{8}$ in acetonitrile followed by (b) re-reduction to the doubly oxidized $\left[\mathrm{L}_{4} \mathrm{Ru}_{2} \mathrm{Co}_{2}\right]\left[\mathrm{BF}_{4}\right]_{6}$ grid by applying a potential of $0.7 \mathrm{~V}$. All potentials are referenced against $\mathrm{Fc} / \mathrm{Fc}^{+}$. 


\section{4 - Synthesis and Characterization of Doubly Oxidized $\left[\mathrm{L}_{4} \mathrm{Ru}_{2} \mathrm{Co}_{2}\right]\left[\mathrm{BF}_{4}\right]_{6}$}

As mentioned earlier, the four oxidative waves observed in the cyclic voltammogram in Figure 3.14 could not be assigned to the specific metal ions at that point. Consequently, in order to determine which metal ions are oxidized first, the doubly oxidized $\left[\mathrm{LH}_{4} \mathrm{Ru}_{2} \mathrm{Co}_{2}\right]\left[\mathrm{BF}_{4}\right]_{6}$ was chemically synthesized by adding two equivalents of [thianthrene $]^{+}\left[\mathrm{BF}_{4}\right]^{-}$into a solution of $\left[\mathrm{L}_{4} \mathrm{Ru}_{2} \mathrm{Co}_{2}\right]\left[\mathrm{BF}_{4}\right]_{4}$ in dry acetonitrile (Scheme 3.3). Upon addition, the brown solution immediately turns dark red and the reaction was stirred at room temperature overnight. Next day, the solution was filtered and precipitated with diethyl ether to give a burgundy solid, which was purified by slow diffusion of diethyl ether into a solution of the product in acetonitrile.

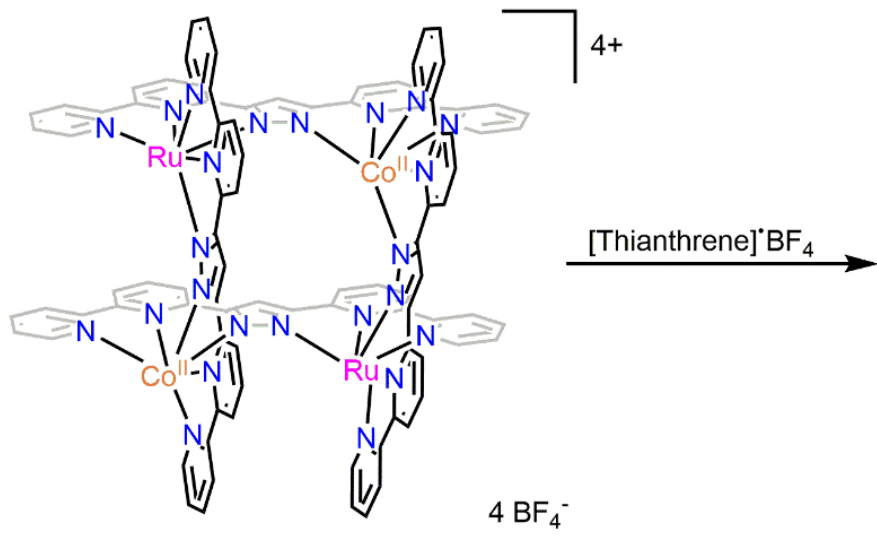

3-2

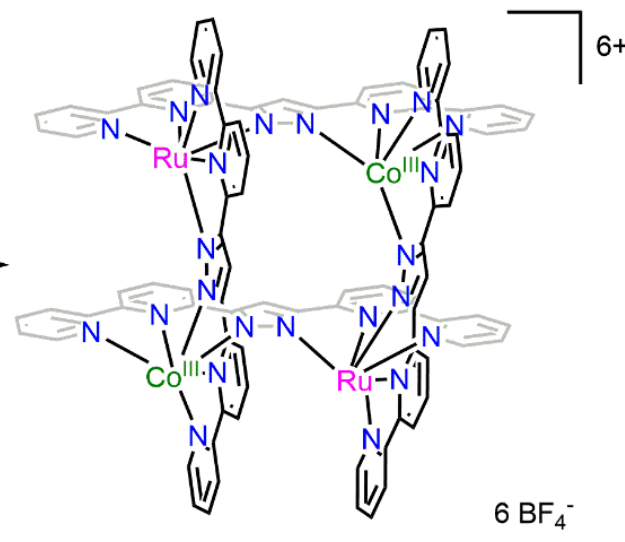

3-3

Scheme 3.3. Synthetic scheme for the doubly oxidized $\left[\mathrm{LH}_{4} \mathrm{Ru}_{2} \mathrm{Co}_{2}\right]\left[\mathrm{BF}_{4}\right]_{6}$ grid.

\subsection{1 - ${ }^{1} \mathrm{H}$ NMR Spectroscopy}

The integrity of the doubly oxidized $\left[\mathrm{L}_{4} \mathrm{Ru}_{2} \mathrm{Co}_{2}\right]\left[\mathrm{BF}_{4}\right]_{6}$ grid was investigated by ${ }^{1} \mathrm{H}$ NMR spectroscopy in $\mathrm{MeCN}-\mathrm{d}_{3}$ at room temperature, displaying 15 peaks in the diamagnetic region (5- $10 \mathrm{ppm}$ ), as illustrated in Figure 3.18. Despite its highly charged nature, the doubly oxidized grid remains intact and only a single isomer is observed based on the simplicity of the ${ }^{1} \mathrm{H}$ NMR spectrum. The hydrogen atom on the pyrazole backbone of $\left[\mathrm{L}^{\mathrm{H}}{ }_{4} \mathrm{Ru}_{2} \mathrm{Co}_{2}\right]\left[\mathrm{BF}_{4}\right]_{6}$ is assigned to the singlet at $8.57 \mathrm{ppm}$. The remaining hydrogen atoms are assigned using 2D NMR spectroscopy (Figure A29). Another important feature entails the shift of all the resonances of $\left[\mathrm{L}_{4} \mathrm{Ru}_{2} \mathrm{Co}_{2}\right]\left[\mathrm{BF}_{4}\right]_{4}(-30$ to $+120 \mathrm{ppm})$ into the diamagnetic regime (5-10 ppm), supporting that the paramagnetic high-spin $\mathrm{Co}^{\mathrm{II}}$ ions are oxidized first to low-spin $\mathrm{Co}^{\mathrm{III}}$ ions. This was further confirmed by X-ray diffraction analysis on the doubly oxidized $\left[\mathrm{LH}_{4} \mathrm{Ru}_{2} \mathrm{Co}_{2}\right]\left[\mathrm{BF}_{4}\right]_{6}$ grid, discussed in the following section. 

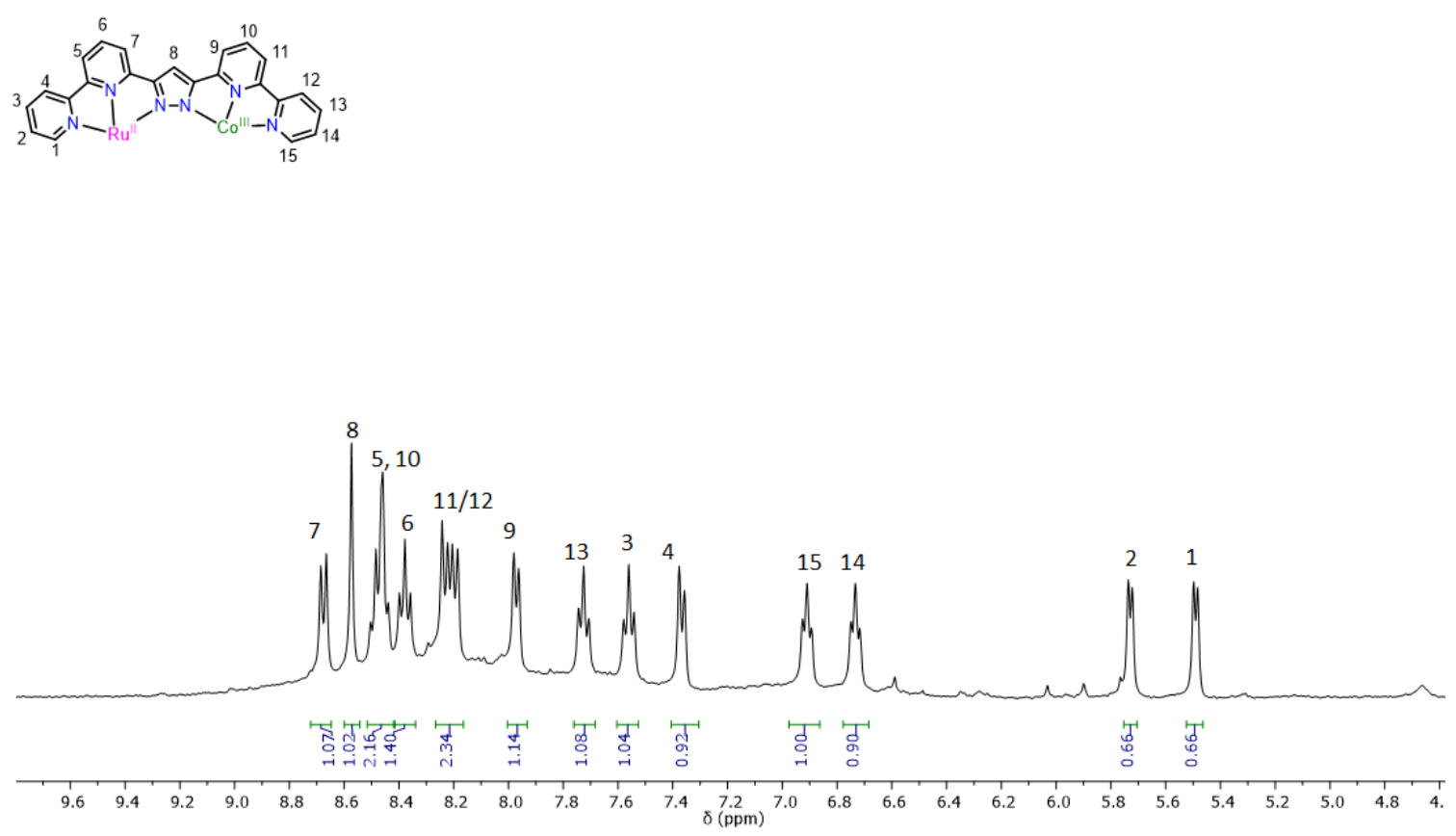

Figure 3.18. ${ }^{1} \mathrm{H}$ NMR spectrum of doubly oxidized $\left[\mathrm{LH}_{4} \mathrm{Ru}_{2} \mathrm{Co}_{2}\right]\left[\mathrm{BF}_{4}\right]_{6}$ in $\mathrm{MeCN}-\mathrm{d}_{3}$ at $298 \mathrm{~K}$.

\subsection{2 - X-ray Diffraction of the Doubly Oxidized $\left[\mathrm{L}_{4} \mathrm{Ru}_{2} \mathrm{Co}_{2}\right]\left[\mathrm{BF}_{4}\right]_{6} \mathrm{Grid}$}

X-ray diffraction analysis on single crystals of $\left[\mathrm{LH}_{4} \mathrm{Ru}_{2} \mathrm{Co}_{2}\right]\left[\mathrm{BF}_{4}\right]_{6}$ revealed the expected gridlike architecture crystallizing in the monoclinic space group $C 2 / c$ (Figure 3.19). The $6^{+}$ charged cation is balanced by six tetrafluoroborate anions and acetonitrile molecules are also present in the crystal lattice. Unlike the $\left[\mathrm{LH}_{4} \mathrm{Ru}_{2} \mathrm{Co}_{2}\right]\left[\mathrm{BF}_{4}\right]_{4}$ congener, both ruthenium and cobalt ions are related by $\mathrm{C}_{2}$ symmetry. The ruthenium and cobalt ions are separated by approximately $4.6 \AA$ with $\mathrm{Ru}-\mathrm{Co}-\mathrm{Ru}$ and $\mathrm{Co}-\mathrm{Ru}-\mathrm{Co}$ angles of $85^{\circ}$ and $95^{\circ}$, respectively. Although $\left[\mathrm{L}_{4} \mathrm{Ru}_{2} \mathrm{Co}_{2}\right]\left[\mathrm{BF}_{4}\right]_{6}$ retains the rhombus geometry observed in the parent $\left[\mathrm{L}_{4} \mathrm{Ru}_{2} \mathrm{Co}_{2}\right]\left[\mathrm{BF}_{4}\right]_{4}$ grid, the $\mathrm{Ru}-\mathrm{Co}-\mathrm{Ru}$ and $\mathrm{Co}-\mathrm{Ru}-\mathrm{Co}$ angles are much closer to an ideal octahedral angle of $90^{\circ}$. Moreover, analysis of the Ru-N and Co-N bond lengths of $2.079 \AA$ and $1.94 \AA$ suggests the presence of low-spin $\mathrm{Ru}^{\mathrm{II}}$ ions and low-spin $\mathrm{Co}^{\mathrm{II}}$ ions, respectively, consistent with the diamagnetic ${ }^{1} \mathrm{H}$ NMR spectrum. 
(a)

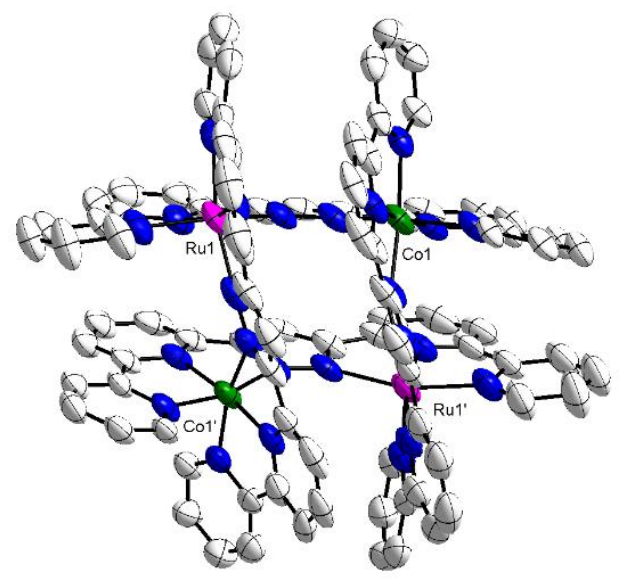

(b)

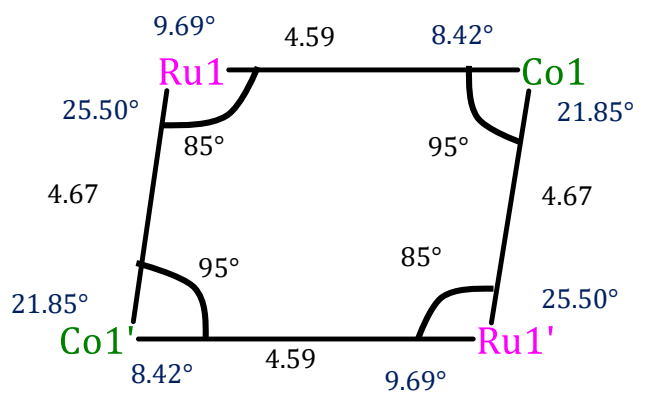

Figure 3.19. (a) Molecular structure (50\% probability thermal ellipsoids) of the doubly oxidized $\left[\mathrm{LH}_{4} \mathrm{Ru}_{2} \mathrm{Co}_{2}\right]\left[\mathrm{BF}_{4}\right]_{6}$ grid. Ruthenium ions are shown in pink and cobalt ions are shown in orange. Hydrogen atoms and solvent molecules are omitted for clarity. (b) Schematic representation of $\left[\mathrm{LH}_{4} \mathrm{Ru}_{2} \mathrm{Co}_{2}\right]\left[\mathrm{BF}_{4}\right]_{6}, \mathrm{Ru} \cdots$ Co distances are shown in black, $\mathrm{Ru}-\mathrm{Co}-\mathrm{Ru}$ and Co$\mathrm{Ru}-\mathrm{Co}$ angles are shown in purple, and torsion angles are shown in blue.

The preference for an octahedral environment can also be determined by analysis of the planarity of the ligand strands. For instance, the torsion angle between the terminal pyridine moiety and the central pyrazole moiety of one ligand strand was calculated revealing minimal differences between one end and the other. This was further confirmed with Continuous Symmetry Measures (CSM), affording small S( $\left.\mathrm{O}_{\mathrm{h}}\right)$ values of 4.09 and 1.57 for Ru1 (Ru1') and Co1(Co1'), respectively. For comparison, the S(itp) for trigonal prism geometry were also computed and tabulated in Table 3.5. Consequently, the combination of the crystal structure and ${ }^{1} \mathrm{H}$ NMR spectroscopy of the doubly oxidized $\left[\mathrm{L}_{4} \mathrm{Ru}_{2} \mathrm{Co}_{2}\right]\left[\mathrm{BF}_{4}\right]_{6}$ grid confirmed that the high-spin $\mathrm{Co}^{\mathrm{II}}$ ions are oxidized first.

Table 3.5. Relevant bond lengths and CSM for $\left[\mathrm{L}_{4} \mathrm{Ru}_{2} \mathrm{Co}_{2}\right]\left[\mathrm{BF}_{4}\right]_{6}$.

\begin{tabular}{lllll}
\hline & $\boldsymbol{d}_{\text {avg }}(\AA)$ & Spin-state & $\mathbf{S}\left(\mathbf{O}_{\mathbf{h}}\right)$ & $\mathbf{S}(\mathbf{i t p})$ \\
\hline Ru1-N & 2.08 & LS & 4.09 & 8.17 \\
Ru1'-N & 2.08 & LS & 4.09 & 7.71 \\
Co1-N & 1.94 & LS & 1.57 & 11.32 \\
Co1'-N & 1.94 & LS & 1.57 & 11.31 \\
\hline
\end{tabular}




\subsection{3 - UV-Vis-NIR on $\left[\mathrm{L}_{4} \mathrm{Ru}_{2} \mathrm{Co}_{2}\right]\left[\mathrm{BF}_{4}\right]_{6}$}

UV-Vis-NIR was conducted on the doubly oxidized $\left[\mathrm{L}_{4} \mathrm{Ru}_{2} \mathrm{Co}_{2}\right]\left[\mathrm{BF}_{4}\right]_{6}$ grid in MeCN illustrating the absence of IVCT bands, as expected since both $\mathrm{Co}^{\mathrm{II}}$ ions are oxidized first. A comparison of the parent $\left[\mathrm{LH}_{4} \mathrm{Ru}_{2} \mathrm{Co}_{2}\right]\left[\mathrm{BF}_{4}\right]_{4}$ grid and doubly oxidized $\left[\mathrm{LH}_{4} \mathrm{Ru}_{2} \mathrm{Co}_{2}\right]\left[\mathrm{BF}_{4}\right]_{6}$ grid is shown in Figure 3.20. Similar to the UV-Vis spectroelectrochemistry of $\left[\mathrm{LH}_{4} \mathrm{Ru}_{2} \mathrm{Co}_{2}\right]\left[\mathrm{BF}_{4}\right]_{4}$ to form the doubly oxidized $\left[\mathrm{L}_{4} \mathrm{Ru}_{2} \mathrm{Co}_{2}\right]\left[\mathrm{BF}_{4}\right]_{6}$ grid, the shoulder at $420 \mathrm{~nm}$ observed in the parent grid disappears after oxidation and the weak band at $450 \mathrm{~nm}$ also decreases in intensity.

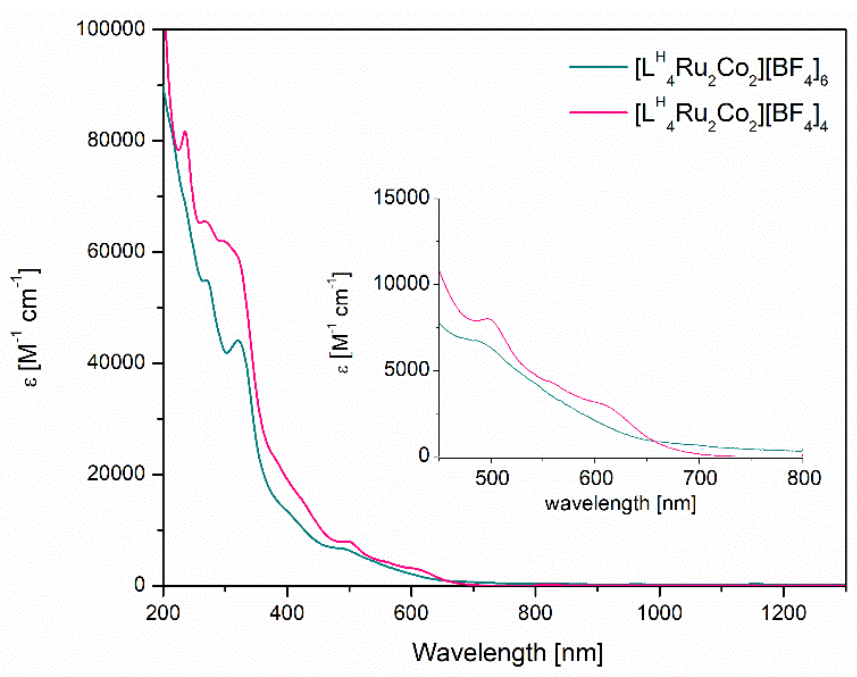

Figure 3.20. UV-Vis-NIR of $\left[\mathrm{L}_{4} \mathrm{Ru}_{2} \mathrm{Co}_{2}\right]\left[\mathrm{BF}_{4}\right]_{4}$ and $\left[\mathrm{L}_{4} \mathrm{Ru}_{2} \mathrm{Co}_{2}\right]\left[\mathrm{BF}_{4}\right]_{6}$ in $\mathrm{MeCN}$ at room temperature.

\section{5 - Summary \& Conclusion}

In conclusion, a new heterometallic $\left[\mathrm{LH}_{4} \mathrm{Ru}_{2} \mathrm{Co}_{2}\right]\left[\mathrm{BF}_{4}\right]_{4}$ grid has been synthesized utilizing a step-wise approach to selectively enforce the anti-isomer. Based on the simplicity of the ${ }^{1} \mathrm{H}$ NMR spectrum, one can conclude that only the anti-isomer is formed. Moreover, the redox properties were investigated illustrating four oxidative waves. Using the so-obtained electrochemical potentials, the doubly oxidized $\left[\mathrm{L}_{4} \mathrm{Ru}_{2} \mathrm{Co}_{2}\right]\left[\mathrm{BF}_{4}\right]_{6}$ grid was chemically synthesized, where X-ray crystallography and ${ }^{1} \mathrm{H}$ NMR spectroscopy confirmed that the first two oxidative waves correspond to the $\mathrm{Co}^{\mathrm{II}} / \mathrm{Co}^{\mathrm{III}}$ couple and thus the latter two anodic waves is assumed to correspond to the $\mathrm{Ru}^{\mathrm{II}} / \mathrm{Ru}^{\mathrm{III}}$ couple. In addition, the magnetic properties of the $\left[\mathrm{L}_{4} \mathrm{Ru}_{2} \mathrm{Co}_{2}\right]\left[\mathrm{BF}_{4}\right]_{4}$ grid were investigated, in which the diagonally positioned high-spin Co ${ }^{\mathrm{II}}$ ions are magnetically isolated, giving rise to unprecedented SMM behaviour in a $3 \mathrm{~d}$ transition metal heterometallic [2 $\times 2]$ grid. 


\section{Chapter 4 - Synthesis and Characterization of Heterometallic $\left[\mathrm{L}_{4} \mathrm{Ru}_{2} \mathrm{Fe}_{2}\right]\left[\mathrm{BF}_{4}\right]_{4} \mathrm{Grid}$}

\section{1 - Introduction}

In the previous chapter, the physical and chemical properties of a new heterometallic $\left[\mathrm{LH}_{4} \mathrm{Ru}_{2} \mathrm{Co}_{2}\right]\left[\mathrm{BF}_{4}\right]_{4}$ grid were presented. Moreover, the synthetic strategy for multicomponent systems, in which the metal ions are sequentially introduced according to their lability was also highlighted. Utilizing this method, a kinetically inert ruthenium "corner complex" was first isolated followed by introduction of Fe ${ }^{\mathrm{II}}$ ions to obtain the desired $\left[\mathrm{L}_{4} \mathrm{Ru}_{2} \mathrm{Fe}_{2}\right][\mathrm{OTf}]_{4}$ grid. Based on the previous chapter as well as other heterometallic and mixed valent [2 x 2] grids, $81,87,124$ it comes to no surprise that the ruthenium "corner complex" selectively enforces a trans configuration, in which the ruthenium and iron ions are situated diagonally across from one another in the $[2 \times 2]$ grid. In the case of the $\left[\mathrm{L}_{4} \mathrm{Ru}_{2} \mathrm{Co}_{2}\right]\left[\mathrm{BF}_{4}\right]_{4}$ grid, the isolated magnetically active high-spin $\mathrm{Co}^{\mathrm{II}}$ ions gave rise to single molecule magnet $(\mathrm{SMM})^{38}$ behaviour. Although SMM behaviour is limited amongst $3 \mathrm{~d}$ transition metals compared to lanthanides, it has been observed with manganese, cobalt, and iron complexes.43,125-127 However, the primarily goal of incorporating Fe ions into the [2 $\mathrm{x} 2]$ grid framework is to investigate the potential for cooperative spin crossover, which has been observed in several polynuclear Fe complexes, ${ }^{19,36,128,129}$ as well as to elucidate IVCT transitions after oxidation to the mixed valent grid.

Although the synthetic strategy follows procedures similar to the $\left[\mathrm{L}_{4} \mathrm{Ru}_{2} \mathrm{Co}_{2}\right]\left[\mathrm{BF}_{4}\right]_{4}$ grid, a series of additional challenges were encountered. With the presence of Fe ions, additional spectroscopic techniques such as Mössbauer spectroscopy can be conducted, which surprisingly revealed the presence of multiple species. Moreover, since the synthesis of the $\left[\mathrm{LH}_{4} \mathrm{Ru}_{2} \mathrm{Fe}_{2}\right][\mathrm{OTf}]_{4}$ grid takes place at much lower temperatures compared to the $\left[\mathrm{L}_{4} \mathrm{Ru}_{2} \mathrm{Co}_{2}\right]\left[\mathrm{BF}_{4}\right]_{4}$ grid, the reaction pathway can be monitored by ${ }^{1} \mathrm{H}$ NMR spectroscopy. Although the linewidths of the resonances for $\left[\mathrm{L}_{4} \mathrm{Ru}_{2} \mathrm{Co}_{2}\right]\left[\mathrm{BF}_{4}\right]_{4}$ were too broad for $2 \mathrm{D} \mathrm{NMR}$ spectroscopy ( $>600 \mathrm{~Hz}$ ), the relaxation time of the protons in $\left[\mathrm{LH}_{4} \mathrm{Ru}_{2} \mathrm{Fe}_{2}\right]\left[\mathrm{BF}_{4}\right]_{4}$ gave rise to resonances with linewidths between $100-200 \mathrm{~Hz}$, and thus 2D NMR spectroscopy was conducted permitting full assignment of the protons. While significant progress has been made to synthesize the $\left[\mathrm{LH}_{4} \mathrm{Ru}_{2} \mathrm{Fe}_{2}\right][\mathrm{OTf}]_{4}$ exclusively, the minor impurity observed in the $\mathrm{MB}$ spectrum was never removed. Nevertheless, the redox and optical properties of the $\left[\mathrm{LH}_{4} \mathrm{Ru}_{2} \mathrm{Fe}_{2}\right][\mathrm{OTf}]_{4}$ grid will be discussed as well as plausible mechanistic pathways deduced from in situ Mössbauer and ${ }^{1} \mathrm{H}$ NMR experiments.

\section{2 - Results}

\subsection{1 - Synthesis}

The synthesis of $\left[\mathrm{LH}_{4} \mathrm{Ru}_{2} \mathrm{Fe}_{2}\right][\mathrm{OTf}]_{4}$ follows procedures similar to $\left[\mathrm{L}_{4}{ }_{4} \mathrm{Ru}_{2} \mathrm{Co}_{2}\right]\left[\mathrm{BF}_{4}\right]_{4}$ discussed in the previous chapter. Although both $\left[\mathrm{LH}_{4} \mathrm{Ru}_{2} \mathrm{Fe}_{2}\right][\mathrm{OTf}]_{4}$ and $\left[\mathrm{LH}_{4} \mathrm{Ru}_{2} \mathrm{Fe}_{2}\right]\left[\mathrm{BF}_{4}\right]_{4}$ were synthesized, only the former with triflate as the anion is shown in scheme 4.1. Initially, $\left[\mathrm{L}_{4} \mathrm{Ru}_{2} \mathrm{Fe}_{2}\right]\left[\mathrm{BF}_{4}\right]_{4}$ was used for X-ray diffraction analysis and magnetic measurements, however, for experiments conducted under inert atmosphere (inside the glove box), $\left[\mathrm{L}_{4} \mathrm{Ru}_{2} \mathrm{Fe}_{2}\right][\mathrm{OTf}]_{4}$ was used instead. A kinetically stable $\mathrm{Ru}^{\text {II }}$ "corner complex" 3-1b with the known compartmental pyrazolate-bridged ligand ${ }^{90}$ was first isolated then reacted with stoichiometric amounts of base $\left(\mathrm{NaO}^{t} \mathrm{Bu}\right.$ or $\left.\mathrm{NaOMe}\right)$ and $\mathrm{Fe}^{\mathrm{II}}(\mathrm{OTf})_{2} \cdot 2 \mathrm{MeCN}$ in dry acetonitrile or dimethylformamide (DMF) and heated at $50{ }^{\circ} \mathrm{C}$ for 16 hours. Crystalline material of 
$\left[\mathrm{L}_{4} \mathrm{Ru}_{2} \mathrm{Fe}_{2}\right]\left[\mathrm{BF}_{4}\right]_{4}$ were obtained by slow diffusion of diethyl ether into a solution of the product in acetonitrile.

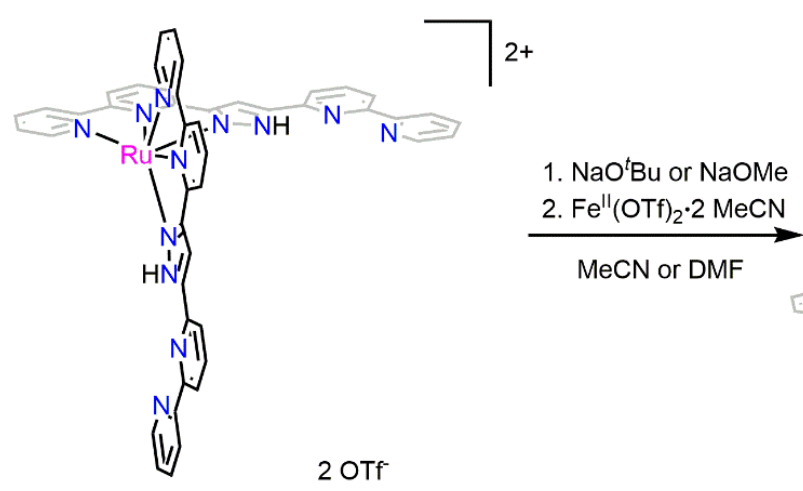

3-1b

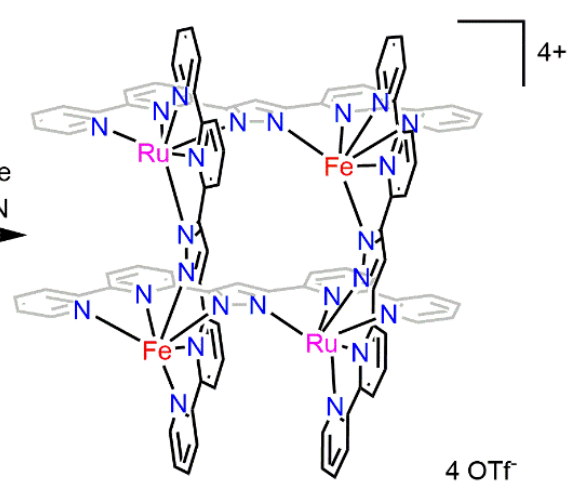

4-1

Scheme 4.1. Synthetic scheme for $\left[\mathrm{LH}_{4} \mathrm{Ru}_{2} \mathrm{Fe}_{2}\right][\mathrm{OTf}]_{4}$, commencing with the ruthenium "corner complex" 3-1b.

\subsection{2 - X-ray Crystallography}

Single crystals of $\left[\mathrm{L}_{4} \mathrm{Ru}_{2} \mathrm{Fe}_{2}\right]\left[\mathrm{BF}_{4}\right]_{4}$ were obtained by slow diffusion of diethyl ether into a solution of the product in MeCN. X-ray diffraction analysis on $\left[\mathrm{LH}_{4} \mathrm{Ru}_{2} \mathrm{Fe}_{2}\right]\left[\mathrm{BF}_{4}\right]_{4}$ revealed the expected [2 $\times 2$ ] grid-like array, crystallizing in the orthorhombic space group Pna $2_{1}$, with the ruthenium and iron atoms situated at opposite corners (Figure 4.1). Despite the symmetric nature of the molecule, the ruthenium and iron atoms are crystallographically independent. Moreover, four tetrafluroborate anions are present to balance the +4 charge of the grid and four acetonitrile molecules are also observed in the crystal lattice.

(a)

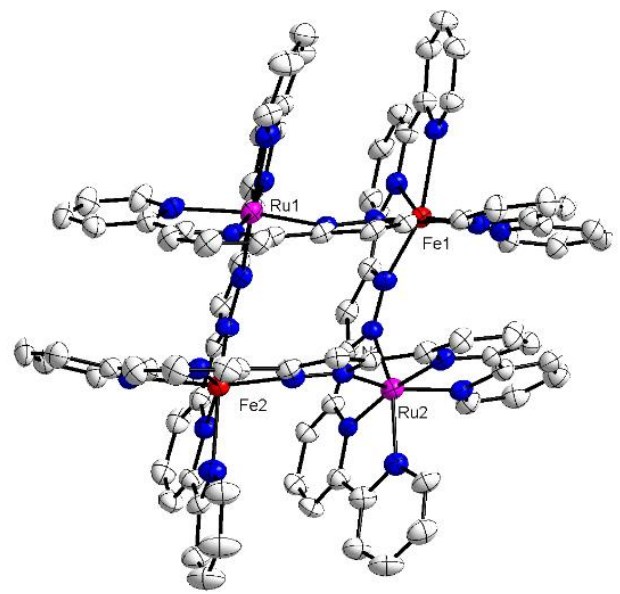

(b)

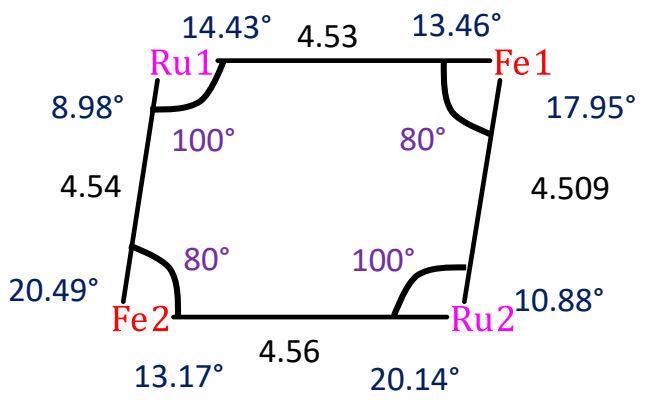

Figure 4.1. (a) Molecular structure of $\left[\mathrm{LH}_{4} \mathrm{Ru}_{2} \mathrm{Fe}_{2}\right]\left[\mathrm{BF}_{4}\right]_{4}$ (50\% probability thermal ellipsoids). Hydrogen atoms and counterions are omitted for clarity. (b) Schematic drawing of the $\mathrm{Ru} \cdots \mathrm{Fe}$ distances (black), Ru-Fe-Ru, and Fe-Ru-Fe angles (purple), and torsion angle defined by the plane of pyrazole moiety and the terminal pyridine (blue). 
Table 4.1. Relevant bond lengths and CSM for $\left[\mathrm{LH}_{4} \mathrm{Ru}_{2} \mathrm{Fe}_{2}\right]\left[\mathrm{BF}_{4}\right]_{4}$.

\begin{tabular}{ccccc}
\hline & $\boldsymbol{d}_{\text {avg }}(\AA)$ & Spin-state & $\mathbf{S}\left(\mathbf{O}_{\mathbf{h}}\right)$ & S(itp) \\
\hline Ru1-N & 2.05 & LS & 3.26 & 9.68 \\
Ru2-N & 2.05 & LS & 3.32 & 10.26 \\
Fe1-N & 2.17 & HS & 7.01 & 5.68 \\
Fe2-N & 2.18 & HS & 6.48 & 6.01 \\
\hline
\end{tabular}

Each metal ion is coordinated by the nitrogen atoms of two orthogonal ligands of the grid structure, forming an $\{\mathrm{N} 6\}$ octahedral environment. Analysis of the $\mathrm{Ru} \cdots \mathrm{Fe}$ distances and angles reveal an average separation of $4.54 \AA$ and $\mathrm{Ru}-\mathrm{Fe}-\mathrm{Ru}$ and Fe-Ru-Fe angles of $80^{\circ}$ and $100^{\circ}$, respectively. Inspection of the Ru-N and Fe-N bond lengths of $2.05 \AA$ and $2.18 \AA$ suggest the presence of LS-RuII ions and HS-FeII ions. Relevant bond lengths are tabulated in Table 4.1.

In order to describe the overall distortion of the ligand (from planarity), the torsion angle between the pyrazole moiety and the terminal pyridine was calculated for each ligand strand (Figure $4.1 \mathrm{~b}$, blue). Based on these values, one end of the ligand strand is typically more twisted relative to the central pyrazole moiety compared to the other end except for the ligand strand coordinated to Ru1 and Fe1, in which a torsion angle of $13^{\circ}$ and $14^{\circ}$ was observed for both terminal pyridine units. The distortion form an ideal octahedral environment was further confirmed using Continuous Symmetry Measures (CSM), ${ }^{83}$ where a small S $\left(\mathrm{O}_{\mathrm{h}}\right)$ value reflects an octahedral environment, while a large $\mathrm{S}\left(\mathrm{O}_{\mathrm{h}}\right)$ value indicates significant distortions from an ideal octahedron. Correlations between the $\mathrm{S}\left(\mathrm{O}_{\mathrm{h}}\right)$ values and $\mathrm{S}(\mathrm{itp})$ values have been well established, in which a high $\mathrm{S}\left(\mathrm{O}_{\mathrm{h}}\right)$ value corresponds to a low $\mathrm{S}(\mathrm{itp})$ value. Using this method, small $\mathrm{S}\left(\mathrm{O}_{\mathrm{h}}\right)$ values of 3.26 and 3.32 were determined for Ru1 and Ru2, respectively, as expected for low-spin $\mathrm{Ru}^{\mathrm{II}}$ ions. On the contrary, large $\mathrm{S}\left(\mathrm{O}_{\mathrm{h}}\right)$ values of 7.01 and 6.48 were obtained for $\mathrm{Fe} 1$ and $\mathrm{Fe} 2$, respectively, confirming the presence of a highly distorted environment. For comparison, the distortion from a trigonal prism geometry, $\mathrm{S}(\mathrm{itp})$, are also tabulated in Table 4.1. Thus, the high $\mathrm{S}\left(\mathrm{O}_{\mathrm{h}}\right)$ values for the Fe centers suggest that the Fe ions are most likely locked in the HS-state and thermal spin crossover is unlikely. 


\subsection{3 - Mass Spectrometry}

In order to confirm the integrity of the $\left[\mathrm{L}_{4} \mathrm{Ru}_{2} \mathrm{Fe}_{2}\right]\left[\mathrm{BF}_{4}\right]_{4}$ grid in solution, ESI-MS of a MeCN solution of 4-1 was conducted revealing a single peak at $m / z=454$ (Figure 4.2), corresponding to the tetracationic $\left[\mathrm{L}_{4} \mathrm{Ru}_{2} \mathrm{Fe}_{2}\right]^{4+}$ ion. This confirms the stability of the grid under ambient conditions in solution. Although ruthenium complexes typically have a very distinct isotopic pattern, ${ }^{130,131}$ only a broad peak was observed due to the limited resolution of the instrument.

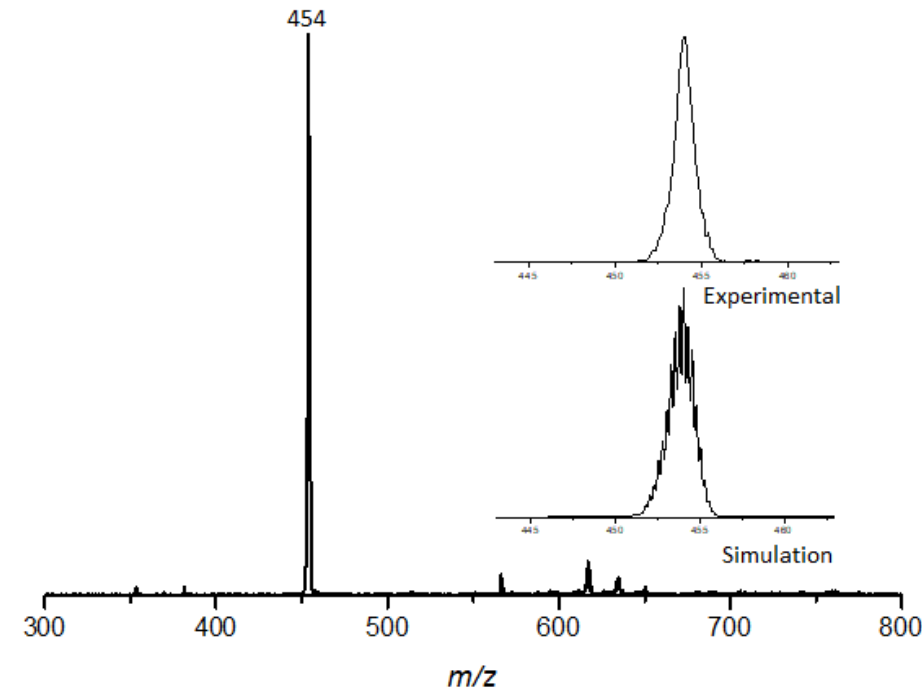

Figure 4.2. ESI mass spectrum of $\left[\mathrm{LH}_{4} \mathrm{Ru}_{2} \mathrm{Fe}_{2}\right]\left[\mathrm{BF}_{4}\right]_{4}$ in acetonitrile, revealing one peak at $m / z=454$ corresponding to $\left[\mathrm{L}_{4} \mathrm{Ru}_{2} \mathrm{Fe}_{2}\right]^{4+}$. Experimental and simulated isotopic distribution patterns are shown in the inset.

\subsection{4 - Cyclic Voltammetry and UV-vis Spectroelectrochemistry}

The redox properties of $\left[\mathrm{LH}_{4} \mathrm{Ru}_{2} \mathrm{Fe}_{2}\right]\left[\mathrm{BF}_{4}\right]_{4}$ in acetonitrile were investigated using cyclic voltammetry with $\left[\mathrm{Bu}_{4} \mathrm{~N}\right] \mathrm{PF}_{6}$ as the electrolyte. As expected, four reversible oxidative waves are observed at $E_{1 / 2}=330 \mathrm{mV}, 505 \mathrm{mV}, 880 \mathrm{mV}$, and $1060 \mathrm{mV}$ versus $\mathrm{Fc} / \mathrm{Fc}^{+}$, which correspond to the step-wise oxidation of each metal ion to form the highly charged $\left[\mathrm{L}_{4} \mathrm{Ru}_{2} \mathrm{Fe}_{2}\right]^{8+}$ grid (Figure 4.3). At this point, the oxidative waves could not be assigned to their respective metal and thus are labelled corresponding to the charge of the complex. With increasing scan rate, $E_{1 / 2}$ changes minimally and peak separations of each process, $\Delta E_{\mathrm{p}}$, fall between $100-110 \mathrm{mV}$, consistent with reversible oxidative behaviour. Similar to the previously reported $\left[\mathrm{LH}_{4} \mathrm{Ru}_{2} \mathrm{Co}_{2}\right]\left[\mathrm{BF}_{4}\right]_{4}$ grid discussed in chapter 3 , a large separation is observed between the $5^{+} / 6^{+}$and $6^{+} / 7^{+}$couples, signifying a pronounced thermodynamic stability for the doubly oxidized $\left[\mathrm{L}_{4}{ }_{4} \mathrm{Ru}_{2} \mathrm{Fe}_{2}\right]^{6+}$ grid $\left(K_{\mathrm{c}}=2.19 \times 10^{6}\right)$. Although the doubly oxidized $\left[\mathrm{L}_{4} \mathrm{Ru}_{2} \mathrm{Fe}_{2}\right]\left[\mathrm{BF}_{4}\right]_{6}$ grid was never isolated, preliminary $\mathrm{MB}$ data revealed the disappearance of the HS-Fe ${ }^{\mathrm{II}}$ species observed in $\left[\mathrm{L}_{4} \mathrm{Ru}_{2} \mathrm{Fe}_{2}\right]\left[\mathrm{BF}_{4}\right]_{4}$ (discussed below), suggesting the first two anodic waves may correspond to oxidation of the $\mathrm{Fe}^{\mathrm{II}}$ ions. However, only a broad non-resolved doublet was observed in the MB spectrum at $80 \mathrm{~K}$. 

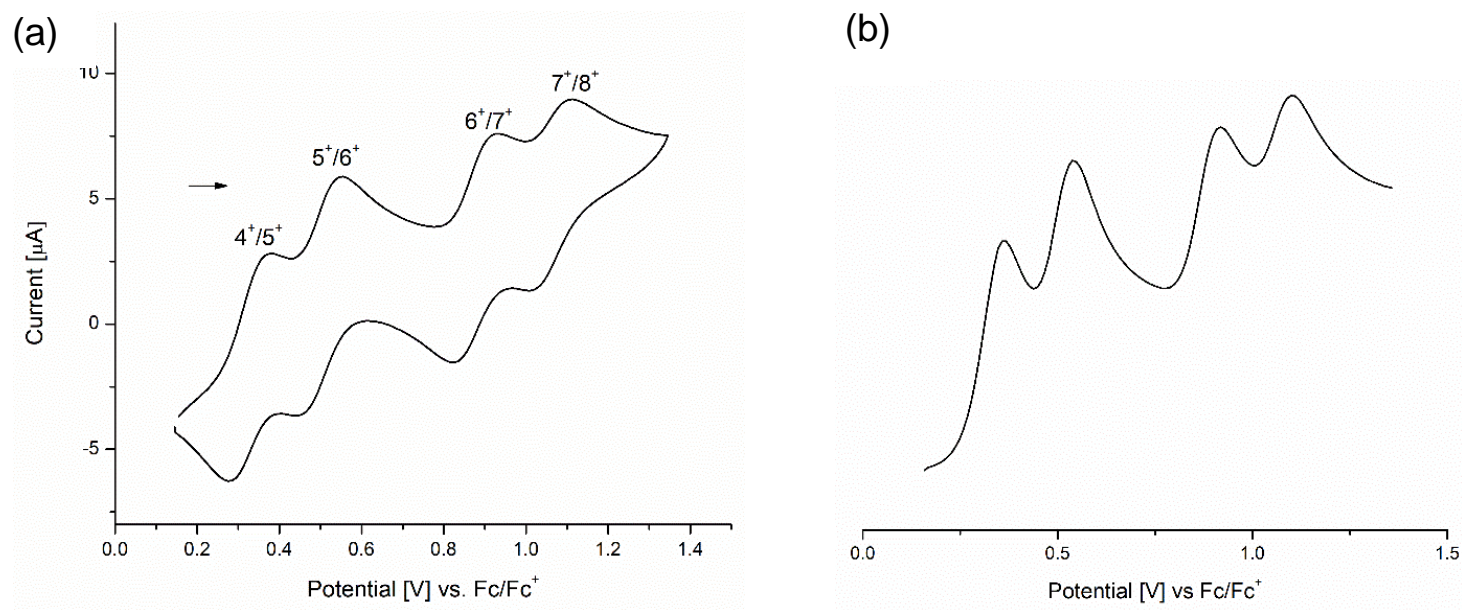

Figure 4.3. (a) Cyclic voltammetry of $\left[\mathrm{LH}_{4} \mathrm{Ru}_{2} \mathrm{Fe}_{2}\right]\left[\mathrm{BF}_{4}\right]_{4}$ in acetonitrile with $\left[\mathrm{Bu}_{4} \mathrm{~N}\right] \mathrm{PF}_{6}$ as the electrolyte. (b) Square wave voltammogram illustrating the four oxidative waves. All potentials are referenced against $\mathrm{Fc} / \mathrm{Fc}^{+}$.

Table 4.2. Electrochemical parameters for $\left[\mathrm{L}_{4} \mathrm{Ru}_{2} \mathrm{Fe}_{2}\right]\left[\mathrm{BF}_{4}\right]_{4}$. All potentials are referenced vs $\mathrm{Fc} / \mathrm{Fc}^{+}$.

\begin{tabular}{cccc}
\hline & $E_{1 / 2}[\mathrm{mV}]$ & $\Delta E_{\mathrm{p}}[\mathrm{mV}]$ & $\Delta E_{1 / 2}-\Delta E_{1 / 2}[\mathrm{mV}]$ \\
\hline $4+/ 5+$ & 330 & 110 & \\
$5+/ 6+$ & 505 & 100 & 175 \\
$6+/ 7+$ & 880 & 110 & 375 \\
$7+/ 8+$ & 1060 & 110 & 180 \\
\hline
\end{tabular}

The UV-vis spectrum of the $\left[\mathrm{L}_{4} \mathrm{Ru}_{2} \mathrm{Fe}_{2}\right]\left[\mathrm{BF}_{4}\right]_{4}$ grid is similar to the UV-Vis spectrum of $\left[\mathrm{LH}_{4} \mathrm{Ru}_{2} \mathrm{Co}_{2}\right]\left[\mathrm{BF}_{4}\right]_{4}$ (Figure 4.4), where a strong band at $388 \mathrm{~nm}$ is observed corresponding to the ligand $\pi-\pi^{*}$ transition also observed in the homonuclear $\left[\mathrm{LR}_{4} \mathrm{Fe}_{4}\right]\left[\mathrm{BF}_{4}\right]_{4}$ grids $(\mathrm{R}=\mathrm{H}, \mathrm{Me}$, $\mathrm{Br}){ }^{36,91,100}$ The weak bands at $495 \mathrm{~nm}$ and $556 \mathrm{~nm}$ most likely correspond to the MLCT transitions from the different metal centers. ${ }^{82}$ In order to monitor the electrochemical changes during oxidation of $\left[\mathrm{LH}_{4} \mathrm{Ru}_{2} \mathrm{Fe}_{2}\right]\left[\mathrm{BF}_{4}\right]_{4}$, UV-vis spectroelectrochemistry was conducted on $\left[\mathrm{LH}_{4} \mathrm{Ru}_{2} \mathrm{Fe}_{2}\right]\left[\mathrm{BF}_{4}\right]_{4}$ in $\mathrm{MeCN}$ by applying a potential at $0.77 \mathrm{~V}$ vs $\mathrm{Fc} / \mathrm{Fc}^{+}$to generate the doubly oxidized $\left[\mathrm{LH}_{4} \mathrm{Ru}_{2} \mathrm{Fe}_{2}\right]^{6+}$ grid followed by application of a potential of $1.4 \mathrm{~V}$ vs $\mathrm{Fc} / \mathrm{Fc}^{+}$to generate the fully oxidized $\left[\mathrm{L}_{4} \mathrm{Ru}_{2} \mathrm{Fe}_{2}\right]^{8+}$ grid. 


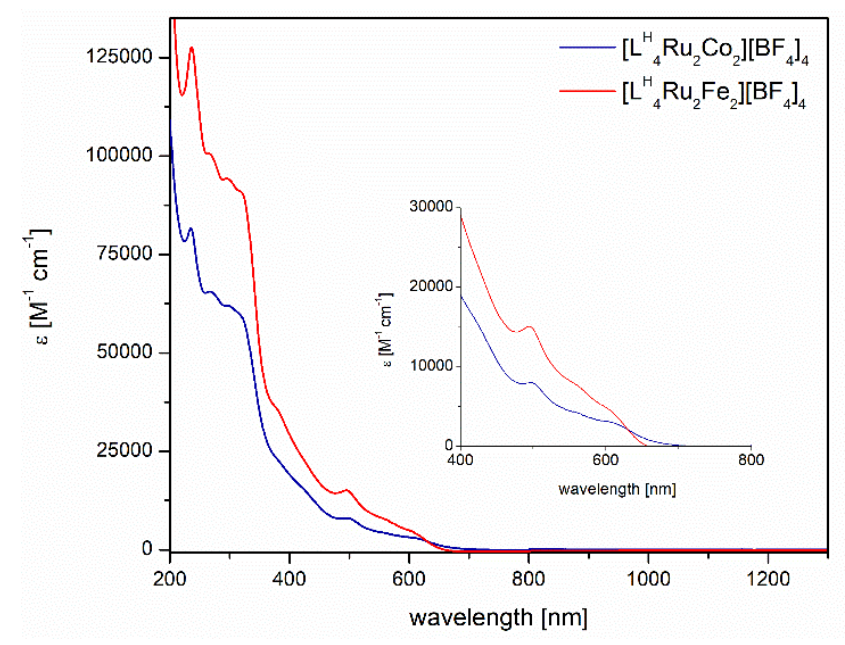

Figure 4.4. UV-Vis spectrum of $\left[\mathrm{L}_{4} \mathrm{Ru}_{2} \mathrm{Fe}_{2}\right]\left[\mathrm{BF}_{4}\right]_{4}$ and $\left[\mathrm{L}_{4} \mathrm{Ru}_{2} \mathrm{Co}_{2}\right]\left[\mathrm{BF}_{4}\right]_{4}$ illustrating minimal differences between the two complexes.

While the UV-vis spectra of $\left[\mathrm{LH}_{4} \mathrm{Ru}_{2} \mathrm{Fe}_{2}\right]\left[\mathrm{BF}_{4}\right]_{4}$ and $\left[\mathrm{LH}_{4} \mathrm{Ru}_{2} \mathrm{Co}_{2}\right][\mathrm{OTf}]_{4}$ are quite similar, electrochemical production of the doubly oxidized $\left[\mathrm{L}_{4} \mathrm{Ru}_{2} \mathrm{Fe}_{2}\right]^{6+}$ and fully oxidized $\left[\mathrm{L}_{4} \mathrm{Ru}_{2} \mathrm{Fe}_{2}\right]^{8+}$ grid (Figure 4.5) revealed several differences compared to the electrochemical oxidation of $\left[\mathrm{LH}_{4} \mathrm{Ru}_{2} \mathrm{Co}_{2}\right]\left[\mathrm{BF}_{4}\right]_{4}$ described in the previous chapter. Firstly, application of a potential of $0.77 \mathrm{~V}$ vs $\mathrm{Fc} / \mathrm{Fc}^{+}$for 20 minutes gave rise to two isosbestic points, whereas electrochemical oxidation to the doubly oxidized $\left[\mathrm{L}_{4} \mathrm{Ru}_{2} \mathrm{Co}_{2}\right]^{6+}$ grid did not display any isosbestic points (instead, a decrease in absorption of all bands were observed). The presence of these isosbestic points suggest a highly localized nature in the optical excitations, which may correspond to the oxidation of two diagonally opposed metal ions (i.e. both Fe ions or both $\mathrm{Ru}$ ions), also observed in the mixed valent $\left[\mathrm{L}^{\mathrm{Br}_{4} \mathrm{Fe}_{4}}\right]^{6+}$ grid. ${ }^{82}$ Secondly, upon electrochemical oxidation to the doubly oxidized $\left[\mathrm{L}_{4} \mathrm{Ru}_{2} \mathrm{Fe}_{2}\right]^{6+}$ grid, the two bands at $386 \mathrm{~nm}$ and $495 \mathrm{~nm}$ gradually decrease, but a new broad band at $654 \mathrm{~nm}$ was observed, which may correspond to a ligand-to-metal-charge transfer (LMCT).

Application of a potential of $1.4 \mathrm{~V}$ to obtain the fully oxidized $\left[\mathrm{L}_{4} \mathrm{Ru}_{2} \mathrm{Fe}_{2}\right]^{8+}$ grid revealed a further decrease in intensity of the broad band centered at $445 \mathrm{~nm}$ and a further increase in absorption of the band at $630 \mathrm{~nm}$ (Figure 4.5b). Based on the UV-vis spectrum of the electrochemically generated $\left[\mathrm{LH}_{4} \mathrm{Ru}_{2} \mathrm{Co}_{2}\right]^{8+}$ grid as well as the $\mathrm{Ru}^{\text {II }}$ "corner complex" (chapter 3), in which a broad band at approximately $650 \mathrm{~nm}$ assigned to the oxidation of $\mathrm{Ru}^{\mathrm{II}}$ to $\mathrm{Ru}^{\mathrm{III}}$, the increase in absorption at $630 \mathrm{~nm}$ can be attributed to the oxidation of the $\mathrm{Ru}^{\mathrm{II}}$ ions. Consequently, the first two anodic waves from the cyclic voltammogram of $\left[\mathrm{LH}_{4} \mathrm{Ru}_{2} \mathrm{Fe}_{2}\right]\left[\mathrm{BF}_{4}\right]_{4}$ can be tentatively assigned to the oxidation of the two Fe ions, while the latter two anodic waves can be assigned to the $\mathrm{Ru}^{\mathrm{II}}$ ions. However, in order to confirm this assumption, synthesis of the doubly oxidized $\left[\mathrm{LH}_{4} \mathrm{Ru}_{2} \mathrm{Fe}_{2}\right]\left[\mathrm{BF}_{4}\right]_{6}$ grid is essential. 
(a)

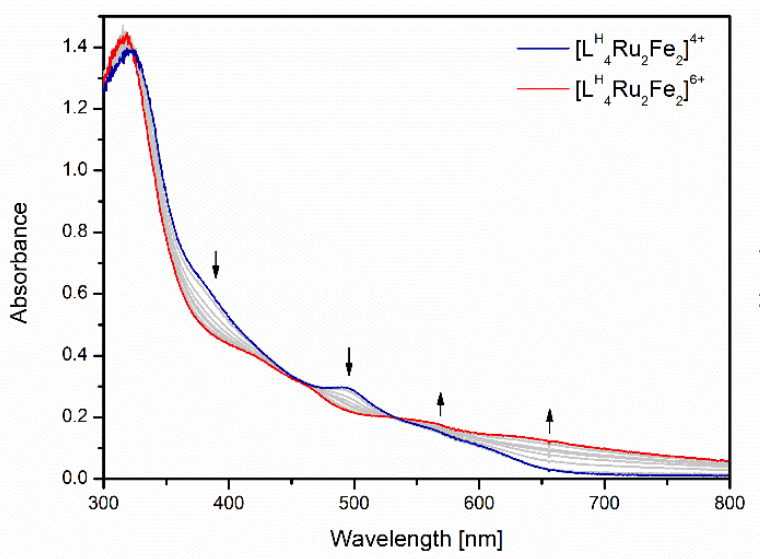

(b)

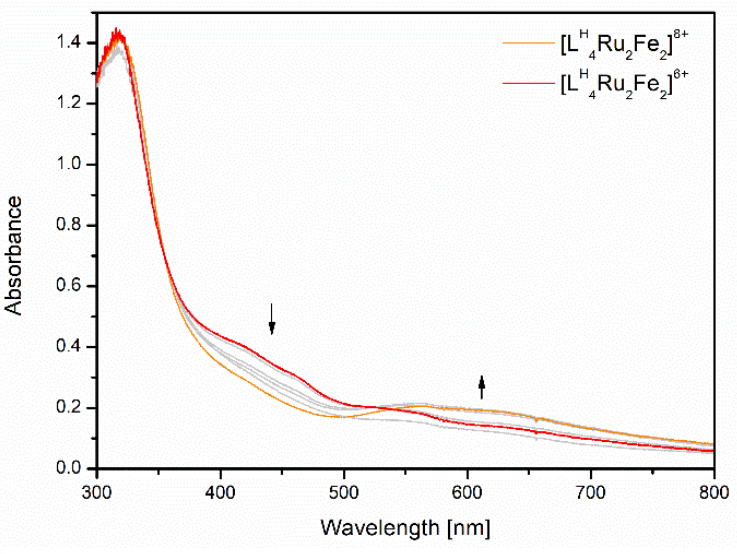

Figure 4.5. UV-vis spectroelectrochemistry of $\left[\mathrm{LH}_{4} \mathrm{Ru}_{2} \mathrm{Fe}_{2}\right]\left[\mathrm{BF}_{4}\right]_{4}$. (a) Application of a potential of $0.77 \mathrm{~V}$ to form the doubly oxidized $\left[\mathrm{L}^{\mathrm{H}}{ }_{4} \mathrm{Ru}_{2} \mathrm{Fe}_{2}\right]^{6+}$ grid and (b) further oxidation to obtain the fully oxidized $\left[\mathrm{L}^{\mathrm{H}}{ }_{4} \mathrm{Ru}_{2} \mathrm{Fe}_{2}\right]^{8+}$ grid by applying a potential of $1.4 \mathrm{~V}$. All potentials are referenced against $\mathrm{Fc} / \mathrm{Fc}^{+}$.

\subsection{5 - Variable Temperature Magnetic Susceptibility}

Variable temperature magnetic susceptibility measurements were conducted on $\left[\mathrm{LH}_{4} \mathrm{Ru}_{2} \mathrm{Fe}_{2}\right]\left[\mathrm{BF}_{4}\right]_{4}$ in the range of $210-2 \mathrm{~K}$, revealing a constant magnetic susceptibility of $\chi_{\mathrm{M}} \mathrm{T}=5.51 \mathrm{~cm}^{3} \mathrm{~mol}^{-1} \mathrm{~K}^{-1}$ above $50 \mathrm{~K}$, consistent with two magnetically uncoupled HS-Fe${ }^{\text {II }}$ ions (Figure 4.6). Below $50 \mathrm{~K}, \chi_{M} \mathrm{~T}$ decreases significantly to $1.94 \mathrm{~cm}^{3} \mathrm{~mol}^{-1} \mathrm{~K}^{-1}$ likely due to zerofield splitting (ZFS) of the HS-Fe ${ }^{\text {II }}$ ions. The data was modelled according to the spin Hamiltonian for two Fe ( $\mathrm{S}=2$ ) ions (eq. 4.1), where $\mu_{\mathrm{B}}$ is the Bohr magneton, and $D, S, B$, are the axial zero-field splitting parameter, the spin, and magnetic field vectors, respectively. Simulation with equation 4.1 afforded g-values of $g_{1}=g_{2}=g_{3}=1.918$ and a ZFS parameter of $D=9.031 \mathrm{~cm}^{-1}$. Based on this measurement, SCO was not observed for $\left[\mathrm{L}_{4} \mathrm{Ru}_{2} \mathrm{Fe}_{2}\right]\left[\mathrm{BF}_{4}\right]_{4}$, as expected with the highly distorted HS-Fe ${ }^{I I}$ environment in the grid.

$$
\widehat{H}=\mu_{B}\left(S_{x} g_{x} B_{x}+S_{y} g_{y} B_{y}+S_{z} g_{z} B_{z}\right)+D\left[\hat{S}_{z}^{2}-\frac{1}{3} S(S+1)+\frac{E}{D}\left(\hat{S}_{x}^{2}-\hat{S}_{y}^{2}\right)\right]
$$

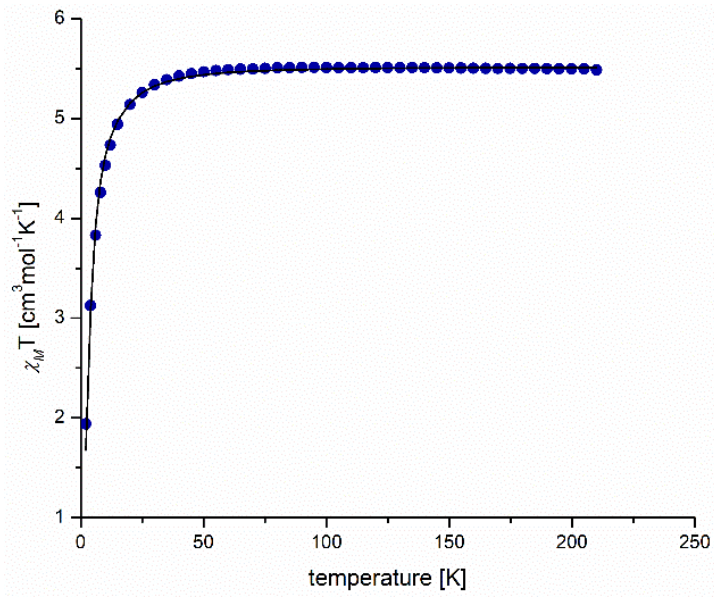

Figure 4.6. Variable magnetic susceptibility measurements for $\left[\mathrm{L}_{4} \mathrm{Ru}_{2} \mathrm{Fe}_{2}\right]\left[\mathrm{BF}_{4}\right]_{4}$ from 210 to $2 \mathrm{~K}$. The data was modelled according to the spin Hamiltonian for two $\mathrm{Fe}(S=2)$ ions (black line). 


\subsection{6 - Mössbauer Spectroscopy}

With the presence of iron, both $\left[\mathrm{L}_{4} \mathrm{Ru}_{2} \mathrm{Fe}_{2}\right][\mathrm{OTf}]_{4}$ and $\left[\mathrm{L}_{4} \mathrm{Ru}_{2} \mathrm{Fe}_{2}\right]\left[\mathrm{BF}_{4}\right]_{4}$ were characterized with ${ }^{57} \mathrm{Fe}$ Mössbauer (MB) spectroscopy. Based on the crystal structure, which revealed the presence of HS-Fe ${ }^{I I}$ ions, a single doublet would be expected by virtue of symmetry. However, Mössbauer spectroscopy on $\left[\mathrm{LH}_{4} \mathrm{Ru}_{2} \mathrm{Fe}_{2}\right]\left[\mathrm{BF}_{4}\right]_{4}$ at $80 \mathrm{~K}$ revealed the presence of two species with a ratio of $94: 6$ (Figure 4.7a). Although this was the best MB spectrum obtained so far, the majority of MB spectra obtained for different samples contain $10-25 \%$ of a second species regardless of the counterion used (Figure $4.7 \mathrm{~b}$ ). The major species with an isomer shift of $\delta=1.04 \mathrm{mms}^{-1}$ and a quadruple splitting of $\Delta E_{\mathrm{Q}}=2.86 \mathrm{mms}^{-1}$, corresponding to high-spin Fe ${ }^{\mathrm{II}}$ ions, is consistent with the $\mathrm{MB}$ parameters also observed in the family of homonuclear pyrazolate-bridged $\left[\mathrm{L}_{4} \mathrm{Fe}_{4}\right]\left[\mathrm{BF}_{4}\right]_{4}$ grids $(\mathrm{R}=\mathrm{H}, \mathrm{Me}, \mathrm{Br}) .{ }^{82,91}$ The minor species with an isomer shift of $\delta=0.36 \mathrm{mms}^{-1}$ and quadruple splitting of $\Delta E_{\mathrm{Q}}=1.04 \mathrm{mms}^{-1}$ corresponds to high-spin Fe ${ }^{I I I}$ ions. All MB parameters are tabulated in Table 4.3.

(a)

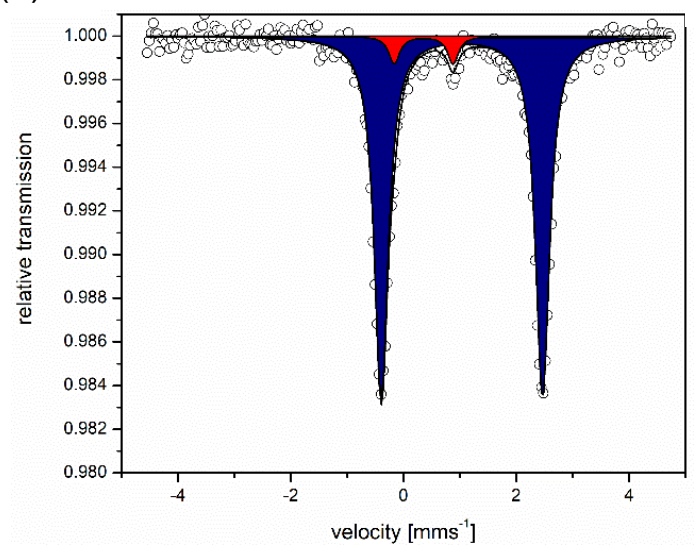

(b)

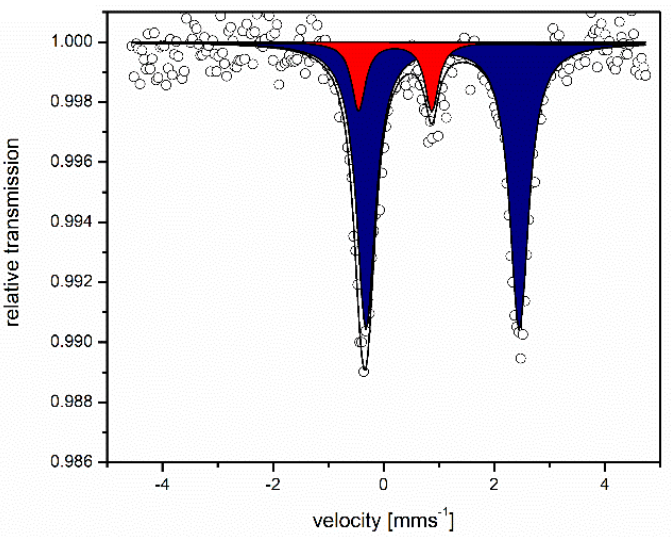

Figure 4.7. (a) Mössbauer spectrum of $\left[\mathrm{LH}_{4} \mathrm{Ru}_{2} \mathrm{Fe}_{2}\right]\left[\mathrm{BF}_{4}\right]_{4}$ at $80 \mathrm{~K}$ with the smallest amount of impurities (94:6). (b) MB spectrum of another sample following the same synthesis affording two species $(87: 13)$.

Table 4.3. Mössbauer parameters for $\left[\mathrm{L}_{4} \mathrm{Ru}_{2} \mathrm{Fe}_{2}\right]\left[\mathrm{BF}_{4}\right]_{4}$ at $80 \mathrm{~K}$.

\begin{tabular}{cccc}
\hline $\mathbf{T}[\mathrm{K}]$ & $\boldsymbol{\delta}\left[\mathbf{m m s}^{-1}\right]$ & $\boldsymbol{\Delta E}_{\mathbf{Q}}\left[\mathbf{m m s}^{-1}\right]$ & Rel. Intensity [\%] \\
\hline \multirow{2}{*}{$80^{\mathrm{a}}$} & 1.04 & 2.86 & 94 \\
& 0.36 & 1.04 & 6 \\
$80^{\mathrm{b}}$ & 1.04 & 2.84 & 87 \\
& 0.39 & 0.98 & 13
\end{tabular}

${ }^{\mathrm{a} M B}$ parameters for Figure $4.7 \mathrm{a} .{ }^{\mathrm{b}} \mathrm{MB}$ parameters for Figure $4.7 \mathrm{~b}$ at $80 \mathrm{~K}$.

Although numerous reaction conditions including different solvents (MeCN, DMF), bases $\left(\mathrm{NaOMe}, \mathrm{NaO}^{t} \mathrm{Bu}\right.$ ) reaction times ( 2 hours -7 days), temperatures (room temperature up to 100 degrees), and crystallization methods were attempted, a minor species ranging from $10-25 \%$ is persistently present. Since the impurity is an $\mathrm{Fe}^{\mathrm{III}}$ species, the $\mathrm{Fe}^{\mathrm{II}}$ salt may be oxidized during the synthesis or, unlike the previously reported pyrazolate-bridge homonuclear $\left[\mathrm{L}_{4} \mathrm{Fe}_{4}\right]\left[\mathrm{BF}_{4}\right]_{4}$ grids $(\mathrm{R}=\mathrm{H}, \mathrm{Br}, \mathrm{Me}),\left[\mathrm{L}_{4} \mathrm{Ru}_{2} \mathrm{Fe}_{2}\right]\left[\mathrm{BF}_{4}\right]_{4}$ may be prone to oxidation and/or decomposition in air. In order to confirm this assumption, the reaction was attempted inside the glove box and monitored with $\mathrm{MB}$ spectroscopy (Figure 4.8). For these experiments, $\left[\mathrm{L}_{4} \mathrm{Ru}_{2} \mathrm{Fe}_{2}\right][\mathrm{OTf}]_{4}$ was used exclusively. Prior to any heating, the $\mathrm{Ru}^{\mathrm{II}}$ "corner 
complex" and NaOMe were dissolved in dry DMF, followed by addition of stoichiometric amounts of ${ }^{57} \mathrm{Fe}^{\mathrm{II}}(\mathrm{OTf})_{2} \cdot 2 \mathrm{MeCN}$ and a $\mathrm{MB}$ spectrum was recorded at $80 \mathrm{~K}$.

(a) Room temperature

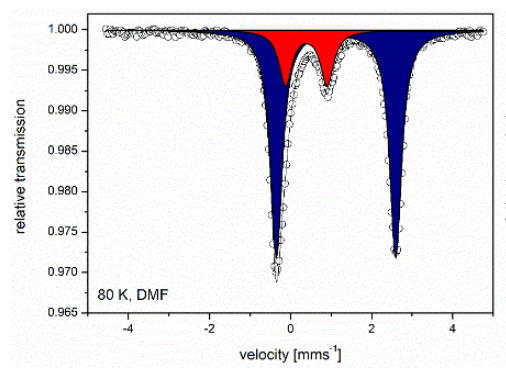

(b) Heated at $50^{\circ} \mathrm{C}$ for $16 \mathrm{~h}$

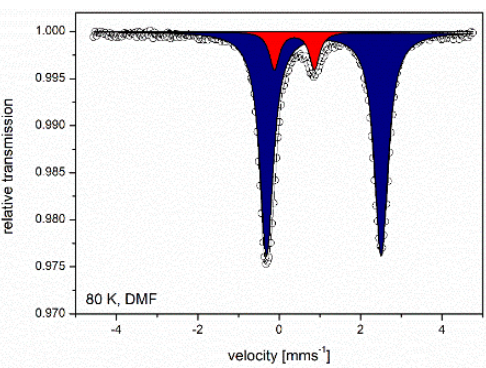

(c) Precipitated after heating

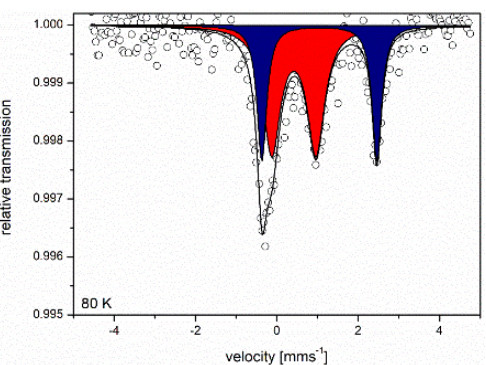

Figure 4.8. (a) $\mathrm{MB}$ spectrum of $\left[\mathrm{L}_{4} \mathrm{Ru}_{2} \mathrm{Fe}_{2}\right][\mathrm{OTf}]_{4}$ prior to heating at $80 \mathrm{~K}$ in frozen DMF. (b) MB spectrum at $80 \mathrm{~K}$ in frozen DMF after heating at $50{ }^{\circ} \mathrm{C}$ for 16 hours. (c) MB spectrum of the solid precipitated with diethyl ether at $80 \mathrm{~K}$.

Table 4.4. Monitoring the synthesis of the $\left[\mathrm{L}_{4} \mathrm{Ru}_{2} \mathrm{Fe}_{2}\right][\mathrm{OTf}]_{4}$ grid with $\mathrm{MB}$ spectroscopy.

\begin{tabular}{ccccc}
\hline $\begin{array}{c}\text { Reaction } \\
\text { Conditions }\end{array}$ & Temperature [K] & $\boldsymbol{\delta}\left[\mathbf{m m s}^{-1}\right]$ & $\boldsymbol{\Delta} \boldsymbol{E}_{\mathbf{Q}}\left[\mathbf{m m s}^{-1}\right]$ & $\begin{array}{c}\text { Rel. Intensity } \\
{[\%]}\end{array}$ \\
\hline \multirow{2}{*}{ Frozen DMFa } & \multirow{2}{*}{80} & 1.12 & 2.94 & 79 \\
& & 0.39 & 1.02 & 21 \\
Frozen DMFb & \multirow{2}{*}{80} & 1.10 & 2.82 & 89 \\
& & 0.38 & 0.98 & 11 \\
Powder & \multirow{2}{*}{80} & 1.09 & 2.86 & 75 \\
& & 0.49 & 0.79 & 25 \\
\hline
\end{tabular}

${ }^{\mathrm{a} M B}$ spectrum in frozen DMF at the start of the reaction prior to heating. ${ }^{\mathrm{b}} \mathrm{MB}$ spectrum in frozen DMF after the reaction was heated at $50{ }^{\circ} \mathrm{C}$.

Surprisingly, even during the initial reaction, two species with an isomer shift of $\delta=1.12 \mathrm{mms}^{-1}\left(\Delta E_{\mathrm{Q}}=2.94 \mathrm{mms}^{-1}\right)$ and $\delta=0.39 \mathrm{mms}^{-1}\left(\Delta E_{\mathrm{Q}}=1.02 \mathrm{mms}^{-1}\right)$ in a ratio of 79:21 were observed (Table 4.4). This suggest that the minor species at $\delta=0.39 \mathrm{mms}^{-1}$ may originate from the complex itself. Moreover, based on the higher isomer shift $\left(\delta=1.12 \mathrm{mms}^{-1}\right)$ compared to the reported $\left[\mathrm{L}_{4} \mathrm{Fe}_{4}\right]\left[\mathrm{BF}_{4}\right]_{4}$ grids $\left(\delta=1.04-1.07 \mathrm{mms}^{-1} ; \mathrm{R}=\mathrm{H}\right.$, $\mathrm{Br}, \mathrm{Me}),\left[\mathrm{LH}_{4} \mathrm{Ru}_{2} \mathrm{Fe}_{2}\right][\mathrm{OTf}]_{4}$ may not be formed immediately or without heating. This may be the result of an intermediate where one or both of the Fe centers are coordinated to DMF molecules prior to self-assembly of the [2 $\mathrm{x} 2]$ grid (Figure 4.9). The electron donating nature of the coordinated DMF molecules provides less d-electron shielding and thus a slightly higher isomer shift is observed. 


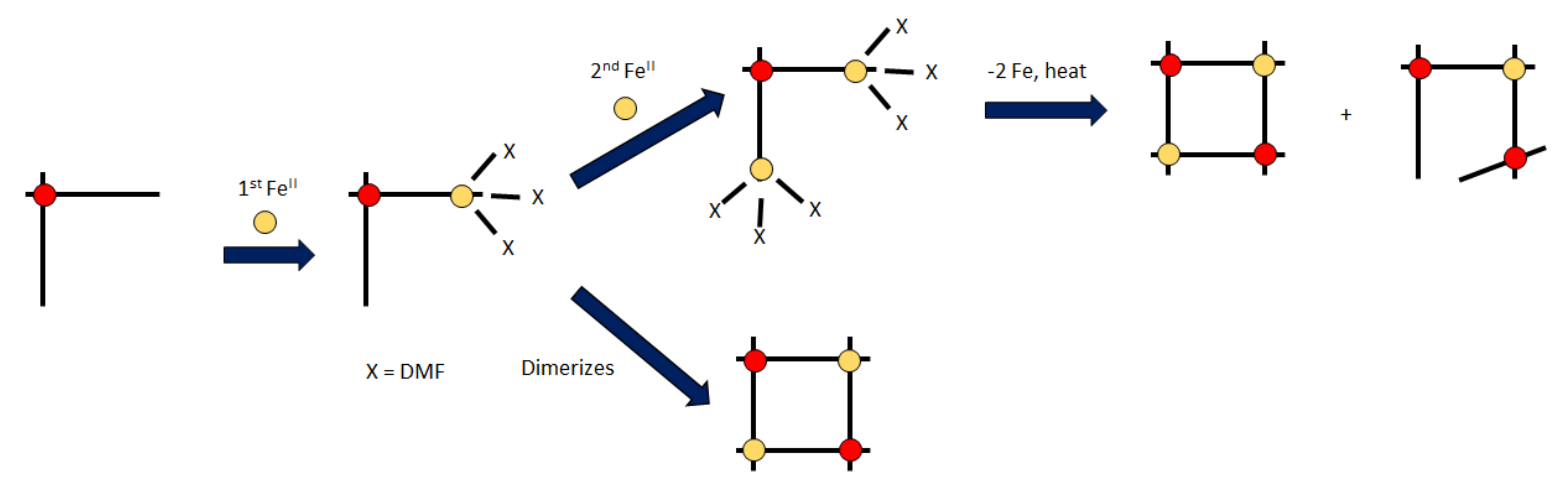

Figure 4.9. Hypothetical mechanism for the assembly of the $\left[\mathrm{LH}_{4} \mathrm{Ru}_{2} \mathrm{Fe}_{2}\right][\mathrm{OTf}]_{4}$ grid. Two potential pathways are possible after coordination of the first $\mathrm{Fe}$ ion: the intermediate dimerizes to form the desired grid, or the reaction is heated to dissociate the Fe ions followed by dimerization to form the desired grid and possibly the defect grid as well.

Following this hypothesis, two potential pathways are possible after the first coordination of an Fe atom to the ligand as well as free solvent molecules (i.e. DMF): (a) the intermediate dimerizes to form the desired grid; or (b) an intermediate with two Fe ions coordinated to the terminal ligand strands is formed, in which the Fe ions dissociate in the presence of heat (i.e. only the $\mathrm{Ru}^{\mathrm{II}}$ corner complex remains after dissociation) followed by dimerization to form the desired grid and/or the defect grid, in which only three metal centers are present. The former hypothetical pathway should be much faster, while the latter may require heat to remove the coordinated iron ions to initiate the self-assembly process of the [2 x 2] grid.

Consequently, in order to determine whether the grid is formed in the presence of heat, the reaction was heated at $50^{\circ} \mathrm{C}$ for 16 hours and another $\mathrm{MB}$ spectrum was measured at $80 \mathrm{~K}$ in frozen DMF (Figure 4.8b). Unfortunately, little changes were observed in the isomer shift and quadruple splitting $\left(\delta=1.10 \mathrm{mms}^{-1}, \Delta E_{\mathrm{Q}}=2.82 \mathrm{mms}^{-1}\right.$ ) for the major species as well as the minor species $\left(\delta=0.38 \mathrm{mms}^{-1}, \Delta E_{\mathrm{Q}}=0.98 \mathrm{mms}^{-1}\right)$. Although the ratio between the two species improved marginally from 79:21 to 89:11 with heating, additional experiments with prolonged heating (seven days) and higher temperatures afforded negligible differences in the conversion between the two species. Since the isomer shift is still significantly higher than the expected isomer shift for the $\left[\mathrm{LR}_{4} \mathrm{Fe}_{4}\right]\left[\mathrm{BF}_{4}\right]_{4}$ grids, $\left[\mathrm{L}_{4} \mathrm{Ru}_{2} \mathrm{Fe}_{2}\right][\mathrm{OTf}]_{4}$ may not be formed until precipitated.

Consequently, the last step involved the precipitation of the reaction in order to determine whether the isomer shift coincides with the typical values observed in the $\left[\mathrm{L}_{4} \mathrm{Fe}_{4}\right]\left[\mathrm{BF}_{4}\right]_{4}$ grids. After 16 hours, the DMF solution of $\left[\mathrm{LH}_{4} \mathrm{Ru}_{2} \mathrm{Fe}_{2}\right][\mathrm{OTf}]_{4}$ was precipitated with diethyl ether and MB spectroscopy was conducted on the solid sample at $80 \mathrm{~K}$ (Figure 4.8c). Once again, two species with isomer shifts of $\delta=1.09 \mathrm{mms}^{-1}\left(\Delta E_{\mathrm{Q}}=2.86 \mathrm{mms}^{-1}\right)$ and $\delta=0.49 \mathrm{mms}^{-1}$ $\left(\Delta E_{\mathrm{Q}}=0.79 \mathrm{mms}^{-1}\right)$ were observed. Although the isomer shift of the major species is reminiscent to the parameters found in the frozen DMF spectra, the isomer shift of the minor species changes quite significantly for the solid powder sample. However, due to the small quantity of the precipitated material, the intensity and resolution of the MB spectrum of the powder material is much lower compared to the MB spectra of the frozen DMF solution.

Several additional experiments were conducted in order to identify the minor Fe ${ }^{\text {III }}$ species including $\mathrm{MB}$ spectroscopy on $\mathrm{Fe}^{\mathrm{II}}(\mathrm{OTf})_{2} \cdot 2 \mathrm{MeCN}$ in the solid-state as well as in frozen DMF. However, the MB parameters of the $\mathrm{Fe}^{\mathrm{II}}$ salts revealed significantly higher isomer shifts of $\delta 1.39 \mathrm{mms}^{-1}$ (solid-state, Figure A40) and $1.27 \mathrm{mms}^{-1}$ (frozen-solution, Figure A41). 
Interestingly, one particular experiment where an excess of ${ }^{57} \mathrm{Fe}^{\mathrm{II}}$ salt was used to generate $\left[\mathrm{L}_{4} \mathrm{Ru}_{2} \mathrm{Fe}_{2}\right][\mathrm{OTf}]_{4}$ in situ in DMF revealed that upon oxidation in air for 16 hours, over $80 \%$ of the Fe ${ }^{\text {II }}$ species is converted to Fe ${ }^{\text {III }}$ ions (Figure 4.10 ). Here, a higher isomer shift for $\left[\mathrm{LH}_{4} \mathrm{Ru}_{2} \mathrm{Fe}_{2}\right][\mathrm{OTf}]_{4}$ was observed $\left(\delta=1.13 \mathrm{mms}^{-1}\right)$, but more importantly, the isomer shift of the second species $\left(\delta=0.46-0.48 \mathrm{mms}^{-1}\right.$, Table 4.5$)$ coincides with the isomer shift of the precipitated powder $\left(\delta=0.49 \mathrm{mms}^{-1}\right)$ from the previous in situ experiments (Table 4.4). Consequently, the origin of the second species may simply be reaction of excess or unreacted $\mathrm{Fe}^{\mathrm{II}}$ salt from solvents that contain marginal amounts of water. Conversely, comparison of the isomer shift of the minor species from $\left[\mathrm{L}_{4} \mathrm{Ru}_{2} \mathrm{Fe}_{2}\right][\mathrm{OTf}]_{4}$ in frozen DMF $\left(\delta=0.37-0.38 \mathrm{mms}^{-1}\right.$, Table 4.4) and the isomer shift of the Fe ${ }^{\text {III }}$ species generated by oxidation of excess Fe ${ }^{\text {II }}$ salt ( $\delta=0.46-0.48 \mathrm{mms}^{-1}$ ), affords a difference of $\delta=0.08 \mathrm{mms}^{-1}$. In terms of MB spectroscopy, this difference is quite significant indicating that the species formed in the two experiments are not identical. Therefore, definitive assignment of the minor species is still inconclusive based on the MB experiments.

(a)

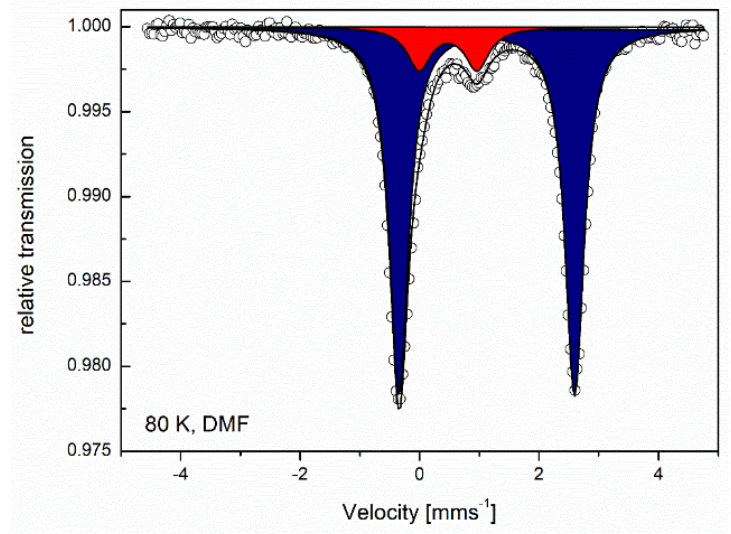

(b)

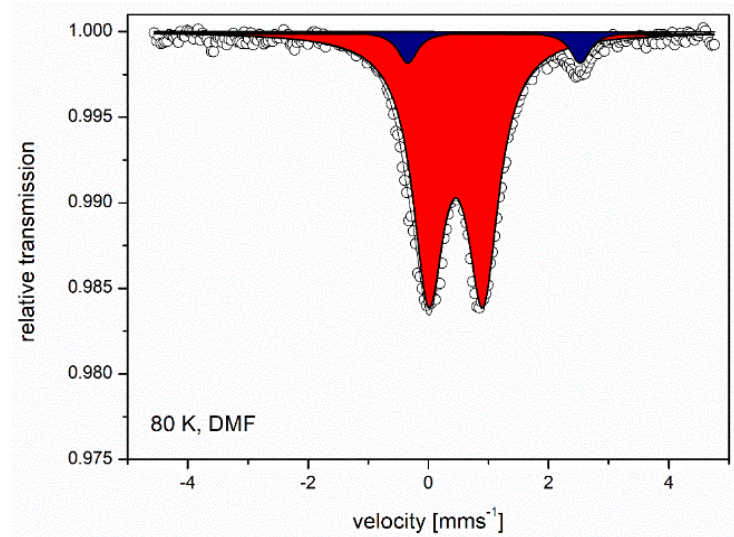

Figure 4.10. (a) MB spectrum of $\left[\mathrm{L}_{4} \mathrm{Ru}_{2} \mathrm{Fe}_{2}\right][\mathrm{OTf}]_{4}$ generated in situ with excess $\mathrm{Fe}^{\mathrm{Il}}$ salt in frozen DMF under inert conditions; the spectrum was recorded in frozen DMF solution. (b) MB spectrum of the same sample left in air after 16 hours.

Table 4.5. MB parameters of spectra recorded in frozen DMF of the in situ synthesis of $\left[\mathrm{L}_{4} \mathrm{Ru}_{2} \mathrm{Fe}_{2}\right][\mathrm{OTf}]_{4}$ with an excess of $\mathrm{Fe}^{\mathrm{II}}$ salt followed by oxidation in air.

\begin{tabular}{ccccc}
\hline $\begin{array}{c}\text { Reaction } \\
\text { Conditions }\end{array}$ & $\mathbf{T}[\mathbf{K}]$ & $\boldsymbol{\delta}\left[\mathbf{m m s}^{-1}\right]$ & $\boldsymbol{\Delta}_{\mathbf{Q}}\left[\mathbf{m m s}^{-1}\right]$ & $\begin{array}{c}\text { Rel. Intensity } \\
{[\%]}\end{array}$ \\
\hline \multirow{2}{*}{ Initial Reaction } & \multirow{2}{*}{80} & 1.13 & 2.94 & 87 \\
& & 0.48 & 0.97 & 13 \\
After oxidation & \multirow{2}{*}{80} & 1.09 & 2.87 & 7 \\
& & 0.46 & 0.90 & 93 \\
\hline
\end{tabular}

Based on the MB experiments discussed above, the presence of two species observed in all of the MB spectra suggest the second minor species (11-25\%) is formed during the addition of the Fe salt and conversion between the two species is unlikely even with heat. 


\subsection{7 - ${ }^{1} \mathrm{H}$ NMR Spectroscopy}

In order to investigate the mechanism of the formation of $\left[\mathrm{L}_{4} \mathrm{Ru}_{2} \mathrm{Fe}_{2}\right][\mathrm{OTf}]_{4}$ as well as the nature of the minor species, ${ }^{1} \mathrm{H}$ NMR spectroscopy was used to monitor the reaction. $\left[\mathrm{LH}_{4} \mathrm{Ru}_{2} \mathrm{Fe}_{2}\right][\mathrm{OTf}]_{4}$ was generated in situ by deprotonation of the ligand $\mathbf{H L}^{\mathbf{H}}$ with $\mathrm{NaOMe}$ followed by addition of stoichiometric amounts of Fell salt in DMF- $\mathrm{d}_{7}$. The reaction was heated at $50{ }^{\circ} \mathrm{C}$ for 16 hours and a ${ }^{1} \mathrm{H}$ NMR spectrum was recorded every hour (Figure 4.11). Initially, numerous broad overlapping peaks are observed arising potentially from a mixture of the $\mathrm{Ru}^{\mathrm{II}}$ "corner complex", defect grid with three metal centers and the full $\left[\mathrm{L}_{4} \mathrm{Ru}_{2} \mathrm{Fe}_{2}\right][\mathrm{OTf}]_{4}$ grid. Upon heating, the resonances between $40-70$ ppm sharpen along with disappearance of many of the peaks in the diamagnetic region. Moreover, the resonance at $27 \mathrm{ppm}$ gradually disappears with heating and a new broad peak at $130 \mathrm{ppm}$ is observed. After 15 hours of heating, the sample was remeasured at room temperature (Figure 4.12) affording the expected resonances for the $\left[\mathrm{LH}_{4} \mathrm{Ru}_{2} \mathrm{Fe}_{2}\right][\mathrm{OTf}]_{4}$ grid.

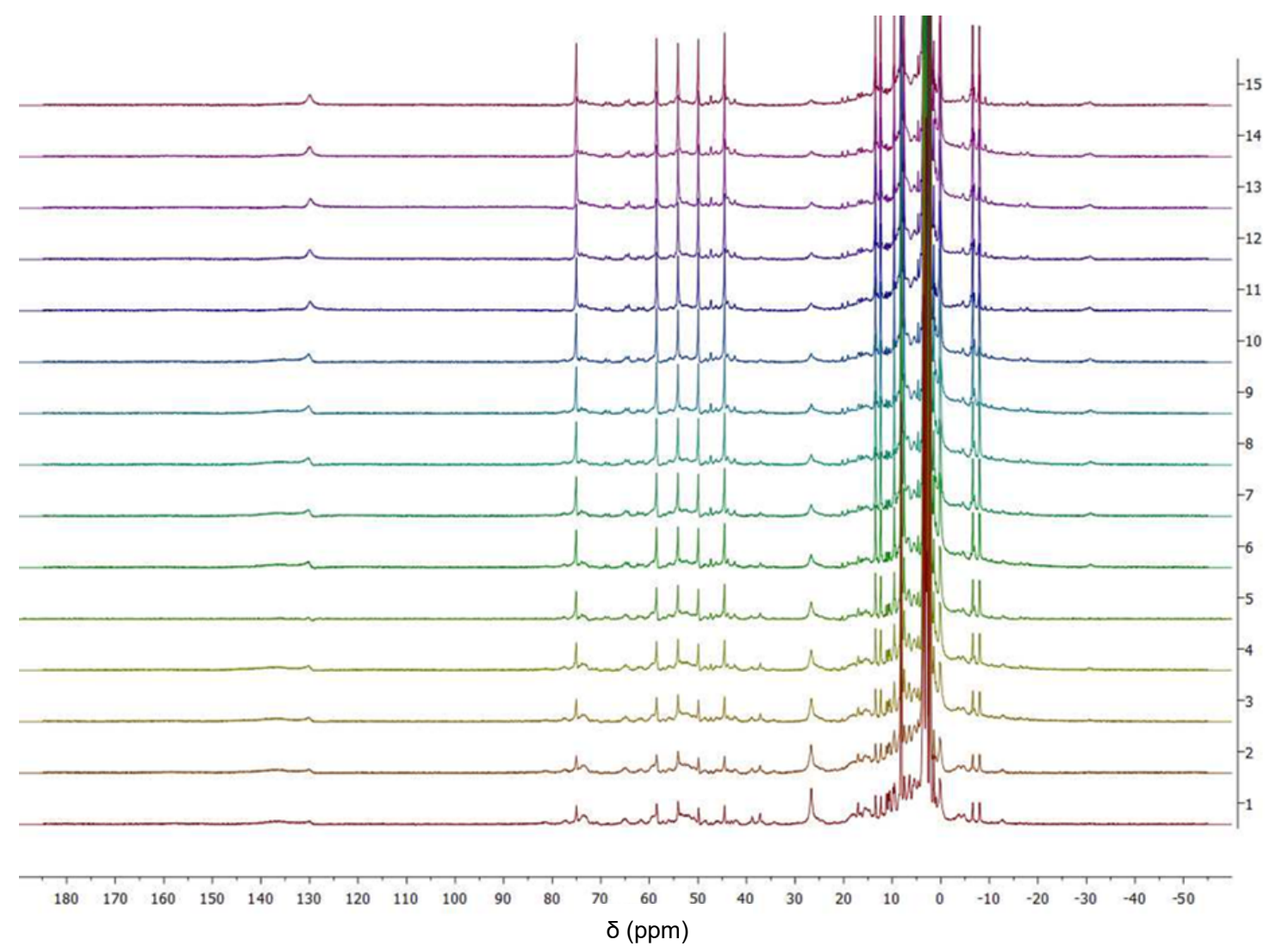

Figure 4.11. ${ }^{1} \mathrm{H}$ NMR spectroscopy monitoring the formation of $\left[\mathrm{L}_{4}{ }_{4} \mathrm{Ru}_{2} \mathrm{Fe}_{2}\right][\mathrm{OTf}]_{4}$ in DMF- $\mathrm{d}_{7}$. A spectrum was recorded every hour for 15 hours at $50{ }^{\circ} \mathrm{C}$.

Similar to the ${ }^{1} \mathrm{H}$ NMR spectrum of $\left[\mathrm{L}_{4} \mathrm{Ru}_{2} \mathrm{Co}_{2}\right]\left[\mathrm{BF}_{4}\right]_{4}$ discussed in the previous chapter, 15 peaks corresponding to the proton resonances of $\left[\mathrm{L}_{4} \mathrm{Ru}_{2} \mathrm{Fe}_{2}\right][\mathrm{OTf}]_{4}$ were observed. Solvent molecules such as DMF and methanol (originating from sodium methoxide), are indicated in asterisks. Since only one set of resonances corresponding to the anti-isomer are present (similar to ${ }^{1} \mathrm{H}$ NMR spectrum of $\left[\mathrm{LH}_{4} \mathrm{Ru}_{2} \mathrm{Co}_{2}\right]\left[\mathrm{BF}_{4}\right]_{4}$ discussed in the previous chapter), only a single isomer is formed. In the case of the syn isomer, in which the ruthenium or iron metal ions are adjacent to one another, 28 resonances would be expected. Consequently, once again, despite the different reaction conditions, the ruthenium corner complex is capable of selectively enforcing the formation of the trans isomer exclusively. 
Due to the fast spin relaxation times associated with HS-FeII systems, resonances with linewidths between $100-200 \mathrm{~Hz}$ were observed in the ${ }^{1} \mathrm{H}$ NMR spectrum of $\left[\mathrm{L}_{4} \mathrm{Ru}_{2} \mathrm{Fe}_{2}\right][\mathrm{OTf}]_{4}$. Consequently, ${ }^{1} \mathrm{H}-{ }^{-1} \mathrm{H}$ COSY experiments were conducted permitting full assignment of the protons of the grid. Surprisingly, the resonance at $54 \mathrm{ppm}$ did not display any correlation to other protons. However, using the other proton-proton correlations and the proton-metal distances determined from the crystal structure, all the proton resonances between 45-77 ppm can be assigned unambiguously. Moreover, the close proximity of $\mathrm{H}^{\mathrm{a}}$ to the paramagnetic Fe center afforded a broad resonance at much lower fields $(\delta=143 \mathrm{ppm})$ than the remaining resonances. The remaining resonances from $-20 \mathrm{ppm}$ to $+20 \mathrm{ppm}$ were assigned based on ${ }^{1} \mathrm{H}^{-1} \mathrm{H}$ COSY experiments, however resonances for $\mathrm{H}^{\mathrm{i}}$ and $\mathrm{H} \mathrm{j}$, could not be unambiguously assigned.

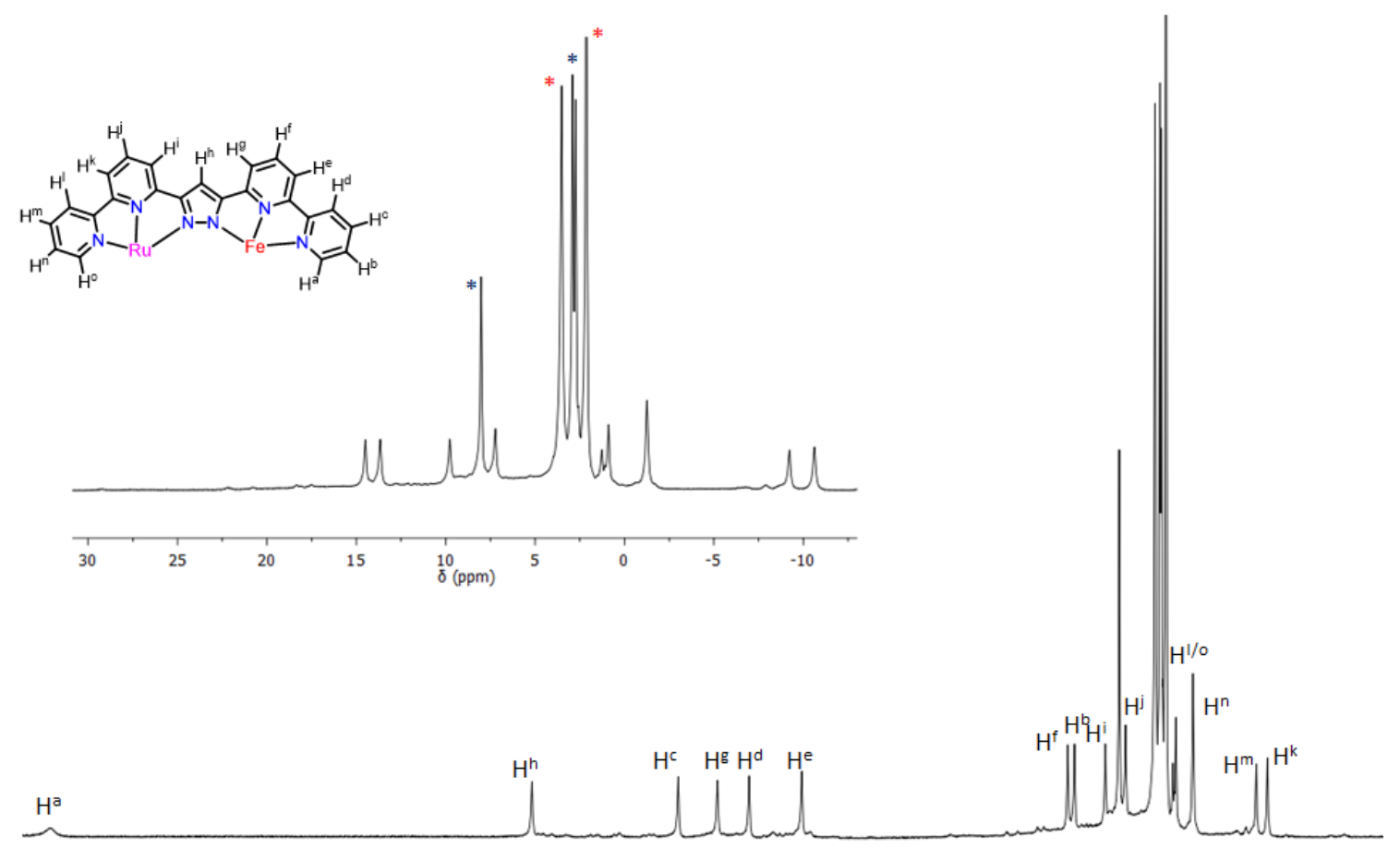

$140 \quad 130 \quad 120 \quad 110 \quad 100 \quad 90$

Figure 4.12. ${ }^{1} \mathrm{H}$ NMR spectrum of $\left[\mathrm{L}^{\mathrm{H}}{ }_{4} \mathrm{Ru}_{2} \mathrm{Fe}_{2}\right][\mathrm{OTf}]_{4}$ after heating at $50{ }^{\circ} \mathrm{C}$ for 16 hours. The spectrum was recorded in DMF- $d_{7}$ at room temperature. Solvent peaks such as DMF $\left(^{*}\right)$ and $\mathrm{MeOH}\left({ }^{*}\right)$ are indicated with asterisks.

Since the ${ }^{1} \mathrm{H}$ NMR experiment was conducted in situ, the remaining solution of $\left[\mathrm{LH}_{4} \mathrm{Ru}_{2} \mathrm{Fe}_{2}\right][\mathrm{OTf}]_{4}$ in DMF- $\mathrm{d}_{7}$ was subsequently precipitated with diethyl ether and a ${ }^{1} \mathrm{H}$ NMR spectrum of the powder in DMF- $\mathrm{d}_{7}$ was recorded at room temperature. The spectrum displayed minor changes in which the methanol peaks are no longer present since the solid was subjected to minor workup and drying in vacuo. The reaction was repeated in larger scales, the product crystallized, and a ${ }^{1} \mathrm{H}$ NMR spectrum of the crystallized $\left[\mathrm{L}^{\mathrm{H}_{4}} \mathrm{Ru}_{2} \mathrm{Fe}_{2}\right][\mathrm{OTf}]_{4}$ grid dissolved in MeCN- $\mathrm{d}_{3}$ is shown in Figure 4.13, illustrating little differences between the in-situ ${ }^{1} \mathrm{H}$ NMR spectrum and the spectrum of the final product. Based on these NMR experiments, $\left[\mathrm{L}_{4} \mathrm{Ru}_{2} \mathrm{Fe}_{2}\right][\mathrm{OTf}]_{4}$ is formed in solution (in contrast to the conclusion of the MB experiments) after heating at $50{ }^{\circ} \mathrm{C}$ for 15 hours. 


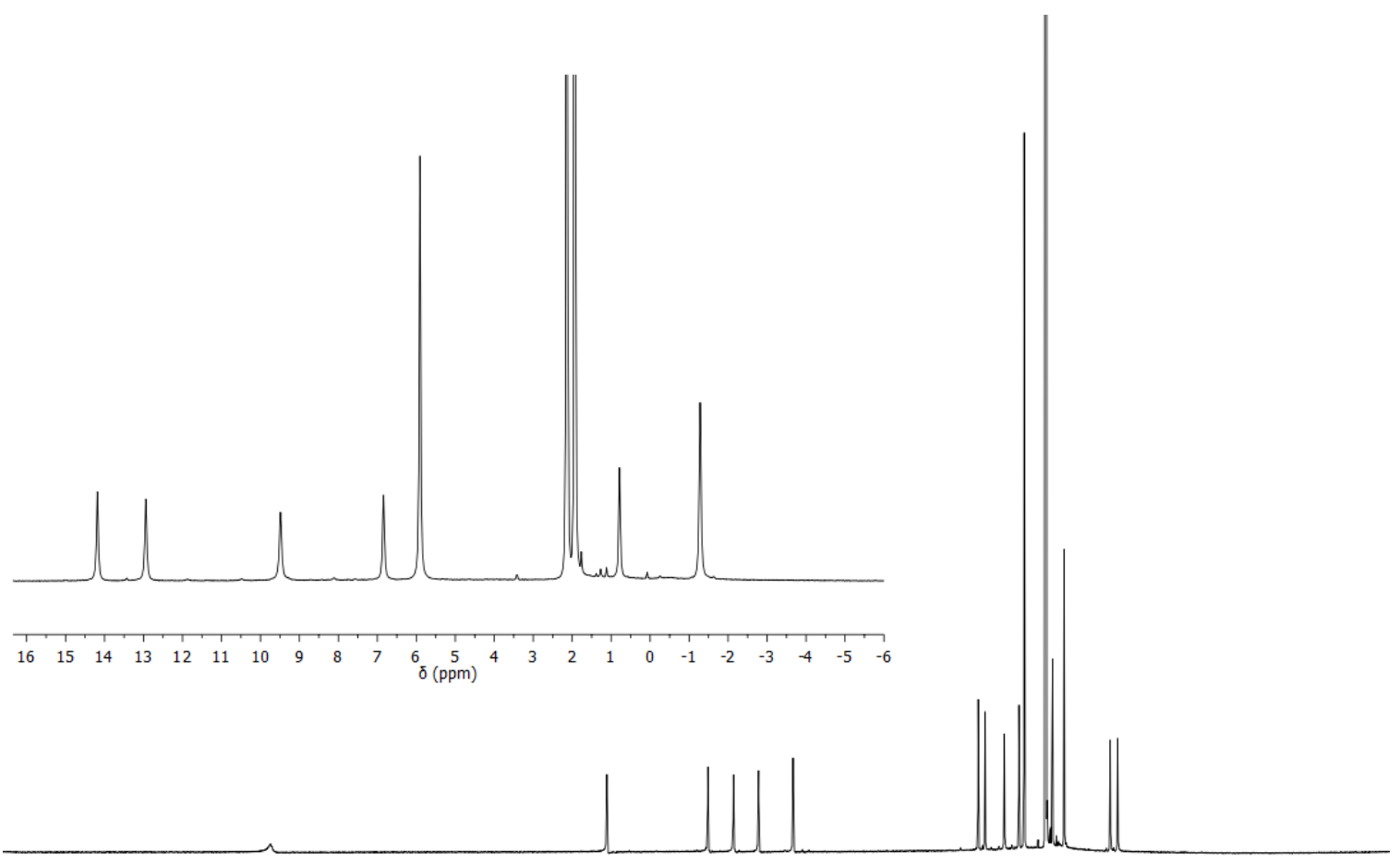

$\begin{array}{lllllllllllllllllllllllllll}180 & 170 & 160 & 150 & 140 & 130 & 120 & 110 & 100 & 90 & 80 & 70 & 60 & 50 & 40 & 30 & 20 & 10 & 0 & -10 & -20 & -30 & -40 & -50\end{array}$

Figure 4.13. ${ }^{1} \mathrm{H}$ NMR spectrum of $\left[\mathrm{L}^{\mathrm{H}}{ }_{4} \mathrm{Ru}_{2} \mathrm{Fe}_{2}\right][\mathrm{OTf}]_{4}$ after crystallization in $\mathrm{MeCN}-\mathrm{d}_{3}$ at room temperature.

\section{3 - Discussion}

Although a variety of experiments including mass spectrometry and ${ }^{1} \mathrm{H}$ NMR spectroscopy suggest the formation of $\left[\mathrm{L}_{4} \mathrm{Ru}_{2} \mathrm{Fe}_{2}\right][\mathrm{OTf}]_{4}$ exclusively, an additional Fe ${ }^{\mathrm{III}}$ species was observed in the majority of the Mössbauer spectra (10-25\%). Based on the MB experiments discussed previously, an additional Fe ${ }^{\mathrm{III}}$ species is present while ${ }^{1} \mathrm{H}$ NMR experiments suggest the presence of an Fe salt without protons or a HS-Fe ${ }^{\text {III }}$ species in which relaxation times are unfavourable and thus not detected in ${ }^{1} \mathrm{H}$ NMR spectrum. Although using stoichiometric amounts of $\mathrm{Fe}^{\mathrm{II}}$ salt afforded two species in the MB spectrum, using less than one equivalent of $\mathrm{Fe}^{\mathrm{II}}$ salt may lead to a mixture of the defect $\left[\mathrm{L}_{2}\left(\mathrm{HL}^{\mathrm{H}}\right)_{2} \mathrm{Ru}_{2} \mathrm{Fe}\right][\mathrm{OTf}]_{4}$ and full $\left[\mathrm{LH}_{4} \mathrm{Ru}_{2} \mathrm{Fe}_{2}\right][\mathrm{OTf}]_{4}$ grid as observed in a multitude of peaks from $-50 \mathrm{ppm}$ to $+180 \mathrm{ppm}$ in the ${ }^{1} \mathrm{H}$ NMR spectrum. In particular, another set of paramagnetic resonances between $30-90$

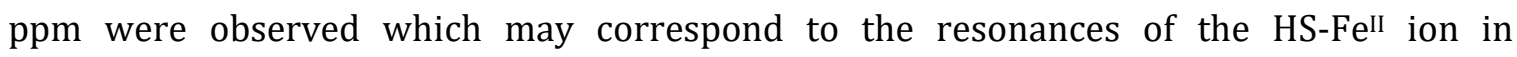
$\left[\mathrm{L}_{2}\left(\mathrm{HL}^{\mathrm{H}}\right)_{2} \mathrm{Ru}_{2} \mathrm{Fe}\right][\mathrm{OTf}]_{4}$. 
In terms of the proposed mechanism of the formation of $\left[\mathrm{L}_{4} \mathrm{Ru}_{2} \mathrm{Fe}_{2}\right][\mathrm{OTf}]_{4}$ discussed in Figure 4.9, the assumption that the grid is not formed in solution is disputable since the reaction was monitored by ${ }^{1} \mathrm{H}$ NMR spectroscopy in DMF- $\mathrm{d}_{7}$. Since all the peaks corresponding to $\left[\mathrm{LH}_{4} \mathrm{Ru}_{2} \mathrm{Fe}_{2}\right][\mathrm{OTf}]_{4}$ are present in solution and after precipitation confirms that the grid is formed in solution (in contrast to the assumption that the grid forms only upon precipitation). The slightly higher isomer shift ( $\left.\delta=1.09-1.12 \mathrm{mms}^{-1}\right)$ observed in the MB spectrum of $\left[\mathrm{L}_{4} \mathrm{Ru}_{2} \mathrm{Fe}_{2}\right][\mathrm{OTf}]_{4}$ generated in situ in frozen DMF compared to the crystallized material may be the result of the simulation. The doublet in the frozen DMF samples are much broader, which may be attributed to two Fe species, namely unreacted Fe ${ }^{\text {II }}$ salt and $\left[\mathrm{LH}_{4} \mathrm{Ru}_{2} \mathrm{Fe}_{2}\right][\mathrm{OTf}]_{4}$. Consequently, simulation of the broad doublet with two species is shown in Figure 4.14. Since the MB spectrum prior to heating and after heating at $50^{\circ} \mathrm{C}$ are relatively similar, only the MB spectrum after heating is shown. All MB parameters are tabulated Table 4.6. Indeed, after fitting the broad doublet with two species, the isomer shift of $1.06 \mathrm{mms}^{-1}$ typical for the grids was observed along with unreacted $\mathrm{Fe}^{\mathrm{II}}(\mathrm{OTf})_{2}$ at $1.18 \mathrm{mms}^{-1}$. According to the relative intensity of the three different species, even after heating at $50{ }^{\circ} \mathrm{C}, 21 \%$ of the $\mathrm{Fe}^{\mathrm{II}}$ salt remains unreacted. Although longer reaction times and higher temperatures have already been attempted, further work to remove the excess $\mathrm{Fe}^{\mathrm{II}}$ salt after precipitation is essential to obtain $\left[\mathrm{L}_{4} \mathrm{Ru}_{2} \mathrm{Fe}_{2}\right][\mathrm{OTf}]_{4}$ exclusively.

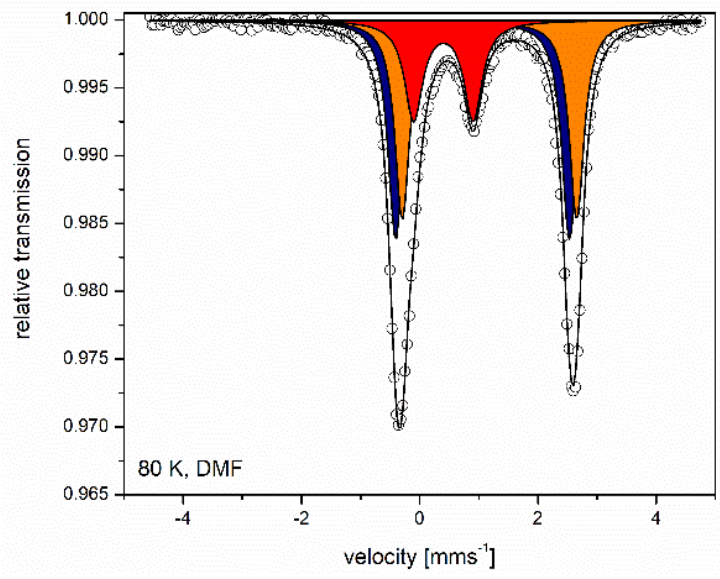

Figure 4.14. Refitted Mössbauer spectrum of $\left[\mathrm{L}_{4} \mathrm{Ru}_{2} \mathrm{Fe}_{2}\right][\mathrm{OTf}]_{4}$ in frozen DMF at $80 \mathrm{~K}$ with three different species: $\left[\mathrm{L}_{4} \mathrm{Ru}_{2} \mathrm{Fe}_{2}\right][\mathrm{OTf}]_{4}$, unreacted $\mathrm{Fe}^{\mathrm{III}}(\mathrm{OTf})_{2}$, and the unknown $\mathrm{Fe}^{\mathrm{III}}$ species.

Table 4.6. Refitted MB parameters of the in situ synthesis of $\left[\mathrm{L}_{4} \mathrm{Ru}_{2} \mathrm{Fe}_{2}\right][\mathrm{OTf}]_{4}$ prior to heating and after heating in frozen DMF at $80 \mathrm{~K}$.

\begin{tabular}{cccc}
\hline $\begin{array}{c}\text { Reaction } \\
\text { Conditions }\end{array}$ & $\boldsymbol{\delta}\left[\mathbf{m m s}^{-\mathbf{1}}\right]$ & $\boldsymbol{\Delta} \boldsymbol{E}_{\mathbf{Q}}\left[\mathbf{m m s} \mathbf{s}^{-\mathbf{1}}\right]$ & Rel. Intensity [\%] \\
\hline \multirow{3}{*}{ Prior to heating } & 1.06 & 2.94 & 41 \\
& 1.18 & 2.95 & 37 \\
& 0.40 & 1.01 & 22 \\
\hline \multirow{2}{*}{ After heating } & 1.06 & 2.81 & 66 \\
& 1.23 & 2.79 & 21 \\
& 0.33 & 1.07 & 13 \\
\hline
\end{tabular}

Unfortunately, although the synthetic issues of $\left[\mathrm{L}_{4} \mathrm{Ru}_{2} \mathrm{Fe}_{2}\right]\left[\mathrm{BF}_{4}\right]_{4}\left(\right.$ or $\left.\left[\mathrm{L}_{4} \mathrm{Ru}_{2} \mathrm{Fe}_{2}\right][\mathrm{OTf}]_{4}\right)$ have been identified along with the identity of the FellI species, there was little success in 
preventing or removing the impurity. Aside from crystallization and optimizing reaction conditions, another possibility involved the synthesis of the doubly oxidized $\left[\mathrm{L}_{4} \mathrm{Ru}_{2} \mathrm{Fe}_{2}\right]\left[\mathrm{BF}_{4}\right]_{6}$ grid, followed by reduction and crystallization. However, preliminary attempts to chemically synthesize $\left[\mathrm{LH}_{4} \mathrm{Ru}_{2} \mathrm{Fe}_{2}\right]\left[\mathrm{BF}_{4}\right]_{6}$ afforded an ill-resolved doublet in the MB spectrum. This may be the result of the impure starting material or unlike the $\left[\mathrm{L}_{4} \mathrm{Ru}_{2} \mathrm{Co}_{2}\right]\left[\mathrm{BF}_{4}\right]_{6}$ congener, $\left[\mathrm{LH}_{4} \mathrm{Ru}_{2} \mathrm{Fe}_{2}\right]\left[\mathrm{BF}_{4}\right]_{6}$ may be prone to decomposition from oxidation.

\section{4 - Summary \& Conclusion}

In conclusion, a new heterometallic $\left[\mathrm{L}_{4} \mathrm{Ru}_{2} \mathrm{Fe}_{2}\right]\left[\mathrm{BF}_{4}\right]_{4}$ grid was synthesized and its physical and chemical properties were thoroughly investigated in solution and in the solid-state. Similar to the $\left[\mathrm{L}_{4} \mathrm{Ru}_{2} \mathrm{Co}_{2}\right]\left[\mathrm{BF}_{4}\right]_{4}$ congener, the ruthenium "corner complex" selectively enforces a trans configuration in which the ruthenium and iron centers are situated diagonally across from one another. The presence of only a single isomer was confirmed with ${ }^{1} \mathrm{H}$ NMR spectroscopy, in which only 15 peaks are observed as expected for a highly symmetric molecule. Surprisingly, the $\mathrm{MB}$ spectrum of $\left[\mathrm{L}_{4} \mathrm{Ru}_{2} \mathrm{Fe}_{2}\right][\mathrm{OTf}]_{4}$ and $\left[\mathrm{LH}_{4} \mathrm{Ru}_{2} \mathrm{Fe}_{2}\right]\left[\mathrm{BF}_{4}\right]_{4}$ gave rise to two distinct Fe species: one species corresponding to the grid and a minor species corresponding to the oxidized Fe ${ }^{\mathrm{II}}$ salt. Unfortunately, as of now, there has been little progress in the removal or prevention of this $\mathrm{Fe}^{\mathrm{III}}$ species. Further work must be done on the optimization and purification of $\left[\mathrm{L}_{4}^{\mathrm{H}} \mathrm{Ru}_{2} \mathrm{Fe}_{2}\right]\left[\mathrm{BF}_{4}\right]_{4}$. Moreover, the doubly oxidized $\left[\mathrm{LH}_{4} \mathrm{Ru}_{2} \mathrm{Fe}_{2}\right]\left[\mathrm{BF}_{4}\right]_{6}$ grid must be characterized in order to definitively assign the four oxidative waves in the cyclic voltammogram to their corresponding metal centers. 


\section{Chapter 5 - Highly Distorted Dinuclear FeII and CoII Complexes}

\section{1 - Introduction}

Photoinduced spin crossover (SCO) has been well documented for Fe"ll complexes. ${ }^{23,94}$ However, applications in photocatalysis and light-to-energy conversion are limited due to the rapid decay of the transient 1,3 MLCT state with a lifetime of less than $20 \mathrm{fs},{ }^{95}$ as observed for numerous mononuclear Fe species discussed in chapter 2. On the contrary, a variety of Ru ${ }^{\mathrm{II}}$ complexes with terpyridine(tpy)-based ligands have exhibited longer ${ }^{3}$ MLCT life times by reducing the radiationless deactivation from the ${ }^{3} \mathrm{MC}$ state. This can be accomplished by incorporation of electron withdrawing groups to lower the energy of the ${ }^{3}$ MLCT state or incorporation of electron donating groups to destabilize the ${ }^{3} \mathrm{MC}$ state. The larger the energy difference between the two states, the less radiationless deactivation from the ${ }^{3} \mathrm{MC}$ state would be observed, and thus longer ${ }^{3}$ MLCT lifetimes. ${ }^{92}$ Another approach involves increasing the Ru-N-Ru bite angles affording better overlap of the Ru $d$-orbitals. Consequently, the ligand field splitting will be larger leading to a greater separation between the ${ }^{3} \mathrm{MLCT}$ and ${ }^{3} \mathrm{MC}$ states. One particular example utilized the combination of a terpyridine (tpy) and a ddpd ligand (dppd = N,N'dimethyl-N,N'dipyridin-2-ylpyridine-2,6-diamine) to enforce large bite angles due to the additional $\mathrm{NCH}_{3}$ group between the pyridine moieties of the dppn ligand. ${ }^{92}$ This complex afforded a remarkable increase in the ${ }^{3}$ MLCT life time of $722 \mathrm{~ns}$ compared to typical mononuclear Fe $\mathrm{II}^{\mathrm{II}}$ complexes.

Since $\mathrm{Ru}^{\mathrm{II}}$ ions are not prone to SCO due to their intrinsically large ligand field splitting, substitution of $\mathrm{Fe}^{\mathrm{II}}$ ions into similar frameworks may provide interesting photochemistry as well as spin crossover. To that end, a new ligand with additional $\mathrm{NCH}_{3}$ groups incorporated between the pyridine moieties was synthesized. Although the initial interest of this work stems from the synthesis of new homometallic and heterometallic [2 $\mathrm{x} 2$ ] grid-like arrays, the increase in flexibility in the ligand strand compromised one of the essential criteria for grid formation - the need for the self-assembly of two mutually perpendicular ligand strands. Instead, a new dinuclear $\mathrm{Fe}_{2}$ complex was synthesized along with the mixed valent congener. The physical properties of the two complexes will be discussed along with preliminary results on the analogous dinuclear $\mathrm{Co}_{2}$ complex.

\section{2 - Results}

\subsection{1 - Ligand Synthesis}

The ligand $\mathbf{H L}^{\mathbf{1}}$ was first developed by Dr. Shao-An Hua and can be synthesized in nine steps according to Scheme 5.1. The precursor 5-3 was synthesized by esterfication of 6bromopicolinic acid 5-1 with sulfuric acid $\left(\mathrm{H}_{2} \mathrm{SO}_{4}\right)$ in methanol, followed by a coupling reaction with 4-methylaminopyridine to obtain the ester 5-3 as a pale yellow solid. The second precursor 5-8 was synthesized in four steps commencing with the acetylation of 2,6dibromopyridine 5-4 with $n$-butyllithium ( $n$-BuLi) and dry dimethylacetamide (DMA) to give white crystalline material. Subsequently, the ketone moiety was protected with a dioxolane group using ethylene glycol and $p$-toluenefulfonic acid monohydrate (PTSA) as a catalyst. The yellow oil 5-6 was subjected to a coupling reaction with 4-methylaminopyridine to give 5-7, which was subsequently hydrolyzed with $2 \mathrm{M} \mathrm{HCl}$ to give an orange oil 5-8. Once both precursors 5-3 and 5-8 were obtained, the diketone 5-9 was easily synthesized by a condensation of the two precursors in the presence of a base to give a bright yellow powder. 
Due to the presence of tautomerization between the ketone and enol forms, 5-9 was not characterized by ${ }^{1} \mathrm{H}$ NMR spectroscopy. The final steps involves the methylation of the diketone 5-9 with KHMDS to give a brown oil which was subsequently treated with hydrazine monohydrate to give $\mathbf{H L}^{\mathbf{1}}$ as a pale yellow powder in good yields.
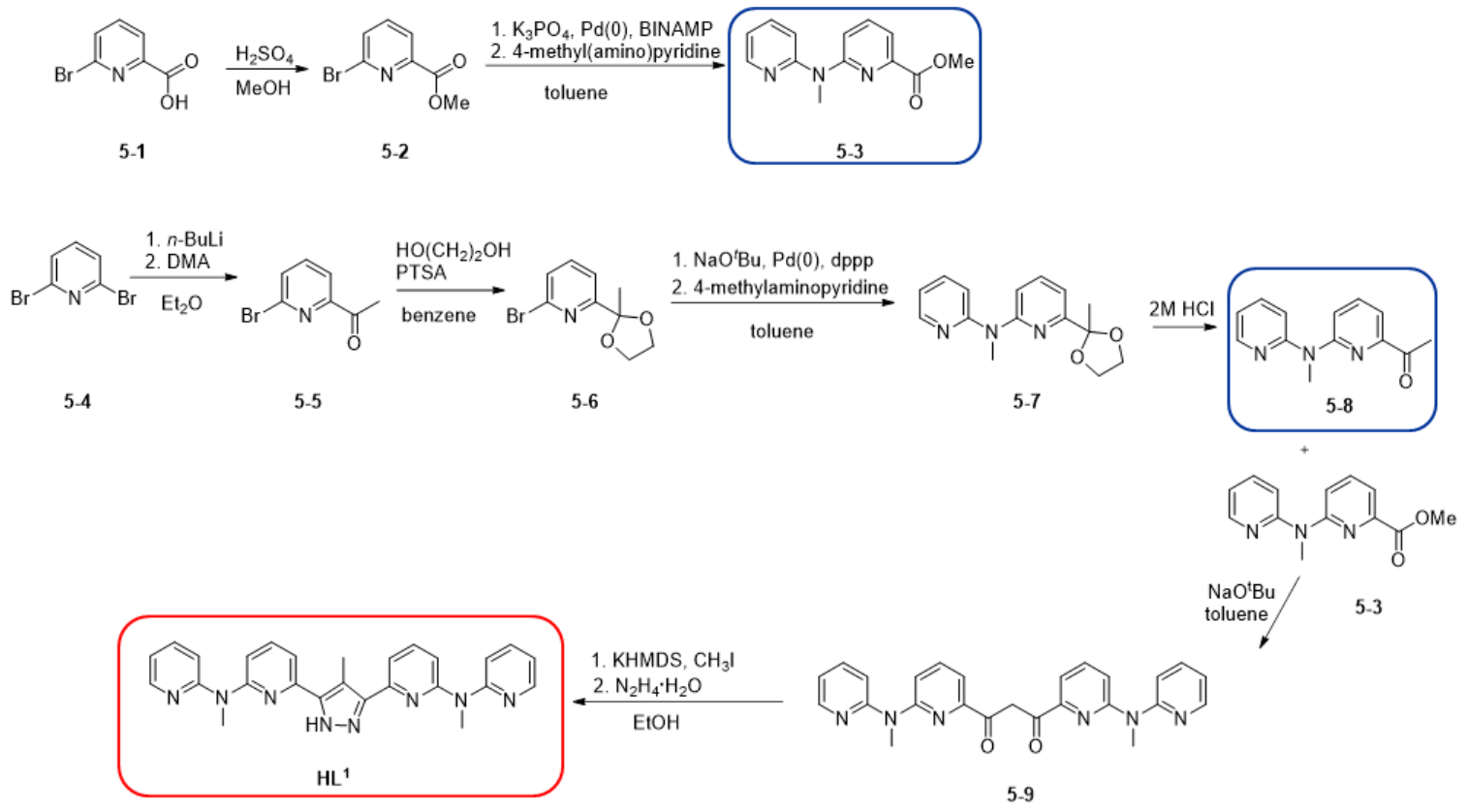

Scheme 5.1. Synthetic scheme for HL1.

\subsection{2 - ${ }^{1}$ H NMR Spectroscopy on HL ${ }^{1}$}

In order to determine the purity of the ligand $\mathbf{H L}^{\mathbf{1}},{ }^{1} \mathrm{H}$ NMR spectroscopy was conducted in dimethyl sulfoxide- $\mathrm{d}_{6}\left(\right.$ DMSO- $\left.\mathrm{d}_{6}\right)$. The ${ }^{1} \mathrm{H}$ NMR spectrum revealed nine peaks suggesting a highly symmetric molecule (Figure 5.1). The signal at $13 \mathrm{ppm}$ corresponds to the proton on the pyrazole moiety, while the methyl groups on the pyrazole backbone and on the bridging nitrogen atoms are observed at $2.61 \mathrm{ppm}$ and $3.61 \mathrm{ppm}$, respectively. The remaining hydrogen atoms are assigned according to ${ }^{1} \mathrm{H}^{-1} \mathrm{H}$ COSY experiments. 

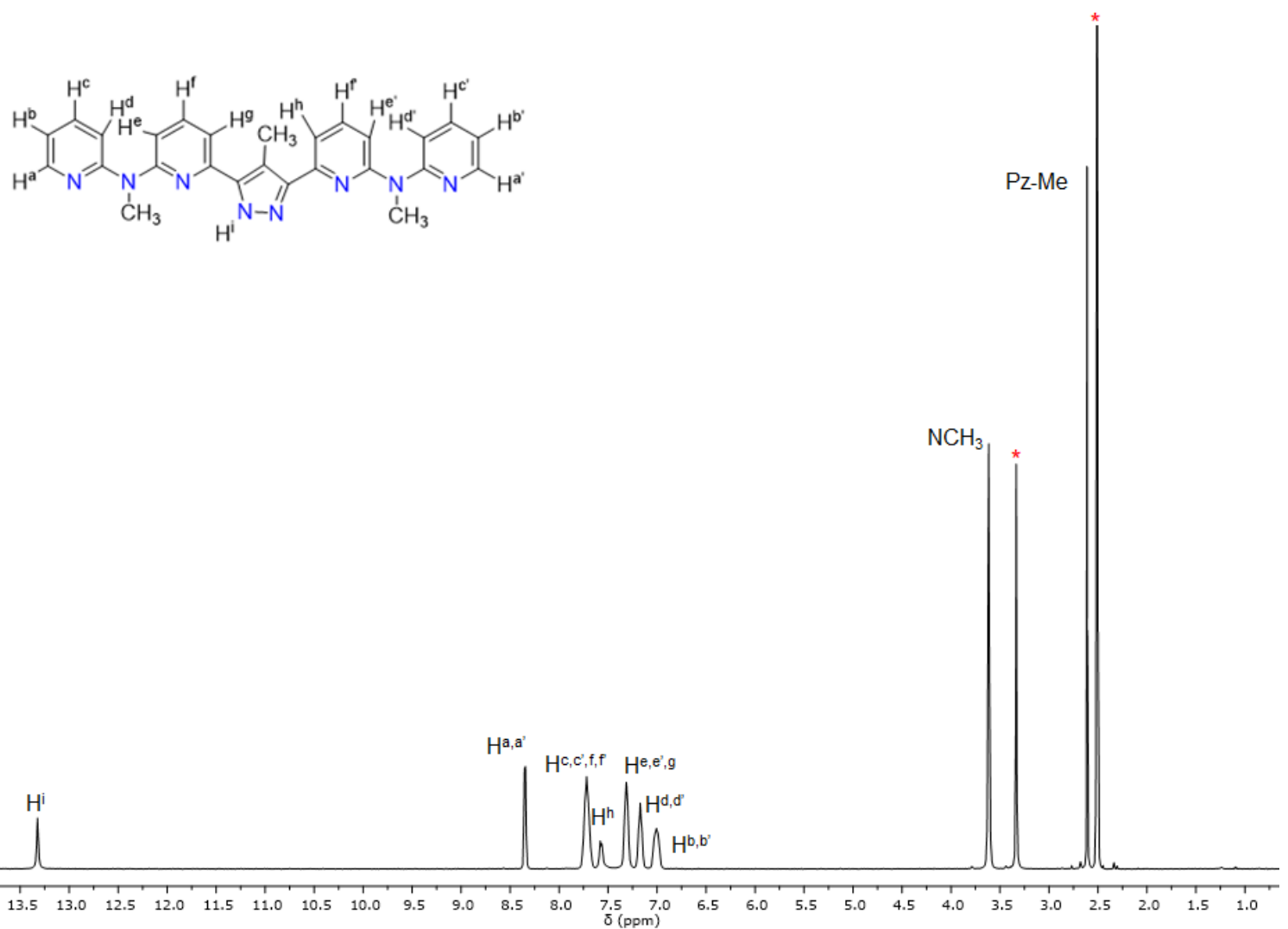

Figure 5.1. ${ }^{1} \mathrm{H}$ NMR spectrum of HL $^{1}{ }^{D M S O}-\mathrm{d}_{6}$. Solvent molecules such as DMSO and water are indicated with asterisks.

\subsection{3 - Complex Synthesis}

As mentioned in the previous chapters, the formation of grid-like arrays requires ligands that preferentially coordinate one metal ion between two mutually perpendicular ligand strands. However, addition of the $\mathrm{NCH}_{3}$ group in between the pyridine rings may have compromised the rigidity required for [ $2 \times 2$ ] grid formation. Instead, a dinuclear $\mathrm{Fe}_{2}$ complex was formed, which was further confirmed by X-ray crystallography. Nevertheless, the dinuclear $\mathrm{Fe}_{2}$ complex with $\mathrm{BF}_{4}-([\mathbf{5 - 1 0 a}])$ and OTf- $([\mathbf{5 - 1 0 b}])$ counterions can be synthesized from $\mathbf{H L}^{\mathbf{1}}$ by addition of stoichiometric amounts of $\mathrm{Fe}^{\mathrm{II}}\left(\mathrm{BF}_{4}\right)_{2} \cdot 6 \mathrm{H}_{2} \mathrm{O}$ or Fe $\mathrm{Fe}^{\mathrm{II}}(\mathrm{OTf})_{2} \cdot 2 \mathrm{MeCN}$ in the presence of sodium $t$-butoxide $\left(\mathrm{NaO}^{t} \mathrm{Bu}\right)$ in dry acetonitrile (Scheme 5.2). After stirring for 16 hours, a yellow precipitate was formed, which was further purified by slow diffusion of diethyl ether into a solution of the product in acetonitrile to give yellow plate-like crystals. 


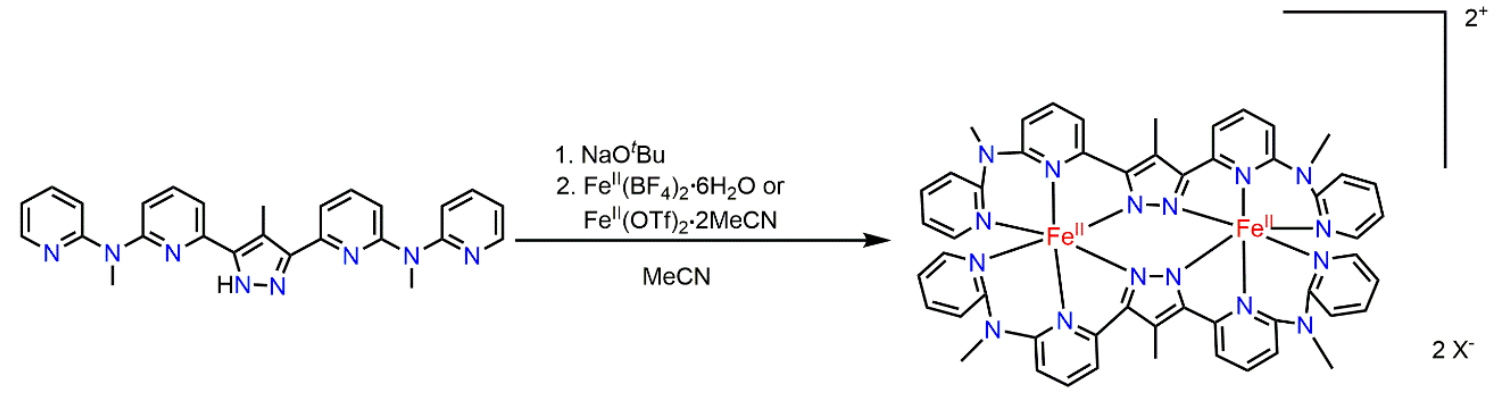

$H L^{1}$

[5-10a]: $\mathrm{X}=\mathrm{BF}_{4}^{-}$

$[5-10 b]: X=O T f$

Scheme 5.2. Synthetic scheme of the dinuclear $\left[\mathrm{L}_{2}{ }_{2} \mathrm{Fe}_{2}\right]\left[\mathrm{BF}_{4}\right]_{2}$ and $\left[\mathrm{L}_{2}{ }_{2} \mathrm{Fe}_{2}\right][\mathrm{OTf}]_{2}$.

\subsection{4 - Single Crystal X-ray Diffraction of $\left[\mathrm{L}_{2}{ }_{2} \mathrm{Fe}_{2}\right][\mathrm{OTf}]_{2}$}

Single crystals of $\left[\mathrm{L}_{2}^{1} \mathrm{Fe}_{2}\right][\mathrm{OTf}]_{2}$ revealed a dinuclear $\mathrm{Fe}_{2}$ complex, in which the nitrogen atoms of both terminal pyridine moieties are twisted inward to coordinate one Fe ion (Figure 5.2). As a result, the planarity required for grid-like arrays is no long present. The molecular structure of $\left[\mathrm{L}_{2}{ }_{2} \mathrm{Fe}_{2}\right][\mathrm{OTf}]_{2}$ is highly symmetric crystallizing in the orthorhombic space group Pnnm, in which both the Fe centers are crystallographically equivalent (Figure 5.2). The overall charge of the molecule is balanced by two triflate (OTf-) molecules. The two Fe ions are in close proximity to one another, separated by $4.346 \AA$, consistent with numerous reported dinuclear $\mathrm{Fe}^{\mathrm{II}}$ complexes with PMRT-type (4-substituted-3,5- bis $\{[(2-$ pyridylmethyl)-amino]methyl\}-4H-1,2,4-triazole) ligands. ${ }^{20}$ Moreover, the average Fe- $\mathrm{N}$ bond lengths of $2.19 \AA$ A suggest the presence of high-spin Fe ${ }^{\text {II }}$ ions at both coordination sites, also observed in various Fe ${ }^{\mathrm{II}}$ complexes with PMAT-based ligands ${ }^{31,132-135}$ as well as in a Fe $\mathrm{Fe}^{\mathrm{II}}$ complex with a binucleating ligand with two $\{\mathrm{PNN}\}$ pincer type subunits. ${ }^{136}$

The overall distortion from an ideal octahedron was described using Continuous Symmetry Measure (CSM), in which a high value of $\mathrm{S}\left(\mathrm{O}_{\mathrm{h}}\right)=11.04$ was determined corresponding to a small trigonal prism geometry value of $S($ itp $)=2.94$. The high deviation from an ideal octahedron stems from the flexibility of the ligand strand which imposes a trigonal twist on the Fe coordination sphere. Although a variety of dinuclear $\mathrm{Fe}_{2}$ complexes displayed SCO behaviour, ${ }^{20}$ the high $\mathrm{S}\left(\mathrm{O}_{\mathrm{h}}\right)$ value obtained for $\left[\mathrm{L}_{2}^{1} \mathrm{Fe}_{2}\right][\mathrm{OTf}]_{2}$ most likely prevents the onset of any thermal spin transitions. 
(a)

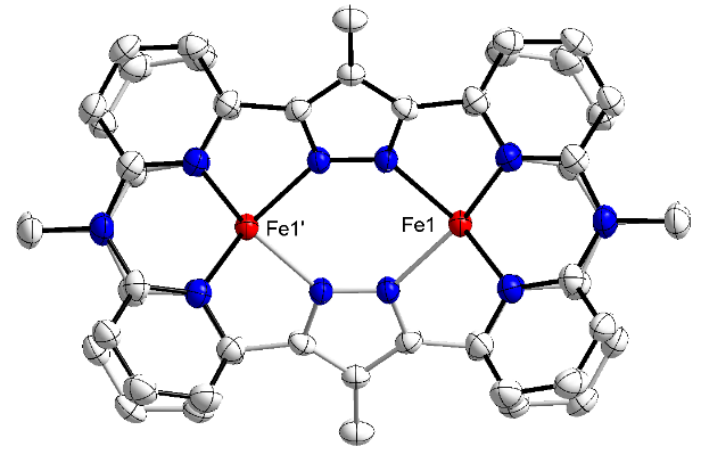

(b)

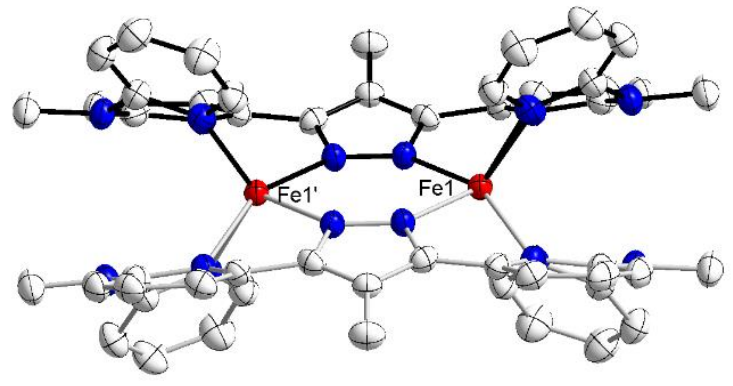

Figure 5.2. (a) Top view and (b) side view of the molecular structure of the dinuclear $\left[\mathrm{L}_{2}{ }_{2} \mathrm{Fe}_{2}\right][\mathrm{OTf}]_{2}(50 \%$ probability thermal ellipsoids). Hydrogen atoms and counterions are omitted for clarity.

\subsection{5 - Mössbauer Spectroscopy \& Magnetic Susceptibility Measurements}

With the presence of iron atoms, the electronic structure of $\left[\mathrm{L}_{2}^{1} \mathrm{Fe}_{2}\right][\mathrm{OTf}]_{2}$ can be further investigated with Mössbauer (MB) spectroscopy (Figure 5.3a). The MB spectrum revealed one doublet with an isomer shift of $\delta=1.10 \mathrm{mms}^{-1}$ and a quadruple splitting of $\Delta E_{\mathrm{Q}}=4.22 \mathrm{mms}^{-1}$, consistent with high-spin $\mathrm{Fe}^{\mathrm{II}}$ ions. The difference in intensity between the two peaks of the doublet can be attributed to the texture of the sample. The relatively large quadruple splitting originates from the highly distorted environment of the Fe centers $\left(\mathrm{S}\left(\mathrm{O}_{\mathrm{h}}\right)=11.04\right)$, giving rise to a large valence and lattice contribution and thus a large electronic field gradient.

(a)

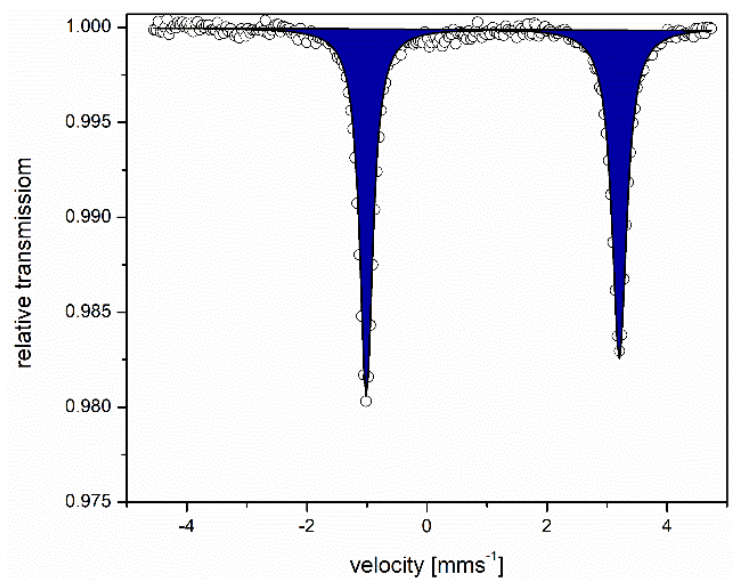

(b)

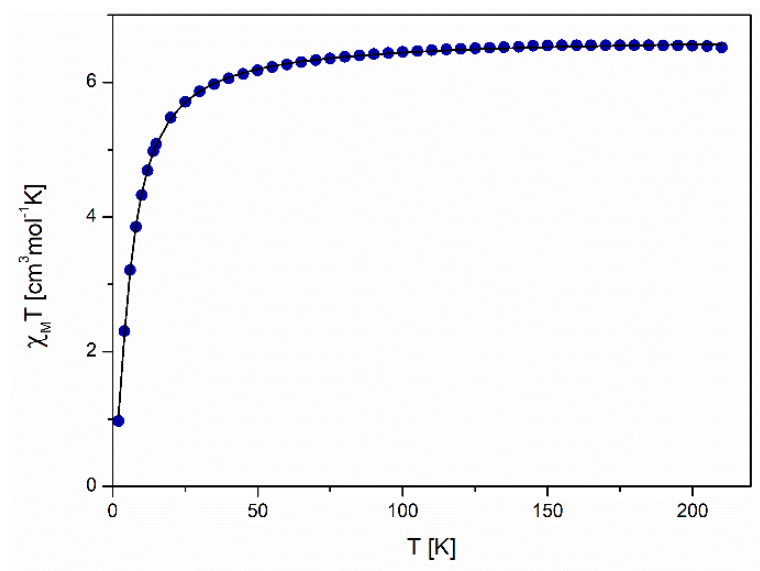

Figure 5.3. (a) Mössbauer spectrum of $\left[\mathrm{L}_{2} \mathrm{Fe}_{2}\right][0 \mathrm{OTf}]_{2}$ revealing a doublet $\left(\delta=1.10 \mathrm{mms}^{-1}\right.$ and $\Delta E_{\mathrm{Q}}=4.22 \mathrm{mms}^{-1}$ ) consistent with $\mathrm{HS}-\mathrm{Fe}^{\mathrm{II}}$ ions. (b) Variable temperature magnetic susceptibility measurement confirming the presence of two antiferromagnetically coupled $\mathrm{Fe}^{\mathrm{II}}(S=2)$ ions. 
The electronic properties were further investigated using magnetic susceptibility measurements from $2-210 \mathrm{~K}$ (Figure 5.3b). Indeed, spin crossover was not observed in this temperature range. Instead, a constant $\chi_{\mathrm{M}} \mathrm{T}$ value of $6.55 \mathrm{~cm}^{3} \mathrm{~mol}^{-1} \mathrm{~K}$ was observed from 210 $50 \mathrm{~K}$, consistent with two uncoupled Fe ${ }^{\mathrm{II}}$ ions $(S=2)$. Below $50 \mathrm{~K}, \chi_{\mathrm{M}} \mathrm{T}$ drastically declines to a minimal at $\chi_{\mathrm{M}} \mathrm{T}=0.970 \mathrm{~cm}^{3} \mathrm{~mol}^{-1} \mathrm{~K}$ due to weak antiferromagnetic coupling between two $\mathrm{Fe}$ II ions. The data was modelled according to the Heisenberg-Dirac-van-Vleck Hamiltonian $(\mathrm{HDvV})$, where $J$ is the coupling constant, $\mu_{\mathrm{B}}$ is the Bohr magneton, and $B$ is the magnetic field (Eq. 5.1). Using this equation, the coupling constant between the two Fe ions was determined to be $J=-0.63 \mathrm{~cm}^{-1}$ with $\mathrm{g}_{1}=\mathrm{g}_{2}=2.11$. Simulation of the data using only the axial zero field splitting (ZFS) without $J$ coupling afforded a lower quality fit. Moreover, although a combination of both $J$ coupling and ZFS can be modelled, the contribution from the ZFS is negligible and thus, the significant decrease of $\chi_{M} T$ below $50 \mathrm{~K}$ can be predominately attributed to weak antiferromagnetic coupling.

$$
\widehat{H}=-2 J\left(\hat{S}_{1} \hat{S}_{2}\right)+g \mu_{B} \sum \vec{B} \vec{S}_{i}
$$

\subsection{6 - Mass Spectrometry}

The integrity of $\left[\mathrm{L}_{2}^{1} \mathrm{Fe}_{2}\right][\mathrm{OTf}]_{2}$ was investigated by mass spectrometry in acetonitrile. One dominant peak at $m / z=503$ was observed corresponding to the doubly charged cation $\left[\mathrm{L}_{2}{ }_{2} \mathrm{Fe}_{2}\right]^{2+}$. The experimental isotopic distribution pattern corresponds well with the simulated pattern (Figure 5.4, inset) confirming $\left[\mathrm{L}_{2}{ }_{2} \mathrm{Fe}_{2}\right][\mathrm{OTf}]_{2}$ remains intact even at low concentrations.

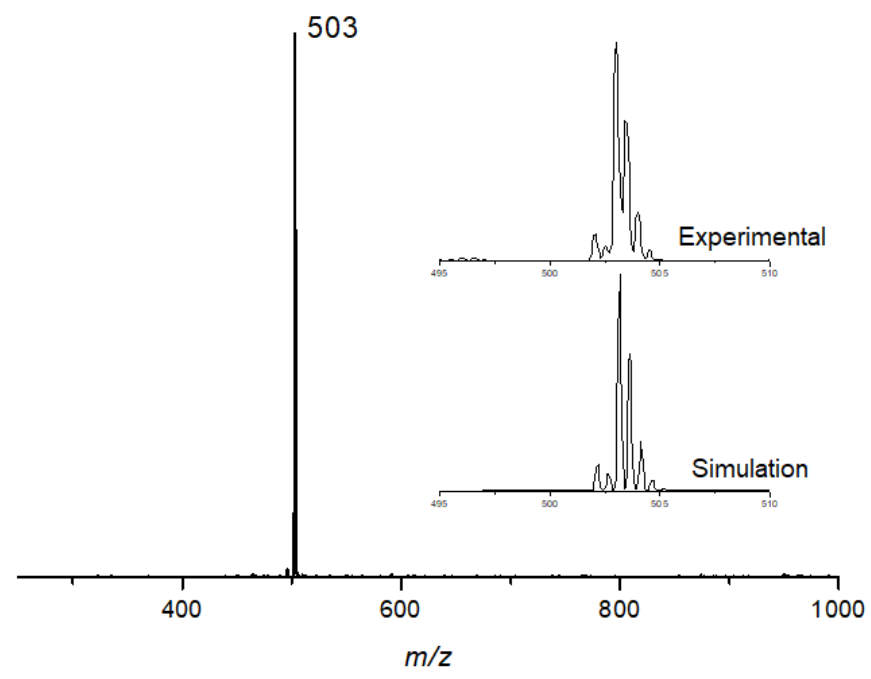

Figure 5.4. ESI-MS of $\left[\mathrm{L}_{2} \mathrm{Fe}_{2}\right][\mathrm{OTf}]_{2}$ in acetonitrile displaying one dominant peak at $m / z=503$, corresponding to the doubly charged cation $\left[\mathrm{L}_{2}{ }_{2} \mathrm{Fe}_{2}\right]^{2+}$. Simulation of the isotopic distribution pattern is shown in the inset. 


\subsection{7 - ${ }^{1} \mathrm{H}$ NMR Spectroscopy}

Similar to the $\left[\mathrm{L}_{4} \mathrm{Ru}_{2} \mathrm{Fe}_{2}\right]\left[\mathrm{BF}_{4}\right]_{4}$ grids discussed in chapter $4, \mathrm{HS}$ - $\mathrm{Fe}^{\mathrm{II}}$ ions have a favourable relaxation time between $10^{-13}$ to $10^{-12} \mathrm{~s}$, and thus paramagnetic ${ }^{1} \mathrm{H}$ NMR was conducted on the $\left[\mathrm{L}_{2}{ }_{2} \mathrm{Fe}_{2}\right][\mathrm{OTf}]_{2}$ in $\mathrm{MeCN}-\mathrm{d}_{3}$. Unfortunately, the linewidths of this spectrum exceed the requirements for $2 \mathrm{D}{ }^{1} \mathrm{H}$ NMR experiments $(<200 \mathrm{~Hz})$. Thus, definitive assignment of the peaks to their corresponding protons could not be made. Nevertheless, the ${ }^{1} \mathrm{H}$ NMR spectrum of $\left[\mathrm{L}_{2} \mathrm{Fe}_{2}\right][\mathrm{OTf}]_{2}$ reveals nine distinct peaks (including the broad bump at $61 \mathrm{ppm}$ ), confirming the symmetric nature of the complex (Figure 5.5). The weak broad peak at $61 \mathrm{ppm}$ may correspond to the proton closest to the paramagnetic center $(3.024 \AA), \mathrm{H}^{\mathrm{a}}$, following the labeling scheme of the ligand $\mathbf{H L}^{\mathbf{1}}$ (Figure 5.1). The distances of the remaining protons from the paramagnetic Fe center range from $4.9 \AA$ to $5.7 \AA$ and may be tentatively assigned based on the proton-metal distances. Based on the integration, the methyl groups on the pyrazole backbone and on the bridging nitrogen atoms can be assigned to the signals at $22.06 \mathrm{ppm}$ and -2.79 ppm, respectively. Although the distance between the Pz-Me groups and the paramagnetic Fe center are larger than the $\mathrm{N}-\mathrm{CH}_{3}$ groups, the Pz-Me groups are situated in close proximity between both paramagnetic Fe centers, and hence are shifted to higher fields compared to the $\mathrm{NCH}_{3}$ resonances.

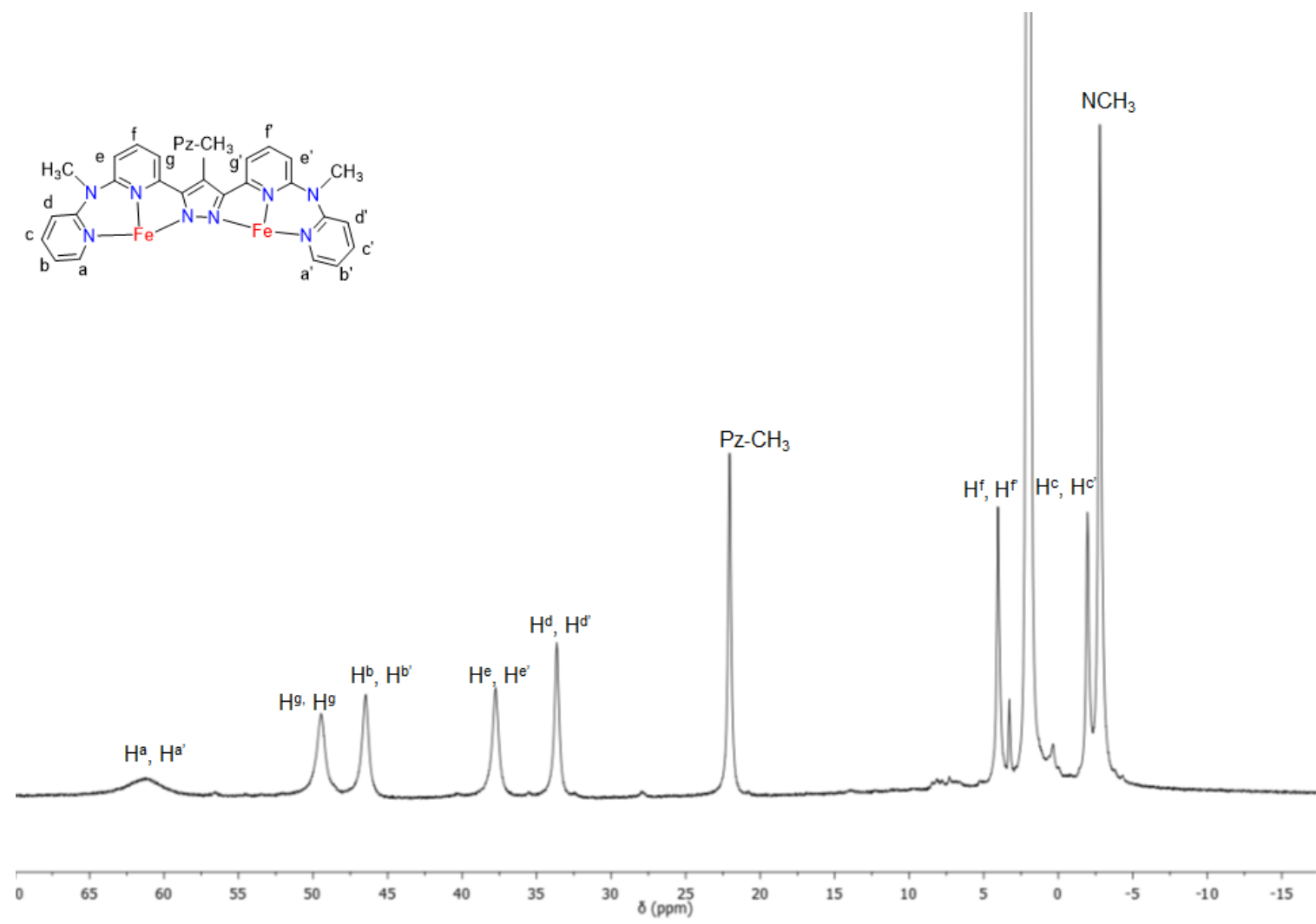

Figure 5.5. ${ }^{1} \mathrm{H}$ NMR spectrum of $\left[\mathrm{L}_{2}^{1} \mathrm{Fe}_{2}\right][\mathrm{OTf}]_{2}$ in $\mathrm{MeCN}-\mathrm{d}_{3}$ at room temperature revealing nine peaks. 


\subsection{8 - Cyclic Voltammetry and Spectroelectrochemistry}

The redox properties of $\left[\mathrm{L}_{2}^{1} \mathrm{Fe}_{2}\right][\mathrm{OTf}]_{2}$ was investigated using cyclic voltammetry and square wave voltammetry in acetonitrile (Figure 5.6). Two reversible waves at $E_{1 / 2}=0.019 \mathrm{~V}$ and $E_{1 / 2}$ $=0.530 \mathrm{~V}$ versus $\mathrm{Fc} / \mathrm{Fc}^{+}$were observed, corresponding to the $\mathrm{Fe}_{2} \mathrm{II} / \mathrm{Fe}^{\mathrm{II}} \mathrm{Fe}^{\mathrm{III}}$ and $\mathrm{Fe}^{\mathrm{II}} \mathrm{Fe}^{\mathrm{III}} / \mathrm{Fe}^{\mathrm{III}}{ }_{2}$ couple, respectively. The difference between the anodic and cathodic peak potentials $(v=50$ $\mathrm{mV} / \mathrm{s}$ ) are $91 \mathrm{mV}$ and $143 \mathrm{mV}$ for the first and second oxidative wave, respectively. While the first redox process displays reversible behaviour based on scan-rate dependent measurements (Figure A39, peak separations $\Delta E_{\mathrm{p}}=80-96 \mathrm{mV} ; i_{\mathrm{pf}} \mathrm{vs} . v^{1 / 2}$ ), the current of the second anodic wave $\left(i_{\mathrm{pr}}\right)$ becomes more irreversible at slower scan rates (i.e. $i_{\mathrm{pr}}$ becomes significantly smaller compared to $i_{\mathrm{pf}}$ ) most likely due to decomposition from the highly charged nature of the molecule. Moreover, a large separation of $511 \mathrm{mV}$ was observed between the first and second anodic wave suggesting a pronounced thermodynamic stability of the mixed valent $\left[\mathrm{L}_{2}{ }_{2} \mathrm{Fe}^{\mathrm{IIF}} \mathrm{Fe}^{\mathrm{III}}\right][\mathrm{OTf}]_{3}$ complex $\left(K_{\mathrm{c}}=4.39 \times 10^{8}\right)$, which was indeed chemically synthesized (discussed in the following sections). All electrochemical parameters are tabulated in Table 5.1.

(a)

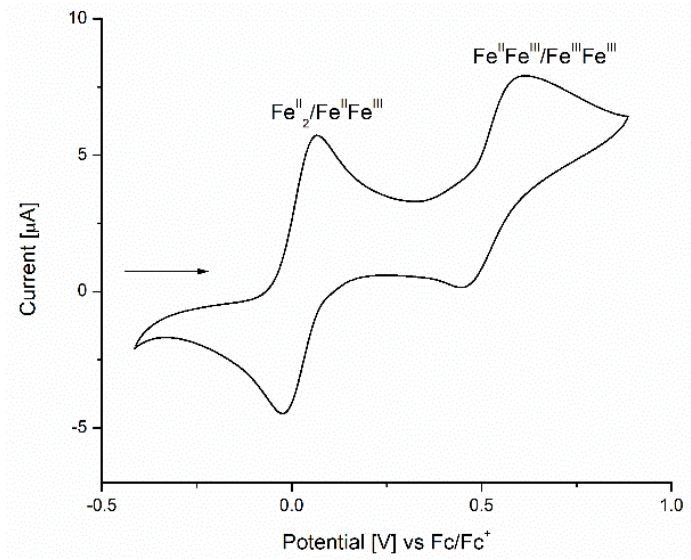

(b)

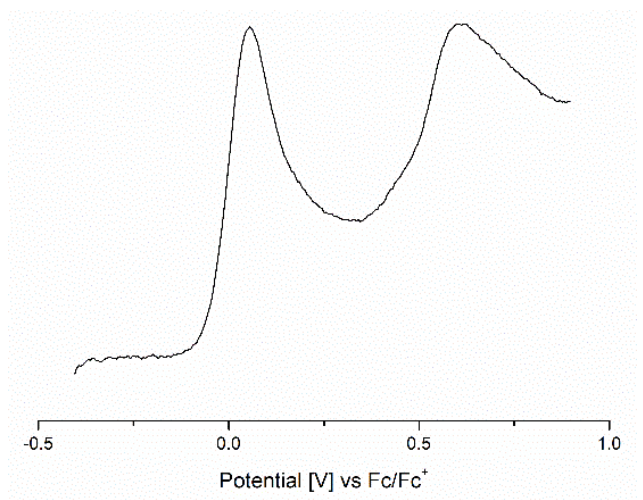

Figure 5.6. (a) Cyclic voltammetry and (b) square wave voltammetry of $\left[\mathrm{L}_{2}^{1} \mathrm{Fe}_{2}\right][\mathrm{OTf}]_{2}$ in acetonitrile at $100 \mathrm{mV} / \mathrm{s}$ with $\left[\mathrm{Bu}_{4} \mathrm{~N}\right] \mathrm{PF}_{6}$ as the electrolyte. All potentials are referenced versus $\mathrm{Fc} / \mathrm{Fc}^{+}$.

Table 5.1. Electrochemical parameters for $\left[\mathrm{L}_{2}{ }_{2} \mathrm{Fe}_{2}\right][\mathrm{OTf}]_{2}$ in acetonitrile at $50 \mathrm{mV} / \mathrm{s}$.

\begin{tabular}{cccc}
\hline & $E_{1 / 2}[\mathrm{mV}]$ & $\Delta E_{\mathrm{p}}[\mathrm{mV}]$ & $\Delta E_{1 / 2}-\Delta E_{1 / 2}[\mathrm{mV}]$ \\
\hline $\mathrm{Fe}^{\mathrm{II}} / \mathrm{Fe}^{\mathrm{II}} \mathrm{Fe}^{\mathrm{III}}$ & 19 & 91 & 511 \\
$\mathrm{Fe}^{\mathrm{II}} \mathrm{Fe}^{\mathrm{IIII}} / \mathrm{Fe}^{\mathrm{III}}{ }_{2}$ & 530 & 143 & \\
\hline
\end{tabular}

In order to monitor the changes in the UV-vis spectrum for the electrochemical formation of the mixed valent $\left[\mathrm{L}_{2}{ }_{2} \mathrm{Fe}^{I I F} \mathrm{Fe}^{\mathrm{III}}\right][\mathrm{OTf}]_{3}$ and doubly oxidized $\left[\mathrm{L}_{2}{ }_{2} \mathrm{Fe}^{\mathrm{III}}{ }_{2}\right][\mathrm{OTf}]_{4}$ complex, UV-vis spectroelectrochemistry was conducted by applying a voltage of $0.53 \mathrm{~V}$ and $1.03 \mathrm{~V}$ vs Fc/Fc+, respectively. Initially, a strong band at $332 \mathrm{~nm}$ corresponding to the ligand $\pi \rightarrow \pi^{*}$ transition concomitant with a weaker broad band at $468 \mathrm{~nm}$, most likely corresponding to the MLCT transition, were observed. Upon oxidation to the mixed valent $\left[\mathrm{L}_{2} \mathrm{Fe}^{\mathrm{II}} \mathrm{Fe}^{\mathrm{III}}\right][\mathrm{OTf}]_{3}$, the broad band at $468 \mathrm{~nm}$ gradually disappears and a new broad band at $634 \mathrm{~nm}$ is observed (Figure 5.7a). This band may correspond to an intervalence charge transfer (IVCT) or ligand-to-metal- 
charge transfer (LMCT). Moreover, two isosbestic points were observed suggesting that oxidation is localized on one Fe atom. Further electrochemical oxidation to the doubly oxidized $\left[\mathrm{L}_{2}^{1} \mathrm{Fe}_{2} \mathrm{III}_{2}\right][\mathrm{OTf}]_{4}$ gave rise to minimal additional changes; the intensity of the broad band at $634 \mathrm{~nm}$ continues to increase marginally. This suggest that this broad band most likely originates from a LMCT.

(a)

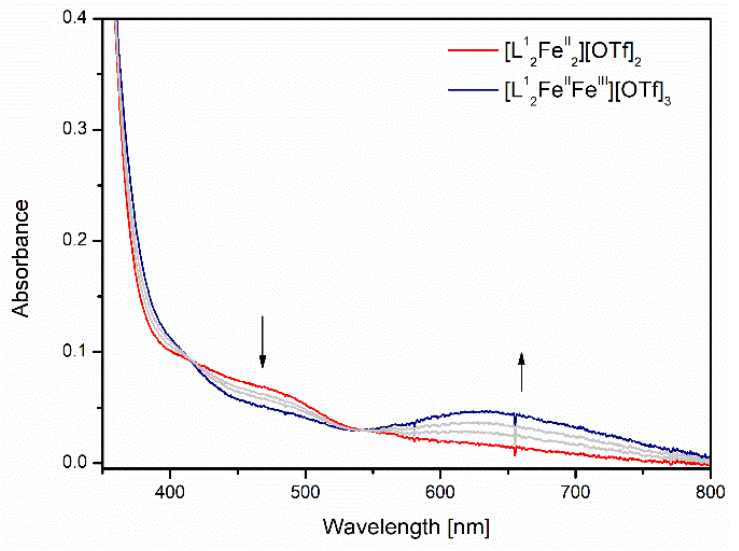

(b)

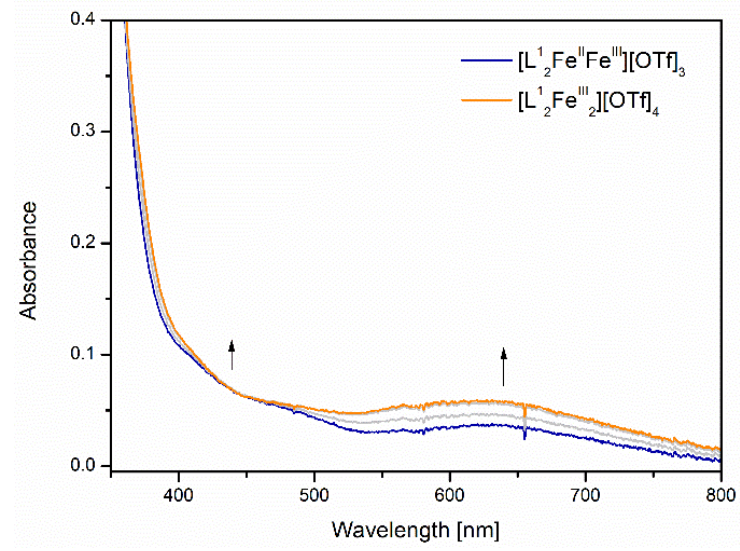

Figure 5.7. UV-vis spectroelectrochemistry of the (a) first and (b) second oxidation of $\left[\mathrm{L}_{2}{ }_{2} \mathrm{Fe}^{\mathrm{II}}\right][\mathrm{OTf}]_{2}$.

\section{3 - Synthesis of mixed valent $\left[\mathrm{L}_{2}{ }_{2} \mathrm{Fe}^{\mathrm{IIF}} \mathrm{Fe}^{\mathrm{III}}\right][\mathrm{OTf}]_{3}$ and Doubly Oxidized $\left[\mathrm{L}_{2}{ }_{2} \mathrm{Fe}^{\mathrm{III}}{ }_{2}\right][\mathrm{OTf}]_{4}$}

Based on the cyclic voltammetry experiments, the mixed valent $\left[\mathrm{L}_{2}^{1} \mathrm{Fe}^{\mathrm{II}} \mathrm{Fe}^{\mathrm{III}}\right][\mathrm{OTf}]_{3}$ and the doubly oxidized $\left[\mathrm{L}_{2}{ }_{2} \mathrm{Fe}^{\mathrm{III}}{ }_{2}\right][\mathrm{OTf}]_{4}$ complex may be chemically synthesized by addition of a suitable oxidizing agent (Scheme 5.3). In this case, $\mathrm{Cu}(\mathrm{OTf})_{2}\left(E_{1 / 2}=0.40 \mathrm{vs} \mathrm{Fc} / \mathrm{Fc}^{+}\right.$in $\mathrm{MeCN}$ ) was used. However, a variety of other oxidizing agents were used including $\mathrm{AgBF}_{4}$ in $\mathrm{THF}$ $\left(E_{1 / 2}=0.41 \mathrm{vs} \mathrm{Fc} / \mathrm{Fc}^{+}\right)$and [thianthrene $]^{+}\left[\mathrm{BF}_{4}\right]^{-}\left(E_{1 / 2}=0.86 \mathrm{vs} \mathrm{Fc} / \mathrm{Fc}^{+}\right)$in MeCN. In the case of $\mathrm{AgBF}_{4}$, the so-obtained $\mathrm{MB}$ spectrum of the product contained several different Fe species, which could not be removed even with repeated crystallizations. On the contrary, the MB spectrum of the mixed valent $\left[\mathrm{L}_{2}^{1} \mathrm{Fe}^{\mathrm{II}} \mathrm{Fe}^{\mathrm{III}}\right][\mathrm{OTf}]_{3}$ obtained with stoichiometric amount of [thianthrene] $]^{+}\left[\mathrm{BF}_{4}\right]^{-}$afforded the expected two different Fe species, but a ratio of 42:58 ( $\left.\mathrm{Fe}^{\mathrm{II}}: \mathrm{Fe}^{\mathrm{III}}\right)$ was obtained due to the higher oxidizing potential of [thianthrene $]^{++}\left[\mathrm{BF}_{4}\right]^{-}$: As a result, both Fe ions in $\left[\mathrm{L}_{2}{ }_{2} \mathrm{Fe}^{\mathrm{II}}{ }_{2}\right][\mathrm{OTf}]_{2}$ may be oxidized. Consequently, $\mathrm{Cu}(\mathrm{OTf})_{2}$ was used as the oxidizing agent. Upon addition of one equivalent of $\mathrm{Cu}(\mathrm{OTf})_{2}$ to a solution of $\left[\mathrm{L}_{2}{ }_{2} \mathrm{Fel}_{2}{ }_{2}\right][\mathrm{OTf}]_{2}$ in dry MeCN, an immediate colour change from a yellow suspension to a dark green solution was observed. The solution was stirred at room temperature for 16 hours, concentrated and filtered. Subsequently, diethyl ether was slowly diffused into the MeCN solution of the product to give green plate-like crystals. It was later deduced that the mixed valent $\left[\mathrm{L}_{2} \mathrm{Fe}^{\mathrm{IIIF}} \mathrm{Fe}^{\mathrm{III}}\right][\mathrm{OTf}]_{3}$ slowly decomposes in the presence of air affording a yellow/brown powder. Thus, it is essential the synthesis and crystallizations are carried out under an inert atmosphere. In contrast, synthesis of the doubly oxidized $\left[\mathrm{L}_{2}{ }_{2} \mathrm{Fe}^{\mathrm{III}}{ }_{2}\right][\mathrm{OTf}]_{4}$ congener required additional time and heat. Starting from $\left[\mathrm{L}_{2}{ }_{2} \mathrm{Fe}^{\mathrm{II}}{ }_{2}\right][\mathrm{OTf}]_{2}$, two equivalents of [thianthrene $]^{+}\left[\mathrm{BF}_{4}\right]^{-}$were added and the reaction was heated at $70{ }^{\circ} \mathrm{C}$ for 48 hours to give a red powder upon precipitation with diethyl ether. Unfortunately, further characterization of $\left[\mathrm{L}_{2} \mathrm{Fe}^{\mathrm{III}}{ }_{2}\right][\mathrm{OTf}]_{4}$ led to decomposition and was not further pursued. The following sections will focus mainly on the properties of the mixed valent $\left[\mathrm{L}_{2}^{1} \mathrm{Fe}^{\mathrm{II}} \mathrm{Fe}{ }^{\mathrm{III}}\right][\mathrm{OTf}]_{3}$ complex. 


\subsection{1 - X-ray Crystallography on the Mixed valent $\left[\mathrm{L}_{2}{ }_{2} \mathrm{Fe}^{\mathrm{IIF}} \mathrm{Fe}^{\mathrm{III}}\right][\mathrm{OTf}]_{3}$ Complex}

Although $\mathrm{Cu}(\mathrm{OTf})_{2}$ was chosen as the primarily oxidizing agent, the crystal structure of the

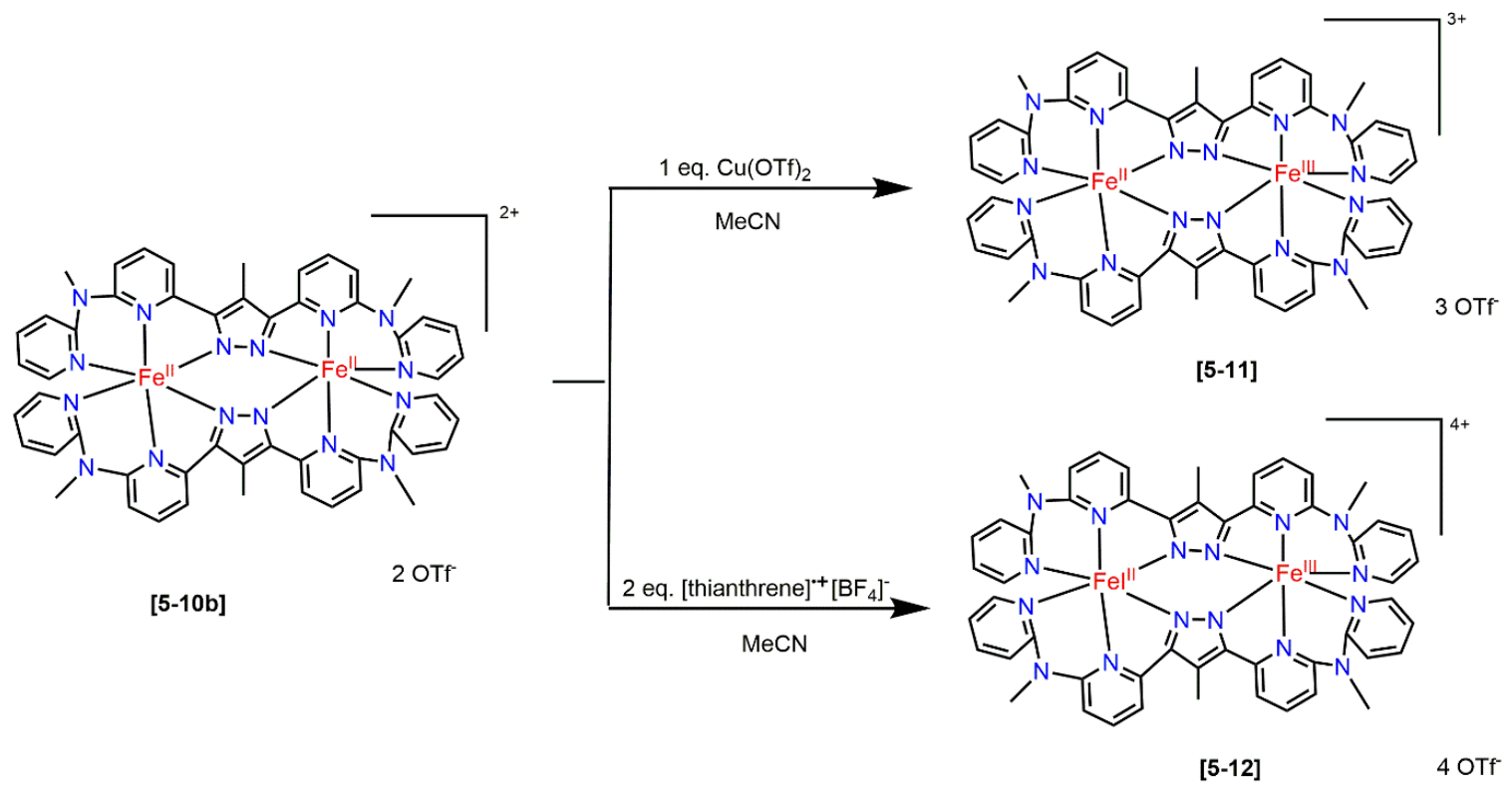

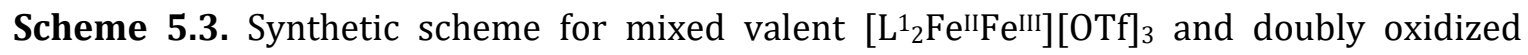
$\left[\mathrm{L}_{2}{ }_{2} \mathrm{Fe}^{\mathrm{III}}{ }_{2}\right][\mathrm{OTf}]_{4}$

mixed valent compound was obtained by oxidation with one equivalent of [thianthrene $]^{+}\left[\mathrm{BF}_{4}\right]^{-}$. Consequently, due to incomplete anion exchange, a mixture of two OTfanions and one $\mathrm{BF}_{4}^{-}$anion are present in the crystal lattice. The molecular structure of the mixed valent $\left[\mathrm{L}_{2}{ }_{2} \mathrm{Fe}^{\mathrm{II}} \mathrm{Fe}^{\mathrm{III}}\right][\mathrm{OTf}]_{2}\left[\mathrm{BF}_{4}\right]$ crystallizes in the triclinic space group $P \overline{1}$ (Figure 5.8 ). Moreover acetonitrile molecules are also present in the crystal lattice. Analysis of the bond lengths of each Fe center afforded average bond lengths of $2.17 \AA$ A for Fe1 and $1.95 \AA$ for Fe2, consistent with high-spin Fe' ${ }^{I I}$ ions and low-spin FeIII ions, respectively. Thus, oxidation to the mixed valent is accompanied by large structural changes in which one of the high-spin Fe ${ }^{\mathrm{II}}$ ions is converted to a low-spin Fe ${ }^{\mathrm{III}}$ ion. To quantify the structural changes, Continuous Symmetry Measure was determined for each of the Fe ions. To that end, the HS-FeII ion retains its highly distorted environment with $\mathrm{S}\left(\mathrm{O}_{\mathrm{h}}\right)$ values of 11.82 and $\mathrm{S}(\mathrm{itp})$ values of 2.63. In contrast, the oxidized LS-Fe'III ion favours an octahedral geometry, in which values an $\mathrm{S}\left(\mathrm{O}_{\mathrm{h}}\right)$ value of 2.21 and $S($ itp) value of 8.48 were determined. All parameters are tabulated in Table 5.2 . 
(a)

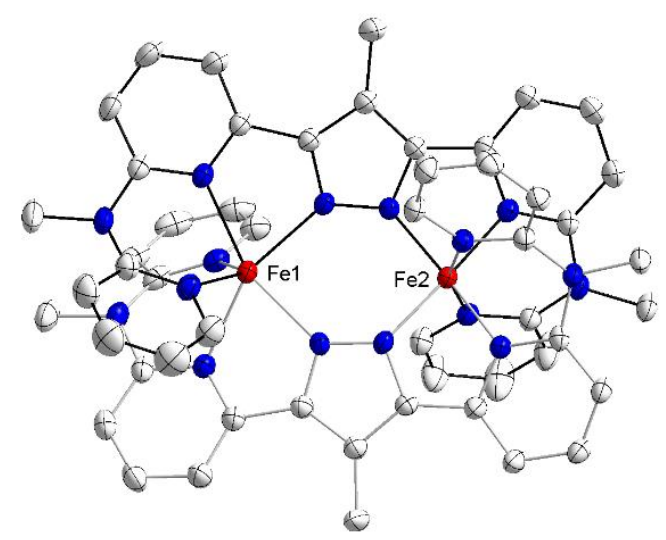

(b)

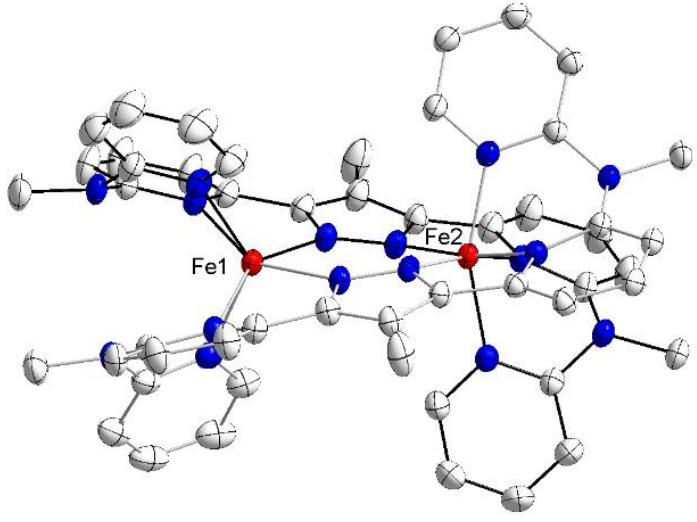

Figure 5.8. (a) Top and (b) side view of the molecular structure of $\left[\mathrm{L}_{2}^{1} \mathrm{Fe}^{\mathrm{II}} \mathrm{Fe}^{\mathrm{III}}\right][\mathrm{OTf}]_{2}\left[\mathrm{BF}_{4}\right]$ complex (50\% probability thermal ellipsoids).

Table 5.2. Selected bond lengths and Continuous Symmetry Measures (CSM) for the parent $\left[\mathrm{L}_{2}{ }_{2} \mathrm{Fe}^{\mathrm{II}}{ }_{2}\right][\mathrm{OTf}]_{2}$ and mixed valent $\left[\mathrm{L}_{2}{ }_{2} \mathrm{Fe}^{\mathrm{II}} \mathrm{Fe}^{\mathrm{III}}\right][\mathrm{OTf}]_{2}\left[\mathrm{BF}_{4}\right]$.

\begin{tabular}{ccccc}
\hline & & $\mathbf{d}_{\text {avg }}(\AA)$ & $\mathbf{S}\left(\mathbf{O}_{\mathbf{h}}\right)$ & S(itp) \\
\hline \multirow{2}{*}[\mathrm{L}_{2}{}_{2}\mathrm{Fe}^{\mathrm{II}}{}_{2}]{$[\mathrm{OTf}]_{2}$} & Fe1-N & 2.19 & 11.04 & 2.96 \\
& $\mathrm{Fe} 1^{\prime}-\mathrm{N}$ & 2.19 & 11.04 & 2.96 \\
\hline \multirow{2}{*}[\mathrm{L}_{2}{}_{2}\mathrm{Fe}^{\mathrm{II}}\mathrm{Fe}^{\mathrm{III}}]{$[\mathrm{OTf}]_{2}\left[\mathrm{BF}_{4}\right]$} & $\mathrm{Fe} 1-\mathrm{N}$ & 2.17 & 11.82 & 2.63 \\
& Fe2-N & 1.95 & 2.21 & 8.48 \\
\hline
\end{tabular}

More interestingly, comparison of the molecular structure of the parent $\left[\mathrm{L}_{2}{ }_{2} \mathrm{Fe}^{\mathrm{II}}{ }_{2}\right][\mathrm{OTf}]_{2}$ and the mixed valent $\left[\mathrm{L}_{2}{ }_{2} \mathrm{Fe}^{\mathrm{II}} \mathrm{Fe}^{\mathrm{III}}\right][\mathrm{OTf}]_{2}\left[\mathrm{BF}_{4}\right]$ revealed stark differences in the coordination geometry. In the former, both ends of the terminal pyridine groups are cis to one another (i.e. related by $\mathrm{C}_{2 \mathrm{v}}$ symmetry). However, in the case of the mixed valent complex, a transconfirmation takes place in which one end of the pyridine groups is twisted in one direction while the other is twisted in the opposite direction forming a helical structure. This was also observed in several dinuclear Fe ${ }^{\mathrm{II}}$ complexes with PMRT-based ligands, namely replacement of the NH linkages in PMRT with sulfur atoms (PSRT ligands) as well as in a thiadiazole analogue of PMRT.134,137 While PMRT ligands are known to coordinate two Fe ions in a cisaxial fashion (similar to $\left[\mathrm{L}_{2}^{1} \mathrm{Fe}_{2}{ }_{2}\right][\mathrm{OTf}]$ ), ${ }^{20}$ the increase in flexibility from the longer $\mathrm{C}-\mathrm{S}$ bond in PSRT ligands compared to the $\mathrm{C}-\mathrm{N}$ bond in PMRT ligands permitted both cis and trans coordination modes for PSRT ligands. Similarly, the flexibility of $\mathbf{H L}^{\mathbf{1}}$ may be favourable for both cis- and trans-binding modes depending on the metal ion. In particular, the cis-binding mode was observed for the parent $\left[\mathrm{L}_{2} \mathrm{Fe}_{2} \mathrm{II}_{2}\right][\mathrm{OTf}]_{2}$, while the trans-binding mode was observed for the mixed valent $\left[\mathrm{L}_{2} \mathrm{Fe}^{\mathrm{II} F e}{ }^{\mathrm{III}}\right]\left[\mathrm{OTf}_{2}\left[\mathrm{BF}_{4}\right]\right.$ complex. This also suggest that during the oxidation process, the dinuclear $\mathrm{Fe}_{2}$ complex may partially dissociate in solution and subsequently recombine to form the desired framework of the mixed valent $\left[\mathrm{L}_{2}{ }_{2} \mathrm{Fe}^{\mathrm{II}} \mathrm{Fe}^{\mathrm{III}}\right][\mathrm{OTf}]_{2}\left[\mathrm{BF}_{4}\right]$ species. This assumption may explain the initial impurities in the Mössbauer spectrum as well as in the ${ }^{1} \mathrm{H}$ NMR spectrum, which can be removed by crystallizations. 


\subsection{2 - Mössbauer Spectroscopy on the Mixed valent $\left[\mathrm{L}^{1}{ }_{2} \mathrm{Fe}^{\mathrm{II}} \mathrm{Fe}^{\mathrm{III}}\right][\mathrm{OTf}]_{3}$}

Based on the bond lengths obtained from the molecular structure of $\left[\mathrm{L}_{2}^{1} \mathrm{Fe}^{I I} \mathrm{Fe}{ }^{I I I}\right][\mathrm{OTf}]_{2}\left[\mathrm{BF}_{4}\right]$, one HS-FeII ion and one LS-Fe ${ }^{\mathrm{III}}$ ion is present. This was further confirmed with MB spectroscopy on a sample obtained by oxidation with $\mathrm{Cu}(\mathrm{OTf})_{2}$, in which two doublets with an isomer shift of $\delta=1.12 \mathrm{mms}^{-1}\left(\Delta E_{\mathrm{Q}}=3.98 \mathrm{mms}^{-1}\right)$ and $\delta=0.15 \mathrm{mms}^{-1}\left(\Delta E_{\mathrm{Q}}=2.26 \mathrm{mms}^{-1}\right)$ were observed (Figure 5.9). Similar to the previous MB spectrum of $\left[\mathrm{L}_{2}^{1} \mathrm{Fe}^{\mathrm{II}}\right][\mathrm{OTf}]_{2}$, the doublet with a large isomer shift $\left(\delta=1.12 \mathrm{mms}^{-1}\right)$ corresponds to a HS-Fe ${ }^{\mathrm{II}}$ species, while the doublet with a significantly smaller isomer shift $\left(\delta=0.15 \mathrm{mms}^{-1}\right)$ corresponds to a low-spin Fe ${ }^{I I I}$ species. Moreover, a ratio of $1: 1$ ( $\mathrm{Fe}^{\mathrm{II}}: \mathrm{Fe}^{\mathrm{III}}$ ) was also observed, consistent with the molecular structure of $\left[\mathrm{L}_{2}{ }_{2} \mathrm{Fe}^{\mathrm{II} F e} \mathrm{FIII}^{\mathrm{III}}\right][\mathrm{OTf}]_{2}\left[\mathrm{BF}_{4}\right]$. Similar to the MB spectrum of the parent $\left[\mathrm{L}_{2}{ }_{2} \mathrm{Fe}_{2}\right][\mathrm{OTf}]_{2}$, the marginal difference in intensity between the peaks of one doublet is attributed to the amorphous texture of the sample. All MB parameters are tabulated in Table 5.3 .

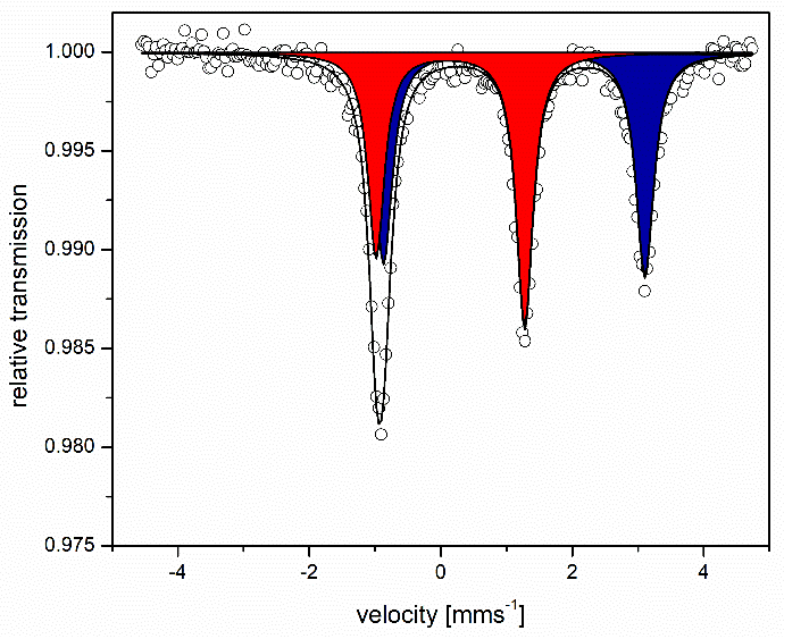

Figure 5.9. MB spectrum of the mixed valent $\left[\mathrm{L}^{1}{ }_{2} \mathrm{Fe}^{\mathrm{II}} \mathrm{Fe}^{\mathrm{III}}\right][\mathrm{OTf}]_{3}$ at $80 \mathrm{~K}$.

Table 5.3. MB parameters for $\left[\mathrm{L}_{2}{ }_{2} \mathrm{Fe}^{\mathrm{II}}{ }_{2}\right][\mathrm{OTf}]_{2}$ and $\left[\mathrm{L}_{2}^{1} \mathrm{Fe}^{\mathrm{II}} \mathrm{Fe}^{\mathrm{III}} \mathrm{L}_{2}{ }_{2}\right][\mathrm{OTf}]_{3}$ at $80 \mathrm{~K}$.

\begin{tabular}{ccccc}
\hline & $\mathbf{T}[\mathrm{K}]$ & $\boldsymbol{\delta}\left[\mathbf{m m s}^{-1}\right]$ & $\boldsymbol{\Delta} \boldsymbol{E}_{\mathbf{Q}}\left[\mathbf{m m s}^{-1}\right]$ & $\begin{array}{c}\text { Rel. Intensity } \\
{[\%]}\end{array}$ \\
\hline$\left[\mathrm{L}_{2}{ }_{2} \mathrm{Fe}^{\mathrm{II}}{ }_{2}\right][\mathrm{OTf}]_{2}$ & 80 & 1.10 & 4.22 & 100 \\
{$\left[\mathrm{~L}_{2}{ }_{2} \mathrm{Fe}^{\mathrm{II}} \mathrm{Fe}^{\mathrm{III}} \mathrm{L}_{2}{ }_{2}\right][\mathrm{OTf}]_{3}$} & 80 & 1.12 & 3.98 & 49 \\
& & 0.15 & 2.26 & 51 \\
\hline
\end{tabular}




\subsection{3 - ${ }^{1} \mathrm{H}$ NMR Spectroscopy on the Mixed valent $\left[\mathrm{L}_{2}{ }_{2} \mathrm{Fe}^{\mathrm{II}} \mathrm{Fe} \mathrm{e}^{\mathrm{III}}\right][\mathrm{OTf}]_{3}$}

The integrity of the mixed valent $\left[\mathrm{L}_{2}{ }_{2} \mathrm{Fe}^{\mathrm{II}} \mathrm{Fe}^{\mathrm{III}} \mathrm{L}_{2}{ }_{2}\right][\mathrm{OTf}]_{3}$ in solution was also investigated by ${ }^{1} \mathrm{H}$ NMR spectroscopy in MeCN- $\mathrm{d}_{3}$ revealing a new set of resonances. For comparison, both ${ }^{1} \mathrm{H}$ NMR spectra of the parent $\left[\mathrm{L}_{2}{ }_{2} \mathrm{Fe}^{\mathrm{II}}{ }_{2}\right][\mathrm{OTf}]_{2}$ and mixed valent $\left[\mathrm{L}_{2} \mathrm{Fe}^{\mathrm{II}} \mathrm{Fe}^{\mathrm{III}}\right][\mathrm{OTf}]_{3}$ are shown in Figure 5.10. Although the peaks cannot be assigned definitively by 2D NMR spectroscopy due to the broad linewidths, comparison with ${ }^{1} \mathrm{H}$ NMR spectrum of the parent complex suggest full conversion between the two species. Excluding the small resonances, originating from diamagnetic impurities, nine peaks are once again observed. Although two sets of resonances are expected for the high-spin Fe'II ion and low-spin Fe III ion, fast IVCT between the two Fe ions may result in only a single set of signals. Consequently, ${ }^{1} \mathrm{H}$ NMR spectroscopy was conducted on the same sample at $233 \mathrm{~K}$ (see below).

$\left[\mathrm{L}_{2}^{1} \mathrm{Fe}_{2}\right][\mathrm{OTf}]_{2}$$$
\text { (1) }
$$

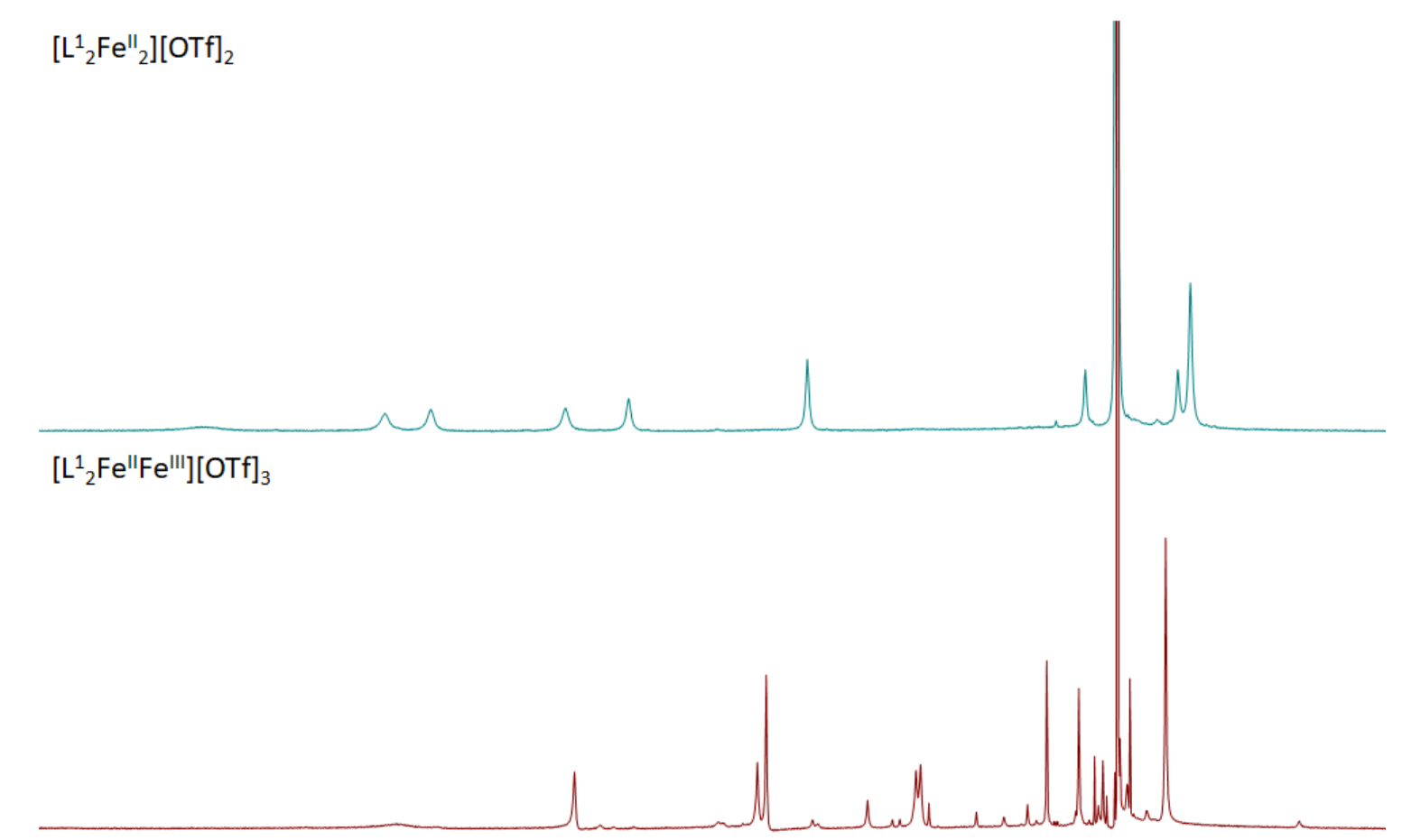

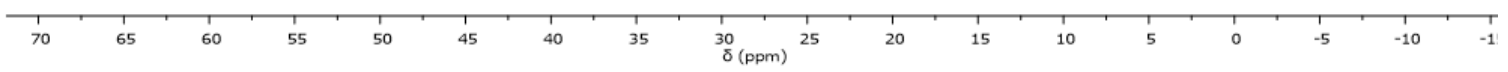

Figure 5.10. ${ }^{1} \mathrm{H}$ NMR spectrum of the parent $\left[\mathrm{L}_{2}^{1} \mathrm{Fe}^{\mathrm{II}}{ }_{2}\right][\mathrm{OTf}]_{2}$ and mixed valent

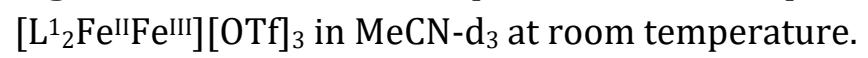

Unfortunately, the ${ }^{1} \mathrm{H}$ NMR spectrum of $\left[\mathrm{L}_{2}{ }_{2} \mathrm{Fe}^{\mathrm{II}} \mathrm{Fe}^{\mathrm{III}}\right][\mathrm{OTf}]_{3}$ in $\mathrm{MeCN}-\mathrm{d}_{3}$ did not reveal 16 different peaks expected for the mixed valent complex (excluding the $\mathrm{CH}_{3}$ group on the pyrazole moiety). Instead, only 9-10 resonances were observed at different chemical shifts compared to the ${ }^{1} \mathrm{H}$ NMR spectrum of $\left[\mathrm{L}_{2}{ }_{2} \mathrm{Fe}^{\mathrm{II}} \mathrm{Fe}{ }^{\mathrm{III}}\right][\mathrm{OTf}]_{3}$ at $298 \mathrm{~K}$. Another ${ }^{1} \mathrm{H}$ NMR spectrum was recorded after warming to $298 \mathrm{~K}$ revealing an identical spectrum prior to cooling. Therefore, the disappearance of resonances can most likely be attributed to the larger linewidths at lower temperatures, and thus, the resonances become much broader or cannot be observed. 


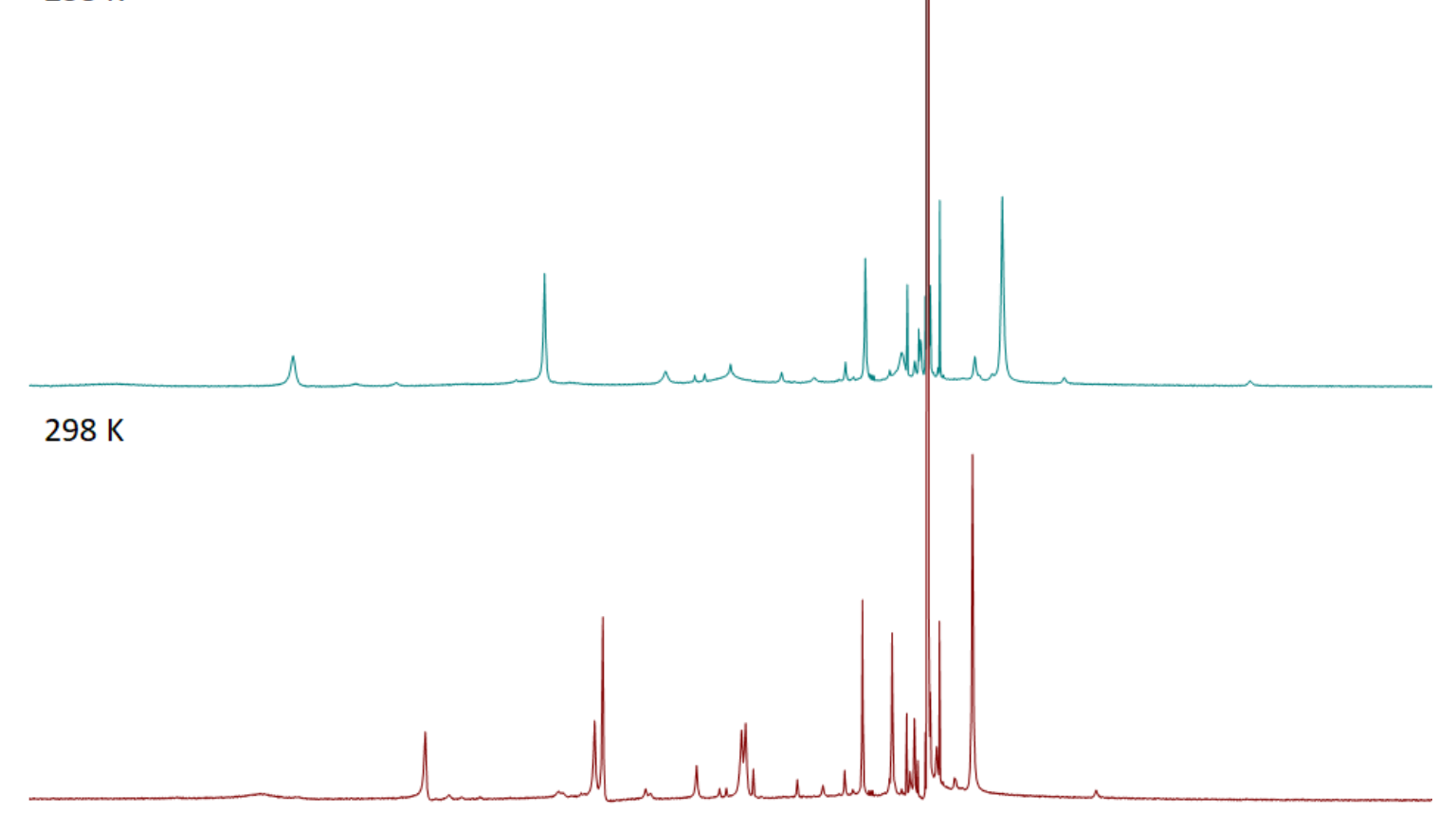

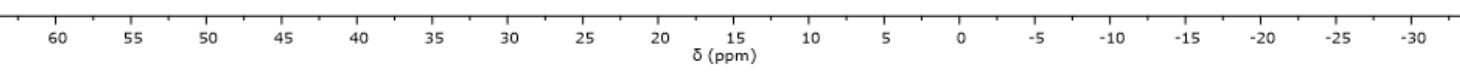

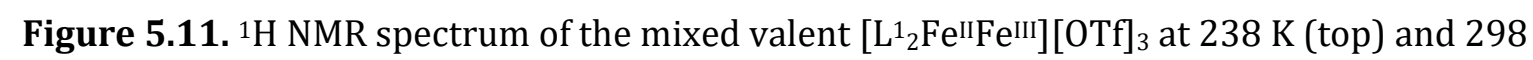
K (bottom).

Based on the results from the previous sections, both the parent $\left[\mathrm{L}_{2} \mathrm{Fe}_{2} \mathrm{II}_{2}\right][\mathrm{OTf}]_{2}$ and mixed valent $\left[\mathrm{L}_{2} \mathrm{Fe}^{\mathrm{II}} \mathrm{Fe}{ }^{\mathrm{III}}\right][\mathrm{OTf}]_{3}$ complex were characterized in the solid-state and in solution using X-ray crystallography, MB spectroscopy, ${ }^{1} \mathrm{H}$ NMR spectroscopy and UV-vis spectroelectrochemistry. While spin crossover was not expected, as evidenced by variable magnetic temperature measurements, the structural changes accompanied by oxidation revealed a clear transformation of one of the HS-Fe ${ }^{\mathrm{II}}$ ions to a LS-Fe ${ }^{\mathrm{III}}$ ion. The structural rearrangements from $\left[\mathrm{L}_{2}{ }_{2} \mathrm{Fe}_{2}{ }_{2}\right][\mathrm{OTf}]_{2}$ to the mixed valent $\left[\mathrm{L}_{2}{ }_{2} \mathrm{Fe}^{\mathrm{II} F e}{ }^{\mathrm{III}}\right][\mathrm{OTf}]_{2}\left[\mathrm{BF}_{4}\right]$ suggest bond dissociation followed by reformation upon addition of the oxidizing agent. Further work on elucidating the mechanism behind the formation of the mixed valent $\left[\mathrm{L}_{2}{ }_{2} \mathrm{Fe}^{\mathrm{II}} \mathrm{Fe}{ }^{\mathrm{III}}\right][\mathrm{OTf}]_{2}\left[\mathrm{BF}_{4}\right]$ is being pursued. At the same time, attempts to chemically synthesize the doubly oxidized $\left[\mathrm{L}_{2}{ }_{2} \mathrm{Fe}^{\mathrm{III}}{ }_{2}\right][\mathrm{OTf}]_{4}$ complex afforded potentially decomposition product(s) based on the obtained ${ }^{1} \mathrm{H}$ NMR spectrum, which displayed resonances predominantly in the diamagnetic region in contrast to the expected paramagnetic nature of $\mathrm{Fe}^{\mathrm{III}}$ ions. 


\section{4 - Preliminary Work on an Analogous Dinuclear $\left[\mathrm{L}_{2}{ }_{2} \mathrm{Co}_{2}\right]\left[\mathrm{BF}_{4}\right]_{2}$ Complex}

An analogous dinuclear $\left[\mathrm{L}_{2} \mathrm{Co}_{2}\right]\left[\mathrm{BF}_{4}\right]_{2}$ complex was synthesized following similar procedures to $\left[\mathrm{L}_{2}{ }_{2} \mathrm{Fe}_{2}\right][\mathrm{OTf}]_{2}$, in which the ligand $\mathbf{H L}^{1}$ was deprotonated with $\mathrm{NaO}{ }^{t} \mathrm{Bu}$ followed by addition of $\mathrm{Co}^{\mathrm{II}}\left(\mathrm{BF}_{4}\right) \cdot 6 \mathrm{H}_{2} \mathrm{O}$ in dry acetonitrile (Scheme 5.4). However, the tendency for the formation of the mononuclear [ $\left.\mathrm{L}_{2}{ }_{2} \mathrm{Co}\right][\mathrm{OTf}]_{2}$ species (confirmed by ESI-MS) is much more prominent such that the reaction must be stirred at room temperature for 36 hours. Subsequently, the red solution was filtered, and the solution precipitated with diethyl ether and further purified by slow diffusion of diethyl ether into a solution of the product in acetonitrile to give beige crystalline material.

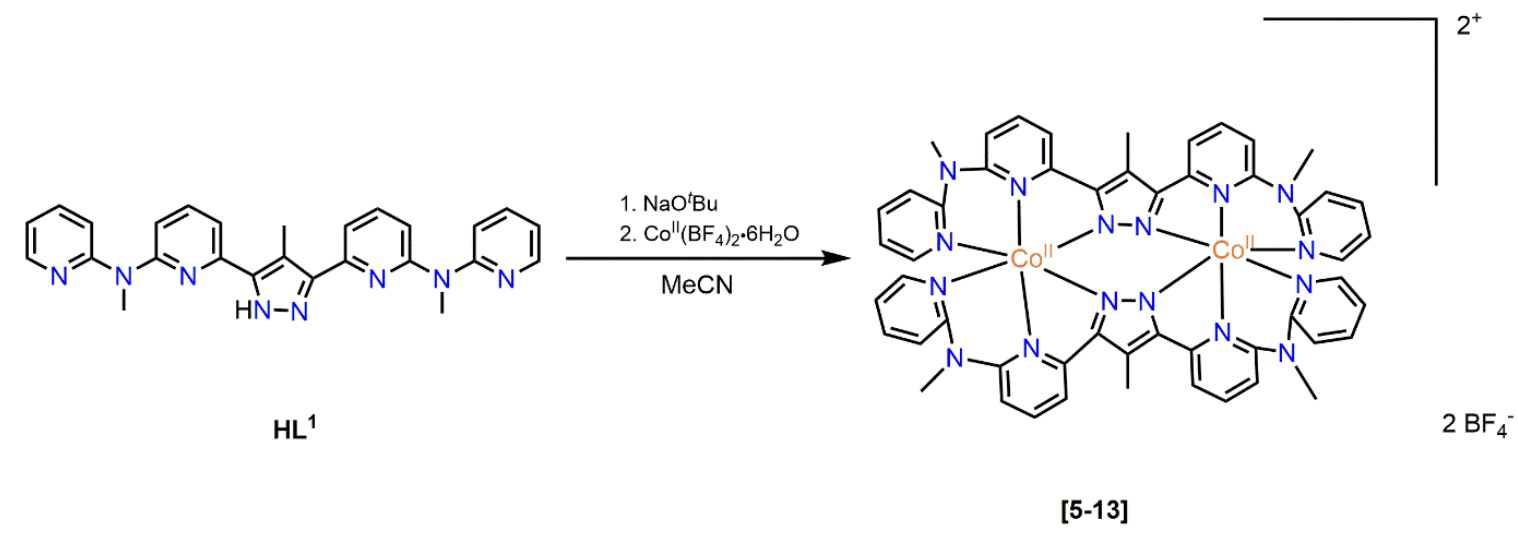

Scheme 5.4. Synthetic scheme for the dinuclear $\left[\mathrm{L}_{2}^{1} \mathrm{Co}_{2}{ }_{2}\right]\left[\mathrm{BF}_{4}\right]_{2}$ complex.

Since the mononuclear $\left[\mathrm{L}_{2}^{1} \mathrm{Co}\right][\mathrm{OTf}]_{2}$ species is strongly preferred with shorter reaction times, heterometallic dinuclear complexes were also pursued. Unfortunately, using a one-pot synthesis with stoichiometric amounts of $\mathrm{Co}^{\mathrm{II}}$ ions, followed by Fe ${ }^{\mathrm{II}}$ ions or $\mathrm{Ru}^{\mathrm{II}}$ ions, gave rise to predominately a mixture of both homonuclear and heterometallic complexes. Based on mass spectrometry, a preference for the homonuclear complexes was observed and thus heterometallic dinuclear systems were not pursued with this ligand.

\subsection{1 - X-ray Crystallography}

The dinuclear complex $\left[\mathrm{L}_{2}{ }_{2} \mathrm{Co}_{2}\right]\left[\mathrm{BF}_{4}\right]_{2}$ crystallizes in the monoclinic space group $P 2 / \mathrm{n}$ with two molecules in the asymmetric unit (Figure 5.11). The overall charge is balanced by two $\mathrm{BF}_{4}{ }^{-}$anions and both cobalt ions are crystallographically equivalent. Interestingly, the crystal structure is reminiscent of the molecular structure of the mixed valent $\left[\mathrm{L}_{2}{ }_{2} \mathrm{Fe}^{\mathrm{II}} \mathrm{Fe}^{\mathrm{III}}\right][\mathrm{OTf}]_{2}\left[\mathrm{BF}_{4}\right]$, in which the terminal pyridines are coordinated trans-axial to one another forming a helical structure. An overlay of the molecular structures of $\left[\mathrm{L}_{2}{ }_{2} \mathrm{Fe}^{\mathrm{II}} \mathrm{Fe}^{\mathrm{III}}\right][\mathrm{OTf}]_{2}\left[\mathrm{BF}_{4}\right]$ and $\left[\mathrm{L}_{2}{ }_{2} \mathrm{Co}^{\mathrm{II}}{ }_{2}\right]\left[\mathrm{BF}_{4}\right]_{2}$ are shown in Figure $5.11 \mathrm{~b}$. As mentioned in the previous section, due to the flexibility of the ligand $\mathbf{H L}^{\mathbf{1}}$, both binding modes (cis and trans) are possible depending on the metal ion. Analysis of the Co- $\mathrm{N}$ distances reveals an average distance of $2.12 \AA$, consistent with high-spin $\mathrm{Co}^{\mathrm{II}}$ ions. Although both $\left[\mathrm{L}_{2}^{1} \mathrm{Co}_{2}\right]\left[\mathrm{BF}_{4}\right]_{2}$ and $\left[\mathrm{L}_{2}{ }_{2} \mathrm{Fe}^{\mathrm{II}} \mathrm{Fe}^{\mathrm{III}}\right]\left[\mathrm{OTf}_{2}\left[\mathrm{BF}_{4}\right]\right.$ adopt the helical structure, both $\mathrm{Co}^{\mathrm{II}}$ ions are in the high-spin configuration, while HS-Fe ${ }^{\mathrm{II}}$ and $\mathrm{LS}-\mathrm{Fe}{ }^{\mathrm{III}}$ ions are present in $\left[\mathrm{L}_{2} \mathrm{Fe}^{\mathrm{IIF}} \mathrm{Fe}^{\mathrm{III}}\right][\mathrm{OTf}]_{2}\left[\mathrm{BF}_{4}\right]$. The 
overall distortion of the molecule was determined by Continuous Symmetry Measures, in which a large $\mathrm{S}\left(\mathrm{O}_{\mathrm{h}}\right)$ value of 6.61 was obtained for each of the $\mathrm{Co}^{\mathrm{II}}$ ions.

(a)

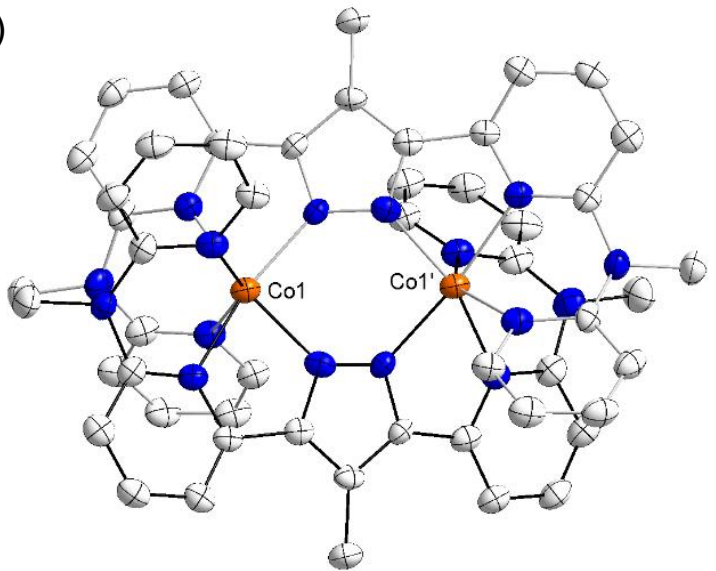

(b)

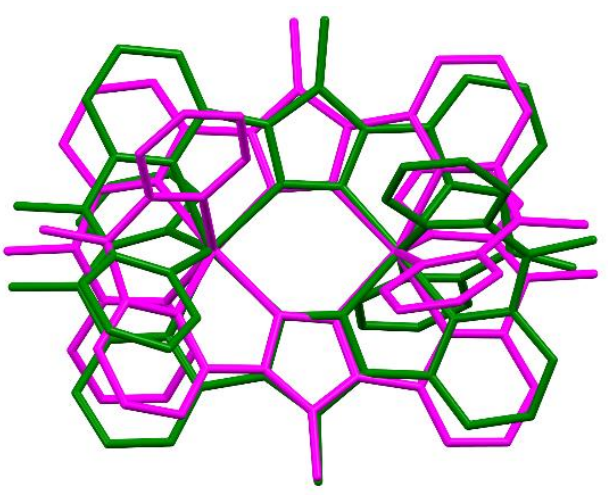

Figure 5.12. (a) Molecule structure of the dinuclear $\left[\mathrm{L}_{2}{ }_{2} \mathrm{Co}_{2}\right]\left[\mathrm{BF}_{4}\right]_{2}$ complex and (b) overlay of $\left[\mathrm{L}_{2}^{1} \mathrm{Fe}^{\mathrm{II}} \mathrm{Fe}^{\mathrm{IIII}}\right][\mathrm{OTf}]_{2}\left[\mathrm{BF}_{4}\right]$ (green) and $\left[\mathrm{L}_{2}{ }_{2} \mathrm{Co}_{2}\right]\left[\mathrm{BF}_{4}\right]_{2}$ (pink) for comparison.

\subsection{2 - Mass Spectrometry}

The integrity of $\left[\mathrm{L}_{2} \mathrm{Co}_{2}\right]\left[\mathrm{BF}_{4}\right]_{2}$ in $\mathrm{MeCN}$ was investigated by mass spectrometry. One dominant peak at $m / z=506$ was observed corresponding to the doubly charged cation $\left[\mathrm{L}_{2}{ }_{2} \mathrm{Co}_{2}\right]^{2+}$ with the expected isotopic pattern as seen in the simulated pattern shown in the inset of Figure 5.13. The small peak at $m / z=337$ corresponds to the $\left[\mathrm{L}_{2}^{1} \mathrm{Co}_{2}\right]^{3+}$ species, which despite subsequent crystallizations, still remains present in the ESI mass spectrum.

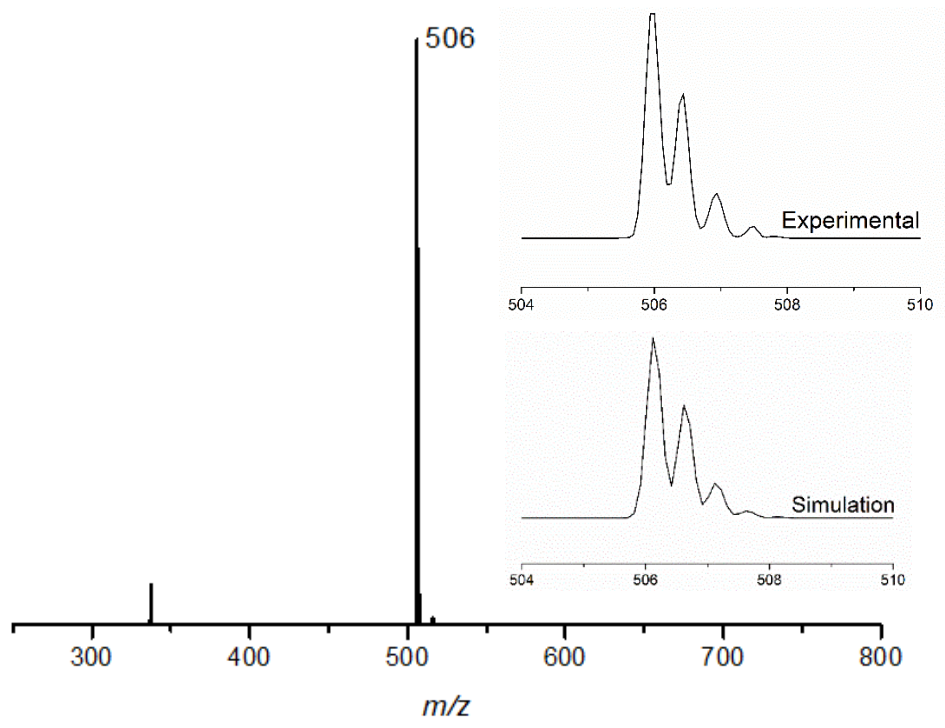

Figure 5.13. ESI-MS of $\left[\mathrm{L}_{2}{ }_{2} \mathrm{Co}_{2}{ }_{2}\right]\left[\mathrm{BF}_{4}\right]_{2}$ in $\mathrm{MeCN}$ displaying one dominant peak at $\mathrm{m} / z=506$, corresponding to the doubly charged cation $\left[\mathrm{L}_{2}{ }_{2} \mathrm{Co}^{\mathrm{II}_{2}}\right]^{2+}$. The simulated isotopic distribution pattern is shown in the inset. 


\subsection{3 - ${ }^{1} \mathrm{H}$ NMR Spectroscopy}

The electronic structure of $\left[\mathrm{L}_{2}^{1} \mathrm{Co}_{2}\right]\left[\mathrm{BF}_{4}\right]_{2}$ was further probed by ${ }^{1} \mathrm{H}$ NMR spectroscopy in MeCN- $\mathrm{d}_{3}$ revealing a set of paramagnetic resonances from $-35 \mathrm{ppm}$ to $+85 \mathrm{ppm}$, as expected for high-spin $\mathrm{Co}^{\mathrm{II}}$ ions. Although the linewidths are much smaller compared to the ${ }^{1} \mathrm{H}$ NMR spectrum of $\left[\mathrm{L}_{2} \mathrm{Fe}_{2}\right][\mathrm{OTf}]_{2}$, 2D NMR spectroscopy was still not obtainable for this complex. Nevertheless, nine major peaks are observed with minor contributions most likely arising from the minor species also observed in the ESI-MS. Unfortunately, even with repeated crystallizations, $10 \%$ of the impurity consistently persist in solution. Integration of the peaks affords the expected 23 protons. The broad resonance at $133 \mathrm{ppm}$ most likely correspond to the proton closest to the paramagnetic HS-CoII ion. The largest peak at $-20.54 \mathrm{ppm}$ may be attributed to the two $\mathrm{NCH}_{3}$ moieties and the methyl group on the pyrazole backbone can be assigned to the resonance at $45.24 \mathrm{ppm}$ based on the integration and proximity to the paramagnetic center.

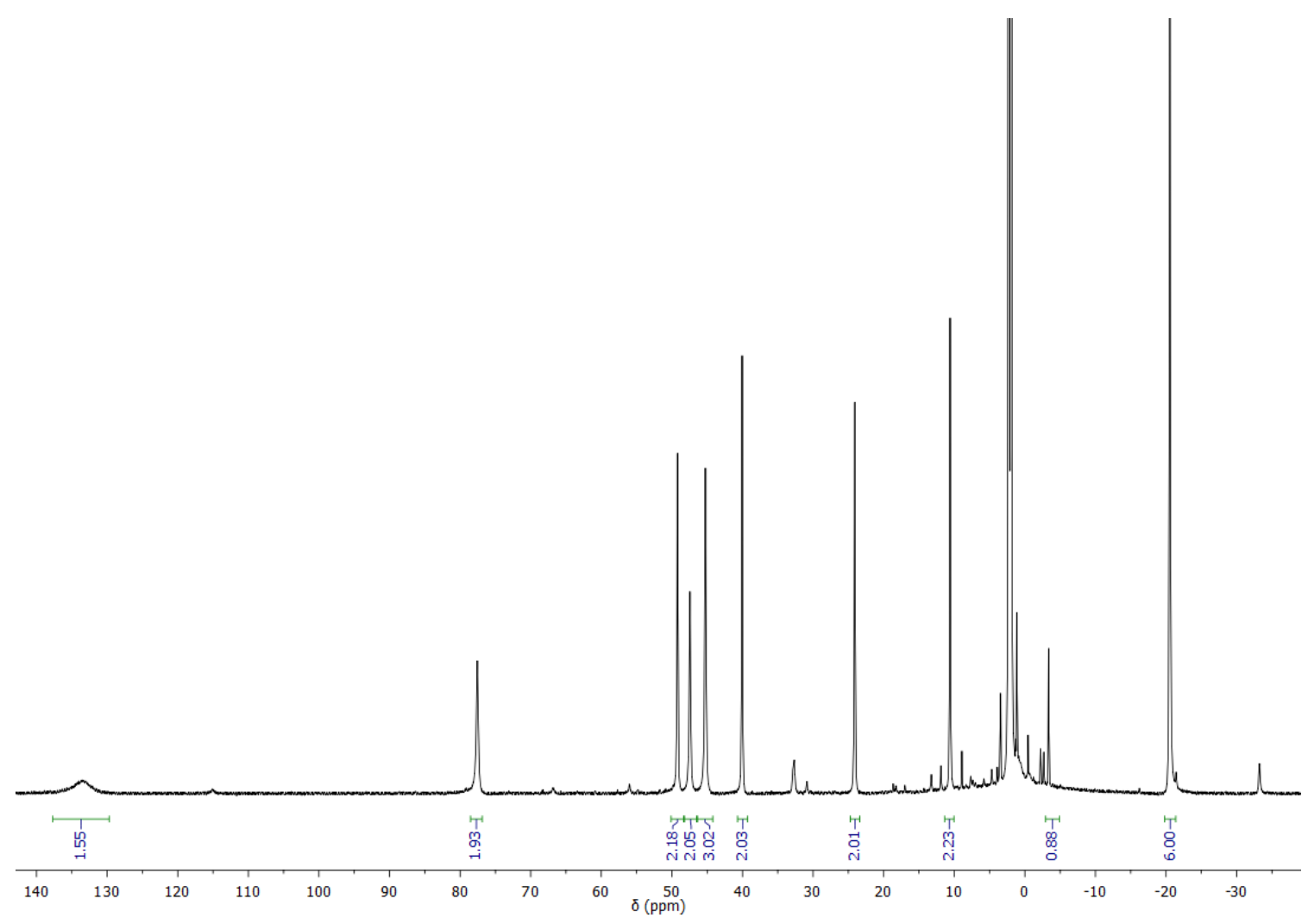

Figure 5.14. ${ }^{1} \mathrm{H}$ NMR spectrum of $\left[\mathrm{L}_{2}{ }_{2} \mathrm{Co}_{2}\right]\left[\mathrm{BF}_{4}\right]_{2}$ in $\mathrm{MeCN}-\mathrm{d}_{3}$. 


\subsection{4 - Preliminary Cyclic Voltammogram of $\left[\mathrm{L}_{2}{ }_{2} \mathrm{Co}{ }_{2}\right]\left[\mathrm{BF}_{4}\right]_{2}$}

The redox properties of $\left[\mathrm{L}_{2}{ }_{2} \mathrm{Co}_{2} \mathrm{II}_{2}\right]\left[\mathrm{BF}_{4}\right]_{2}$ was investigated by cyclic voltammetry in acetonitrile with $\left[\mathrm{NBu}_{4}\right] \mathrm{PF}_{6}$ as the electrolyte (Figure 5.15). Unlike $\left[\mathrm{L}_{2}^{1} \mathrm{Fe}^{\mathrm{II}}{ }_{2}\right][\mathrm{OTf}]_{2}$ in which two anodic waves were observed, one anodic peak $\left(E_{\mathrm{pf}}\right)$ at $1.33 \mathrm{~V}$ and one cathodic peak $\left(E_{\mathrm{pr}}\right)$ at $-0.02 \mathrm{~V}$ were observed. The large separation between the two redox events may be due to the slow electron transfer also observed in several other $\mathrm{Co}^{\mathrm{II}}$ complexes discussed in Chapter 3. Moreover, structural reorganization of one $\mathrm{HS}-\mathrm{Co}^{\mathrm{II}}$ to $\mathrm{LS}-\mathrm{Co}^{\mathrm{III}}$ may be unfavourable in the helical structure resulting in much larger reorganization energies and thus, slower electron transfer. In comparison to $\left[\mathrm{L}_{2}^{1} \mathrm{Fe}_{2}{ }_{2}\right][\mathrm{OTf}]_{2}$, in which the terminal pyridine moieties are cis to one another, oxidation to $\left[\mathrm{L}_{2}{ }_{2} \mathrm{Fe}^{\mathrm{II} F} \mathrm{Fe}^{\mathrm{III}}\right]\left[\mathrm{OTf}_{2}\left[\mathrm{BF}_{4}\right]\right.$ then leads to formation of the helical structure. However, the molecular structure of $\left[\mathrm{L}_{2}{ }_{2} \mathrm{Co}_{2}{ }_{2}\right]\left[\mathrm{BF}_{4}\right]_{2}$ is already in the helical structure and thus oxidation may be more difficult, resulting in the large separation between the redox events as well as a much higher potential for the first anodic peak $\left(E_{\mathrm{pf}}=1.33 \mathrm{~V}\right.$ in $\left[\mathrm{L}_{2}{ }_{2} \mathrm{Co}_{2}{ }_{2}\right]\left[\mathrm{BF}_{4}\right]_{2}$ compared to $E_{1 / 2}=0.019 \mathrm{~V}$ in $\left.\left[\mathrm{L}_{2}{ }_{2} \mathrm{Fe}{ }^{I I}\right][\mathrm{OTf}]_{2}\right)$. Further work on obtaining the UV-Vis spectroelectrochemistry of $\left[\mathrm{L}_{2}^{1} \mathrm{Co}^{{ }^{I I}}{ }_{2}\right]\left[\mathrm{BF}_{4}\right]_{2}$ may provide information regarding the nature of the two redox processes (i.e. assignment of the anodic and cathodic peak to oxidation from $\mathrm{Co}^{\mathrm{II}}$ to $\mathrm{Co}^{\mathrm{III}}$ ). Moreover, preliminary magnetic susceptibility measurements revealed additional impurities and the data could not be fitted to the expected two HS-Co ${ }^{\text {II }}$ ions. Further work on obtaining the dinuclear $\left[\mathrm{L}_{2}^{1} \mathrm{Co}_{2}{ }_{2}\right]\left[\mathrm{BF}_{4}\right]_{2}$ exclusively is essential for further measurements.

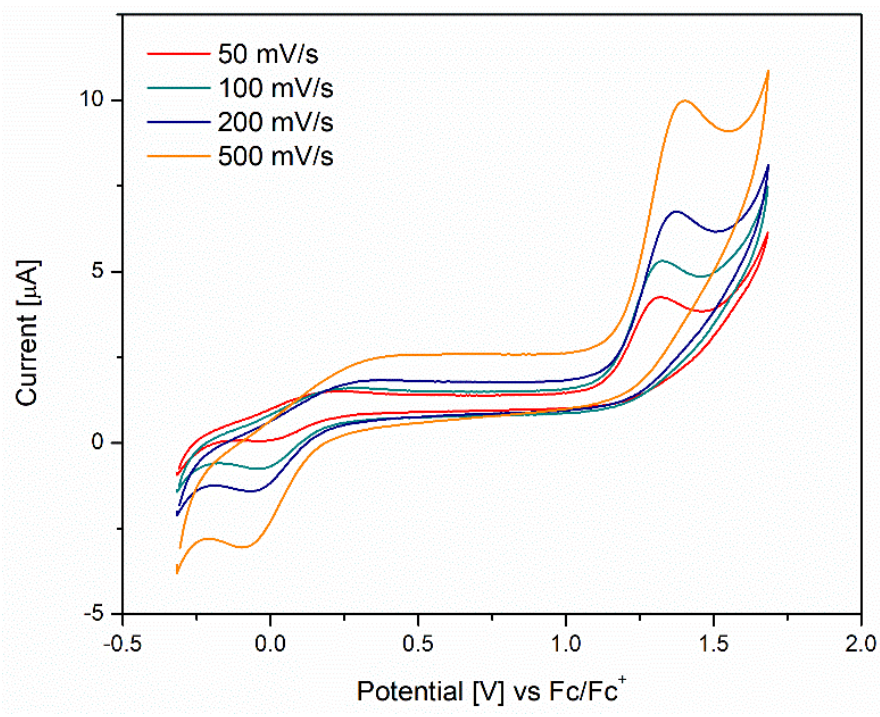

Figure 5.15. Cyclic voltammogram of $\left[\mathrm{L}_{2}{ }_{2} \mathrm{Co}_{2}\right]\left[\mathrm{BF}_{4}\right]_{2}$ at different scan rates in MeCN with $\left[\mathrm{NBu}_{4}\right] \mathrm{PF}_{6}$ as the electrolyte.

\section{5 - Summary \& Conclusion}

In summary, a new family of dinuclear $\mathrm{Fe}_{2}$ and $\mathrm{Co}_{2}$ complexes was developed using a new bis(tridentate) pyrazolate-bridged ligand with flexible terminal pyridine moieties. With the presence of two metal centers, the redox properties of the dinuclear $\left[\mathrm{L}_{2}{ }_{2} \mathrm{Fe}_{2}\right][\mathrm{OTf}]_{2}$ complex were investigated revealing a large thermodynamic stability of the mixed valent $\left[\mathrm{L}_{2}{ }_{2} \mathrm{Fe}^{\mathrm{II}} \mathrm{Fe}^{\mathrm{III}}\right][\mathrm{OTf}]_{2}\left[\mathrm{BF}_{4}\right]$ complex. The structural and electronic changes between the two complexes were investigated using ${ }^{1} \mathrm{H}$ NMR spectroscopy and MB spectroscopy confirming the presence of large structural reorganization upon oxidation from the HS-FeII-HS-FeII species to a HS-Fe ${ }^{\mathrm{II}}-\mathrm{LS}-\mathrm{Fe}^{\mathrm{III}}$ species. At the same time, an analogous dinuclear $\left[\mathrm{L}^{1}{ }_{2} \mathrm{Co}_{2}\right]\left[\mathrm{BF}_{4}\right]_{2}$ 
complex was synthesized, which crystallized with the same trans-binding mode as the mixed valent $\left[\mathrm{L}_{2}^{1} \mathrm{Fe}^{\mathrm{II}} \mathrm{Fe}^{\mathrm{III}}\right][\mathrm{OTf}]_{2}\left[\mathrm{BF}_{4}\right]$ species. However, both $\mathrm{Co}^{\mathrm{II}}$ ions adopt a high-spin configuration. Further work on the mixed valent $\left[\mathrm{L}_{2}^{1} \mathrm{Fe}^{\mathrm{II}} \mathrm{Fe}{ }^{\mathrm{III}}\right][\mathrm{OTf}]_{2}[\mathrm{BF} 4]$ including magnetic measurements are currently being pursued. Moreover, full characterization for $\left[\mathrm{L}^{1} \mathrm{Co}_{2}\right]\left[\mathrm{BF}_{4}\right]_{2}$ including UV-Vis spectroelectrochemistry and magnetic measurements are also currently being pursued. 


\section{Chapter 6 - [2 x 2] Grid-like Arrays with Asymmetric Pyrazolate-bridged Ligands}

\section{1 - Introduction}

[2 x 2] grid-like arrays have attracted significant interest due to their potential as molecular storage devices and applications in Quantum Cellular Automata (QCA).17,18 In particular, heterometallic grids with several different metal ions embedded in a matrix-like array hold potential for multiple stable redox states as well as site-specific responses to an external stimulus such as light irradiation. As already mentioned in chapter 1, heterometallic [2 x 2] grids can be synthesized following two methods: a step-wise approach involving sequential introduction of metal ions or designing ligands with site-specific binding pockets. The former approach was pioneered by Lehn, in which one site of the ligand was protected, followed by addition of a kinetically stable $\mathrm{Ru}^{\mathrm{II}}$ ion to form the so-called "corner complex". ${ }^{87}$ Once this was isolated, the other end of the ligand was deprotected and a second metal ion was introduced to spontaneously form the heterometallic [2 $\mathrm{x} 2$ ] grid. Following a similar strategy, we have also developed two new heterometallic $\left[\mathrm{LH}_{4} \mathrm{Ru}_{2} \mathrm{Co}_{2}\right]\left[\mathrm{BF}_{4}\right]_{4}$ and $\left[\mathrm{LH}_{4} \mathrm{Ru}_{2} \mathrm{Fe}_{2}\right]\left[\mathrm{BF}_{4}\right]_{4}$ grids, as discussed in chapters 3 and 4, respectively.

The second strategy involves designing asymmetric ligands with different binding pockets. For instance, ligands containing both bidentate and tridentate sites have demonstrated to effectively incorporate metal ions with different coordination affinities. ${ }^{138}$ This strategy was also implemented for larger [ $3 \times 3$ ] grids, in which a bidentate-tridentate-bidentate ligand scaffold was used to assemble heterometallic [3 $\left[\begin{array}{ll}3 & 3\end{array}\right]$ grids in the form of $\left[\mathrm{M}^{\mathrm{II}} \mathrm{Cu}_{4} \mathrm{Cu} \mathrm{Cu}_{4} \mathrm{~L}_{6}\right] \mathrm{Cl}_{2} \cdot n \mathrm{H}_{2} \mathrm{O}(\mathrm{M}=\mathrm{Fe}, \mathrm{Ni}, \mathrm{Cu}, \mathrm{Zn}) \cdot{ }^{107}$ More recently, in pursuit of heterometallic [2 $\mathrm{x} 2$ ] grids with single molecule magnet behaviour, ligands with a much larger cavity were designed for coordination of lanthanide ions. ${ }^{79,80}$

The focus of this chapter follows the latter strategy in which an asymmetric ligand was designed for developing new homonuclear and heterometallic [2 $\mathrm{x} 2]$ grids. The ligand $\mathbf{H L}^{2}$ stems from combining the precursors of the previous ligands $\mathbf{H L}^{\mathbf{H}}$ and $\mathbf{H L}^{\mathbf{1}}$ (chart 6.1) introduced in chapters 2 and 5 , respectively. Although the increased in flexibility of $\mathbf{H L}^{\mathbf{1}}$ due to the additional $\mathrm{NCH}_{3}$ groups between the pyridine moieties favours the formation of dinuclear complexes, a hybrid of a rigid bipyridine on one end and a flexible terminal pyridine group afforded a [2 x 2] grid-like array. Although a molecular structure was obtained, other spectroscopic measurements suggest the presence of multiple species potentially arising from different isomers as well as impurities including the dinuclear $\left[\mathrm{L}_{2}^{2} \mathrm{Fe}_{2}\right]\left[\mathrm{BF}_{4}\right]_{2}$. Moreover, preliminary results on enforcing the formation of a single isomer by following a step-wise approach will also be discussed.

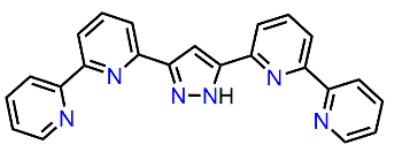

$\mathrm{HL}^{\mathrm{H}}$

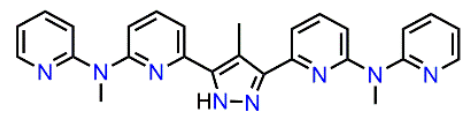

$H L^{1}$

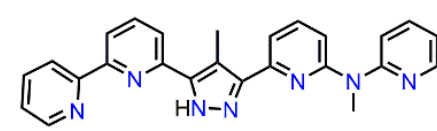

$\mathrm{HL}^{2}$

Chart 6.1. Schematic drawing comparing the different ligands used in this work. 


\subsection{1 - Ligand Synthesis and Characterization}

The ligand $\mathbf{H L}^{2}$ was prepared by using the ketone 5-8 and methylester 2-7 precursors from the previous reported ligands $\mathbf{H L}^{\mathbf{1}}$ and $\mathbf{H L}^{\mathbf{H}}$, respectively (Scheme 6.1). Sodium $t$-butoxide $(\mathrm{NaO} t \mathrm{Bu})$ was added to a solution of 5-8 in 1,4-dioxane, followed by addition of a solution of 2-7 in 1,4-dioxane. The reaction was stirred at room temperature for 16 hours then treated with acetic acid $(\mathrm{AcOH})$ to give a fine yellow powder 6-1, which was used without further purification. Hydrazine monohydrate $\left(\mathrm{N}_{2} \mathrm{H}_{4} \cdot \mathrm{H}_{2} \mathrm{O}\right)$ was added to a suspension of 6-1 in ethanol and heated at reflux for three hours affording an orange oil, which crystallized by dissolving the oil in acetone and layering with water to give $\mathbf{H L}^{2}$ as a white powder.<smiles>CC(=O)c1cccc(N(C)c2ccccn2)n1</smiles>

5-8

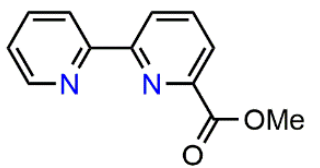

2-7
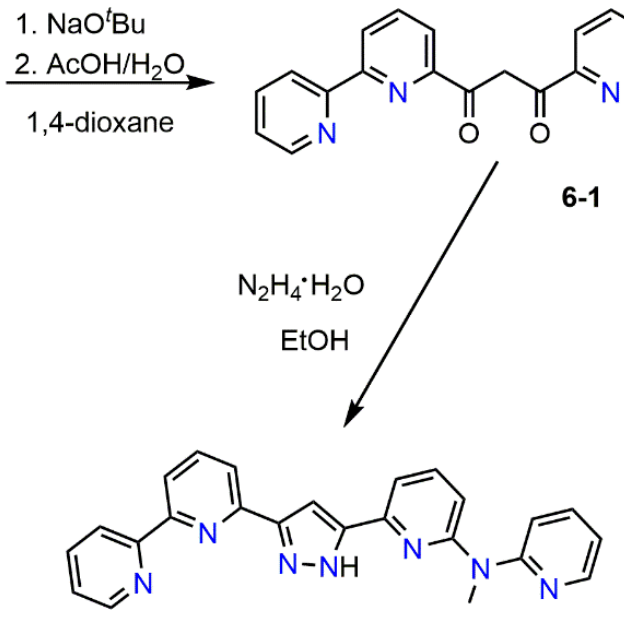

$\mathrm{HL}^{2}$

Scheme 6.1. Synthetic scheme for the asymmetric ligand $\mathbf{H L}^{2}$.

The purity of the ligand was investigated with ${ }^{1} \mathrm{H}$ NMR spectroscopy in $\mathrm{CDCl}_{3}$. The hydrogen atom on the pyrazole backbone is assigned to the peak at $7.46 \mathrm{ppm}$ which is shifted to slightly lower fields compared to the symmetric bipyridine $\mathbf{H L}^{\mathbf{H}}(8.05 \mathrm{ppm})$, due to the additional electron donating $\mathrm{NCH}_{3}$ group. The $\mathrm{NCH}_{3}$ resonance can also be assigned to the resonance at $3.73 \mathrm{ppm}$. All remaining protons are assigned according to ${ }^{1} \mathrm{H}-{ }^{-1} \mathrm{H}$ COSY experiments. 


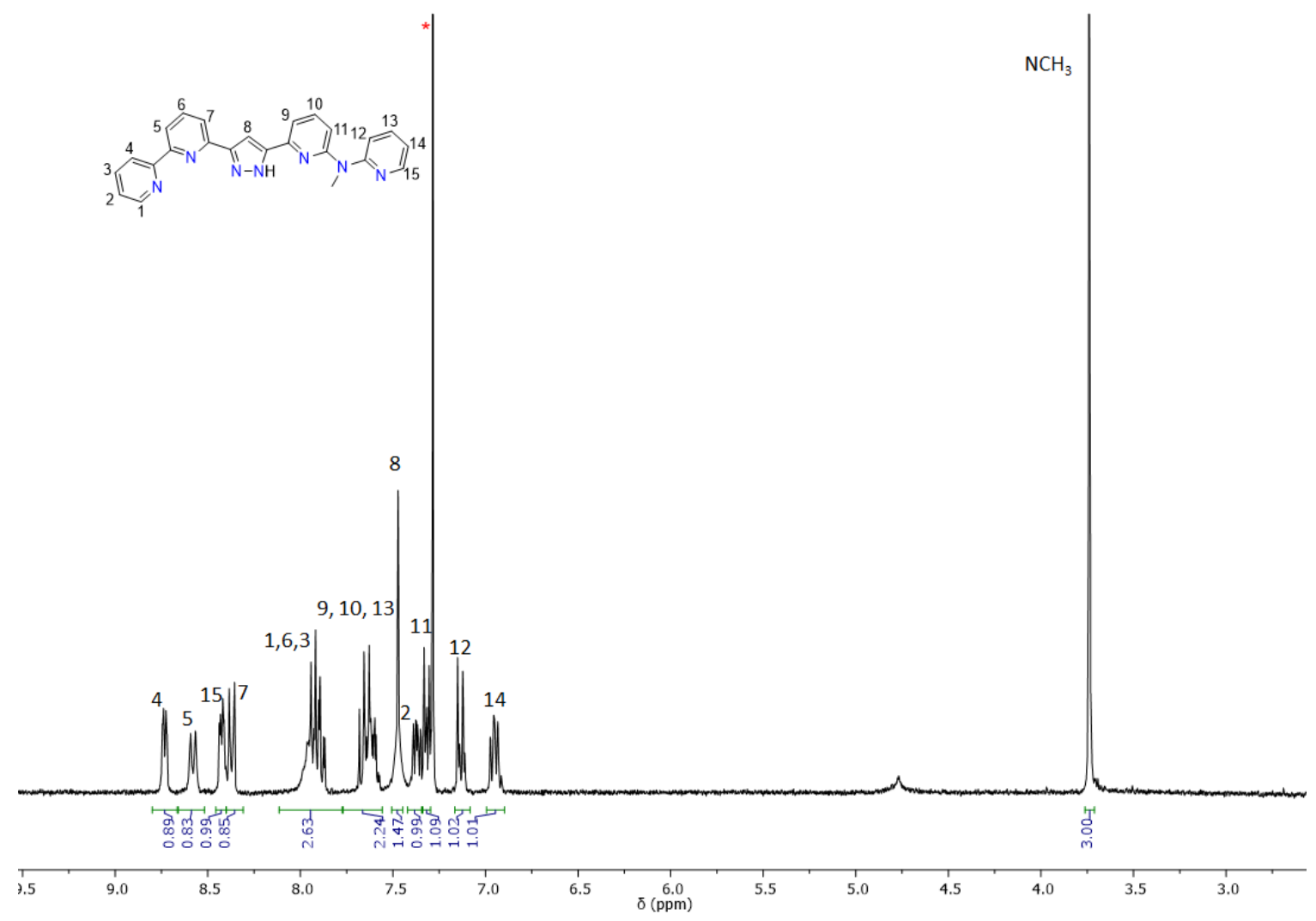

Figure 6.1. ${ }^{1} \mathrm{H}$ NMR spectrum of $\mathbf{H L}^{2}$ in $\mathrm{CDCl}_{3}$. Solvent molecules $\left(\mathrm{CDCl}_{3}\right)$ are indicated with asterisks $(*)$.

\subsection{2 - Complex Synthesis}

The synthesis of the $\left[\mathrm{L}_{4}^{2} \mathrm{Fe}_{4}\right]\left[\mathrm{BF}_{4}\right]_{4}$ grid follows similar procedures for the reported homonuclear $\mathrm{Fe}_{4}$ grids, in which a stoichiometric amount of $\mathrm{NaO}{ }^{t} \mathrm{Bu}$ and $\mathrm{Fe}^{\mathrm{II}}\left(\mathrm{BF}_{4}\right)_{2}$ were combined in dry acetonitrile (Scheme 6.2). Although $\mathbf{H L}^{2}$ is adequately soluble in acetonitrile, deprotonation with $\mathrm{NaO}^{t} \mathrm{Bu}$ afforded a yellow insoluble species, which dissolved only upon addition of the $\mathrm{Fe}^{\mathrm{II}}$ salt. The reaction was stirred at room temperature for 16 hours, the solution filtered, and crystallized by slow diffusion of diethyl ether into a solution of the product in acetonitrile. Although a molecule structure was obtained, various other species or isomers are also present in the ${ }^{1} \mathrm{H}$ NMR spectrum as well as in the Mössbauer (MB) spectrum, which will be discussed in the following sections. Other crystallization methods including using different solvents (methanol) and temperatures afforded similar results. 


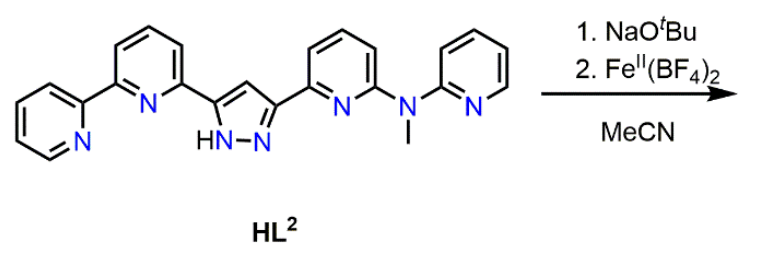

$\mathrm{HL}^{2}$

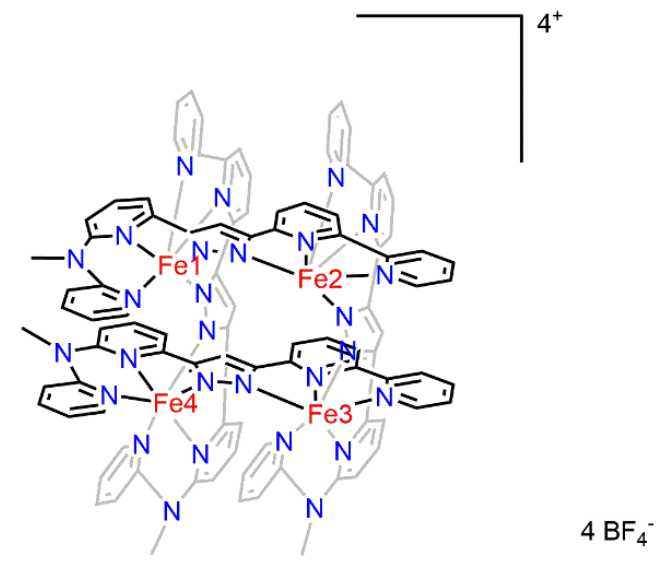

6-2

Scheme 6.2. Synthetic scheme for $\left[\mathrm{L}^{2}{ }_{4} \mathrm{Fe}_{4}\right]\left[\mathrm{BF}_{4}\right]_{4}$.

\subsection{3 - X-ray Crystallography}

In chapter 5 , the flexibility imparted by the additional $\mathrm{NCH}_{3}$ moiety in between the pyridine groups of $\mathbf{H L}^{\mathbf{1}}$ prevented [2 $\mathrm{x} 2$ 2] grid formation, but instead facilitated new dinuclear complexes exclusively. In $\mathbf{H L}^{2}$, only one end has a high degree of flexibility implemented by the $\mathrm{NCH}_{3}$ moiety, while the other end retains a degree of rigidity. Consequently, whether the typical grid-like array would be obtained was uncertain. Surprisingly, single crystals of $\left[\mathrm{L}_{4}{ }_{4} \mathrm{Fe}_{4}\right]\left[\mathrm{BF}_{4}\right]_{4}$ revealed the typical [2 $\left.\mathrm{x} 2\right]$ grid-like architecture, crystallizing in the monoclinic space group $P 2_{1}$. Moreover, four tetrafluroborate $\left(\mathrm{BF}_{4}^{-}\right)$ions are present to balance the overall tetracationic charge of the complex. Each Fe ion is situated in an $\{\mathrm{N} 6\}$ coordination sphere, with bond lengths between $2.124 \AA-2.334 \AA$, corresponding to HS-Fe ${ }^{I I}$ ions. Relevant bond lengths are tabulated in Table 6.1.

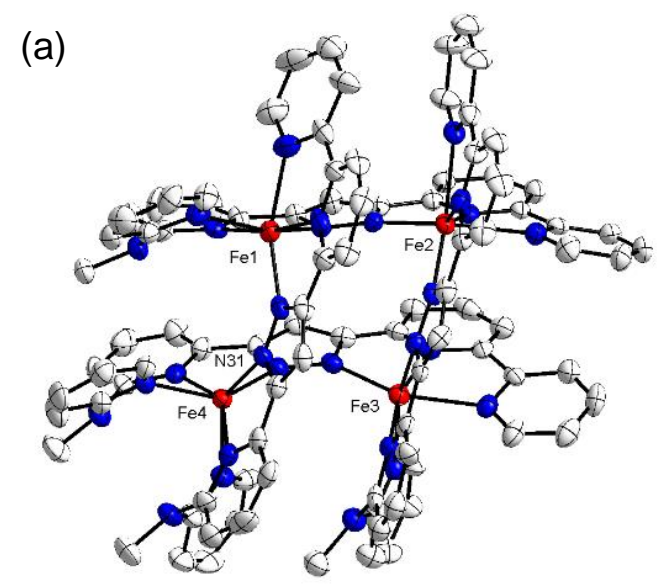

(b)

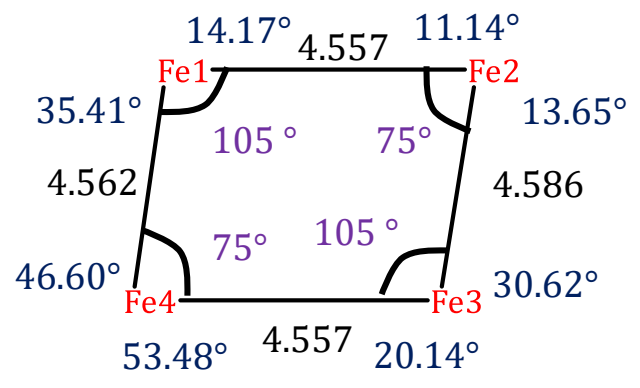

Figure 6.2. (a) Molecular structure of $\left[\mathrm{L}_{4} \mathrm{Fe}_{4}\right]\left[\mathrm{BF}_{4}\right]_{4}$. Counterions and hydrogen atoms are omitted for clarity. (b) Fe...Fe distances (black) and Fe-Fe-Fe angles (purple), and torsion angles defined by the plane of the pyrazole moiety and terminal pyridine ring of the same ligand strand (blue). 
Table 6.1. Relevant bond lengths and Continuous Symmetry Measures for $\left[\mathrm{L}_{4}{ }_{4} \mathrm{Fe}_{4}\right]\left[\mathrm{BF}_{4}\right]_{4}$.

\begin{tabular}{ccccc}
\hline & $\boldsymbol{d}_{\text {avg }}(\AA)$ & Spin-state & $\mathbf{S}\left(\mathbf{O}_{\mathbf{h}}\right)$ & $\mathbf{S}(\mathbf{i t p})$ \\
\hline Fe1-N & 2.15 & HS & 3.42 & 11.92 \\
Fe2-N & 2.20 & HS & 8.14 & 5.14 \\
Fe3-N & 2.16 & HS & 3.30 & 11.57 \\
Fe4-N & 2.19 & HS & 11.39 & 1.56 \\
\hline
\end{tabular}

The four Fe ions are separated by an average distance of $4.565 \AA$, and Fe-Fe-Fe angles of $105^{\circ}$ and $75^{\circ}$ were observed, similar to the literature known homonuclear $\left[\mathrm{L}_{4} \mathrm{Fe}_{4}\right]\left[\mathrm{BF}_{4}\right]_{4}$ grids $(\mathrm{R}=\mathrm{H}, \mathrm{Me}, \mathrm{Br})$, as well as the heterometallic $\left[\mathrm{L}_{4} \mathrm{Ru}_{2} \mathrm{Co}_{2}\right]\left[\mathrm{BF}_{4}\right]_{4}$ and $\left[\mathrm{L}_{4} \mathrm{Ru}_{2} \mathrm{Fe}_{2}\right]\left[\mathrm{BF}_{4}\right]_{4}$ grids discussed in the previous chapters. Although three distinctly different $\mathrm{Fe}$ atoms would be expected in the molecular structure, all four Fe ions are crystallographically independent. The $\mathrm{NCH}_{3}$ moiety of the ligand is consistently situated parallel to one another in this isomer in order to optimize the spatial organization of the ligand strands in the [2 x 2] grid. While this may be one of the few thermodynamically stable isomers, the potential for several other isomers with a different arrangement of ligands may also be possible.

The planarity of the ligand strands in $\left[\mathrm{L}_{4}^{2} \mathrm{Fe}_{4}\right]\left[\mathrm{BF}_{4}\right]_{4}$ can be described by examining the torsion angle between the central pyrazole group and the terminal pyridine moiety of each ligand strand (Figure 6.2b). The largest torsion angle was observed in the ligands coordinated to Fe4 with torsion angles between $46-54^{\circ}$ while small torsion angles of $11-14^{\circ}$ were observed in the ligands coordinated to Fe2. In order to describe the overall distortion from an ideal octahedron, Continuous Symmetry Measures (CSM) can be employed. Based on these calculations, $\mathrm{S}\left(\mathrm{O}_{\mathrm{h}}\right)$ values of 3.42, 8.14, 3.30, and 11.39 were obtained for $\mathrm{Fe} 1, \mathrm{Fe} 2, \mathrm{Fe} 3$, and $\mathrm{Fe} 4$, respectively. The high $\mathrm{S}\left(\mathrm{O}_{\mathrm{h}}\right)$ value of $\mathrm{Fe} 2$ and $\mathrm{Fe} 4$ suggest a high distortion from an ideal octahedron while small $\mathrm{S}\left(\mathrm{O}_{\mathrm{h}}\right)$ values for $\mathrm{Fe} 1$ and $\mathrm{Fe} 3$ suggest only minor distortions. Comparison of the $\mathrm{S}\left(\mathrm{O}_{\mathrm{h}}\right)$ values of $\left[\mathrm{L}_{4} \mathrm{Fe}_{4}\right]\left[\mathrm{BF}_{4}\right]_{4}$ to $\left[\mathrm{L}_{4} \mathrm{Fe}_{4}\right]\left[\mathrm{BF}_{4}\right]_{4}$ reveal slightly higher $\mathrm{S}\left(\mathrm{O}_{\mathrm{h}}\right)$ values most likely attributed to the additional $\mathrm{NCH}_{3}$ group in the ligand strands. In the $\left[\mathrm{L}_{4} \mathrm{Fe}_{4}\right]\left[\mathrm{BF}_{4}\right]_{4}$ grids $(\mathrm{R}=\mathrm{H}, \mathrm{Me}, \mathrm{Br})$, the $\mathrm{S}\left(\mathrm{O}_{\mathrm{h}}\right)$ values range between $6-8$. This was also observed in $\mathrm{Fe} 2$, which is coordinated to two ligand strands with terminal bipyridine subunits. At the opposite corner, $\mathrm{Fe} 4$ is coordinated to two ligand strands with the additional $\mathrm{NCH}_{3}$ moiety, which displayed similar $\mathrm{S}\left(\mathrm{O}_{\mathrm{h}}\right)$ values to the dinuclear $\left[\mathrm{L}_{2}{ }_{2} \mathrm{Fe}_{2}\right][\mathrm{OTf}]_{2}$ $\left(\mathrm{S}\left(\mathrm{O}_{\mathrm{h}}\right)=11.04\right)$ described in Chapter 5 . For comparison, values for the distortion from an ideal trigonal geometry S(itp) were also calculated for each Fe atom and are tabulated in Table 6.1. 


\subsection{4 - Mössbauer Spectroscopy}

With the presence of Fe atoms, Mössbauer (MB) spectroscopy was conducted both on a sample in the solid-state and in frozen MeCN at $80 \mathrm{~K}$. Since the MB spectrum of the solid-state and frozen MeCN sample afforded similar MB parameters, only the frozen solution MB spectrum is shown due to the higher resolution (Figure 6.3). The spectrum revealed four different quadruple doublets. The major species with an isomer shift of $\delta=0.35 \mathrm{mms}^{-1}$ $\left(\Delta E_{\mathrm{Q}}=0.85 \mathrm{mms}^{-1}\right)$ is assumed to be a LS-Fe ${ }^{\mathrm{II}}$ or HS-Fe ${ }^{\mathrm{III}}$ species, which does not correspond to the molecular structure of $\left[\mathrm{L}_{2} \mathrm{Fe}_{4}\right]\left[\mathrm{BF}_{4}\right]_{4}$. Consequently, this peak may be attributed to impurities or decomposition products. The three well resolved doublets at higher isomer shifts of $\delta=1.32 \mathrm{mms}^{-1}\left(\Delta E_{\mathrm{Q}}=3.01 \mathrm{mms}^{-1}\right), 1.11 \mathrm{mms}^{-1}\left(\Delta E_{\mathrm{Q}}=4.22 \mathrm{mms}^{-1}\right), 0.87 \mathrm{mms}^{-1}$ $\left(\Delta E_{\mathrm{Q}}=3.01 \mathrm{mms}^{-1}\right)$ correspond to HS-Fe"I ions. Although different simulations of the doublets may give rise to slightly different isomer shifts and quadruple splittings, the high isomer shift $\left(\delta>0.7 \mathrm{mms}^{-1}\right)$ can only correspond to HS-Fell ions. The three doublets may correspond to the three different Fe species found in the molecular structure; one Fe ion (Fe2) coordinated to two ligands with two bipyridine units, a second $\mathrm{Fe}$ ion (Fe4) coordinated to two ligands with the $\mathrm{NCH}_{3}$ moieties at the opposite corner, and the remaining $\mathrm{Fe} 1$ and $\mathrm{Fe} 3$ ions which are coordinated to ligands consisting of a hybrid of bipyridine and pyridine subunits. The large quadruple splitting observed in the doublet at $\delta=1.11 \mathrm{mms}^{-1}\left(\Delta E_{\mathrm{Q}}=4.22 \mathrm{mms}^{-1}\right)$ is also very similar to the MB parameters of the dinuclear $\left[\mathrm{L}_{2}^{1} \mathrm{Fe}_{2}\right][\mathrm{OTf}]_{2}\left(\delta=1.10 \mathrm{mms}^{-1}, \Delta E_{\mathrm{Q}}=4.22 \mathrm{mms}^{-}\right.$ 1) reported in Chapter 5. Consequently, this doublet may correspond to the Fe coordinated to the ligands with the additional $\mathrm{NCH}_{3}$ moieties. Although a relative intensity of 1:1:2 is expected, surprisingly a ratio of approximately 1:1:1 was observed for the three doublets at high isomer shifts. All MB parameters are tabulated in Table 6.2. Further work on optimizing the synthesis of this specific isomer is essential for further spectroscopic measurements.

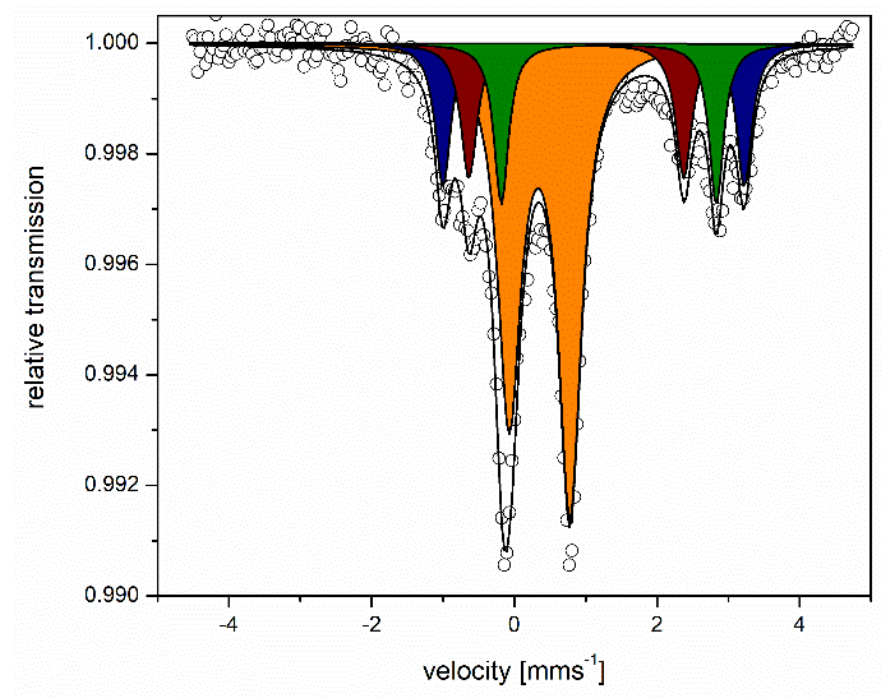

Figure 6.3. Mössbauer spectrum of $\left[\mathrm{L}_{4}^{2} \mathrm{Fe}_{4}\right]\left[\mathrm{BF}_{4}\right]_{4}$ in frozen acetonitrile at $80 \mathrm{~K}$. 
Table 6.2. Mössbauer parameters for $\left[\mathrm{L}_{4}^{2} \mathrm{Fe}_{4}\right]\left[\mathrm{BF}_{4}\right]_{4}$ in frozen acetonitrile at $80 \mathrm{~K}$.

\begin{tabular}{ccc}
\hline $\boldsymbol{\delta}\left[\mathbf{m m s}^{-1}\right]$ & $\boldsymbol{\Delta} \boldsymbol{E}_{\mathbf{Q}}\left[\mathbf{m m s}^{-1}\right]$ & Rel. intensity \\
\hline 1.32 & 3.01 & 14.40 \\
1.11 & 4.22 & 13.53 \\
0.87 & 3.01 & 13.49 \\
0.35 & 0.85 & 58.58 \\
\hline
\end{tabular}

\subsection{5 - Investigations in Solution: Mass Spectrometry \& 1H NMR Spectroscopy}

The integrity as well as the identity of $\left[\mathrm{L}_{2}^{2} \mathrm{Fe}_{4}\right]\left[\mathrm{BF}_{4}\right]_{4}$ in $\mathrm{MeCN}$ was determined by mass spectrometry (Figure 6.4). The dominant peak at $m / z=460$ and $m / z=493$ corresponds to $\left[\mathrm{L}_{4} \mathrm{Fe}_{4}\right]^{4+}$ and $\left[\mathrm{L}_{4} \mathrm{Fe}_{4} \mathrm{NaF}_{2}+\mathrm{nNa}\right]^{4+}$ (from $\mathrm{BF}_{4}^{-}$anions), respectively, confirming the grid remains intact in solution. However, another peak at $m / z=865$ can be attributed to $\left[\mathrm{Fe}_{2} \mathrm{~L}_{4}\right]^{2+}$, in which only two Fe ions are present in the grid-like array. Based on the mass spectra, the species present in the $\mathrm{MB}$ spectrum may originate from $\left[\mathrm{L}_{4}^{2} \mathrm{Fe}_{4}\right]\left[\mathrm{BF}_{4}\right]_{4}$, as well as $\left[\mathrm{L}_{4} \mathrm{Fe}_{2}\right]\left[\mathrm{BF}_{4}\right]_{4}$.

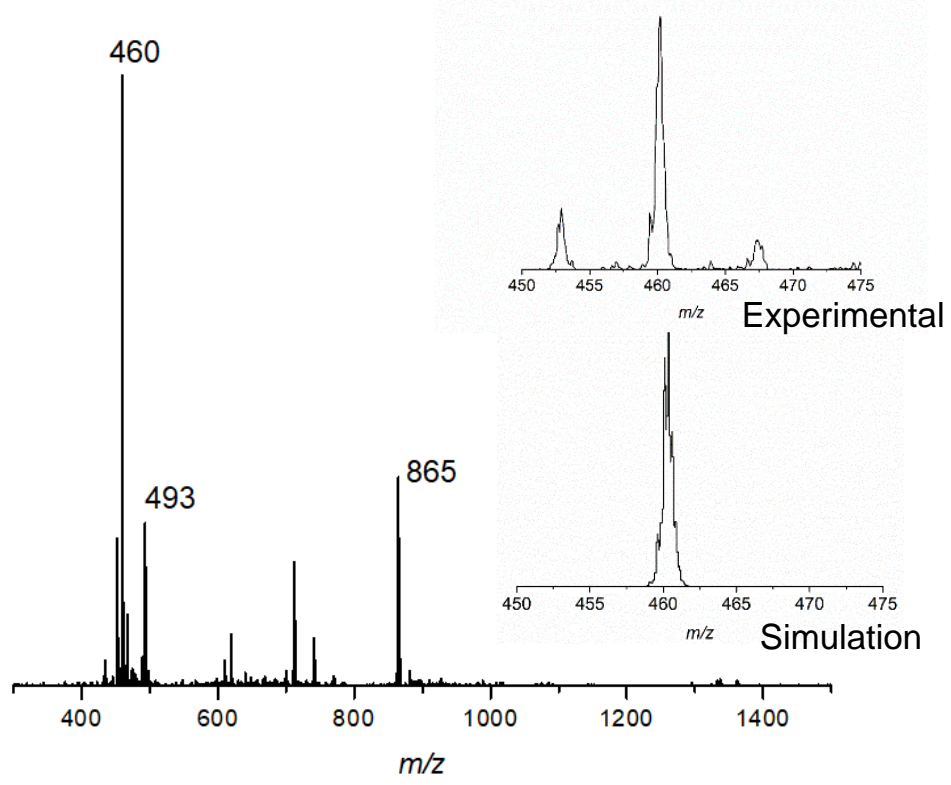

Figure 6.4. ESI mass spectrum of $\left[\mathrm{L}_{4}{ }_{4} \mathrm{Fe}_{4}\right]\left[\mathrm{BF}_{4}\right]_{4}$ in acetonitrile.

${ }^{1} \mathrm{H}$ NMR spectroscopy was conducted on 6-2 in MeCN- $\mathrm{d}_{3}$ revealing a multitude of signals from -25 to $+85 \mathrm{ppm}$, as expected with the presence of $\mathrm{HS}-\mathrm{Fe}^{\mathrm{II}}$ ions (Figure 6.5). Despite repeated crystallizations as well as carrying out the reaction inside the glove box, little change was observed in the ${ }^{1} \mathrm{H}$ NMR spectrum. As already mentioned, the complexity of the spectrum may originate from the presence of multiple isomers (in this case, four isomers are possible) as well as other impurities. For $\left[\mathrm{L}_{4}{ }_{4} \mathrm{Fe}_{4}\right]\left[\mathrm{BF}_{4}\right]_{4}, 49$ signals would be expected, which coincides with the approximate number of signals observed in the ${ }^{1} \mathrm{H}$ NMR spectrum ( $~ 45-50$ signals). Due to the poor resolution of the resonances, the purity of $\left[\mathrm{L}_{4}{ }_{4} \mathrm{Fe}_{4}\right]\left[\mathrm{BF}_{4}\right]_{4}$ cannot be determined. 


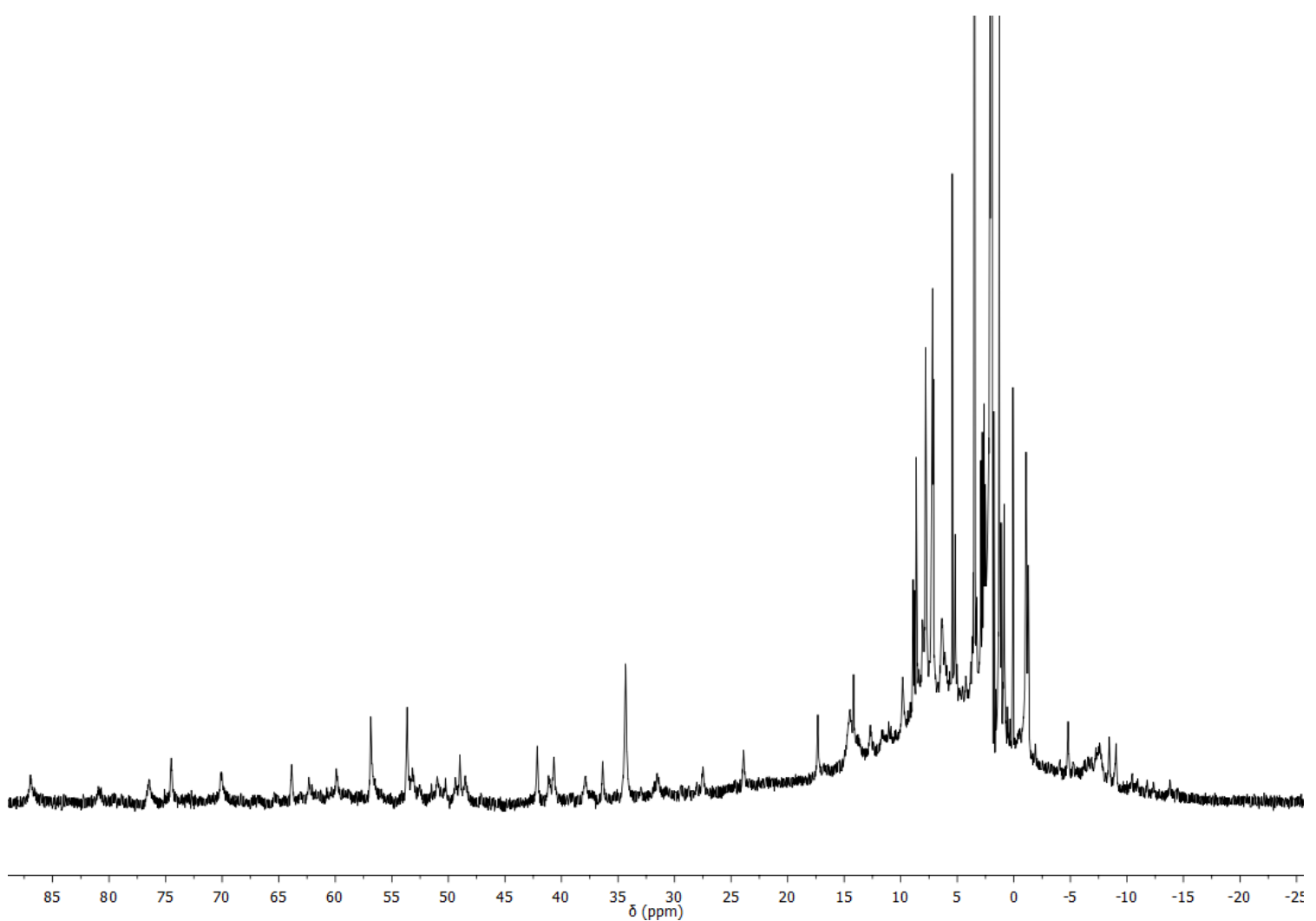

Figure 6.5. ${ }^{1} \mathrm{H}$ NMR spectrum of $\left[\mathrm{L}^{2}{ }_{4} \mathrm{Fe}_{4}\right]\left[\mathrm{BF}_{4}\right]_{4}$ in $\mathrm{MeCN}-\mathrm{d}_{3}$ at room temperature.

\subsection{6 - Cyclic Voltammetry}

The redox properties of crystallized samples of $\left[\mathrm{L}_{4}^{2} \mathrm{Fe}_{4}\right]\left[\mathrm{BF}_{4}\right]_{4}$ were investigated using cyclic voltammetry (CV) in acetonitrile with $\left[\mathrm{Bu}_{4} \mathrm{~N}\right] \mathrm{PF}_{6}$ as the electrolyte (Figure 6.6a). Preliminary $\mathrm{CV}$ experiments afforded four oxidative waves, which are tentatively assigned to the charge of the complex $\left(4^{+} / 5^{+}, 5^{+} / 6^{+}, 6^{+} / 7^{+}, 7^{+} / 8^{+}\right)$. Square wave voltammetry was also conducted confirming the presence of four oxidative waves (Figure $6.6 \mathrm{~b}$ ). The $5^{+} / 6^{+}$and $6^{+} / 7^{+}$processes are much broader, potentially arising from oxidation of two different $\mathrm{Fe}^{\mathrm{II}}$ species from different isomers or from other impurities. Moreover, the last oxidative wave $\left(7^{+} / 8^{+}\right)$ displayed a significantly smaller current relative to the former oxidative waves. This occasionally occurs in step-wise oxidation in elastically coupled [2 $\mathrm{x} 2$ ] grids, in which further oxidation to the four-fold oxidized species is kinetically hampered due to the high charge of the complex. 
(a)

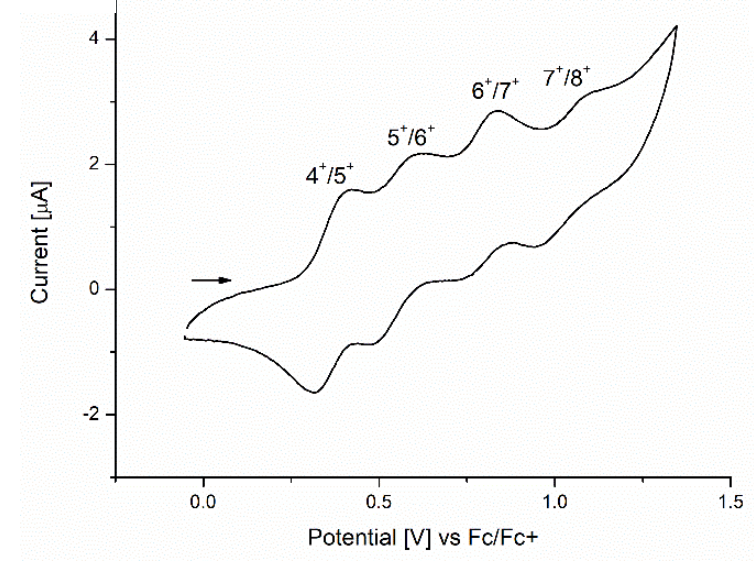

(b)

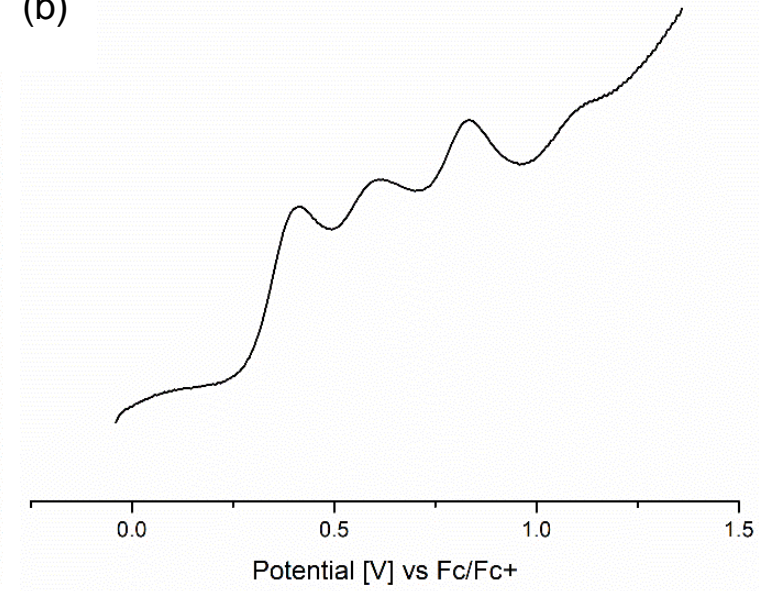

Figure 6.6. (a) Cyclic voltammogram and (b) square wave voltammogram of $\left[\mathrm{L}_{4}{ }_{4} \mathrm{Fe}_{4}\right][\mathrm{BF} 4]_{4}$ with $\left[\mathrm{Bu}_{4} \mathrm{~N}\right] \mathrm{PF}_{6}$ as the electrolyte in acetonitrile at $100 \mathrm{mV} / \mathrm{s}$. All potentials are referenced against $\mathrm{Fc} / \mathrm{Fc}^{+}$.

Table 6.3. Electrochemical parameters for $\left[\mathrm{L}_{4} \mathrm{Fe}_{4}\right]\left[\mathrm{BF}_{4}\right]_{4}$ in acetonitrile at $100 \mathrm{mV} / \mathrm{s}$.

\begin{tabular}{cccc}
\hline & $E_{1 / 2}[\mathrm{mV}]$ & $\Delta E_{\mathrm{p}}[\mathrm{mV}]$ & $E_{1 / 2}-E_{1 / 2}[\mathrm{mV}]$ \\
\hline $4^{+} / 5^{+}$ & 368 & 108 & 177 \\
$5^{+} / 6^{+}$ & 545 & 138 & 240 \\
$6^{+} / 7^{+}$ & 785 & 114 & 234 \\
$7^{+} / 8^{+}$ & 1019 & 126 & \\
\hline
\end{tabular}

The four waves display quasi-reversible behaviour with peak separations between $108-138$ $\mathrm{mV}$ (Table 6.3). Comparison of the electrochemical parameters of the homonuclear $\mathrm{Fe}_{4}$ grids afforded slightly higher potentials for all oxidative waves arising from the different ligand scaffold. Interestingly, unlike the previously reported $\left[\mathrm{L}_{4} \mathrm{Fe}_{4}\right]\left[\mathrm{BF}_{4}\right]_{4}$ grids $(\mathrm{R}=\mathrm{H}, \mathrm{Me}, \mathrm{Br})$ in which a large separation between the $5^{+} / 6^{+}$and $6+/ 7+$ couple was observed $(\sim 400-500 \mathrm{~V})$, $\left[\mathrm{L}_{4} \mathrm{Fe}_{4}\right]\left[\mathrm{BF}_{4}\right]_{4}$ displayed only a marginally large separation of $255 \mathrm{~V}$ between the $5^{+} / 6^{+}$and $6^{+} / 7^{+}$couple. Generally, a large separation suggests the first two oxidative waves correspond to two metal centers at opposite corners. However, in this case, the oxidative waves cannot be unambiguously assigned.

\section{3 - Synthesis of $\left[\mathrm{L}_{2}{ }_{2} \mathrm{Ru}\right]\left[\mathrm{BF}_{4}\right]_{2}$ "Corner Complex"}

For the homometallic $\left[\mathrm{L}_{4}^{2} \mathrm{Fe}_{4}\right]\left[\mathrm{BF}_{4}\right]_{4}$ grids, a variety of species were detected in the ${ }^{1} \mathrm{H} \mathrm{NMR}$, $\mathrm{MB}$, and ESI mass spectra. Although both binding pockets of $\mathbf{H L}^{2}$ prefer the coordination of six-coordination metal ions, the rigidity of the bipyridine ring may be utilized to selectively enforce a stable $\mathrm{Ru}^{\mathrm{II}}$ "corner complex", as previously described for the synthesis of the heterometallic $\left[\mathrm{L}_{4} \mathrm{Ru}_{2} \mathrm{Co}_{2}\right]\left[\mathrm{BF}_{4}\right]_{4}$ and $\left[\mathrm{L}_{4} \mathrm{Ru}_{2} \mathrm{Fe}_{2}\right]\left[\mathrm{BF}_{4}\right]_{4}$ grids. The remaining sections will highlight the preliminary results on the $\left[\mathrm{L}_{2} \mathrm{Ru} \mathrm{B}\left[\mathrm{BF}_{4}\right]_{2}\right.$ "corner complex". 
The kinetically inert $\mathrm{Ru}^{\mathrm{II}}$ "corner complex" was synthesized by addition of $\mathrm{Ru}(\mathrm{DMSO})_{4} \mathrm{Cl}_{2}$ to a degassed solution of $\mathbf{H L}^{2}$ in ethanol:acetone (1:1). The solution was heated at reflux for 16 hours followed by metathesis with $\mathrm{NH}_{4} \mathrm{BF}_{4}$. The solution was filtered, then crystallized by slow diffusion of diethyl ether into a solution of the product 6-3 in acetonitrile to give dark brown crystalline material (Scheme 6.3). Unfortunately, the crystalline material was not suitable for X-ray diffraction and thus the molecular structure of $\left[\mathrm{L}_{2} \mathrm{Ru}_{[}\left[\mathrm{BF}_{4}\right]_{4}\right.$ has not been determined.
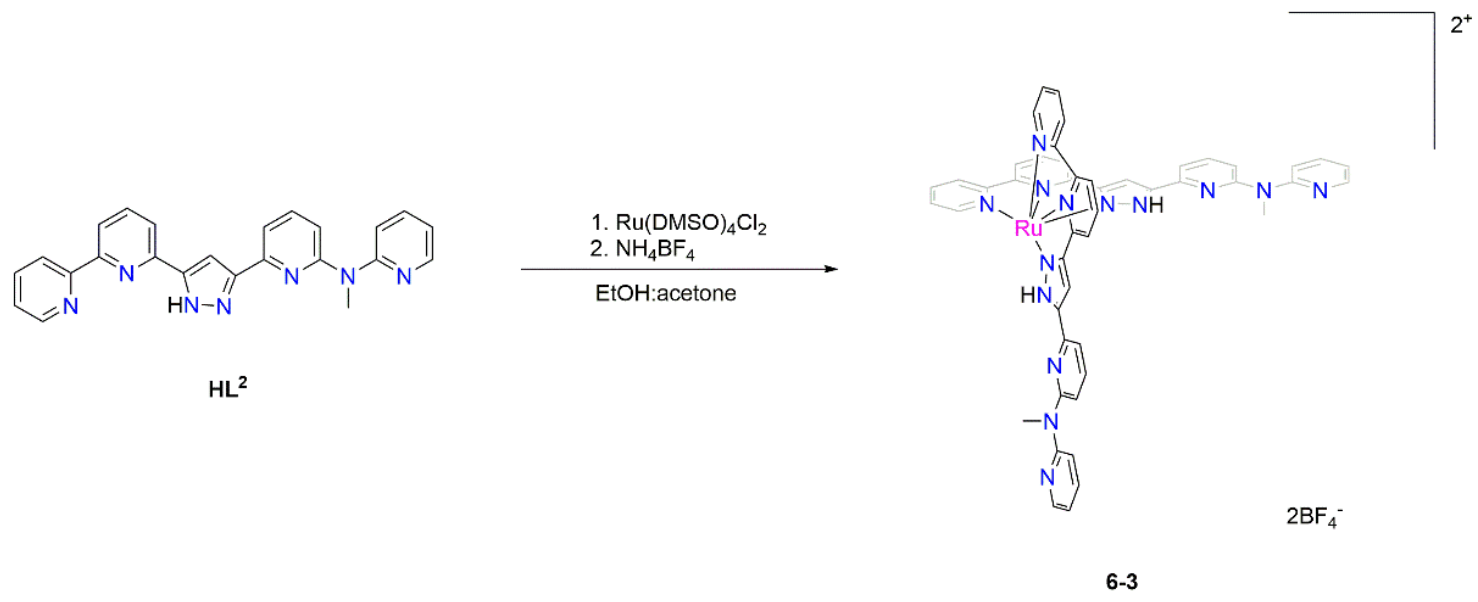

Scheme 6.3. Synthetic scheme for the $\mathrm{Ru}^{\mathrm{II}}$ corner complex $\left[\mathrm{L}^{2}{ }_{2} \mathrm{Ru}\right]\left[\mathrm{BF}_{4}\right]_{2}$.

\subsection{1 - Solution Investigations: Mass Spectrometry \& ${ }^{1}$ H NMR Spectroscopy}

ESI mass spectrometry was conducted on a MeCN solution of $\left[\mathrm{L}_{2}{ }_{2} \mathrm{Ru}\right]\left[\mathrm{BF}_{4}\right]_{2}$ illustrating one dominant peak at $m / z=911$, corresponding to the monomeric $\left[\mathrm{L}_{2} \mathrm{Ru}^{2+}\right.$ species (Figure 6.7) However, in more concentrated solutions, the tetracationic $\left[\mathrm{Ru}_{2} \mathrm{~L}_{4}^{2}\right]^{4+}$ is also present in solution (Figure A22). This was also observed for the $\left[\mathrm{L}_{2} \mathrm{H}_{2} \mathrm{Ru}\left[\mathrm{BF}_{4}\right]_{2}\right.$ "corner complex" described in chapter 3 , in which at high concentrations $\left(>10^{-5} \mathrm{M}\right)$, the $\mathrm{Ru}^{\mathrm{II}}$ "corner complex" persists as a dimer, while at low concentrations $\left(<10^{-5} \mathrm{M}\right)$, both the monomer and dimer coexist in solution.

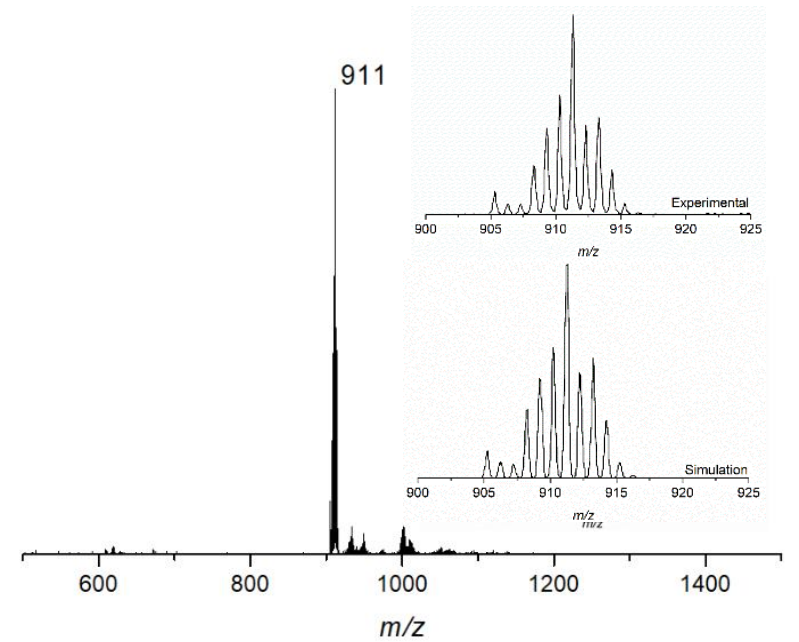

Figure 6.7. ESI mass spectrum of a $\mathrm{MeCN}$ solution of $\left[\mathrm{L}_{2}^{2} \mathrm{Ru}\right]\left[\mathrm{BF}_{4}\right]_{2}$ illustrating the presence of the doubly charged monomer $\left[\mathrm{L}_{2}{ }_{2} \mathrm{Ru}\right]^{2+}$. Simulated and experimental isotopic distribution patterns are shown in the inset. 
In order to confirm the purity as well as the selectivity of the $\mathrm{Ru}^{\mathrm{II}}$ ions, ${ }^{1} \mathrm{H}$ NMR spectroscopy was conducted in MeCN- $\mathrm{d}_{3}$ at room temperature (Figure 6.8). Remarkably, the simplicity of the ${ }^{1} \mathrm{H}$ NMR spectrum with 15 peaks signifies the formation of only a single isomer. The distinctive singlet at $7.72 \mathrm{ppm}$ corresponds to the hydrogen on the pyrazole backbone and the $\mathrm{NCH}_{3}$ moiety on one end of the ligand strand is shown at $3.44 \mathrm{ppm}$. However, definitively assignment of the $\left[\mathrm{L}_{2}{ }_{2} \mathrm{Ru}\right]\left[\mathrm{BF}_{4}\right]_{2}$ "corner complex" could not be obtained even with $2 \mathrm{D}{ }^{1} \mathrm{H}$ NMR spectroscopy (Figure A33). In particular, whether the $\mathrm{Ru}^{\mathrm{II}}$ ion preferentially binds to two mutually perpendicular bipyridine subunits or on the flexible $\mathrm{NCH}_{3}$ end cannot be determined definitively by ${ }^{1} \mathrm{H}$ NMR spectroscopy. Thus, molecular structure determination by X-ray diffraction is essential for elucidating its preferential coordination environment. Nevertheless, based on the ${ }^{1} \mathrm{H}$ NMR spectrum of 6-3, it is indeed possible to isolate a single isomer. Naturally, further characterization including X-ray crystallography, cyclic voltammetry and UV-vis spectroelectrochemistry must be conducted. Furthermore, new heterometallic grids with interesting magnetic and redox properties may be realized.

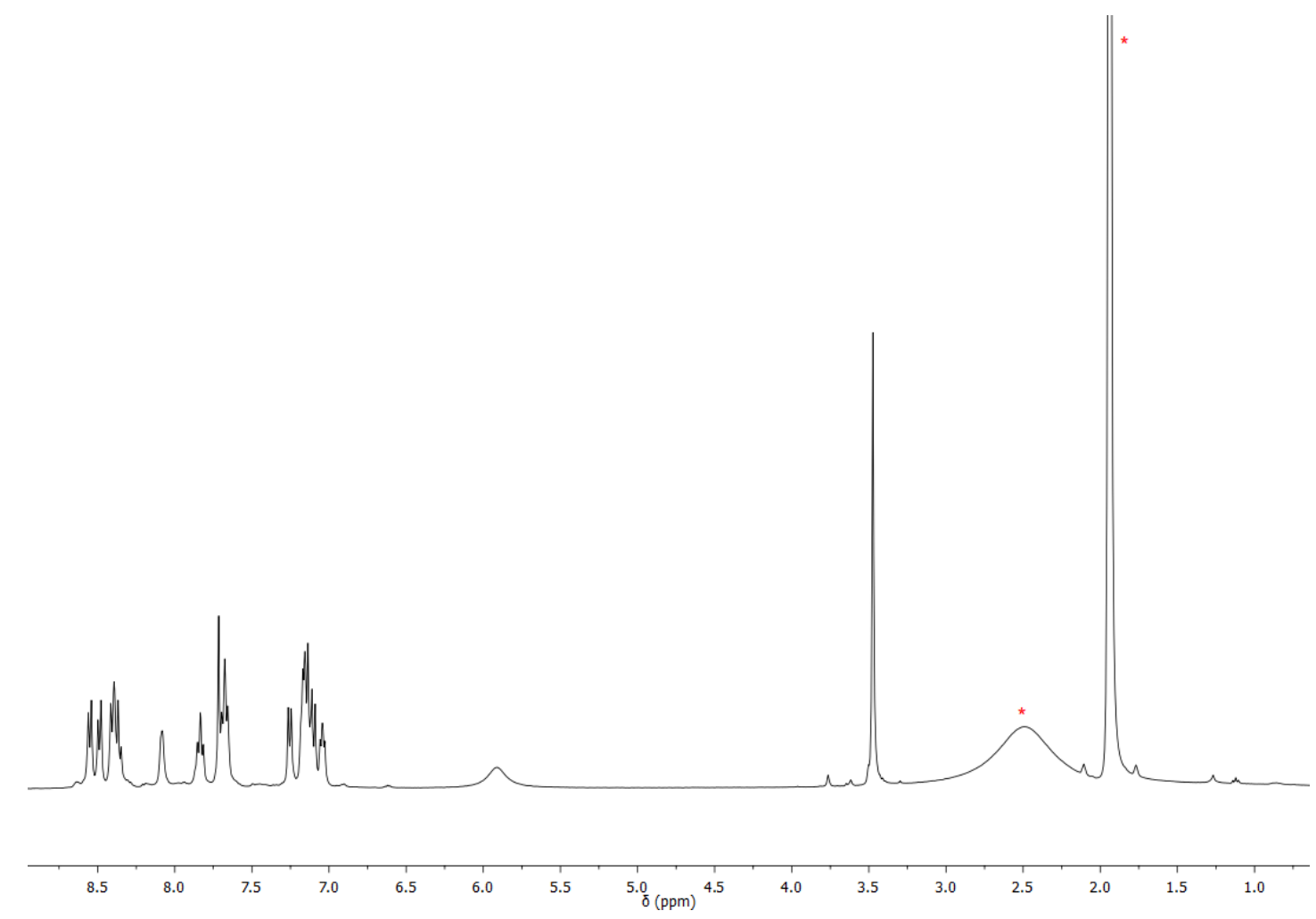

Figure 6.8. ${ }^{1} \mathrm{H}$ NMR spectrum of $\left[\mathrm{L}^{2}{ }_{4} \mathrm{Ru}\right]\left[\mathrm{BF}_{4}\right]_{2}$ in $\mathrm{MeCN}-\mathrm{d}_{3}$ at room temperature. Solvent molecules ( $\mathrm{MeCN}$ and $\mathrm{H}_{2} \mathrm{O}$ ) are indicated with asterisks $\left({ }^{*}\right)$. 


\section{4 - Summary and Conclusion}

In conclusion, a new asymmetric ligand with a rigid bipyridine moiety on one end and a flexible terminal pyridine group on the other end has been developed. Despite the plasticity of half of the ligand strand, a new [2 x 2] homometallic $\mathrm{Fe}_{4}$ grid was synthesized, confirmed by X-ray crystallography. However, the asymmetric nature of the ligand also provides the potential for different arrangements of the ligand within the [2 x 2] grid-like array affording several isomers. MB and ${ }^{1} \mathrm{H}$ NMR experiments on $\left[\mathrm{L}_{4}{ }_{4} \mathrm{Fe}_{4}\right]\left[\mathrm{BF}_{4}\right]_{4}$ suggest the presence of either different isomers and/or impurities. Nevertheless, cyclic voltammetry was conducted on the $\left[\mathrm{L}_{4}{ }_{4} \mathrm{Fe}_{4}\right]\left[\mathrm{BF}_{4}\right]_{4}$ displaying four oxidative waves, which may correspond to the step-wise oxidation the grid. Definitive assignment will require chemical oxidation of $\left[\mathrm{L}_{4}^{2} \mathrm{Fe}_{4}\right]\left[\mathrm{BF}_{4}\right]_{4}$. In pursuit of isolating a single isomer, a stable $\mathrm{Ru}^{\mathrm{II}}$ "corner complex" was synthesized. The simplicity of the ${ }^{1} \mathrm{H}$ NMR spectrum confirmed the presence of a single isomer, which can be used for the synthesis of heterometallic [2 $\mathrm{x} 2$ ] grid-like arrays. 


\section{Chapter 7 - Summary and Conclusion}

The work in this thesis presented new heterometallic $\left[\mathrm{LH}_{4} \mathrm{Ru}_{2} \mathrm{Co}_{2}\right]\left[\mathrm{BF}_{4}\right]_{4}$ and $\left[\mathrm{L}_{4} \mathrm{Ru}_{2} \mathrm{Fe}_{2}\right]\left[\mathrm{BF}_{4}\right]_{4}$ grids based on the known compartamental pyrazolate-bridged ligand (chapters 3 and 4). The field of single molecule magnets in [2 x 2] grids are quite rare, especially with $3 \mathrm{~d}$ transition metals due to antiferromagnetic coupling between the two metal ions affording a diamagnetic ground-state. Now, a new method was employed to overcome this barrier by enforcing diamagnetic spacers such that metal ions with a large magnet anisotropy are introduced at opposing corners. Using this method, we have introduced magnetically active Co ${ }^{\text {II }}$ ions isolated in a highly distorted environment, which gave rise to localized single molecule magnet behaviour (SMM) typically not observed in $3 \mathrm{~d}$ transition metal grid-like arrays. At the same time, the redox properties of the $\left[\mathrm{LH}_{4} \mathrm{Ru}_{2} \mathrm{Co}_{2}\right]\left[\mathrm{BF}_{4}\right]_{4}$ were also investigated, which illustrated a high thermodynamic stability between the $5^{+} / 6^{+}$and $6^{+} / 7^{+}$couple permitting the chemical isolation of the doubly oxidized $\left[\mathrm{LH}_{4} \mathrm{Ru}_{2} \mathrm{Co}_{2}\right]\left[\mathrm{BF}_{4}\right]_{6}$ grid. Indeed, analysis of the molecular structure of $\left[\mathrm{LH}_{4} \mathrm{Ru}_{2} \mathrm{Co}_{2}\right]\left[\mathrm{BF}_{4}\right]_{6}$ revealed the first two oxidations are associated to the oxidation of the $\mathrm{Co}^{\mathrm{II}}$ ions. Therefore, the first two anodic waves observed in the CV of $\left[\mathrm{L}_{4} \mathrm{Ru}_{2} \mathrm{Co}_{2}\right]\left[\mathrm{BF}_{4}\right]_{4}$ correspond to the $\mathrm{Co}^{\mathrm{II}} / \mathrm{Co}^{\mathrm{III}}$ couple and the latter two anodic waves are assumed to correspond to the $\mathrm{Ru}^{\mathrm{II}} / \mathrm{Ru}^{\mathrm{III}}$ couple.

For $\left[\mathrm{LH}_{4} \mathrm{Ru}_{2} \mathrm{Fe}_{2}\right]\left[\mathrm{BF}_{4}\right]_{4}$, despite the synthetic challenges, the results are remarkably similar to $\left[\mathrm{LH}_{4} \mathrm{Ru}_{2} \mathrm{Co}_{2}\right]\left[\mathrm{BF}_{4}\right]_{4}$. Once again, the $\mathrm{Ru}^{\mathrm{II}}$ ions are situated at opposite corners of the grid affording the expected trans-isomer. Unfortunately, due to the high distortion of the FeII environment, thermal SCO and SMM behaviour were not observed, confirmed by variable temperature magnetic measurements. Cyclic voltammetry afforded four oxidative waves with a large electrochemical separation between the $5^{+} / 6^{+}$and $6^{+} / 7^{+}$waves, suggesting the potential for isolation of the doubly oxidized $\left[\mathrm{L}_{4} \mathrm{Ru}_{2} \mathrm{Fe}_{2}\right]^{6+}$ grid. Although this has yet to be achieved due to persistent impurities (10-25\%) in $\left[\mathrm{LH}_{4} \mathrm{Ru}_{2} \mathrm{Fe}_{2}\right]\left[\mathrm{BF}_{4}\right]_{4}$, the first two oxidations may correspond to the $\mathrm{Fe}$ II $/ F e^{\mathrm{III}}$ couple, as observed in the $\left[\mathrm{LH}_{4} \mathrm{Ru}_{2} \mathrm{Co}_{2}\right]\left[\mathrm{BF}_{4}\right]_{4}$ grid.

Although only preliminary results on the excited state structure were obtained for the previously reported homonuclear $\mathrm{Fe}_{4}$ grids using femtosecond (fs) transient absorption spectroscopy in collaboration with the Max Planck Institute, heterometallic grids are also prime candidates for transient absorption spectroscopy. From the homonuclear $\mathrm{Fe}_{4}$ grids, a tentative mechanistic pathway was derived by the life time of the transient species, in which a short-lived species of $\tau_{1^{\prime}}<<100 \mathrm{fs}$, followed by another species with $\tau_{1}=15-20$ ps and finally a long-lived species with $\tau_{2}>1$ ns (chapter 2). However, definitive assignment of the transient excited species requires extensive theoretical calculations on the optical excited states as well as vibrational band assignments. In the case of $\left[\mathrm{LH}_{4} \mathrm{Ru}_{2} \mathrm{Co}_{2}\right]\left[\mathrm{BF}_{4}\right]_{4}$, optical excitation may occur either at the $\mathrm{Ru}^{\mathrm{II}}$ or $\mathrm{Co}$ II ion, which may give rise to more distinct bands for monitoring the changes upon optical excitation. Based on the UV-vis spectroelectrochemistry experiment, electrochemical oxidation from $\mathrm{Co}^{\mathrm{II}}$ to $\mathrm{Co}^{\mathrm{III}}$ afforded a significant decrease in intensity of all bands, while a new broad band centered at $650 \mathrm{~nm}$ was observed for the $\mathrm{Ru}{ }^{\mathrm{II}} / \mathrm{Ru}^{\mathrm{III}}$ couple. Moreover, the ground-state of $\left[\mathrm{LH}_{4} \mathrm{Ru}_{2} \mathrm{Co}_{2}\right]\left[\mathrm{BF}_{4}\right]_{4}$ in solution may be more stable permitting a definitive assignment of the ground-state at room temperature compared to the gradual SCO observed in the homonuclear Fe grids in solution.

The last two chapters focuses on designing new ligands for new homonuclear as well as heterometallic grids. Although addition of $\mathrm{NCH}_{3}$ groups between the pyridine groups of $\mathbf{H L}^{\mathbf{H}}$ imparted a high degree of flexibility at the terminal pyridines rings which prevented [2 $\mathrm{x} 2]$ 
grid formation, a highly distorted dinuclear $\mathrm{Fe}^{\mathrm{II}}$ complex was obtained. The high distortion of the $\mathrm{Fe}^{\mathrm{II}}$ ions favouring the trigonal prism geometry prevented the presence of thermal SCO, and thus the $\mathrm{Fe}^{\mathrm{II}}$ ions are locked in the high-spin state, confirmed by variable temperature magnetic measurements. Interestingly, one-time oxidation to the mixed valent $\left[\mathrm{Fe}^{\mathrm{II}} \mathrm{Fe}^{\mathrm{III}} \mathrm{L}_{2}\right]^{3+}$ afforded significant structural changes, in which one of the HS-Fe"I ions is converted to a LSFeIII ion, while the other Fe ion remains HS-Fe'I. Analysis of the molecular structure suggest large structural rearrangements within the ligand strands upon oxidation, suggesting bond dissociation and reformation. However, further experiments must be conducted to elucidate the mechanistic pathway for the formation of the mixed valent species.

The last chapter involves combining both parts of the parent ligand $\mathbf{H L}^{\mathbf{H}}$ and the additional $\mathrm{NCH}_{3}$ moiety in $\mathbf{H L}^{\mathbf{1}}$ to form the hybrid ligand $\mathbf{H L}^{2}$. Surprisingly, despite the increase in flexibility at one end, a [2 $\mathrm{x}$ 2] $\mathrm{Fe}_{4}$ grid was obtained confirmed by X-ray crystallography. However, bulk analysis including mass spectrometry, MB spectroscopy, and ${ }^{1} \mathrm{H}$ NMR spectroscopy indicate the presence of multiple species/impurities as well as isomers in the solid-state and in solution. In order to facilitate the formation of a single isomer, a step-wise approach was used in which a stable $\mathrm{Ru}^{\mathrm{II}}$ corner complex was isolated. Based on the ${ }^{1} \mathrm{H}$ NMR spectrum of the $\mathrm{Ru}^{\mathrm{II}}$ corner complex, only a single isomer is formed. Further work on synthesis of heterometallic [2 $\mathrm{x} 2]$ grids using this hybrid ligand may be pursued.

Overall, the thesis highlights the synthetic challenges associated with homonuclear and heterometallic [2 $\mathrm{x} 2]$ grid-like arrays. The need for rigid robust ligands with site-specific binding pockets, especially in the case of heterometallic grids, are essential in order for $[2 \mathrm{x}$ 2] grid formation. While a new strategy to incorporate SMM behaviour in [2 $\mathrm{x} 2$ ] grids was introduced, other methods involving incorporation of lanthanide ions have also been recently reported, which gave rise to a remarkable enhancement in magnetic properties of [2 x 2] grids. Moreover, incorporation of $\mathrm{Fe}^{\mathrm{II}}$ and $\mathrm{Co}^{\mathrm{II}}$ ions into these new $4 \mathrm{f}-3 \mathrm{~d}$ heterometallic grids may also induce thermal or photoinduced SCO coupled with SMM behaviour. While there is no systematic method to design ligands with SCO and SMM properties, the goal of many chemists in this field strive to design new ligands with the appropriate $\Delta_{\mathrm{o}}$ for SCO and desired crystal packing motif through the use of strong and weak bonding interactions. 


\section{Chapter 8 - Experimental Section}

\section{1 - Materials and Methods}

\section{General Considerations}

All syntheses were carried out under anaerobic and anhydrous atmosphere of dry nitrogen by standard Schlenk techniques or in an MBraun glovebox unless otherwise stated. Solvents were dried and degassed following standard procedures before use. MeCN, DCM, and DMF were dried with $\mathrm{CaH}_{2} ; \mathrm{Et}_{2} \mathrm{O}$ and THF were dried with sodium in the presence of benzophenone; $\mathrm{MeOH}$ was dried with magnesium turnings activated with iodine. All solvents were distilled prior to use.

\section{Chemicals}

Chemicals were purchased from commercial sources and used without further purification. The following chemicals were prepared following literature procedures: $\mathrm{Ru}(\mathrm{DMSO})_{4} \mathrm{Cl}_{2}{ }^{139}$ and $\mathrm{Fe}^{\mathrm{II}}(\mathrm{OTf})_{2} \cdot 2 \mathrm{MeCN},{ }^{140}$ and $[\text { thianthrene }]^{+}\left[\mathrm{BF}_{4}\right]^{-141}$

\section{UV-vis/NIR Spectroscopy}

UV-vis experiments were carried out using an Agilent $8453 \mathrm{UV}$-vis spectrophotometer as well as the Varian Cary 5000 instrument with an Agilent A453 spectrometer using quartz cuvettes. Spectra were analyzed by Cary Win UV software.

\section{${ }^{1} \mathrm{H} \&{ }^{13} \mathrm{C}$ NMR Spectroscopy}

${ }^{1} \mathrm{H}$ NMR spectra of the complexes were recorded on a Bruker Avance $400 \mathrm{MHz}$ and Bruker Avance $500 \mathrm{MHz}$ instrument. ${ }^{1} \mathrm{H}$ NMR spectra of the ligands were recorded on the Bruker Avance $300 \mathrm{MHz}$ instrument. All chemical shifts are reported relative to the respective residual proton signals used: $\mathrm{CDCl} 3(\delta=7.26 \mathrm{ppm}), \mathrm{DMSO} \mathrm{d}_{6}(\delta=2.50 \mathrm{ppm}), \mathrm{CD}_{3} \mathrm{CN}(\delta=1.94$ ppm). ${ }^{142}{ }^{13} \mathrm{C}$ NMR spectra were recorded using the Avance $500 \mathrm{MHz}$ instrument and the Avance $300 \mathrm{MHZ}$ instrument. Splitting assignments are given as follows: s (singlet), d (doublet), $\mathrm{t}$ (triplet), dt (doublet of a triplet), td (triplet of a doublet), and m (multiplet).

\section{Cyclic Voltammetry and UV-vis Spectroelectrochemistry}

Cyclic voltammetry experiments were performed with the Gamry Interface 1010 and analyzed using the Gamry Framework program. A three electrode setup was used: glassy carbon as the working electrode, $\mathrm{Ag}$ wire as the reference electrode and Pt wire as the counter electrode. For $\left[\mathrm{L}_{4} \mathrm{Ru}_{2} \mathrm{Co}_{2}\right]\left[\mathrm{BF}_{4}\right]_{4}$ and $\left[\mathrm{L}_{4} \mathrm{Ru}_{2} \mathrm{Fe}_{2}\right]\left[\mathrm{BF}_{4}\right]_{4}$, the MeCN solution with $0.1 \mathrm{mM}$ $\mathrm{NBu}_{4} \mathrm{PF}_{6}$ were degassed before each measurement. All resulting data were referenced to $\mathrm{Fc} / \mathrm{Fc}^{+}$.

UV-vis spectroelectrochemistry was performed with the Gamry Interface 1000B inside a glovebox.

\section{Mass spectrometry}

Electro ionization (EI) and Electrospray ionization (ESI) mass spectra were collected on an Applied Biosystems API 2000 device or on a Bruker HCTultra instrument. 


\section{Mössbauer Spectroscopy}

Mössbauer (MB) spectra were recorded with a ${ }^{57}$ Co source in a Rh matrix using an alternating constant acceleration Wissel Mössbauer spectrometer operated in the transmission mode and equipped with a Janis closed-cycle helium cryostat. Isomer shifts $(\delta)$ and quadrupole splitting $\left(\Delta E_{Q}\right)$ are given relative to iron metal at ambient temperature. Simulation of the experimental data was performed with the Mfit program using Lo-rentzian line doublets: E. Bill, Max-Planck Institute for Chemical Energy Conversion, Mülheim/Ruhr, Germany.

\section{Magnetic Susceptibility Measurements}

Temperature-dependent magnetic susceptibility data were measured using a QuantumDesign MPMS-XL-5 SQUID magnetometer equipped with a 5 Tesla magnet in the range from $2.0-400 \mathrm{~K}$. The powdered or crystalline samples were contained in a teflon bucket and fixed in a non-magnetic sample holder. Each raw data file for the measured magnetic moment was corrected for the diamagnetic contribution of the sample holder and the teflon bucket. The molar susceptibility data were corrected for the diamagnetic contribution.

\section{Electron Paramagnetic Resonance}

X-band EPR spectra were measured using a Bruker E500 ELEXSYS spectrometer equipped with a standard cavity (ER4102ST, $9.45 \mathrm{GHz}$ ). The sample temperature was controlled using an Oxford Instruments Helium flow cryostat (ESP910) and an Oxford temperature controller (ITC-4). The microwave frequency was measured with the built-in frequency counter and the magnetic field was calibrated using an NMR field probe (Bruker ER035M). EPR spectra were simulated using Easy-Spin.

\section{2 - Syntheses of Ligands and Complexes}

Synthesis of the ligand $\mathbf{H L}^{\mathbf{H}}$ was adopted from the literature with minimal changes. ${ }^{90}$

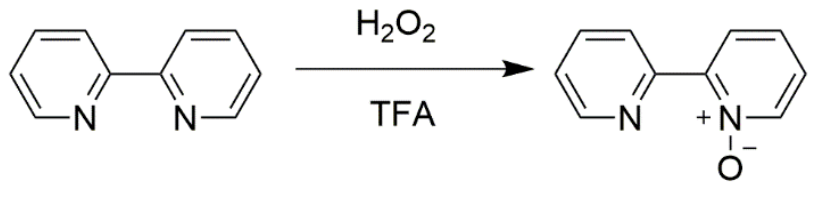

Preparation of [2,2'-bipyridine] 1-oxide 2-5.143 2,2'-Bipyridine 2-4 (28 g, 0.179 mol. 1 eq.) was dissolved in TFA $(100 \mathrm{~mL})$ then $\mathrm{H}_{2} \mathrm{O}_{2}(27 \mathrm{~mL},(1.15 \mathrm{~mol}, 6.42 \mathrm{eq})$ was added dropwise at $0{ }^{\circ} \mathrm{C}$. The reaction was stirred overnight then $6 \mathrm{M} \mathrm{NaOH}$ was added $(250 \mathrm{~mL})$ until a pH of 10 was obtained. The clear solution was stirred for 1 hour, then extracted $3 \times 100 \mathrm{~mL}$ with $\mathrm{CHCl}_{3}$, dried with $\mathrm{MgSO}_{4}$ then the solvent removed to give a yellow oil. The oil was dried in vacuo overnight to give $\mathbf{2 - 5}$ as a white solid (21.85 g, $127 \mathrm{mmol}, 71 \%$ yield).

1 H NMR $\left(300 \mathrm{MHz}, \mathrm{CDCl}_{3}\right): \delta(\mathrm{ppm})=8.85(\mathrm{~d}, J=8.2 \mathrm{~Hz}, 1 \mathrm{Ar}-\mathrm{H}), 8.66(\mathrm{dd}, J=1.5 \mathrm{~Hz}, 1 \mathrm{Ar}-\mathrm{H})$, $8.24(\mathrm{~d}, J=6.9 \mathrm{~Hz}, 1 \mathrm{Ar}-\mathrm{H}), 8.13(\mathrm{dd}, J=2.2 \mathrm{~Hz}, 1 \mathrm{Ar}-\mathrm{H}), 7.76(\mathrm{dt}, J=1.8 \mathrm{~Hz}, 1 \mathrm{Ar}-\mathrm{H}$ ), $7.25-7.32$ (m, 2 Ar-H), $7.17-7.21(\mathrm{~m}, 1 \mathrm{H})$. 


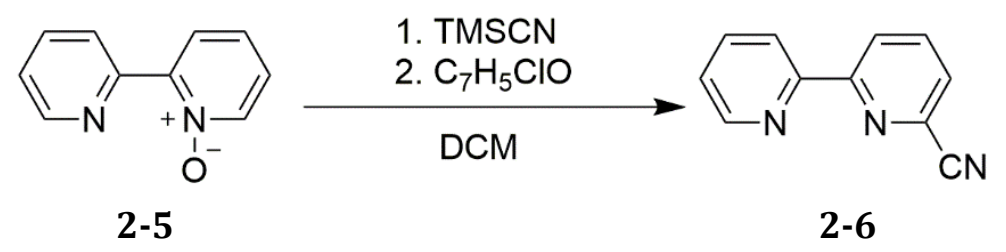

Preparation of [2,2'-bipyridine]-6-carbonitrile $2-6 .{ }^{90}$ The $N$-oxide $\mathbf{2 - 5}(10.613 \mathrm{~g}, 61.6$ mmol, 1 eq.) and TMSCN (30.80 mL, 247 mmol, 4 eq.) were dissolved in dry DCM (100 mL) then

benzoyl chloride ( $14.32 \mathrm{~mL}, 123 \mathrm{mmol}, 2 \mathrm{eq}$ ) was added dropwise to the solution at $0{ }^{\circ} \mathrm{C}$. The reaction was stirred for one hour at $0{ }^{\circ} \mathrm{C}$ then at room temperature for 16 hours. Subsequently,

$\mathrm{NaHCO}_{3}(350 \mathrm{~mL})$ was added slowly and the organic layer was separated. The aqueous phase was extracted with DCM $(2 \times 100 \mathrm{~mL})$ then the solvent removed to give a white solid, which was washed with pentane ( $4 \times 50 \mathrm{~mL}$ ), and dried in vacuo to give 2-6 as a white solid (yield: $5.614 \mathrm{~g}, 310 \mathrm{mmol}, 50 \%)$.

1H NMR (300 MHz, CDCl $): \delta(\mathrm{ppm})=8.64(\mathrm{~m}, 2 \mathrm{Ar}-\mathrm{H}), 8.42(\mathrm{dt}, J=8.1,1.0 \mathrm{~Hz}, 1 \mathrm{Ar}-\mathrm{H}), 7.90(\mathrm{t}$, $J=8.1 \mathrm{~Hz}, 1 \mathrm{Ar}-\mathrm{H}$ ), 7.82 (td, $J=7.7,1.8 \mathrm{~Hz}, 1 \mathrm{Ar}-\mathrm{H}$ ), 7.64 (dd, $J=7.7,1.1 \mathrm{~Hz}, 1 \mathrm{Ar}-\mathrm{H}$ ), 7.33 (ddd, $J=11.2,4.9,1.2 \mathrm{~Hz}, 1 \mathrm{Ar}-\mathrm{H})$.

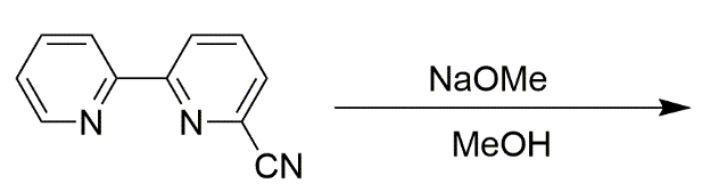

2-6

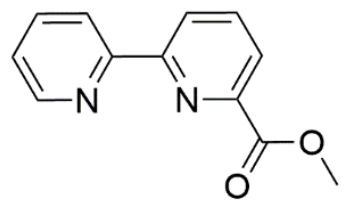

$2-7$

Preparation of methyl [2,2'-bipyridine]-6-carboxylate 2-7.90 6-cyano-2,2'bipyridine 2-6 (7.5 g, $41.39 \mathrm{mmol}, 1$ eq.) was added to a solution of $\mathrm{NaOMe}$ in $\mathrm{MeOH}$ generated in situ with Na metal (1.14 g, $49.61 \mathrm{mmol}, 1.2 \mathrm{eq}$.) and stirred at room temperature for 16 hours to give a deep red solution. Next day, $\mathrm{AcOH}(12 \mathrm{~mL})$ and $\mathrm{NaHCO}_{3}(12 \mathrm{~g}, 142.86 \mathrm{mmol}, 3.4$ eq.) were added and stirred for 1.5 hour at room temperature. After the alloted reaction time, the solid was filtered, washed with $\mathrm{MeOH}$ and the solvent removed to give a yellow solid. The intermediate was redissolved in ethyl acetate $(100 \mathrm{~mL})$ and washed with $\mathrm{NaHCO}_{3}(100 \mathrm{~mL})$. Subsequently, the organic layer was separated, then the aq. phase extracted with ethyl acetate $(3 \times 100 \mathrm{~mL})$ and the solvent removed to give a dark yellow oil, which was redissolved in $\mathrm{MeOH}: \mathrm{H}_{2} \mathrm{O}(1: 1,200 \mathrm{~mL})$. Concentrated $\mathrm{HCl}$ was added until a $\mathrm{pH}$ of 2 was obtained and the reaction was left to stir for 16 hours followed by addition of $6 \mathrm{M} \mathrm{NaOH}$ until the solution is neutralized. The aq. phase was extracted with ethyl acetate $(3 \times 100 \mathrm{~mL})$, dried with $\mathrm{MgSO}_{4}$ and the solvent removed to give $\mathbf{2 - 7}$ as a yellow solid (4.415 g, $20.61 \mathrm{mmol}, 50 \%$ ).

1H NMR (300 MHz, $\left.\mathrm{CDCl}_{3}\right): \delta(\mathrm{ppm})=8.61$ (ddd, $\left.J=4.8,1.8,0.9 \mathrm{~Hz}, 1 \mathrm{Ar}-\mathrm{H}\right), 8.53$ (dd, $J=7.9,1.1 \mathrm{~Hz}, 1 \mathrm{H}), 8.46(\mathrm{dt}, J=8.0,1.0 \mathrm{~Hz}, 1 \mathrm{Ar}-\mathrm{H}), 8.06(\mathrm{dd}, J=7.7,1.1 \mathrm{~Hz}, 1 \mathrm{Ar}-\mathrm{H}), 7.88$ (t, $J=7.8 \mathrm{~Hz}, \mathrm{Ar}-\mathrm{H}), 7.76$ (td, $J=7.5,1.9 \mathrm{~Hz}, 1 \mathrm{Ar}-\mathrm{H}), 7.26(\mathrm{~m}, 1 \mathrm{H}), 3.96\left(\mathrm{~s}, 1 \mathrm{CH}_{3}\right)$. 


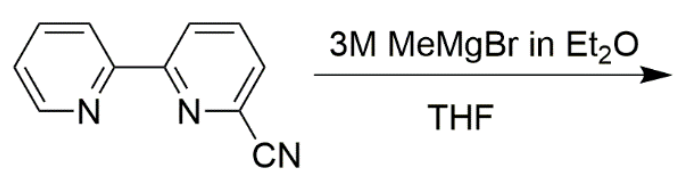

$2-6$

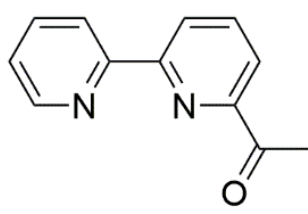

2-8

Preparation of 1-([2,2'-bipyridin]-6-yl)ethan-1-one 2-8. ${ }^{90} 3 \mathrm{M} \mathrm{MeMgBr}$ (36.24 mL, 314.56 mmol, 5.7 eq.) was added dropwise into a solution of $2-6$ (10.0 g, $55.15 \mathrm{mmol}, 1$ eq.) in dry THF $(150 \mathrm{~mL})$ at $0{ }^{\circ} \mathrm{C}$. After 45 minutes, the reaction was allowed to stir at rt for two additional hours then saturated $\mathrm{NH}_{4} \mathrm{Cl}(150 \mathrm{~mL})$ was added and allowed to stir for 20 minutes. Subsequently, the organic phase was separated, the aq. phase extracted with DCM $(3 \times 100 \mathrm{~mL})$ and the solvent removed to give a brown oil. The oil was extracted with pentane ( $3 \times 50 \mathrm{~mL}$ ) to give $\mathbf{2 - 8}$ as a bright yellow solid (9.71 $\mathrm{g} 48.98 \mathrm{mmol}$, 93\% yield).

${ }^{1} \mathrm{H}$ NMR $\left(300 \mathrm{MHz}, \mathrm{CDCl}_{3}\right): \delta(\mathrm{ppm})=8.63(\mathrm{ddd}, J=4.8,1.8,0.9 \mathrm{~Hz}, 1 \mathrm{Ar}-\mathrm{H}), 8.56(\mathrm{dd}, J=7.8$, $1.2 \mathrm{~Hz}, 1 \mathrm{Ar}-\mathrm{H}$ ), 8.48 (dt, $J=8.0,1.1 \mathrm{~Hz}, 1 \mathrm{Ar}-\mathrm{H}), 7.99$ (dd, $J=7.7,1.2 \mathrm{~Hz}, 1 \mathrm{Ar}-\mathrm{H}), 7.89$ (t, $J=7.7$ $\mathrm{Hz}, 1 \mathrm{Ar}-\mathrm{H}$ ), 7.80 (ddd, $J=8.0,7.5,1.8 \mathrm{~Hz}, 1 \mathrm{Ar}-\mathrm{H}), 7.24-7.34(\mathrm{~m}, 1 \mathrm{Ar}-\mathrm{H}), 2.77\left(\mathrm{~s}, 1 \mathrm{CH}_{3}\right)$.

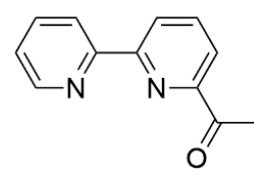

2-8

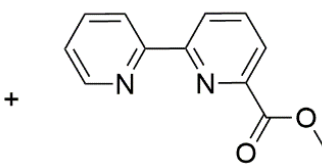

$2-7$

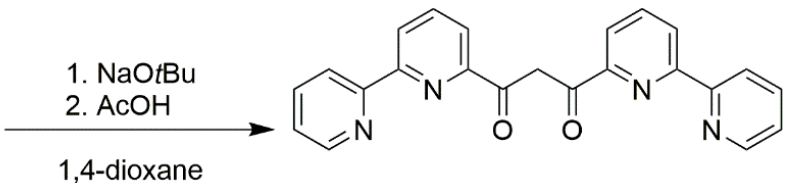

$2-9$

Preparation of 1,3-di([2,2'-bipyridin]-6-yl)propane-1,3-dione 2-9.90,91 The ketone precursor 2-8 (2.085 g, $10.52 \mathrm{mmol}, 1.1 \mathrm{eq})$ and methyl ester precursor 2-7 (2.00 g, 9.33 mmol, 1 eq) was dissolved in dry 1,4-dioxane ( $25 \mathrm{~mL})$. Subsequently, $\mathrm{NaO}{ }^{t} \mathrm{Bu}(1.415 \mathrm{~g}, 14.72$ mmol, 1.58 eq) was added to give a heavy brown precipitate which was stirred at room temperature for 16 hours. Subsequently, AcOH (0.595 mL, $10.41 \mathrm{mmol}, 1.2 \mathrm{eq}$.) was diluted to $10 \mathrm{~mL}$ and added into the reaction slowly to give a beige solid. The solid was filtered, washed with $\mathrm{H}_{2} \mathrm{O}$ and further crystallized in $\mathrm{MeOH}$ or toluene to give 2-9 as a white powder (1.8 g, $4.73 \mathrm{mmol}, 45 \%$ yield). Due to tautomerization, assignment of the protons was not feasible.<smiles>O=C(CC(=O)c1cccc(-c2ccccn2)n1)c1cccc(-c2ccccn2)n1</smiles>

2-9<smiles>c1ccc(-c2cc(-c3cccc(-c4ccccn4)n3)[nH]n2)nc1</smiles>

$\mathrm{HL}^{\mathrm{H}}$

Preparation of 6,6"-(1H-pyrazole-3,5-diyl)di-2,2'-bipyridine HLH.90 Hydrazine monohydrate $(0.500 \mathrm{~mL}, 16.05 \mathrm{mmol}, 3 \mathrm{eq}$.) was added to a suspension of the diketone $\mathbf{2 - 9}$ 
( $2.00 \mathrm{~g}, 5.25 \mathrm{mmol}, 3.1 \mathrm{eq}$.) in EtOH $\left(20 \mathrm{~mL}\right.$ ). The reaction was heated at $80^{\circ} \mathrm{C}$ for three hours, cooled to room temperature, and the solvent removed to give a yellow solid. The yellow solid was redissolved in minimal amounts of acetone and layered with $\mathrm{H}_{2} \mathrm{O}$ to give $\mathbf{H L}^{\mathbf{H}}$ as a white powder $(1.49 \mathrm{~g}, 4.0 \mathrm{mmol}, 76 \%)$.

${ }^{1}$ H NMR $\left(300 \mathrm{MHz}, \mathrm{CDCl}_{3}\right): \delta(\mathrm{ppm})=11.65(\mathrm{~s}, 1 \mathrm{NH}), 8.74(\mathrm{ddd}, J=4.9,1.9,0.9 \mathrm{~Hz}, 1 \mathrm{Ar}-\mathrm{H})$, 8.62-8.53 (m, 1H), 8.42-8.36 (m, 1 Ar-H), 8.04-7.82 (m, 3 Ar-H), 7.60 (s, 1 Ar-H), 7.36 (ddd, $J$ $=7.5,4.8,1.2 \mathrm{~Hz}, 1 \mathrm{Ar}-\mathrm{H})$.<smiles>O=C(O)c1cccc(Br)n1</smiles>

5-1<smiles>COC(=O)c1cccc(Br)n1</smiles>

5-2

Preparation of methyl 6-bromopicolinate 5-2.144 6-bromopyridine-2-carboxylic acid 5-1 (30 g, $148.51 \mathrm{mmol} 1$ eq.) was suspended in $\mathrm{MeOH}(150 \mathrm{~mL})$. Subsequently, $\mathrm{H}_{2} \mathrm{SO}_{4}$ (1 mL, $18.76 \mathrm{mmol}, 0.12$ eq.) was added and the reaction was heated at reflux for 16 hours. After the allocated reaction time, the reaction was neutralized with $\mathrm{NaHCO}_{3}$ and the organic phase separated. The aqueous phase was extracted with DCM ( $3 \times 100 \mathrm{~mL})$ and the solvent removed to give a white solid (17.5 g, $87.06 \mathrm{mmol}, 73 \%$ yield).

${ }^{1}$ H NMR $\left(300 \mathrm{MHz}, \mathrm{CDCl}_{3}\right): \delta(\mathrm{ppm})=8.11(\mathrm{dd}, J=6.6,1.9 \mathrm{~Hz}, 1 \mathrm{Ar}-\mathrm{H}), 7.77-7.66(\mathrm{~m}, 2 \mathrm{Ar}-\mathrm{H})$, 8.11(dd, $J=6.6,1.9 \mathrm{~Hz}, 1 \mathrm{H}), 4.01\left(\mathrm{~s}, 1 \mathrm{CH}_{3}\right)$.<smiles>COC(=O)c1cccc(Br)n1</smiles>

5-2

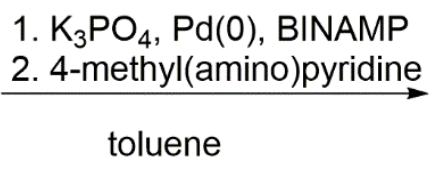<smiles>COC(=O)c1cccc(N(C)c2ccccn2)n1</smiles>

5-3

Preparation of methyl 6-(methyl(pyridin-2-yl)amino)picolinate 5-3. Compound 5-2 (1.0 g, $4.63 \mathrm{mmol}, 1$ eq.) was dissolved in dry toluene $(15 \mathrm{~mL})$ then $\mathrm{K}_{3} \mathrm{PO}_{4}(1.18 \mathrm{~g}, 5.56$ mmol, 1.2 eq.), Pd(0) (0.085 g, $0.093 \mathrm{mmol}, 0.02$ eq.), and BINAMP ( $0.115 \mathrm{~g}, 0.185 \mathrm{mmol}, 0.04$ eq.) were added to give a red suspension. After 15 minutes, 4-methyl(amino)pyridine $(0.475$ $\mathrm{mL}, 4.62 \mathrm{mmol}, 1$ eq.) was added to immediately give an orange solution. The reaction was heated at reflux for 16 hours, filtered, and the solvent removed to give a yellow oil, which was further purified by column chromatography (silica gel) with hexane:ethylacetate (3:1) as the eluent to give 5-3 as a yellow solid ( $0.544 \mathrm{~g}, 2.23 \mathrm{mmol}, 48 \%$ yield).

${ }^{1}$ H NMR $\left(300 \mathrm{MHz}, \mathrm{CDCl}_{3}\right): \delta(\mathrm{ppm})=8.42-8.34(\mathrm{~m}, 1 \mathrm{Ar}-\mathrm{H}), 7.75-7.58(\mathrm{~m}, 3 \mathrm{Ar}-\mathrm{H}), 7.43$ (dd, $J=7.8,1.5 \mathrm{~Hz} 1 \mathrm{Ar}-\mathrm{H}$ ), 7.24 (dt, $J=8.5,1.0 \mathrm{~Hz} 1 \mathrm{Ar}-\mathrm{H}$ ), 6.95 (ddd, $J=7.3,5.0,0.9 \mathrm{~Hz} 1 \mathrm{Ar}-\mathrm{H}$ ), $3.99\left(\mathrm{~s}, 1 \mathrm{CH}_{3}\right), 3.74\left(\mathrm{~s}, 1 \mathrm{CH}_{3}\right)$.

${ }^{13} \mathrm{C}$ NMR $(300 \mathrm{MHz}, \mathrm{CDCl} 3): \delta(\mathrm{ppm})=200.4(1 \mathrm{C}, \mathrm{C}=0), 157.4(1 \mathrm{C}, \mathrm{Ar}-\mathrm{C}), 156.5$ (1C, Ar-C), 151.8 (1C, Ar-C), 148.1 (1C, Ar-C), 137.6 (1C, Ar-C), 137.5(1C, Ar-C), 117.8 (1C, Ar-C), 116.9 (1C, Ar-C), 115.2 (1C, Ar-C), $114.2(1 \mathrm{C}, \mathrm{CH}), 36.0$ (1C, $\left.\mathrm{NCH}_{3}\right), 25.8\left(1 \mathrm{C}, \mathrm{CH}_{3}\right)$. 


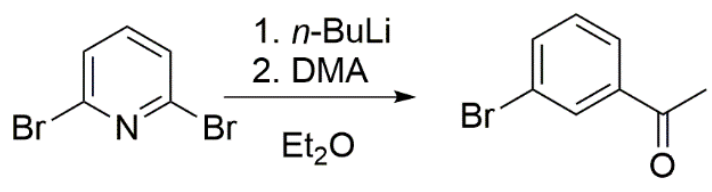

Preparation of 1-(3-bromophenyl)ethan-1-one 5-5.145 $\mathrm{n}$-BuLi (25 mL, $62 \mathrm{mmol}, 1.1 \mathrm{eq})$ was added dropwise to a suspension of 2,6-dibromopyridine (13.3 g, $56.27 \mathrm{mmol}, 1 \mathrm{eq}$.) in dry $\mathrm{Et}_{2} \mathrm{O}(150 \mathrm{~mL})$ at $-78^{\circ} \mathrm{C}$ for 30 minutes. Subsequently, the reaction was warmed to $-40{ }^{\circ} \mathrm{C}$ and stirred for one hour. The reaction was cooled to $-78{ }^{\circ} \mathrm{C}$, then DMA was added and the reaction was warmed to room temperature. Subsequently, saturated $\mathrm{NH}_{4} \mathrm{Cl}(100 \mathrm{~mL})$ was added, and the organic phase was separated. The aq. phase was extracted with $\mathrm{Et}_{2} \mathrm{O}(3 \times 100$ $\mathrm{mL}$ ) then the combined organic layers were concentrated in vacuo to give 5-5 as white crystalline material ( $8.1 \mathrm{~g}, 40.50 \mathrm{mmol}, 72 \%$ yield).

${ }_{1}^{1}$ H NMR $\left(300 \mathrm{MHz}, \mathrm{CDCl}_{3}\right): \delta(\mathrm{ppm})=8.01(\mathrm{dd}, J=6.9,1.7 \mathrm{~Hz}, 1 \mathrm{Ar}-\mathrm{H}), 7.75-7.64(\mathrm{~m}, 2 \mathrm{Ar}-\mathrm{H})$, $2.72\left(\mathrm{~s}, 1 \mathrm{CH}_{3}\right)$.<smiles>CC(=O)c1cccc(Br)c1</smiles>

5-5

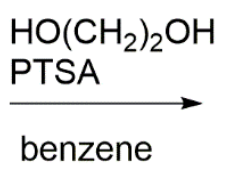

benzene<smiles>CC1(c2cccc(Br)n2)OCCO1</smiles>

5-6

Preparation of N-methyl-6-(2-methyl-1,3-dioxolan-2-yl)-N-(pyridin-2-yl)pyridin-2amine 5-6. ${ }^{146}$ Compound 5-5 (15.495 g, 77.46 mmol, 1 eq.) was dissolved in benzene (130 $\mathrm{mL}$ ) then ethylene glycol ( $4.8 \mathrm{~mL}, 85.84 \mathrm{mmol}, 1.1 \mathrm{eq}$.) and PTSA (1.47 g, $7.73 \mathrm{mmol}, 0.5 \mathrm{eq}$.) was added. The reaction was heated with a Diens-stalk apparatus for 32 hours at 120 degrees. Subsequently, the reaction was cooled to room temperature, and $1 \mathrm{M} \mathrm{NaOH}$ was added until the reaction was neutralized. The organic phase was separated then the aq. phase was extracted with $\mathrm{Et}_{2} \mathrm{O}(3 \times 100 \mathrm{~mL})$ and the solvent removed to give a dark purple oil, which was further purified by column chromatography (silica gel) with EtOAc: $\mathrm{C}_{6} \mathrm{H}_{14}$ (1:3) as the eluent to give an orange oil ( $6.09 \mathrm{~g}, 24.95 \mathrm{mmol}, 32 \%$ yield).

1H NMR $\left(300 \mathrm{MHz}, \mathrm{CDCl}_{3}\right): \delta(\mathrm{ppm})=7.61-7.47(\mathrm{~m}, 2 \mathrm{Ar}-\mathrm{H}), 7.42(\mathrm{dd}, J=7.0,1.9 \mathrm{~Hz} 1 \mathrm{Ar}-\mathrm{H})$, 4.19-4.02 (m, $\left.2 \mathrm{CH}_{2}\right), 3.99-3.80\left(\mathrm{~m}, 2 \mathrm{CH}_{2}\right), 1.73\left(\mathrm{~s}, 1 \mathrm{CH}_{3}\right)$. 
<smiles>CC1(c2cccc(Br)n2)OCCO1</smiles>

5-6
1. $\mathrm{NaO}{ }^{t} \mathrm{Bu}, \mathrm{Pd}(0)$, dppp

2. 4-methylaminopyridine

toluene<smiles>CN(c1ccccn1)c1cccc(C2(C)OCCO2)n1</smiles>

5-7

Preparation of N-methyl-6-(2-methyl-1,3-dioxolan-2-yl)-N-(pyridin-2-yl)pyridin-2amine 5-7. $\mathrm{NaO}^{t} \mathrm{Bu}(2.83 \mathrm{~g}, 29.49 \mathrm{mmol} 1.2$ eq.), $\mathrm{Pd}(0)$ ( $0.450 \mathrm{~g}, 0.491 \mathrm{mmol}, 0.02$ eq.), and dppp ( $0.405 \mathrm{~g}, 0.983 \mathrm{mmol}, 0.04$ eq.) were dissolved in dry toluene $(100 \mathrm{~mL})$ then a solution of 5-6 (6.00 g, $24.59 \mathrm{mmol}, 1 \mathrm{eq})$ in dry toluene $(10 \mathrm{~mL})$ was added. After 5 minutes, 4methyl(amino)pyridine $(2.50 \mathrm{~mL}, 24.55 \mathrm{mmol}, 1 \mathrm{eq}$.) was added and the reaction was heated at reflux for 48 hours. Subsequently, the reaction was cooled to room temperature, filtered, and the solvent removed to give a dark blue/purple oil, which was purified by column chromotagraphy (silica gel) with ethylaetate:hexane (1:6) as the first eluent followed by ethylacetate:hexane (1:1) to give a yellow oil ( $4.3 \mathrm{~g}, 15.79 \mathrm{mmol}, 64 \%$ yield).

${ }_{1}^{1} \mathbf{H}$ NMR $\left(300 \mathrm{MHz}, \mathrm{CDCl}_{3}\right): \delta(\mathrm{ppm})=8.35(\mathrm{ddd}, J=5.0,2.0,0.9 \mathrm{~Hz}, 1 \mathrm{Ar}-\mathrm{H}), 7.63-7.45(\mathrm{~m}, 2$ Ar-H), 7.31 (m, 1 Ar-H), 7.18-7.06 (m, 2 Ar-H), 6.86 (ddd, J = 7.2, 5.0, 1.0 Hz, 1 Ar-H), 4.20-4.04 (m, $\left.2 \mathrm{CH}_{2}\right), 4.04-3.86\left(\mathrm{~m}, 2 \mathrm{CH}_{2}\right), 3.68\left(\mathrm{~s}, 1 \mathrm{NCH}_{3}\right), 1.77\left(\mathrm{~s}, 1 \mathrm{CH}_{3}\right)$.

13C NMR (300 MHz, CDCl3): $\delta(\mathrm{ppm})=159.1(1 \mathrm{C}, \mathrm{Ar}-\mathrm{C}), 157.6$ (1C, Ar-C), 157.1 (1C, Ar-C), 147.1 (1C, Ar-C), 137.4 (1C, Ar-C), 137.1 (1C, Ar-C), 116.8 (1C, Ar-C), 114.2 (1C, Ar-C), 113.4 (1C, Ar-C), 112.0 (1C, Ar-C), 108.7 (1C, CH), 157.1 (1C, Ar-C), 65 (2C, $\left.\mathrm{CH}_{2}\right), 35.9$ (1C, $\mathrm{NCH}_{3}$ ), $24.7\left(1 \mathrm{C}, \mathrm{CH}_{3}\right)$.<smiles>CN(c1ccccn1)c1cccc(C2(C)OCCO2)n1</smiles>

$5-7$

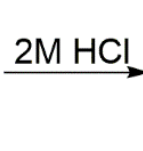<smiles>CC(=O)c1cccc(N(C)c2ccccn2)n1</smiles>

5-8

Preparation of 1-(6-(methyl(pyridin-2-yl)amino)pyridin-2-yl)ethan-1-one 5-8. Compound 5-7 (16.54 g, $60.96 \mathrm{mmol}, 1$ eq.) was redissolved in acetone then 6M HCl (60 mL) was added and the reaction was stirred at room temperature for 16 hours. Subsequently, the solution was neutralized with $\mathrm{Na}_{2} \mathrm{CO}_{3}$ then extracted with DCM $(3 \times 100 \mathrm{~mL})$. The solvent was removed to give an orange oil, which was purified by coloumn chromatography (silica gel) with ethylacetate:hexane (2:3) as the eluent to give an orange oil $(10.357 \mathrm{~g}, 45,55 \mathrm{mmol}, 75 \%$ yield).

${ }_{1} \mathbf{H}$ NMR $\left(300 \mathrm{MHz}, \mathrm{CDCl}_{3}\right): \delta(\mathrm{ppm})=8.39(\mathrm{ddd}, J=5.0,2.0,0.8 \mathrm{~Hz}, 1 \mathrm{Ar}-\mathrm{H}), 7.70-7.50(\mathrm{~m}, 3$ Ar-H), 7.38 (dd, , J = 8.1, 1.1 Hz, 1 Ar-H), 7.32-7.19 (m, 1 Ar-H), 6.96 (ddd, $J=7.3,4.9,0.9$ Hz, 1 Ar-H), 3.70 (s, $1 \mathrm{NCH}_{3}$ ), 2.67 (s, $1 \mathrm{CH}_{3}$ ). 
${ }^{13} \mathrm{C}$ NMR $(300 \mathrm{MHz}, \mathrm{CDCl} 3): \delta(\mathrm{ppm})=200.4(1 \mathrm{C}, \mathrm{C}=0), 157.5$ (1C, Ar-C), $151.8(1 \mathrm{C}, \mathrm{Ar}-\mathrm{C})$, 148.1 (1C, Ar-C), 137.6 (1C, Ar-C), 137.5 (1C, Ar-C), 117.9 (1C, Ar-C), 116.9 (1C, Ar-C), 115.2 (1C, Ar-C), 114.1 (1C, Ar-C), $36.0\left(1 \mathrm{C}, \mathrm{NCH}_{3}\right), 25.8\left(1 \mathrm{C}, \mathrm{CH}_{3}\right)$.<smiles>CC(=O)c1cccc(N(C)c2ccccn2)n1</smiles>

5-8<smiles>COC(=O)c1cccc(N(C)c2ccccn2)n1</smiles>

5-3

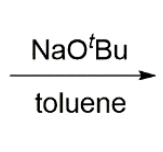<smiles>CN(c1ccccn1)c1cccc(C(=O)CC(=O)c2cccc(N(C)c3ccccn3)n2)n1</smiles>

5-9

Preparation of 1,3-bis(6-(methyl(pyridin-2-yl)amino)pyridin-2-yl)propane-1,3-dione 5-9. The ketone ( $6.12 \mathrm{~g}, 26.93 \mathrm{mmol} 1$ eq.) and methyl ester ( $6.2 \mathrm{~g} 25.48 \mathrm{mmol}, 1.05$ eq.) were suspended in dry toluene then $\mathrm{NaO}^{t} \mathrm{Bu}(2.72 \mathrm{~g}, 28.27 \mathrm{mmol}, 1.5 \mathrm{eq}$.) was added to give a red solution. The reaction was heated to $80^{\circ} \mathrm{C}$ for 3 hours. Subsequently, the reaction was cooled to room temperature, precipitated with $\mathrm{EtOH}: \mathrm{AcOH}(20 \mathrm{~mL}, 1: 1)$ to give a bright yellow solid. The solid was filtered, redissolved in DCM and washed with $\mathrm{H}_{2} \mathrm{O}$. The aq. phase was extracted with DCM $(3 \times 100 \mathrm{~mL})$, then the solvent was removed to give a bright yellow solid (8.48 $\mathrm{g}$, $19.33 \mathrm{mmol}, 72 \%)$.

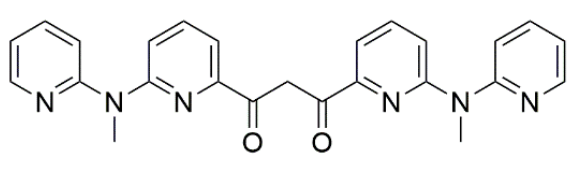

5-9
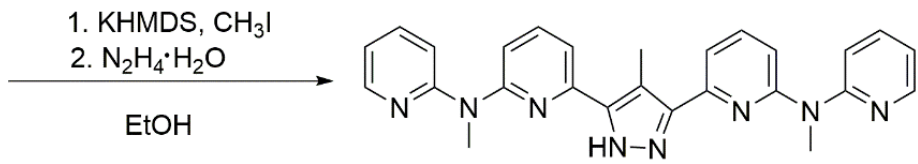

HL $^{1}$

Preparation of 6,6'-(4-methyl-1H-pyrazole-3,5-diyl)bis(N-methyl-N-(pyridin-2yl)pyridin-2-amine) HL1. Compound 5-9 (3.348 g, 7.64 mmol 1 eq.) was suspended in dry toluene $(50 \mathrm{~mL})$ then KHMDS $(1.80 \mathrm{~g}, 9.02 \mathrm{mmol}, 1.2 \mathrm{eq}$.) was added to give a green solution. The reaction was heated to $80^{\circ} \mathrm{C} \mathrm{s}$ for one hour then $\mathrm{CH}_{3} \mathrm{I}(1.90 \mathrm{~mL}, 30.52 \mathrm{mmol}$, 4 eq.) was added to give a yellow precipitate. The reaction was heated for an additional three hours, cooled to room temperature and the yellow solid filtered, washed with toluene. The organic layer was washed with sat. $\mathrm{Na}_{2} \mathrm{CO}_{3}$, separated, then the aq. phase was extracted with chloroform $(3 \times 100 \mathrm{~mL})$ and the solvent removed to give a brown oil, which was used without purification.

The brown oil ( $3.43 \mathrm{~g}, 7.58 \mathrm{mmol}, 1$ eq.) was redissolved in EtOH (30 mL) then hydrazine $(0.71 \mathrm{~mL}, 22.79 \mathrm{mmol}, 3 \mathrm{eq})$ was added and the solution was heated at reflux for 4 hours affording a fine yellow precipitate. The reaction was stirred at room temperature for an additional 16 hours, the yellow solid filtered, then washed with EtOH to give $\mathbf{H L}^{\mathbf{1}}(2.2 \mathrm{~g}, 4.90$ mmol, $64 \%$ yield).

${ }^{1} \mathbf{H}$ NMR (500 MHz, DMSO-d 6 ): $\delta(\mathrm{ppm})=8.33(\mathrm{dd}, J=7.8,2.0 \mathrm{~Hz}, 2$ Ar-H), 7.71 (m, 4 Ar-H), 7.42 (d, $J=7.5 \mathrm{~Hz}, 2 \mathrm{Ar}-\mathrm{H}$ ), 7.30 (d, $J=8.4 \mathrm{~Hz}, 2 \mathrm{Ar}-\mathrm{H}$ ), 7.16 (dd, $J=8.3 \mathrm{~Hz}$, $0.7 \mathrm{~Hz}, 2 \mathrm{Ar}-\mathrm{H}$ ), 7.00 (ddd, $J=7.2 \mathrm{~Hz}, 4.9 \mathrm{~Hz}, 0.9 \mathrm{~Hz}, 2 \mathrm{Ar}-\mathrm{H}$ ), 3.60 (s, $2 \mathrm{NCH}_{3}$ ), 2.59 (s, $1 \mathrm{CH}_{3}$ ). 
${ }^{13} \mathrm{C}$ NMR $(300 \mathrm{MHz}, \mathrm{CDCl} 3): \delta(\mathrm{ppm})=157.3$ (4C, Ar-C), 156.7 (2C, Ar-C), 156.3 (4C, Ar-C), 148.0 (2C, Ar-C), 147.9 (2C, Ar-C), 138.3 (2C, Ar-C), 138.0 (2C, Ar-C), 137.7 (2C, Ar-C), 117.5 (2C, Ar-C), 114.9 (2C, Ar-C), 113.8 (1C, Ar-C), 111.9 (2C, Ar-C), 35.9 (1C, $\left.\mathrm{NCH}_{3}\right), 10.8\left(1 \mathrm{C}, \mathrm{CH}_{3}\right.$ ).<smiles>CC(=O)c1cccc(N(C)c2ccccn2)n1</smiles>

$5-8$

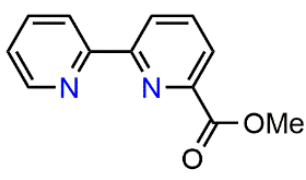

2-7

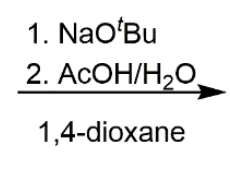<smiles>CN(c1ccccn1)c1cccc(C(=O)CC(=O)c2cccc(-c3ccccn3)n2)n1</smiles>

6-1

Preparation of 1-([2,2'-bipyridin]-6-yl)-3-(6-(methyl(pyridin-2-yl)amino)pyridin-2yl)propane-1,3-dione 6-1. The methylester 2-7 (2.00 g, 9.34 mmol, 1 eq.) and ketone 5-8 ( $2.12 \mathrm{~g}, 9.32 \mathrm{mmol}, 1.00 \mathrm{eq}$.) were suspended in dry 1,4-dioxane. NaOtBu (1.07 g, $11.20 \mathrm{mmol}$, 1.2 eq.) was added to give a brown solution and the solution was stirred at room temperature for 16 hours. Subsequently, a mixture of $\mathrm{AcOH}$ ( $0.534 \mathrm{~mL}, 9.32 \mathrm{mmol}, 1$ eq.) was diluted to 10 $\mathrm{mL}$ then added slowly to give a yellow solid ( $1.42 \mathrm{~g}, 3.47 \mathrm{mmol}, 37 \%$ yield). The solid was used without further purification.<smiles>CCN(CC)CCO</smiles>

$6-1$<smiles>CN(c1ccccn1)c1cccc(-c2cc(-c3cccc(-c4ccccn4)n3)n[nH]2)n1</smiles>

$\mathrm{HL}^{2}$

Preparation of 6-(3-([2,2'-bipyridin]-6-yl)-1H-pyrazol-5-yl)-N-methyl-N-(pyridin-2yl)pyridin-2-amine $\mathrm{HL}^{2}$.

The diketone 6-1 (1.00 g, $2.44 \mathrm{mmol}, 1$ eq.) was suspended in EtOH ( $30 \mathrm{~mL}$ ) then $\mathrm{N}_{2} \mathrm{H}_{4} \cdot \mathrm{H}_{2} \mathrm{O}$ $(0.288 \mathrm{~mL}, 9.24 \mathrm{mmol}, 3.8 \mathrm{eq}$.) was added and the solution was heated at reflux for three hours. Subsequently, the solution was cooled to room temperature and the solvent was removed to give an orange oil. Acetone was added to the oil then layered with $\mathrm{H}_{2} \mathrm{O}$ to give $\mathbf{H L}^{2}$ as a white powder $(0.73 \mathrm{~g}, 0.180 \mathrm{mmol}, 73 \%$ yield $)$.

${ }_{1}^{1} \mathbf{H}$ NMR $\left(300 \mathrm{MHz}, \mathrm{CDCl}_{3}\right): \delta(\mathrm{ppm})=8.73$ (ddd, $\left.J=4.8,1.8,0.9 \mathrm{~Hz}, 1 \mathrm{Ar}-\mathrm{H}\right)$, $8.58(\mathrm{~d}, J=8.0 \mathrm{~Hz}, 1 \mathrm{Ar}-\mathrm{H}), 8.40-8.46(\mathrm{~m}, 1 \mathrm{Ar}-\mathrm{H}), 8.36$ - 8.48 (m, $1 \mathrm{Ar}-\mathrm{H}), 7.91$ (ddt, J = 9.6, 7.6, 3.3 Hz, 2 Ar-H), 7.55-7.71 (m, 2 Ar-H), 7.47 (s, Pz-H), 7.37 (ddd, J = 7.6, 4.9, 1.2 Hz, 1 ArH), 7.30-7.34 (m, $1 \mathrm{Ar}-\mathrm{H}), 7.19-7.09(\mathrm{~m}, 1 \mathrm{Ar}-\mathrm{H}), 6.85$ (ddd, J = 7.3, 5.0, 0.9 Hz, $1 \mathrm{Ar}-\mathrm{H}), 3.74$ $\left(\mathrm{s}, \mathrm{NCH}_{3}\right)$. 
<smiles>c1ccc(-c2cc(-c3cccc(-c4ccccn4)n3)[nH]n2)nc1</smiles><smiles></smiles>

$\mathrm{HL}^{\mathrm{H}}$

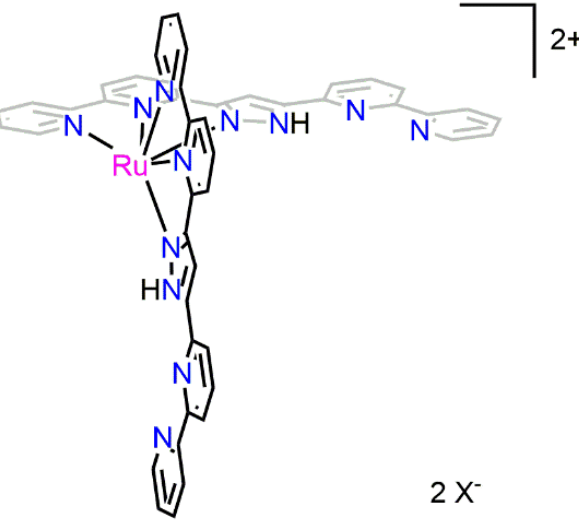

3-1a: $\mathrm{X}=\mathrm{BF}_{4}^{-}$

3-1b: $X=\mathrm{OTf}^{-}$

Preparation of [Ru( $\left.\left.\mathbf{L}^{\mathrm{H}}\right)_{2}\right][\mathrm{BF} 4]_{2}$ 3-1a. $\mathbf{H L}^{\mathrm{H}}\left(0.378 \mathrm{~g}, 1.06 \mathrm{mmol}, 3\right.$ eq.) and $\mathrm{Ru}(\mathrm{DMSO})_{4} \mathrm{Cl}_{2}$ $(0.162 \mathrm{~g}, 0.334 \mathrm{mmol}, 1 \mathrm{eq}$.) were suspended in degassed EtOH:acetone ( $24 \mathrm{~mL})$ then heated at reflux for 16 hours to give a deep brown solution. Subsequently, the reaction was cooled to room temperature, $\mathrm{NH}_{4} \mathrm{BF}_{4}(0.112 \mathrm{~g}, 1.07 \mathrm{mmol}, 3.2 \mathrm{eq}$.) was added and the crude product was obtained by precipitation with $\mathrm{Et}_{2} \mathrm{O}$. Red crystalline material was obtained by slow diffusion of $\mathrm{Et}_{2} \mathrm{O}$ into a solution of 3-1a ( $0.293 \mathrm{~g}, 0.277 \mathrm{mmol}, 78 \%$ yield). For the synthesis of 3-1b, the procedure is identical except NaOTf $(0.184 \mathrm{~g}, 1.06 \mathrm{mmol}, 3.2 \mathrm{eq}$.) was used instead of $\mathrm{NH}_{4} \mathrm{BF}_{4}$.

${ }^{1} \mathrm{H}$ NMR $\left(300 \mathrm{MHz}, \mathrm{CD}_{3} \mathrm{CN}\right): \delta(\mathrm{ppm})=15.08(\mathrm{~s}, 1 \mathrm{NH}), 8.60(\mathrm{dd},, J=5.3,3.7 \mathrm{~Hz}, 1 \mathrm{Ar}-\mathrm{H}), 8.10$ (s, $1 \mathrm{Ar}-\mathrm{H}), 8.03$ (t, $J=7.9,1 \mathrm{Ar}-\mathrm{H}), 7.86(\mathrm{dd}, J=7.9,0.9 \mathrm{~Hz}, 1 \mathrm{Ar}-\mathrm{H}), 7.82-7.70(\mathrm{~m}, 3 \mathrm{Ar}-\mathrm{H}), 2.67$ (s, $\left.1 \mathrm{CH}_{3}\right), 7.54(\mathrm{~d}, J=8.0 \mathrm{~Hz}, 1 \mathrm{Ar}-\mathrm{H}), 7.48(\mathrm{~d}, J=8.0,1 \mathrm{Ar}-\mathrm{H}), 7.42(\mathrm{td}, J=7.7,1.8 \mathrm{~Hz}, 1 \mathrm{Ar}-\mathrm{H})$, 7.24 (td, $J=7.9,1.5 \mathrm{~Hz}, 1 \mathrm{Ar}-\mathrm{H}$ ), 6.84 (dt, $J=4.5,1.5 \mathrm{~Hz}, 1 \mathrm{Ar}-\mathrm{H}$ ), 6.78 (ddd, $J=7.5,5.6,1.3 \mathrm{~Hz}$, $1 \mathrm{Ar}-\mathrm{H}), 6.72-6.59$ (m, $2 \mathrm{Ar}-\mathrm{H})$.

MS (ESI(+), MeCN): $m / z=853\left[\mathrm{HL}_{2} \mathrm{Ru}\right]^{2+}, m / z=458\left[\mathrm{~L}_{4}{ }_{4} \mathrm{Ru}_{2} \mathrm{NaF}\right]^{4+}$

UV-Vis (MeCN, $298 \mathrm{~K}): \lambda[\mathrm{nm}]\left(\varepsilon\left[\mathrm{M}^{-1} \mathrm{~cm}^{-1}\right]\right)=434$ (27660), 457 (26700), 514 (11250), 554 (7290). 


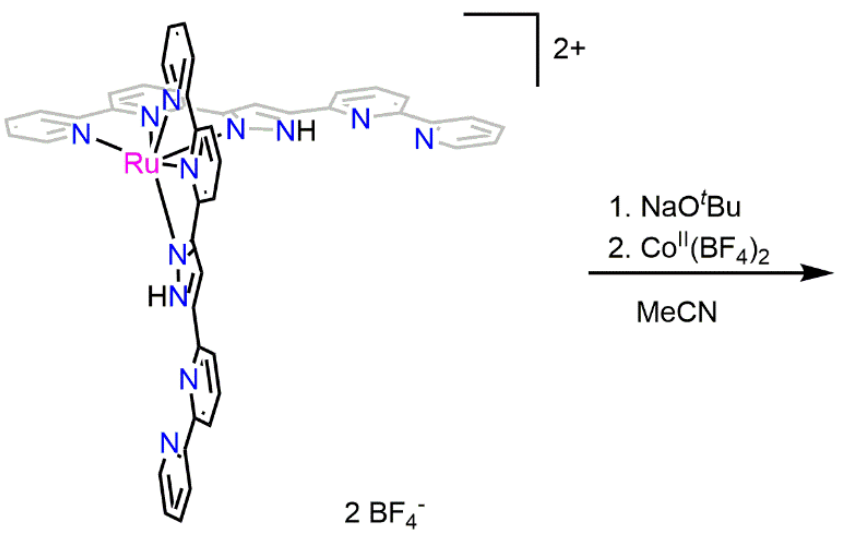

3-1a

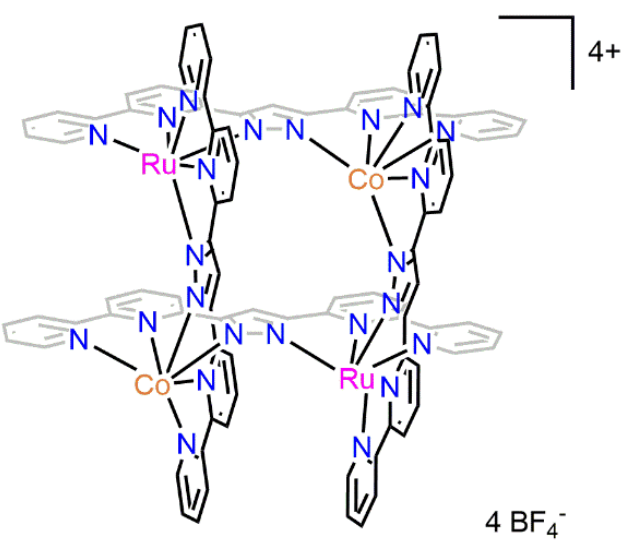

3-2

Preparation of $\left[\mathbf{L}_{4} \mathrm{Ru}_{2} \mathrm{Co}_{2}\right]\left[\mathrm{BF}_{4}\right]_{4}$ 3-2. The $\mathrm{Ru}^{\mathrm{II}}$ corner complex 3-1a $(0.118 \mathrm{~g}, 0.114 \mathrm{mmol}$, 1 eq.) and $\mathrm{NaO}^{t} \mathrm{Bu}(0.029 \mathrm{~g}, 0.301 \mathrm{mmol}, 2.6 \mathrm{eq}$.) was dissolved in dry $\mathrm{MeCN}(8 \mathrm{~mL})$. After one hour, $\mathrm{Co}^{\mathrm{II}}\left(\mathrm{BF}_{4}\right)_{2}(0.078 \mathrm{~g}, 0.229 \mathrm{mmol}, 2$ eq.) was added and the solution was heated at reflux for two days. Subsequently, the brown solution was cooled to room temperature, filtered, and the crude product was obtained by precipitation with $\mathrm{Et}_{2} \mathrm{O}$. Crystalline material was obtained by slow diffusion of $\mathrm{Et}_{2} \mathrm{O}$ into a solution of 3-2 in $\mathrm{MeCN}(0.067 \mathrm{~g}, 0.031 \mathrm{mmol}, 27 \%$ yield).

${ }^{1}$ H NMR (400 MHz, CD $\left.{ }_{3} \mathrm{CN}\right): \delta(\mathrm{ppm})=120.28(\mathrm{~s}, 1 \mathrm{Ar}-\mathrm{H}), 97.69$ (s, $\left.1 \mathrm{Ar}-\mathrm{H}\right), 66.67(\mathrm{~s}, 1 \mathrm{Ar}-\mathrm{H})$, 48.49 (s, 1 Ar-H), 29.64 (s, 1 Ar-H), 27.15 (s, 1 Ar-H), 25.91 (s, 1 Ar-H), 11.90 (s, 1 Ar-H), 6.34 (s, 1 Ar-H), -2.91 (s, 1 Ar-H), -4.14 (s, 1 Ar-H), -9.38 (s, 1 Ar-H), -17.10 (s, 1 Ar-H), -22.44 (s, 1 Ar-H).

MS (ESI(+), MeCN): $m / z=455\left[\mathrm{LH}_{4} \mathrm{Ru}_{2} \mathrm{Co}_{2}\right]^{4+}$

UV-vis (MeCN, $298 \mathrm{~K}): \lambda$ [nm] $\left(\varepsilon\left[\mathrm{M}^{-1} \mathrm{~cm}^{-1}\right]\right)=235$ (81670), 267 (65560), 299 (61930), 408 (17770), 496 (8010), 608 (3040).

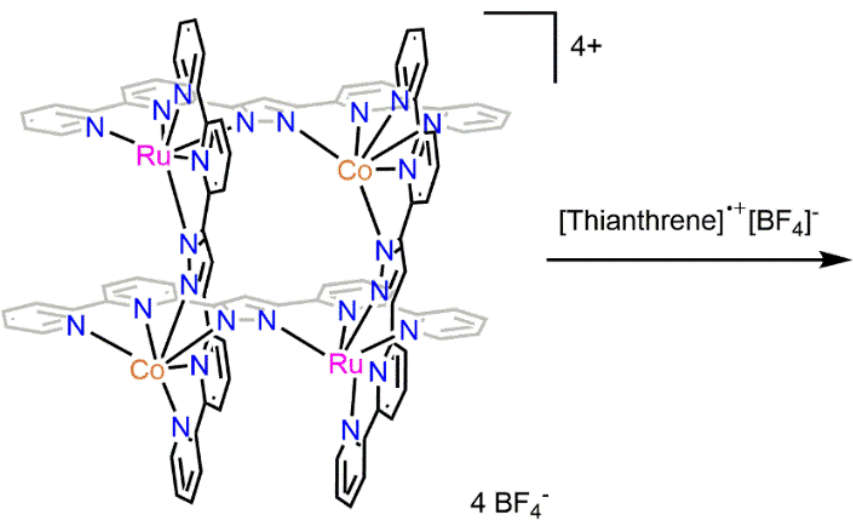

3-2

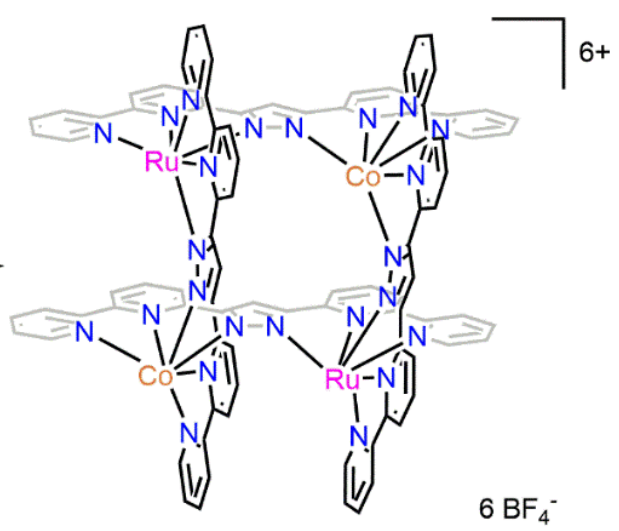

3-3

Preparation of $\left[\mathrm{L}_{4} \mathrm{Ru}_{2} \mathrm{Co}_{2}\right]\left[\mathrm{BF}_{4}\right]_{6}$ 3-3. $\left[\mathrm{LH}_{4} \mathrm{Ru}_{2} \mathrm{Co}_{2}\right]\left[\mathrm{BF}_{4}\right]_{4}$ 3-2 (0.020 g, 0.009 mmol, 1 eq.) was dissolved dry MeCN (3 ml). Subsequently, a solution of [thianthrene] ${ }^{++}\left[\mathrm{BF}_{4}\right]^{-}(0.006 \mathrm{~g}$, $0.018 \mathrm{mmol}, 2$ eq.) was added to immediately give a red solution. The reaction was stirred at 
room temperature for 16 hours, filtered and precipitated with $\mathrm{Et}_{2} \mathrm{O}$ to give a red powder. Crystalline material was obtained by slow diffusion of $\mathrm{Et}_{2} \mathrm{O}$ into a solution of 3-3 in $\mathrm{MeCN}$ (0.005 g, $0.002 \mathrm{mmol}, 25 \%$ yield).

${ }^{1} \mathrm{H}$ NMR $\left(400 \mathrm{MHz}, \mathrm{CD}_{3} \mathrm{CN}\right): \delta(\mathrm{ppm})=8.68(\mathrm{~d}, J=8.0 \mathrm{~Hz}, 1 \mathrm{Ar}-\mathrm{H}), 8.57(\mathrm{~s}, 1 \mathrm{Pyz}-\mathrm{H}), 8.47(\mathrm{~m}, 2$ Ar-H), 8.38 (t, $J=8.0,1 \mathrm{Ar}-\mathrm{H}), 8.21(\mathrm{~m}, 2 \mathrm{Ar}-\mathrm{H}), 7.97(\mathrm{~d}, J=7.2 \mathrm{~Hz}, 1 \mathrm{Ar}-\mathrm{H}), 7.73(\mathrm{t}, J=7.7 \mathrm{~Hz}$, $1 \mathrm{Ar}-\mathrm{H}), 7.56(\mathrm{t}, J=7.8,1 \mathrm{Ar}-\mathrm{H}), 7.37(\mathrm{~d}, J=7.8 \mathrm{~Hz}, 1 \mathrm{Ar}-\mathrm{H}), 6.91(\mathrm{t}, J=6.9 \mathrm{~Hz}, 1 \mathrm{Ar}-\mathrm{H}), 6.73(\mathrm{t}$, $J=6.8 \mathrm{~Hz}, 1 \mathrm{Ar}-\mathrm{H}), 5.73(\mathrm{~d}, J=6.0,1 \mathrm{Ar}-\mathrm{H}), 5.49$ (d, J = 6.1, $1 \mathrm{Ar}-\mathrm{H})$.

UV-vis (MeCN, $298 \mathrm{~K}): \lambda[\mathrm{nm}]\left(\varepsilon\left[\mathrm{M}^{-1} \mathrm{~cm}^{-1}\right]\right)=270$ (55010), 320 (44190), 401 (12833), 497 (6427).

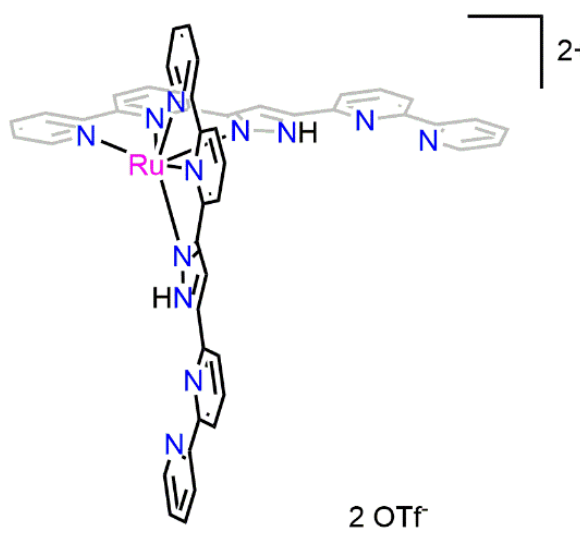

3-1b

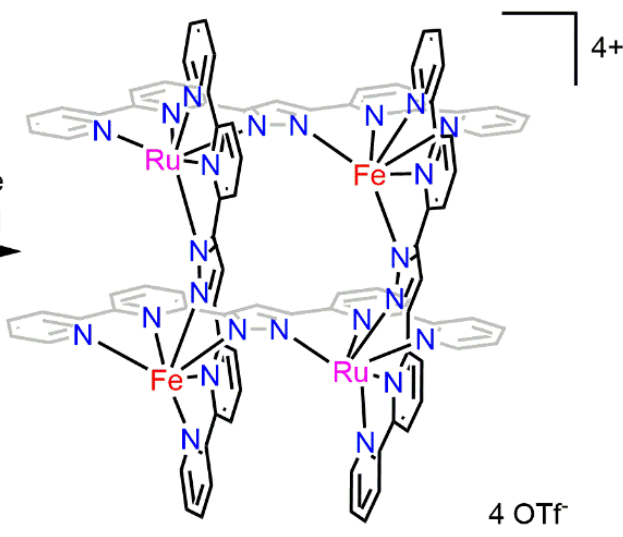

4-1

Preparation of [ $\left.\mathbf{L}_{4} \mathbf{R u}_{2} \mathbf{F e}_{2}\right][\mathbf{O T f}]_{4}$ 4-1. [HL $\left.{ }_{2} \mathrm{Ru}\right][\mathrm{OTf}]_{2} \mathbf{3 - 1} \mathbf{b}(0.104 \mathrm{~g}, 0.101 \mathrm{mmol}, 1$ eq.) and $\mathrm{NaO}{ }^{t} \mathrm{Bu}(0.019 \mathrm{~g}, 0.198 \mathrm{mmol} 2 \mathrm{eq}$.) were dissolved in dry MeCN (6 mL) and stirred at room temperature for one hour. Subsequently, $\mathrm{Fe}^{\mathrm{II}}\left(\mathrm{BF}_{4}\right)_{2}(0.033 \mathrm{~g}, 0.098 \mathrm{mmol}, 1$ eq. $)$ was added and the reaction was heated at reflux for 16 hours. The reaction was filtered, precipitated with $\mathrm{Et}_{2} \mathrm{O}$ and further crystallized by slow diffusion of $\mathrm{Et}_{2} \mathrm{O}$ into a solution of 4-2 in $\mathrm{MeCN}$ (0.070 g, $0.032 \mathrm{mmol}, 33 \%$ yield).

${ }_{1}^{1}$ H NMR (400 MHz, CD $\left.{ }_{3} \mathrm{CN}\right): \delta(\mathrm{ppm})=140.38(\mathrm{~s}, 1 \mathrm{Ar}-\mathrm{H}), 81.27(\mathrm{~s}, 1 \mathrm{Ar}-\mathrm{H}), 62.86(\mathrm{~s}, 1 \mathrm{Ar}-\mathrm{H})$, 58.25 (s, 1 Ar-H), 53.75 (s, 1 Ar-H), 47.53 (s, 1 Ar-H), 14.18 (s, 1 Ar-H), 12.94 (s, 1 Ar-H), 9.49 (s, 1 Ar-H), 6.84 (s, 1 Ar-H), 0.79 (s, 1 Ar-H), -1.28 (s, 1 Ar-H), -9.58 (s, 1 Ar-H), -10.93 (s, 1 Ar$\mathrm{H})$.

MS (ESI(+), MeCN): $m / z=454\left[\mathrm{LH}_{4} \mathrm{Ru}_{2} \mathrm{Fe}_{2}\right]^{4+}$

UV-vis (MeCN, $298 \mathrm{~K}): \lambda[\mathrm{nm}]\left(\varepsilon\left[\mathrm{M}^{-1} \mathrm{~cm}^{-1}\right]\right)=386$ (33860), 497 (15040), 560 (7730), 608 (4410).

MB: $\delta=1.04 \mathrm{mms}^{-1} ; \Delta E_{\mathrm{Q}}=2.84 \mathrm{mms}^{-1}$ 


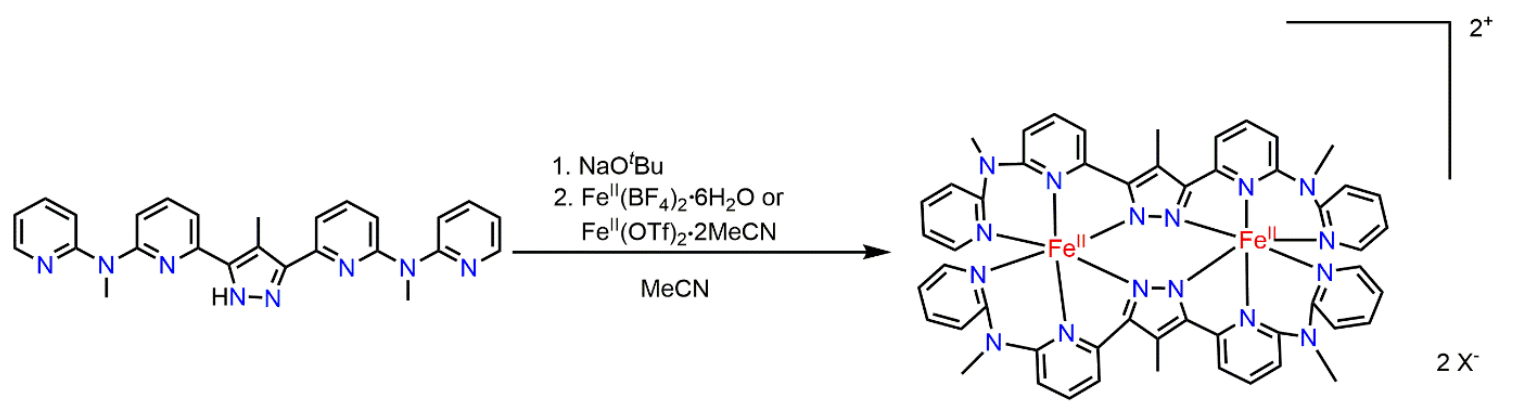

$\mathrm{HL}^{1}$

[5-10a]: $\mathrm{X}=\mathrm{BF}_{4}-$

[5-10b]: $X=\mathrm{OTf}^{-}$

Preparation of [ $\left.\mathbf{L}^{\mathbf{L}_{2}} \mathbf{F e}_{2}\right][\mathbf{O T f}]_{2} \cdot \mathbf{H L}^{\mathbf{1}}(0.100 \mathrm{~g}, 0.223 \mathrm{mmol}, 2$ eq. $)$ and $\mathrm{NaO}{ }^{t} \mathrm{Bu}(0.087 \mathrm{~g}, 0.905$ mmol, 4 eq.) were dissolved in dry $\mathrm{MeCN}(10 \mathrm{~mL})$ and stirred at room temperature for 45 minutes. Subsequently, Fe ${ }^{\mathrm{II}}(\mathrm{OTf})_{2} \cdot 2 \mathrm{MeCN}(0.116 \mathrm{~g}, 0.266 \mathrm{mmol}, 1.14$ eq.) was added to give a deep red solution, which was stirred at room temperature. After 16 hours, bright yellow crystalline material of $\left[\mathrm{L}_{2}^{1} \mathrm{Fe}_{2}\right][\mathrm{OTf}]_{2}$ was obtained $(0.102 \mathrm{~g}, 0.085 \mathrm{mmol}, 38 \%$ yield). Since ESI-MS, ${ }^{1} \mathrm{H}$ NMR spectroscopy and MB spectroscopy of the crude product confirm the purity of the complex, most analytical measurements were conducted on the non-crystallized material. For X-ray diffraction analysis, single crystals of $\left[\mathrm{L}_{2}^{1} \mathrm{Fe}_{2}\right][\mathrm{OTf}]_{2}$ were obtained by slow diffusion of Et2O into a solution of [L12Fe2][OTf]2 in MeCN. The same procedure was repeated for [5-10a] except $\mathrm{Fe}\left(\mathrm{BF}_{4}\right)_{2} \cdot 6 \mathrm{H}_{2} \mathrm{O}(0.091 \mathrm{~g}, 0.270 \mathrm{mmol}, 1.2 \mathrm{eq}$.) was used instead of $\mathrm{Fe}^{\mathrm{II}}(\mathrm{OTf})_{2} \cdot 2 \mathrm{MeCN}$.

${ }^{1}$ H NMR (400 MHz, CD $\left.{ }_{3} \mathrm{CN}\right): \delta(\mathrm{ppm})=60.99(\mathrm{~s}, 1 \mathrm{Ar}-\mathrm{H}), 49.41(\mathrm{~s}, 1 \mathrm{Ar}-\mathrm{H}), 46.57(\mathrm{~s}, 1 \mathrm{Ar}-\mathrm{H})$, 37.75(s, $1 \mathrm{Ar}-\mathrm{H}$ ), 33.55 (s, $1 \mathrm{Ar}-\mathrm{H}), 22.05$ (s, $1 \mathrm{Ar}-\mathrm{H}), 4.04$ (s, $1 \mathrm{Ar}-\mathrm{H}),-1.96$ (s, $1 \mathrm{Ar}-\mathrm{H})$, $-2.79(\mathrm{~s}, 1 \mathrm{Ar}-\mathrm{H})$.

MS (ESI(+), MeCN): $m / z=503\left[\mathrm{~L}_{2}{ }_{2} \mathrm{Fe}_{2}\right]^{2+}$

UV-vis (MeCN, $298 \mathrm{~K}): \lambda$ [nm] $\left(\varepsilon\left[\mathrm{M}^{-1} \mathrm{~cm}^{-1}\right]\right)=260$ (72172), 303 (47554), 332 (45495), 839 (130).

MB: $\delta=1.10 \mathrm{mms}^{-1} ; \Delta E_{\mathrm{Q}}=4.10 \mathrm{mms}^{-1}$ 

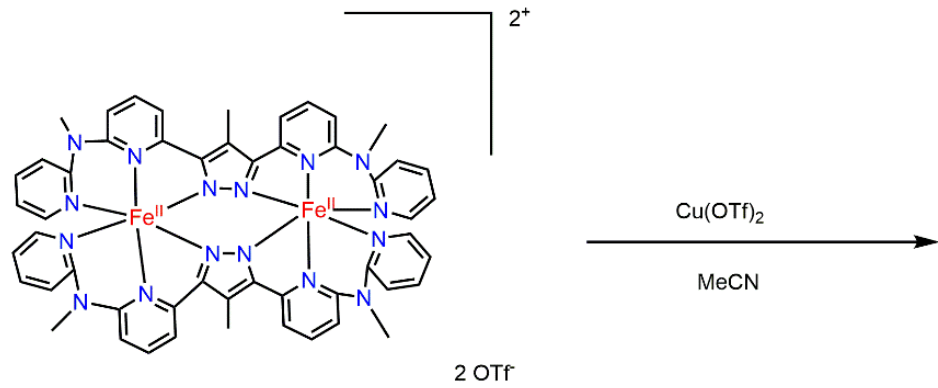

[5-10b]

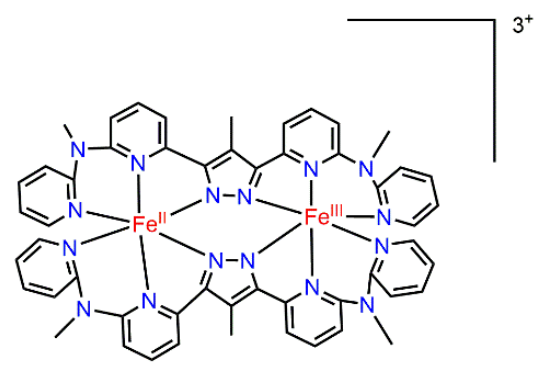

$30 \mathrm{OTf}^{-}$

Preparation of $\left[\mathbf{L}_{2}{ }_{2} \mathrm{Fe}^{\mathrm{II}} \mathrm{Fe}^{\mathrm{III}}\right][\mathrm{OTf}]_{3}$ [5-11]. The dinuclear $\left[\mathrm{L}^{1}{ }_{2} \mathrm{Fe}^{\mathrm{II}}{ }_{2}\right][\mathrm{OTf}]_{2}(0.050 \mathrm{~g}, 0.038$ mmol, 1 eq.) was suspended in dry $\mathrm{MeCN}(6 \mathrm{~mL})$ then a solution of $\mathrm{Cu}(\mathrm{OTf})_{2}(0.014 \mathrm{~g}, 0.038$ mmol, 1 eq.) in $\mathrm{MeCN}$ was added to give a green solution. The reaction was stirred at room temperature for 16 hours under inert conditions, filtered then crystallized by slow diffusion of Et2O into a solution of the product in MeCN $(0.011 \mathrm{~g}, 0.008 \mathrm{mmol}, 20 \%$ yield).

1H NMR (400 MHz, CD $\left.{ }_{3} \mathrm{CN}\right): \delta(\mathrm{ppm})=48.46(\mathrm{~s}, 1 \mathrm{Ar}-\mathrm{H}), 37.12(\mathrm{~s}, 1 \mathrm{Ar}-\mathrm{H}), 25.27(\mathrm{~s}, 1 \mathrm{Ar}-\mathrm{H})$, 24.70 (s, $1 \mathrm{Ar}-\mathrm{H}), 15.00$ (s, $1 \mathrm{Ar}-\mathrm{H}), 14.70$ (s, $1 \mathrm{Ar}-\mathrm{H}), 6.52$ (s, $1 \mathrm{Ar}-\mathrm{H}), 4.44$ (s, $\left.1 \mathrm{CH}_{3}\right),-1.18$ (s, $\left.2 \mathrm{CH}_{3}\right)$.

MB: $\delta=1.12 \mathrm{mms}^{-1} ; \Delta E_{\mathrm{Q}}=3.98 \mathrm{mms}^{-1}$

$\delta=0.15 \mathrm{mms}^{-1} ; \Delta E_{\mathrm{Q}}=2.26 \mathrm{mms}^{-1}$

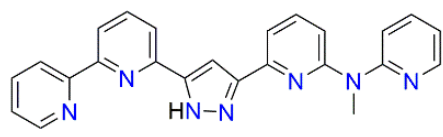

$\mathrm{HL}^{2}$

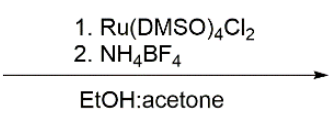

EtOH:acetone
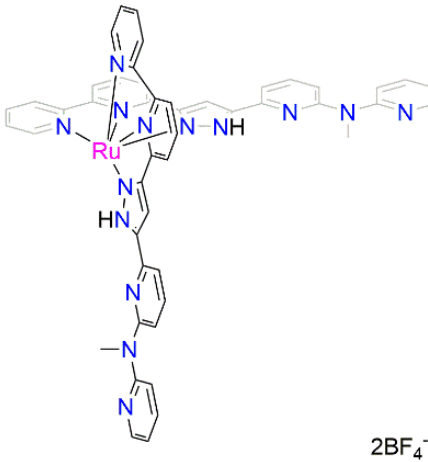

6-3

Preparation of [ $\left.\mathbf{L}^{22} \mathbf{R u}\right]\left[\mathrm{BF}_{4}\right]_{2} . \mathbf{H L}^{2}(0.080 \mathrm{~g}, 0.197 \mathrm{mmol}, 3$ eq. $)$ and $\mathrm{Ru}(\mathrm{DMSO})_{4} \mathrm{Cl}_{2}(0.030 \mathrm{~g}$, $0.061 \mathrm{mmol}, 1$ eq.) was dissolved in degassed $\mathrm{EtOH}$ :acetone $(1: 1,30 \mathrm{~mL})$. The reaction was heated at reflux overnight then $\mathrm{NH}_{4} \mathrm{BF}_{4}(0.020 \mathrm{~g}, 0.191 \mathrm{mmol}, 3.1 \mathrm{eq}$.) was added. The solution was stirred for an additional 3 hours then the solution filtered and precipitated with Et20 to give a brown powder, which was further purified by slow diffusion of Et20 into a MeCN solution of the product ( $0.012 \mathrm{~g}, 0.011 \mathrm{mmol}, 18 \%$ yield).

${ }^{1} \mathrm{H}$ NMR $\left(400 \mathrm{MHz}, \mathrm{CD}_{3} \mathrm{CN}\right): \delta(\mathrm{ppm})=8.55(\mathrm{~d}, J=8.1 \mathrm{~Hz}, 1 \mathrm{Ar}-\mathrm{H}), 8.49(\mathrm{~d}, J=7.9 \mathrm{~Hz}, 1 \mathrm{Ar}-\mathrm{H})$, $8.31-8.45(\mathrm{~m}, 2 \mathrm{Ar}-\mathrm{H}), 8.08(\mathrm{~d}, J=5.1 \mathrm{~Hz}, 1 \mathrm{Ar}-\mathrm{H}), 7.93(\mathrm{t}, J=7.8 \mathrm{~Hz}, 1 \mathrm{Ar}-\mathrm{H}), 7.69(\mathrm{~m}, 3 \mathrm{Ar}-\mathrm{H})$, $7.25(\mathrm{~d}, J=7.6 \mathrm{~Hz}, 1 \mathrm{Ar}-\mathrm{H}), 7.09-7.18(\mathrm{~m}, 3 \mathrm{Ar}-\mathrm{H}), 7.04\left(\mathrm{t}, J=6.0 \mathrm{~Hz}, 1 \mathrm{CH}_{3}\right), 3.47\left(\mathrm{~s}, 1 \mathrm{NCH}_{3}\right)$ 


\section{Appendix}

A1 - NMR Spectroscopy for the Ligands and Ligand Precursors
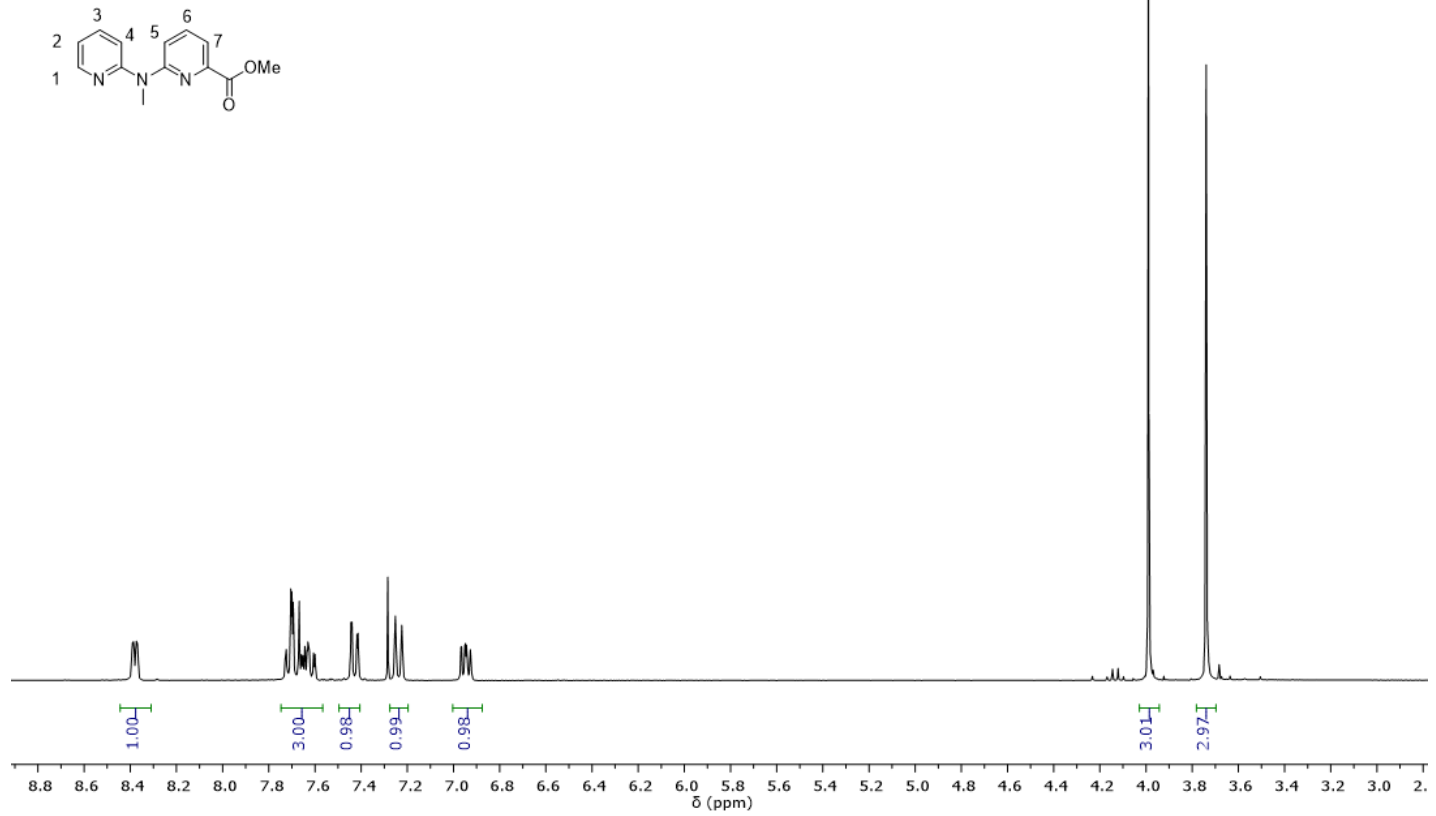

Figure A1. ${ }^{1} \mathrm{H}$ NMR spectrum of 5-3 in $\mathrm{CDCl}_{3}$ at room temperature.
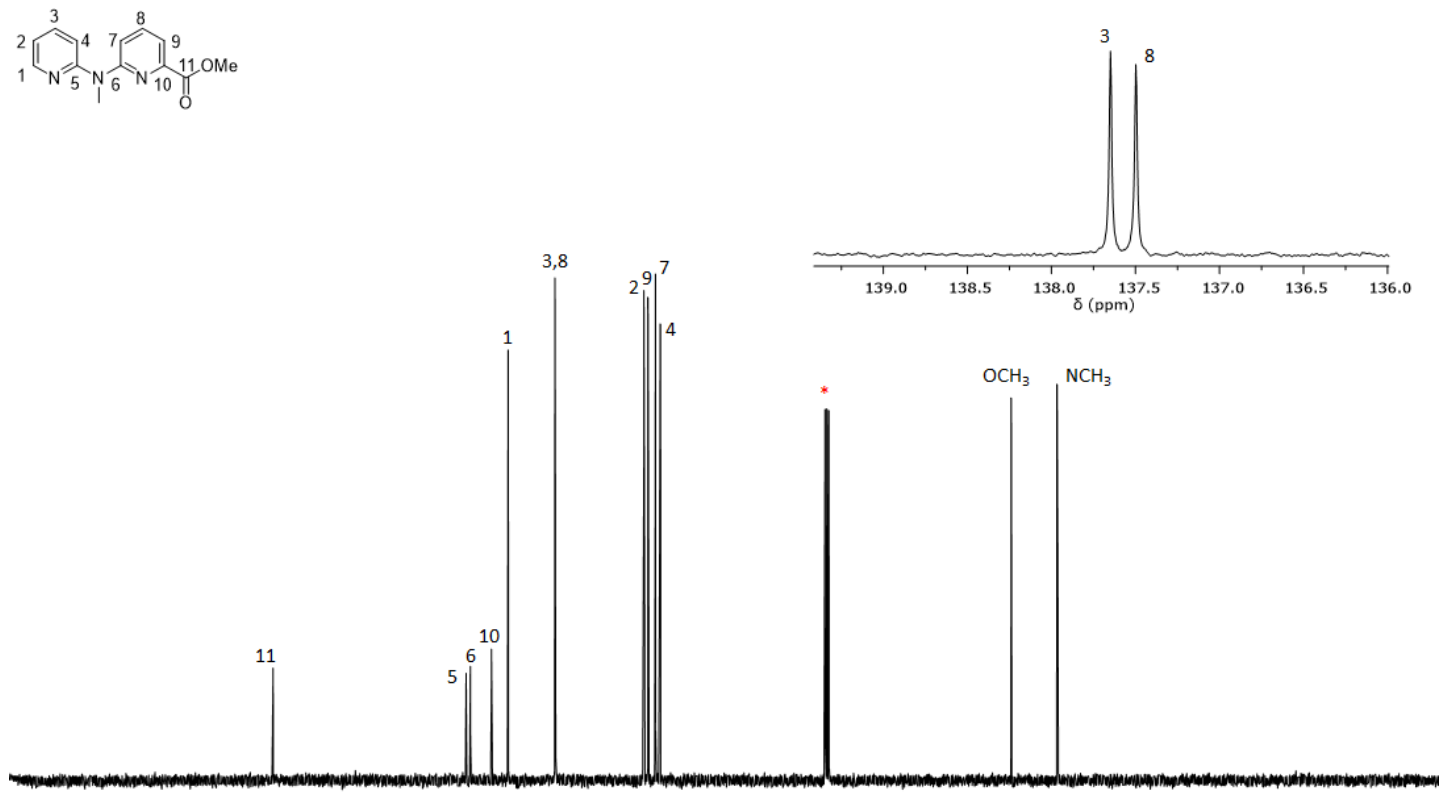

$\begin{array}{llllllllllllllllllllllllllllllllllll}1250 & 240 & 230 & 220 & 210 & 200 & 190 & 180 & 170 & 160 & 150 & 140 & 130 & 120 & 110 & 100 & 90 & 80 & 70 & 60 & 50 & 40 & 30 & 20 & 10 & 0 & -10 & -20 & -30 & -40 & -50\end{array}$

Figure A2. ${ }^{13} \mathrm{C}$ NMR spectrum of 5-3 in $\mathrm{CDCl}_{3}$ at room temperature. 


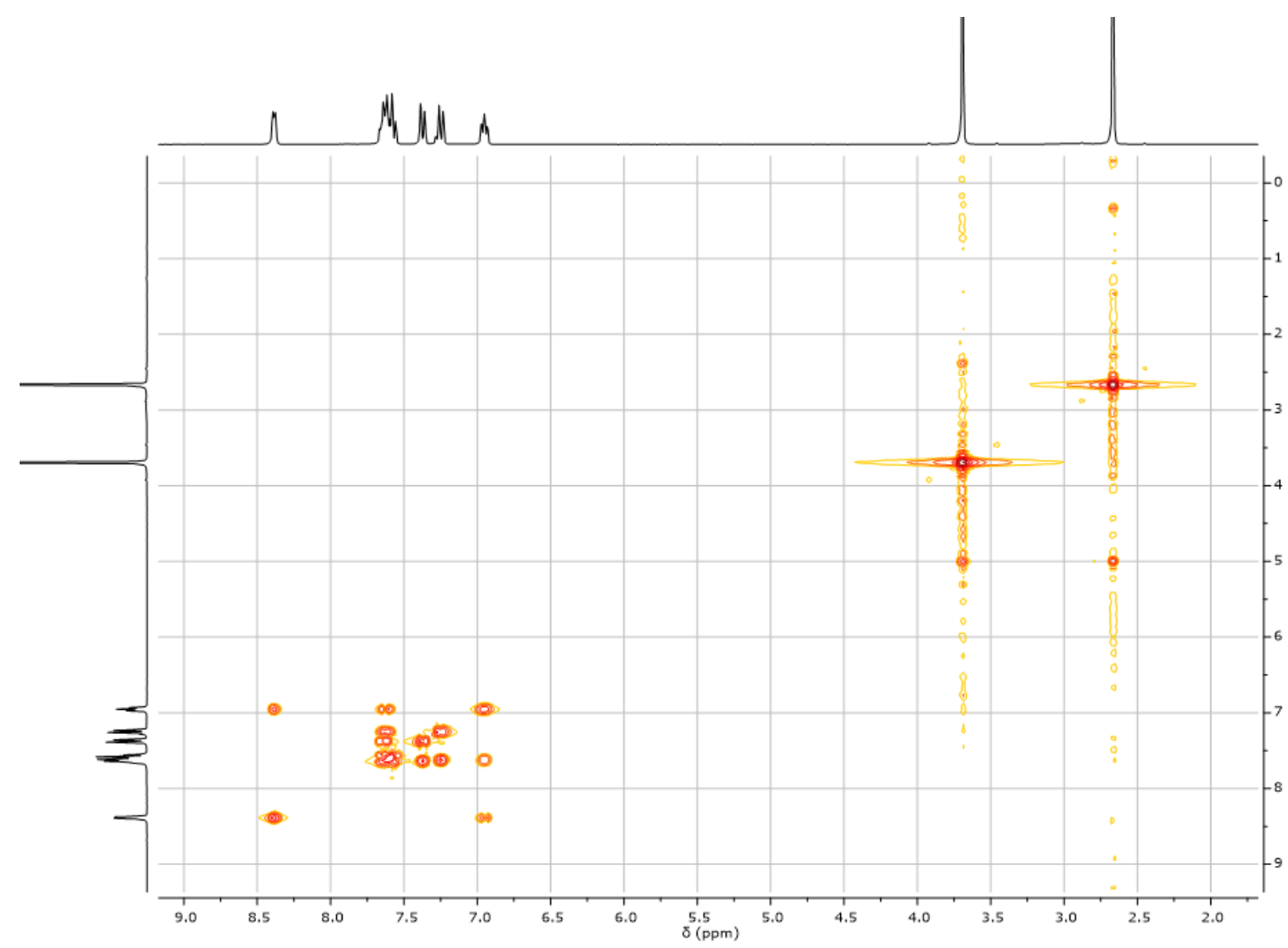

Figure A3. ${ }^{1} \mathrm{H}-{ }^{-1} \mathrm{H}$ COSY spectrum of $\mathbf{5 - 3}$ in $\mathrm{CDCl}_{3}$ at room temperature.

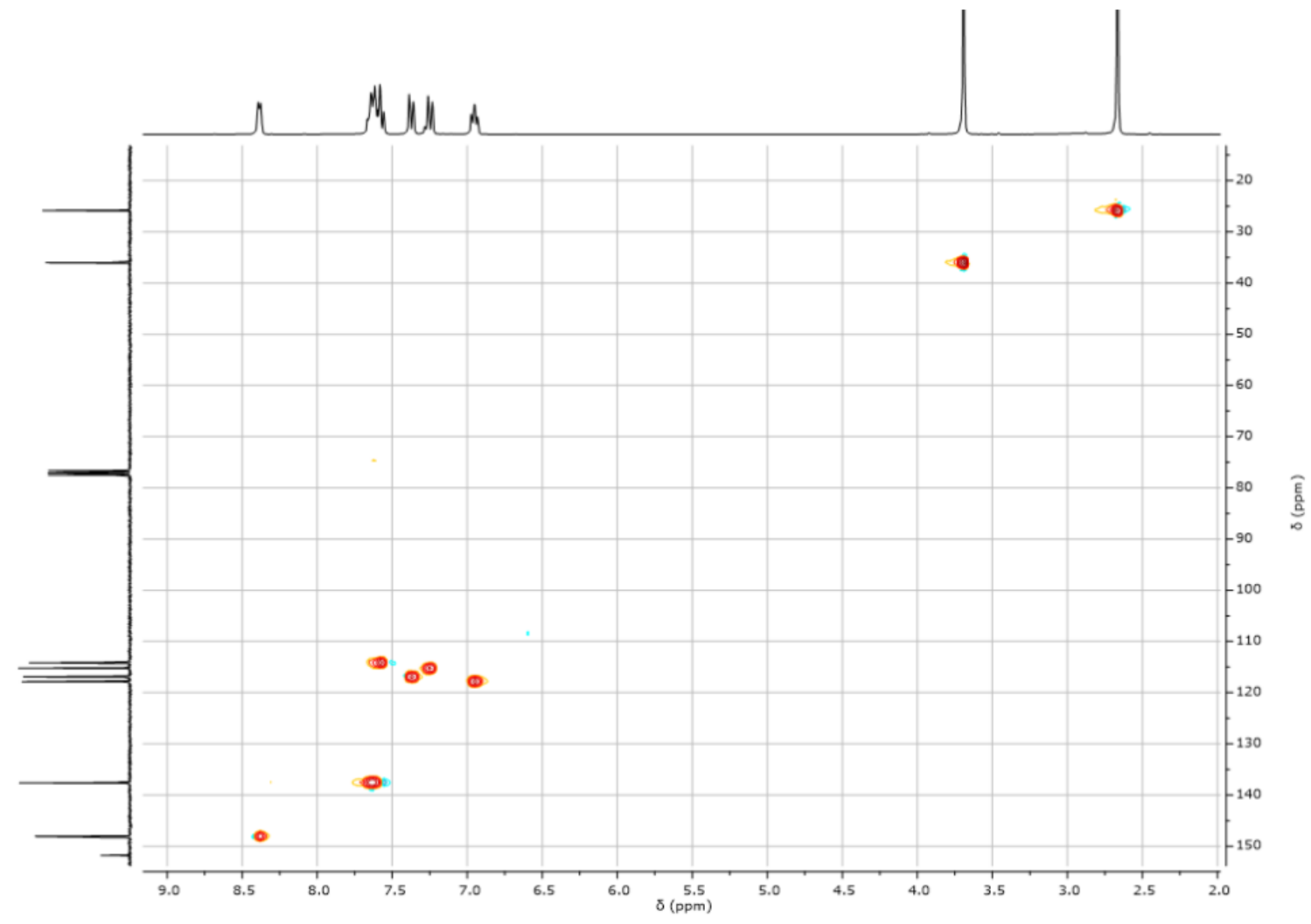

Figure A4. ${ }^{1} \mathrm{H}-13 \mathrm{C} \mathrm{HSQC}$ spectrum of $5-3$ in $\mathrm{CDCl}_{3}$ at room temperature. 


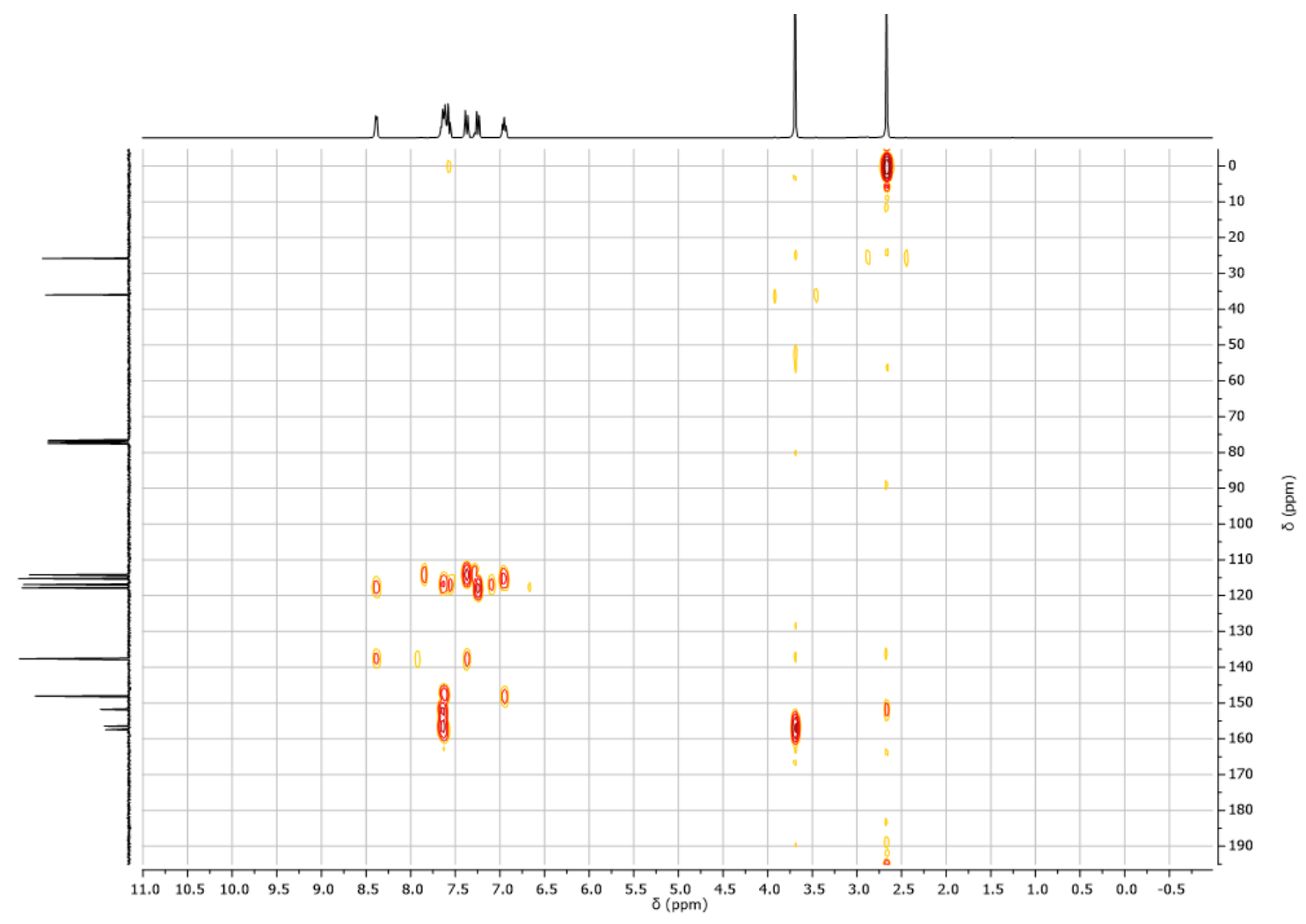

Figure A5. ${ }^{1} \mathrm{H}-{ }^{13} \mathrm{C}$ HMBC spectrum of 5-3 in $\mathrm{CDCl}_{3}$ at room temperature.

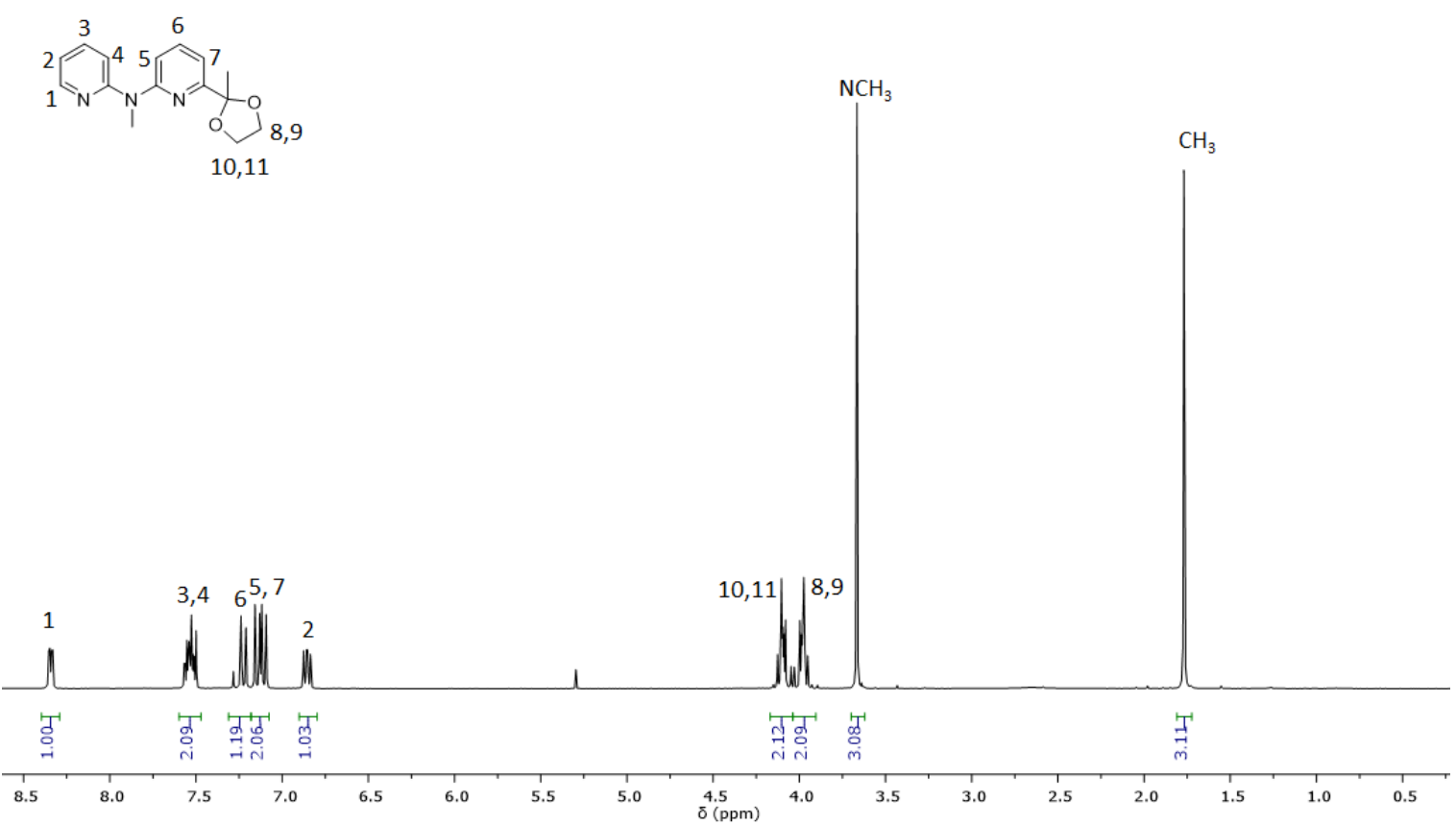

Figure A6. ${ }^{1} \mathrm{H}$ NMR spectrum of 5-7 in $\mathrm{CDCl}_{3}$ at room temperature. 

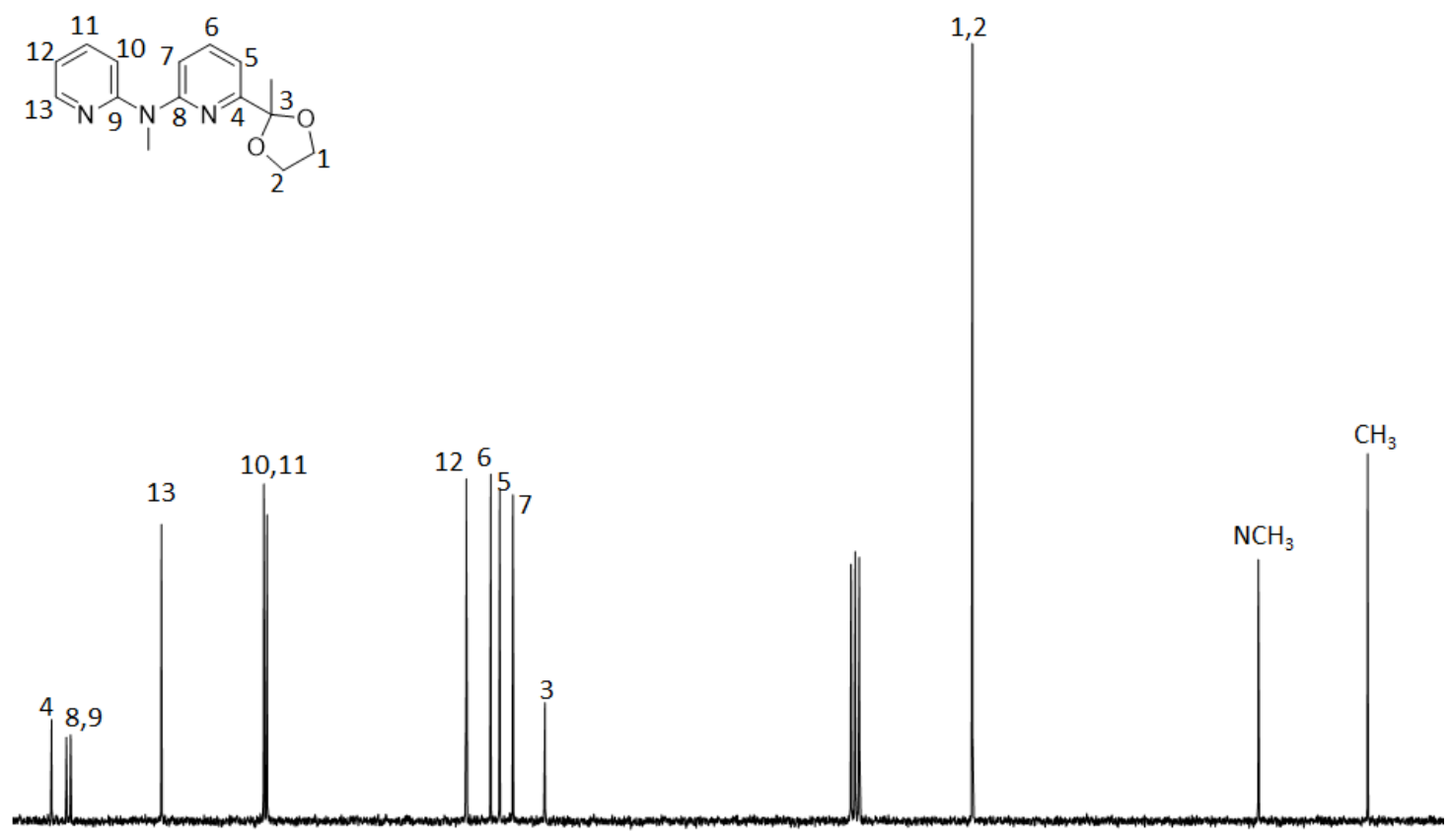

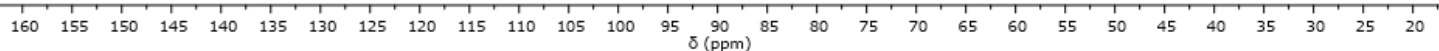

Figure A7. ${ }^{13} \mathrm{C}$ NMR spectrum of 5-7 in $\mathrm{CD}_{3} \mathrm{Cl}$ at room temperature.

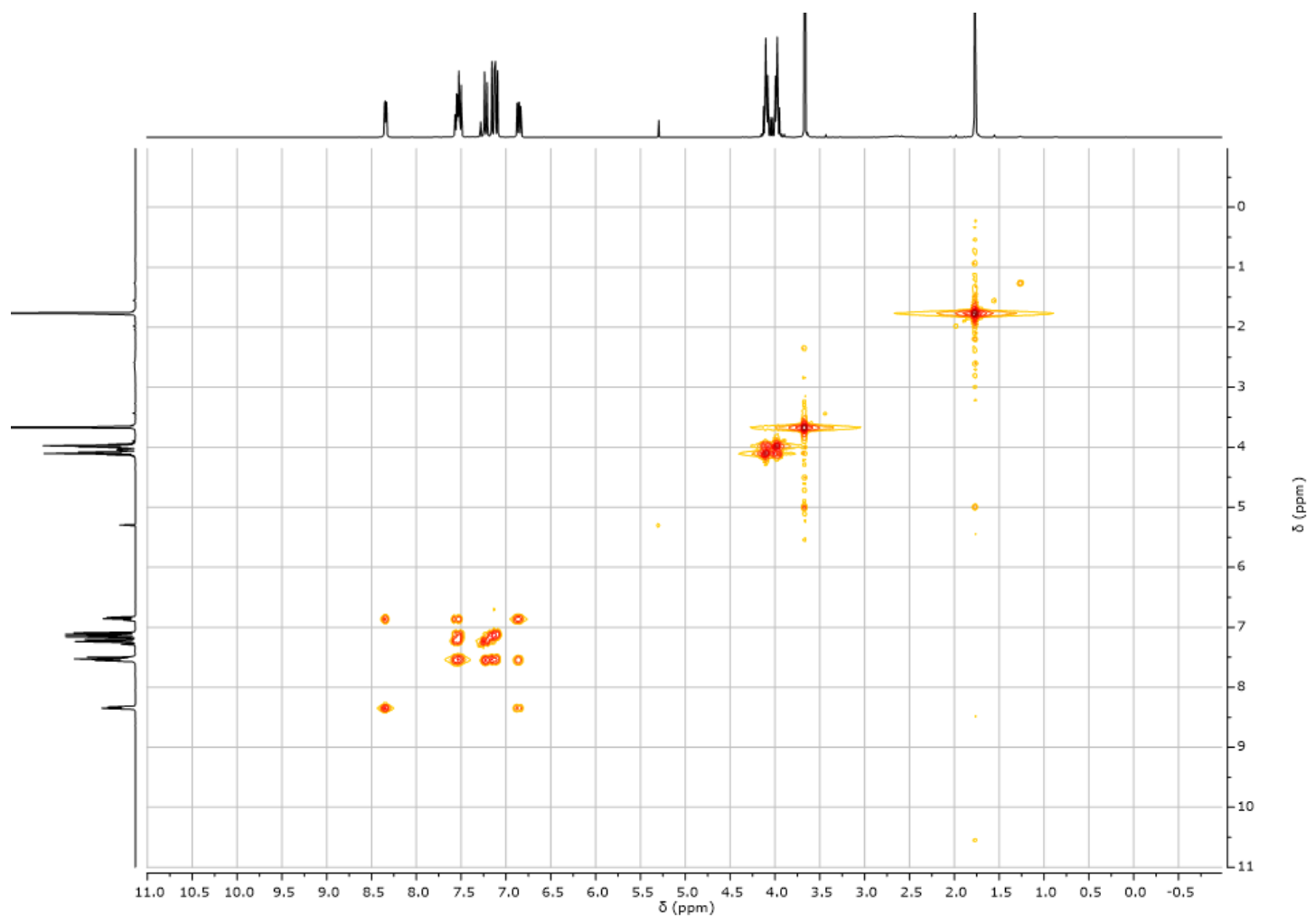

Figure A8. ${ }^{1} \mathrm{H}-1{ }^{-1} \mathrm{COSY}$ spectrum of $\mathbf{5 - 7}$ in $\mathrm{CD}_{3} \mathrm{Cl}$ at room temperature. 


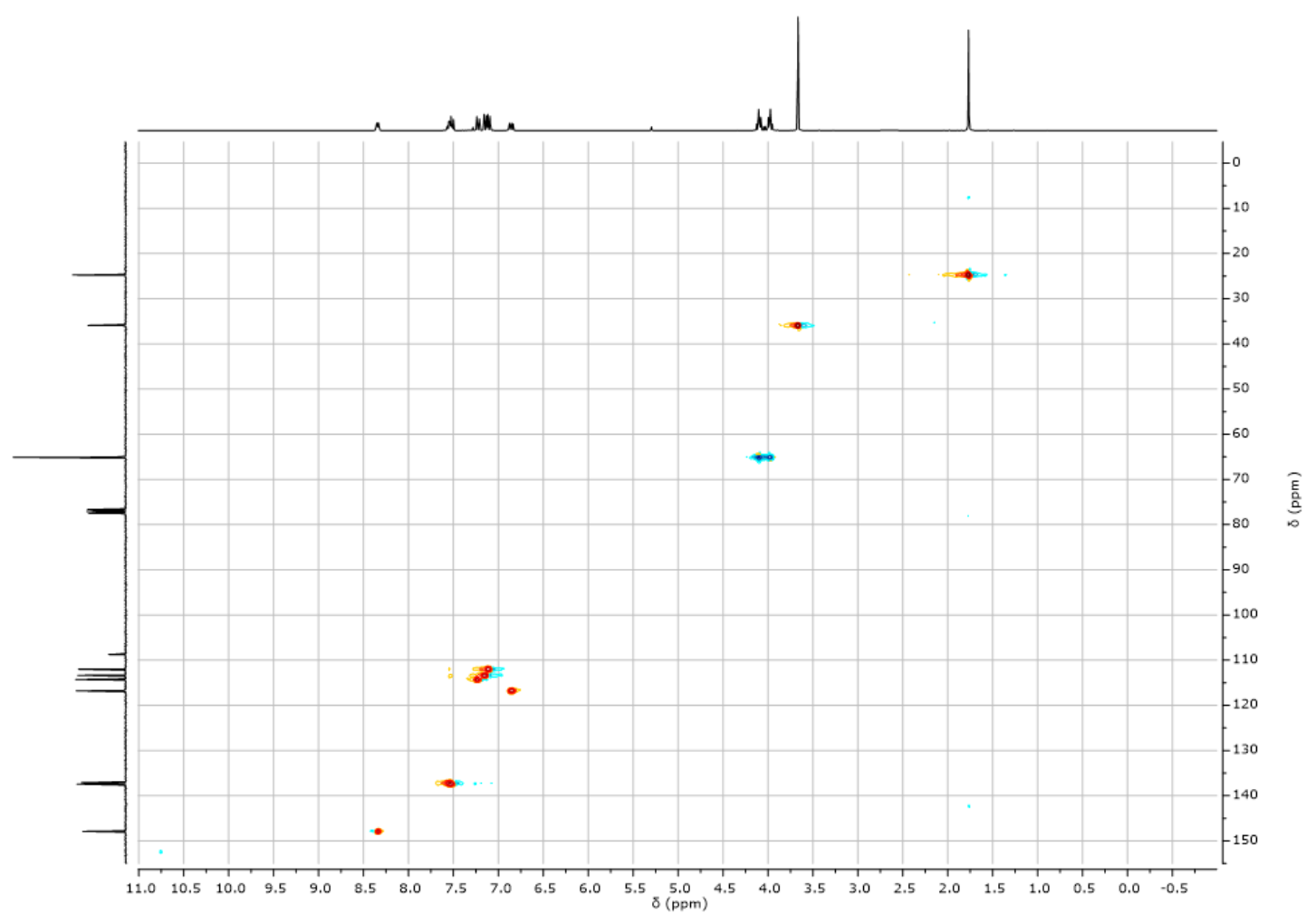

Figure A9. $^{1} \mathrm{H}^{-13} \mathrm{C}$ HSQC spectrum of 5-7 in $\mathrm{CD}_{3} \mathrm{Cl}$ at room temperature.

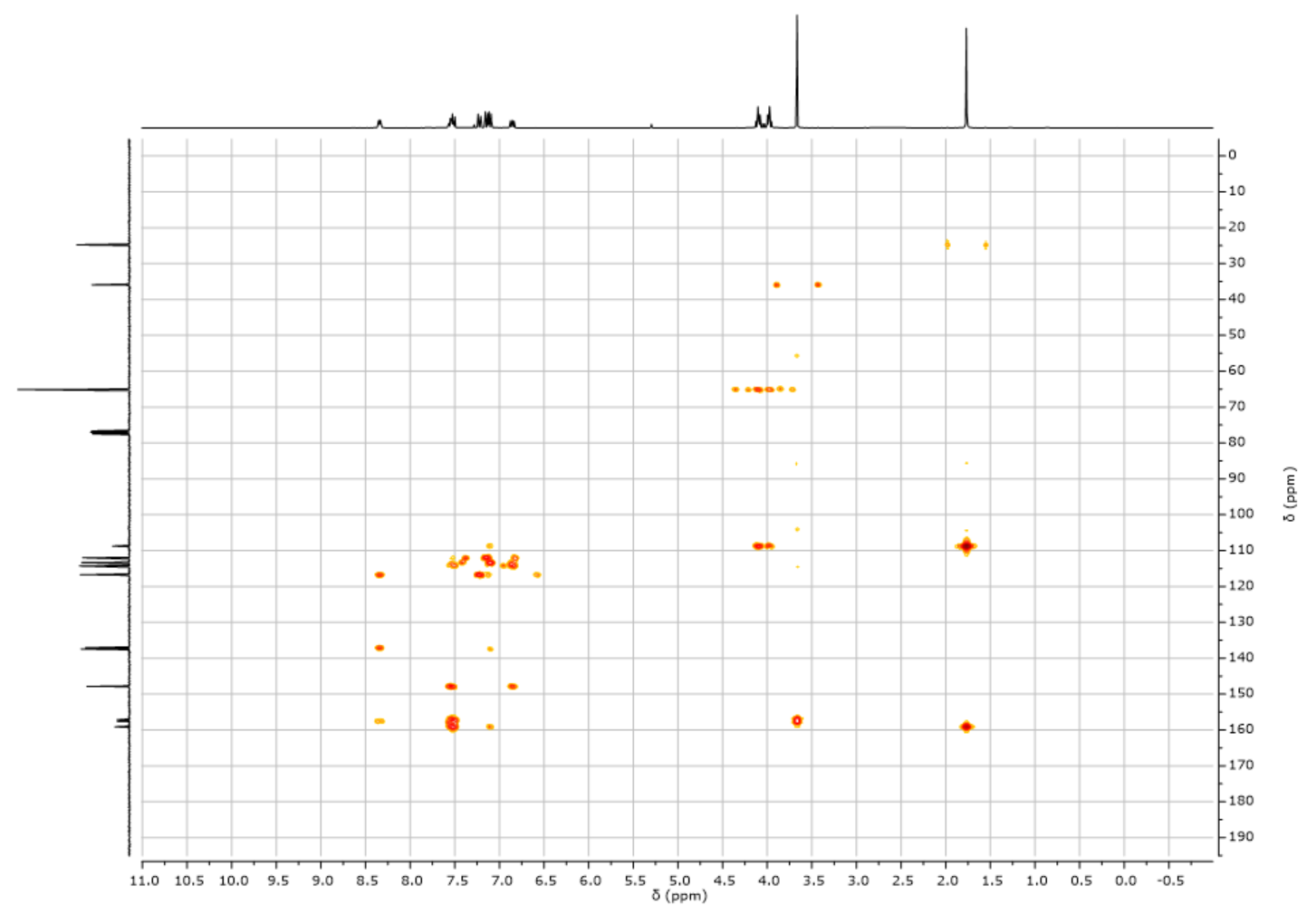

Figure A10. ${ }^{1} \mathrm{H}-{ }^{13} \mathrm{C}$ HMBC spectrum of 5-7 in $\mathrm{CD}_{3} \mathrm{Cl}$ at room temperature. 


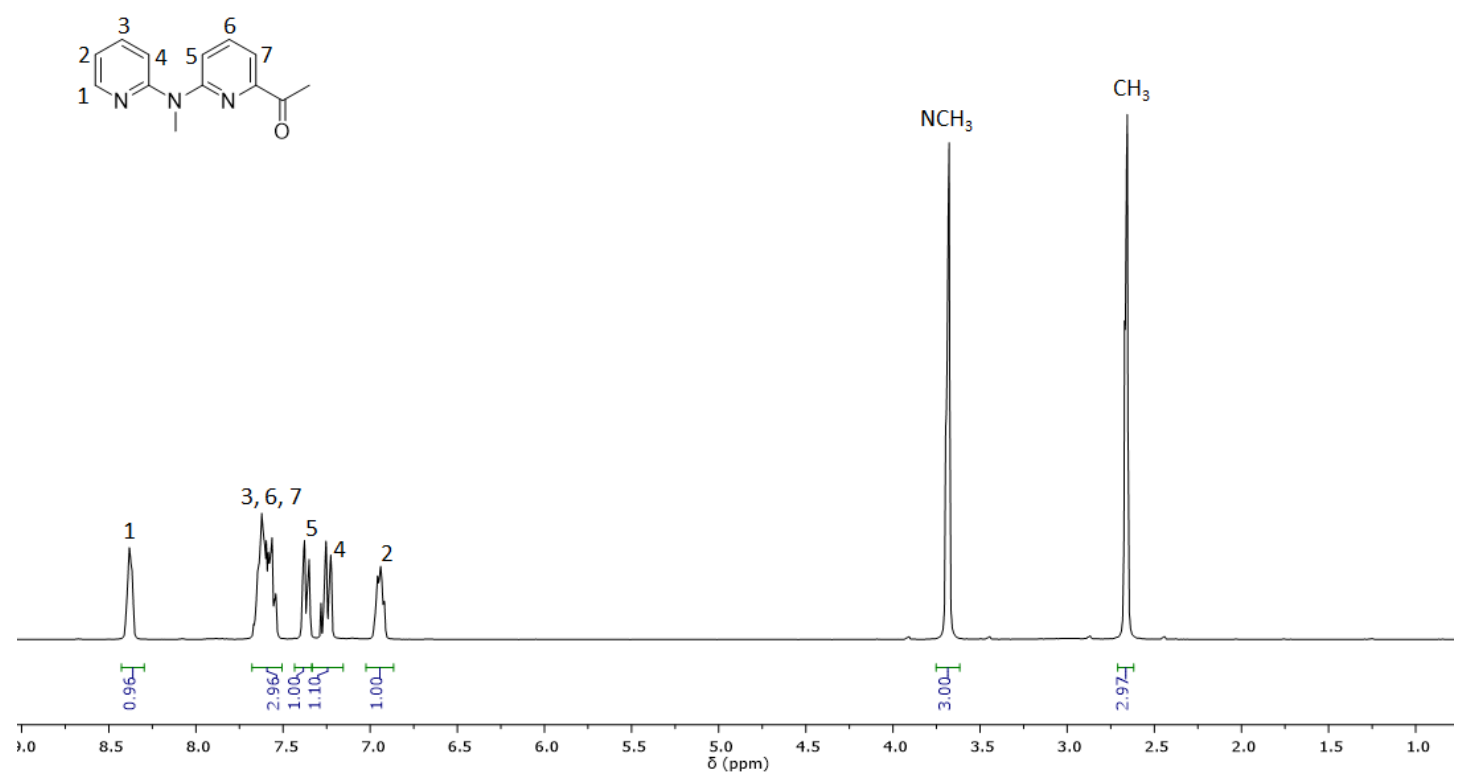

Figure A11. ${ }^{1} \mathrm{H}$ NMR spectrum of 5-8 in $\mathrm{CDCl}_{3}$ at room temperature.

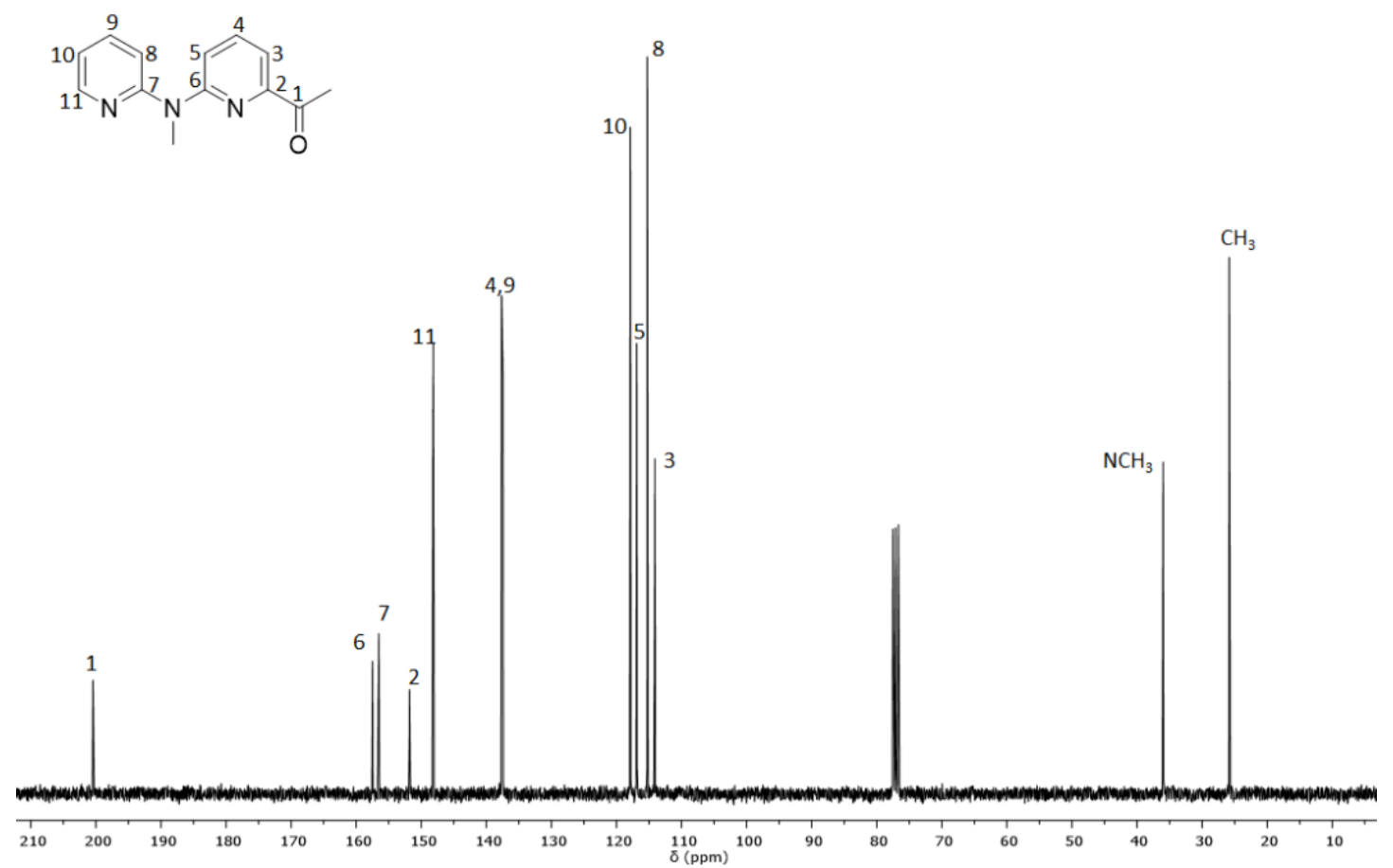

Figure $\mathbf{A 1 2} .{ }^{13} \mathrm{C}$ NMR spectrum of $\mathbf{5 - 8}$ in $\mathrm{CDCl}_{3}$ at room temperature. 


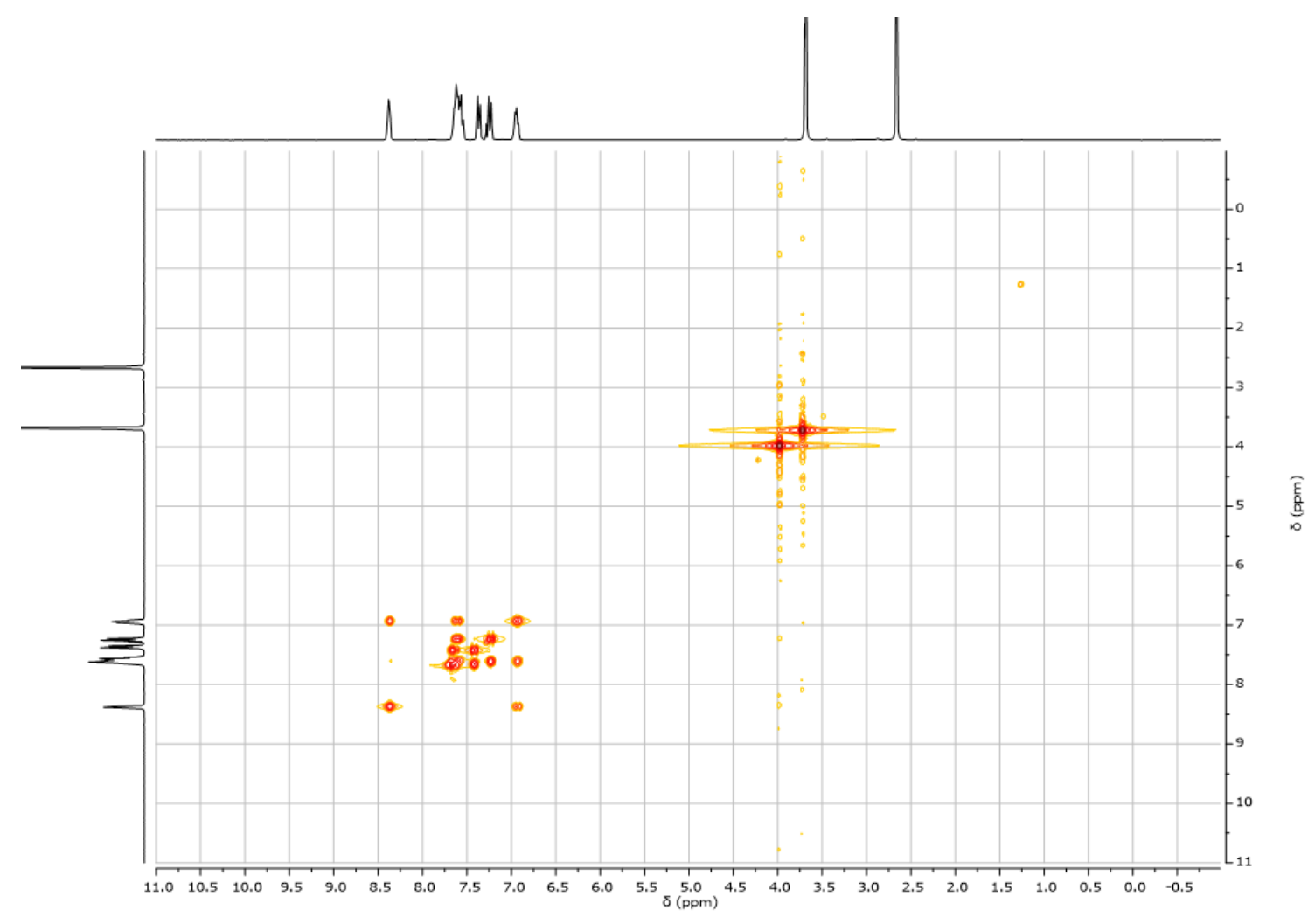

Figure A13. ${ }^{1} \mathrm{H}-1 \mathrm{H}$ COSY spectrum of 5-8 in $\mathrm{CDCl}_{3}$ at room temperature.

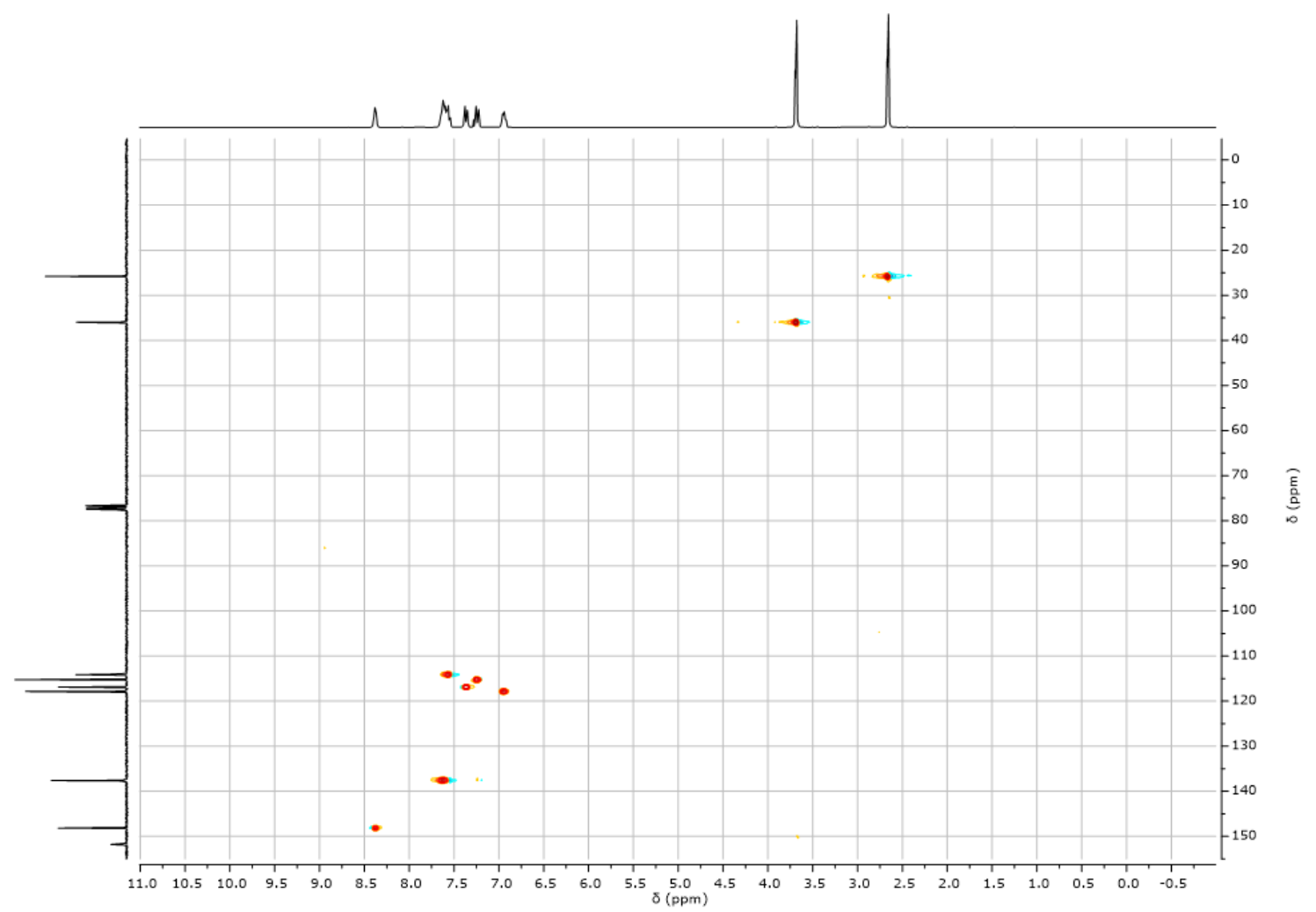

Figure A14. ${ }^{1} \mathrm{H}^{-13} \mathrm{C}$ HSQC spectrum of 5-8 in $\mathrm{CDCl}_{3}$ at room temperature. 


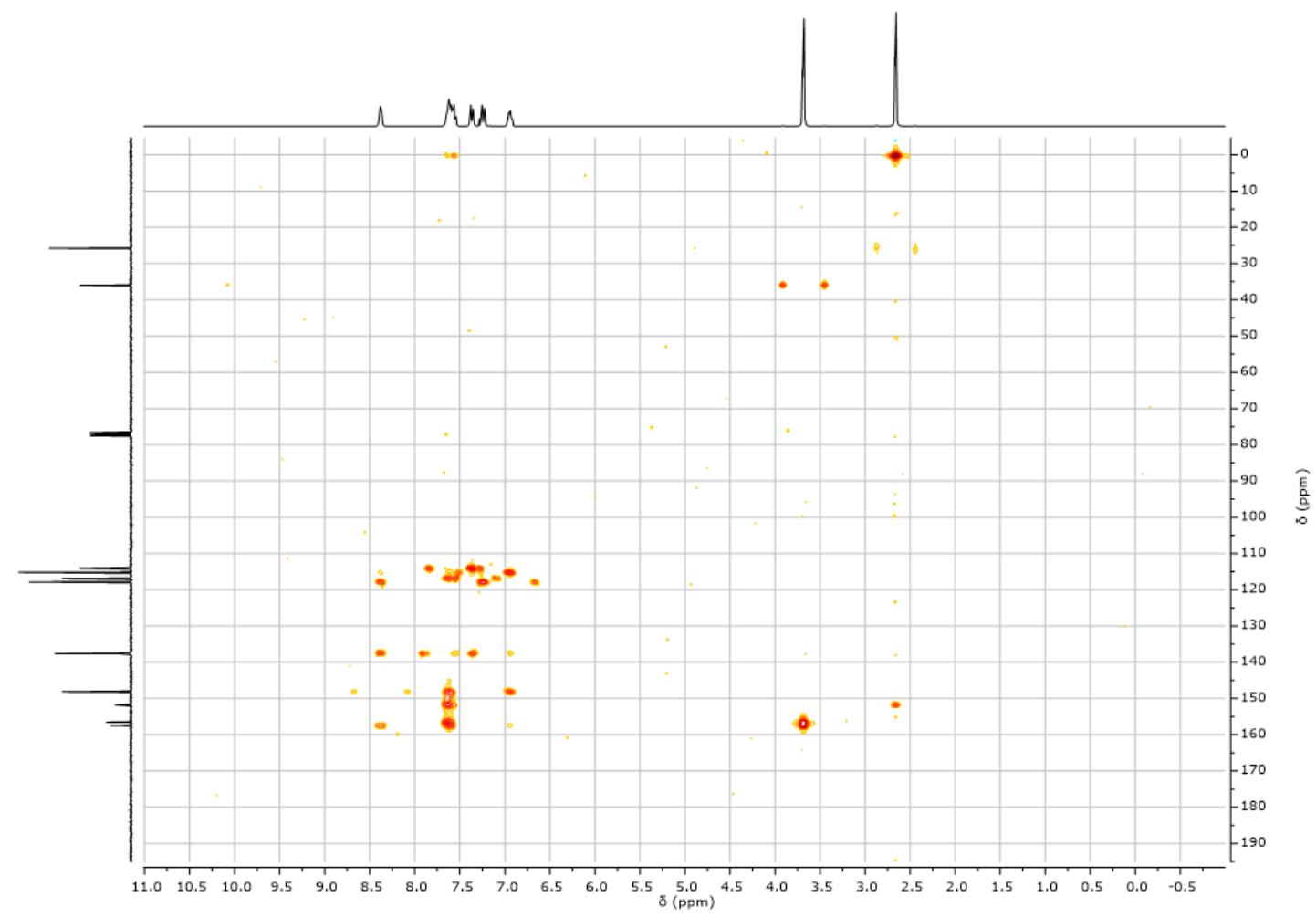

Figure A15. ${ }^{1} \mathrm{H}-13 \mathrm{C}$ HMBC spectrum of 5-8 in $\mathrm{CDCl}_{3}$ at room temperature.

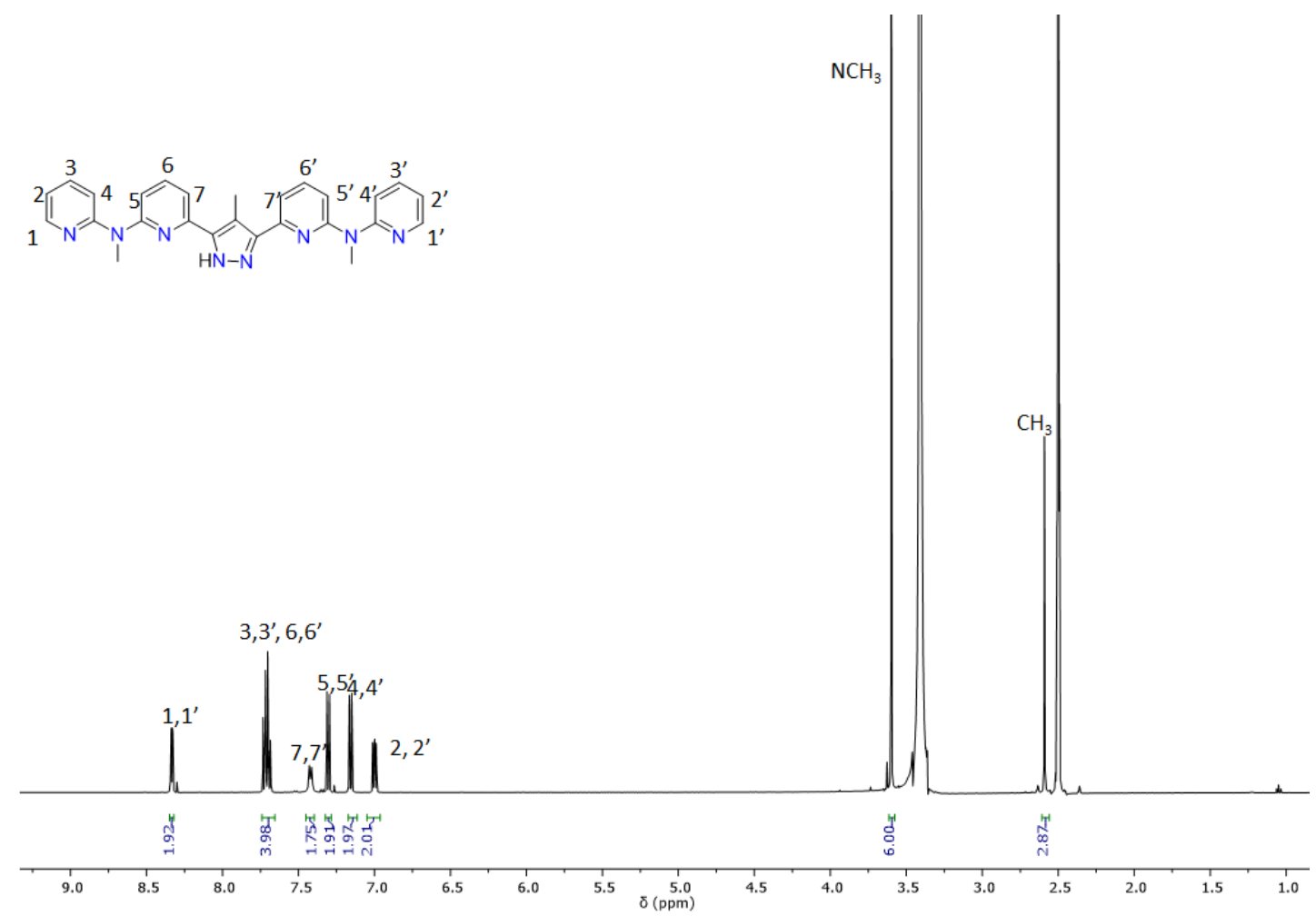

Figure A16. ${ }^{1} \mathrm{H}$ NMR spectrum of $\mathbf{H L}^{1}$ in DMSO- $\mathrm{d}_{6}$ at room temperature. 

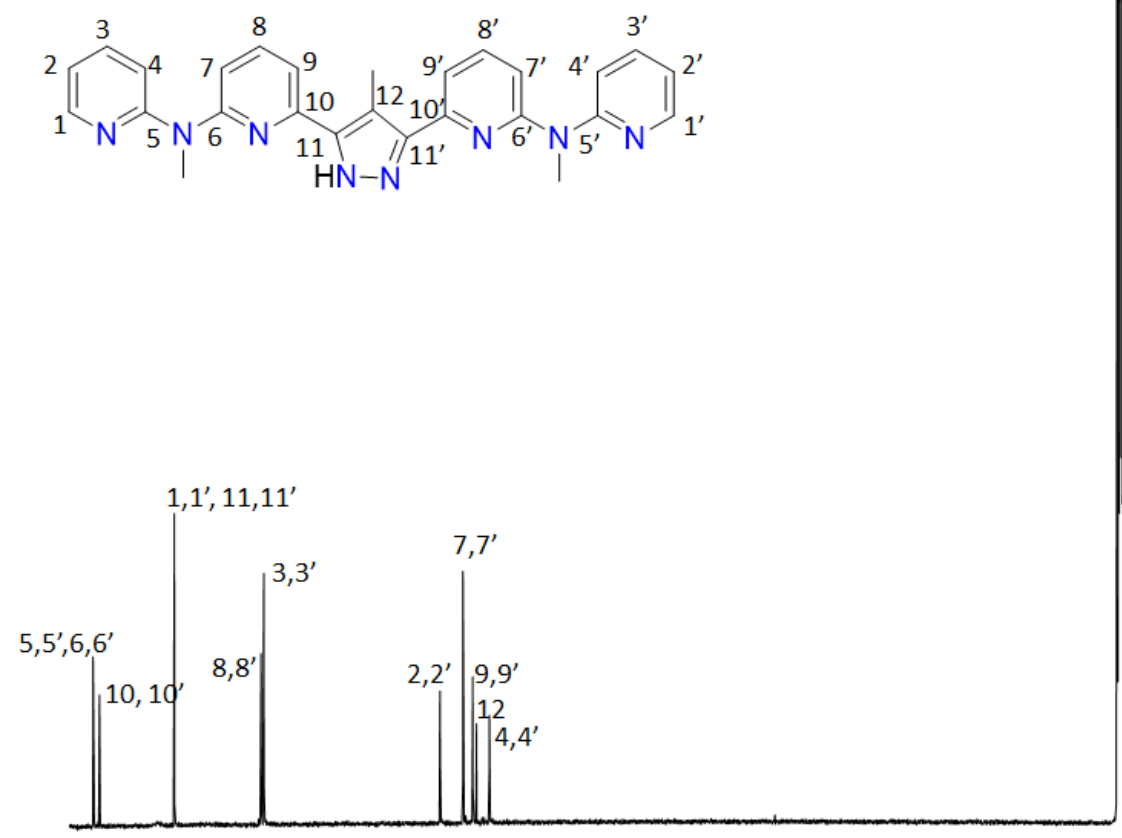

$\mathrm{NCH}_{3}$

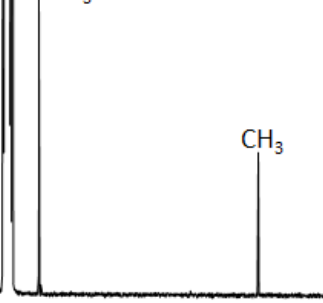

$150 \quad 140 \quad 130 \quad 120$

Figure A17. ${ }^{13} \mathrm{C}$ NMR spectrum of $\mathbf{H L}^{\mathbf{1}}$ in DMSO-d ${ }_{6}$ at room temperature.

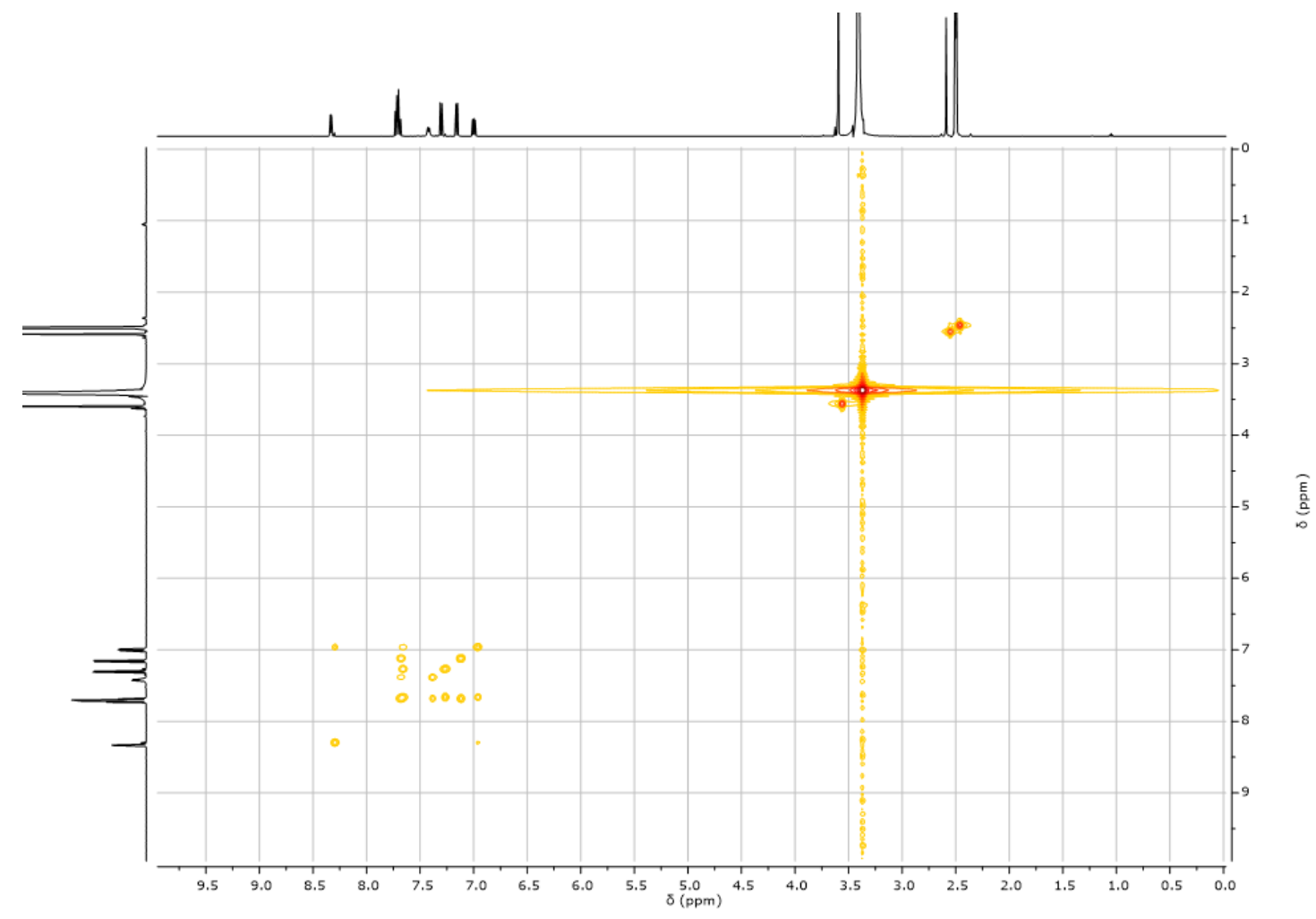

Figure A18. ${ }^{1} \mathrm{H}^{-1} \mathrm{H}$ COSY spectrum of $\mathbf{H L}^{1}$ in DMSO- $\mathrm{d}_{6}$ at room temperature. 


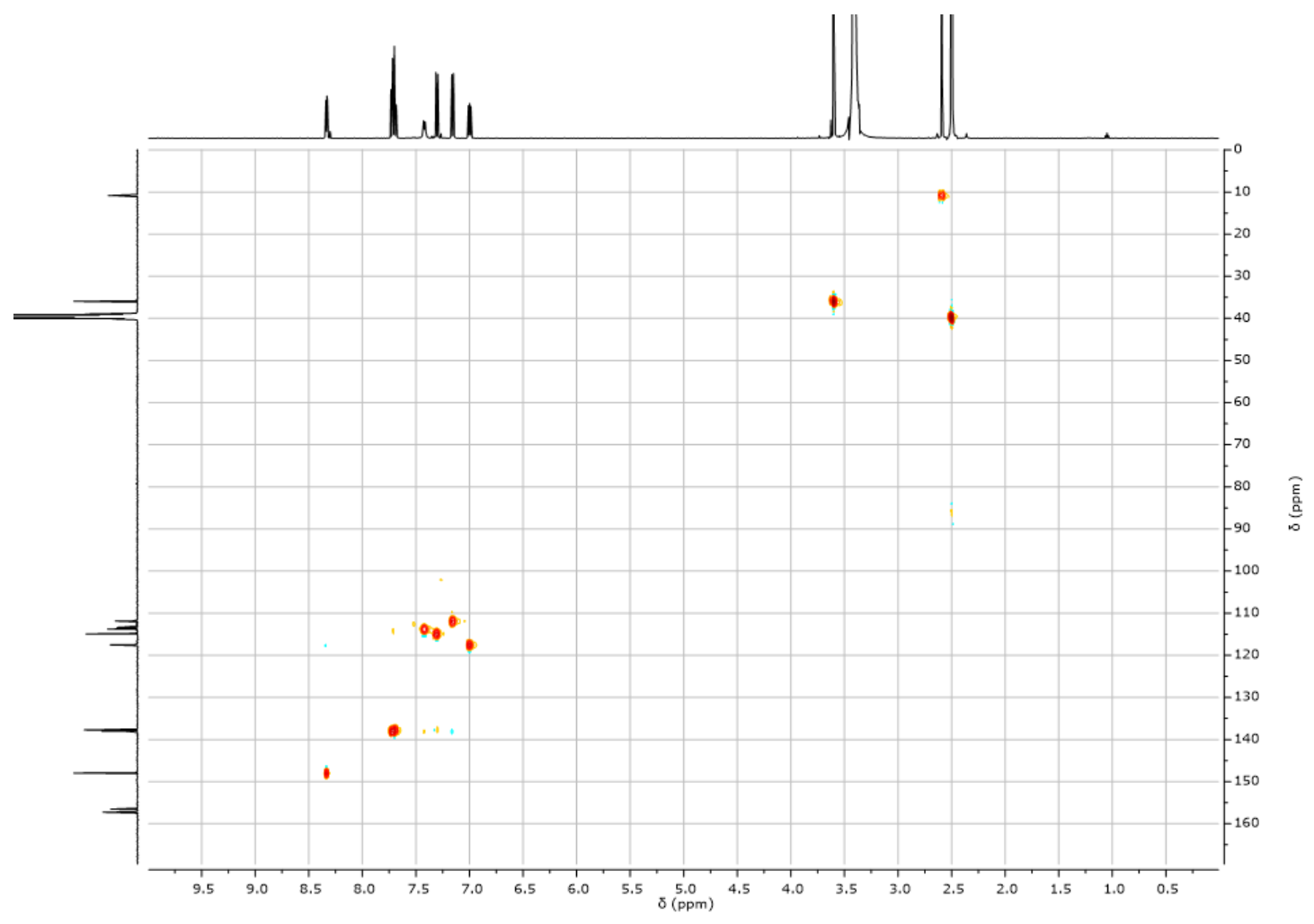

Figure A19. ${ }^{1} \mathrm{H}^{-13} \mathrm{C}$ HSQC spectrum of $\mathbf{H L}^{1}$ in DMSO- $\mathrm{d}_{6}$ at room temperature.

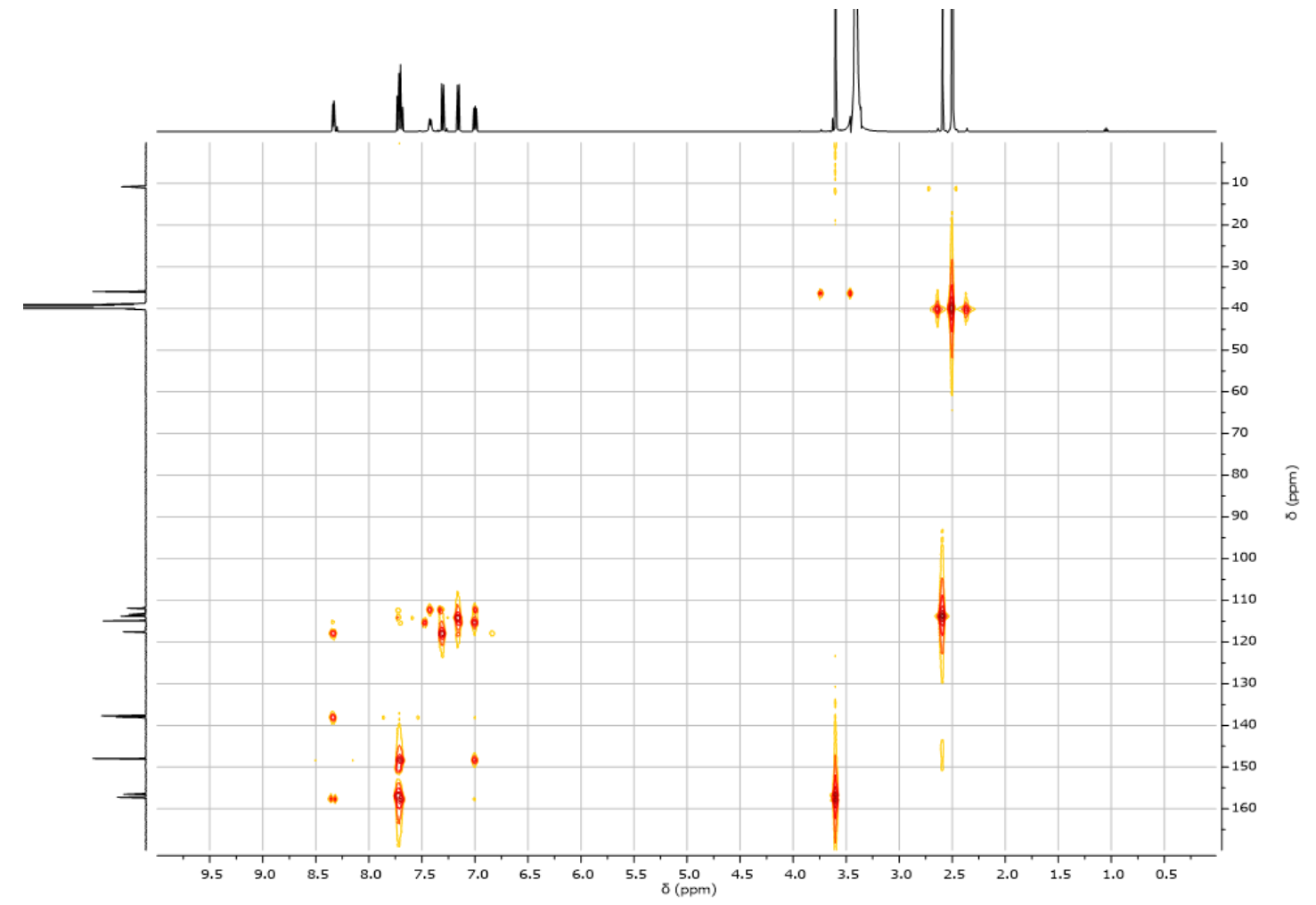

Figure A20. ${ }^{1} \mathrm{H}^{-13} \mathrm{C} \mathrm{HMBC}$ spectrum of $\mathbf{H L}^{1}$ in DMSO- $\mathrm{d}_{6}$ at room temperature. 


\section{Appendix for Complexes}

\section{A2 - Mass spectrometry}

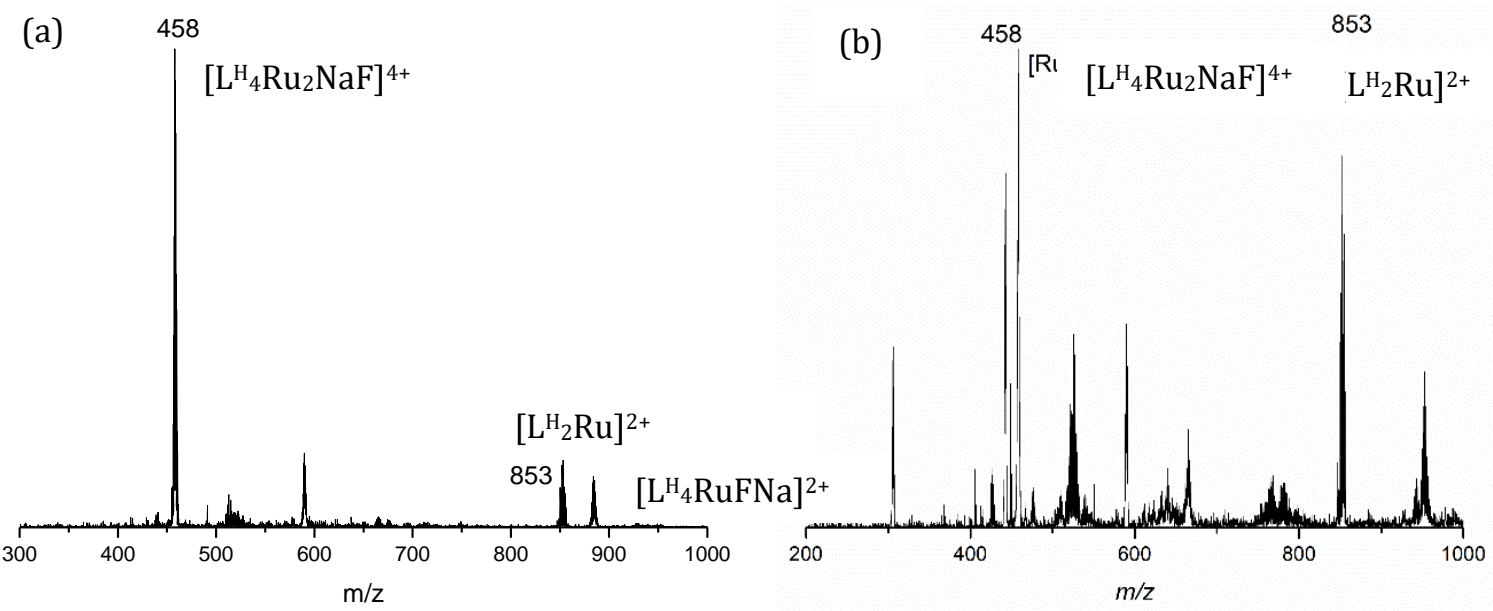

Figure A21. ESI-MS of the $\mathrm{Ru}^{\mathrm{II}}$ "corner complex" $\left[\mathrm{LH}_{4} \mathrm{Ru}_{2}\right]\left[\mathrm{BF}_{4}\right]_{4}$ in (a) concentrated and (b) dilute MeCN.

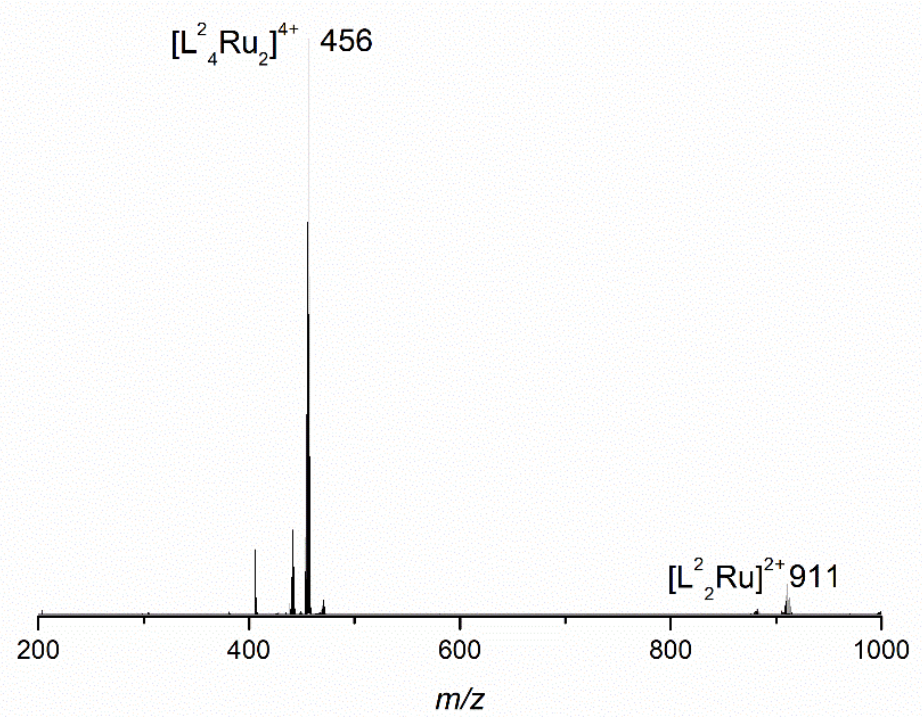

Figure A22. ESI-MS of $\left[\mathrm{L}^{2}{ }_{4} \mathrm{Ru}_{2}\right]\left[\mathrm{BF}_{4}\right]_{4}$ in $\mathrm{MeCN}$ at higher concentrations $\left(>10^{-5} \mathrm{M}\right)$ revealing one dominant peak at $\mathrm{m} / \mathrm{z}=455$ corresponding to $\left[\mathrm{L}_{4}{ }_{4} \mathrm{Ru}_{2}\right]^{4+}$. 

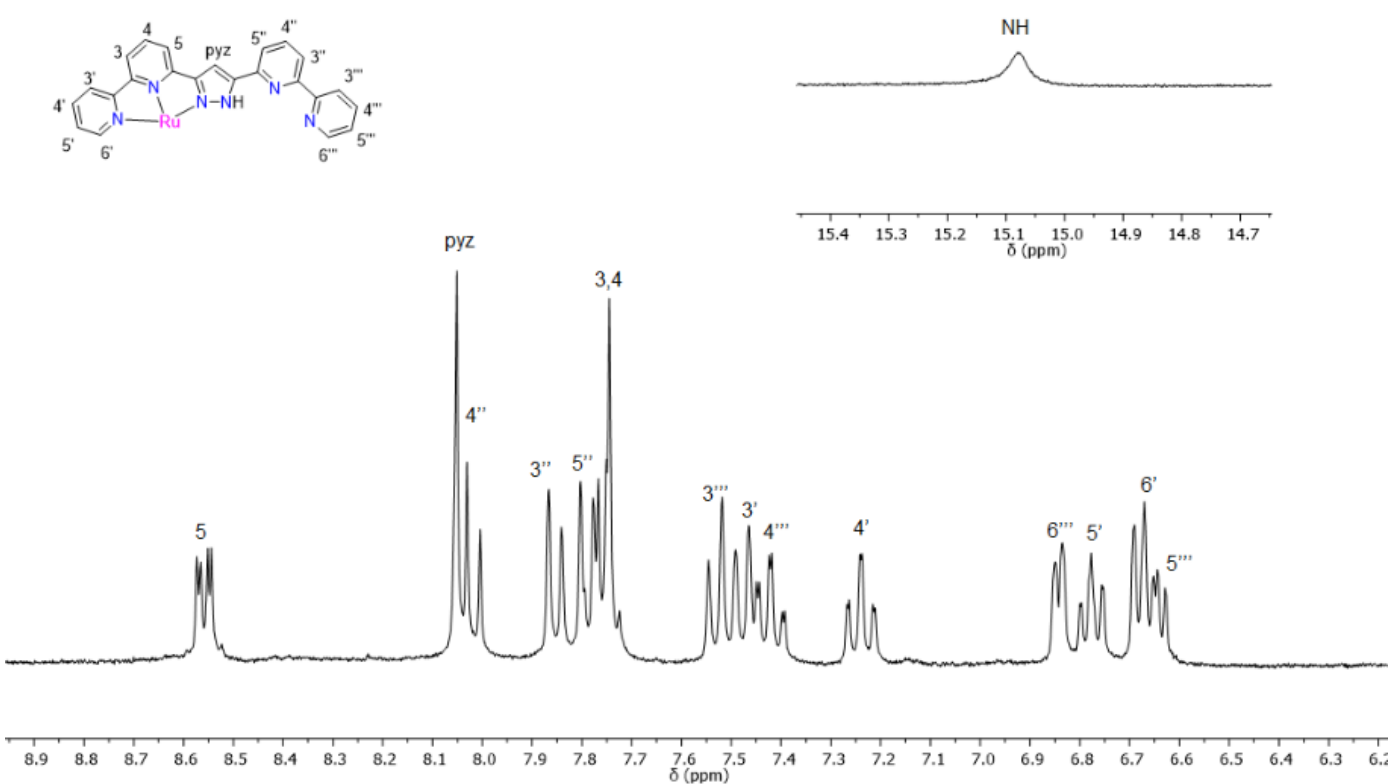

Figure A23. ${ }^{1} \mathrm{H}$ NMR spectrum of $\left[\mathrm{L}_{2} \mathrm{H}_{2} \mathrm{Ru}\right]\left[\mathrm{BF}_{4}\right]_{2}$ in $\mathrm{MeCN}-\mathrm{d}_{3}$.

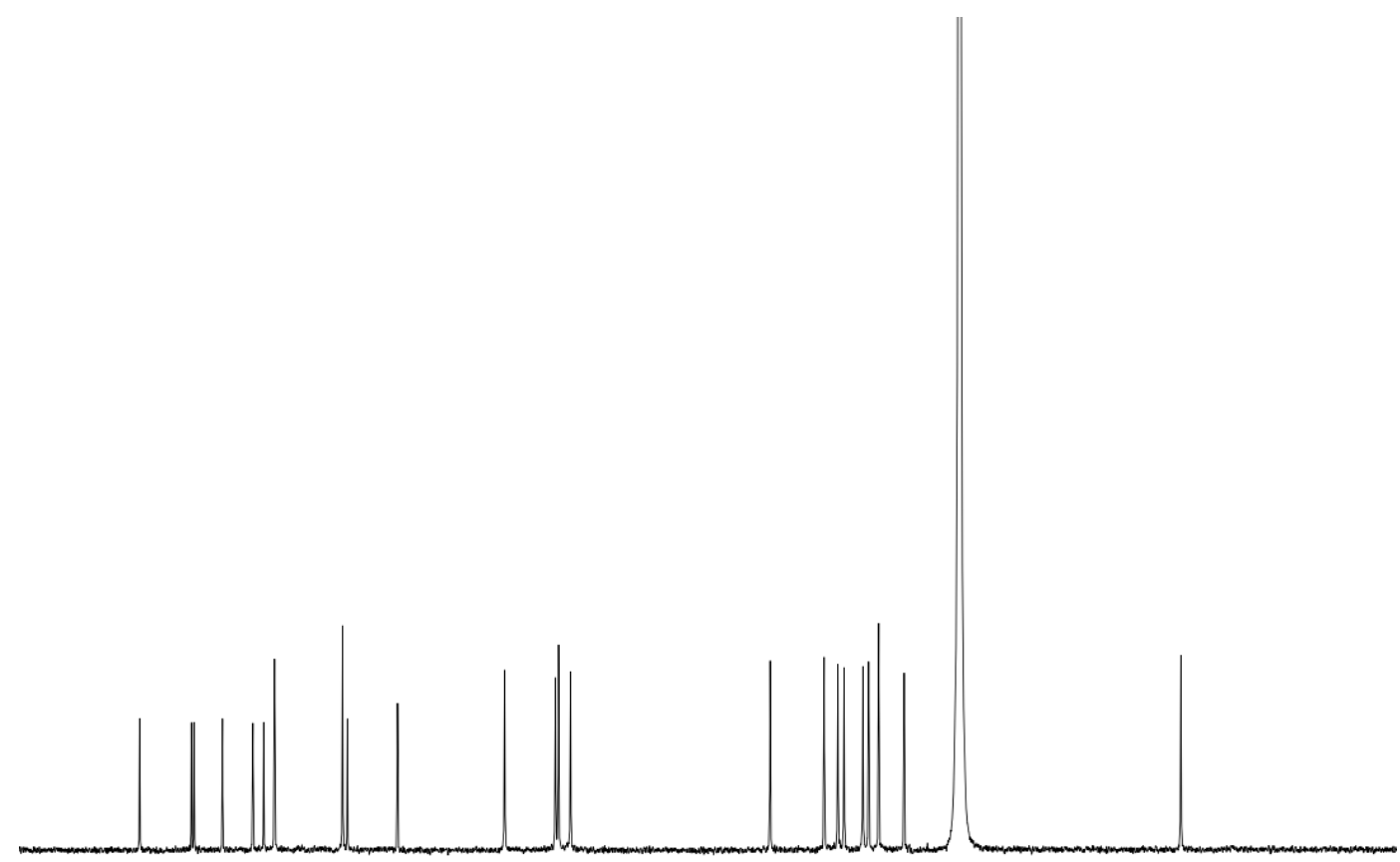

$\begin{array}{lllllllllllllllllllllllllllllllllllll}164 & 162 & 160 & 158 & 156 & 154 & 152 & 150 & 148 & 146 & 144 & 142 & 140 & 138 & 136 & 134 & 132 & 130 & 128 & 126 & 124 & 122 & 120 & 118 & 116 & 114 & 112 & 110 & 108 & 106 & 104 & 102 & 100 & 98\end{array}$

Figure A24. ${ }^{13} \mathrm{C}$ NMR spectrum of $\left[\mathrm{LH}_{2} \mathrm{Ru}\right]\left[\mathrm{BF}_{4}\right]_{2}$ in $\mathrm{MeCN}-\mathrm{d}_{3}$. 


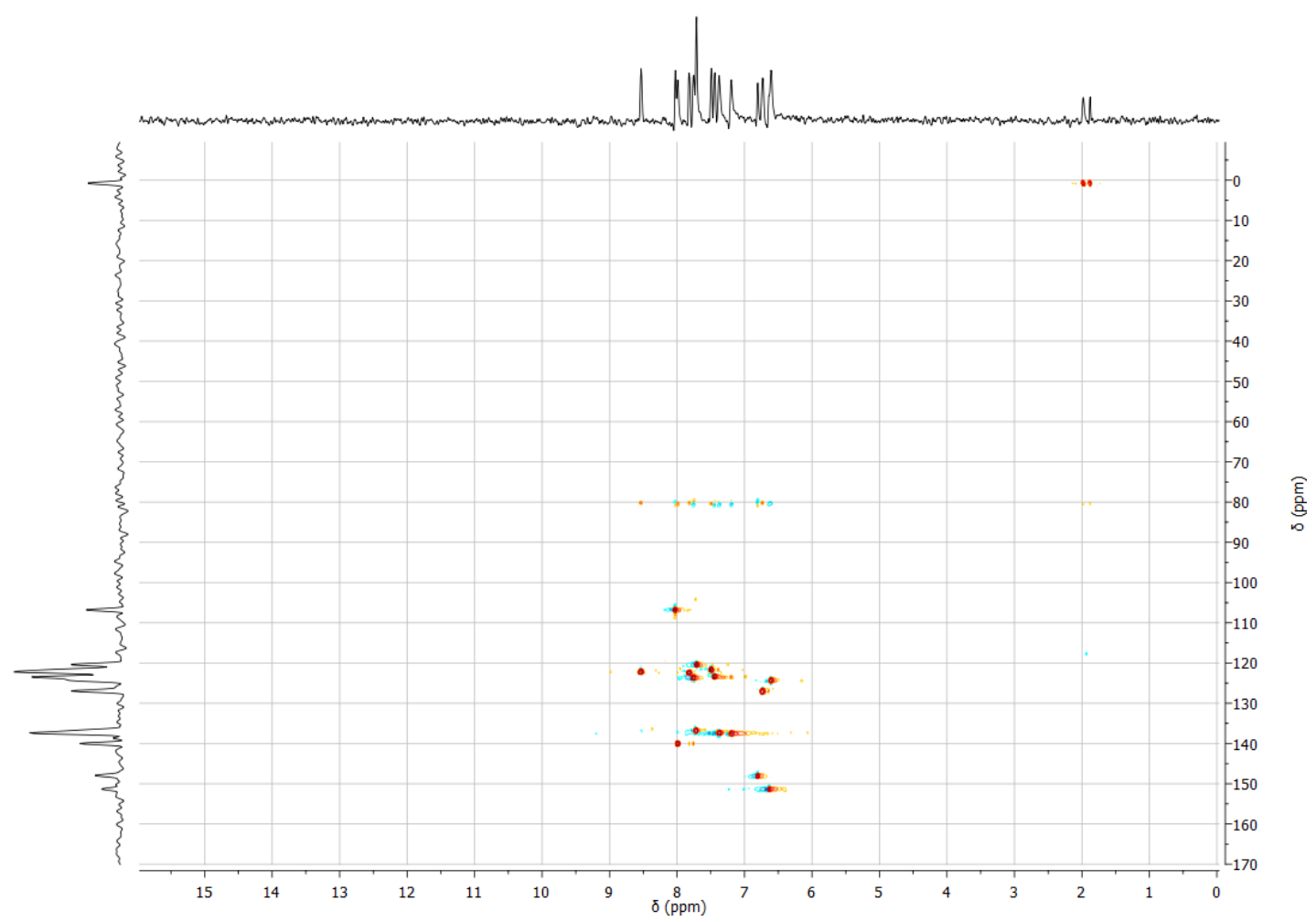

Figure A25. ${ }^{1} \mathrm{H}^{-13} \mathrm{C} \mathrm{HMBC}$ spectrum of $\left[\mathrm{L}^{\mathrm{H}} \mathrm{Ru}^{\mathrm{Ru}}\left[\mathrm{BF}_{4}\right]_{2}\right.$ in $\mathrm{MeCN}-\mathrm{d}_{3}$.

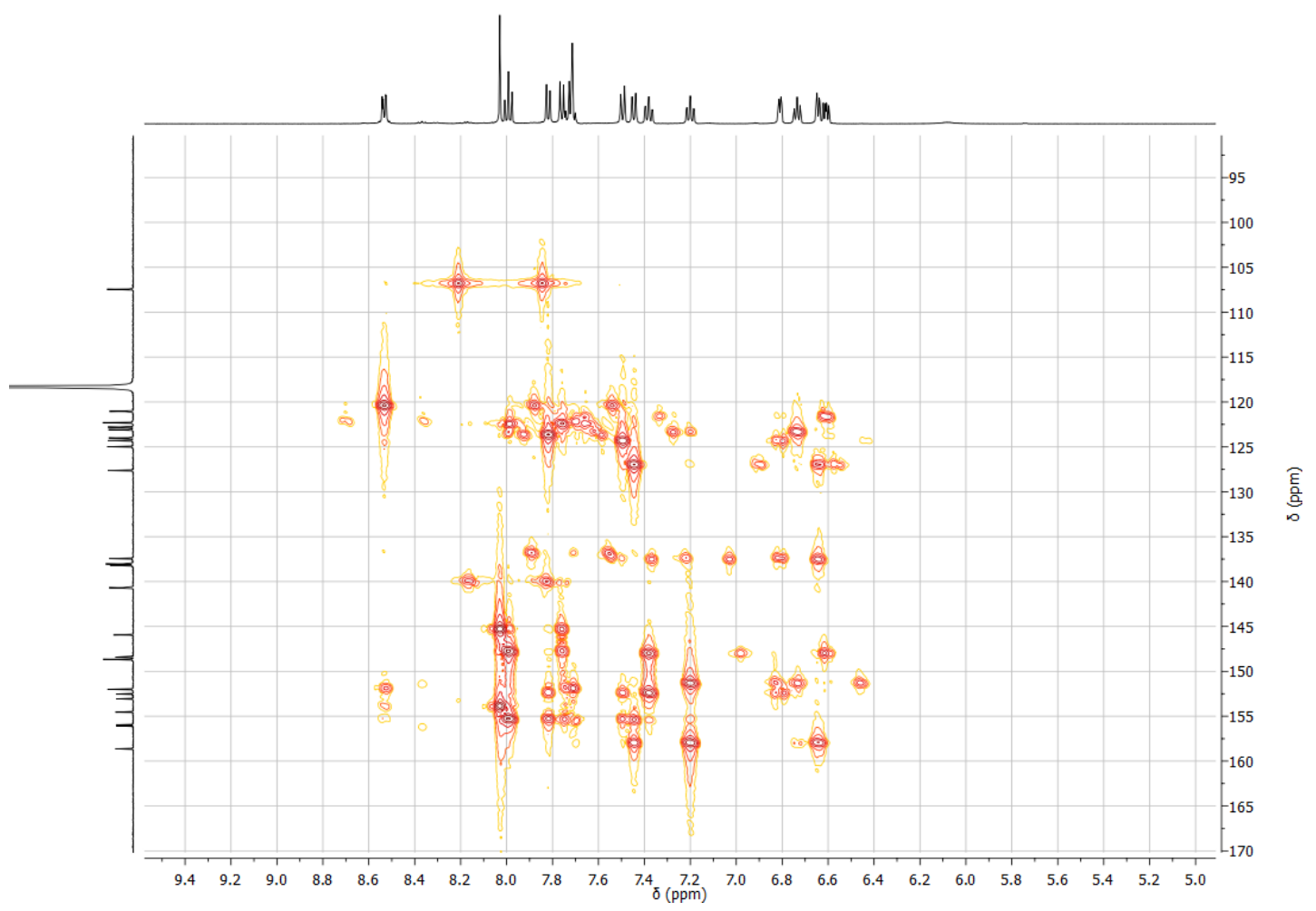

Figure A26. ${ }^{1} \mathrm{H}^{-13} \mathrm{C}$ HSQC spectrum of $\left[\mathrm{L}_{2} \mathrm{Ru}\right]\left[\mathrm{BF}_{4}\right]_{2}$ in $\mathrm{MeCN}-\mathrm{d}_{3}$. 


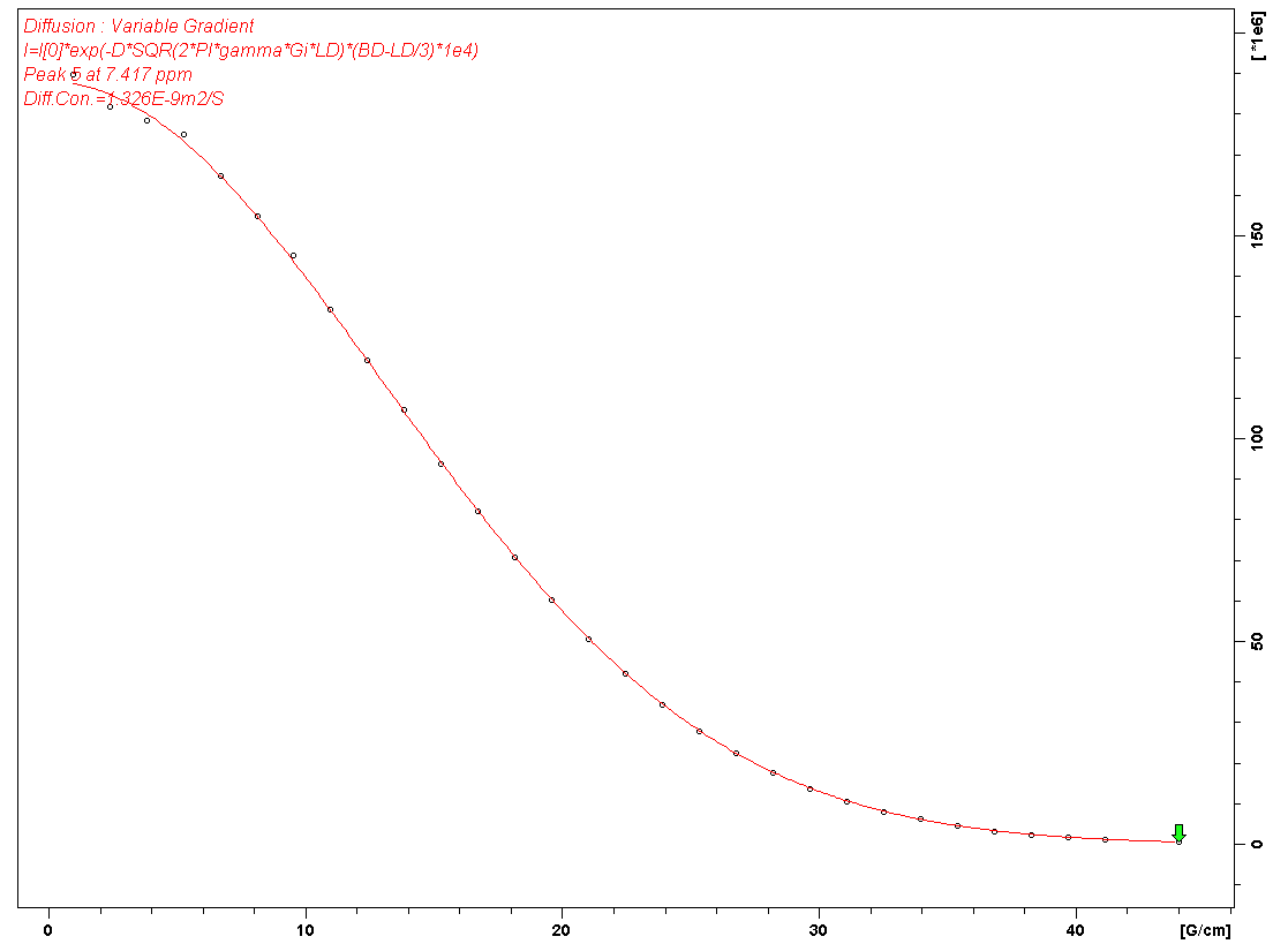

Figure A27. Simulation of the diffusion coefficient at one peak $(7.417 \mathrm{ppm})$ for $\mathbf{H L}^{\mathbf{H}}$. The diffusion coefficients for $\mathbf{H L}^{\mathbf{H}}$ and $\left[\mathrm{LH}_{2} \mathrm{Ru}\right]\left[\mathrm{BF}_{4}\right]_{2}$ reported in Chapter 3 were obtained by averaging the diffusion coefficients for all selected resonances.
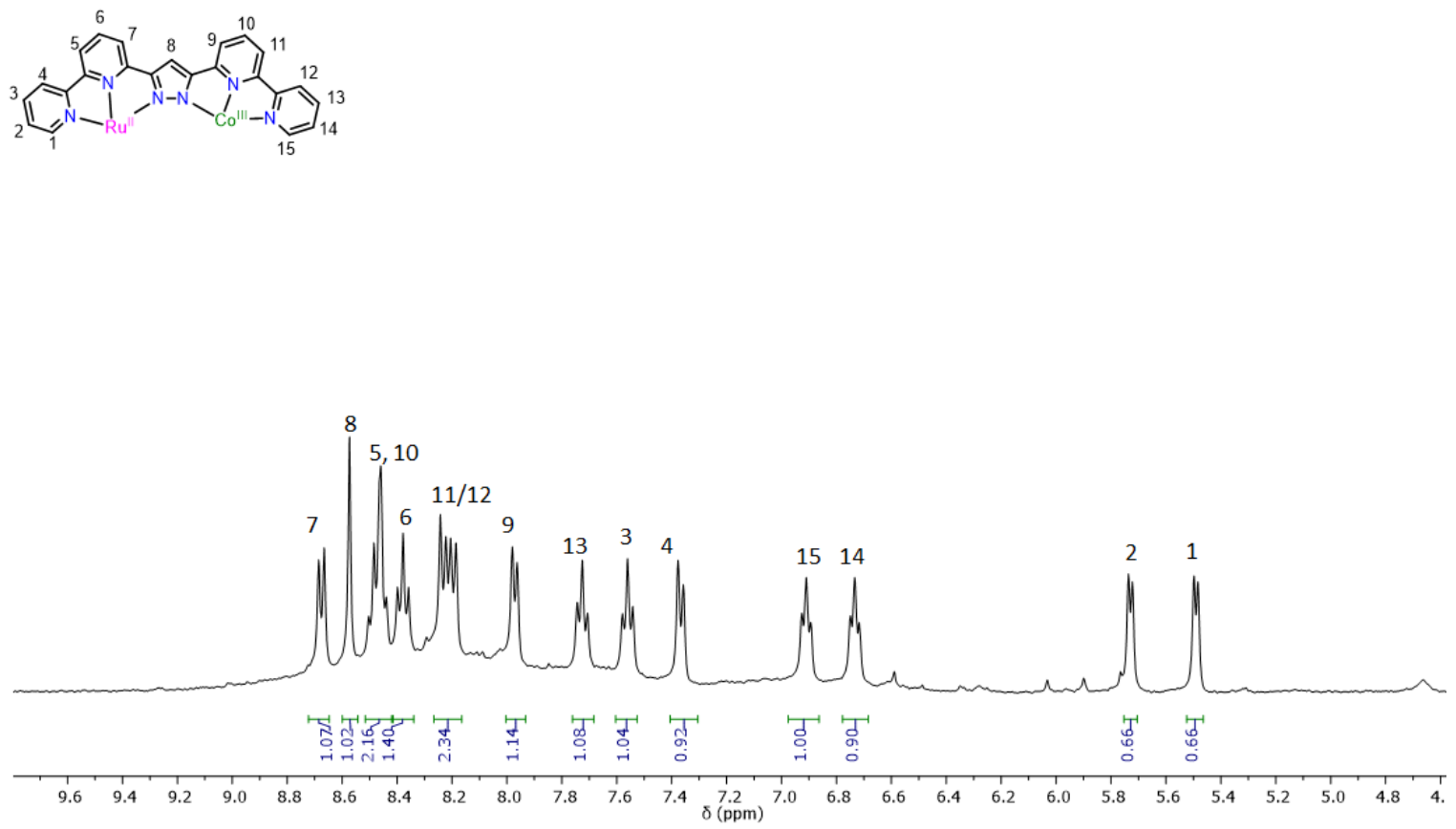

Figure A28. ${ }^{1} \mathrm{H}$ NMR spectrum of $\left[\mathrm{L}^{\mathrm{H}} \mathrm{Ru}_{2} \mathrm{Co}_{2}\right]\left[\mathrm{BF}_{4}\right]_{6}$ in $\mathrm{MeCN}-\mathrm{d}_{3}$. 


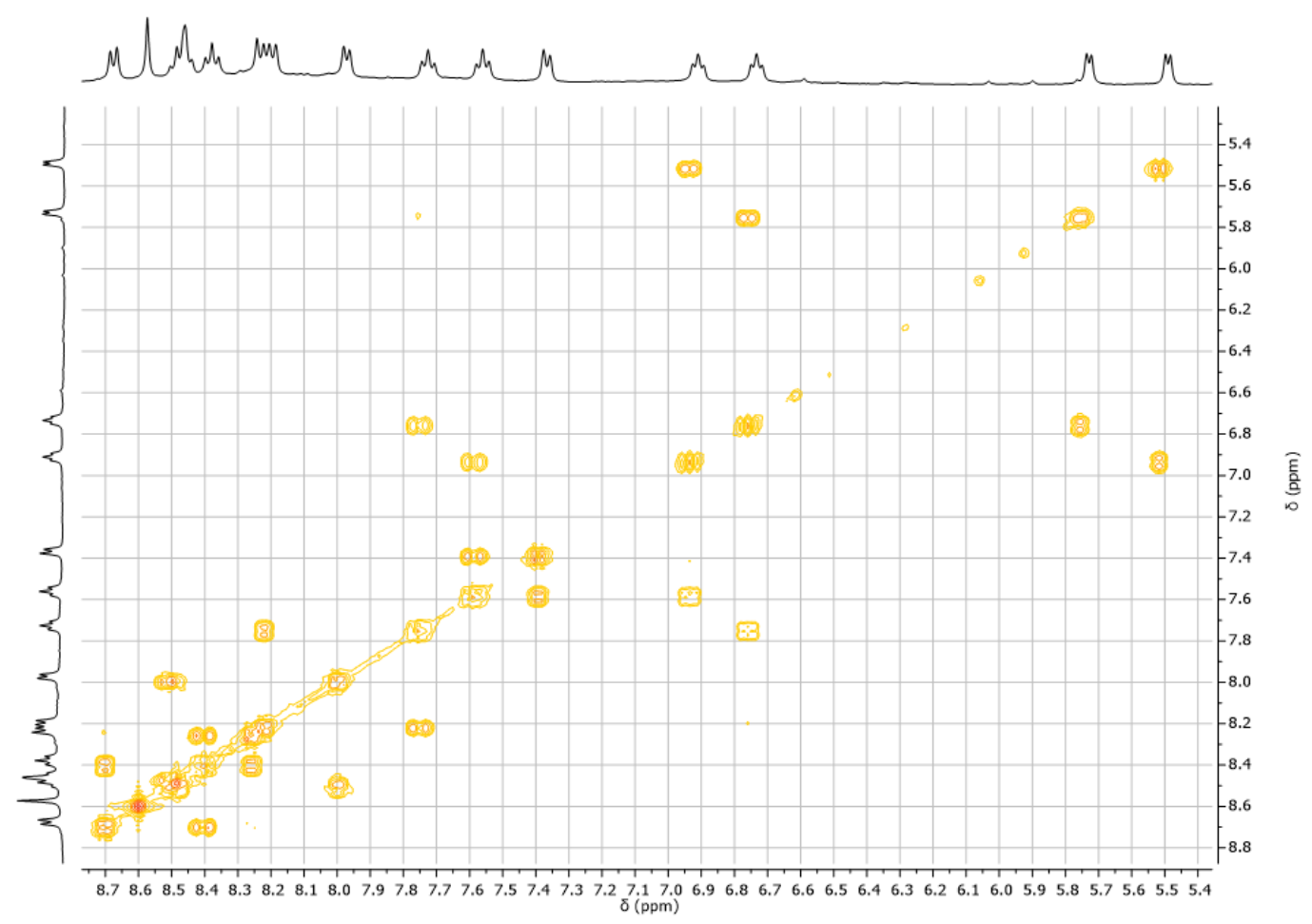

Figure A29. ${ }^{1} \mathrm{H}-{ }^{1} \mathrm{H}$ COSY spectrum of the doubly oxidized $\left[\mathrm{L}_{4} \mathrm{Ru}_{2} \mathrm{Co}_{2}\right]\left[\mathrm{BF}_{4}\right]_{6}$ grid in $\mathrm{MeCN}-\mathrm{d}_{3}$ at room temperature.

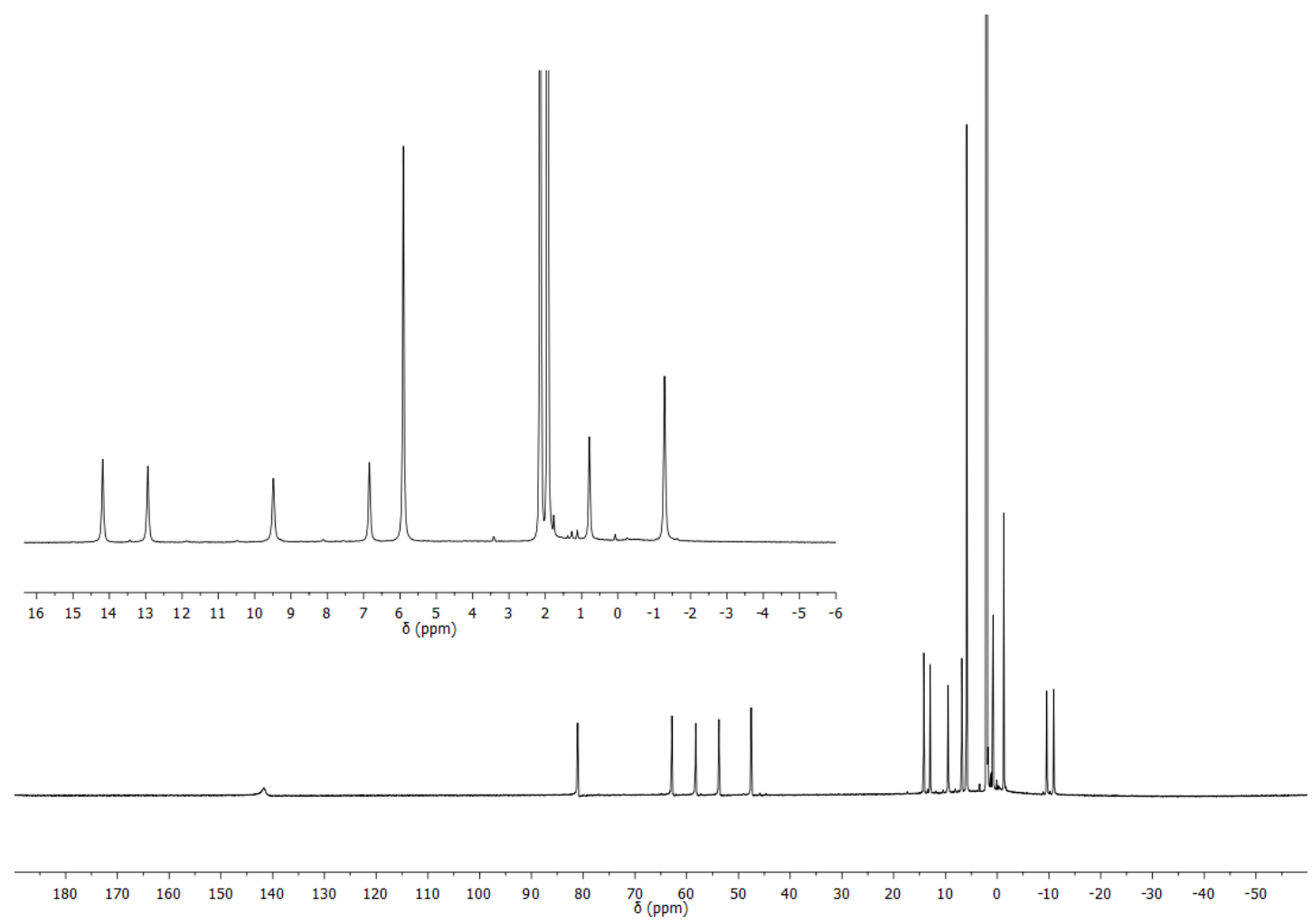

Figure A30. ${ }^{1} \mathrm{H}$ NMR spectrum pf $\left[\mathrm{LH}_{4} \mathrm{Ru}_{2} \mathrm{Fe}_{2}\right]\left[\mathrm{BF}_{4}\right]_{4}$ in $\mathrm{MeCN}-\mathrm{d}_{3}$. 


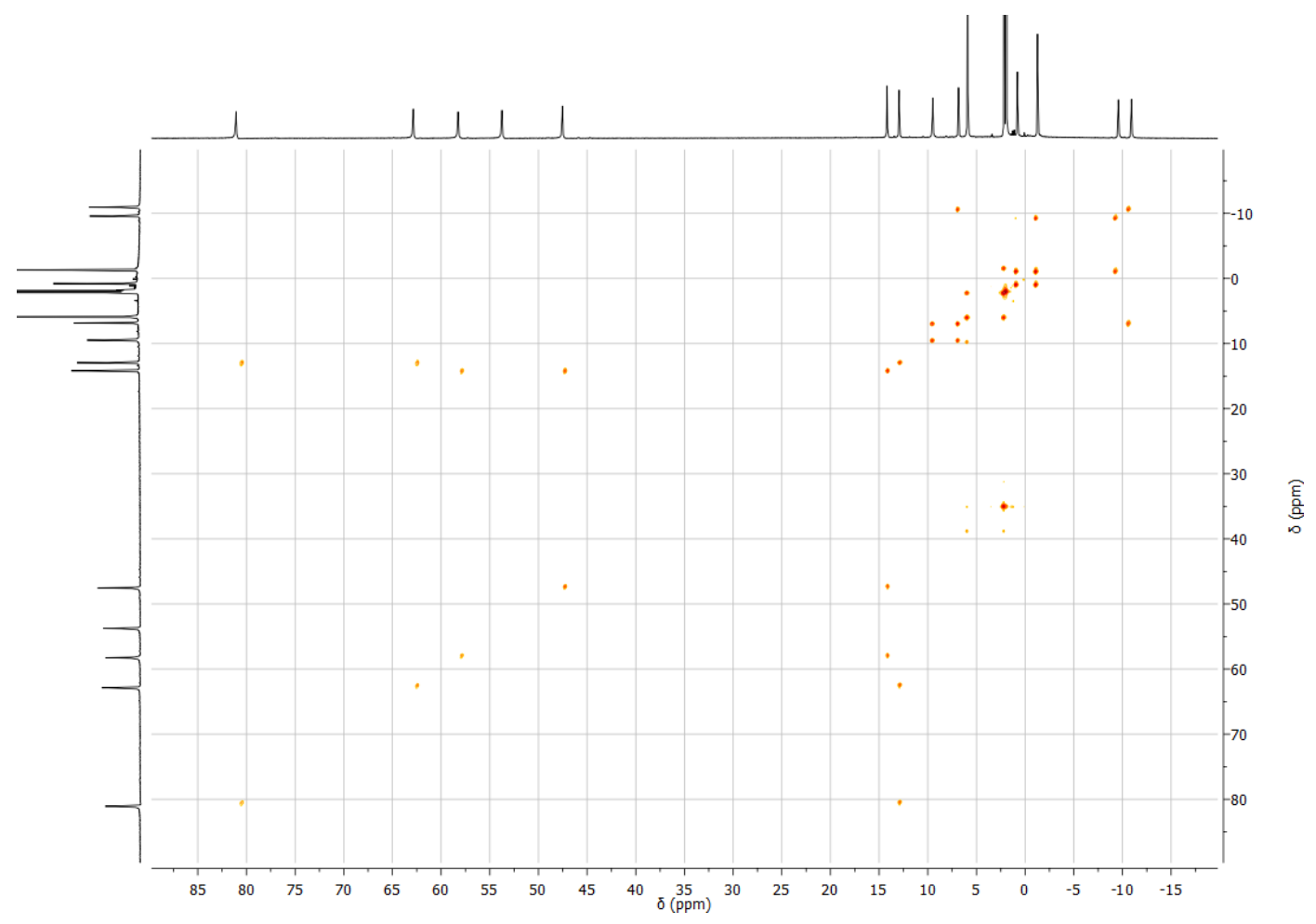

Figure A31. ${ }^{1} \mathrm{H}-1 \mathrm{H}$ COSY spectrum pf $\left[\mathrm{L}_{4} \mathrm{Ru}_{2} \mathrm{Fe}_{2}\right]\left[\mathrm{BF}_{4}\right]_{4}$ in $\mathrm{MeCN}-\mathrm{d}_{3}$ at room temperature.

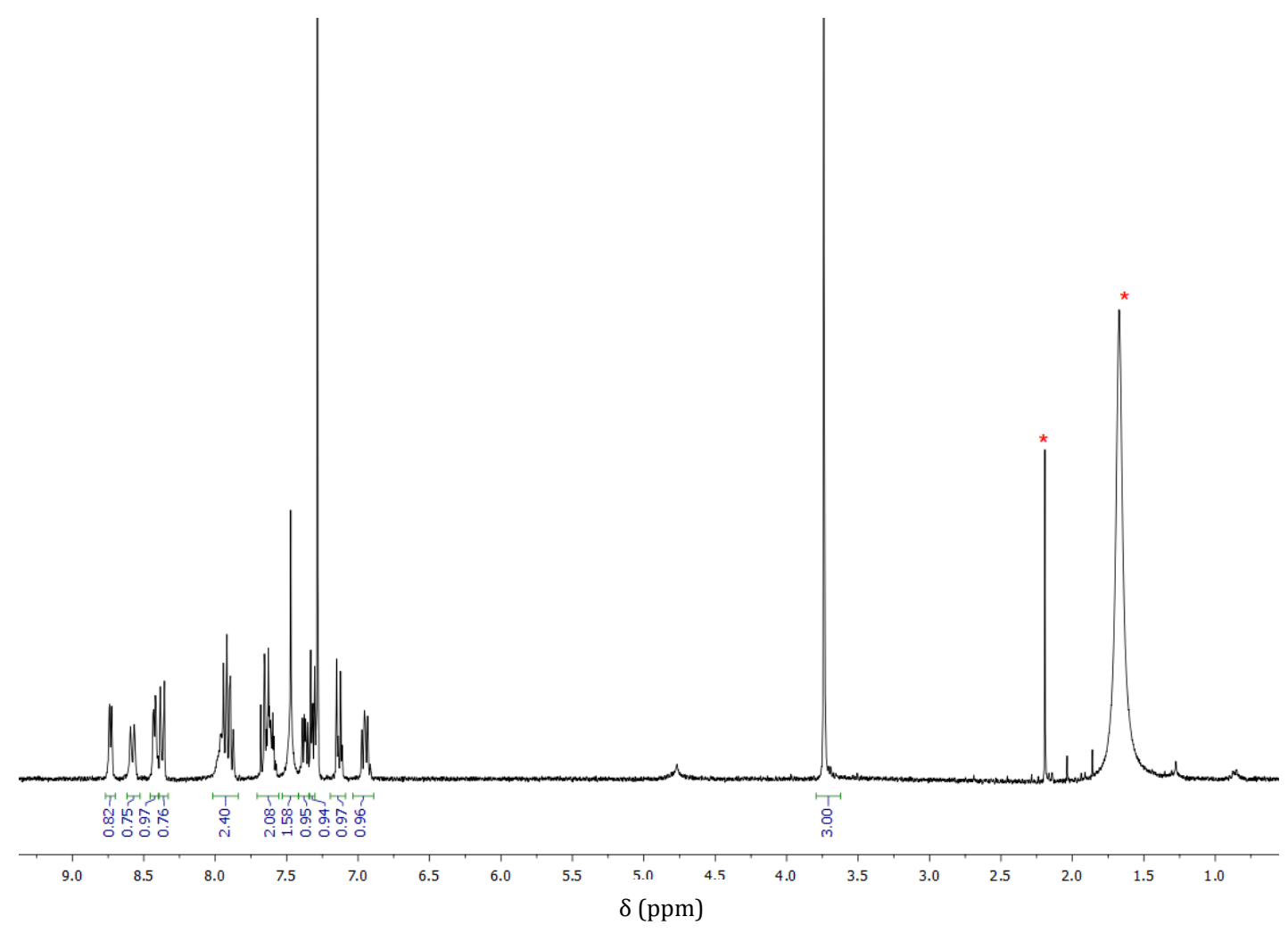

Figure A32. ${ }^{1} \mathrm{H}$ NMR spectrum of $\left[\mathrm{L}_{2}{ }_{2} \mathrm{Ru}\right]\left[\mathrm{BF}_{4}\right]_{2}$ in $\mathrm{MeCN}-\mathrm{d}_{3}$. Solvent molecules (MeCN and $\mathrm{H}_{2} \mathrm{O}$ ) are indicated with asterisks $\left({ }^{*}\right)$. 


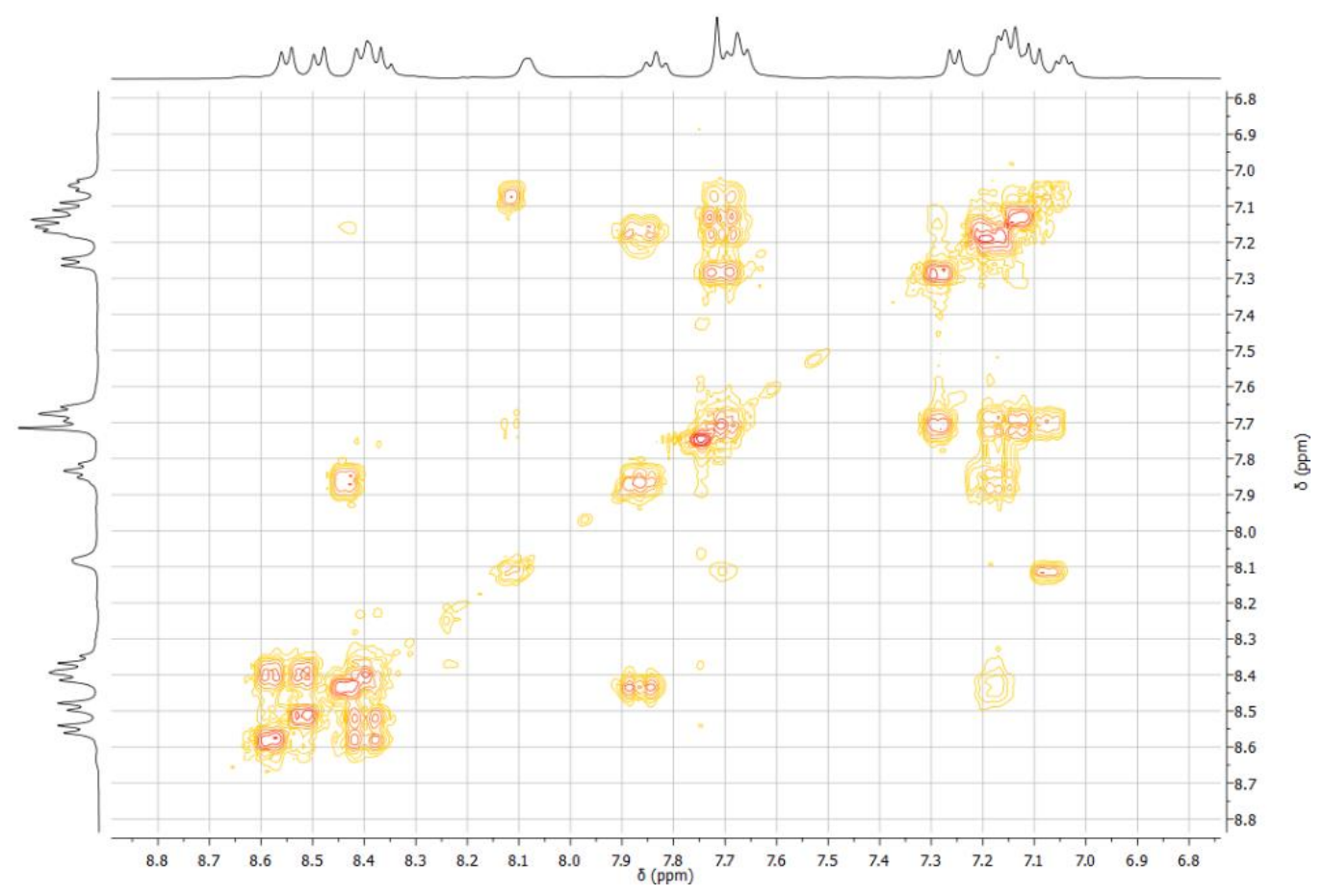

Figure A33. ${ }^{1} \mathrm{H}-1 \mathrm{H}$ COSY on the $\left[\mathrm{L}_{2}^{2} \mathrm{Ru}\right]\left[\mathrm{BF}_{4}\right]_{2}$ corner complex in the range of $\delta=6-9 \mathrm{ppm}$ in $\mathrm{MeCN}-\mathrm{d}_{3}$ at room temperature. 


\section{A4 - Electrochemical Measurements}

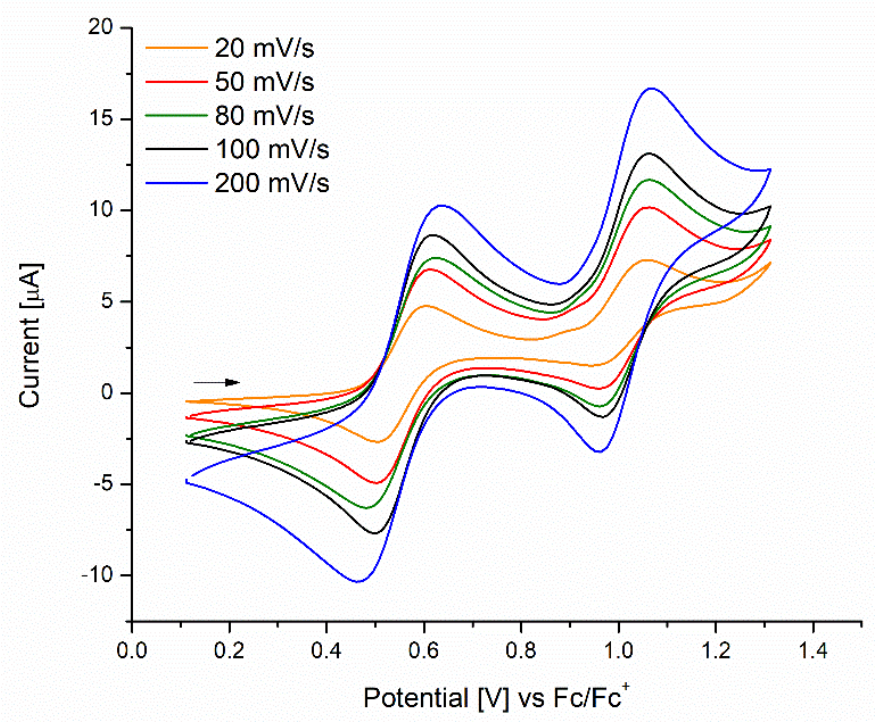

Figure A34. Cyclic voltammogram of $\left[\mathrm{L}_{2} \mathrm{Ru}\right]\left[\mathrm{BF}_{4}\right]_{2}$ in $\mathrm{MeCN}$ with $\left[\mathrm{NBu}_{4}\right] \mathrm{PF}_{6}$ as the electrolyte at various scan rates. All potentials are referenced against $\mathrm{Fc} / \mathrm{FC}^{+}$.

Table A1. Electrochemical parameters for the first oxidative wave of $\left[\mathrm{L}_{2} \mathrm{Ru}\right]\left[\mathrm{BF}_{4}\right]_{2}$ at different scan rates.

\begin{tabular}{|c|c|c|c|c|c|c|}
\hline $\begin{array}{c}\text { Scan rate } \\
{\left[\mathrm{mms}^{-1}\right]}\end{array}$ & $E_{\text {ox }}[\mathrm{mV}]$ & $E_{\text {red }}[\mathrm{mV}]$ & $E_{1 / 2}[\mathrm{mV}]$ & & $\Delta E_{\mathrm{p}}[\mathrm{mV}]$ & \\
\hline & 20 & & 604 & 502 & 553 & 102 \\
\hline & 50 & & 616 & 504 & 560 & 112 \\
\hline & 80 & & 624 & 488 & 556 & 136 \\
\hline & 100 & & 616 & 496 & 556 & 120 \\
\hline & 200 & & 640 & 460 & 550 & 180 \\
\hline
\end{tabular}

Table A2. Electrochemical parameters for the second oxidative wave of $\left[\mathrm{LH}_{2} \mathrm{Ru}\right]\left[\mathrm{BF}_{4}\right]_{2}$ at different scan rates.

\begin{tabular}{ccccc}
\hline Scan rate $\left[\mathrm{mms}^{-1}\right]$ & $E_{\text {ox }}[\mathrm{mV}]$ & $E_{\text {red }}[\mathrm{mV}]$ & $E_{1 / 2}[\mathrm{mV}]$ & $\Delta E_{\mathrm{p}}[\mathrm{mV}]$ \\
\hline 20 & 1060 & 965 & 1012 & 96 \\
50 & 1064 & 961 & 1013 & 104 \\
80 & 1064 & 961 & 1013 & 104 \\
100 & 1064 & 969 & 1017 & 96 \\
200 & 1072 & 965 & 1018 & 108 \\
\hline
\end{tabular}



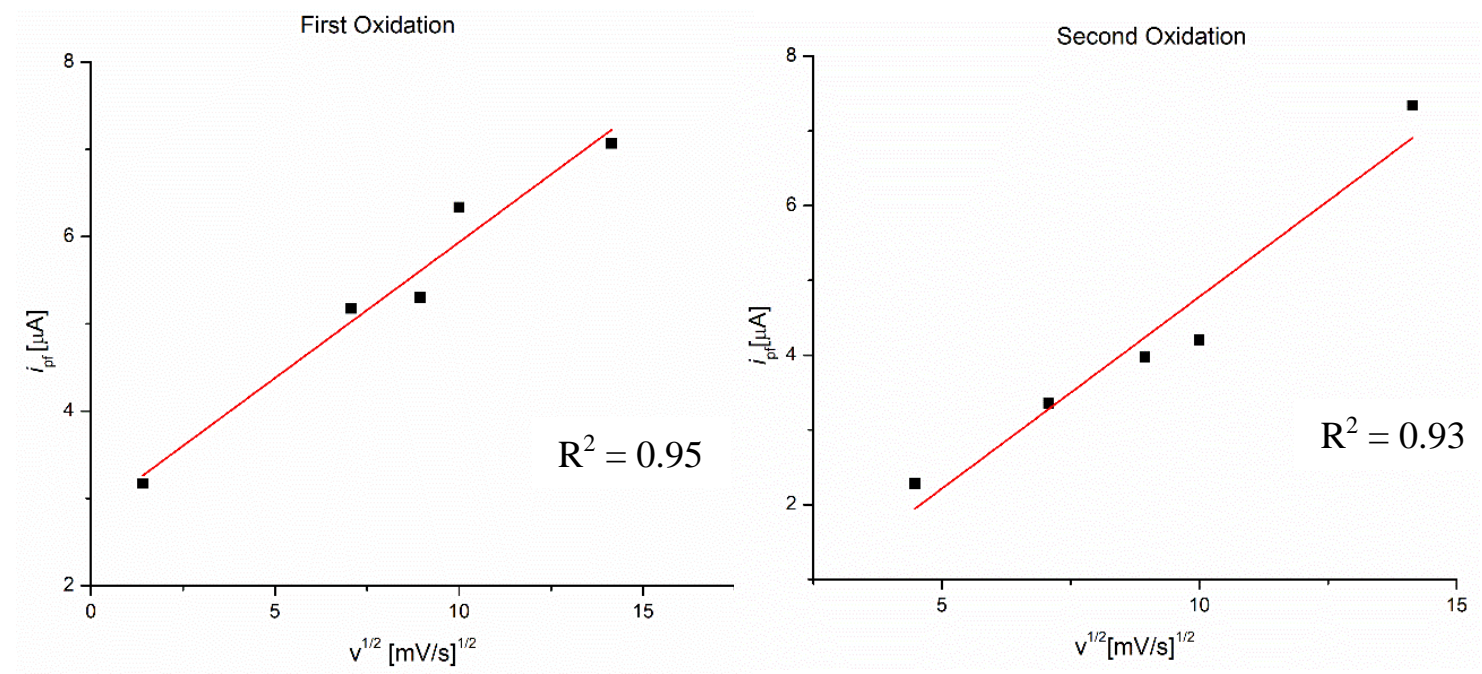

Figure A35. A plot of the peak current $\left(i_{\mathrm{pf}}\right)$ versus $v^{1 / 2}$ of $\left[\mathrm{LH}_{2} \mathrm{Ru}\right]\left[\mathrm{BF}_{4}\right]_{2}$ suggesting quasireversible behaviour for both oxidative waves.

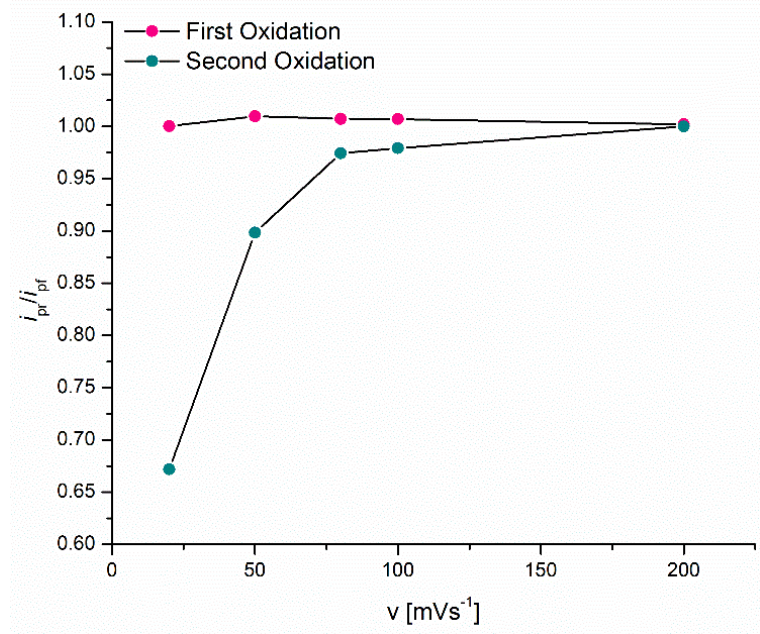

Figure A36. Plot of $\mathrm{i}_{\mathrm{pr}} / \mathrm{i}_{\mathrm{pf}} \mathrm{VS} \mathrm{v}^{1 / 2}$ for $\left[\mathrm{L}_{2} \mathrm{Ru}\right]\left[\mathrm{BF}_{4}\right]_{2}$ illustrating the increase in irreversibility at slower scan rates.

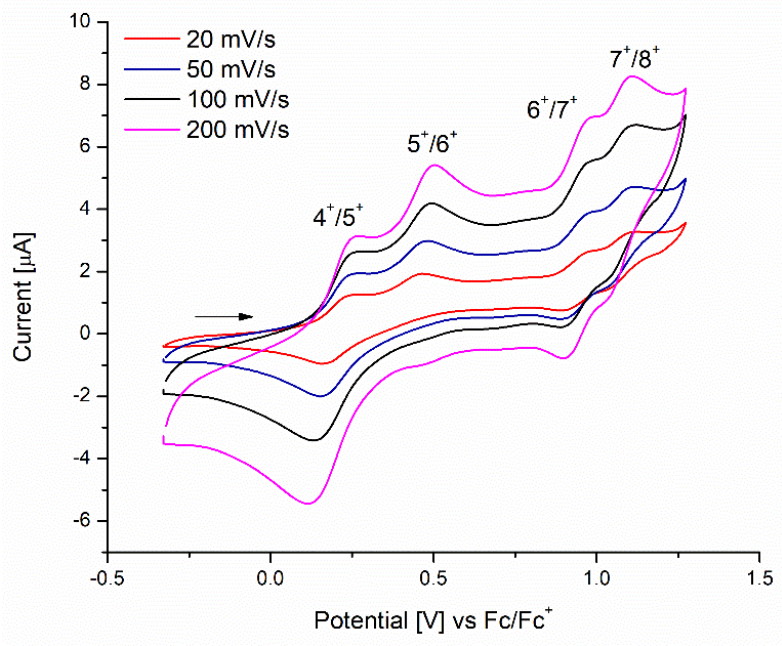

Figure A37. Cyclic voltammetry of $\left[\mathrm{L}_{4} \mathrm{Ru}_{2} \mathrm{Co}_{2}\right]\left[\mathrm{BF}_{4}\right]_{4}$ in $\mathrm{MeCN}$ with $\left[\mathrm{NBu}_{4}\right] \mathrm{PF}_{6}$ as the electrolyte at various scan rates. All potentials are referenced against $\mathrm{Fc} / \mathrm{Fc}^{+}$. 


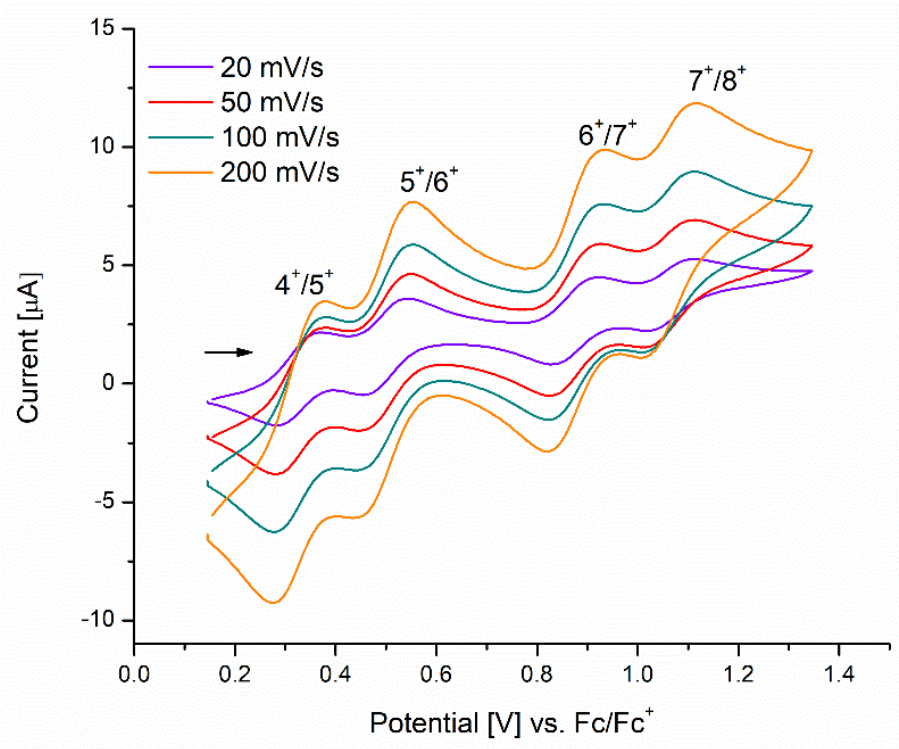

Figure A38. Cyclic voltammetry of $\left[\mathrm{L}_{4} \mathrm{Ru}_{2} \mathrm{Fe}_{2}\right]\left[\mathrm{BF}_{4}\right]_{4}$ in $\mathrm{MeCN}$ with $\left[\mathrm{NBu}_{4}\right] \mathrm{PF}_{6}$ as the electrolyte at various scan rates. All potentials are referenced against $\mathrm{Fc} / \mathrm{Fc}^{+}$.

(a)

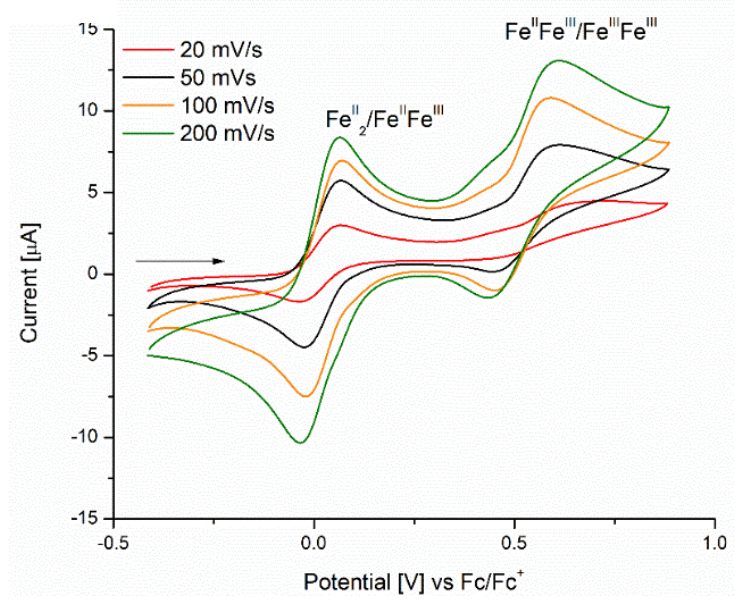

(b)

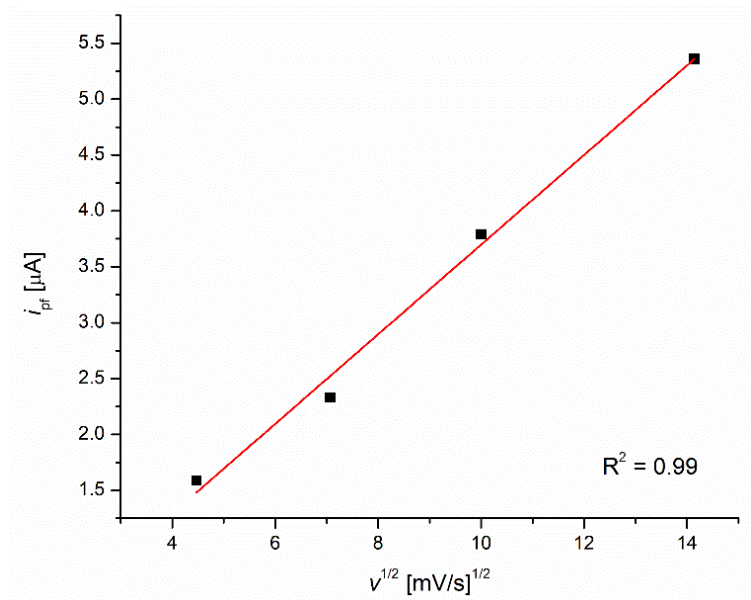

Figure A39. (a)Cyclic voltammogram of $\left[\mathrm{L}_{2}{ }_{2} \mathrm{Fe}_{2}\right][\mathrm{OTf}]_{2}$ in $\mathrm{MeCN}$ at different scan rates with $\left[\mathrm{Bu}_{4} \mathrm{~N}\right] \mathrm{PF}_{6}$ as the electrolyte (left). All potentials are referenced against $\mathrm{Fc} / \mathrm{Fc}^{+}$. (b) Plot of $i_{\mathrm{pf}}$ $\mathrm{vs} \mathrm{V}^{1 / 2}$ for the first anodic wave of $\left[\mathrm{L}_{2}{ }_{2} \mathrm{Fe}_{2}\right][\mathrm{OTf}]_{2}$ suggesting reversible behaviour. 


\section{A5 - Mössbauer Spectroscopy}

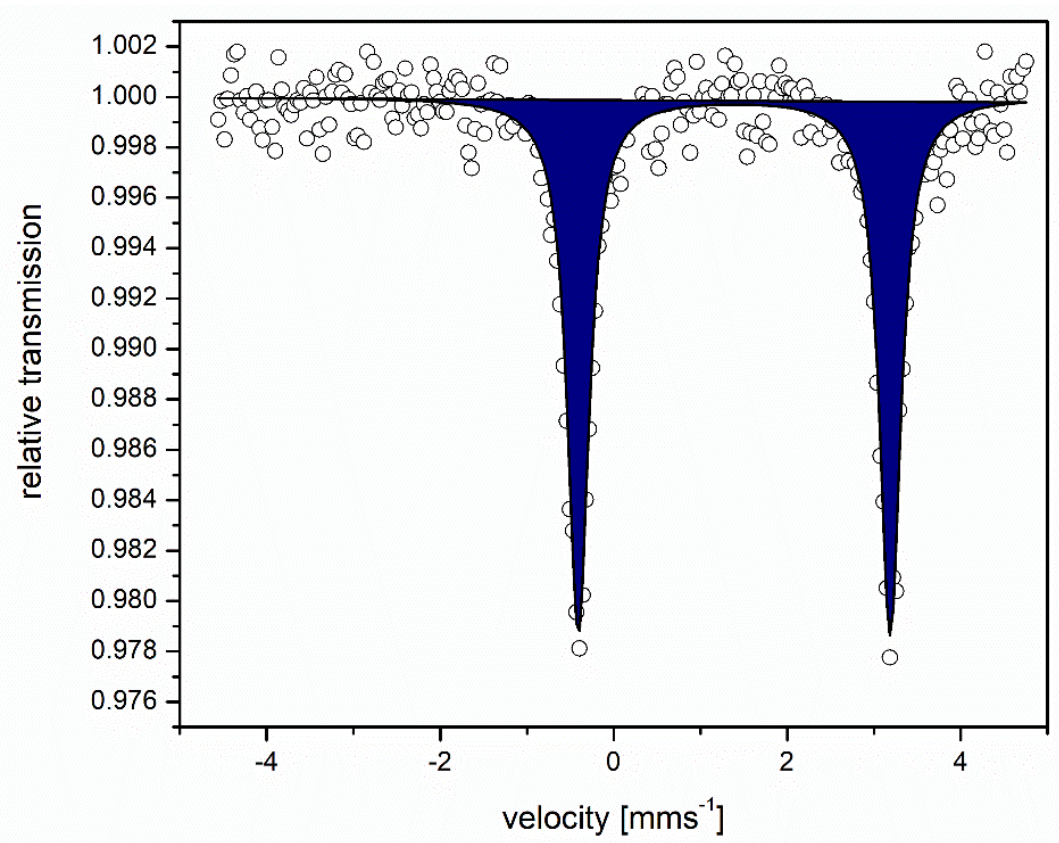

Figure A40. $\mathrm{MB}$ spectrum of $\mathrm{Fe}(\mathrm{OTf})_{2} \cdot 2 \mathrm{MeCN}$ at $80 \mathrm{~K}$. One doublet with an isomer shift of $\delta=1.39 \mathrm{mms}^{-1}$ and quadruple splitting $\Delta \mathrm{E}_{\mathrm{Q}}=3.60 \mathrm{mms}^{-1}$ was observed.

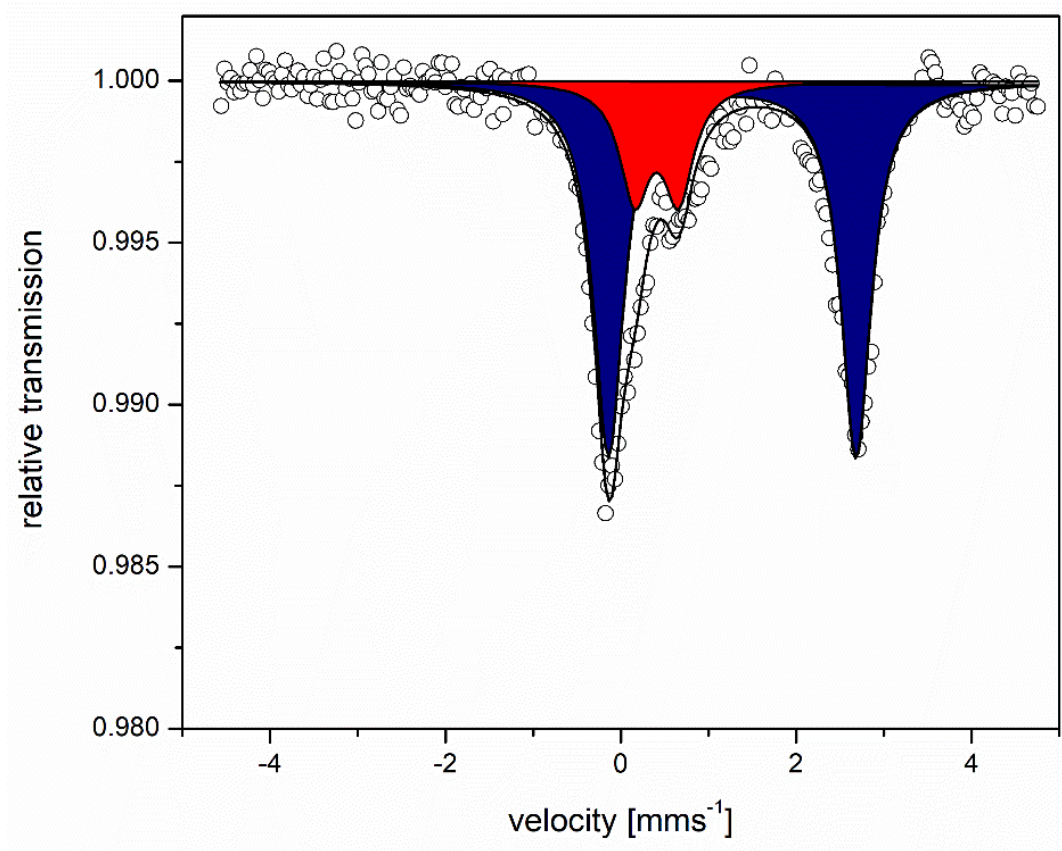

Figure A41. Frozen solution $\mathrm{MB}$ spectrum of $\mathrm{Fe}(\mathrm{OTf})_{2} \cdot 2 \mathrm{MeCN}$ in $\mathrm{DMF}$ at $80 \mathrm{~K}$. One doublet with an isomer shift of $\delta=1.27 \mathrm{mms}^{-1}$ and quadruple splitting $\Delta E_{\mathrm{Q}}=282 \mathrm{mms}^{-1}$ was observed, corresponding the $\mathrm{HS}-\mathrm{Fe}^{\mathrm{II}}$ ion in $\mathrm{Fe}(\mathrm{OTf})_{2} \cdot 2 \mathrm{MeCN}$. The second species with an isomer shift of $\delta=0.41 \mathrm{mms}^{-1}\left(\Delta E_{\mathrm{Q}}=0.50 \mathrm{mms}^{-1}\right)$ may be attributed to oxidation of the Fe ${ }^{\mathrm{II}}$ salt in DMF. 


\section{A6 - Magnetic Measurements}

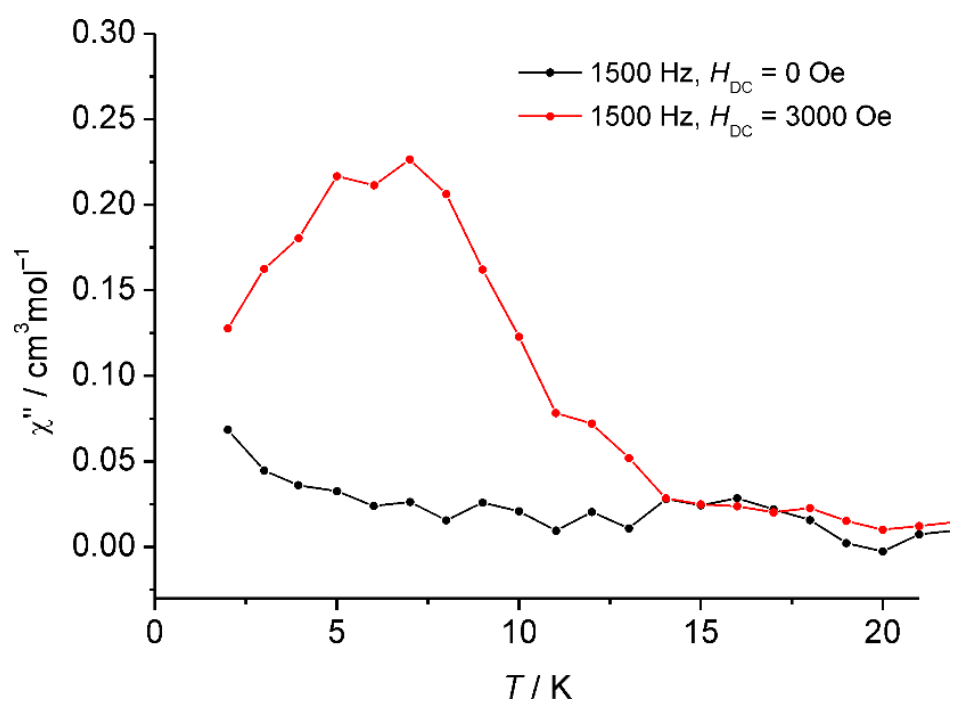

Figure A42. Temperature dependence of $\chi^{\prime \prime}$ at $1500 \mathrm{~Hz}$ in the absence of a dc field and with the applied dc field $H_{\mathrm{dc}}=3000$ Oe.

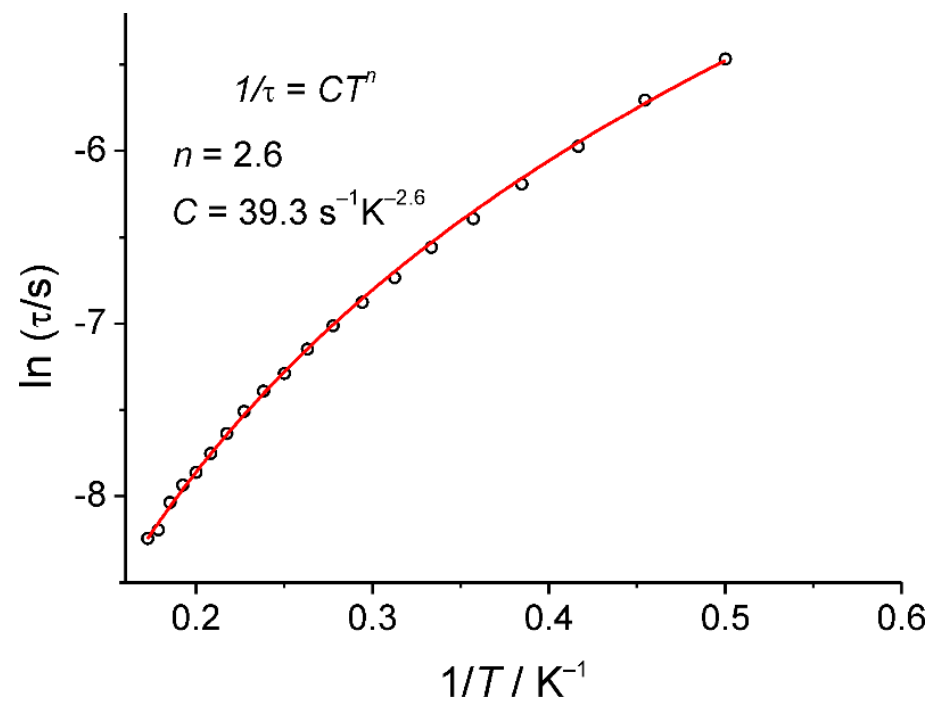

Figure A43. Relaxation times vs. inverse temperature for $\left[\mathrm{LH}_{4} \mathrm{Ru}_{2} \mathrm{Co}_{2}\right]\left[\mathrm{BF}_{4}\right]$, fitted according to Raman relaxation $1 / \tau=C T^{n}$. 


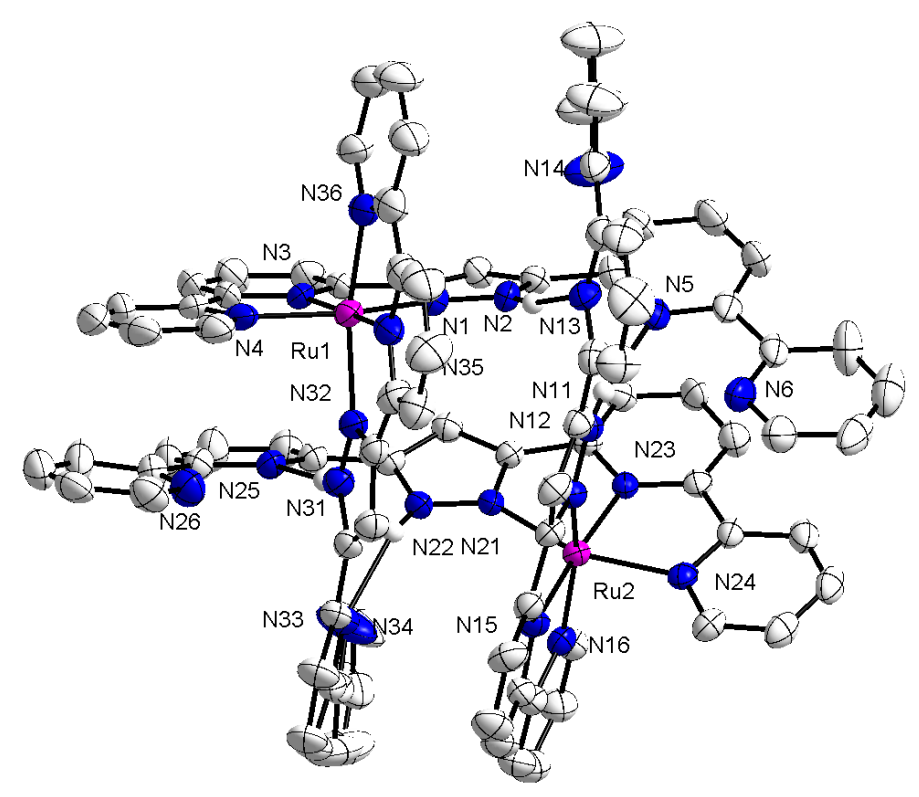

Figure A44. ORTEP plot (50\% probability thermal ellipsoids) of $\left[\mathrm{L}_{4} \mathrm{Ru}_{2}\right]\left[\mathrm{BF}_{4}\right]_{4}$. Hydrogen atoms, counterions and solvent molecules are omitted for clarity. Selected bond lengths $[\AA]$ : $\mathrm{Ru}(1)-\mathrm{N}(35)$ 1.979(7), Ru(1)-N(3) 1.982(7), Ru(1)-N(36) 2.047(7), Ru(1)-N(1) 2.068(7), $\mathrm{Ru}(1)-\mathrm{N}(32)$ 2.071(6), Ru(1)-N(4) 2.076(7), Ru(2)-N(23) 1.985(6), Ru(2)-N(15) 1.999(6), $\mathrm{Ru}(2)-\mathrm{N}(16) 2.047(6), \mathrm{Ru}(2)-\mathrm{N}(24)$ 2.060(6), $\mathrm{Ru}(2)-\mathrm{N}(12)$ 2.069(6), $\mathrm{Ru}(2)-\mathrm{N}(21) 2.073(6)$. 
Table A3. Crystal metrics and refinement data for $\left[\mathrm{L}_{4} \mathrm{Ru}_{2}\right]\left[\mathrm{BF}_{4}\right]_{4}$

\begin{tabular}{|c|c|}
\hline & {$\left[\mathbf{L}_{4} \mathbf{R u}_{2}\right]\left[\mathrm{BF}_{4}\right]_{4}$} \\
\hline Empirical Formula & $\mathrm{C}_{94} \mathrm{H}_{67} \mathrm{~B}_{4} \mathrm{~F}_{16} \mathrm{~N}_{25} \mathrm{Ru}_{2}$ \\
\hline Formula Weight & 2096.10 \\
\hline Temperature $[\mathrm{K}]$ & $133(2)$ \\
\hline Crystal size $\left[\mathrm{mm}^{3}\right]$ & $0.500 \times 0.120 \times 0.100$ \\
\hline Crystal system & Triclinic \\
\hline Space group & $\mathrm{P}-1$ \\
\hline $\mathrm{a}[\AA ̊]$ & $13.8944(5)$ \\
\hline $\mathrm{b}[\AA]$ & $17.1622(6)$ \\
\hline c $[\AA]$ & $21.3058(8)$ \\
\hline$\alpha\left[^{\circ}\right]$ & $84.379(3)$ \\
\hline$\beta\left[^{\circ}\right]$ & $80.806(3)$ \\
\hline$\gamma\left[^{\circ}\right]$ & $82.125(3)$ \\
\hline Volume $\left[\AA^{3}\right]$ & $4952.9(3)$ \\
\hline$Z$ & 2 \\
\hline$\rho\left[\mathrm{Mg} / \mathrm{m}^{3}\right]$ & 1.405 \\
\hline$\mu\left[\mathrm{mm}^{-1}\right]$ & 0.393 \\
\hline$F(000)$ & 2116 \\
\hline$\theta$ range $\left[{ }^{\circ}\right]$ & $1.201-26.176$ \\
\hline$h k l$ range & $\pm 17, \pm 21, \pm 26$ \\
\hline Measured refl. & 84099 \\
\hline Unique refl. [Rint] & 84099 \\
\hline$T_{\min } / T_{\max }$ & $0.9059,0.7094$ \\
\hline data / restr. / param. & $84099 / 472 / 1336$ \\
\hline goodness-of-fit on $\left(\mathrm{F}^{2}\right)$ & 1.042 \\
\hline $\mathrm{R} 1, \mathrm{wR} 2(\mathrm{I}>2 \sigma(\mathrm{I}))$ & $0.0755,0.1945$ \\
\hline $\mathrm{R} 1, \mathrm{wR} 2$ (all data) & $0.1490,0.2314$ \\
\hline res. el. dens. $\left[\mathrm{e} / \AA^{3}\right]$ & $1.200 /-0.886$ \\
\hline
\end{tabular}




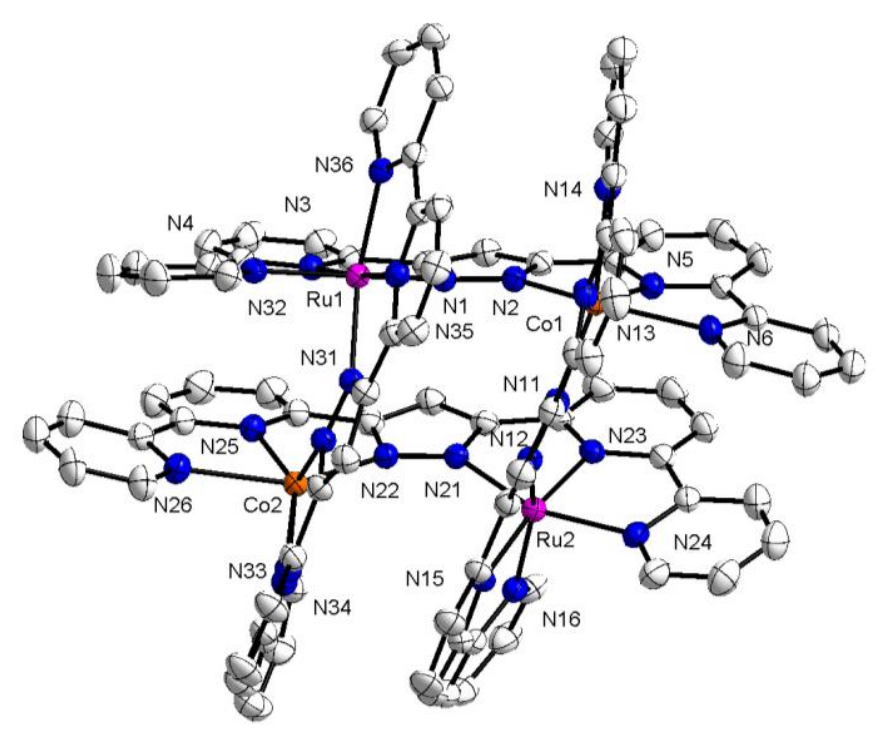

Figure A45. ORTEP plot (50\% probability thermal ellipsoids) of $\left[\mathrm{LH}_{4} \mathrm{Ru}_{2} \mathrm{Co}_{2}\right]\left[\mathrm{BF}_{4}\right]_{4}$. Hydrogen atoms, counterions and solvent molecules are omitted for clarity. Selected bond lengths $[\AA]$ : $\mathrm{Ru}(1)-\mathrm{N}(35)$ 1.982(3), $\mathrm{Ru}(1)-\mathrm{N}(3)$ 1.986(3), $\mathrm{Ru}(1)-\mathrm{N}(4)$ 2.059(3), $\mathrm{Ru}(1)-\mathrm{N}(36)$ 2.065(3), $\mathrm{Ru}(1)-\mathrm{N}(32)$ 2.069(3), $\mathrm{Ru}(1)-\mathrm{N}(1) 2.075(3), \mathrm{Ru}(2)-\mathrm{N}(15)$ 1.999(3), $\mathrm{Ru}(2)-\mathrm{N}(23)$ 2.004(3), $\mathrm{Ru}(2)-\mathrm{N}(16)$ 2.086(3), Ru(2)-N(24) 2.087(3), Ru(2)-N(21) 2.098(3), Ru(2)-N(12) 2.105(3), Co(1)-N(13) 2.077(3), Co(1)-N(5) 2.079(3), Co(1)-N(11) 2.098(3), Co(1)-N(2) 2.123(3), Co(1)-N(14) 2.249(3), Co(1)-N(6) 2.307(3), Co(2)-N(33) 2.068(3), Co(2)-N(25) 2.082(3), Co(2)-N(22) 2.098(3), Co(2)-N(31) 2.116(3), Co(2)-N(34) 2.305(3), Co(2)-N(26) 2.313(3).

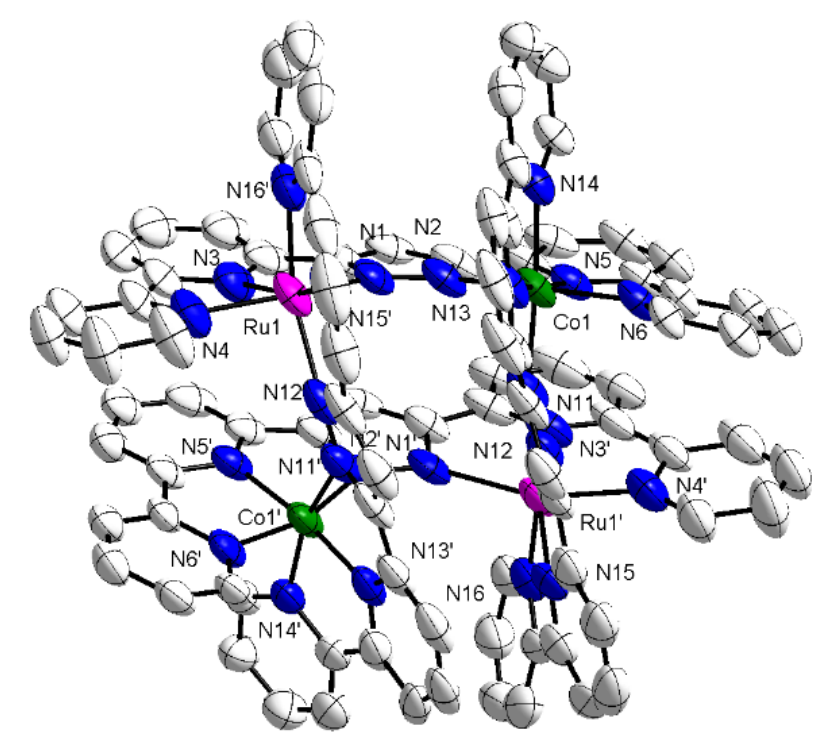

Figure A46. ORTEP plot (50\% probability thermal ellipsoids) of $\left[\mathrm{L}_{4} \mathrm{Ru}_{2} \mathrm{Co}_{2}\right]\left[\mathrm{BF}_{4}\right]_{6}$. Hydrogen atoms, counterions and solvent molecules are omitted for clarity. Selected bond lengths $[\AA]$ : $\mathrm{Ru}(1)-\mathrm{N}(3)$ 2.011(9), Ru(1)-N(15)\#1 2.015(9), Ru(1)-N(16)\#1, 2.055(10), Ru(1)-N(4) 2.077(10), Ru(1)-N(12)\#1 2.142(10), Ru(1)-N(1) 2.176(9), Co(1)-N(13) 1.865(8), Co(1)$\mathrm{N}(5) 1.888(8), \operatorname{Co}(1)-\mathrm{N}(11) 1.952(9), \operatorname{Co}(1)-\mathrm{N}(2)$ 1.971(8), Co(1)-N(14) 1.973(8), Co(1)-N(6) $1.992(8)$. 


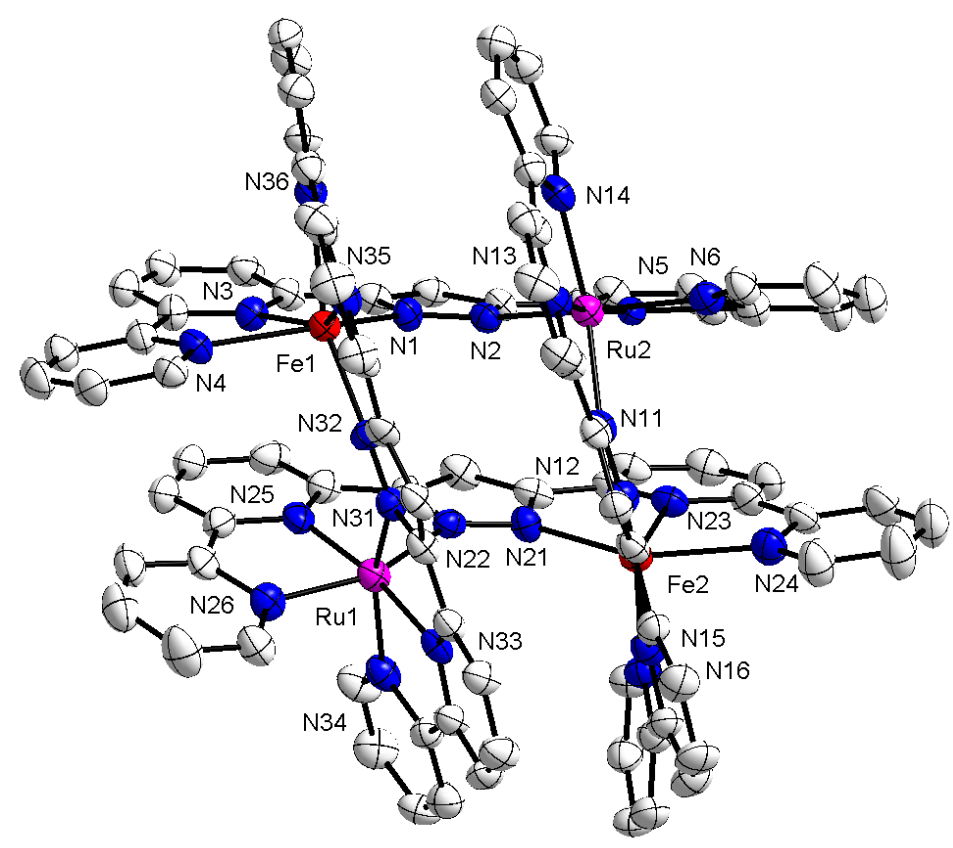

Figure A47. ORTEP plot (50\% probability thermal ellipsoids) of $\left[\mathrm{L}_{4} \mathrm{Ru}_{2} \mathrm{Fe}_{2}\right]\left[\mathrm{BF}_{4}\right]_{4}$. Hydrogen atoms, counterions and solvent molecules are omitted for clarity. Selected bond lengths $[\AA]$ : $\mathrm{Ru}(1)-\mathrm{N}(25)$ 1.979(9), Ru(1)-N(33) 1.990(8), Ru(1)-N(34) 2.055(9), Ru(1)-N(26) 2.070(8), $\mathrm{Ru}(1)-\mathrm{N}(22)$ 2.096(8), Ru(1)-N(31) 2.097(8), Ru(2)-N(5) 1.976(9), Ru(2)-N(13) 1.999(9), $\mathrm{Ru}(2)-\mathrm{N}(6)$ 2.065(9), Ru(2)-N(14) 2.068(8), Ru(2)-N(11) 2.090(8), Ru(2)-N(2) 2.096(8), $\mathrm{Fe}(1)-\mathrm{N}(35)$ 2.118(8), $\mathrm{Fe}(1)-\mathrm{N}(3)$ 2.123(8), $\mathrm{Fe}(1)-\mathrm{N}(1)$ 2.128(8), $\mathrm{Fe}(1)-\mathrm{N}(32)$ 2.133(8), $\mathrm{Fe}(1)-\mathrm{N}(36)$ 2.301(8), Fe(1)-N(4) 2.302(7), Fe(2)-N(23) 2.106(8), Fe(2)-N(15) 2.119(8), $\mathrm{Fe}(2)-\mathrm{N}(21)$ 2.124(8), $\mathrm{Fe}(2)-\mathrm{N}(12)$ 2.130(9), $\mathrm{Fe}(2)-\mathrm{N}(24)$ 2.262(9), $\mathrm{Fe}(2)-\mathrm{N}(16)$ 2.303(9). 
Table A4. Crystal metrics and refinement data for $\left[\mathrm{LH}_{4} \mathrm{Ru}_{2} \mathrm{Co}_{2}\right]\left[\mathrm{BF}_{4}\right]_{4},\left[\mathrm{LH}_{4} \mathrm{Ru}_{2} \mathrm{Co}_{2}\right]\left[\mathrm{BF}_{4}\right]_{6}$, and $\left[\mathrm{L}_{4} \mathrm{Ru}_{2} \mathrm{Fe}_{2}\right]\left[\mathrm{BF}_{4}\right]_{4}$.

\begin{tabular}{|c|c|c|c|}
\hline & {$\left[\mathrm{LH}_{4} \mathrm{Ru}_{2} \mathrm{Co}_{2}\right]\left[\mathrm{BF}_{4}\right]_{4}$} & {$\left[\mathrm{LH}_{4} \mathrm{Ru}_{2} \mathrm{Co}_{2}\right]\left[\mathrm{BF}_{4}\right]_{6}$} & {$\left[\mathrm{LH}_{4} \mathrm{Ru}_{2} \mathrm{Fe}_{2}\right]\left[\mathrm{BF}_{4}\right]_{4}$} \\
\hline Empirical Formula & $\begin{array}{c}\mathrm{C}_{92} \mathrm{H}_{60} \mathrm{~B}_{4} \mathrm{Co}_{2} \mathrm{~F}_{16} \mathrm{~N}_{24} \\
\mathrm{Ru}_{2}\end{array}$ & $\mathrm{C}_{96} \mathrm{H}_{66} \mathrm{~B}_{6} \mathrm{Co}_{2} \mathrm{~F}_{24} \mathrm{~N}_{26} \mathrm{Ru}_{2}$ & $\mathrm{C}_{100} \mathrm{H}_{72} \mathrm{~B}_{4} \mathrm{~F}_{16} \mathrm{Fe}_{2} \mathrm{~N}_{28} \mathrm{Ru}_{2}$ \\
\hline Formula Weight & 2168.88 & 2424.60 & 2326.93 \\
\hline Temperature $[\mathrm{K}]$ & $133(2)$ & $133(2)$ & $133(2)$ \\
\hline Crystal size $\left[\mathrm{mm}^{3}\right]$ & $\begin{array}{c}0.500 \times 0.480 \times \\
0.390\end{array}$ & $0.240 \times 0.200 \times 0.170$ & $0.360 \times 0.220 \times 0.060$ \\
\hline Crystal system & monoclinic & monoclinic & Orthorhombic \\
\hline Space group & $P 22_{1} / n$ & $C 2 / c$ & $\mathrm{Pna}_{1}$ \\
\hline $\mathrm{a}[\AA]$ & $19.6906(3)$ & $22.2361(10)$ & $30.4627(7)$ \\
\hline $\mathrm{b}[\AA]$ & $23.6818(3)$ & $21.1294(10)$ & $14.0563(2)$ \\
\hline$c[\AA]$ & $22.1913(3)$ & $20.3095(8)$ & $22.2083(4)$ \\
\hline$\alpha\left[^{\circ}\right]$ & 90 & 90 & 90 \\
\hline$\beta\left[^{\circ}\right]$ & $96.8020(10)$ & $91.847(3)$ & 90 \\
\hline$\gamma\left[{ }^{\circ}\right]$ & 90 & 90 & 90 \\
\hline Volume $\left[\AA^{3}\right]$ & $10275.2(2)$ & $9537.2(7)$ & $9509.4(3)$ \\
\hline$Z$ & 4 & 4 & 4 \\
\hline$\rho\left[\mathrm{Mg} / \mathrm{m}^{3}\right]$ & 1.402 & 1.689 & 1.625 \\
\hline$\mu\left[\mathrm{mm}^{-1}\right]$ & 0.690 & 0.765 & 0.709 \\
\hline$F(000)$ & 4344 & 4848 & 4688 \\
\hline$\theta$ range $\left[^{\circ}\right]$ & $1.351-5.859$ & $1.330-25.795$ & $1.337-25.825$ \\
\hline$h k l$ range & $\pm 23, \pm 28, \pm 27$ & $\pm 27, \pm 25, \pm 24$ & $\pm 36, \pm 17, \pm 27$ \\
\hline Measured refl. & 127085 & 57798 & 81899 \\
\hline Unique refl. [Rint] & $19400[0.0333]$ & $9061[0.1566]$ & $17944[0.0640]$ \\
\hline$T_{\min } / T_{\max }$ & $0.6660,0.5360$ & $0.9829,0.8693$ & $0.9712,0.6687$ \\
\hline $\begin{array}{l}\text { data / restr. / } \\
\text { param. }\end{array}$ & $\begin{array}{c}19400 / 354 / \\
1321\end{array}$ & $9061 / 141 / 764$ & 17944 / 268 / 1447 \\
\hline $\begin{array}{l}\text { goodness-of-fit } \\
\left(\mathrm{F}^{2}\right)\end{array}$ & 1.057 & 1.054 & 1.088 \\
\hline $\begin{array}{l}\mathrm{R} 1, \mathrm{wR} 2(\mathrm{I}> \\
2 \sigma(\mathrm{I}))\end{array}$ & $0.0461,0.1266$ & $0.0805,0.2087$ & $0.0551,0.1225$ \\
\hline R1, wR2 (all data) & $0.0563,0.1361$ & $0.1852,0.2837$ & $0.0778,0.1394$ \\
\hline $\begin{array}{l}\text { res. el. dens. } \\
{\left[\mathrm{e} / \AA^{3}\right]}\end{array}$ & $0.777 /-0.806$ & $0.893 /-0.716$ & $1.008 /-0.924$ \\
\hline
\end{tabular}




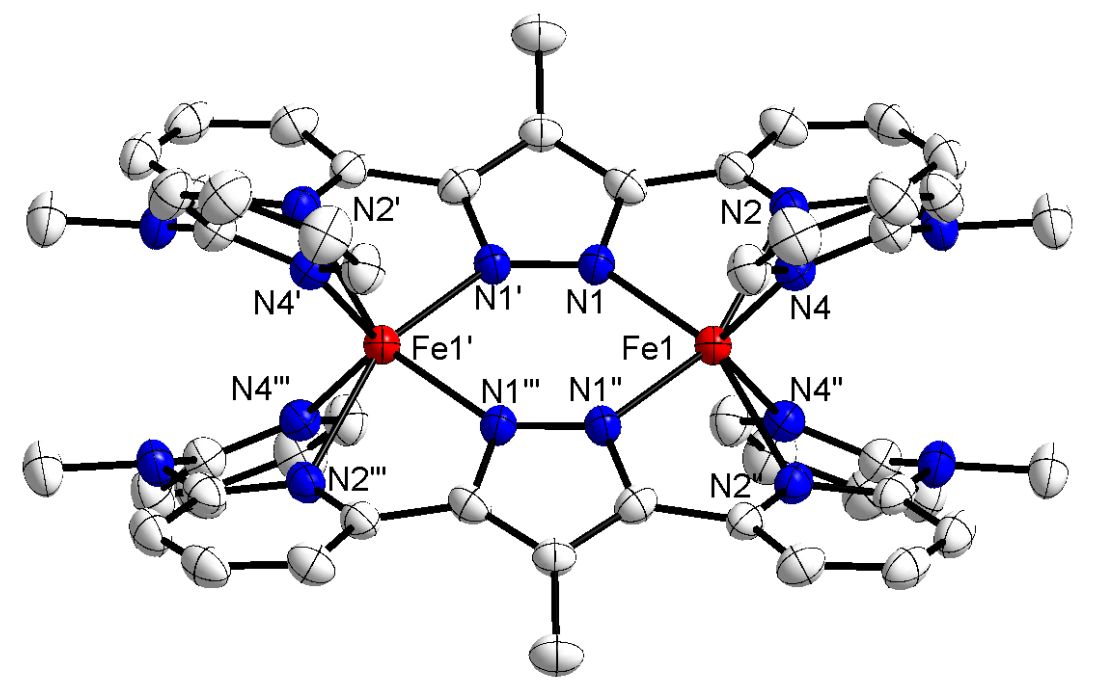

Figure A48. ORTEP plot (50\% probability thermal ellipsoids) of $\left[\mathrm{L}_{2}^{1} \mathrm{Fe}_{2} \mathrm{~L}_{2}{ }_{2}\right][\mathrm{OTf}]_{2}$. Hydrogen atoms, counterions and solvent molecules are omitted for clarity. Selected bond lengths $[\AA]$ : $\mathrm{Fe}(1)-\mathrm{N}(1)$ 2.120(4), Fe(1)-N(1)\#1 2.120(4), Fe(1)-N(4)\#1 2.200(5), Fe(1)-N(4) 2.201(5), $\mathrm{Fe}(1)-\mathrm{N}(2)$ 2.248(5), $\mathrm{Fe}(1)-\mathrm{N}(2) \# 12.248(5)$.

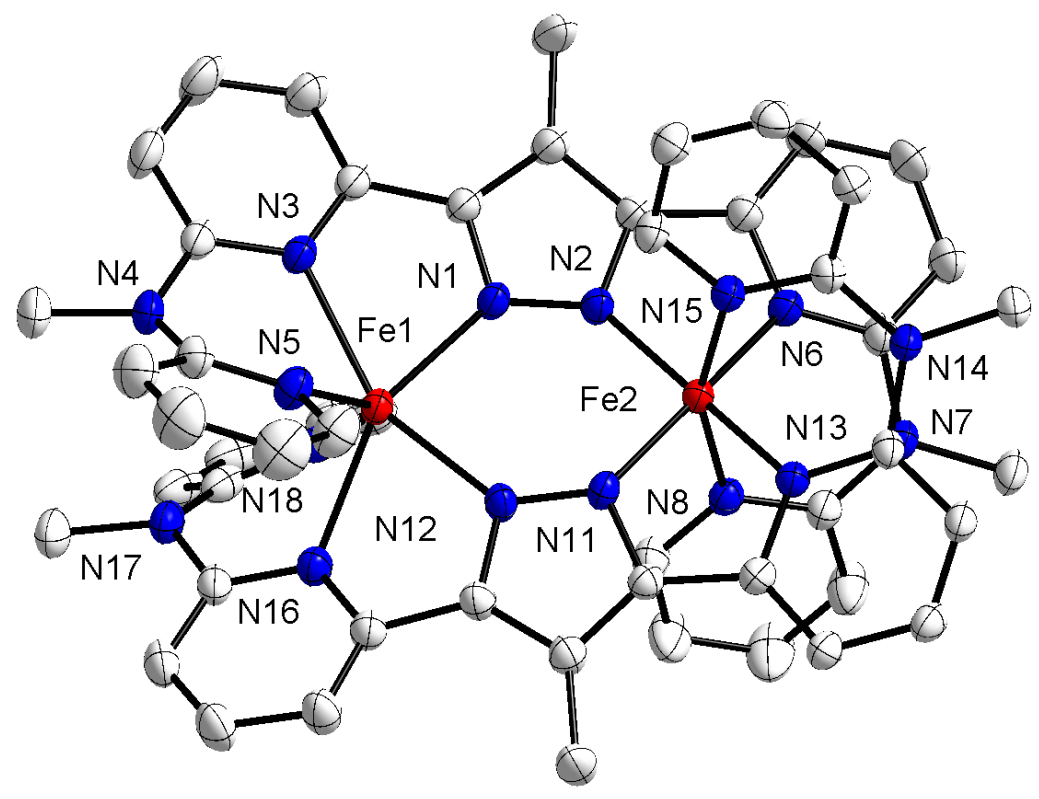

Figure A49. ORTEP plot (50\% probability thermal ellipsoids) of $\left[\mathrm{L}_{2}{ }_{2} \mathrm{Fe}^{\mathrm{II}} \mathrm{Fe}^{\mathrm{III}}\right][\mathrm{OTf}]_{2}\left[\mathrm{BF}_{4}\right]$. Hydrogen atoms, counterions and solvent molecules are omitted for clarity. Selected bond lengths [Å]: $\mathrm{Fe}(1)-\mathrm{N}(1)$ 2.125(3), $\mathrm{Fe}(1)-\mathrm{N}(12)$ 2.134(3), $\mathrm{Fe}(1)-\mathrm{N}(5)$ 2.155(3), $\mathrm{Fe}(1)-\mathrm{N}(18)$ 2.182(3), Fe(1)-N(16) 2.215(3), Fe(1)-N(3) 2.221(3), Fe(2)-N(11) 1.902(3), Fe(2)-N(2) 1.912(3), Fe(2)-N(13) 1.954(2), Fe(2)-N(6) 1.966(3), Fe(2)-N(8) 1.995(3), Fe(2)-N(15) $1.997(3)$. 


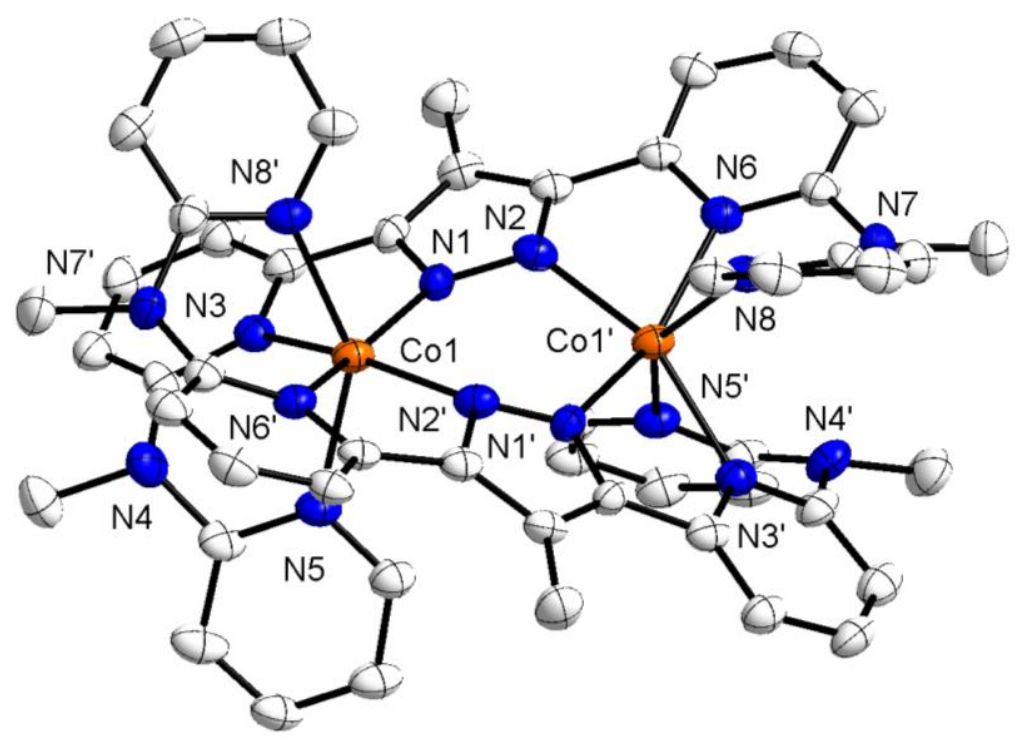

Figure A50. ORTEP plot (50\% probability thermal ellipsoids) of $\left[\mathrm{L}_{2}^{1} \mathrm{Co}_{2}\right]\left[\mathrm{BF}_{4}\right]_{2}$. Hydrogen atoms, counterions and solvent molecules are omitted for clarity. Selected bond lengths $[\AA]$ : Fe(1)-N(1) 2.125(3), Fe(1)-N(12) 2.134(3), Fe(1)-N(5) 2.155(3), Fe(1)-N(18) 2.182(3), $\mathrm{Fe}(1)-\mathrm{N}(16)$ 2.215(3), Fe(1)-N(3) 2.221(3), Fe(2)-N(11) 1.902(3), Fe(2)-N(2) 1.912(3), $\mathrm{Fe}(2)-\mathrm{N}(13)$ 1.954(2), $\mathrm{Fe}(2)-\mathrm{N}(6)$ 1.966(3), Fe(2)-N(8) 1.995(3), Fe(2)-N(15) 1.997(3). 
Table A5. Crystal metrics and refinement data for $\left[\mathrm{L}_{2}^{1} \mathrm{Fe}_{2}\right][\mathrm{OTf}]_{2}$, mixed valent $\left[\mathrm{L}_{2}{ }_{2} \mathrm{Fe}^{\mathrm{IIIF}} \mathrm{Fe}^{\mathrm{III}}\right][\mathrm{OTf}]_{2}\left[\mathrm{BF}_{4}\right]$ and $\left[\mathrm{L}_{2}{ }_{2} \mathrm{Co}_{2}\right]\left[\mathrm{BF}_{4}\right]_{2}$.

\begin{tabular}{|c|c|c|c|}
\hline & {$\left[\mathrm{L}_{2}{ }_{2} \mathrm{Fe}_{2}\right][\mathrm{OTf}]_{2}$} & {$\left[\mathrm{~L}_{2}{ }_{2} \mathrm{Fe}^{\mathrm{II}} \mathrm{Fe}^{\mathrm{III}}\right][\mathrm{OTf}]_{2}\left[\mathrm{BF}_{4}\right]$} & {$\left[\mathrm{L}_{2} \mathrm{CO}_{2}\right]\left[\mathrm{BF}_{4}\right]_{2}$} \\
\hline $\begin{array}{l}\text { Empirical } \\
\text { Formula }\end{array}$ & $\mathrm{C}_{54} \mathrm{H}_{45} \mathrm{~F}_{6} \mathrm{Fe}_{2} \mathrm{~N}_{16} \mathrm{O}_{6} \mathrm{~S}_{2}$ & $\mathrm{C}_{61} \mathrm{H}_{56.50} \mathrm{~B}_{6} \mathrm{BF}_{10} \mathrm{Fe}_{2} \mathrm{~N}_{19.50} \mathrm{O}_{6} \mathrm{~S}_{2}$ & $\mathrm{C}_{52} \mathrm{H}_{46} \mathrm{~B}_{2} \mathrm{Co}_{2} \mathrm{~F}_{8} \mathrm{~N}_{16}$ \\
\hline Formula Weight & 1304.89 & 1535.38 & 1186.53 \\
\hline $\begin{array}{l}\text { Temperature } \\
{[\mathrm{K}]}\end{array}$ & $133(2)$ & $133(2)$ & $133(2)$ \\
\hline $\begin{array}{l}\text { Crystal size } \\
{\left[\mathrm{mm}^{3}\right]}\end{array}$ & $\begin{array}{c}0.489 \times 0.078 \times \\
0.077\end{array}$ & $0.500 \times 0.180 \times 0.130$ & $\begin{array}{c}0.415 \times 0.111 \times \\
0.106\end{array}$ \\
\hline Crystal system & Orthorhombic & triclinic & Monoclinic \\
\hline Space group & Pnnm & $P-1$ & $P 2 / n$ \\
\hline $\mathrm{a}[\AA]$ & 20.948 & $12.6118(4)$ & $16.0258(11)$ \\
\hline $\mathrm{b}[\AA \AA]$ & 8.026 & $16.4277(5)$ & $8.7007(4)$ \\
\hline c $[\AA]$ & 17.289 & $18.3350(6)$ & $18.2124(12)$ \\
\hline$\alpha\left[^{\circ}\right]$ & 90 & $66.359(2)$ & 90 \\
\hline$\beta\left[^{\circ}\right]$ & 90 & $85.141(3)$ & $90.178(6)$ \\
\hline$\gamma\left[^{\circ}\right]$ & 90 & $88.888(3)$ & 90 \\
\hline Volume $\left[\AA^{3}\right]$ & 2906.9 & $3466.9(2)$ & $2539.4(3)$ \\
\hline$Z$ & 2 & 2 & 2 \\
\hline$\rho\left[\mathrm{Mg} / \mathrm{m}^{3}\right]$ & 1.491 & 1.471 & 1.552 \\
\hline$\mu\left[\mathrm{mm}^{-1}\right]$ & 0.654 & 0.570 & 0.739 \\
\hline$F(000)$ & 1336 & 1572 & 1212 \\
\hline$\theta$ range $\left[{ }^{\circ}\right]$ & $1.527-25.743$ & $1.353-26.911$ & $1.271-25.899$ \\
\hline$h k l$ range & $\pm 25,-8-9,-21-20$ & $\pm 15, \pm 20,-22-23$ & $\pm 19, \pm 10, \pm 22$ \\
\hline Measured refl. & 27298 & 49557 & 25413 \\
\hline $\begin{array}{l}\text { Unique refl. } \\
\text { [Rint] }\end{array}$ & $2855[0.0853]$ & $14679[0.0452]$ & $4825[0.0654]$ \\
\hline$T_{\min } / T_{\max }$ & $0.8960,0.7270$ & $0.8860,0.7100$ & $0.8549,0.5906$ \\
\hline $\begin{array}{l}\text { data / restr. / } \\
\text { param. }\end{array}$ & 2855 / $241 / 263$ & 14679 / 50 / 1003 & 4835 / 16 / 381 \\
\hline $\begin{array}{l}\text { goodness-of-fit } \\
\left(\mathrm{F}^{2}\right)\end{array}$ & 1.088 & 1.031 & 1.085 \\
\hline $\begin{array}{l}\mathrm{R} 1, \mathrm{wR} 2(\mathrm{I}> \\
2 \sigma(\mathrm{I}))\end{array}$ & $0.0816,0.2278$ & $0.0530,0.1471$ & $0.0479,0.1169$ \\
\hline $\begin{array}{l}\text { R1, wR2 (all } \\
\text { data) }\end{array}$ & $0.1103,0.2540$ & $0.0780,0.1626$ & $0.0670,0.1415$ \\
\hline $\begin{array}{l}\text { res. el. dens. } \\
{\left[\mathrm{e} / \AA^{3}\right]}\end{array}$ & $2.255 /-1.623$ & $1.058 /-0.771$ & $0.539 /-1.133$ \\
\hline
\end{tabular}




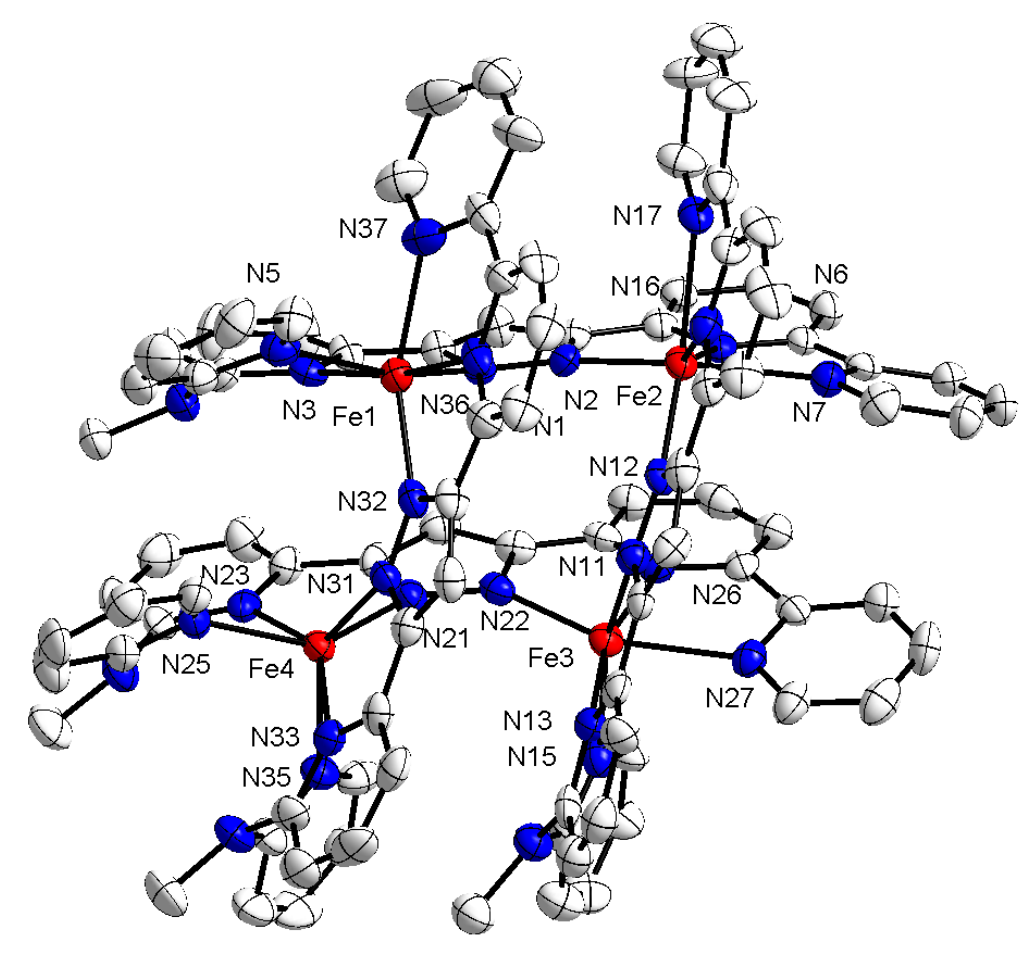

Figure A51. ORTEP plot (50\% probability thermal ellipsoids) of $\left[\mathrm{L}_{4}{ }_{4} \mathrm{Fe}_{4}\right]\left[\mathrm{BF}_{4}\right]_{2}$. Hydrogen atoms, counterions and solvent molecules are omitted for clarity. Selected bond lengths $[\AA]$ : $\mathrm{Fe}(1)-\mathrm{N}(1)$ 2.096(7), Fe(1)-N(36) 2.124(8), Fe(1)-N(32) 2.127(8), Fe(1)-N(3) 2.130(7), Fe(1)-N(5) 2.206(8), Fe(1)-N(37) 2.227(8), Fe(2)-N(6) 2.124(7), Fe(2)-N(2) 2.126(7), Fe(2)$\mathrm{N}(16)$ 2.127(7), Fe(2)-N(12) 2.133(7), Fe(2)-N(7) 2.334(7), Fe(2)-N(17) 2.349(8), Fe(3)$\mathrm{N}(26) 2.109(6), \mathrm{Fe}(3)-\mathrm{N}(22)$ 2.114(7), $\mathrm{Fe}(3)-\mathrm{N}(11)$ 2.128(8), Fe(3)-N(13) 2.146(7), Fe(3)$\mathrm{N}(15)$ 2.203(8), Fe(3)-N(27) 2.278(7), Fe(4)-N(21) 2.155(7), Fe(4)-N(23) 2.160(8), Fe(4)$\mathrm{N}(31)$ 2.170(7), Fe(4)-N(33) 2.171(7), Fe(4)-N(25) 2.234(7), Fe(4)-N(35) 2.261(8) 
Table A6. Crystal metrics and refinement data for $\left[\mathrm{L}_{4}^{2} \mathrm{Fe}_{4}\right]\left[\mathrm{BF}_{4}\right]_{4}$

\begin{tabular}{|c|c|}
\hline & {$\left[\mathrm{L}_{4} \mathrm{Fe}_{4}\right]\left[\mathrm{BF}_{4}\right]_{4}$} \\
\hline Empirical Formula & $\mathrm{C}_{102} \mathrm{H}_{81} \mathrm{~B}_{4} \mathrm{~F}_{16} \mathrm{~N}_{31} \mathrm{Fe}_{4}$ \\
\hline Formula Weight & 2311.61 \\
\hline Temperature [K] & $133(2)$ \\
\hline Crystal size $\left[\mathrm{mm}^{3}\right]$ & $0.480 \times 0.200 \times 0.170$ \\
\hline Crystal system & Monoclinic \\
\hline Space group & $P 2_{1}$ \\
\hline a $[\AA]$ & $14.1008(4)$ \\
\hline $\mathrm{b}[\AA]$ & $23.9119(7)$ \\
\hline c $[\AA]$ & $14.9708(4)$ \\
\hline$\alpha\left[^{\circ}\right]$ & 90 \\
\hline$\beta\left[^{\circ}\right]$ & $101.014(2)$ \\
\hline$\gamma\left[^{\circ}\right]$ & 90 \\
\hline Volume $\left[\AA^{3}\right]$ & $4954.8(2)$ \\
\hline$Z$ & 2 \\
\hline$\rho\left[\mathrm{Mg} / \mathrm{m}^{3}\right]$ & 1.549 \\
\hline$\mu\left[\mathrm{mm}^{-1}\right]$ & 0.672 \\
\hline$F(000)$ & 2356 \\
\hline$\theta$ range $\left[{ }^{\circ}\right]$ & $1.386-25.715$ \\
\hline$h k l$ range & $\pm 17, \pm 29,-18-15$ \\
\hline Measured refl. & 45939 \\
\hline Unique refl. [Rint] & $18549[0.0823]$ \\
\hline$T_{\min } / T_{\max }$ & $0.9572,0.8065$ \\
\hline data / restr. / param. & $84549 / 211 / 1481$ \\
\hline goodness-of-fit on $\left(\mathrm{F}^{2}\right)$ & 1.036 \\
\hline 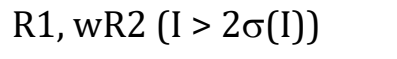 & $0.0594,0.1191$ \\
\hline $\mathrm{R} 1, \mathrm{wR} 2$ (all data) & $0.1037,0.1367$ \\
\hline res. el. dens. $\left[\mathrm{e} / \AA^{3}\right]$ & $0.616 /-0.325$ \\
\hline
\end{tabular}




\section{Structures of Ligands and Molecules}

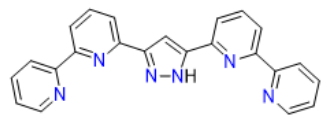

$\mathrm{HL}^{\mathrm{H}}$

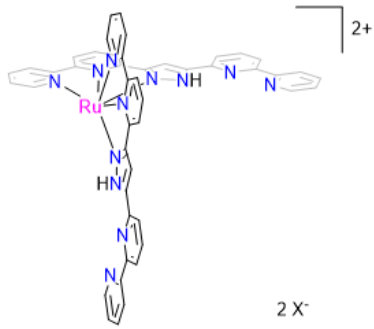

3-1a: $\mathrm{X}=\mathrm{BF}_{4}^{-}$

3-1b: $X=O T f$

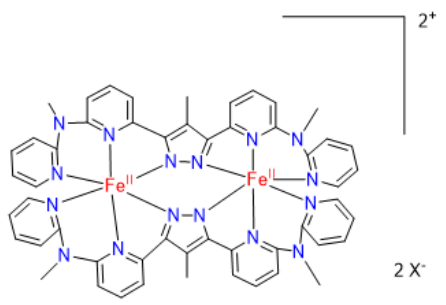

[5-10a]: $\mathrm{X}=\mathrm{BF}_{4}$ -
[5-10b]: $\mathrm{X}=\mathrm{OTf}$

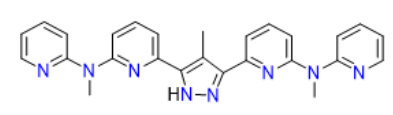

$\mathrm{HL}^{1}$

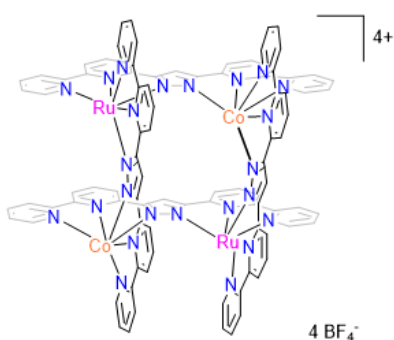

3-2

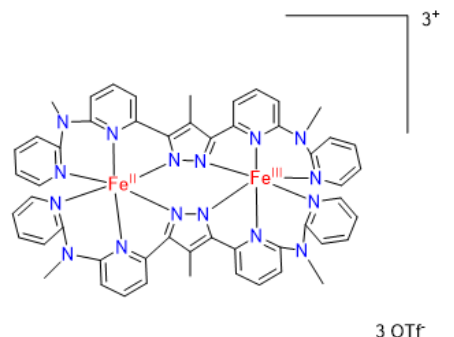

[5-11]

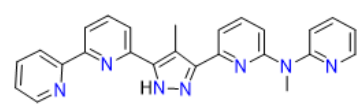

$\mathrm{HL}^{2}$

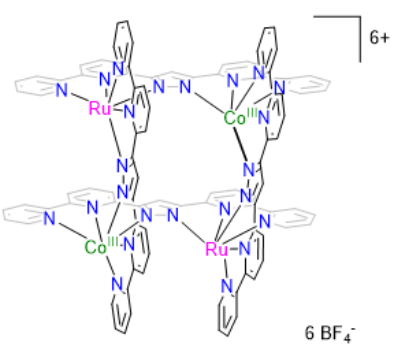

3-3

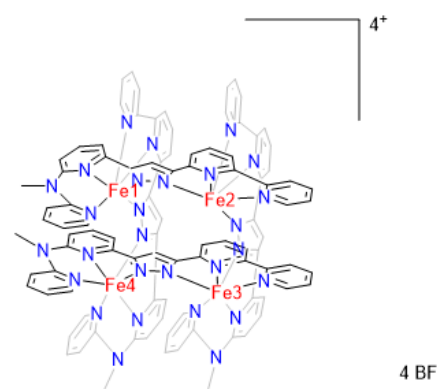

6-2 


\begin{tabular}{|c|c|}
\hline \multicolumn{2}{|c|}{ List of Abbreviations } \\
\hline \multicolumn{2}{|r|}{ tetrabutylammonium hexafluorophosphate } \\
\hline $2 \mathrm{D}$ & two dimension \\
\hline $\mathrm{ac}$ & alternating current \\
\hline $\mathrm{BF}_{4}$ & tetrafluroborate \\
\hline $\mathrm{CN}$ & cyanide \\
\hline COSY & correlation spectroscopy \\
\hline CSM & continuous symmetry measures \\
\hline CT & charge transfer \\
\hline CTIST & charge transfer induced spin transitions \\
\hline $\mathrm{CV}$ & cyclic voltammetry \\
\hline DCM & dichlormethane \\
\hline DMA & dimethylacetamide \\
\hline DMSO & dimethylsulfoxide \\
\hline DMF & $\mathrm{N}, \mathrm{N}$-dimethylformamide \\
\hline DOSY & diffusion ordered spectroscopy \\
\hline EFG & electron field gradient \\
\hline EPR & electron paramagnetic resonance \\
\hline ESI & electron spray ionization \\
\hline eq & equivalent \\
\hline fs & femtosecond \\
\hline $\mathrm{Fc}$ & ferrocene \\
\hline hfpip & $\begin{array}{l}\text { hexafluoro-4-(4-tert-butylphenylimino)-2- } \\
\text { pentanoate }\end{array}$ \\
\hline HDvV & Heisenberg-Dirac-van-Vleck \\
\hline HS & high-spin \\
\hline IR & Infrared \\
\hline IVCT & Invervalent charge transfer \\
\hline LS & low-spin \\
\hline MB & Mössbauer \\
\hline $\mathrm{MeCN}$ & acetonitrile \\
\hline MLCT & metal-to-ligand-charge-transfer \\
\hline MS & mass spectrometry \\
\hline $\mathrm{Na}_{2} \mathrm{CO}_{3}$ & sodium carbonate \\
\hline $\mathrm{Na}^{t} \mathrm{OBu}$ & sodium $t$-butoxide \\
\hline $\mathrm{NaOMe}$ & sodium methoxide \\
\hline NaOTf & sodium triflate \\
\hline $\mathrm{NH}_{4} \mathrm{BF}_{4}$ & ammonium tetrafluoroborate \\
\hline NMR & nuclear magnetic resonance \\
\hline $\mathrm{NOBF}_{4}$ & nitrosonium tetrafluoroborate \\
\hline ns & nanosecond \\
\hline OTf & triflate $\left(\mathrm{CF}_{3} \mathrm{SO}_{3}\right)$ \\
\hline ps & picosecond \\
\hline ppm & parts per million \\
\hline PTSA & $p$-toluenesulfonic acid monohydrate \\
\hline QCA & quantum celluar automata \\
\hline rt & room temperature \\
\hline SIM & single ion magnet \\
\hline SMM & single molecule magnet \\
\hline SCO & spin crossover \\
\hline SQUID & superconducting quantum interference device \\
\hline UV-vis & ultraviolet and visible \\
\hline VTVH & Variable temperature variable field \\
\hline ZFS & zero-field splitting \\
\hline
\end{tabular}




\section{References}

1. Lehn, J.-M. Supra-molecular chemistry. Science. 1993, 260, 1762-1764.

2. Pedersen, C. J. Expediting the study on the role of stem cells in wound treatment. Angew. Chem. Int. Ed. Engl. 1988, 27, 1021-1027.

3. Lehn, J. M. Cryptates: inclusion complexes of macropolycyclic receptor molecules. Pure Appl. Chem. 1978, 50, 871-892.

4. Lehn, J. -M. Supramolecular Chemistry-Scope and Perspectives Molecules, Supermolecules, and Molecular Devices (Nobel Lecture). Angew. Chemie Int. Ed. English 1988, 27, 89-112.

5. Lehn, J. M.; Rigault, A.; Siegel, J.; Harrowfield, J.; Chevrier, B.; Moras, D. Spontaneous assembly of double-stranded helicates from oligobipyridine ligands and copper(I) cations: structure of an inorganic double helix. Proc. Natl. Acad. Sci. 2006, 84, 25652569.

6. Piguet, C.; Bernardinelli, G.; Hopfgartner, G. Helicates as Versatile Supramolecular Complexes. Chem. Rev. 1997, 97, 2005-2062.

7. Hasenknopf, B.; Lehn, J. M.; Boumediene, N.; Dupont-Gervais, A.; Van Dorsselaer, A.; Kneisel, B.; Fenske, D. Self-assembly of tetra- and hexanuclear circular helicates. J. Am. Chem. Soc. 1997, 119, 10956-10962.

8. Hanan, G. S.; Arana, C. R.; Lehn, J. M.; Baum, G.; Fenske, D. Coordination arrays: Synthesis and characterisation of rack-type dinuclear complexes. Chem. Eur. J. 1996, 2, 1292-1302.

9. Dawe, L. N.; Shuvaev, K. V; Thompson, L. K. Magnetic [ $\mathrm{n} \times \mathrm{n}$ ] $(\mathrm{n}=2-5)$ Grids by Directed Self-Assembly. 2009, 48, 3323-3341.

10. Yang, Q.; Tang, J. Heterometallic grids: synthetic strategies and recent advances. Dalt. Trans. 2019, 48, 769-778.

11. Ruben, M.; Rojo, J.; Romero-Salguero, F. J.; Uppadine, L. H.; Lehn, J. M. Grid-type metal ion architectures: Functional metallosupramolecular arrays. Angew. Chem. Int. Ed. 2004, 43, 3644-3662.

12. Chakrabarty, R.; Mukherjee, P. S.; Stang, P. J. Supramolecular Coordination: SelfAssembly of Finite Two- and Three-Dimensional Ensembles. Chem. Rev. 2011, 111, 6810-6918.

13. Kahn, O.; Martinez, C. J. Spin-Transition Polymers: From Molecular Materials Toward Memory Devices. Science. 1998, 279, 44-48.

14. Dawe, L. N.; Abedin, T. S. M.; Thompson, L. K. Ligand directed self-assembly of polymetallic $[\mathrm{n} \times \mathrm{n}]$ grids: Rational routes to large functional molecular subunits? Dalt. Trans. 2008, 2, 1661-1675.

15. Lieber, C. M.; Zhong, Z. H.; Wang, D. L.; Cui, Y.; Bockrath, M. W. Nanowire crossbar arrays as address decoders for integrated nanosystems. Science.. 2003, 302, 1377-1379.

16. Lent, C. S.; Tougaw, P. D. A device architecture for computing with quantum dots. Proc. IEEE 1997, 85, 541-557.

17. Isaksen, B.; Lent, C. S. Molecular quantum-dot cellular automata. Proc. IEEE Conf. Nanotechnol. 2003, 1, 5-8.

18. Orlov, A. O.; Amlani, I.; Bernstein, G. H.; Lent, C. S.; Snider, G. L. Realization of a 
Functional Cell for Quantum-Dot Cellular Automata. 2012, 277, 928-930.

19. Gütlich, P.; Gaspar, A. B.; Garcia, Y. Spin state switching in iron coordination compounds. Beilstein J. Org. Chem. 2013, 9, 342-391.

20. Hogue, R. W.; Singh, S.; Brooker, S. Spin crossover in discrete polynuclear iron(ii) complexes. Chem. Soc. Rev. 2018, 47, 7303-7338.

21. Gütlich, P.; Garcia, Y.; Goodwin, H. A. Spin crossover phenomena in Fe(II) complexes. Chem. Soc. Rev. 2000, 29, 419-427.

22. Olguín, J.; Brooker, S. Spin crossover active iron(II) complexes of selected pyrazolepyridine/pyrazine ligands. Coord. Chem. Rev. 2011, 255, 203-240.

23. Hauser A. Ligand Field Theoretical Considerations. In: Gütlich P., Goodwin H. (eds) Spin Crossover in Transition Metal Compounds I. Topics in Current Chemistry, 233. Springer, Berlin, Heidelberg

24. Schläfer, H. L.; Gliemann, G. Einführung in die Ligandenfeldtheorie; Akademische Verlagsgesellschaft: Einführung die Ligandenfeldtheorie; Akad. Verlagsgesellschaft 1980, Wiesbaden, Germany.

25. Shriver, D. F.; Atkins, P. W.; Langford, C. H. Inorganic Chemistry, 2nd ed. 1994, Oxford University Press.

26. Gütlich, P. Fifty Years of Mössbauer Spectroscopy in Solid State Research - Remarkable Achievements, Future Perspectives. Zeitschrift für Anorg. und Allg. Chemie 2012, 638, 15-43.

27. Gütlich, P.; Garcia, Y. Mössbauer spectroscopy: Elegance and versatility in chemical diagnostics. J. Phys. Conf. Ser. 2010, 217, 1-21.

28. Nicolazzi, W.; Bousseksou, A. Thermodynamical aspects of the spin crossover phenomenon. Comptes Rendus Chim. 2018, 21, 1060-1074.

29. Cambi, L.; Szegö, L. Ber. Dtsch. Chem. Ges. B 1931, 64, 2591-2598.

30. Knoll, C.; Müller, D.; Seifried, M.; Giester, G.; Welch, J. M.; Artner, W.; Hradil, K.; Reissner, M.; Weinberger, P. Cooperativity in spin crossover materials as ligand's responsibilityinvestigations of the Fe(ii)-1,3-bis((1: H -tetrazol-1-yl)methyl)bicyclo[1.1.1]pentane system. Dalt. Trans. 2018, 47, 5553-5557.

31. Klingele, J.; Kaase, D.; Klingele, M. H.; Lach, J.; Demeshko, S. Two-step spin crossover in the mononuclear iron(II) complex [Fe"l(L) $\left.2(\mathrm{NCS})_{2}\right]$ ( $\mathrm{L}=$ 2,5-di-(2-pyridyl)-1,3,4thiadiazole). Dalt. Trans. 2010, 39, 1689-1691.

32. Kepenekian, M.; Costa, J. S.; Le Guennic, B.; Maldivi, P.; Bonnet, S.; Reedijk, J.; Gamez, P.; Robert, V. Reliability and storage capacity: A compromise illustrated in the two-step spin-crossover system [Fe(bapbpy)(NCS) 2]. Inorg. Chem. 2010, 49, 11057-11061.

33. Chernyshov, D.; Hostettler, M.; Törnroos, K. W.; Bürgi, H. B. Ordering phenomena and phase transitions in a spin-crossover compound - Uncovering the nature of the intermediate phase of $\left[\mathrm{Fe}(2-\mathrm{pic})_{3}\right] \mathrm{Cl}_{2} \cdot \mathrm{EtOH}$. Angew. Chemie - Int. Ed. 2003, 42, 38253830.

34. Zhong, Z. J.; Tao, J. Q.; Zhi, Y.; Dun, C. Y.; Liu, Y. J.; You, X. Z. A stacking spin-crossover iron(n) compound with a large hysteresis. J. Chem. Soc. - Dalt. Trans. 1998, 2, 327-328.

35. P. Gütlich; Bill, B.; Trautwein, A. X. Mössbauer Spectroscopy and Transition Metal Chemistry. 2011. 
36. Schneider, B.; Demeshko, S.; Dechert, S.; Meyer, F. A Double-Switching Multistable $\mathrm{Fe}_{4}$ Grid Complex with Stepwise Spin-Crossover and Redox Transitions. Angew. Chemie Int. Ed. 2010, 49, 9274-9277.

37. Sessoli, R.; Gatteschi, D.; Tsai, H. L.; Hendrickson, D. N.; Schake, A. R.; Wang, S.; Vincent, J. B.; Christou, G.; Folting, K. High-Spin Molecules: $\left[\mathrm{Mn}_{12} \mathrm{O}_{12}\left(\mathrm{O}_{2} \mathrm{CR}\right)_{16}\left(\mathrm{H}_{2} \mathrm{O}\right)_{4}\right]$. J. Am. Chem. Soc. 1993, 115, 1804-1816.

38. Sessoli, R.; Gatteschi, D.; Caneschi, A.; Novakt, M. A. Magnetic bistability in a metal-ion cluster. Nature 1993, 365, 141-143.

39. Leuenberger, M. N.; Loss, D. Quantum computing in molecular magnets. Nature 2001, 410, 789-793.

40. Ardavan, A.; Rival, O.; Morton, J. J. L.; Blundell, S. J.; Tyryshkin, A. M.; Timco, G. A.; Winpenny, R. E. P. Will spin-relaxation times in molecular magnets permit quantum information processing? Phys. Rev. Lett. 2007, 98, 1-4.

41. Woodruff, D. N.; Winpenny, R. E. P.; Layfield, R. A. Lanthanide Single-Molecule Magnets. Chem. Rev. 2013, 113, 5110-5148.

42. Lis, T. Preparation, crystal structure, and magnetic properties of a trinuclear mixedvalence manganese carboxylate. Acta. Cryst. 1980, 2042-2046.

43. Frost, J. M.; Harriman, K. L. M.; Murugesu, M. The rise of 3-d single-ion magnets in molecular magnetism: Towards materials from molecules? Chem. Sci. 2016, 7, 24702491.

44. Zadrozny, J. M.; Xiao, D. J.; Atanasov, M.; Long, G. J.; Grandjean, F.; Neese, F.; Long, J. R. Magnetic blocking in a linear iron(I) complex. Nat. Chem. 2013, 5, 577-581.

45. Guo, Y. N.; Xu, G. F.; Gamez, P.; Zhao, L.; Lin, S. Y.; Deng, R.; Tang, J.; Zhang, H. J. Two-step relaxation in a linear tetranuclear dysprosium(III) aggregate showing single-molecule magnet behavior. J. Am. Chem. Soc. 2010, 132, 8538-8539.

46. Gupta, S. K.; Rajeshkumar, T.; Rajaraman, G.; Murugavel, R. An air-stable Dy(III) singleion magnet with high anisotropy barrier and blocking temperature. Chem. Sci. 2016, 7, 5181-5191.

47. Lin, P. H.; Burchell, T. J.; Ungur, L.; Chibotaru, L. F.; Wernsdorfer, W.; Murugesu, M. A polinuclear lanthanide single-molecule magnet with a record anisotropic barrier. Angew. Chemie - Int. Ed. 2009, 48, 9489-9492.

48. Aldamen, M. A.; Cardona-serra, S.; Clemente-juan, J. M.; Coronado, E.; Gaita-arin, A.; Martı, C. Mononuclear Lanthanide Single Molecule Magnets Based on the ( Ln III) Tb, Dy , Ho , Er , Tm , and Yb ). Society 2009, 48, 3467-3479.

49. Guo, F. S.; Day, B. M.; Chen, Y. C.; Tong, M. L.; Mansikkamäki, A.; Layfield, R. A. A Dysprosium Metallocene Single-Molecule Magnet Functioning at the Axial Limit. Angew. Chemie - Int. Ed. 2017, 56, 11445-11449.

50. Chaboussant, G.; Sieber, A.; Ochsenbein, S.; Güdel, H. U.; Murrie, M.; Honecker, A.; Fukushima, N.; Normand, B. Exchange interactions and high-energy spin states in Mn12- acetate. Phys. Rev. B - Condens. Matter Mater. Phys. 2004, 70, 1-16.

51. Ferguson, A.; Lawrence, J.; Parkin, A.; Sanchez-Benitez, J.; Kamenev, K. V.; Brechin, E. K.; Wernsdorfer, W.; Hill, S.; Murrie, M. Synthesis and characterisation of a Ni 4 singlemolecule magnet with S 4 symmetry. J. Chem. Soc. Dalt. Trans. 2008, 6409-6414.

52. Yang, E. C.; Wernsdorfer, W.; Zakharov, L. N.; Karaki, Y.; Yamaguchi, A.; Isidro, R. M.; Lu, 
G. Di; Wilson, S. A.; Rheingold, A. L.; Ishimoto, H.; Hendrickson, D. N. Fast magnetization tunneling in tetranickel(II) single-molecule magnets. Inorg. Chem. 2006, 45, 529-546.

53. Waldmann, O. A criterion for the anisotropy barrier in single-molecule magnets. Inorg. Chem. 2007, 46, 10035-10037.

54. Ruiz, E.; Cirera, J.; Cano, J.; Alvarez, S.; Loose, C.; Kortus, J. Can large magnetic anisotropy and high spin really coexist? Chem. Commun. 2008, 2, 52-54.

55. Layfield, R. A. Organometallic Single-Molecule Magnets. Organometallics 2014, 33, 1084-1099.

56. Rinehart, J. D.; Long, J. R. Exploiting single-ion anisotropy in the design of f-element single-molecule magnets. Chem. Sci. 2011, 2, 2078-2085.

57. Vallejo, J.; Castro, I.; Cañadillas-Delgado, L.; Ruiz-Pérez, C.; Ferrando-Soria, J.; RuizGarcía, R.; Cano, J.; Lloret, F.; Julve, M. Ferromagnetic coupling and magnetic anisotropy in oxalato-bridged trinuclear chromium(iii)-cobalt(ii) complexes with aromatic diimine ligands. Dalt. Trans. 2010, 39, 2350-2358.

58. Murrie, M. Cobalt(II) single-molecule magnets. Chem. Soc. Rev. 2010, 39, 1986-1995.

59. Kahn, O. Molecular Magnetism. 1993, Wiley-VCH. Publishers Inc, New York.

60. Yoshihara, D.; Karasawa, S.; Koga, N. Cyclic single-molecule magnet in heterospin system. J. Am. Chem. Soc. 2008, 130, 10460-10461.

61. Zadrozny, J. M.; Atanasov, M.; Bryan, A. M.; Lin, C. Y.; Rekken, B. D.; Power, P. P.; Neese, F.; Long, J. R. Slow magnetization dynamics in a series of two-coordinate iron(ii) complexes. Chem. Sci. 2013, 4, 125-138.

62. Yang, E. C.; Hendrickson, D. N.; Wernsdorfer, W.; Nakano, M.; Zakharov, L. N.; Sommer, R. D.; Rheingold, A. L.; Ledezma-Gairaud, M.; Christou, G. Cobalt single-molecule magnet. J. Appl. Phys. 2002, 91, 7382-7384.

63. Moubaraki, B.; Murray, K. S.; Hudson, T. A.; Robson, R. Tetranuclear and octanuclear cobalt(II) citrate cluster single molecule magnets. Eur. J. Inorg. Chem. 2008, 45254529.

64. Wernsdorfer, W.; Whyte, A. M.; Kamenev, K. V.; Murrie, M.; Parkin, A.; Sanchez-Benitez, J.; Galloway, K. W.; Peacock, R. D. Cobalt(II) Citrate Cubane Single-Molecule Magnet. Inorg. Chem. 2008, 47, 7438-7442.

65. Lui, J.; Datta, S.; Bolin, E.; J., L.; Beedle, C. C.; Yang, E.-C.; Goy., P.; Hendrickson, D. N.; Hill, S. Anisotropic exchange in a tetranuclear Co ${ }^{\mathrm{II}}$ complex. Polyhedron 2009, 28, 1922.

66. Wu, D.; Guo, D.; Song, Y.; Huang, W.; Duan, C.; Meng, Q.; Sato, O. CoII Molecular Square with Single-Molecule Magnet Properties. Inorg. Chem. 2009, 48, 854-860.

67. Zhang, Y. Z.; Wernsdorfer, W.; Pan, F.; Wang, Z. M.; Gao, S. An azido-bridged disc-like heptanuclear cobalt(II) cluster: Towards a single-molecule magnet. Chem. Commun. 2006, 3302-3304.

68. Moragues-Canovás, M.; Talbot-Eeckelaers, C. E.; Catala, L.; Lloret, F.; Wernsdorfer, W.; Brechin, E. K.; Mallah, T. Ferromagnetic cobalt metallocycles. Inorg. Chem. 2006, 45, 7038-7040.

69. Alley, K. G.; Bircher, R.; Waldmann, O.; Ochsenbein, S. T.; Gudel, H. U.; Moubaraki, B.; Murray, K. S.; Fernandez-Alonso, F.; Abrahams, B. F.; Boskovic, C. Mixed-valent cobalt 
spin clusters: A hexanuclear complex and a one-dimensional coordination polymer comprised of alternating hepta- and mononuclear fragments. Inorg. Chem. 2006, 45, 8950-8957.

70. Ungur, L.; Pilet, G.; Luneau, D.; Chibotaru, L. F.; Elmoll, H.; Aronica, C. Structure, Magnetism, and Theoretical Study of a Mixed-Valence $\mathrm{Co}_{3} \mathrm{Co}^{\mathrm{III}}{ }_{4}$ Heptanuclear Wheel: Lack of SMM Behavior despite Negative Magnetic Anisotropy . J. Am. Chem. Soc. 2008, $130,12445-12455$.

71. Klöwer, F.; Lan, Y.; Nehrkorn, J.; Waldmann, O.; Anson, C. E.; Powell, A. K. modelling the magnetic behaviour of square-pyramidal coII5 aggregates: tuning SMM behaviour through variations in the ligand shell. Chem. Eur. J. 2009, 15, 7413-7422.

72. Waldmann, O.; Ruben, M.; Ziener, U.; Muller, P.; Lehn, J. M. Supramolecular Co ( II ) - [ 2 $\times 2$ ] Grids : Metamagnetic Behavior in a Single Molecule. 2006, 45, 6535-6540.

73. Dawe, L. N.; Shuvaev, K. V; Thompson, L. K. Magnetic $[\mathrm{n} \times \mathrm{n}](\mathrm{n}=2-5)$ Grids by Directed Self-Assembly. Inorg. Chem. 2009, 48, 3323-3341.

74. Shuvaev, K. V.; Dawe, L. N.; Thompson, L. K. Formation of unusual molecular rectangles and squares containing low spin and high spin Co(II) and Fe(II) centers†. Dalt. Trans. 2010, 39, 4693-4707.

75. Youinou, B. M.-T.; Rahmouni, N.; Fisclwr, J.; Osborn, J. A. Self-Assembly of a Cu, Complex with Coplanar and Electrochemical Properties **. Angew. Chem. Int. Ed. Engl. 1992, 31, 733-735.

76. Randell, N. M.; Anwar, M. U.; Drover, M. W.; Dawe, L. N.; Thompson, L. K. Self-Assembled Ln(III) 4 (Ln = Eu, Gd, Dy, Ho, Yb) [2 × 2] Square Grids: a New Class of Lanthanide Cluster. Inorg. Chem. 2013, 6731-6742.

77. Anwar, M. U.; Thompson, L. K.; Dawe, L. N.; Habib, F.; Murugesu, M. Predictable selfassembled [2 $[2] \mathrm{Ln}(\mathrm{III}) 4$ square grids $(\mathrm{Ln}=\mathrm{Dy}, \mathrm{Tb})-\mathrm{SMM}$ behaviour in a new lanthanide cluster motif. Chem. Commun. 2012, 48, 4576.

78. Gusev, A.; Herchel, R.; Nemec, I.; Shul, V.; Eremenko, I. L.; Lyssenko, K.; Linert, W. Tetranuclear Lanthanide Complexes Containing a Hydrazone-type. Inorg. Chem. 2016, $55,12470-12476$.

79. Wu, J.; Zhao, L.; Zhang, L.; Li, X.; Guo, M.; Tang, J. Metallosupramolecular Coordination Complexes: The Design of Heterometallic 3d - 4f Gridlike Structures. Inorg. Chem. 2016, 55, 5514-5519.

80. Wu, J.; Zhao, L.; Guo, M.; Tang, J. Constructing supramolecular grids: From 4f square to 3d-4f grid. Chem. Commun. 2015, 51, 17317-17320.

81. Tong, J.; Demeshko, S.; John, M.; Dechert, S.; Meyer, F. Redox-Induced Single-Molecule Magnetism in Mixed-Valent [2 $\times 2$ 2] $\mathrm{Co}_{4}$ Grid Complexes. Inorg. Chem. 2016, 55, 43624372.

82. Steinert, M.; Schneider, B.; Dechert, S.; Demeshko, S.; Meyer, F. Spin-State Versatility in a Series of Fe4 [2 $\times 2$ ] Grid Complexes: Effects of Counteranions, Lattice Solvent, and Intramolecular Cooperativity. Inorg. Chem. 2016, 55, 2363-2373.

83. Alvarez, S.; Avnir, D.; Llunell, M.; Pinsky, M. Continuous symmetry maps and shape classification. The case of six-coordinated metal compounds. New J. Chem. 2002, 26, 996-1009.

84. Petitjean, A.; Kyritsakas, N.; Lehn, J. M. Programmed single step self-assembly of a [2 $\times$ 
2] grid architecture built on metallic centers of different coordination geometries. Chem. Commun. 2004, 4, 1168-1169.

85. Xu, Z.; Thompson, L. K.; Matthews, C. J.; Miller, D. O.; Goeta, A. E.; Howard, J. A. K. Synthesis of a spin-coupled, mixed-metal double square grid complex [(poap$\left.\mathrm{H})_{4} \mathrm{Cu}(\mathrm{II})_{3} \mathrm{Fe}(\mathrm{III})-\quad\left(\mathrm{NO}_{3}\right)\right]_{2}\left(\mathrm{ClO}_{4}\right)_{4} \quad\left(\mathrm{NO}_{3}\right)_{4} \cdot 12 \mathrm{H}_{2} \mathrm{O} \quad$ (poap $=$ N3-(2-pyridoyl)-2pyridinecarboxamidrazone) with an $\mathrm{S}=3$ ground state, from a mononuclear Fe(III) precursor complex. Inorg. Chem. 2001, 40, 2446-2449.

86. Moroz, Y. S.; Szyrwiel, L.; Demeshko, S.; Kozowski, H.; Meyer, F.; Fritsky, I. O. One-Pot Synthesis of a New Magnetically Coupled Heterometallic $\mathrm{Cu}_{2} \mathrm{Mn}_{2}[2 \times 2]$ Molecular Grid. Inorg. Chem. 2010, 49, 4750-4752.

87. Bassani, D. M.; Lehn, J.; Fromm, K.; Fenske, D. Toposelective and Chiroselective SelfAssembly of [ $2 \times 2$ ] Grid-Type Inorganic Arrays Con- taining Different Octahedral Metallic Centers. Angew. Chem. Int. Ed. 1998, 55, 2364-2367.

88. Uppadine, L. H.; Lehn, J. M. Three-Level Synthetic Strategy Towards Mixed-Valence and Heterometallic [2 x 2] Gridlike Arrays. Angew. Chemie - Int. Ed. 2003, 43, 240-243.

89. Thompson, L. K.; Zhao, L.; Xu, Z.; Miller, D. O.; Reiff, W. M. Self-assembled supramolecular $\mathrm{M}_{9}$ (Mn(II), Fe(III), $\mathrm{Zn}(\mathrm{II})$ ), $\mathrm{M}_{5}$ (Fe(III)), and [ $\left.\mathrm{M}_{3}\right]_{2}$ (Pb(II)) Complexes: Structural, Magnetic, and Mössbauer Properties. Inorg. Chem. 2003, 42, 128-139.

90. Vlugt, J. I. Van Der; Demeshko, S.; Dechert, S.; Meyer, F. Tetranuclear CoII, Mn"I, and CuII Complexes of a Novel Binucleating Pyrazolate Ligand Preorganized for the SelfAssembly of Compact [ $2 \times 2$ ] -Grid Structures. Inorg. Chem. 2008, 47, 1576-1585.

91. Schneider, B.; Demeshko, S.; Neudeck, S.; Dechert, S.; Meyer, F. Mixed-Spin [2 $\times 2] \mathrm{Fe}_{4}$ Grid Complex Optimized for Quantum Cellular Automata. Inorg. Chem. 2013, 52, 13230-13237.

92. Breivogel, A.; Meister, M.; Förster, C.; Laquai, F.; Heinze, K. Excited state tuning of bis(tridentate) ruthenium(II) polypyridine chromophores by push-pull effects and bite angle optimization: A comprehensive experimental and theoretical study. Chem. Eur. J. 2013, 19, 13745-13760.

93. Förster, C.; Mack, K.; Carrella, L. M.; Ksenofontov, V.; Rentschler, E.; Heinze, K. Coordination of expanded terpyridine ligands to cobalt. Polyhedron 2013, 52, 576581.

94. Cannizzo, a.; Milne, C. J.; Consani, C.; Gawelda, W.; Bressler, C.; van Mourik, F.; Chergui, M. Light-induced spin crossover in Fe(II)-based complexes: The full photocycle unraveled by ultrafast optical and X-ray spectroscopies. Coord. Chem. Rev. 2010, 254, 2677-2686.

95. Auböck, G.; Chergui, M. Sub-50-fs photoinduced spin crossover in [Fe(bpy)3] ${ }^{2+}$. Nat. Chem. 2015, 7, 629-633.

96. Smeigh, A. L.; Creelman, M.; Mathies, R. A.; McCusker, J. K. Femtosecond Time-Resolved Optical and Raman Spectroscopy of Photoinduced Spin Crossover: Temporal Resolution of Low-to-High Spin Optical Switching. J. Am. Chem. Soc 2008, 1410514107.

97. Zhang, W.; Alonso-Mori, R.; Bergmann, U.; Bressler, C.; Chollet, M.; Galler, A.; Gawelda, W.; Hadt, R. G.; Hartsock, R. W.; Kroll, T.; Kjær, K. S.; Kubiek, K.; Lemke, H. T.; Liang, H. W.; Meyer, D. A.; Nielsen, M. M.; Purser, C.; Robinson, J. S.; Solomon, E. I.; et al. Tracking excited-state charge and spin dynamics in iron coordination complexes. Nature 2014, $509,345-348$. 
98. Robin, M. B. The Color and Electronic Configurations of Prussian Blue. Inorg. Chem. 1962, 1, 337-342.

99. Aguilà, D.; Prado, Y.; Koumousi, E. S.; Mathonière, C.; Clérac, R. Switchable Fe/Co Prussian blue networks and molecular analogues. Chem. Soc. Rev. 2016, 45, 203-224.

100. Steinert, M.; Schneider, B.; Dechert, S.; Demeshko, S.; Meyer, F. A Trinuclear Defect-Grid Iron(II) Spin Crossover Complex with a Large Hysteresis Loop that is Readily Silenced by Solvent Vapor. Angew. Chemie Int. Ed. 2014, 53, 6135-6139.

101. Berera, R.; van Grondelle, R.; Kennis, J. T. M. Ultrafast transient absorption spectroscopy: Principles and application to photosynthetic systems. Photosynth. Res. 2009, 101, 105-118.

102. Ahrens, J. PhD Thesis. 2018.

103. Franke, P. L.; Haasnoot, J. G.; Zuur, A. P. Tetrazoles as ligands. Part IV. Iron(II) complexes of monofunctional tetrazole ligands, showing high-spin $\leftrightharpoons$ low-spin transitions. Inorganica Chim. Acta 1982, 59, 5-9.

104. Decurtins, S.; Gütlich, P.; Köhler, C. P.; Spiering, H.; Hauser, A. Light-induced excited spin state trapping in a transition-metal complex: The hexa-1-propyltetrazole-iron (II) tetrafluoroborate spin-crossover system. Chem. Phys. Lett. 1984, 105, 1-4.

105. Parsons, S. R.; Thompson, L. K.; Dey, S. K.; Wilson, C.; Howard, J. A. K. High-spin [2 x 2] [FeIII2NiII2] heterometallic square grid with an $\mathrm{S}=3$ ground state. Inorg. Chem. 2006, $45,8832-8834$.

106. Newton, G. N.; Onuki, T.; Shiga, T.; Noguchi, M.; Matsumoto, T.; Mathieson, J. S.; Nihei, M.; Nakano, M. Mapping the Sequential Self-Assembly of Heterometallic Clusters: From a Helix to a Grid. Angew. Chem. Int. Ed. 2011, 50, 4844-4848.

107. Bao, X.; Liu, W.; Mao, L.-L.; Jiang, S.-D.; Liu, J.-L.; Chen, Y.-C.; Tong, M.-L. Programmed Self-Assembly of Heterometallic [3 $\times 3$ ] Grid $\left[\mathrm{M}^{\mathrm{II}} \mathrm{Cu}^{{ }^{I I}}{ }_{4} \mathrm{Cu}_{4}{ }_{4}\right](\mathrm{M}=\mathrm{Fe}, \mathrm{Ni}, \mathrm{Cu}$, and $\mathrm{Zn})$. Inorg. Chem. 2013, 52, 6233-6235.

108. Bassani, D. M.; Lehn, J. M.; Serroni, S.; Puntoriero, F.; Campagna, S. Homo- and Heterometallic [2 $\times 2$ ] Grid Arrays Containing $\mathrm{Ru}^{\mathrm{II}}$, OsII, and Fe ${ }^{\mathrm{II}}$ Subunits and their Mononuclear RuII and Os ${ }^{\mathrm{II}}$ Precursors: Synthesis, Absorption Spectra, Redox Behavior, and Luminescence Properties. Chem. Eur. J. 2003, 9, 5936-5946.

109. Waldmann, O.; Ruben, M.; Ziener, U.; Muller, P.; Lehn, J. M. Supramolecular Co( II )-[ 2 $\times 2$ ] Grids : Metamagnetic Behavior in a Single Molecule. Inorg. Chem. 2006, 45, 65356540 .

110. Nedelko, N.; Kornowicz, A.; Justyniak, I.; Aleshkevych, P.; Prochowicz, D.; Krupiński, P.; Dorosh, 0.; Ślawska-Waniewska, A.; Lewiński, J. Supramolecular Control over Molecular Magnetic Materials: $\gamma$ - Cyclodextrin-Templated Grid of Cobalt(II) Single-Ion Magnets. Inorg. Chem. 2014, 53, 12870-12876.

111. Wong, J. W. L.; Demeshko, S.; Dechert, S.; Meyer, F. Heterometallic $\mathrm{Ru}_{2} \mathrm{Co}_{2}[2 \mathrm{x}$ 2] Grid with Localized Single Molecule Magnet Behavior. Inorg. Chem. 2019. manuscript accepted. 
112. A. Nayak, S. Patra, B. Sarkar, S. Ghumaan, V. G. Puranik, W. Kaim, G. K. L. Tetrazine derived mononuclear RuII(acac)2(L) (1), [RuII(bpy)2(L)](ClO4)2 (2) and $\left[\mathrm{Ru}^{\mathrm{II}}(\mathrm{bpy})(\mathrm{L})_{2}\right]\left(\mathrm{ClO}_{4}\right) 2(3) \quad(\mathrm{L}=3$-amino-6-(3,5-dimethylpyrazol-1-yl)-1,2,4,5tetrazine, acac $=$ acetylacetonate, bpy $=2,2^{\prime}$-bipyridine): syntheses, structures, spectra and redox properties. Polyhedron 2005, 24, 333-342.

113. Ceroni, P.; Paolucci, F.; Paradisi, C.; Juris, A.; Roffia, S.; Serroni, S.; Campagna, S.; Bard, A. J. Dinuclear and Dendritic Polynuclear Ruthenium(II) and Ssmium(II) Polypyridine Complexes: Electrochemistry at very Positive Potentials in Liquid SO2. J. Am. Chem. Soc. 1998, $120,5480-5487$.

114. Di Giovanni, C.; Vaquer, L.; Sala, X.; Benet-Buchholz, J.; Llobet, A. New Dinuclear Ruthenium Complexes: Structure and Oxidative Catalysis. Inorg. Chem. 2013, 52, $4335-4345$.

115. Serroni, S.; Campagna, S.; Nascone, R. P.; Hanan, G. S.; Davidson, G. J. E.; Lehn, J. M. Controlling the Direction of Photoinduced Energy Transfer in Multicomponent Species. Chem. Eur. J. 1999, 5, 3523-3527.

116. Kojima, T.; Hayashi, K. I.; Iizuka, S. Y.; Tani, F.; Naruta, Y.; Kawano, M.; Ohashi, Y.; Hirai, Y.; Ohkubo, K.; Matsuda, Y.; Fukuzumi, S. Synthesis and Characterization of Mononuclear Ruthenium(III) Pyridylamine Complexes and Mechanistic Insights into their Catalytic Alkane Functionalization with m-Chloroperbenzoic Acid. Chem. Eur. J. 2007, 13, 8212-8222.

117. Juris, A.; Balzani, V. Ru(II) Polypyridine Complexes: Photophysics, Photochemistry, Electrochemistry, and Chemiluminescence. Coord. Chem. Rev. 1988, 84, 85-277.

118. Webb, M. I.; Walsby, C. J. Control of ligand-exchange processes and the oxidation state of the antimetastatic Ru(III) complex NAMI-A by interactions with human serum albumin. Dalt. Trans. 2011, 40, 1322-1331.

119. De Paula, Q. A.; Batista, A. A.; Nascimento, O. R.; Da Costa-Filho, A. J.; Schultz, M. S.; Bonfadini, M. R.; Oliva, G. EPR and Electrochemistry of [ $\left.\mathrm{NH}_{4}\right]$ trans-[ $\mathrm{RuCl}_{4}\left(\mathrm{DMSO}_{\mathrm{C}}(\mathrm{L})\right]$ complexes (L = DMSO, py). X-Ray Molecular Structure of [pyH][RuCl $\left.{ }_{4}(\mathrm{DMSO})(\mathrm{py})\right] . J$. Braz. Chem. Soc. 2000, 11, 530-536.

120. Griffith, J. S. Octahedral Complexes Part 1. Magnetic Susceptibilities in the First Transition Series. Trans. Faraday Soc. 1958, 54, 1109-1116.

121. Ruben, M.; Breuning, E.; Barboiu, M.; Gisselbrecht, J. P.; Lehn, J. M. Functional Supramolecular Devices: $\left[\mathrm{M}_{4}{ }^{\mathrm{II}} \mathrm{L}_{4}\right]^{8+}[2 \times 2]$-Grid-Type Complexes as Multilevel Molecular Electronic Species. Chem. Eur. J. 2003, 9, 291-299.

122. Ruben, M.; Breuning, E.; Gisselbrecht, J. P.; Lehn, J. M. Multilevel Molecular Electronic Species: Electrochemical Reduction of a. Angew Chem Int Ed Engl 2000, 39, 4139-4142.

123. Sapp, S. A.; Elliott, C. M.; Contado, C.; Caramori, S.; Bignozzi, C. A. Substituted Polypyridine Complexes of Cobalt(II/III) as Efficient Electron-transfer Mediators in Dye-sensitized solar cells. J. Am. Chem. Soc. 2002, 124, 11215-11222.

124. Parsons, S. R.; Howard, J. A. K.; Wilson, C.; Dey, S. K.; Thompson, L. K. High-Spin [2 × 2] $\left[\mathrm{Fe} \mathrm{III}_{2} \mathrm{Ni}^{\mathrm{II}}{ }_{2}\right]$ Heterometallic Square Grid with an $\mathrm{S}=3$ Ground State . Inorg. Chem. 2006, $45,8832-8834$.

125. Yao, X. N.; Du, J. Z.; Zhang, Y. Q.; Leng, X. B.; Yang, M. W.; Jiang, S. Da; Wang, Z. X.; Ouyang, Z. W.; Deng, L.; Wang, B. W.; Gao, S. Two-coordinate Co(II) imido complexes as 
outstanding single-molecule magnets. J. Am. Chem. Soc. 2017, 139, 373-380.

126. Maniaki, D.; Pilichos, E.; Perlepes, S. P. Coordination Clusters of 3d-Metals That Behave as Single-Molecule Magnets (SMMs): Synthetic Routes and Strategies. Front. Chem. 2018, 6, 1-28.

127. Abbasi, P.; Quinn, K.; Alexandropoulos, D. I.; Damjanović, M.; Wernsdorfer, W.; Escuer, A.; Mayans, J.; Pilkington, M.; Stamatatos, T. C. Transition Metal Single-Molecule Magnets: A $\{$ Mn31\} Nanosized Cluster with a Large Energy Barrier of $\sim 60 \mathrm{~K}$ and Magnetic Hysteresis at 5 K. J. Am. Chem. Soc. 2017, 139, 15644-15647.

128. Ruben, M.; Breuning, E.; Lehn, J. M.; Ksenofontov, V.; Renz, F.; Gütlich, P.; Vaughan, G. B. M. Supramolecular spintronic devices: Spin transitions and magnetostructural correlations in [Fe4IIL4]8+ [2 $\times 2$ 2]-grid-type complexes. Chem. Eur. J. 2003, 9, 44224429.

129. Ruben, M.; Ziener, U.; Lehn, J. M.; Ksenofontov, V.; Gütlich, P.; Vaughan, G. B. M. Hierarchical self-assembly of supramolecular spintronic modules into 1D- and 2Darchitectures with emergence of magnetic properties. Chem. Eur. J. 2005, 11, 94-100.

130. Moucheron, C.; Kirsch-De Mesmaeker, A.; Dupont-Gervais, A.; Leize, E.; Van Dorsselaer, A. Synthesis and characterization by electrospray mass spectrometry of a novel dendritic heptanuclear complex of ruthenium(II). J. Am. Chem. Soc. 1996, 118, 1283412835.

131. Wang, F.; Weidt, S.; Xu, J.; Mackay, C. L.; Langridge-Smith, P. R. R.; Sadler, P. J. Identification of Clusters from Reactions of Ruthenium Arene Anticancer Complex with Glutathione Using Nanoscale Liquid Chromatography Fourier Transform Ion Cyclotron Mass Spectrometry Combined with 180-Labeling. J. Am. Soc. Mass Spectrom. 2008, 19, 544-549.

132. Kitchen, J. A.; Olguín, J.; Kulmaczewski, R.; White, N. G.; Milway, V. A.; Jameson, G. N. L.; Tallon, J. L.; Brooker, S. Effect of N 4-substituent choice on spin crossover in dinuclear iron(II) complexes of bis-terdentate 1,2,4-triazole-based ligands. Inorg. Chem. 2013, $52,11185-11199$.

133. Hogue, R. W.; Feltham, H. L. C.; Miller, R. G.; Brooker, S. Spin Crossover in Dinuclear N4S2 Iron(II) Thioether-Triazole Complexes: Access to [HS-HS], [HS-LS], and [LS-LS] States. Inorg. Chem. 2016, 55, 4152-4165.

134. Köhler, C.; Rentschler, E. The First 1,3,4-Oxadiazole Based Dinuclear Iron(II) Complexes Showing Spin Crossover Behavior with Hysteresis. Eur. J. Inorg. Chem. 2016, 2016, 1955-1960.

135. Kulmaczewski, R.; Olguín, J.; Kitchen, J. A.; Feltham, H. L. C.; Jameson, G. N. L.; Tallon, J. L.; Brooker, S. Remarkable scan rate dependence for a highly constrained dinuclear iron(ii) spin crossover complex with a wide thermal hysteresis loop. J. Am. Chem. Soc. 2014, 136, 878-881.

136. Samanta, S.; Demesko, S.; Dechert, S.; Meyer, F. A Two-in-one Pincer Ligand and its Diiron(II) Complex Showing Spin State Switching in Solution through Reversible Ligand Exchange. Angew. Chemie 2015, 127, 593-597.

137. Herold, C. F.; Carrella, L. M.; Rentschler, E. A Family of Dinuclear Iron(II) SCO Compounds Based on a 1,3,4-Thiadiazole Bridging Ligand. Eur. J. Inorg. Chem. 2015, 3632-3636.

138. Yang, Q.; Tang, J. Heterometallic grids: synthetic strategies and recent advances. 2019, 769-778. 
139. Tarighi, S.; Abbasi, A. Crystal Structure of cis $-\mathrm{Ru}(\mathrm{DMSO})_{4} \mathrm{Cl}_{2}$ in different synthetic methods. 2007, 33, 19-21.

140. Hagen, K. S. Iron(II) triflate salts as convenient substitutes for perchlorate salts: Crystal structures of $\left[\mathrm{Fe}\left(\mathrm{H}_{2} \mathrm{O}\right)_{6}\right]\left(\mathrm{CF}_{3} \mathrm{SO}_{3}\right)_{2}$ and $\mathrm{Fe}(\mathrm{MeCN})_{4}\left(\mathrm{CF}_{3} \mathrm{SO}_{3}\right)$ 2. Inorg. Chem. 2000, 39, 5867-5869.

141. Boduszek, B.; Shine, H. J. Preparation of Solid Thianthrene Cation Radical Tetrafluoroborate. J. Org. Chem. 1988, 53, 5142-5143.

142. Gottlieb, H. E.; Kotlyar, V.; Nudelman, A. NMR chemical shifts of common laboratory solvents as trace impurities. J. Org. Chem. 1997, 62, 7512-7515.

143. Joachim Demnitz, F. W.; D'heni, M. B. A high yielding preparation of 2,2'-bipyridine-1oxide. Org. Prep. Proced. Int. 1998, 30, 467-469.

144. Chiarelli, L. R.; Mori, M.; Barlocco, D.; Beretta, G.; Gerlain, A.; Pini, E.; Porcino, M.; Mori, G.; Stelitano, G.; Costantino, L.; Lapillo, M.; Bonanni, D.; Polo, G.; Tuccinardi, T.; Villa, S.; Meneghetti, F. Discovery and development of novel salicylate synthase (MbtI) furanic inhibitors as antitubercular agents. Eur. J. Med. Chem. 2018, 155, 754-763.

145. Zong, R.; Wang, D.; Hammitt, R.; Thummel, R. P. Synthetic approaches to polypyridyl bridging ligands with proximal multidentate binding sites. J. Org. Chem. 2006, 71, 167175.

146. Lyubov, D. M.; Fukin, G. K.; Cherkasov, A. V.; Shavyrin, A. S.; Trifonov, A. A.; Luconi, L.; Bianchini, C.; Meli, A.; Giambastiani, G. Selective $\sigma$-bond metathesis in alkyl - aryl and alkyl - benzyl yttrium complexes. new aryl - and benzyl - hydrido yttrium derivatives supported by amidopyridinate ligands. Organometallics 2009, 28, 1227-1232. 


\section{Acknowledgements}

Firstly, I would like to thank my supervisor Prof. Franc Meyer for the continuous support, scientific discussions and motivation over the years. I am grateful for the scientific freedom and abundance of laboratory equipment and instruments, which allowed me to gain immense knowledge in certain areas (i.e. Mössbauer spectroscopy and electrochemistry). Furthermore, I would also like to express my gratitude to my second and third supervisor Prof. Sven Schneider and Prof. Dirk Schwarzer for sharing insightful suggestions.

Apart from my supervisors, I would like to thank my collaborators and colleagues including Dr. Jennifer Ahrens for the transient absorption measurements on the Fe grids, Dr. Serhiy Demeshko for his patience, encouragement, and expertise in Mössbauer spectroscopy and magnetism. I could not have asked for a better mentor to enhance my knowledge in these two fields. I am also grateful to Dr. Sebastian Dechert for finding and measuring the smallest crystals, which wouldn't have been possible without his expertise. I would also like to thank Dr. Claudia Stückl for the EPR measurement, Dr. Michael John and Ralf Schöne for the NMR measurements and discussions. Furthermore, I am grateful to Britta Müller, Carmen Kasper, and Dr. Claudia Stückl for handling all administrative tasks.

I am grateful to Dr. Eleonora Ferretti, Dr. Allyssa Massie, Dr. Munmun Ghosh, Dr. Shao-an Hua, Hendrik Stevens, and Thomas Kothe, for taking the time to proofread my thesis.

I would like to thank my labmate, Anna Kölpin, who has provided endless support inside and outside of the lab since the first day. Thank you for all the encouragement, endless translations, and the wonderful working environment. I would like to extend my gratitude to all of AK Meyer, past and present, for the wonderful times both in and outside of the lab. In particular, I'd like to mention Eleonora, Anna, Sheida, Thomas, Hendrik, Munmun, Sara (bambina), Giuseppe, Allyssa, Vandana, and Marten for the wonderful memories during the last years. This PhD wouldn't have been the same without all of you. Furthermore, I would like to extend my thanks to all my friends in Canada who woke up early or stayed up late to listen to my stories. A special mention to Dorothy Lui for always trying to understand the life of a phD student. Last but not least, I would like to extend my thanks to my family for their unwavering support and encouragement even from the other side of the world. 